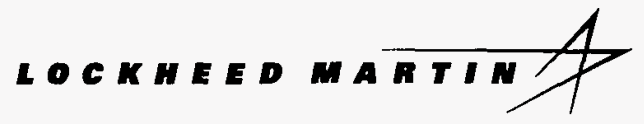

\section{ENVIRONMENTAL RESTORATION PROGRAM}

\section{RECEIVED}

MAR 201998

ORNL/ER-436

\title{
Groundwater Quality Assessment Report for Solid Waste Storage Area 6 at Oak Ridge National Laboratory, Oak Ridge, Tennessee
}

1997

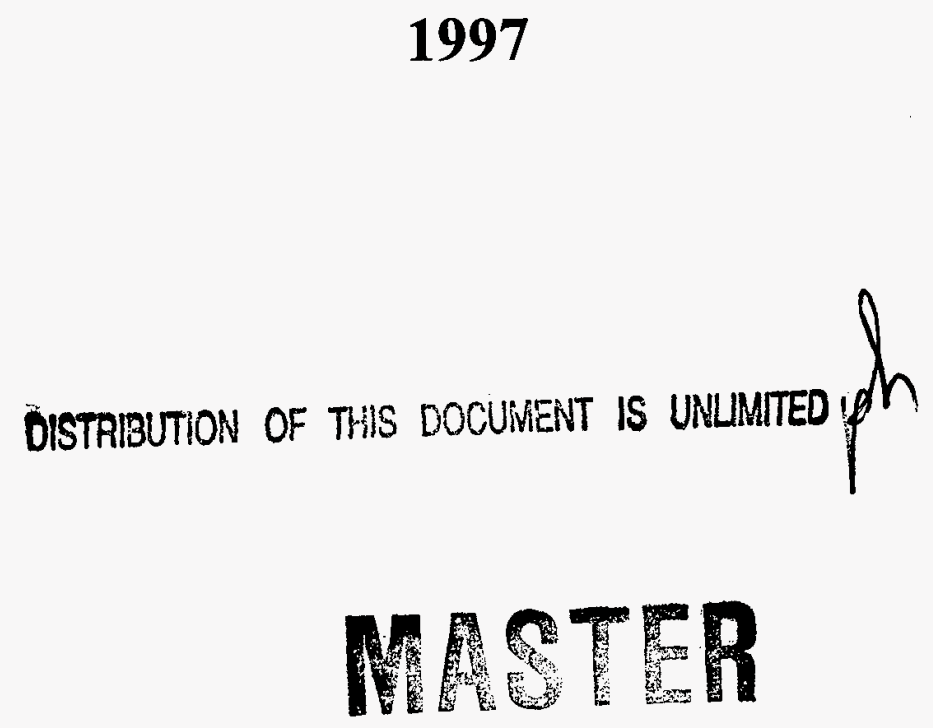




\section{Science Applications International Corporation}

contributed to the preparation of this document and should not be considered an eligible contractor for its review. 


\section{DISCLAIMER}

This report was prepared as an account of work sponsored by an agency of the United States Government. Neither the United States Government nor any agency thereof, nor any of their employees, makes any warranty, express or implied, or assumes any legal liability or responsibility for the accuracy, completeness, or usefulness of any information, apparatus, product, or process disclosed, or represents that its use would not infringe privately owned rights. Reference herein to any specific commercial product, process, or service by trade name, trademark, manufacturer, or otherwise does not necessarily constitute or imply its endorsement, recommendation, or favoring by the United States Government or any agency thereof. The views and opinions of authors expressed herein do not necessarily state or reflect those of the United States Government or.any agency thereof. 


\section{Groundwater Quality Assessment Report for Solid Waste Storage Area 6 at Oak Ridge National Laboratory, Oak Ridge, Tennessee}

\section{7}

Date Issued-February 1998

Prepared by the

Science Applications International Corporation

Oak Ridge, Tennessee

under subcontract 78B-99346C

Prepared for the

U.S. Department of Energy

Office of Environmental Management

under budget and reporting code EW 20

Environmental Management Activities at

OAK RIDGE NATIONAL LABORATORY

Oak Ridge, Tennessee 37831

managed by

LOCKHEED MARTIN ENERGY SYSTEMS, INC.

for the

U.S. DEPARTMENT OF ENERGY

under contract DE-AC05-84OR21400 


\section{PREFACE}

This document has been prepared as required under Subparts 1200-1-11-.05(5)(f), 1200-1-11$.05(5)(\mathrm{h})$, and 1200-1-11-.05(6)(e)2 of the "Rules of Tennessee Department of Environmental and Conservation (TDEC) Division of Solid Waste Management Chapter 1200-1-11, Hazardous Waste Management." This work was performed under Work Breakdown Structure 1.4.12.4.1.05.10 (Cost Center Activity Data Sheet 4349). Information provided in this document reflects the results of 1997 sampling of Resource Conservation and Recovery Act monitoring wells at Solid Waste Storage Area 6 at Oak Ridge National Laboratory. 


\section{CONTENTS}

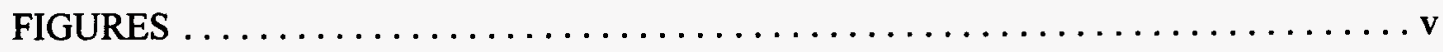

TABLES $\ldots \ldots \ldots \ldots \ldots \ldots \ldots \ldots \ldots \ldots \ldots \ldots \ldots \ldots \ldots \ldots \ldots \ldots \ldots \ldots$, vii

ACRONYMS AND ABBREVIATIONS $\ldots \ldots \ldots \ldots \ldots \ldots \ldots \ldots \ldots \ldots \ldots \ldots \ldots \ldots$

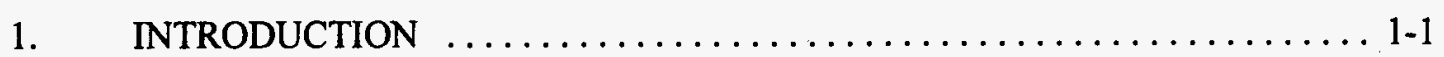

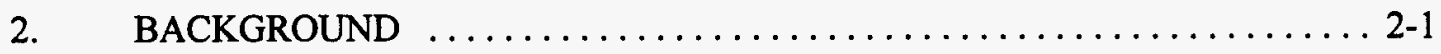

$2.1 \quad$ FACILITY DESCRIPTION $\ldots \ldots \ldots \ldots \ldots \ldots \ldots \ldots \ldots \ldots \ldots \ldots \ldots \ldots \ldots \ldots, 2-1$

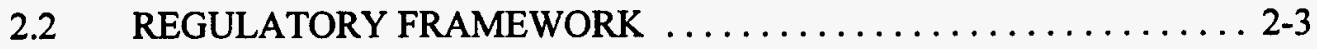

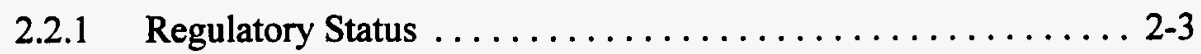

2.2.2 RCRA/CERCLA Integration $\ldots \ldots \ldots \ldots \ldots \ldots \ldots \ldots \ldots, 2-4$

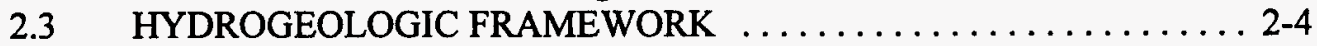

$2.4 \quad 1997$ ASSESSMENT MONITORING PROGRAM $\ldots \ldots \ldots \ldots \ldots \ldots .2-4$

2.4.1 Monitoring Network ....................... 2-4

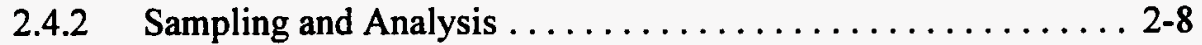

2.4.3 Quality Assurance/Quality Control $\ldots \ldots \ldots \ldots \ldots \ldots \ldots .2-8$

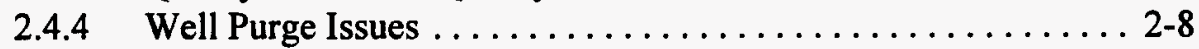

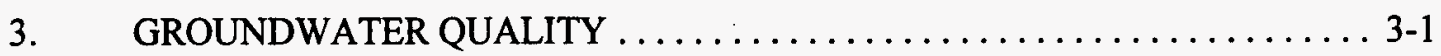

3.1 GROUNDWATER DATA ASSESSMENT $\ldots \ldots \ldots \ldots \ldots \ldots \ldots .3-1$

3.1.1 Data Quality Assessment .................... 3-1

3.1.2 Analysis of Well Purging Techniques .............. 3-1

3.1.3 1997 Assessment Results ...................... 3-1

3.2 NATURE AND EXTENT OF CONTAMINATION $\ldots \ldots \ldots \ldots \ldots .3-18$

3.2.1 Lateral Extent of Contamination ................ 3-18

3.2.2 Vertical Extent of Contamination $\ldots \ldots \ldots \ldots \ldots \ldots \ldots, 3-18$

3.3 CONTAMINANT MIGRATION $\ldots \ldots \ldots \ldots \ldots \ldots \ldots \ldots \ldots \ldots \ldots \ldots \ldots .21$

4. RECOMMENDATIONS $\ldots \ldots \ldots \ldots \ldots \ldots \ldots \ldots \ldots \ldots \ldots \ldots \ldots \ldots, 4,1$

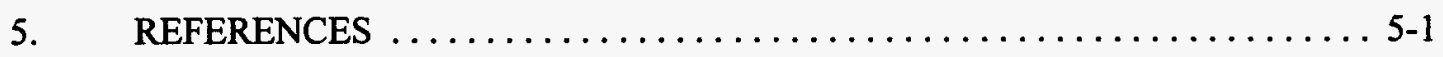

\section{APPENDICES}

A MICROPURGE PROCEDURE $\ldots \ldots \ldots \ldots \ldots \ldots \ldots \ldots \ldots \ldots \ldots \ldots \ldots \ldots \ldots$

B 1997 SWSA 6 DATA SUMMARY STATISTICS ................. B-1

C DATA QUALITY ASSESSMENT $\ldots \ldots \ldots \ldots \ldots \ldots \ldots \ldots \ldots \ldots \ldots \ldots \ldots \ldots \ldots$

D LEAD SAMPLE RESULTS FOR HILLCUT TEST FACILITY 1988-1997 _. . D-1 


\section{FIGURES}

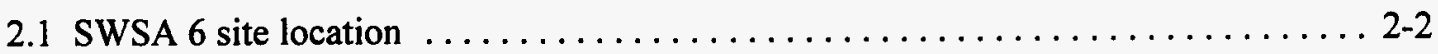

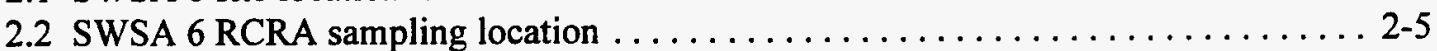

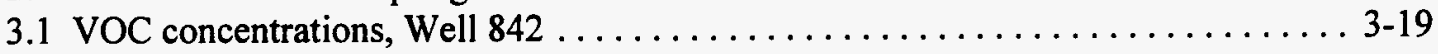

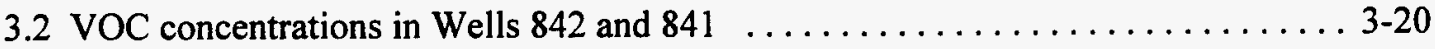

3.3 SWSA 6 water levels $\ldots \ldots \ldots \ldots \ldots \ldots \ldots \ldots \ldots \ldots \ldots \ldots \ldots \ldots \ldots \ldots \ldots \ldots \ldots \ldots \ldots \ldots \ldots \ldots .22$ 


\section{TABLES}

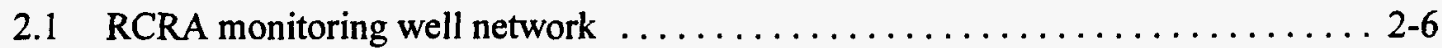

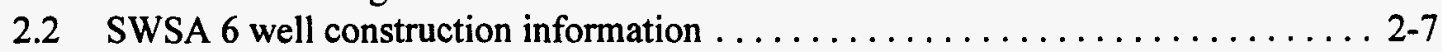

2.3 SWSA 6 Target compound list, regulatory limits and detection limits . . . . . . . 2-9

3.1 Comparison of three-volume purge and micropurge results $\ldots \ldots \ldots \ldots \ldots \ldots .2$

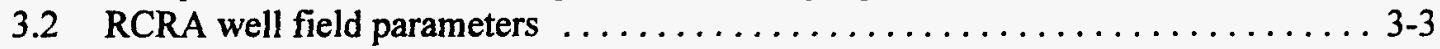

3.3 Target compound monitoring results Well $835-1997 \ldots \ldots \ldots \ldots \ldots \ldots \ldots$ 3-4

3.4 Target compound monitoring results Well $837-1997 \ldots \ldots \ldots \ldots \ldots \ldots \ldots .5 .5$

3.5 Target compound monitoring results Well $841-1997 \ldots \ldots \ldots \ldots \ldots \ldots \ldots$ 3-6

3.6 Target compound monitoring results Well $842-1997 \ldots \ldots \ldots \ldots \ldots \ldots \ldots$.

3.7 Target compound monitoring results Well $843-1997 \ldots \ldots \ldots \ldots \ldots \ldots \ldots$ 3-8

3.8 Target compound monitoring results Well $844-1997 \ldots \ldots \ldots \ldots \ldots \ldots \ldots . . .6$

3.9 Target compound monitoring results Well $846-1997 \ldots \ldots \ldots \ldots \ldots \ldots \ldots$ 3-10

3.10 Target compound monitoring results Well $857-1997 \ldots \ldots \ldots \ldots \ldots \ldots$ 3-11

3.11 Target compound monitoring results Well $858-1997 \ldots \ldots \ldots \ldots \ldots \ldots \ldots$ 3-12

3.12 Target compound monitoring results Well $4315-1997 \ldots \ldots \ldots \ldots \ldots \ldots \ldots$ 3-13

3.13 Target compound monitoring results Well $4316-1997 \ldots \ldots \ldots \ldots \ldots \ldots$ 3-14

3.14 Target compound monitoring results Well $4317-1997 \ldots \ldots \ldots \ldots \ldots \ldots \ldots$. . . . . . . . . .

3.15 Comparison of VOC detections to action levels, June $1997 \ldots \ldots \ldots \ldots \ldots$. . . . 16

3.16 Comparison of VOC detections to action levels, November $1997 \ldots \ldots \ldots \ldots$. . . 17 


\section{ACRONYMS}

\begin{tabular}{ll} 
bgs & below ground surface \\
CEI & Compliance Evaluation Investigation \\
CERCLA & Comprehensive Environmental Response, Compensation, and Liability Act \\
DOE & U.S. Department of Energy \\
EDT & Explosives Detonation Trench \\
EMP & Environmental Monitoring Plan for Waste Area Grouping 6 \\
EPA & U.S. Environmental Protection Agency \\
EWB & Emergency Waste Basin \\
GWQAP & Groundwater Quality Assessment Plan \\
GWQAR & Groundwater Quality Assessment Report \\
HDPE & high-density polyethylene \\
HTF & Hillcut Test Facility \\
ICM & Interim Corrective Measure \\
IDL & instrument detected limit \\
IWQP & Integrated Water Quality Program \\
LLW & low-level radioactive waste \\
msl & mean sea level \\
NPL & National Priority List \\
ORNL & Oak Ridge National Laboratory \\
ORR & Oak Ridge Reservation \\
PCPA & Post-Closure Permit Application \\
QA & quality assurance \\
QC & quality control \\
RCRA & Resource Conservation and Recovery Act \\
RFI & RCRA Facility Investigation \\
RI & Remedial Investigation \\
SWMU & Solid Waste Management Unit \\
SWSA & Solid Waste Storage Area \\
TDEC & Tennessee Department of Environment and Conservation \\
TOC & Total Organics Carbon \\
TOX & Total Organics Halide \\
VOC & volatile organic compound \\
WAG & Waste Area Grouping \\
& \\
\hline
\end{tabular}




\section{INTRODUCTION}

Solid Waste Storage Area (SWSA) 6, located at the U.S. Department of Energy (DOE) Oak Ridge National Laboratory (ORNL) facility, is a shallow land burial site for low-level radioactive waste (LLW) and other waste types. Wastes were disposed of in unlined trenches and auger holes from 1969 until May 1986, when it was determined that Resource Conservation and Recovery Act (RCRA) regulated wastes were being disposed of there. DOE closed SWSA 6 until changes in operating procedures prevented the disposal of RCRA wastes at SWSA 6. The site, which reopened for waste disposal activities in July 1986, is the only currently operating disposal area for low-level radioactive waste at ORNL.

In addition to SWSA 6, it was determined that hazardous wastes were treated at the Explosives Detonation Trench (EDT). Explosives and shock-sensitive chemicals such as picric acid, phosphorous, and ammonium nitrate were detonated; debris from the explosions was backfilled into the trench.

The Hillcut Test Facility (HTF) is an experimental facility designed to demonstrate a new method of subsurface disposal for solid LLW. In August and September 1986, 27 concrete casks were stacked on a subterranean concrete pad and covered with backfill material. In 1995, an investigation of sources of waste placed in HTF concluded that at least two casks contained lead that was not used as shielding, and that as many as 20 casks could contain RCRA components (lead, solvents, or aerosol cans). A groundwater collection system collects groundwater coming into contact with the casks.

Because of the disposal of RCRA-regulated wastes at SWSA 6 prior to May 1986, treatment of hazardous wastes at the EDT, and now because of the presence of RCRA-regulated waste in the HTF, the facility is considered a RCRA interim status site awaiting final closure. Groundwater quality monitoring at SWSA 6 was initiated in 1988. In 1989, initial data indicated that hazardous waste constituents had entered the groundwater in the eastern portion of the SWSA in the vicinity of Wells 841 and 842. Accordingly, the Groundwater Quality Assessment Plan (GWQAP) was developed and submitted to TDEC in December 1989. The first annual Groundwater Quality Assessment Report (GWQAR) was submitted to the Tennessee Department of Environment and Conservation (TDEC) for 1990, Groundwater Quality Assessment Report for Solid Waste Storage Area at the Oak Ridge National Laboratory (ORNL 1991). Data from the 1989 monitoring was submitted as part of the Hazardous Waste Management Annual Report, 1989 (ORNL 1990).

In 1988 and 1989, 8 Interim Corrective Measure (ICM) caps were placed over approximately 10 acres of SWSA 6. The caps were made of $80-\mathrm{mil}$ high density polyethylene (HDPE), and were designed to last for $\sim 5$ years (BNI 1988).

TDEC never approved the GWQAP, but agreed to proposed monitoring that was recommended in the GWQAR for 1990. The report recommended quarterly sampling of 10 assessment wells at SWSA 6, along with semiannual sampling of 16 perimeter wells. This plan was implemented in 1991 and the results were submitted in the 1991 GWQAR. The 1991 report was never approved by TDEC.

The 1992 monitoring was conducted per recommendations in the 1991 GWQAR and per verbal instruction provided by TDEC during the TDEC Compliance Evaluation Investigation (CEI) 
(Burroughs 1992). Based on the CEI, the analytical parameters for the 16 semi-annual monitoring wells were amended to include 10 target SWSA 6 assessment volatile organic compounds (VOCs), rather than Total Organic Halide (TOX) and Total Organic Carbon (TOC). The revisions were initiated in the third quarter of 1992 and continued through 1995.

Since 1992, monitoring has been conducted as part of the CERCLA Environmental Monitoring Plan For Waste Area Grouping (WAG) 6 (EMP) (DOE 1995). WAG 6 is composed of the RCRA interim status units plus other radioactive waste disposal units regulated under CERCLA. The EMP was developed to meet the requirements of a letter of agreement signed by the DOE, TDEC, and the U.S. Environmental Protection Agency (EPA) in June 1994. The letter specified that (1) no active source control measures would be implemented at WAG 6 in the near term under CERCLA authority, (2) monitoring would occur to track off-WAG releases, and (3) DOE would develop and demonstrate new environmental restoration technologies that could be used at other sites.

The WAG 6 EMP incorporated compliance monitoring at the RCRA groundwater quality assessment monitoring locations. The CERCLA plan recommended that monitoring be performed in two phases: baseline and routine (DOE 1995). After the baseline monitoring was conducted from October 1994 through September 1995, recommendations were made to reduce the number of RCRA monitoring locations and the frequency of monitoring (DOE 1995). TDEC verbally agreed to the plan, but never formally approved the routine monitoring approach. The routine monitoring approach was implemented beginning in December 1995 and involved monitoring nine downgradient RCRA wells on a semiannual basis, and three upgradient locations on an annual basis. The nine downgradient wells were located based on disposal history and on monitoring results collected since 1989.

Shortly after this, agreements were made to (1) continue RCRA groundwater assessment activities at SWSA 6, deferring closure and post-closure activities until a time when CERCLA remediation activities would be implemented, and (2) discontinue maintenance of the ICM caps.

This report provides the results of the 1997 RCRA groundwater assessment monitoring. The monitoring was performed in accordance with the proposed routine monitoring plan recommended in the $1996 \mathrm{EMP}$.

Section 2 provides pertinent background on SWSA 6. Section 3 presents the 1997 monitoring results and discusses the results in terms of any significant changes from previous monitoring efforts. Section 4 provides recommendations for changes in monitoring based on the 1997 results. References are provided in Section 5. Appendix A provides the field procedure for the micropurge technique. Appendix B provides the data summary statistics for the wells and Appendix C provides the 1997 water level data. Appendix D lists lead sample results for the Hillcut Test Facility from 1988 through 1997. 


\section{BACKGROUND}

\subsection{FACILITY DESCRIPTION}

WAG 6 is 68-acre LLW disposal facility at the western end of Melton Valley approximately 1.8 miles southwest of the main ORNL plant area (see Figure 2.1). WAG 6 is comprised of five Solid Waste Management Units (SWMUs): SWSA 6, the Emergency Waste Basin (EWB), the Explosives Detonation Trench (EDT), the SWSA 6 Staging Facility (7878), and the SWSA 6 Waste Storage Facility (7842). WAG 6 is bounded by State Highway 95 on the west, White Oak Lake on the south, the West Seep Tributary on the east that separates WAG 6 from WAG 7, and Lagoon Road to the north. Access to the WAG is controlled and monitored and enclosed within an 8-ft chain link security fence topped with barbed wire.

Ground surface elevations within the SWSA range from $745 \mathrm{ft}$ mean sea level (msl) at White Oak Lake along the southern boundary to $>850 \mathrm{ft}$ msl along a crest of knobs on the northwest boundary. Maximum topographic relief across the site is $105 \mathrm{ft}$. Slopes within the SWSA are variable and range from 10 to $57 \%$. The highest topographic relief and steepest slopes occur in the northeastern and eastern site perimeter. The major surface drainages at the WAG are shown on Figure 2.1 and flow from the northern portions of the site to White Oak Lake.

SWSA 6 opened for limited waste disposal operations in 1969 and began full-scale operations in 1973. It has received LLW as well as chemicals, biological wastes, and a variety of other wastes resulting from operations conducted at $\mathrm{ORNL}$ (e.g., solvents, scintillation liquids, laboratory glassware and equipment, and protective clothing). The EDT lies within the southern portion of the site (Figure 2.1), where explosives and shock-sensitive chemicals such as picric acid, phosphorous, and ammonium nitrate were detonated with small plastic charges. Debris from the explosives was left in the EDT, which was subsequently backfilled and is no longer in use. The EWB, located in the northeastern corner of the site, received groundwater seepage from several LLW units within the WAG.

Several types of waste disposal techniques were used in SWSA 6, including trenches, auger holes, silos, and above-ground container disposal units. Waste packing varied from complete lack of containerization to plastic bags to stainless steel drums.

Wastes buried before May 1986 were placed in unlined and large-diameter auger holes and concrete-lined disposal casks, quadrex boxes, and greater confinement disposal silos and auger holes. The trenches were generally $50 \mathrm{ft}$ long, $10 \mathrm{ft}$ wide, and 14-18 ft deep, depending on depth to the water table. When the disposal trenches were filled to within $2 \mathrm{ft}$ of ground surface, the trench was backfilled with soils, compacted with heavy equipment, and seeded to retard erosion. LLW was also disposed of in auger holes that were typically 1 to $4 \mathrm{ft}$ in diameter (and were as much as $9 \mathrm{ft}$ in diameter), $18 \mathrm{ft}$ deep, and spaced a minimum of $3 \mathrm{ft}$ apart. Wastes were disposed of in various sized containers up to 55-gallon drums. Following waste disposal, the auger holes were sealed with a concrete plug overlain by 1 to $2 \mathrm{ft}$ of soil cover. Waste inventory records indicate that $>85 \%$ of the total SWSA 6 chemical and radionuclide activity was disposed of in the 2-acre high activity solvent auger holes in the northeastern portion of the site. 
LEGEND:
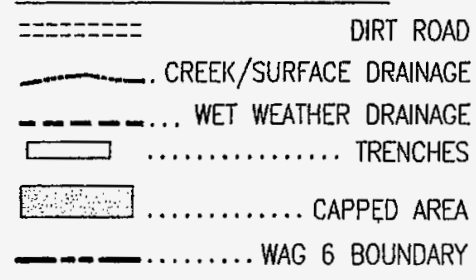

M51

SURFACE WATER

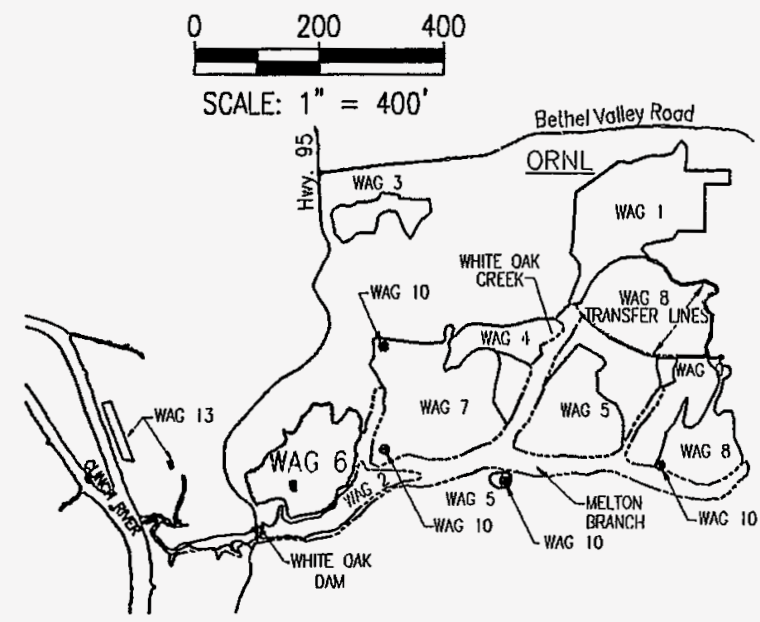

$\frac{\text { Location of WAG } 6 \text { at ORNL Site }}{\text { not to scale }}$

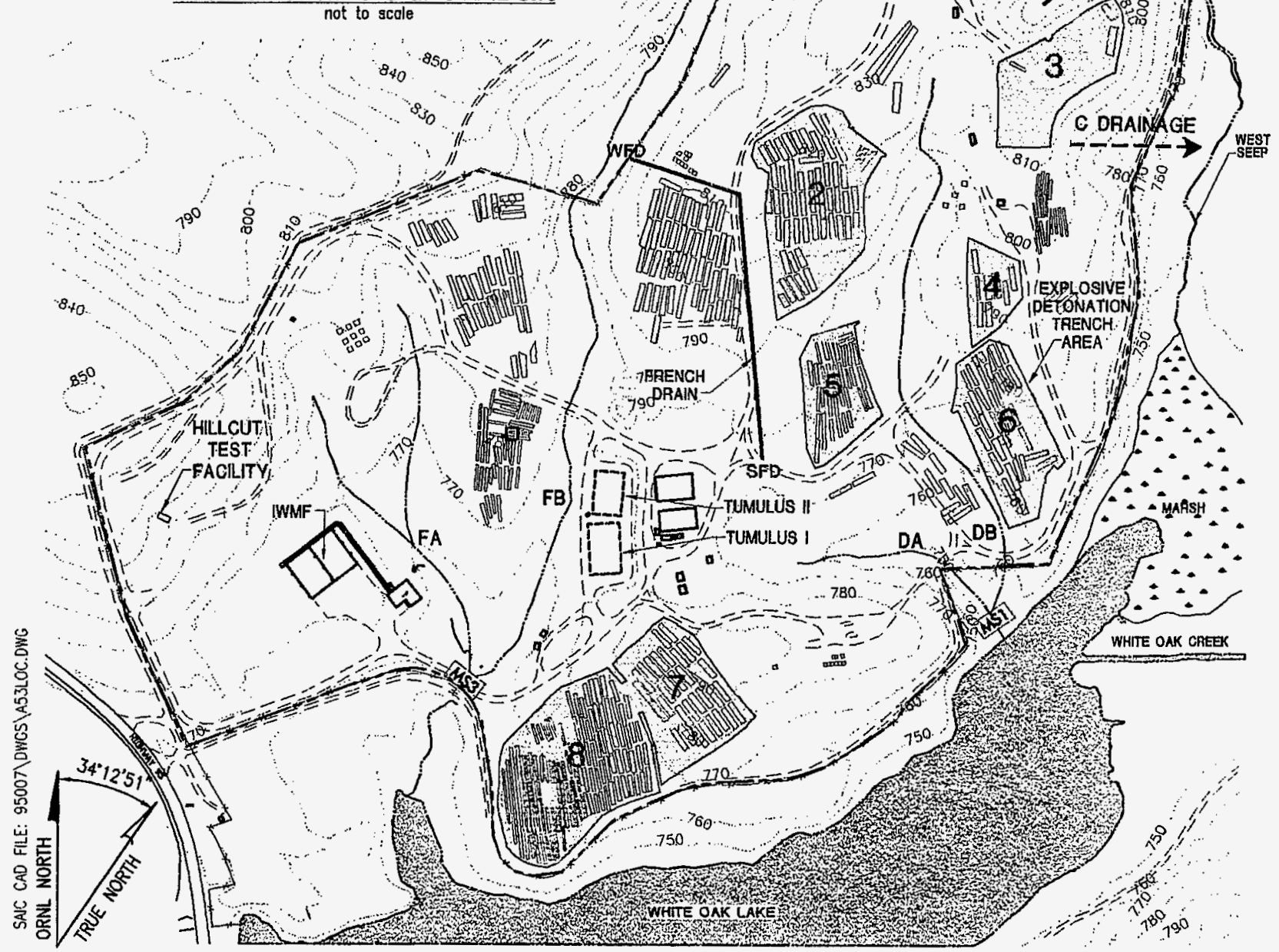

Fig. 2.1. SWSA 6 site location.
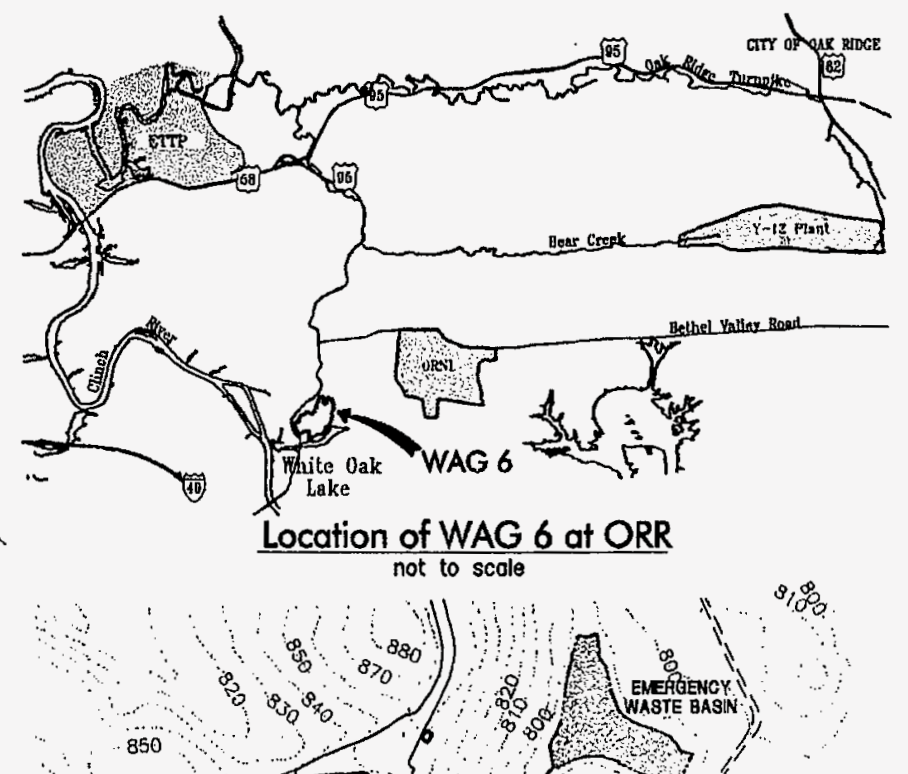
In May 1986, it was determined that about $25 \%$ of the landfilled area had received RCRAregulated wastes. The disposal operations were halted by DOE and later reopened with revised operating procedures and allowed only LLW waste disposal

Greater confinement auger holes and silos were used after May 1986. The silos were concrete lined, $10 \mathrm{ft}$ in diameter and 14 to $20 \mathrm{ft}$ deep. Auger holes were typically $20 \mathrm{ft}$ deep, 20 to $25 \mathrm{ft}$ in diameter, and lined with 3- to 4-in. thick iron pipe casings and 2-ft concrete seals at the base.

In 1988 and 1989, 8 ICM caps were placed over $\sim 10$ acres of SWSA 6 (Figure 2.1). The caps were made of 80-ml HDPE and were designed to last for $\sim 5$ years (BNI 1988).

The HTF is located $\sim 800 \mathrm{ft}$ northwest of White Oak Lake within SWSA 6. In August and September 1986, 27 concrete casks (dimensions $3.5 \times 4.5 \times 4.5 \mathrm{ft}$ ) were stacked on a subterranean concrete pad in a $3 \times 3 \times 3$ arrangement and covered with a multilayer cap. A groundwater collection system was designed to collect groundwater that could come into contact with the casks.

\subsection{REGULATORY FRAMEWORK}

\subsubsection{Regulatory Status}

SWSA 6 has been operated under the authority of the Atomic Energy Act of 1954, as amended, and administered under the guidance of the DOE for the purpose of managing LLW. In May 1986, DOE temporarily discontinued disposal of waste at SWSA 6 to address RCRA issues at the site. Although it was found that RCRA-regulated waste had been stored at the SWSA, new disposal procedures were developed to prevent further disposal of RCRA wastes.

SWSA 6 units that received RCRA-regulated wastes after November 8, 1980 are classified as RCRA-regulated units. A RCRA closure plan was submitted to TDEC in August 1988 and approved that September. The plan required that SWSA 6 stop receiving RCRA wastes and the ICM caps be built, and described a series of activities, including a RCRA Facility Investigation (RFI), leading to final closure.

The ICM caps were placed on the RCRA-regulated units and run-on/run-off controls were installed to minimize direct infiltration of precipitation into the RCRA-regulated disposal sites. This interim action allowed time to develop a total comprehensive plan for final RCRA closure.

In December 1989 the Oak Ridge Reservation (ORR), including ORNL, was placed on the National Priority List (NPL) to be remediated under the Comprehensive Environmental Response, Compensation, and Liability Act (CERCLA). Inclusion into CERCLA meant that final closure also had to comply with CERCLA.

DOE entered into a Federal Facility Agreement with EPA and TDEC, effective January 1, 1992. As a result of this agreement, WAG 6 is regulated under CERCLA. SWSA 6 is also regulated under RCRA as an interim status site.

The RFI/Remedial Investigation (RI) for WAG 6 was completed in 1992. Based on the findings of this and related CERCLA characterization efforts at ORNL, it was determined that the preferred alternative for WAG 6 was to defer action on the site, allowing remediation resources to be applied 
to other sites at ORNL with more immediate release concerns. As a result of this decision, final RCRA closure has not been completed. The current plan is to complete closure in conjunction with CERCLA actions associated with the remediation of Melton Valley. A revision of the 1988 RCRA Closure Plan was submitted to TDEC in July 1995, which outlined the deferred action approach. The 1995 Closure Plan deferred the groundwater assessment approach to the EMP.

A Post-Closure Permit Application (PCPA) was to have been submitted to TDEC in October 1995, stating that the preferred alternative was deferred action and that monitoring would continue as outlined in the EMP. However, on August 31, 1995, TDEC requested that DOE not submit the PCPA pending the resolution of the impact of draft EPA regulation dealing with issuance of PCPAs at federal facility NPL sites (Burroughs 1992).

\subsubsection{RCRA/CERCLA Integration}

The TDEC, DOE and EPA agreed that RCRA authority at SWSA 6, EDT, and HTF would be accomplished through future remedial actions under CERCLA. DOE is currently monitoring SWSA 6 and HTC under the EMP. Comprehensive baseline monitoring under the EMP was conducted from October 1994 through September 1995. Twenty-four RCRA wells were monitored, including the 8 wells sampled quarterly and 16 wells sampled semi-annually (Figure 2.2). Results of that sampling were reported in DOE 1995 and both the 1995 and 1996 GWQARs.

Based on the results of the baseline monitoring, a routine monitoring program was proposed (DOE 1995). The RCRA assessment monitoring portion of the EMP routine monitoring covers nine downgradient and three upgradient wells. The routine monitoring was initiated in May 1996.

\subsection{HYDROGEOLOGIC FRAMEWORK}

A complete summary of the hydrogeologic conditions at SWSA 6 has been presented in several reports (ORNL 1993, BNI 1991; DOE 1995).

\subsection{ASSESSMENT MONITORING PROGRAM}

\subsubsection{Monitoring Network}

During 1997, 12 groundwater wells were monitored at SWSA 6. The wells are described in Table 2.1 and shown in Figure 2.2. Three of the wells are upgradient wells: 858, 857, and 846. The remaining nine are located downgradient/along strike of the RCRA-regulated units, as indicated in the table. The wells were sampled in both June and November. Table 2.2 summarizes the key well construction information for the wells. Detailed well construction diagrams and drilling logs for the original 24 RCRA wells are contained in Monitor Well Data Packages for SWSA 6, ORNL/RAP-44 (Mortimore and Ebers 1988). Three sets of wells pairs are part of the routine monitoring program: $843 / 844,841 / 842$, and $857 / 858$ (upgradient wells). These wells help define the vertical extent of contamination at SWSA 6 . 


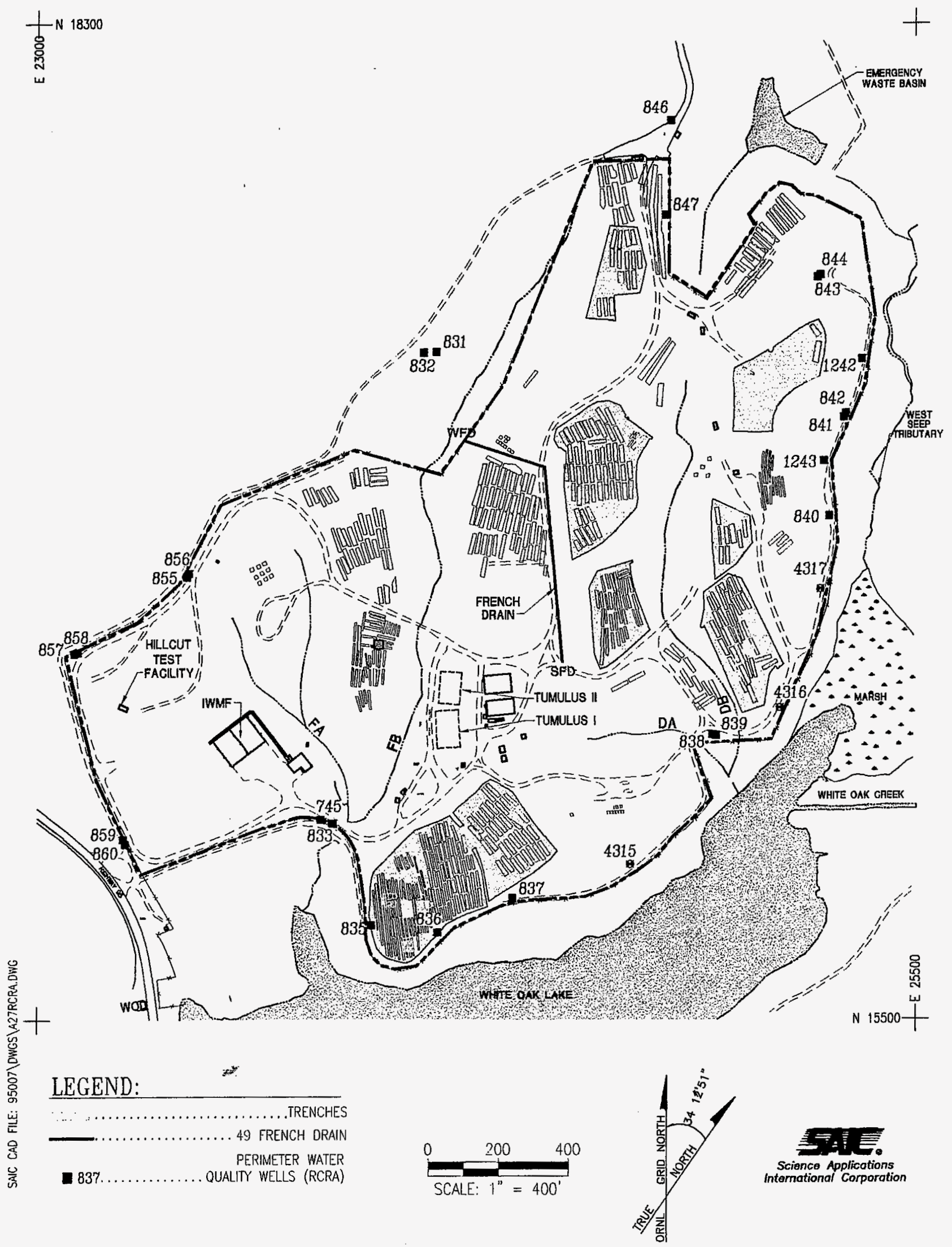

Fig. 2.2. SWSA 6 RCRA sampling location. 
Table 2.1. RCRA monitoring well network

\begin{tabular}{|c|c|}
\hline Well ID & Rationale \\
\hline \multicolumn{2}{|r|}{ Downgradient wells } \\
\hline 835 & Provides coverage downgradient of RCRA ICM Cap 8 \\
\hline 837 & Downgradient of RCRA ICM Cap 7; provides coverage \\
\hline 841 & $\begin{array}{l}\text { Historical VOC detections; provides coverage downgradient of RCRA ICM Cap } \\
\text { 3/Solvent Auger Holes and along eastern WAG perimeter; deep well of } \\
\text { downgradient shallow/deep well pair }\end{array}$ \\
\hline 842 & $\begin{array}{l}\text { Historical VOC detections; provides coverage downgradient of RCRA ICM Cap } \\
\text { 3/Solvent Auger Holes and along eastern WAG perimeter; shallow well of } \\
\text { downgradient shallow/deep well pair }\end{array}$ \\
\hline 843 & $\begin{array}{l}\text { Historical VOC detections; provides coverage along eastern WAG perimeter; } \\
\text { shallow well of downgradient shallow/deep well pair }\end{array}$ \\
\hline 844 & $\begin{array}{l}\text { Historical VOC detections; provides coverage along eastern WAG perimeter; deep } \\
\text { well of downgradient shallow/deep well pair }\end{array}$ \\
\hline 4315 & $\begin{array}{l}\text { Potentially downgradient, along strike of RCRA ICM Cap 7; recent lead detections; } \\
\text { provides coverage of southern WAG perimeter }\end{array}$ \\
\hline 4316 & $\begin{array}{l}\text { Downgradient of RCRA ICM Cap 6; potentially downgradient of Cap 4; provides } \\
\text { coverage of southern WAG perimeter }\end{array}$ \\
\hline 4317 & Downgradient of RCRA ICM Cap 4 \\
\hline \multicolumn{2}{|r|}{ Upgradient wells } \\
\hline 846 & $\begin{array}{l}\text { RCRA reference well; most upgradient well at WAG; historically clean of VOCs and } \\
\text { radionuclides }\end{array}$ \\
\hline 857 & $\begin{array}{l}\text { RCRA reference wells; help provide full coverage of upgradient perimeter; } \\
\text { historically clean; shallow well of upgradient shallow/deep pair }\end{array}$ \\
\hline 858 & $\begin{array}{l}\text { RCRA reference wells deep well of upgradient shallow/deep pair; help provide full } \\
\text { coverage of upgradient perimeter and deep zone; historically clean; deep well of } \\
\text { upgradient shallow/deep pair }\end{array}$ \\
\hline HCTANK1P & Hillcut Test Facility (HTF) Pad 1 Drain \\
\hline HCTANK2UP & HTF Pad 2 Underpad Drain \\
\hline
\end{tabular}




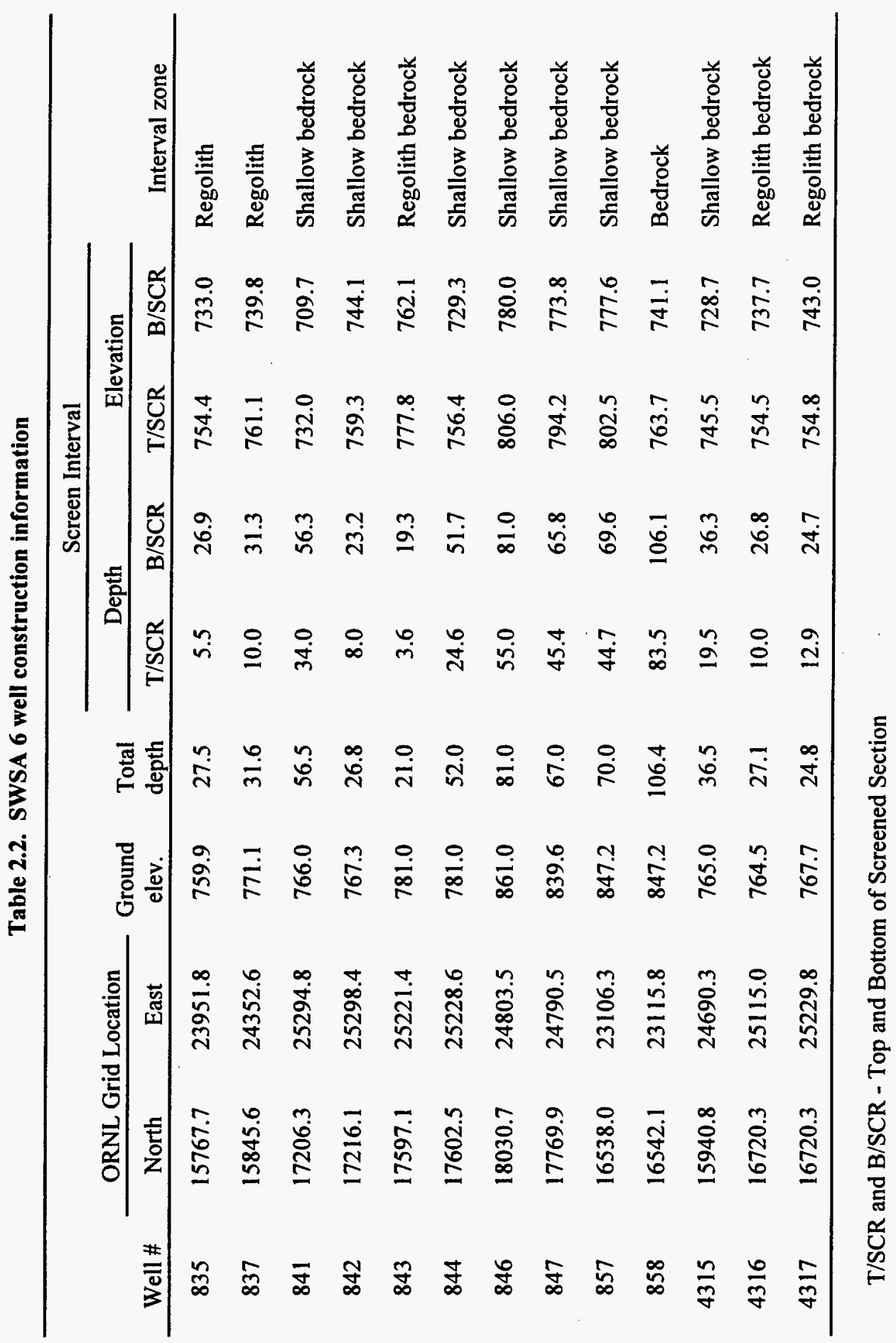


Under the routine monitoring program, the RCRA wells are sampled less frequently than they were prior to 1995. In 1997, the downgradient RCRA wells were sampled semi-annually, in June and November/December. The semiannual approach was determined to be the most cost-effective approach of tracking changes in releases at the wells. It also ensured that contaminant concentrations were evaluated for both the wet and dry season.

\title{
2.4.2 Sampling and Analysis
}

During 1997, all 12 RCRA wells were monitored for SW-8240 VOCs, field parameters, radiological parameters, and water levels. Radiological parameters are CERCLA parameters and are reported by the ORR Integrated Water Quality Program (IWQP) and are not discussed as part of the RCRA reporting. The analytical detection limit requirements are presented in the EMP.

Beginning in 1991, a "target compound list" of VOCs was developed for SWSA 6 based on historical sampling results. This list is provided in Table 2.3 along with regulatory levels and detection levels for each chemical. An additional analyte was added to the list as part of developing the EMP. Lead is a target analyte for two monitoring locations, Well 4315 and the HTF. Lead is known to have been stored in the HTF. Historical results from Well 4315 indicate the presence of low levels of lead $(\sim 10$ to $30 \mathrm{ug} / \mathrm{L})$, and thus lead will continue to be analyzed for in this well. The regulatory level for lead is $15 \mathrm{ug} / \mathrm{L}$.

The groundwater sampling was conducted by the ORR IWQP as part of the reservation-wide monitoring activities. The IWQP was developed to integrate CERCLA and RCRA monitoring efforts across the reservation and across the various monitoring programs within the reservation, to the extent possible. The following standard procedures were followed:

\author{
SOP-ESP-302-2 Guideline for Well Purging \\ SOP-ESP-302-1 Measurement Using a Water Level Indicator \\ SOP-ESP-302-1 Using a Bladder Pump \\ SOP-ESP-801 Cleaning and Decontaminating Sample Containers and Sampling Devices \\ SOP-ESP-307-x Field Measurement Procedures - (1) Temperature, (2) pH, and (8) Specific \\ Conductance \\ SOP-ESP-003.012 Use of the Horiba U-10 Water Quality Checker
}

\subsubsection{Quality Assurance/Quality Control}

Standard field and laboratory quality control procedures were followed to ensure the analytical quality of the sampling results. Data were validated following the same procedures as used for other IWQP monitoring locations; this level of validation follows CERCLA guidelines and is more extensive than what is routinely required for RCRA monitoring. The procedure called for collecting field blanks, field duplicates, and standard laboratory quality control (QC) procedures, including lab blanks and matrix spikes/matrix spike duplicates.

\subsubsection{Well Purge Issues}

In addition to the sample collected using the standard WAG 6 RCRA three-volume purge method, a sample was collected from several wells using a micropurge, or low-flow purge method. This was done to compare conventional and micropurge results to provide some technical support for 
Table 2.3. SWSA 6 Target compound list, regulatory limits, and detection limits

\begin{tabular}{lcc}
\hline \multicolumn{1}{c}{ Target compound } & Regulatory limit (ug/L) & Detection limit (ug/L) \\
\hline 1,1-dichloroethane & $7^{a}$ & 5 \\
1,2-dichloroethane & $5^{b}$ & 5 \\
1,2-dichloroethene (total cis and trans) & - & 5 \\
carbon disulfide & $5^{b}$ & 5 \\
carbon tetrachloride & $100^{a}$ & 5 \\
chloroform & - & 5 \\
chloromethane & $5^{a}$ & 5 \\
tetrachloroethene & $5^{a}$ & 5 \\
trichloroethene & $10,000^{a}$ & 5 \\
total xylenes & $15^{c}$ & 5 \\
Lead &
\end{tabular}

${ }^{\circ} 40$ CFR Part 414 - National Primary Drinking Water Regulations, Subparts B and G as amended.

${ }^{b}$ Rules of Tennessee Department of Environmental and Conservation, Division of Water Pollution Control, Chapter 1200-4-3, General Water Quality Criteria, as amended.

'Technology action level for lead, defined by 40 CFR Part 141. 
a possible recommended switch to the micropurge technique. In all cases, the micropurge sample was collected first since it was assumed the micropurge would disrupt the water less. This is part of an effort to standardize well purging techniques across the IWQP program and across the ORR. This purge technique has been adopted for several reasons, including (1) there is less disruption of the water and thus less assumed volatilization and less introduction of particulates in samples and (2) micropurging results in significantly less waste water generated that must be treated and disposed of as hazardous material. During the November 1997 sampling event, conventional purging yielded 311 gal of purge water, compared to 9 gal generated using micropurge techniques. It is desirable that the SWSA 6 RCRA sampling also be performed using this technique. The micropurge procedure used by the IWQP is provided in Appendix A. 


\section{GROUNDWATER QUALITY}

This chapter presents the 1997 RCRA groundwater assessment monitoring results for SWSA 6, identifies any changes in the nature and extent of VOC contamination, and provides a calculation of the rate of groundwater movement at the WAG. A complete compilation of RCRA monitoring results for the 12 RCRA wells is provided in Appendix B of this report.

\subsection{GROUNDWATER DATA ASSESSMENT}

\subsubsection{Data Quality Assessment}

This section provides a summary of the 1997 RCRA well sampling results. Appendix B provides a complete list of the monitoring results of RCRA wells for all years. The 1997 data were collected and analyzed as described in Section 2.4. Trip blanks and field duplicates were collected in the field and matrix spike and matrix spike duplicates were run in the laboratory, as per the QC procedures developed for the IWQP.

Data Quality Assessment Summary packages are provided in Appendix C. No data quality issues were identified for VOCs. Methylene chloride was detected frequently in blank samples; associated analyses were qualified with a "B." A data quality issue was identified for lead analysis. The instrument detected limit (IDL) for both the June and November samples was greater than the lead regulatory limit of $15 \mathrm{ug} / \mathrm{L}$. The IDL was $22.7 \mathrm{ug} / \mathrm{L}$ in June and $36.2 \mathrm{ug} / \mathrm{L}$ in November. This IDL is greater than IDLs achieved in the past because the Inductively Coupled Plasma (ICP) method was inadvertently used instead of the Graphite Furnace/Atomic Absorption (GF/AA). Well 4315 will be resampled in early spring and GF/AA will be used to achieve the $5 \mu \mathrm{g} / \mathrm{L}$ detection limit requirement.

\subsubsection{Analysis of Well Purging Techniques}

In addition to QC issues, additional samples were collected from several wells in order to make recommendations pertaining to the use of low-flow purging techniques for future sampling. During the first sampling effort in June, two samples were collected from selected wells, one using the threevolume purge technique, and one using the low-flow purge technique. Two samples were collected in wells with historical records of VOC contamination, including Wells 841, 842, and 843. In both cases, samples received separate sample IDs and were sent to the same laboratory for VOC analysis. A comparison of the results for key VOC parameters is presented in Table 3.1. These results indicate that the results obtained from either purge technique are comparable. In most cases, the quantitative results for the detected VOC are off by only a fraction.

\subsubsection{Assessment Results}

A complete listing the analytical results for each well over time is provided in Appendix A. This section provides summaries of the 1997 results. Summaries of the 1997 results are provided in Table 3.2 for field parameters and in Tables 3.3 through 3.14 for the SWSA 6 target compounds.

Outside of a few of the 11 target analytes, no additional VOCs were detected in any of the RCRA wells during either sampling event in 1997. Tables 3.15 and 3.16 provide a summary of positive detections in wells for June and November, respectively, and indicate which detections are greater 


$$
\text { 3-2 }
$$

Table 3.1. Comparison of three-volume purge and micropurge results

\begin{tabular}{lllccc}
\hline Well & $\begin{array}{c}\text { Sample } \\
\text { date }\end{array}$ & \multicolumn{1}{c}{ Analyte } & $\begin{array}{c}\text { Three- } \\
\text { volume } \\
\text { purge } \\
\text { results } \\
(\mu \mathrm{g} / \mathrm{L})\end{array}$ & $\begin{array}{c}\text { Micro- } \\
\text { purge } \\
\text { results } \\
(\mu \mathrm{\mu g} / \mathrm{L})\end{array}$ & $\begin{array}{c}\text { Ratio: } \\
\text { RCRA } \\
\text { method/ } \\
\text { micro- } \\
\text { purge }\end{array}$ \\
\hline 841 & $8 / 26 / 97$ & Trichloroethene & 8 & 10 & 0.80 \\
842 & $6 / 97$ & 1,2-Dichloroethane & 8 & 11 & 0.73 \\
& & Carbon Tetrachloride & 28 & 20 & 1.4 \\
& & Chloroform & 30 & 34 & 0.88 \\
& Trichloroethene & 110 & 140 & 0.78 \\
\hline
\end{tabular}




$$
\text { 3-3 }
$$

Table 3.2. RCRA well field parameters

\begin{tabular}{|c|c|c|c|c|c|}
\hline Well & $\begin{array}{c}\text { Measurement } \\
\text { date }\end{array}$ & $\begin{array}{c}\begin{array}{c}\text { Conductivity } \\
(\mathrm{ms} / \mathrm{cm})\end{array} \\
\end{array}$ & $\begin{array}{c}\text { Depth to } \\
\text { water (ft) }\end{array}$ & $\begin{array}{c}\text { pH (std } \\
\text { unit) }\end{array}$ & $\begin{array}{c}\text { Temperature } \\
\text { (deg C) }\end{array}$ \\
\hline 835 & 23-Jun & 0.12 & 15.12 & 6.04 & 18.3 \\
\hline 835 & $11-$ Nov & 0.166 & 16.42 & 5.65 & 17.12 \\
\hline 837 & 23-Jun & 0.015 & 26 & 5.58 & 18.6 \\
\hline 837 & $10-\mathrm{Nov}$ & 0.04 & 27.98 & 5.39 & 14.99 \\
\hline 841 & 26-Jun & 0.336 & 11.61 & 7.54 & 17.2 \\
\hline 841 & $12-\mathrm{Nov}$ & 0.55 & 12.66 & 7.3 & 12.37 \\
\hline 842 & 18-Jun & 0.391 & 8.93 & 7.11 & 15.4 \\
\hline 842 & $11-\mathrm{Nov}$ & 0.673 & 10.58 & 7 & 14.87 \\
\hline 843 & 25-Jun & 0.613 & 2.98 & 6.88 & 15.3 \\
\hline 843 & $20-\mathrm{Nov}$ & 1.002 & 7.23 & 6.57 & 13.3 \\
\hline 844 & 25-Jun & 0.481 & 11.9 & 7.37 & 18.4 \\
\hline 844 & $20-\mathrm{Nov}$ & 0.829 & 14.08 & 6.97 & 14.2 \\
\hline 846 & 24-Jun & 0.546 & 42.16 & 7.17 & 18.2 \\
\hline 846 & 19-Nov & 0.984 & 50.45 & 6.77 & 14.5 \\
\hline 857 & 17-Jun & 0.007 & 49.89 & 5.55 & 16 \\
\hline 857 & 13-Nov & 0.028 & 56.6 & 5.32 & 12.82 \\
\hline 858 & 16-Jun & 0.138 & 51.54 & 8.3 & 18.2 \\
\hline 858 & $17-\mathrm{Nov}$ & 0.229 & 57.45 & 8.09 & 13.06 \\
\hline 4315 & 23-Jun & 0.08 & 21.64 & 6.36 & 17.9 \\
\hline 4315 & $18-\mathrm{Nov}$ & 0.192 & 22.71 & 6.42 & 15.18 \\
\hline 4316 & 24-Jun & 0.436 & 20.36 & 7.19 & 18.3 \\
\hline 4316 & $18-\mathrm{Nov}$ & 0.812 & 20.25 & 6.73 & 16.5 \\
\hline 4317 & 17-Jun & 0.173 & 15.62 & 6.53 & 16.5 \\
\hline 4317 & 18-Nov & 0.567 & 18.2 & 6.09 & 10.7 \\
\hline
\end{tabular}




\section{$3-4$}

Table 3.3. Target compound monitoring results Well 835 - 1997

\begin{tabular}{|c|c|c|c|c|}
\hline Sample ID & Sample date & Target compound & Results $(\mu \mathrm{g} / \mathrm{L})$ & Data qualifier $^{a}$ \\
\hline M00823 & 23-Jun-97 & 1,1-Dichloroethene & 5 & $\mathrm{U}$ \\
\hline M01225 & 11-Nov-97 & 1,1-Dichloroethene & 5 & $\mathrm{U}$ \\
\hline M00823 & 23-Jun-97 & 1,1-Dichloroethene & 5 & $\mathrm{U}$ \\
\hline M01225 & 11-Nov-97 & 1,1-Dichloroethene & 5 & $\mathrm{U}$ \\
\hline M00823 & 23-Jun-97 & 1,2-Dichloroethane & 5 & $U$ \\
\hline M01225 & 11-Nov-97 & 1,2-Dichloroethane & 5 & $\mathrm{U}$ \\
\hline M00823 & 23-Jun-97 & Carbon disulfide & 5 & $\mathrm{U}$ \\
\hline M01225 & 11-Nov-97 & Carbon disulfide & 5 & $\mathrm{U}$ \\
\hline M00823 & 23-Jun-97 & Carbon tetrachloride & 5 & $\mathrm{U}$ \\
\hline M01225 & $11-$ Nov-97 & Carbon tetrachloride & 5 & $\mathrm{U}$ \\
\hline M00823 & 23-Jun-97 & Chloroform & 5 & $\mathrm{U}$ \\
\hline M01225 & 11-Nov-97 & Chloroform & 5 & $\mathrm{U}$ \\
\hline M00823 & 23-Jun-97 & Chloromethane & 10 & $\mathrm{U}$ \\
\hline M01225 & $11-$ Nov-97 & Chloromethane & 10 & $\mathrm{U}$ \\
\hline M00823 & 23-Jun-97 & cis-1,2-Dichloroethene & 5 & $\mathrm{U}$ \\
\hline M01225 & 11-Nov-97 & cis-1,2-Dichloroethene & 5 & $\mathrm{U}$ \\
\hline M00823 & 23-Jun-97 & m,p-Xylene & 5 & U \\
\hline M01225 & 11-Nov-97 & m,p-Xylene & 5 & $\mathrm{U}$ \\
\hline M00823 & 23-Jun-97 & Tetrachloroethene & 5 & $\mathbf{U}$ \\
\hline M01225 & 11-Nov-97 & Tetrachloroethene & 5 & $\mathbf{U}$ \\
\hline M00823 & 23-Jun-97 & trans-1,2-Dichloroethene & 5 & $\mathrm{U}$ \\
\hline M01225 & $11-$ Nov-97 & trans-1,2-Dichloroethene & 5 & $\mathbf{U}$ \\
\hline M00823 & 23-Jun-97 & Trichloroethene & 5 & $\mathbf{U}$ \\
\hline M01225 & 11-Nov-97 & Trichloroethene & 5 & $\mathrm{U}$ \\
\hline
\end{tabular}

a " =" = detected at reported value; $\mathrm{U}$ - not detected; $\mathrm{J}=$ estimated value 


\section{3-5}

Table 3.4. Target compound monitoring results Well 837 - 1997

\begin{tabular}{|c|c|c|c|c|}
\hline Sample ID & Sample date & Target compound & Results $(\mu \mathrm{g} / \mathrm{L})$ & Data qualifier ${ }^{\mathrm{a}}$ \\
\hline M00823 & 23-Jun-97 & 1,1-Dichloroethene & 5 & $\mathrm{U}$ \\
\hline M01225 & 11-Nov-97 & 1,1-Dichloroethene & 5 & $\mathrm{U}$ \\
\hline M00823 & 23-Jun-97 & 1,1-Dichloroethene & 5 & $\mathrm{U}$ \\
\hline M01225 & 11-Nov-97 & 1,1-Dichloroethene & 5 & $\mathrm{U}$ \\
\hline M00823 & 23-Jun-97 & 1,2-Dichloroethane & 5 & $\mathrm{U}$ \\
\hline M01225 & 11-Nov-97 & 1,2-Dichloroethane & 5 & $\mathrm{U}$ \\
\hline M00823 & 23-Jun-97 & Carbon disulfide & 5 & $\mathrm{U}$ \\
\hline M01225 & 11-Nov-97 & Carbon disulfide & 5 & $\mathrm{U}$ \\
\hline M00823 & 23-Jun-97 & Carbon tetrachloride & 5 & U \\
\hline M01225 & 11-Nov-97 & Carbon tetrachloride & 5 & $\mathrm{U}$ \\
\hline M00823 & 23-Jun-97 & Chloroform & 5 & $\mathrm{U}$ \\
\hline M01225 & 11-Nov-97 & Chloroform & 5 & $\mathrm{U}$ \\
\hline M00823 & 23-Jun-97 & Chloromethane & 10 & $\mathrm{U}$ \\
\hline M01225 & 11-Nov-97 & Chloromethane & 10 & $\mathrm{U}$ \\
\hline M00823 & 23-Jun-97 & cis-1,2-Dichloroethene & 5 & $\mathrm{U}$ \\
\hline M01225 & 11-Nov-97 & cis-1,2-Dichloroethene & 5 & $\mathrm{U}$ \\
\hline M00823 & 23-Jun-97 & m,p-Xylene & 5 & $\mathrm{U}$ \\
\hline M01225 & 11-Nov-97 & $\mathrm{m}, \mathrm{p}$-Xylene & 5 & $\mathrm{U}$ \\
\hline M00823 & 23-Jun-97 & Tetrachloroethene & 5 & $\mathrm{U}$ \\
\hline M01225 & 11-Nov-97 & Tetrachloroethene & 5 & $\mathrm{U}$ \\
\hline M00823 & 23-Jun-97 & trans-1,2-Dichloroethene & 5 & $\mathbf{U}$ \\
\hline M01225 & 11-Nov-97 & trans-1,2-Dichloroethene & 5 & U \\
\hline M00823 & 23-Jun-97 & Trichloroethene & 5 & $\mathrm{U}$ \\
\hline M01225 & 11-Nov-97 & Trichloroethene & 5 & $\mathrm{U}$ \\
\hline
\end{tabular}

o" =" = detected at reported value; $\mathrm{U}$ - not detected; $\mathrm{J}=$ estimated value 


\section{$3-6$}

Table 3.5. Target compound monitoring results Well $841-1997$

\begin{tabular}{|c|c|c|c|c|}
\hline Sample ID & Sample date & Target compound & Results $(\mu \mathrm{g} / \mathrm{L})$ & Data qualifier \\
\hline M00823 & 23-Jun-97 & 1,1-Dichloroethene & 5 & $\mathrm{U}$ \\
\hline M01225 & 11-Nov-97 & 1,1-Dichloroethene & 5 & $\mathrm{U}$ \\
\hline M00823 & 23-Jun-97 & 1,1-Dichloroethene & 5 & $\mathrm{U}$ \\
\hline M01225 & $11-$ Nov-97 & 1,1-Dichloroethene & 5 & $\mathrm{U}$ \\
\hline M00823 & 23-Jun-97 & 1,2-Dichloroethane & 5 & $\mathrm{U}$ \\
\hline M01225 & 11-Nov-97 & 1,2-Dichloroethane & 5 & $\mathrm{U}$ \\
\hline M00823 & 23-Jun-97 & Carbon disulfide & 5 & $\mathrm{U}$ \\
\hline M01225 & 11-Nov-97 & Carbon disulfide & 5 & $\mathrm{U}$ \\
\hline M00823 & 23-Jun-97 & Carbon tetrachloride & 5 & $\mathrm{U}$ \\
\hline M01225 & 11-Nov-97 & Carbon tetrachloride & 5 & $\mathrm{U}$ \\
\hline M00823 & 23-Jun-97 & Chloroform & 5 & $\mathrm{U}$ \\
\hline M01225 & 11-Nov-97 & Chloroform & 5 & $\mathrm{U}$ \\
\hline M00823 & 23-Jun-97 & Chloromethane & 10 & $\mathrm{U}$ \\
\hline M01225 & 11-Nov-97 & Chloromethane & 2 & B \\
\hline M00823 & 23-Jun-97 & cis-1,2-Dichloroethene & 5 & $\mathrm{U}$ \\
\hline M01225 & 11-Nov-97 & cis-1,2-Dichloroethene & 5 & $\mathrm{U}$ \\
\hline M00823 & 23-Jun-97 & m,p-Xylene & 5 & $\mathrm{U}$ \\
\hline M01225 & 11-Nov-97 & m,p-Xylene & 5 & $\mathrm{U}$ \\
\hline M00823 & 23-Jun-97 & Tetrachloroethene & 5 & $\mathrm{U}$ \\
\hline M01225 & 11-Nov-97 & Tetrachloroethene & 5 & U \\
\hline M00823 & 23-Jun-97 & trans-1,2-Dichloroethene & 5 & $\mathrm{U}$ \\
\hline M01225 & 11-Nov-97 & trans-1,2-Dichloroethene & 5 & $\mathrm{U}$ \\
\hline M00823 & 23-Jun-97 & Trichloroethene & 5 & $=$ \\
\hline M01225 & 11-Nov-97 & Trichloroethene & 5 & $=$ \\
\hline
\end{tabular}

${ }^{a c}="$ = detected at reported value; $\mathrm{U}$ - not detected; $\mathrm{J}=$ estimated value 


\section{3-7}

Table 3.6. Target compound monitoring results Well 842 - 1997

\begin{tabular}{|c|c|c|c|c|}
\hline Sample ID & Sample date & Target compound & Results $(\mu \mathrm{g} / \mathbf{L})$ & Data qualifier $^{a}$ \\
\hline M00823 & 23-Jun-97 & 1,1-Dichloroethene & 1 & $\mathrm{U}$ \\
\hline M01225 & 11-Nov-97 & 1,1-Dichloroethene & 2 & $\mathrm{~J}$ \\
\hline M00823 & 23-Jun-97 & 1,1-Dichloroethene & 5 & $\mathrm{U}$ \\
\hline M01225 & 11-Nov-97 & 1,1-Dichloroethene & 5 & $\mathrm{U}$ \\
\hline M00823 & 23-Jun-97 & 1,2-Dichloroethane & 8 & $=$ \\
\hline M01225 & 11-Nov-97 & 1,2-Dichloroethane & 10 & $=$ \\
\hline M00823 & 23-Jun-97 & Carbon disulfide & 5 & $\mathrm{U}$ \\
\hline M01225 & 11-Nov-97 & Carbon disulfide & 5 & $\mathrm{U}$ \\
\hline M00823 & 23-Jun-97 & Carbon tetrachloride & 28 & $=$ \\
\hline M01225 & 11-Nov-97 & Carbon tetrachloride & 29 & $=$ \\
\hline M00823 & 23-Jun-97 & Chloroform & 30 & $=$ \\
\hline M01225 & 11-Nov-97 & Chloroform & 29 & $=$ \\
\hline M00823 & 23-Jun-97 & Chloromethane & 10 & $\mathrm{U}$ \\
\hline M01225 & 11-Nov-97 & Chloromethane & 10 & $\mathrm{U}$ \\
\hline M00823 & 23-Jun-97 & cis-1,2-Dichloroethene & 7 & $=$ \\
\hline M01225 & 11-Nov-97 & cis-1,2-Dichloroethene & 10 & $=$ \\
\hline M00823 & 23-Jun-97 & m,p-Xylene & 5 & $\mathrm{U}$ \\
\hline M01225 & 11-Nov-97 & $\mathrm{m}, \mathrm{p}$-Xylene & 5 & $\mathrm{U}$ \\
\hline M00823 & 23-Jun-97 & Tetrachloroethene & 5 & $\mathrm{U}$ \\
\hline M01225 & 11-Nov-97 & Tetrachloroethene & 5 & $\mathrm{U}$ \\
\hline M00823 & 23-Jun-97 & trans-1,2-Dichloroethene & 5 & $\mathrm{U}$ \\
\hline M01225 & 11-Nov-97 & trans-1,2-Dichloroethene & 5 & $\mathrm{U}$ \\
\hline M00823 & 23-Jun-97 & Trichloroethene & 110 & $=$ \\
\hline M01225 & 11-Nov-97 & Trichloroethene & 150 & $=$ \\
\hline
\end{tabular}

${ }^{a}="=$ detected at reported value; $\mathrm{U}$ - not detected; $\mathrm{J}=$ estimated value 


$$
\text { 3-8 }
$$

Table 3.7. Target compound monitoring results Well 843 - 1997

\begin{tabular}{|c|c|c|c|c|}
\hline Sample ID & Sample date & Target compound & Results $(\mu \mathrm{g} / \mathrm{L})$ & Data qualifier ${ }^{a}$ \\
\hline M00823 & 23-Jun-97 & 1,1-Dichloroethene & 5 & $\mathrm{U}$ \\
\hline M01225 & 11-Nov-97 & 1,1-Dichloroethene & 5 & $\mathrm{U}$ \\
\hline M00823 & 23-Jun-97 & 1,1-Dichloroethene & 5 & $\mathrm{U}$ \\
\hline M01225 & 11-Nov-97 & 1,1-Dichloroethene & 5 & $\mathrm{U}$ \\
\hline M00823 & 23-Jun-97 & 1,2-Dichloroethane & 5 & $\mathrm{U}$ \\
\hline M01225 & 11-Nov-97 & 1,2-Dichloroethane & 5 & $\mathrm{U}$ \\
\hline M00823 & 23-Jun-97 & Carbon disulfide & 5 & $\mathrm{U}$ \\
\hline M01225 & 11 -Nov-97 & Carbon disulfide & 5 & $\mathrm{U}$ \\
\hline M00823 & 23-Jun-97 & Carbon tetrachloride & 5 & $\mathrm{U}$ \\
\hline M01225 & $11-$ Nov-97 & Carbon tetrachloride & 5 & $\mathrm{U}$ \\
\hline M00823 & 23-Jun-97 & Chloroform & 5 & $\mathrm{U}$ \\
\hline M01225 & $11-$ Nov-97 & Chloroform & 5 & $\mathrm{U}$ \\
\hline M00823 & 23-Jun-97 & Chloromethane & 10 & $\mathrm{U}$ \\
\hline M01225 & $11-$ Nov-97 & Chloromethane & 10 & $\mathrm{U}$ \\
\hline M00823 & 23-Jun-97 & cis-1,2-Dichloroethene & 13 & $=$ \\
\hline M01225 & 11-Nov-97 & cis-1,2-Dichloroethene & 6 & $=$ \\
\hline M00823 & 23-Jun-97 & $\mathrm{m}, \mathrm{p}$-Xylene & 5 & $\mathrm{U}$ \\
\hline M01225 & 11-Nov-97 & $\mathrm{m}, \mathrm{p}$-Xylene & 5 & $\mathrm{U}$ \\
\hline M00823 & 23-Jun-97 & Tetrachloroethene & 5 & $\mathrm{U}$ \\
\hline M01225 & 11-Nov-97 & Tetrachloroethene & 5 & $\mathrm{U}$ \\
\hline M00823 & 23-Jun-97 & trans-1,2-Dichloroethene & 5 & $\mathrm{U}$ \\
\hline M01225 & 11-Nov-97 & trans-1,2-Dichloroethene & 5 & $\mathrm{U}$ \\
\hline M00823 & 23-Jun-97 & Trichloroethene & 2 & $\mathrm{U}$ \\
\hline M01225 & 11-Nov-97 & Trichloroethene & 5 & $\mathrm{U}$ \\
\hline
\end{tabular}

a "=" = detected at reported value; $\mathrm{U}$ - not detected; $\mathrm{J}=$ estimated value 
Table 3.8. Target compound monitoring results Well 844 - 1997

\begin{tabular}{|c|c|c|c|c|}
\hline Sample ID & Sample date & Target compound & Results $(\mu \mathrm{g} / \mathrm{L})$ & Data qualifier ${ }^{n}$ \\
\hline M00823 & 23-Jun-97 & 1,1-Dichloroethene & 5 & $\mathrm{U}$ \\
\hline M01225 & 11-Nov-97 & 1,1-Dichloroethene & 5 & $\mathrm{U}$ \\
\hline M00823 & 23-Jun-97 & 1,1-Dichloroethene & 5 & $\mathrm{U}$ \\
\hline M01225 & 11-Nov-97 & 1,1-Dichloroethene & 5 & $\mathrm{U}$ \\
\hline M00823 & 23-Jun-97 & 1,2-Dichloroethane & 5 & $\mathrm{U}$ \\
\hline M01225 & 11-Nov-97 & 1,2-Dichloroethane & 5 & $\mathbf{U}$ \\
\hline M00823 & 23-Jun-97 & Carbon disulfide & 5 & $\mathrm{U}$ \\
\hline M01225 & 11-Nov-97 & Carbon disulfide & 5 & U \\
\hline M00823 & 23-Jun-97 & Carbon tetrachloride & 5 & $\mathrm{U}$ \\
\hline M01225 & 11-Nov-97 & Carbon tetrachloride & 5 & $\mathrm{U}$ \\
\hline M00823 & 23-Jun-97 & Chloroform & 5 & $\mathrm{U}$ \\
\hline M01225 & 11-Nov-97 & Chloroform & 5 & $\mathrm{U}$ \\
\hline M00823 & 23-Jun-97 & Chloromethane & 10 & $\mathrm{U}$ \\
\hline M01225 & 11-Nov-97 & Chloromethane & 10 & $\mathrm{U}$ \\
\hline M00823 & 23-Jun-97 & cis-1,2-Dichloroethene & 5 & $\mathrm{U}$ \\
\hline M01225 & 11-Nov-97 & cis-1,2-Dichloroethene & 5 & $\mathrm{U}$ \\
\hline M00823 & 23-Jun-97 & m,p-Xylene & 5 & $\mathrm{U}$ \\
\hline M01225 & 11-Nov-97 & m,p-Xylene & 5 & $\mathbf{U}$ \\
\hline M00823 & 23-Jun-97 & Tetrachloroethene & 5 & $\mathrm{U}$ \\
\hline M01225 & 11-Nov-97 & Tetrachloroethene & 5 & $\mathbf{U}$ \\
\hline M00823 & 23-Jun-97 & trans-1,2-Dichloroethene & 5 & $\mathrm{U}$ \\
\hline M01225 & 11-Nov-97 & trans-1,2-Dichloroethene & 5 & $\mathrm{U}$ \\
\hline M00823 & 23-Jun-97 & Trichloroethene & 5 & $\mathrm{U}$ \\
\hline M01225 & 11-Nov-97 & Trichloroethene & 5 & $\mathrm{U}$ \\
\hline
\end{tabular}

a "=" = detected at reported value; $\mathrm{U}$ - not detected; $\mathrm{J}=$ estimated value 
Table 3.9. Target compound monitoring results Well 846 - 1997

\begin{tabular}{|c|c|c|c|c|}
\hline Sample ID & Sample date & Target compound & Results (ug/L) & Data qualifier ${ }^{a}$ \\
\hline M00823 & 23-Jun-97 & 1,1-Dichloroethene & 5 & $\mathrm{U}$ \\
\hline M01225 & $11-$ Nov-97 & 1,1-Dichloroethene & 5 & $\mathrm{U}$ \\
\hline M00823 & 23-Jun-97 & 1,1-Dichloroethene & 5 & $\mathrm{U}$ \\
\hline M01225 & 11-Nov-97 & 1,1-Dichloroethene & 5 & $\mathrm{U}$ \\
\hline M00823 & 23-Jun-97 & 1,2-Dichloroethane & 5 & $\mathrm{U}$ \\
\hline M01225 & 11-Nov-97 & 1,2-Dichloroethane & 5 & $\mathrm{U}$ \\
\hline M00823 & 23-Jun-97 & Carbon disulfide & 5 & $\mathrm{U}$ \\
\hline M01225 & 11-Nov-97 & Carbon disulfide & 5 & $\mathrm{U}$ \\
\hline M00823 & 23-Jun-97 & Carbon tetrachloride & 5 & $\mathrm{U}$ \\
\hline M01225 & $11-$ Nov-97 & Carbon tetrachloride & 5 & $\mathrm{U}$ \\
\hline M00823 & 23-Jun-97 & Chloroform & 5 & $\mathrm{U}$ \\
\hline M01225 & 11-Nov-97 & Chloroform & 5 & $\mathrm{U}$ \\
\hline M00823 & 23-Jun-97 & Chloromethane & 10 & $\mathrm{U}$ \\
\hline M01225 & 11-Nov-97 & Chloromethane & 10 & $\mathrm{U}$ \\
\hline M00823 & 23-Jun-97 & cis-1,2-Dichloroethene & 5 & $U$ \\
\hline M01225 & 11-Nov-97 & cis-1,2-Dichloroethene & 5 & $\mathrm{U}$ \\
\hline $\mathrm{M} 00823$ & 23-Jun-97 & $\mathrm{m}, \mathrm{p}$-Xylene & 5 & $\mathrm{U}$ \\
\hline M01225 & 11-Nov-97 & m,p-Xylene & 5 & $\mathrm{U}$ \\
\hline M00823 & 23-Jun-97 & Tetrachloroethene & 5 & $\mathrm{U}$ \\
\hline M01225 & 11-Nov-97 & Tetrachloroethene & 5 & $\mathrm{U}$ \\
\hline M00823 & 23-Jun-97 & trans-1,2-Dichloroethene & 5 & $\mathrm{U}$ \\
\hline M01225 & 11-Nov-97 & trans-1,2-Dichloroethene & 5 & $\mathrm{U}$ \\
\hline M00823 & 23-Jun-97 & Trichloroethene & 5 & $\mathrm{U}$ \\
\hline M01225 & 11-Nov-97 & Trichloroethene & 5 & $\mathrm{U}$ \\
\hline
\end{tabular}

a "=" = detected at reported value; $\mathrm{U}$ - not detected; $\mathrm{J}=$ estimated value 
$3-11$

Table 3.10. Target compound monitoring results Well 857 - 1997

\begin{tabular}{|c|c|c|c|c|}
\hline Sample ID & Sample date & Target compound & Results $(\mu \mathbf{g} / \mathbf{L})$ & Data qualifier ${ }^{a}$ \\
\hline M00823 & 23-Jun-97 & 1,1-Dichloroethene & 5 & $U$ \\
\hline M01225 & 11-Nov-97 & 1,1-Dichloroethene & 5 & $\mathrm{U}$ \\
\hline M00823 & 23-Jun-97 & 1,1-Dichloroethene & 5 & $\mathrm{U}$ \\
\hline M01225 & $11-$ Nov-97 & 1,1-Dichloroethene & 5 & $\mathrm{U}$ \\
\hline M00823 & 23-Jun-97 & 1,2-Dichloroethane & 5 & $\mathrm{U}$ \\
\hline M01225 & 11-Nov-97 & 1,2-Dichloroethane & 5 & $\mathrm{U}$ \\
\hline M00823 & 23-Jun-97 & Carbon disulfide & 5 & $\mathrm{U}$ \\
\hline M01225 & 11-Nov-97 & Carbon disulfide & 5 & $\mathrm{U}$ \\
\hline M00823 & 23-Jun-97 & Carbon tetrachloride & 5 & $\mathrm{U}$ \\
\hline M01225 & $11-N o v-97$ & Carbon tetrachloride & 5 & U \\
\hline M00823 & 23-Jun-97 & Chloroform & 5 & $\mathbf{U}$ \\
\hline M01225 & 11-Nov-97 & Chloroform & 5 & $\mathrm{U}$ \\
\hline M00823 & 23-Jun-97 & Chloromethane & 10 & $\mathrm{U}$ \\
\hline M01225 & 11-Nov-97 & Chloromethane & 10 & $\mathbf{U}$ \\
\hline M00823 & 23-Jun-97 & cis-1,2-Dichloroethene & 5 & $\mathrm{U}$ \\
\hline M01225 & $11-$ Nov-97 & cis-1,2-Dichloroethene & 5 & $\mathrm{U}$ \\
\hline M00823 & 23-Jun-97 & m,p-Xylene & 5 & $\mathrm{U}$ \\
\hline M01225 & 11-Nov-97 & m,p-Xylene & 5 & $\mathrm{U}$ \\
\hline M00823 & 23-Jun-97 & Tetrachloroethene & 5 & $\mathrm{U}$ \\
\hline M01225 & 11-Nov-97 & Tetrachloroethene & 5 & $\mathrm{U}$ \\
\hline M00823 & 23-Jun-97 & trans-1,2-Dichloroethene & 5 & $\mathrm{U}$ \\
\hline M01225 & 11-Nov-97 & trans-1,2-Dichloroethene & 5 & $\mathrm{U}$ \\
\hline M00823 & 23-Jun-97 & Trichloroethene & 5 & $\mathrm{U}$ \\
\hline M01225 & 11-Nov-97 & Trichloroethene & 5 & $\mathrm{U}$ \\
\hline
\end{tabular}

a "=" = detected at reported value; $\mathrm{U}$ - not detected; $\mathrm{J}=$ estimated value 
Table 3.11. Target compound monitoring results Well 858 - 1997

\begin{tabular}{|c|c|c|c|c|}
\hline Sample ID & Sample date & Target compound & Results $(\mu \mathrm{g} / \mathrm{L})$ & Data qualifier \\
\hline M00823 & 23-Jun-97 & 1,1-Dichloroethene & 5 & $\mathrm{U}$ \\
\hline M01225 & 11-Nov-97 & 1,1-Dichloroethene & 5 & $\mathrm{U}$ \\
\hline M00823 & 23-Jun-97 & 1,1-Dichloroethene & 5 & $\mathrm{U}$ \\
\hline M01225' & 11-Nov-97 & 1,1-Dichloroethene & 5 & $\mathrm{U}$ \\
\hline M00823 & 23-Jun-97 & 1,2-Dichloroethane & 5 & $\mathrm{U}$ \\
\hline M01225 & 11-Nov-97 & 1,2-Dichloroethane & 5 & $\mathrm{U}$ \\
\hline M00823 & 23-Jun-97 & Carbon disulfide & 5 & $\mathrm{U}$ \\
\hline M01225 & 11-Nov-97 & Carbon disulfide & 5 & $\mathrm{U}$ \\
\hline M00823 & 23-Jun-97 & Carbon tetrachloride & 5 & $U$ \\
\hline M01225 & 11-Nov-97 & Carbon tetrachloride & 5 & $\mathrm{U}$ \\
\hline M00823 & 23-Jun-97 & Chloroform & 5 & $\mathrm{U}$ \\
\hline M01225 & 11-Nov-97 & Chloroform & 5 & $\mathrm{U}$ \\
\hline M00823 & 23-Jun-97 & Chloromethane & 10 & $\mathrm{U}$ \\
\hline M01225 & 11-Nov-97 & Chloromethane & 10 & $U$ \\
\hline M00823 & 23-Jun-97 & cis-1,2-Dichloroethene & 5 & U \\
\hline M01225 & 11-Nov-97 & cis-1,2-Dichloroethene & 5 & $\mathrm{U}$ \\
\hline M00823 & 23-Jun-97 & m,p-Xylene & 5 & $\mathrm{U}$ \\
\hline M01225 & 11-Nov-97 & m,p-Xylene & 5 & $\mathrm{U}$ \\
\hline M00823 & 23-Jun-97 & Tetrachloroethene & 5 & $\mathrm{U}$ \\
\hline M01225 & 11-Nov-97 & Tetrachloroethene & 5 & $\mathrm{U}$ \\
\hline M00823 & 23-Jun-97 & trans-1,2-Dichloroethene & 5 & $\mathrm{U}$ \\
\hline M01225 & 11-Nov-97 & trans-1,2-Dichloroethene & 5 & $\mathrm{U}$ \\
\hline M00823 & 23-Jun-97 & Trichloroethene & 5 & $\mathrm{U}$ \\
\hline M01225 & 11-Nov-97 & Trichloroethene & 5 & $\mathrm{U}$ \\
\hline
\end{tabular}

$a$ "=" = detected at reported value; $\mathrm{U}$ - not detected; $\mathrm{J}=$ estimated value 


\section{3-13}

Table 3.12. Target compound monitoring results Well $4315-1997$

\begin{tabular}{|c|c|c|c|c|}
\hline Sample ID & Sample date & Target compound & Results $(\mu \mathrm{g} / \mathrm{L})$ & Data qualifier $^{a}$ \\
\hline M00823 & 23-Jun-97 & 1,1-Dichloroethene & 5 & $\mathrm{U}$ \\
\hline M01225 & 11-Nov-97 & 1,1-Dichloroethene & 5 & $\mathrm{U}$ \\
\hline M00823 & 23-Jun-97 & 1,1-Dichloroethene & 5 & $\mathrm{U}$ \\
\hline M01225 & 11-Nov-97 & 1,1-Dichloroethene & 5 & $\mathrm{U}$ \\
\hline M00823 & 23-Jun-97 & 1,2-Dichloroethane & 5 & $\mathrm{U}$ \\
\hline M01225 & 11-Nov-97 & 1,2-Dichloroethane & 5 & $\mathrm{U}$ \\
\hline M00823 & 23-Jun-97 & Carbon disulfide & 5 & $\mathrm{U}$ \\
\hline M01225 & 11-Nov-97 & Carbon disulfide & 5 & $\mathrm{U}$ \\
\hline M00823 & 23-Jun-97 & Carbon tetrachloride & 5 & $\mathrm{U}$ \\
\hline M01225 & 11-Nov-97 & Carbon tetrachloride & 5 & $\mathrm{U}$ \\
\hline M00823 & 23-Jun-97 & Chloroform & 5 & $\mathrm{U}$ \\
\hline M01225 & 11-Nov-97 & Chloroform & 5 & $\mathrm{U}$ \\
\hline M00823 & 23-Jun-97 & Chloromethane & 10 & $\mathrm{U}$ \\
\hline M01225 & $11-$ Nov-97 & Chloromethane & 10 & $\mathrm{U}$ \\
\hline M00823 & 23-Jun-97 & cis-1,2-Dichloroethene & 5 & $\mathrm{U}$ \\
\hline M01225 & 11-Nov-97 & cis-1,2-Dichloroethene & 5 & $\mathrm{U}$ \\
\hline M1017 & 23-Jun-97 & Lead & 36.2 & $\mathbf{U}$ \\
\hline M1289 & 18-Nov-97 & Lead & 22.7 & $\mathrm{U}$ \\
\hline M00823 & 23-Jun-97 & m,p-Xylene & 5 & $\mathrm{U}$ \\
\hline M01225 & $11-$ Nov-97 & m,p-Xylene & 5 & $\mathrm{U}$ \\
\hline M00823 & 23-Jun-97 & Tetrachloroethene & 5 & $\mathrm{U}$ \\
\hline M01225 & 11-Nov-97 & Tetrachloroethene & 5 & $\mathrm{U}$ \\
\hline M00823 & 23-Jun-97 & trans-1,2-Dichloroethene & 5 & $\mathrm{U}$ \\
\hline M01225 & 11-Noy-97 & trans-1,2-Dichloroethene & 5 & $\mathrm{U}$ \\
\hline M00823 & 23-Jun-97 & Trichloroethene & 5 & $\mathrm{U}$ \\
\hline M01225 & 11-Nov-97 & Trichloroethene & 5 & $\mathrm{U}$ \\
\hline
\end{tabular}

$a$ " =" = detected at reported value; $\mathrm{U}$ - not detected; $\mathrm{J}=$ estimated value 


\section{$3-14$}

Table 3.13. Target compound monitoring results Well 4316 - 1997

\begin{tabular}{|c|c|c|c|c|}
\hline Sample ID & Sample date & Target compound & Results $(\mu \mathrm{g} / \mathrm{L})$ & Data qualifier $^{n}$ \\
\hline M00823 & 23-Jun-97 & 1,1-Dichloroethene & 5 & $\mathrm{U}$ \\
\hline M01225 & 11-Nov-97 & 1,1-Dichloroethene & 5 & $\mathrm{U}$ \\
\hline M00823 & 23-Jun-97 & 1,1-Dichloroethene & 5 & $\mathrm{U}$ \\
\hline M01225 & 11-Nov-97 & 1,1-Dichloroethene & 5 & $\mathrm{U}$ \\
\hline M00823 & 23-Jun-97 & 1,2-Dichloroethane & 5 & $\mathrm{U}$ \\
\hline M01225 & 11-Nov-97 & 1,2-Dichloroethane & 5 & $\mathrm{U}$ \\
\hline M00823 & 23-Jun-97 & Carbon disulfide & 5 & $\mathrm{U}$ \\
\hline M01225 & 11-Nov-97 & Carbon disulfide & 5 & $\mathrm{U}$ \\
\hline M00823 & 23-Jun-97 & Carbon tetrachloride & 5 & $\mathrm{U}$ \\
\hline M01225 & 11-Nov-97 & Carbon tetrachloride & 5 & $\mathrm{U}$ \\
\hline M00823 & 23-Jun-97 & Chloroform & 5 & U \\
\hline M01225 & 11-Nov-97 & Chloroform & 5 & $\mathrm{U}$ \\
\hline M00823 & 23-Jun-97 & Chloromethane & 10 & $\mathrm{U}$ \\
\hline M01225 & 11-Nov-97 & Chloromethane & 10 & $\mathrm{U}$ \\
\hline M00823 & 23-Jun-97 & cis-1,2-Dichloroethene & 5 & $\mathrm{U}$ \\
\hline M01225 & 11-Nov-97 & cis-1,2-Dichloroethene & 5 & $\mathrm{U}$ \\
\hline M00823 & 23-Jun-97 & $\mathrm{m}, \mathrm{p}$-Xylene & 5 & $\mathbf{U}$ \\
\hline M01225 & 11-Nov-97 & $\mathrm{m}, \mathrm{p}$-Xylene & 5 & $\mathrm{U}$ \\
\hline M00823 & 23-Jun-97 & Tetrachloroethene & 5 & $\mathrm{U}$ \\
\hline M01225 & 11-Nov-97 & Tetrachloroethene & 5 & $\mathrm{U}$ \\
\hline M00823 & 23-Jun-97 & trans-1,2-Dichloroethene & 5 & $\mathrm{U}$ \\
\hline M01225 & 11-Nov-97 & trans-1,2-Dichloroethene & 5 & $\mathrm{U}$ \\
\hline M00823 & 23-Jun-97 & Trichloroethene & 5 & $\mathrm{U}$ \\
\hline M01225 & 11-Nov-97 & Trichloroethene & 5 & $\mathrm{U}$ \\
\hline
\end{tabular}

$a$ " =" = detected at reported value; $\mathrm{U}$ - not detected; $\mathrm{J}=$ estimated value 


\section{3-15}

Table 3.14. Target compound monitoring results Well $4317-1997$

\begin{tabular}{|c|c|c|c|c|}
\hline Sample ID & Sample date & Target compound & Results $(\mu \mathrm{g} / \mathrm{L})$ & Data qualifier" \\
\hline M00823 & 23-Jun-97 & 1,1-Dichloroethene & 5 & $\mathrm{U}$ \\
\hline M01225 & 11-Nov-97 & 1,1-Dichloroethene & 5 & $\mathrm{U}$ \\
\hline M00823 & 23-Jun-97 & 1,1-Dichloroethene & 5 & $\mathrm{U}$ \\
\hline M01225 & 11-Nov-97 & 1,1-Dichloroethene & 5 & $\mathrm{U}$ \\
\hline M00823 & 23-Jun-97 & 1,2-Dichloroethane & 5 & $\mathrm{U}$ \\
\hline M01225 & 11-Nov-97 & 1,2-Dichloroethane & 5 & $\mathrm{U}$ \\
\hline M00823 & 23-Jun-97 & Carbon disulfide & 5 & $\mathrm{U}$ \\
\hline M01225 & 11-Nov-97 & Carbon disulfide & 5 & $\mathrm{U}$ \\
\hline M00823 & 23-Jun-97 & Carbon tetrachloride & 5 & $\mathrm{U}$ \\
\hline M01225 & 11-Nov-97 & Carbon tetrachloride & 5 & $\mathrm{U}$ \\
\hline M00823 & 23-Jun-97 & Chloroform & 5 & $\mathrm{U}$ \\
\hline M01225 & 11-Nov-97 & Chloroform & 5 & $\mathrm{U}$ \\
\hline $\mathrm{M} 00823$ & 23-Jun-97 & Chloromethane & 10 & $\mathrm{U}$ \\
\hline M01225 & 11-Nov-97 & Chloromethane & 10 & $\mathrm{U}$ \\
\hline M00823 & 23-Jun-97 & cis-1,2-Dichloroethene & 5 & $\mathrm{U}$ \\
\hline M01225 & 11-Nov-97 & cis-1,2-Dichloroethene & 5 & $\mathrm{U}$ \\
\hline M00823 & 23-Jun-97 & m,p-Xylene & 5 & $\mathbf{U}$ \\
\hline M01225 & $11-$ Nov-97 & m,p-Xylene & 5 & $\mathrm{U}$ \\
\hline M00823 & 23-Jun-97 & Tetrachloroethene & 5 & $U$ \\
\hline M01225 & $11-$ Nov-97 & Tetrachloroethene & 5 & $\mathrm{U}$ \\
\hline M00823 & 23-Jun-97 & trans-1,2-Dichloroethene & 5 & $\mathrm{U}$ \\
\hline $\mathrm{M} 01225$ & $11-$ Nov-97 & trans-1,2-Dichloroethene & 5 & $\mathrm{U}$ \\
\hline M00823 & 23-Jun-97 & Trichloroethene & 5 & $U$ \\
\hline M01225 & 11-Nov-97 & Trichloroethene & 5 & $\mathrm{U}$ \\
\hline
\end{tabular}

a "=" = detected at reported value; $\mathrm{U}$ - not detected; $\mathrm{J}=$ estimated value 
Table 3.15. Comparison of VOC detections to action levels, June 1997 (underline represents detections above the regulatory level)

\begin{tabular}{|c|c|c|c|c|c|}
\hline \multirow[b]{2}{*}{ Analyte } & \multirow{2}{*}{$\begin{array}{l}\text { Regulatory } \\
\text { level }(\mu \mathrm{g} / \mathrm{L})\end{array}$} & \multicolumn{4}{|c|}{ Detections $(\mu \mathrm{g} / \mathrm{L}) /$ Well number } \\
\hline & & 841 & 842 & 843 & 4315 \\
\hline 1,1-dichloroethane & - & & & & \\
\hline 1,2-dichloroethane & 7 & & $\underline{8}$ & & \\
\hline 1,2-dichloroethene & 5 & & 1 & $\underline{13}$ & \\
\hline carbon disulfide & - & & & & \\
\hline carbon tetrachloride & 5 & & $\underline{28}$ & & \\
\hline chloroform & 100 & & 30 & & \\
\hline chloromethane & - & & & & \\
\hline tetrachloroethene & 5 & & & & \\
\hline trichloroethene & 5 & $\underline{8}$ & 110 & & \\
\hline total xylenes & 10,000 & & & & \\
\hline lead & 15 & & & & $36(\mathrm{U})$ \\
\hline
\end{tabular}




\section{3-17}

Table 3.16. Comparison of VOC detections to action levels, November 1997 (underline represents hits above the regulatory level)

\begin{tabular}{|c|c|c|c|c|c|}
\hline \multirow[b]{2}{*}{ Analyte } & \multirow{2}{*}{$\begin{array}{l}\text { Regulatory } \\
\text { level }(\mu \mathrm{g} / \mathrm{L})\end{array}$} & \multicolumn{4}{|c|}{ Detections $(\mu \mathrm{g} / \mathrm{L}) /$ Well number } \\
\hline & & 841 & 842 & 843 & 4315 \\
\hline 1,1-dichloroethane & - & & $2(J)$ & & \\
\hline 1,2-dichloroethane & 7 & & $\underline{10}$ & & \\
\hline 1,2-dichloroethene & 5 & & $\underline{10}$ & $\underline{6}$ & \\
\hline carbon disulfide & - & & & & \\
\hline carbon tetrachloride & 5 & & 29 & & \\
\hline chloroform & 100 & & 29 & & \\
\hline chloromethane & - & & & & \\
\hline tetrachloroethene & 5 & & & & \\
\hline trichloroethene & 5 & 7 & 150 & & \\
\hline total xylenes & 10,000 & & & & \\
\hline lead & 15 & & & & $\underline{22(U)}$ \\
\hline
\end{tabular}


than the respective regulatory limits. Only three of the wells had VOC detections above background, with the exception of several low level $(2$ to $8 \mu \mathrm{g} / \mathrm{L})$ detections of methylene chloride. Almost all of the methylene chloride detections were qualified by the laboratory with a " $\mathrm{B}$ " qualifier, indicating the methylene chloride was also detected in the laboratory blanks samples. Methylene chloride is a common laboratory contaminant.

Although the HTF is not formally part of the SWSA 6 RCRA monitoring requirements, 1997 results for the HTF are provided in Appendix D. These results indicate that lead is not leaching from the casks at the facility.

\subsection{NATURE AND EXTENT OF CONTAMINATION}

\subsubsection{Lateral Extent of Contamination}

The 1997 data indicate that the greatest RCRA issue is still associated with releases along the eastern boundary of the site, downgradient of Solvent Auger Hole area on the adjacent hilltop. These releases are measured in Wells 841 and 842, as well as a few detections in Well 843 located in the opposite downgradient direction of the auger holes. In 1990, the WAG 6 RFI installed four wells in the area of 842 to determine the extent of the VOC plume in the area (BNI 1991). Wells were located along strike (Well 1244), downgradient (Well 1245), and to the north (Well 1242) and south (Well 1243). All of these wells were placed within $150 \mathrm{ft}$ of each other. VOCs were not detected in the wells (BNI 1991), indicating that groundwater contaminant migration occurs along discreet fractures at SWSA 6 and cannot really be defined as plumes.

Figures 3.1 and 3.2 present a summary of the nature of the VOC concentrations in Wells 841 and 842 over time. Historical concentrations of trichloroethylene in Well 842 have been as high as 550 $\mu \mathrm{g} / \mathrm{L}$ in 1988 . However, they have continually decreased over time.

\subsubsection{Vertical Extent of Contamination}

Much has been reported about the groundwater flow patterns in the Melton Valley. In general, hydraulic conductivity and fracture density decrease over depth in the Nolichucky Shale and Maryville Limestone. It is estimated that $>90 \%$ of infiltrated rainwater flows along the shallow water table and emerges in nearby surface waters (DOE 1995).

Wells 842 and 841 are a shallow/intermediate well pair and can be used to assess the vertical extent of VOC contamination. Well 842 is screened from 8 to $23.2 \mathrm{ft}$ below ground surface (bgs). Well 841 is screened from 34 to $56.3 \mathrm{ft}$ bgs. Water levels in Well 842 were as high as $10 \mathrm{ft}$ bgs for both sampling events, indicating that the shallow water table was high for both the wet and dry season sampling event. VOC concentrations in Well 842 are significantly higher than in $841,150 \mu \mathrm{g} / \mathrm{L}$ in 842 as opposed to $8 \mu \mathrm{g} / \mathrm{L}$ in 841 . TCE is the highest concentration VOC in Well 842 and the only detected VOC in Well 841. Therefore, although there is an indication that TCE is migrating vertically, it is either diluted in the intermediate groundwater zone, or a majority of it migrates laterally in the shallow zone. In 1994-1995, as part of the EMP baseline monitoring, seeps along the West Seep Tributary, downgradient of wells 841/842, were monitored and analyzed for VOCs. No target compounds were detected in the seeps. 


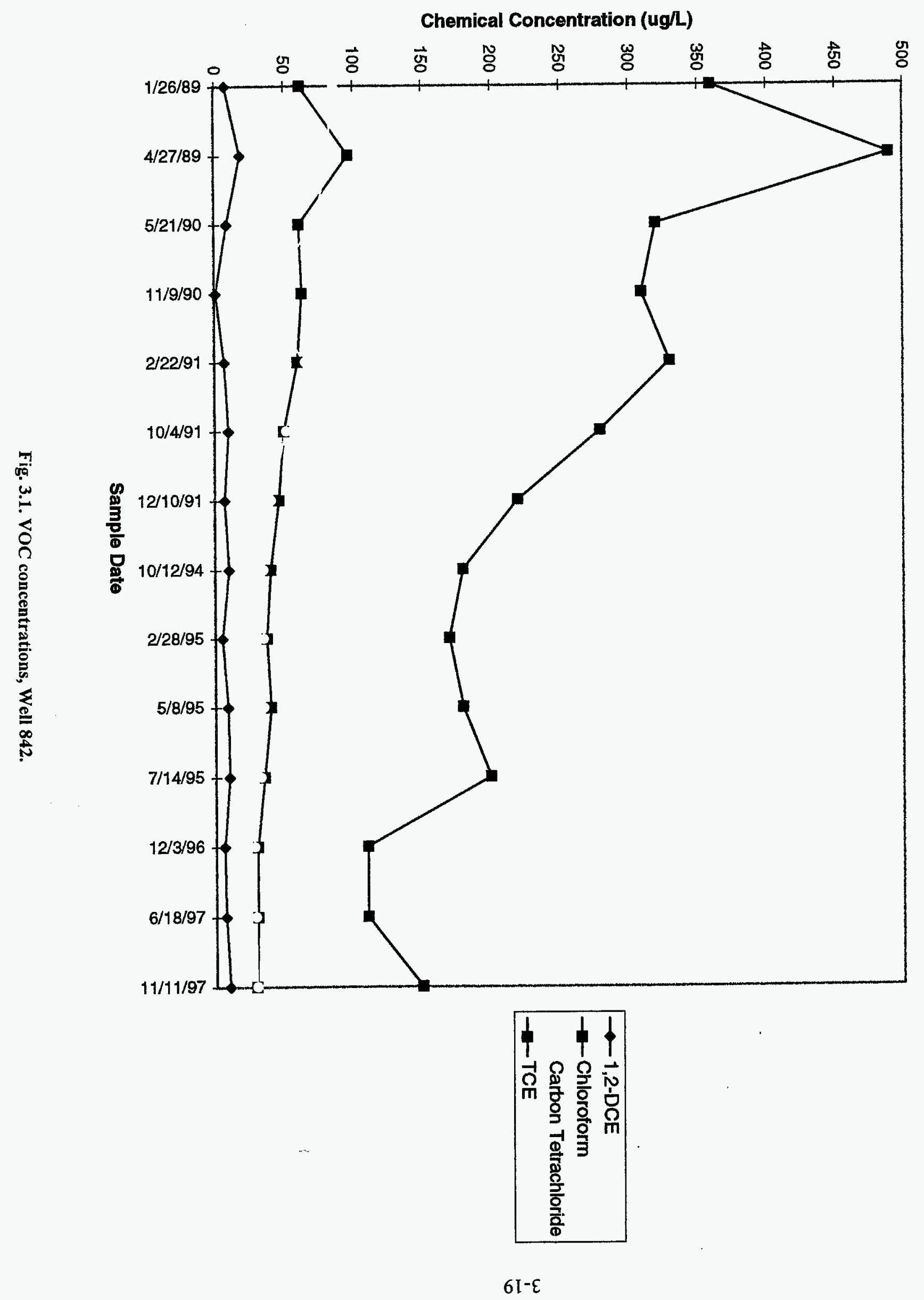




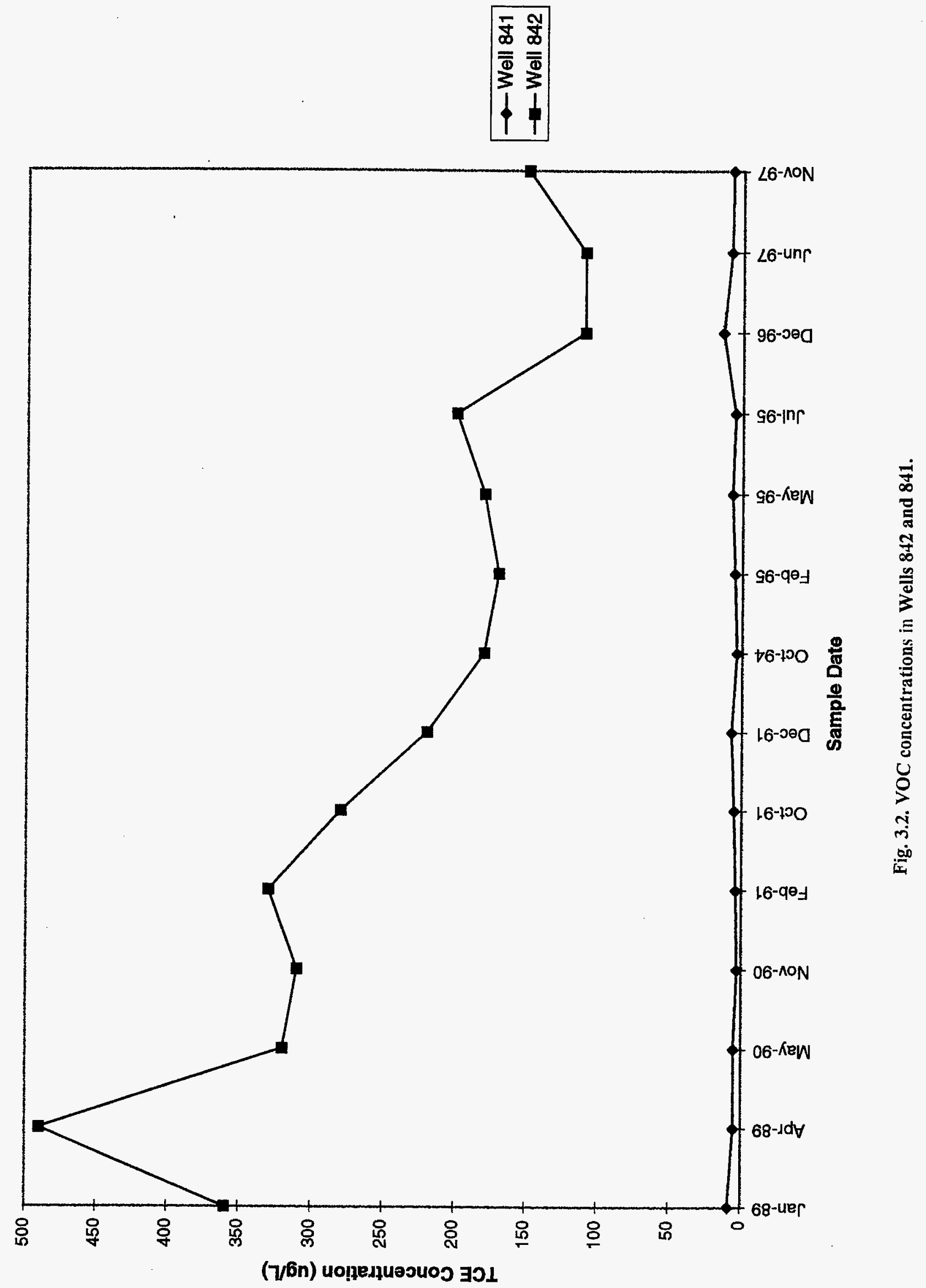




\subsection{CONTAMINANT MIGRATION}

No new studies were performed in 1997 to better define and quantify contaminant migration characteristics at SWSA 6. Several past studies have been performed, including the RFI, the 1994 and 1996 WAG 6 Annual Reports (DOE 1995; DOE 1995), and several valley groundwater modeling efforts. Information from these past studies, and from the 1996 GWQAR, is provided to help define contaminant migration pathways and rates for SWSA 6.

Figure 3.3 shows the shallow water table for WAG 6 as reported in the 1996 WAG 6 Annual Report. The water table and water flow direction does not change from the wet to the dry season (DOE 1995). Most of the water that infiltrates the ground surface exits the WAG at two surface water monitoring stations, MS1 and MS3. Storm flow is the primary mechanism of flow in the unsaturated zone to these discharge points. Flow velocities can vary greatly in storm vs nonstorm conditions. Much of the water flux leaving the WAG occurs during a few heavy storm events per year.

In general, the majority of groundwater flow at SWSA 6 occurs through fractures, particularly in the bedrock. A small amount of matrix diffusion into the rock can occur in cases where contaminant concentration gradients exit between water in fractures and water in the rock matrix. Diffusion out of the rock matrix is slow, primarily because of the mechanisms of sorption, degradation, and chemical precipitation.

Although much of the flow occurs through fractures, average velocities can be estimated using the standard equation for estimating groundwater flow velocity in porous media:

$$
V=\frac{(K)(i)}{n}
$$

where

$\mathrm{v}=$ the average linear seepage velocity,

$\mathrm{K}=$ the hydraulic conductivity,

$\mathrm{i}=$ the hydraulic gradient,

$\mathrm{n}=$ the effective porosity of the aquifer media.

Values for each of the parameters as well as flow velocities were provided in the 1996 GWQAR. Velocities for the regolith range from 0.55 to $1.37 \mathrm{ft} / \mathrm{d}$ and for the bedrock 13.32 to $64 \mathrm{ft} / \mathrm{d}$ for the bedrock (ORNL 1997). 


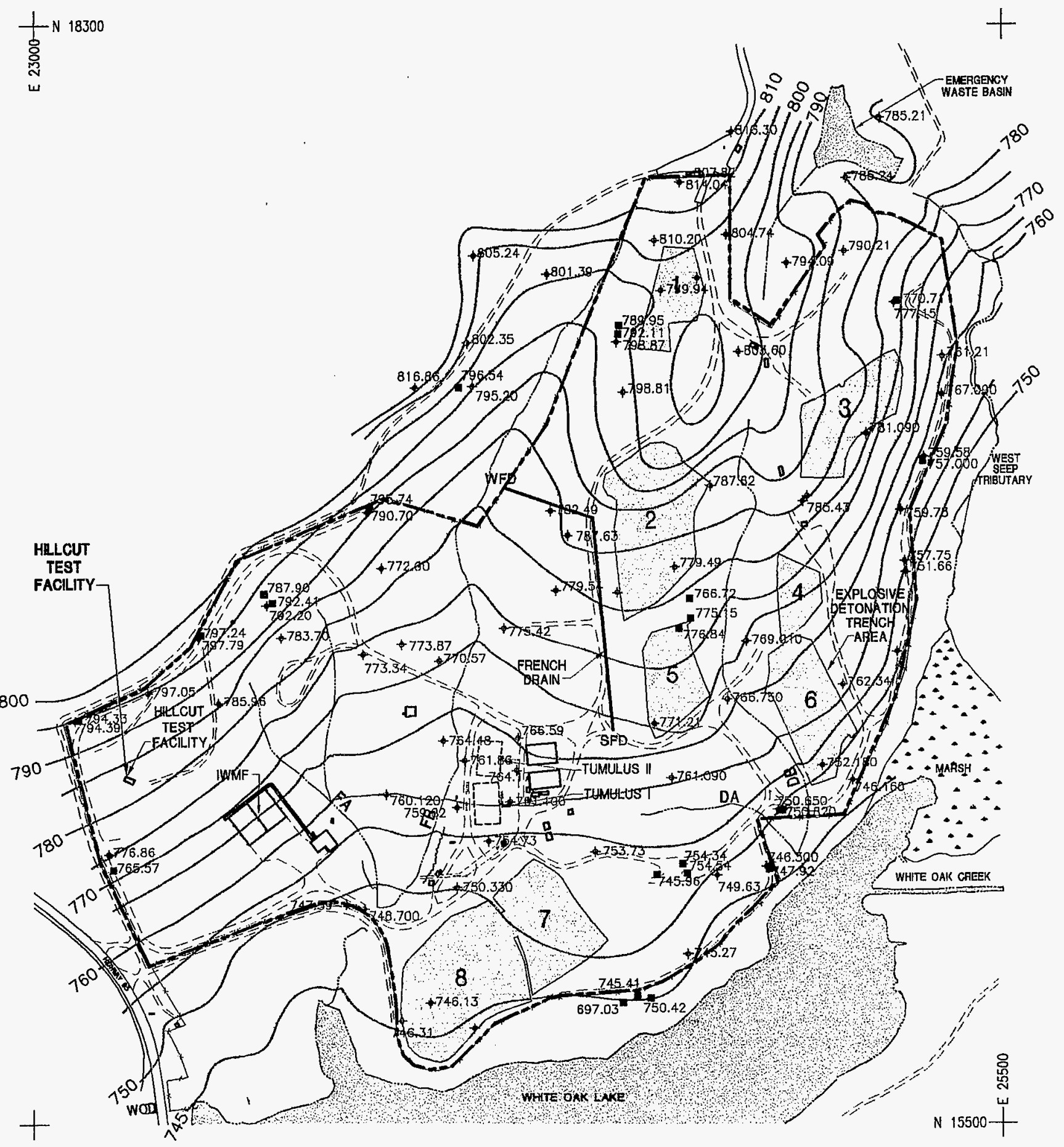

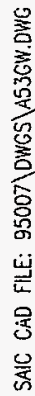

LEGEND:

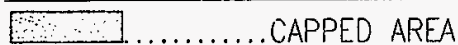

_....WAG 6 BOUNDARY

OCT. 1994

\$ 760.00........WATER LEVEL
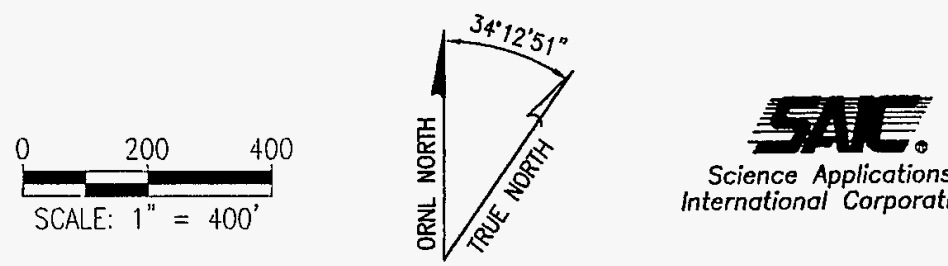

Science Applications

Internotional Corporation

DEEP WELL- not used
- 760.00.........in contouring)

WATER TABLE

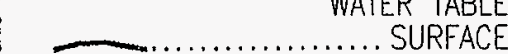

Fig. 3.3. SWSA 6 water levels. 


\section{RECOMMENDATIONS}

The following recommendations are made for 1998 monitoring of SWSA 6:

- Change the well purging technique from a three-volume purge to a micropurge technique. This will bring the SWSA 6 RCRA protocol in line with RCRA sampling at the Y-12 Plant ${ }^{1}$ and with the purge technique being used for all of the CERCLA groundwater sampling at ORNL.

- Because of continued lack of lead detections in the HTF pad drains, it is recommended that the frequency of sampling the HTF be reduced to two samples per year. No formal agreement was made on the frequency of sampling the HTF. This recommendation will be incorporated into the IWQP monitoring requirements.

- Change the semiannual monitoring from June and November/December, to February/March and August. This change will facilitate preparation of the GWQAR since the November/December monitoring results often cause delays in preparing the report. It is also believed that these dates will provide a more distinct wet season vs dry season evaluation of the groundwater conditions.

${ }^{1}$ Modifications to RCRA Post-Closure Permits for the Bear Creek Hydrogeological Regime (TN HW-087), Chestnut Ridge Hydrogeological Regime (TN HW-0088), and Upper East Fork Poplar Creek Hydrogeological Regime (TN HW-089) issued on $7 / 28 / 98,7 / 22 / 97$, and 6/25/97, respectively. 


\section{REFERENCES}

BNI. 1991. RCRA Facility Investigation Report for Waste Area Grouping 6 at Oak Ridge National Laboratory, Oak Ridge, Tennessee, IRA \#910930.a015, ES/ER-22/V1\&D2, ORNL/ER/SUB87/99053/5/VI.

BNI. 1988. Closure Plan for Solid Waste Storage Area 6 ORNL/RAP Sub-87/99053/9\&V2, Oak Ridge, TN.

Burroughs, C.E. 1992. Personal communication to ORNL Environmental Surveillance and Protection Section staff during the RCRA Compliance Evaluation Investigation, April 27-29, 1992

DOE. 1995. 1994 Annual Report on Activities at Waste Area Grouping 6 From February Through September at Oak Ridge National Laboratory, Oak Ridge,Tennessee, DOE/OR/01-1377 \& D1.

Mortimore and Ebers. 1988. Groundwater Quality Monitoring Well Installation for WAG 6, ORNL/RAP-44, Oak Ridge National Laboratory, Oak Ridge, TN

ORNL. 1997. Groundwater Quality Assessment Report for the Solid Waste Storage Area 6 at the Oak Ridge National Laboratory.

ORNL. 1993. Groundwater Quality Assessment Report for the Solid Waste Storage Area 6 at the Oak Ridge National Laboratory. 1992.

ORNL. 1991. Groundwater Quality Assessment Report for solid Waste Storage Area at the Oak Ridge National Laboratory, 1990.

ORNL. 1990. Hazardous Waste Management Annual Report, 1989. 
APPENDIX A

MICROPURGE PROCEDURE 
A-3

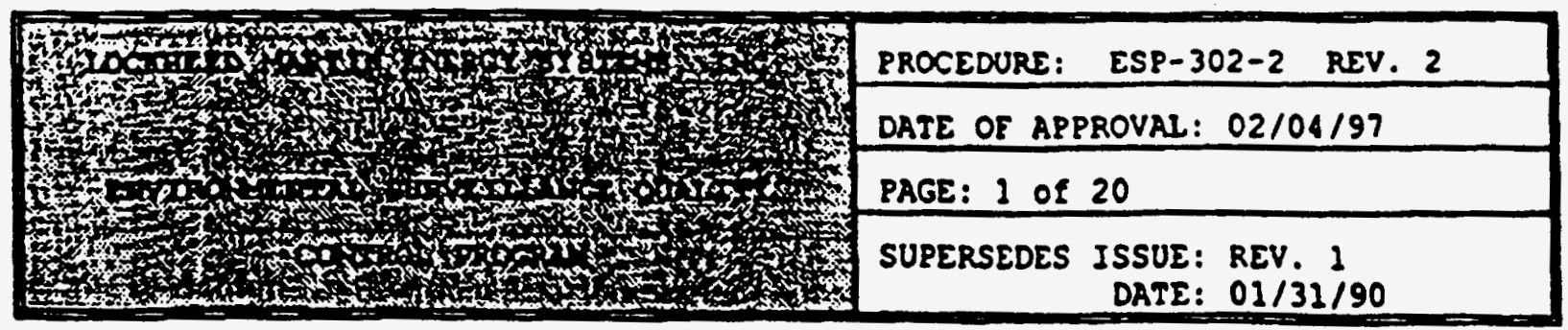

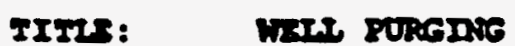

12 20RPoser.

This procedure provides protocols for the purging of woter from a well or borebole prior to sampling to ensure that the sample is representative of the water quality of the subsurface geological formation. Two approaches to purging, the conventional, three-five volume removal method and the low-flow/volume method, and applicable equipnent are described.

$2 \operatorname{coses}$

This procodure is applicable to Lockheed Martin Energy Systens, Ine. (Energy Systans) and subcontractors who are conducting enviromental field activities.

3.2 A Compendium of Superfund Fieid Operations Methods, ERA 540/P87/001, O.S. Environental Protection Agency (ERA), Washington, DC. 1987.

3.2 Environmental Sorvicos Division. Environmental Investigations standard gerating Procedures and Duality assurance Manual, O.S. EPA, Region 4, May 2996. (EISOPQAY)

3.3 Ruls, R.W., and Barcelona, M.:., Low-Flow (Mininal Drawdown) Groundwater Sampling Procecture, U.S. EPA Groundwater Issue, ERA/540/5-95/504, 1995. 


\begin{tabular}{|c|c|c|}
\hline 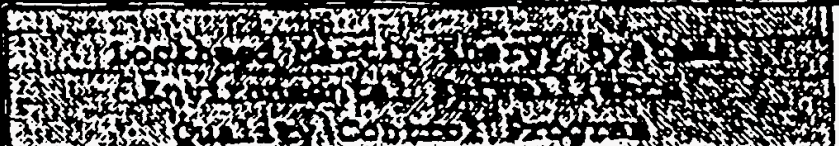 & $\begin{array}{l}\text { Procedure Number: } \\
\text { Revision Number: } \\
\text { Page: } 2 \text { of } 20\end{array}$ & $\begin{array}{l}\text { ESP-302-2 } \\
2\end{array}$ \\
\hline
\end{tabular}

3.4 RCRA Groundwater Monitoring: Draft Technical Guidance, EPA/530-R-93-001, U.S. Environmental Protection Agency, 1992.

3.5 Standard Gulde for Sampling Groundwater Monitoring Wells, ASTM

D 4448-85a, American Society for Testing and Mater1als, 1985.

3.6 Specifications and ouldellnes for Quallty Systems for

Environmental Data Collection and Environmental Technology Prograns, MNSI/ASDC E4-1994.

3.7 Procedures Manuel for Ground Water Monltoring at Solid Waste Disposal Facilit1es, EPA 530/SW-611, O.S. Environmental Protection Agency. August 1977.

\section{Rrspoystathitras}

\subsection{Project/Program Manager/Destonee}

1.1.1 Includes the reguirements of this procedure in the project/program sampling and analysis plan (SAP).

4.1.2 Directs the field tean leader in implementation of the requirements found in this procedure.

4.1.3 Ensures that applicable training and safety requirements are verified and documented, as required for this procecture.

4.1.4 Verifies that the requirements for Quality Assurance/quality Control (OA/OC) of this procedure are properly sat1sfled.

4.1.5 Authorizes devlations from this procecture.

1.2 Eield Term/eader/Superpisor

4.2.1 Directs the activities of the field team for the correct application of this procedure.

4.2.2 Verifies that field events of the specific project/program meet the applicable requirements presented in this procedure. 
4.2.3 Records deviations from requirements of this procedure.

4.2.4 Assures field activities are belng performed in a safe manzer.

\subsection{Fleld Team Members}

4.3.1 Obtain training on, and have knowledge of, the requirements of this procedure prior to start of the applicable activities.

4.3.2 Follow the requirements of this procedure.

1.3.3 Maintain appropriate field documentation li.e.. logbooks, data charts).

4.3.4 Document deviations during performance of this procedure.

4.3.5 Observe health and safety reguirements and project docunentation guidelines when porforming activities.

5.1 Baller - Weighted bottle, hollow cyllndrical tube lor sone modification thereof) that is lowered and raised generally by hand to collect water samples. Ballers should be constructed of materials appropriate for the intended analyais of the samples (generally Toflon or stainless steel).

5.2 Culibration - Comparison and standardization of measurement devices against reference standards of known and documented intensity, concentration, length, etc., to detect and guantify inaccuracies.

5.3 DNAPL - Dense non-agueous phase liquid.

5.4 LNAPI - Light non-aqueous phase 11quid. 
5.5 Buralig - Removal of water from a well until the remaining groundwater is determined to be representative of the subsurface geologic formation based on a set of criteria le.g. three well volunes removed and/or indicator parametor stabllization).

5.6 Specific Conductance - Measure of a material's ability to conduct electric current, the reciprocal of resistivity.

5.7 Turbidity - Turbidity is a measure of the clarity of water. It is caused by suspended matter, such as clay, silt, finely divided organic and inorganic matter, and soluble colored organic compounds. Turbidity is measured in nophelomotric turbidity units (NTU).

6.1 A well is purged before taking samples in order to clear the well or monitored interval of water that is not representative of the formation groundwater.

6.2 Using the conventional mothod, three to five timas the volume of standing water in the woll is removed fron the well during purging and indicator parameters are periodically neasured.

6.3 Using the low-flow/volume method, a minimal volume of water is purged from the monitored interval of the well at a rate equal to the well's recharge rate. Indicator parabeters are periodically measured during the purge evont. 
6.4 Indicator parameters such as, specific conductance, temperature, pH, dissolved oxygen, oxidation/reduction potential, and turbldity may be measured in the purged water. When their ralues have stabilized within definod ranges over consecutive messuroments, it may be assumed that the water now coring fron the well is representative of the subsurface formation of interest.

7.1 This procedure becomes effective 30 days after approval.

7.2 The volume of water to be purged and the equipment to be used depends upon aquifer properties, specifics of well construction, and project data quality objectives.

7.3 The method used to purge each well and the criteria for determining when to stop purging, if different frow the guidelines presented in this procedure, will be included in the project SAP.

7.1 Any specific neods beyond the scope of this procedure must be covered in project/progran documentation (e.g., SAP).

30rz: The use of ballers to purge monitoring wells should generally be avoided as the "plunger" effect created by continually raising and lowering the bailer into the well can result in continual development or overdovelopment of the well. The use of bailers for purging and sampling is discouraged because the correct technique is highly operator dependent. 
8.1 Pump or bailer, as required by the SAP to meet project/program needs (See ESP-302-4 and 302-3, latest rev1sions).

WOT: A variety of pump types may bo used for groundwater purging and sampling. Gonorally, pumps should be able operate at a consistent flow rate and not cause undue pressure or temperature changes, or physical disturbance to the sample. Gas-driven punps should be of a type that does not allow the gas to be in direct contact with the water. Pumps for lowflow/volune purging must be easily adjustable and operate reliably at flow rates < $0.5 \mathrm{~L} /$ ain. Bailers are inappropriate for low-flow/rolume purging.

B.2 Water level measurement equipment (See ESP-302-1, latest revision).

8.3 Specific conductance, PR, temperature (See ESP-307-1, latest revision), and turbidity meters, as reguired by SAP.

8.4 Oxidation/reduction potentiel and/or dissolved oxygen meters. as required by SAP (See ESP-307-5 and 307-3, respectively).

8.5 See Appendix A for additional suggested naterials and equipment. 
9 HATBDE

9.1 It is Energy Systens policy to maintain an effective program for control of employee exposure to chemical, radiological, and physical stress that is consistent with Lockheed Martin Corporation, Dos, and the Occupational Safety and Health Administration (OSAA) standards and requirements.

9.2 noloyee contarination and exposure.

9.3 The plastle sheeting used as a ground cloth may pose a sipping hazard.

9.4 Refer to a site-specific health and safety plan for detailed health and safety procedures.

10 Brocapors

10.1 Review the project/progran SAP for the well location, well construction le.g., diameter of well, depth of well, monitoring intervals, required analyses, and management of purge waste watex.

BOz: Review project/progran SAP to determine if INAPLS or DNAPIs nay have boen previously detected in the well. Adjust water/MAPL level measurement (See ESP-302-1, latest revision) and purging methods (see Appendix B) accordingly. If LNAPL is present and to be collected, the well will not be purged before sampling.

10.2 Identify the method, conventional (three-to-five volume removal) or low-flow/volume, to be used to purge the well. 
Norr: Regulatory agreement on the purge method and volume should be obtained before field work begins. The minimum volume of water to be purged and the "stop purge" criteria should be specifled in the project/program SAP.

10.3 Determine the apparatus to be used to purge the well. For lowflow sampling, the pump must have an adjustable flow rate in the range of $0.1-0.5 \mathrm{w} / \mathrm{min}$.

10.4 Decontaninate purging equipment, as needed, according to the manufacturer's instructions and/Or ESP-801 and ESP-802, latest revisions.

10.5 Identify indicator paraneters to be measured.

10.6 Verify that required accuracy checks 11.e., calibration, standardization, or certification) have been completed in accordance with nanufacture's instrument calibration and maintenance manual.

10.7 Locate well and record well number. slte, date, and well condition in logbook or date form (See ESP-503, latest. revision).

10.8 Don personal protection equipnent.

10.9 Place plastic sheeting on ground around the base of the well, as required, unlock, and opes well.

10.10 Check the well with an organic vapor analyzer, photoionization detector or flame ionization dotector (S↔ ESP-307-6, latest revision), and/or radiation survey meter (see ESP-307-7, latest revision), as directed by health and safety plan. 
10.11 Determine depth to water, depth of well, and calculate volume of water in the well.

10.11.1 Measure the depth to water according to ESP-302-1, latest revision.

1roxt: Dopth of woll and water volune calculation are not routinely needed for low-flow/volune purge method.

10.11.2 Obtain known depth of well value from SAP or, if unknown, measure the depth to the bottom of well according to ESP302-1, latest revision.

10.11.3 If siltation is known to be a problem in the weil, or if purging indicates that siltation is a problen, then measure the dapth to the botton of well according to ESP302-1, latest revision.

10.11.4 Calculate the height of the water column (subtract depth to water from the well depth) and the volume of water in the well (see Appendix B).

10.12 Record all information in field logbook or on field data forms. 10.13 Purge well.

rork: If a packer assenbly has been installed in the monitoring well. inflate with the use of the air compressor before the start of purging. Refor to the manufacturer's instructions for information regarding adequate packer inflation, detecting packer leakage, and maximur packer inflation pressure. 
10.13 .1 Conventional Purging -- Removal of three to five well volumes using a mechanlcal pump.

NOYs: Follow pump oampling procodure ESP-302-4, latest revision, for operation of equipment to purge well.

10.13.1.1 Place inlet line of the pump just below the surface of the well water. Begin purping.

10.13.1.2 Take initial measurements of selected indlcator parameters (e.g.. pH, specific conductance, temperature, turbidity, oxidation/reduction potential, and dissolved oxygen) to baseline parameter values.

10.13.1.3 Continue purging well and neasuring indicator parameters every one-half to one well volime. Record volumes of water purged and parameter values.

10.13.1.4 Lower punp or Intake 11 ne to remain below water surface as water level in well decreases. Always chase the water level during drawdown. Never draw down a well from the bottom.

305x: Aroid purging a well to dryness if the recharge causes the formation water to cascade down the screen.

10.13.1.5 Stop purging whon either (1) the well is ory or (2) the indicator parameters have stabilized over consecutive recording intervals and at least three well volumes have been purged. 


\begin{tabular}{|c|c|}
\hline 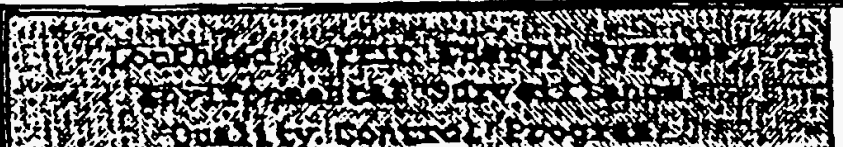 & $\begin{array}{l}\text { Procedure Number: ESP-302-2 } \\
\text { Revision Number: } 2 \\
\text { Page: } 11 \text { of } 20\end{array}$ \\
\hline
\end{tabular}

Nore: The recommended number of consecutive readings to determine stabllity is three. The recommended ranges for indlcator parameters to be considered stabilized are: when $\mathrm{PH}$ measurements agree within \pm 0.1 standard units, temperature measurements agree within $\pm 2^{\circ} \mathrm{C}$, specific conductance measurements are within \pm 10 (i.e., 100 units for readings over 1000 umhos $/ \mathrm{cm}$ ), oxidation/reduction potential measurements are within $\pm 10 \mathrm{mV}$, dissolved oxygen measurements are within \pm 108 . and turbidity has elther stabillzed (e.g., 5 NTOS \pm 2 ) or is below 10 NrOs.

10.13.1.6 If indicator parameter values do not stabilize after five well volumes for the number specified in the profect/program SAP), obtain additional guidance from project/program manager or designee.

10.13.2 Conventional Purging -- Renoval of three to five well volumes using a bailex.

10.13.2.1 Follow bailer sampling procedure ESP-302-3, latest revision, for operation and handing of bailer to purge well.

10.13.2.2 Take initial measurements of selected indicator parameters (e.g., PH, specific conductance, temperature, turbidity, oxidation/reduction potential, and dissolved oxygen) to baseline parameter values.

10.13.2.3 Continue purging well and measure indicator paraneters every one-half to one well volume. Record volumes of water purged and parameter values. 


\begin{tabular}{|c|c|}
\hline 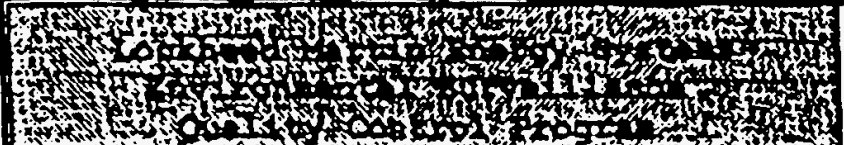 & $\begin{array}{l}\text { Procedure Number: ESP-302-2 } \\
\text { Revision Number: } 2 \\
\text { Page: } 12 \text { of } 20\end{array}$ \\
\hline
\end{tabular}

NOTr: Avoid purging a well to dryness if the recharge causes the formation water to cascade down the screen.

10.13.2.4 Stop purging when either (1) the well is dry or (2) the Indicator parameters have stabilized over consecutive recording intervals and at least three well volumes have been purged.

Norx: The recomended number of consecutive readings to determine stability is three. The recomended ranges for indicator parameters to be considered stabilized are: when pll measurements agree within \pm 0.1 standard units, temperature measurenents agree within $\pm 1{ }^{2} \mathrm{C}$, specific conductance neasurements are within \pm 10 (1.e., 100 units for readings over 1000 whos/cal, oxidation/reduction potential measuresents are within $\pm 10 \mathrm{nv}$, dissolved oxygen measurenents are within \pm 10 , and turbidity has either stablized (e.g.. 5 NTUs \pm 2$)$ or 18 below 10 MTOs.

10.13.2.5 If indicator parameter values do not stabilize after five well volumes lor the number specified in the project/program SAP), obtain additional guidance from project/program manager or designee.

10.13 .3 Low-Flow/Volune Purging 


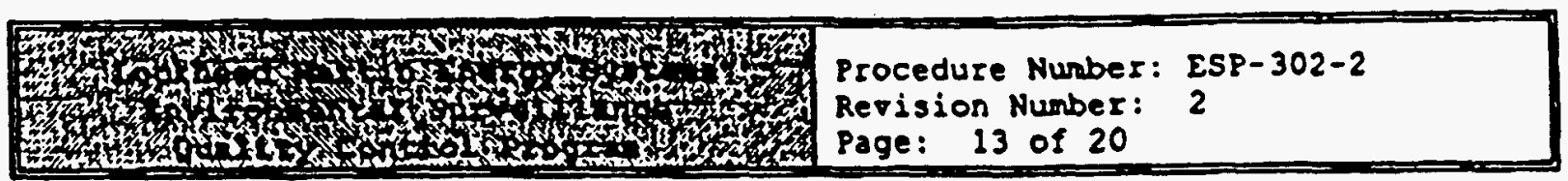

Norr: Follow the manufactures instructions or pump sampling procedure ESP-302-4, latest revision, for operation of equipment to purge weld.

Morz: The rate at which groundwater is rexoved from the woll should bo at or below the well's recharge rate so that water in the well casing above the monitored interval does not mix with the water in the monitored interval. There should be a minimal drawdown of the well's water level.

10.13.3.1 Place inlet line of the sampling pump adjacent to water ylelding interval, if known, or just above the middle of monitored interval of the well. Mininize disturbance of the water.

1025: If the pump is being installed/inserted in the well, allow the well to stabilize for an appropriate tine period (e.g., 12 - 24 nrs is recomended) before beginning to purge.

10.13.3.2 Place dopth to water level indicator back into the well and leave for the duration of the purging and sampling event.

10.13.3.3 Begin purging the well at the pump's lowest setting then gradually Increase the pumping rate until the rato matches the well's recharge rate. The depth to water measurement should not increase by more than $0.3-0.4 \mathrm{ft}$. As a general rule, punp the well at a rate not greater than $0.3 \mathrm{~L} / \mathrm{min}$. The maximum rate for this method is 0.5 I/min. 


\begin{tabular}{|c|c|}
\hline 1) & $\begin{array}{l}\text { Procedure Number: ESP-302-2 } \\
\text { Revision Number: } 2 \\
\text { Page: } 14 \text { of } 20\end{array}$ \\
\hline
\end{tabular}

10.13.3.4 Take initial measurements of selected indicator parameters le.g.. pH, specific conductance, temperature, turbidity, oxidation/reduction potential, and dissolved oxygen) to baseline paranoter palues.

20.13.3.5 Measure depth to water in the well at set intervals (e.g.. every 3-5 nin) during the purge event and adjust punp rate to minimize drawdown. Record time and depth to water.

10.13.3.6 Heasure the 1ndicator parameters at set intervals le.g., every 3-5 min) during the purge event. Record time and paraseter values.

10.13.3.7 Stop purging when (1) the depth to water measurement values have stabilized (e.g.. changing <0.1 ft over 15 min) and (2) the indicator paraneters have stabilized over consecutive recording intervals.

snors: The recomended number of consecutive readings to determine stability is three. The recomended ranges for indicator paranoters to be considered stabilized are: when pB measurements agree within \pm 0.1 standard units, tenperature measurements agree within $\pm 1^{\circ} \mathrm{C}$, specific conductance measurements are within $\$ 10$ percent li.e., 100 units for readings over 1000 mhos/crl, oxidation/reduction potential messurements are within $\$ 10 \mathrm{mV}$, dissolved oxygon reasurements are within \pm 10 percent, and turbidity has either stabilized (e.g.. 5 NTOs \pm 2 ) or is below 10 NTOs. 
10.13.4 Low-volume purging (passive sampling) of wells with very low recharge rates with a pump.

kors: If the known yield of a well is too low to maintain minimal drawdown in the woll at a very low punping rate $10.1 \mathrm{~L} / \mathrm{min}$ or less) then use this "passive sampling" approach.

10.13.4.1 Place inlet line of the sampling pump adjacent to water yielding interval, if known, or just abore the middle of monitored interval of the well, if possible. Minimize disturbance of the water.

w02x: If the pump is being installed/inserted in the well, allow an approprlate time period le.g., 12 - $24 \mathrm{hrs}$ is recomnended) for the well to stabilize before beginning to purge. If high. turbidity is observed/measured during purging, allow the well to stabilize 48 - 96 hrs before attenpting to purge again.

10.13.4.2 Purge only the volme of the saspling device and tubing.

HOSL: This method, termed "passive sampling", is considered to be at least as good as the traditional method of "evacuating" the well and sampling upon recovery, and would be considerably better than bailing a well that contains very little water.

10.14 Record all purge times, volume purged, rates of well evacuation, and indicator paraneter values. The use of a purging form to record data is recoumended to aroid the onisesion of critical data. 
10.15 Following the successful purging of the well, sampling activities may begin and should be completed within 3 hrs (optinum) to 24 hrs (maximum, for low-recharge conditions). See ESP-302-4 or ESP-302-3.

rowe: The pumping rate during sampling should not exceed the pumping rate during purging.

10.16 When all necessary procedures are complete, lock the well. clean the area, and dispose of the refuse in accordance with guldellnes set in ESP-800 series, latest revision.

10.27 Dispose of purge waste water per project/program SAP or waste wanagemont plan.

\section{RTPORLARscosps}

11.1 Record data as required in the field logbook or on a field data sheet.

11.2 All records becowe a part of the progran/project OA files.

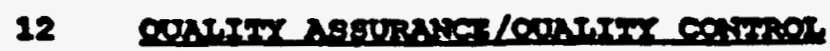

12.1 In addition to adhering to the specific requirements of this procedure, the ninimum $O A / O C$ requirements for this activity are found in ESP-102 (Latest revision).

12.2 Deviations require appropriate project managerent approval and must be sufficiently docwented to sllow repetition of the actirity as actually porformed. 
12.3 QC data are collected as specified in the governing plans and procedures.

12.4 Verification activities are regulred of the above practices, Including periodic survelllance and record audits.

12.5 Verification activities are documented and become part of the project and program records.

12.6 Training to this ESP is required.

13 APPATIOTER

13.1 Appendix A - Example of Eield Checklist.

13.2 Appendix B - Calculating Water Volume for Rurging Wells.

13.3 Appendix C - Well Purging of Honitoring Wells Containing LuAL.

APpronas:

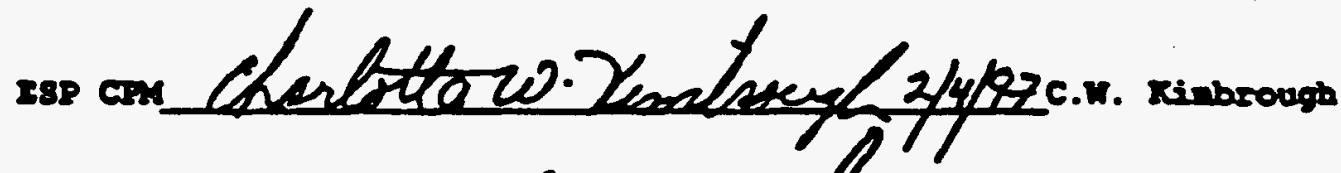

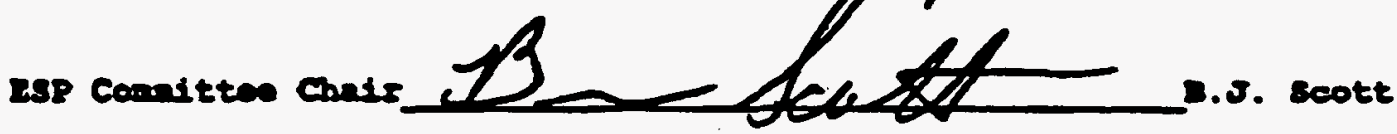




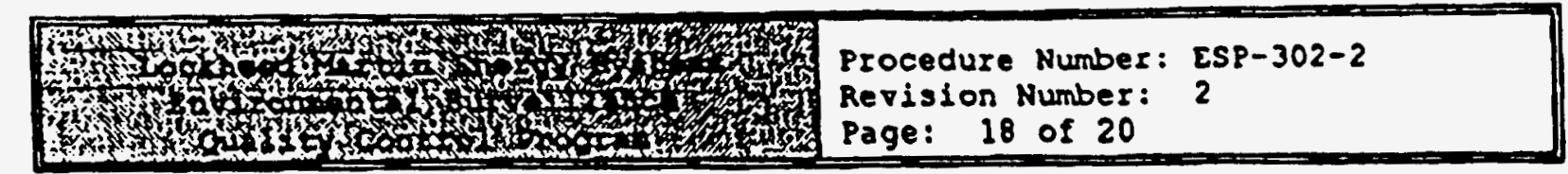

\section{APPENDIX $A$}

Exanple of Field Chockliet

Sampling and Analysis Plan

Health and Safety Pian

Safety equipment le.g.. gloves, safety shoes, eye

protection, etc.)

Logbook and Data Forms

Black Indelible Pen

Keys for Well Lock

Plast1c Sheeting

Punping or Balling Equipment

Hater Level Measurement Eguipment

Cord of Approved Material

Container with Volume Markings

PH, Conductivity, Dissolved Oxygen, Turbidity,

Oxidation/Reduction Potential, and Temperature Measuring

Devices

Manufacturers' Instrumont Calibration and Maintenance Manual

Docontamination Equipment*

Container for Purge Water

- See ESP-802 (latest revision) for decontamination procedures. 


\section{APRHDDX B \\ Culculating Volum of Water for Purging Welle}

B1 The formula for calculating the volume in gallons of water in the well casing or sections of telescoping well casing is as follows:

$7.481\left(\pi x^{2} h\right)=$ gallons:

where $\pi=3.142$,

$I$ - radius of the well pipe in $\mathrm{Et}$.

$h$ - linear $f t$ of water in well (subtract depth to water from well depthl, and

7.481 - gallons per cubic foot of water.

B2 Calculation of the volume of water in typical well casings may be done as follows:

2 in. diameter well:

$0.1632 \mathrm{gal} / \mathrm{ft} x$ __ (1inear ft of water) - __ gal

4 in. diameter well:

0.6528 gal/ft $x$ (linear ft of water) = gal

6 in. dianeter well:

$1.4688 \mathrm{gal} / \mathrm{ft} \mathrm{x}$ (IInear ft of water) = gal

10 in. diameter well:

$4.0800 \mathrm{gal} / \mathrm{ft} x$ (Iinear ft of water) gal 
NOT: Follow these instructions in cases where a light non-agueous phase liqquid (LNAPL) has been detected and the goal is to sample the aqueous phase (groundwater) while preventing the LNAPL from entering the sampling device.

C1 Select a decontaminated stilling tube, constructed of materials meeting performance guidelines for sampling devices, and cover the end of the stilling tube with a membrane or material that will be rupture by the weight of the purp used for purging.

3OF: The menbrane or material must be fastened firmly so that it renains attached to the otilling tube when ruptured. The membrane nust also retain its integrity following the rupture.

Lower the stilling tube slowly into the well so that the bottom is at the approprlate depth (below the luhpl/water interface) and then attach firmly to the top of tho well casing.

C3 Insert the pump so that the weight of the pump breaks the covering on the stilling tube, then the well can be purged and sampled below the INAPL layer. 
APPENDIX B

1997 SWSA 6 DATA SUMMARY STATISTICS 
Station Sample ID Sample Date Sample Type Chemical

\begin{tabular}{|c|c|c|c|c|c|c|c|}
\hline 835 & M01225 & $11 / 11 / 97$ & REG & 1,1,1-Trichloroethane & 5 & ug/L & $\mathrm{U}$ \\
\hline 835 & W00806 & $6 / 23 / 97$ & REG & 1,1,1-Trichloroethane & 5 & $\mathrm{ug} / \mathrm{L}$ & $\mathrm{U}$ \\
\hline 835 & M00041 & $12 / 3 / 96$ & REG & 1,1,1-Trichloroethane & 5 & $\mathrm{ug} / \mathrm{L}$ & $\mathrm{U}$ \\
\hline 835 & M00823 & $6 / 23 / 97$ & REG & 1,1,1-Trichloroethane & 5 & $\mathrm{ug} / \mathrm{L}$ & $\mathrm{U}$ \\
\hline 835 & S02553 & $2 / 24 / 95$ & REG & 1,1,1-Trichloroethane & 5 & $\mathrm{ug} / \mathrm{L}$ & $\mathrm{U}$ \\
\hline 835 & S03495 & $5 / 11 / 95$ & REG & 1,1,1-Trichloroethane & 1 & $\mathrm{ug} / \mathrm{L}$ & $\mathrm{U}$ \\
\hline 835 & S04714 & $7 / 19 / 95$ & REG & 1,1,1-Trichloroethane & 5 & $\mathrm{ug} / \mathrm{L}$ & $\mathrm{U}$ \\
\hline 835 & 835227REGUF & $2 / 27 / 91$ & REG & 1,1,1-Trichloroethane & 5 & $\mathrm{ug} / \mathrm{L}$ & $\mathrm{U}$ \\
\hline 835 & 8356IREGUF & $6 / 1 / 90$ & REG & 1,1,1-Trichloroethane & 5 & $\mathrm{ug} / \mathrm{L}$ & $\mathrm{U}$ \\
\hline 835 & 8351113REGUF & $11 / 13 / 90$ & REG & 1,1,1-Trichloroethane & 5 & $\mathrm{ug} / \mathrm{L}$ & $\mathrm{U}$ \\
\hline 835 & 835130REGUF & $1 / 30 / 89$ & REG & 1,1,1-Trichloroethane & 5 & $\mathrm{ug} / \mathrm{L}$ & $\mathrm{U}$ \\
\hline 835 & 83558REGUF & $5 / 8 / 89$ & REG & 1,1,1-Trichloroethane & 5 & ug/L & $\mathrm{U}$ \\
\hline 835 & 835722REGUF & $7 / 22 / 88$ & REG & 1,1,1-Trichloroethane & 5 & $\mathrm{ug} / \mathrm{L}$ & $\mathrm{U}$ \\
\hline 835 & 8351028REGUF & $10 / 28 / 88$ & REG & 1,1,1-Trichloroethane & 5 & ug/L & $\mathrm{U}$ \\
\hline 835 & M01225 & $11 / 11 / 97$ & REG & 1,1,2,2-Tetrachloroeth & 5 & ug/L & $\mathrm{U}$ \\
\hline 835 & W00806 & $6 / 23 / 97$ & REG & 1,1,2,2-Tetrachloroethane & 5 & $\mathrm{ug} / \mathrm{L}$ & $\mathrm{U}$ \\
\hline 835 & M00041 & $12 / 3 / 96$ & REG & 1,1,2,2-Tetrachloroethane & 5 & ug/L & $\mathrm{U}$ \\
\hline 835 & M00823 & $6 / 23 / 97$ & REG & 1,1,2,2-Tetrachloroethane & 5 & ug/L & $\mathrm{U}$ \\
\hline 835 & S02553 & $2 / 24 / 95$ & REG & 1,1,2,2-Tetrachloroethane & 5 & $\mathrm{ug} / \mathrm{L}$ & $\mathrm{U}$ \\
\hline 835 & S03495 & $5 / 11 / 95$ & REG & 1,1,2,2-Tetrachloroethane & 1 & $\mathrm{ug} / \mathrm{L}$ & $\mathrm{U}$ \\
\hline 835 & S04714 & $7 / 19 / 95$ & REG & 1,1,2,2-Tetrachloroethane & 5 & $\mathrm{ug} / \mathrm{L}$ & $\mathrm{U}$ \\
\hline 835 & 835227REGUF & $2 / 27 / 91$ & REG & 1,1,2,2-Tetrachloroethane & 5 & $\mathrm{ug} / \mathrm{L}$ & $\mathrm{U}$ \\
\hline 835 & 83561REGUF & $6 / 1 / 90$ & REG & 1,1,2,2-Tetrachloroethane & 5 & $\mathrm{ug} / \mathrm{L}$ & $\mathrm{U}$ \\
\hline 835 & 8351113REGUF & $11 / 13 / 90$ & REG & $1,1,2,2$-Tetrachloroethane & 5 & $\mathrm{ug} / \mathrm{L}$ & $\mathrm{U}$ \\
\hline 835 & 835130REGUF & $1 / 30 / 89$ & REG & 1,1,2,2-Tetrachloroethane & 5 & $\mathrm{ug} / \mathrm{L}$ & $\mathrm{U}$ \\
\hline 835 & 83558REGUF & $5 / 8 / 89$ & REG & 1,1,2,2-Tetrachloroethane & 5 & $\mathrm{ug} / \mathrm{L}$ & $\mathrm{U}$ \\
\hline 835 & 835722REGUF & $7 / 22 / 88$ & REG & 1,1,2,2-Tetrachloroethane & 5 & $u g / L$ & $\mathrm{U}$ \\
\hline 835 & 8351028REGUF & $10 / 28 / 88$ & REG & 1,1,2,2-Tetrachloroethane & 5 & $\mathrm{ug} / \mathrm{L}$ & $\mathrm{U}$ \\
\hline 835 & W00806 & $6 / 23 / 97$ & REG & 1,1,2-Trichloro-1,2,2-trifluo & 10 & $\mathrm{ug} / \mathrm{L}$ & $\mathrm{U}$ \\
\hline 835 & M01225 & $11 / 11 / 97$ & REG & 1,1,2-Trichloroethane & 5 & $\mathrm{ug} / \mathrm{L}$ & $\mathrm{U}$ \\
\hline 835 & W00806 & $6 / 23 / 97$ & REG & 1,1,2-Trichloroethane & 5 & $\mathrm{ug} / \mathrm{L}$ & $\mathrm{U}$ \\
\hline 835 & M00041 & $12 / 3 / 96$ & REG & 1,1,2-Trichloroethane & 5 & ug/L & $\mathrm{U}$ \\
\hline 835 & M00823 & $6 / 23 / 97$ & REG & 1,1,2-Trichloroethane & 5 & ug/ $/$ & $\mathrm{U}$ \\
\hline 835 & S02553 & $2 / 24 / 95$ & REG & 1,1,2-Trichloroethane & 5 & $\mathrm{ug} / \mathrm{L}$ & $\mathrm{U}$ \\
\hline 835 & S03495 & $5 / 11 / 95$ & REG & 1,1,2-Trichloroethane & 1 & ug/L & $U$ \\
\hline 835 & S04714 & $7 / 19 / 95$ & REG & 1,1,2-Trichloroethane & 5 & $\mathrm{ug} / \mathrm{L}$ & $\mathrm{U}$ \\
\hline 835 & 835227REGUF & $2 / 27 / 91$ & REG & 1,1,2-Trichloroethane & 5 & $\mathrm{ug} / \mathrm{L}$ & $\mathrm{U}$ \\
\hline 835 & 83561REGUF & $6 / 1 / 90$ & REG & 1,1,2-Trichloroethane & 5 & $\mathrm{ug} / \mathrm{L}$ & $\mathrm{U}$ \\
\hline 835 & 8351113REGUF & $11 / 13 / 90$ & REG & 1,1,2-Trichloroethane & 5 & $\mathrm{ug} / \mathrm{L}$ & $\mathrm{U}$ \\
\hline 835 & 835130REGUF & $1 / 30 / 89$ & REG & 1,1,2-Trichloroethane & 5 & $\mathrm{ug} / \mathrm{L}$ & $\mathrm{U}$ \\
\hline 835 & 83558REGUF & $5 / 8 / 89$ & REG & 1,1,2-Trichloroethane & 5 & $\mathrm{ug} / \mathrm{L}$ & $\mathrm{U}$ \\
\hline 835 & 835722REGUF & $7 / 22 / 88$ & REG & 1,1,2-Trichloroethane & 5 & $\mathrm{ug} / \mathrm{L}$ & $\mathrm{U}$ \\
\hline 835 & 8351028REGUF & $10 / 28 / 88$ & REG & 1,1,2-Trichloroethane & 5 & $\mathrm{ug} / \mathrm{L}$ & $\mathrm{U}$ \\
\hline 835 & M01225 & $11 / 11 / 97$ & REG & 1,1-Dichloroethane & 5 & $\mathrm{ug} / \mathrm{L}$ & $\mathrm{U}$ \\
\hline 835 & W00806 & $6 / 23 / 97$ & REG & 1,1-Dichloroethane & 5 & $\mathrm{ug} / \mathrm{L}$ & $\mathrm{U}$ \\
\hline 835 & M00041 & $12 / 3 / 96$ & REG & 1,1-Dichloroethane & 5 & $\mathrm{ug} / \mathrm{L}$ & $\mathrm{U}$ \\
\hline 835 & M00823 & $6 / 23 / 97$ & REG & 1,1-Dichloroethane & 5 & $\mathrm{ug} / \mathrm{L}$ & $\mathrm{U}$ \\
\hline 835 & S02553 & $2 / 24 / 95$ & REG & 1,1-Dichloroethane & 5 & ug $/ \mathrm{L}$ & $\mathrm{U}$ \\
\hline 835 & S03495 & $5 / 11 / 95$ & REG & 1,1-Dichloroethane & 1 & $\mathrm{ug} / \mathrm{L}$ & $\mathrm{U}$ \\
\hline 835 & S04714 & $7 / 19 / 95$ & REG & 1,1-Dichloroethane & 5 & $\mathrm{ug} / \mathrm{L}$ & $\mathrm{U}$ \\
\hline
\end{tabular}




\begin{tabular}{|c|c|c|c|c|c|c|c|}
\hline Station & Sample ID & Sample Date & Sample Type & Chemical & Results & Units & Qualifier \\
\hline$\overline{835}$ & 835227REGUF & $2 / 27 / 91$ & REG & 1,1-Dichloroethane & 5 & $\mathrm{ug} / \mathrm{L}$ & $\mathrm{U}$ \\
\hline 835 & 83561REGUF & $6 / 1 / 90$ & REG & 1,1-Dichloroethane & 5 & $\mathrm{ug} / \mathrm{L}$ & $\mathrm{U}$ \\
\hline 835 & 8351113REGUF & $11 / 13 / 90$ & REG & 1,1-Dichloroethane & 5 & $\mathrm{ug} / \mathrm{L}$ & $\mathrm{U}$ \\
\hline 835 & 835130REGUF & $1 / 30 / 89$ & REG & 1.1-Dichloroethane & 5 & $\mathrm{ug} / \mathrm{L}$ & $\mathrm{U}$ \\
\hline 835 & 83558REGUF & $5 / 8 / 89$ & REG & 1,1-Dichloroethane & 5 & $\mathrm{ug} / \mathrm{L}$ & $\mathrm{U}$ \\
\hline 835 & 835722REGUF & $7 / 22 / 88$ & REG & 1,1-Dichloroethane & 5 & $\mathrm{ug} / \mathrm{L}$ & $\mathrm{U}$ \\
\hline 835 & 8351028REGUF & $10 / 28 / 88$ & REG & 1,1-Dichloroethane & 5 & $\mathrm{ug} / \mathrm{L}$ & $\mathrm{U}$ \\
\hline 835 & M01225 & $11 / 11 / 97$ & REG & 1,1-Dichloroethene & 5 & $\mathrm{ug} / \mathrm{L}$ & $\mathrm{U}$ \\
\hline 835 & W00806 & $6 / 23 / 97$ & REG & 1,1-Dichloroethene & 5 & $\mathrm{ug} / \mathrm{L}$ & $\mathrm{U}$ \\
\hline 835 & M00041 & $12 / 3 / 96$ & REG & 1,1-Dichloroethene & 5 & $\mathrm{ug} / \mathrm{L}$ & U \\
\hline 835 & M00823 & $6 / 23 / 97$ & REG & 1,1-Dichloroethene & 5 & $\mathrm{ug} / \mathrm{L}$ & $\mathrm{U}$ \\
\hline 835 & $\mathrm{~S} 02553$ & $2 / 24 / 95$ & REG & 1,1-Dichloroethene & 5 & $\mathrm{ug} / \mathrm{L}$ & $\mathrm{U}$ \\
\hline 835 & S03495 & $5 / 11 / 95$ & REG & 1,1-Dichloroethene & 1 & ug/L & $\mathrm{U}$ \\
\hline 835 & S04714 & $7 / 19 / 95$ & REG & 1,1-Dichloroethene & 5 & $\mathrm{ug} / \mathrm{L}$ & U \\
\hline 835 & 835227REGUF & $2 / 27 / 91$ & REG & 1,1-Dichloroethene & 5 & $\mathrm{ug} / \mathrm{L}$ & $\mathrm{U}$ \\
\hline 835 & 8356lREGUF & $6 / 1 / 90$ & REG & 1.1-Dichloroethene & 5 & $\mathrm{ug} / \mathrm{L}$ & $\mathrm{U}$ \\
\hline 835 & 8351113REGUF & $11 / 13 / 90$ & REG & 1,1-Dichloroethene & 5 & $\mathrm{ug} / \mathrm{L}$ & $\mathrm{U}$ \\
\hline 835 & 835130REGUF & $1 / 30 / 89$ & REG & 1,1-Dichloroethene & 5 & $u g / L$ & $\mathrm{U}$ \\
\hline 835 & 83558REGUF & $5 / 8 / 89$ & REG & 1,1-Dichloroethene & 5 & $\mathrm{ug} / \mathrm{L}$ & $\mathrm{U}$ \\
\hline 835 & 835722REGUF & $7 / 22 / 88$ & REG & 1,1-Dichloroethene & 5 & $\mathrm{ug} / \mathrm{L}$ & $\mathrm{U}$ \\
\hline 835 & 8351028REGUF & $10 / 28 / 88$ & REG & 1,1-Dichloroethene & 5 & $\mathrm{ug} / \mathrm{L}$ & $\mathrm{U}$ \\
\hline 835 & S03495 & $5 / 11 / 95$ & REG & 1,2-Dibromo-3-chloropropane & 1 & $\mathrm{ug} / \mathrm{L}$ & $\mathrm{U}$ \\
\hline 835 & S03495 & $5 / 11 / 95$ & REG & 1,2-Dibromoethane & 1 & $\mathrm{ug} / \mathrm{L}$ & $\mathrm{U}$ \\
\hline 835 & M00041 & $12 / 3 / 96$ & REG & 1,2-Dichlorobenzene & 5 & $\mathrm{ug} / \mathrm{L}$ & $\mathrm{U}$ \\
\hline 835 & M01225 & $11 / 11 / 97$ & REG & 1,2-Dichloroethane & 5 & $\mathrm{ug} / \mathrm{L}$ & $\mathrm{U}$ \\
\hline 835 & W00806 & $6 / 23 / 97$ & REG & 1,2-Dichloroethane & 5 & $\mathrm{ug} / \mathrm{L}$ & $\mathrm{U}$ \\
\hline 835 & M00041 & $12 / 3 / 96$ & REG & 1,2-Dichloroethane & 5 & $\mathrm{ug} / \mathrm{L}$ & $\mathrm{U}$ \\
\hline 835 & M00823 & $6 / 23 / 97$ & REG & 1,2-Dichloroethane & 5 & ug/L & U \\
\hline 835 & $\mathrm{~S} 02553$ & $2 / 24 / 95$ & REG & 1,2-Dichloroethane & 5 & $\mathrm{ug} / \mathrm{L}$ & $\mathrm{U}$ \\
\hline 835 & S03495 & $5 / 11 / 95$ & REG & 1,2-Dichloroethane & 1 & $\mathrm{ug} / \mathrm{L}$ & $\mathrm{U}$ \\
\hline 835 & S04714 & $7 / 19 / 95$ & REG & 1,2-Dichloroethane & 5 & $\mathrm{ug} / \mathrm{L}$ & $\mathrm{U}$ \\
\hline 835 & 835227REGUF & $2 / 27 / 91$ & REG & 1,2-Dichloroethane & 5 & $\mathrm{ug} / \mathrm{L}$ & $U$ \\
\hline 835 & 8356IREGUF & $6 / 1 / 90$ & REG & 1,2-Dichloroethane & 5 & $u g / L$ & $\mathrm{U}$ \\
\hline 835 & 8351113REGUF & $11 / 13 / 90$ & REG & 1,2-Dichloroethane & 5 & $\mathrm{ug} / \mathrm{L}$ & $\mathrm{U}$ \\
\hline 835 & 835130REGUF & $1 / 30 / 89$ & REG & 1,2-Dichloroethane & 5 & $\mathrm{ug} / \mathrm{L}$ & $\mathrm{U}$ \\
\hline 835 & 83558REGUF & $5 / 8 / 89$ & REG & 1,2-Dichloroethane & 5 & $\mathrm{ug} / \mathrm{L}$ & $\mathrm{U}$ \\
\hline 835 & 835722REGUF & $7 / 22 / 88$ & REG & 1,2-Dichloroethane & 5 & $\mathrm{ug} / \mathrm{L}$ & $\mathrm{U}$ \\
\hline 835 & 8351028REGUF & $10 / 28 / 88$ & REG & 1,2-Dichloroethane & 5 & $\mathrm{ug} / \mathrm{L}$ & $\mathrm{U}$ \\
\hline 835 & W00806 & $6 / 23 / 97$ & REG & 1,2-Dichloroethene & 5 & $\mathrm{ug} / \mathrm{L}$ & $\mathrm{U}$ \\
\hline 835 & M00041 & $12 / 3 / 96$ & REG & 1,2-Dichloroethene & 5 & $\mathrm{ug} / \mathrm{L}$ & $\mathrm{U}$ \\
\hline 835 & S02553 & $2 / 24 / 95$ & REG & 1,2-Dichloroethene & 5 & $\mathrm{ug} / \mathrm{L}$ & $\mathrm{U}$ \\
\hline 835 & S04714 & $7 / 19 / 95$ & REG & 1,2-Dichloroethene & 5 & $\mathrm{ug} / \mathrm{L}$ & $\mathrm{U}$ \\
\hline 835 & 835227REGUF & $2 / 27 / 91$ & REG & 1,2-Dichloroethene & 5 & ug/L & $\mathrm{U}$ \\
\hline 835 & 8356IREGUF & $6 / 1 / 90$ & REG & 1,2-Dichloroethene & 5 & $\mathrm{ug} / \mathrm{L}$ & $\mathrm{U}$ \\
\hline 835 & 8351113REGUF & $11 / 13 / 90$ & REG & 1,2-Dichloroethene & 5 & ug/ & $\mathrm{U}$ \\
\hline 835 & 835I30REGUF & $1 / 30 / 89$ & REG & 1,2-Dichloroethene & 5 & $\mathrm{ug} / \mathrm{L}$ & $\mathrm{U}$ \\
\hline 835 & 83558REGUF & $5 / 8 / 89$ & REG & 1,2-Dichloroethene & 5 & $\mathrm{ug} / \mathrm{L}$ & $\mathrm{U}$ \\
\hline 835 & 835722REGUF & $7 / 22 / 88$ & REG & 1,2-Dichloroethene & 5 & $\mathrm{ug} / \mathrm{L}$ & $\mathrm{U}$ \\
\hline 835 & 8351028REGUF & $10 / 28 / 88$ & REG & 1,2-Dichloroethene & 5 & $\mathrm{ug} / \mathrm{L}$ & U \\
\hline 835 & M01225 & $11 / 11 / 97$ & REG & 1,2-Dichloropropane & 5 & $\mathrm{ug} / \mathrm{L}$ & $\mathrm{U}$ \\
\hline
\end{tabular}




\begin{tabular}{|c|c|c|c|c|c|c|c|}
\hline Station & Sample ID & Sample Date & Sample Type & Chemical & Results & Units & Qualifier \\
\hline 835 & W00806 & $6 / 23 / 97$ & REG & 1,2-Dichloropropane & 5 & $u g / L$ & $\mathrm{U}$ \\
\hline 835 & M0004l & $12 / 3 / 96$ & REG & 1,2-Dichloropropane & 5 & $\mathrm{ug} / \mathrm{L}$ & $\mathrm{U}$ \\
\hline 835 & M00823 & $6 / 23 / 97$ & REG & 1,2-Dichloropropane & 5 & $\mathrm{ug} / \mathrm{L}$ & $\mathrm{U}$ \\
\hline 835 & $\mathrm{~S} 02553$ & $2 / 24 / 95$ & REG & 1,2-Dichloropropane & 5 & $\mathrm{ug} / \mathrm{L}$ & $\mathrm{U}$ \\
\hline 835 & S03495 & $5 / 11 / 95$ & REG & 1,2-Dichloropropane & 1 & $\mathrm{ug} / \mathrm{L}$ & $\mathrm{U}$ \\
\hline 835 & S04714 & $7 / 19 / 95$ & REG & 1,2-Dichloropropane & 5 & $\mathrm{ug} / \mathrm{L}$ & $\mathrm{U}$ \\
\hline 835 & 835227REGUF & $2 / 27 / 91$ & REG & 1,2-Dichloropropane & 5 & $\mathrm{ug} / \mathrm{L}$ & $\mathrm{U}$ \\
\hline 835 & 8356IREGUF & $6 / 1 / 90$ & REG & 1,2-Dichloropropane & 5 & $\mathrm{ug} / \mathrm{L}$ & U \\
\hline 835 & 8351113REGUF & $11 / 13 / 90$ & REG & 1.2-Dichloropropane & 5 & $\mathrm{ug} / \mathrm{L}$ & $\mathrm{U}$ \\
\hline 835 & 835130REGUF & $1 / 30 / 89$ & REG & 1,2-Dichloropropane & 5 & $\mathrm{ug} / \mathrm{L}$ & $\mathrm{U}$ \\
\hline 835 & 83558REGUF & $5 / 8 / 89$ & REG & 1,2-Dichloropropane & 5 & ug/L & $\mathrm{U}$ \\
\hline 835 & 835722REGUF & $7 / 22 / 88$ & REG & 1,2-Dichloropropane & 5 & $u g / L$ & U \\
\hline 835 & 8351028REGUF & $10 / 28 / 88$ & REG & 1,2-Dichloropropane & 5 & $\mathrm{ug} / \mathrm{L}$ & $\mathrm{U}$ \\
\hline 835 & M00041 & $12 / 3 / 96$ & REG & 1,2-Dimethylbenzene & 5 & $\mathrm{ug} / \mathrm{L}$ & $U$ \\
\hline 835 & S02553 & $2 / 24 / 95$ & REG & 1,2-Dimethylbenzene & 5 & $\mathrm{ug} / \mathrm{L}$ & U \\
\hline 835 & S04714 & $7 / 19 / 95$ & REG & 1,2-Dimethylbenzene & 5 & $u g / L$ & $\mathrm{U}$ \\
\hline 835 & M00041 & $12 / 3 / 96$ & REG & 1,3-Dichlorobenzene & 5 & $\mathrm{ug} / \mathrm{L}$ & U \\
\hline 835 & M00041 & $12 / 3 / 96$ & REG & 1,4-Dichlorobenzene & 5 & $\mathrm{ug} / \mathrm{L}$ & $\mathrm{U}$ \\
\hline 835 & M01225 & $11 / 11 / 97$ & REG & 2-Butanone & 10 & ug/L & $\mathrm{U}$ \\
\hline 835 & W00806 & $6 / 23 / 97$ & REG & 2-Butanone & 10 & $\mathrm{ug} / \mathrm{L}$ & $\mathrm{U}$ \\
\hline 835 & M0004l & $12 / 3 / 96$ & REG & 2-Butanone & 10 & $\mathrm{ug} / \mathrm{L}$ & $\mathrm{U}$ \\
\hline 835 & M00823 & $6 / 23 / 97$ & REG & 2-Butanone & 10 & $\mathrm{ug} / \mathrm{L}$ & $\mathrm{U}$ \\
\hline 835 & S02553 & $2 / 24 / 95$ & REG & 2-Butanone & 3 & $\mathrm{ug} / \mathrm{L}$ & $\mathrm{J}$ \\
\hline 835 & S03495 & $5 / 11 / 95$ & REG & 2-Butanone & 5 & $\mathrm{ug} / \mathrm{L}$ & $\mathrm{U}$ \\
\hline 835 & S04714 & $7 / 19 / 95$ & REG & 2-Butanone & 10 & $\mathrm{ug} / \mathrm{L}$ & $\mathrm{U}$ \\
\hline 835 & 835227REGUF & $2 / 27 / 91$ & REG & 2-Butanone & 10 & $\mathrm{ug} / \mathrm{L}$ & $\mathrm{U}$ \\
\hline 835 & 83561REGUF & $6 / 1 / 90$ & REG & 2-Butanone & 10 & $\mathrm{ug} / \mathrm{L}$ & $\mathrm{U}$ \\
\hline 835 & 8351113REGUF & $11 / 13 / 90$ & REG & 2-Butanone & 10 & $\mathrm{ug} / \mathrm{L}$ & $\mathrm{U}$ \\
\hline 835 & 835130REGUF & $1 / 30 / 89$ & REG & 2-Butanone & 10 & $\mathrm{ug} / \mathrm{L}$ & $\mathrm{U}$ \\
\hline 835 & 83558REGUF & $5 / 8 / 89$ & REG & 2-Butanone & 10 & $\mathrm{ug} / \mathrm{L}$ & $\mathrm{U}$ \\
\hline 835 & 835722REGUF & $7 / 22 / 88$ & REG & 2-Butanone & 10 & $\mathrm{ug} / \mathrm{L}$ & $\mathrm{U}$ \\
\hline 835 & 8351028REGUF & $10 / 28 / 88$ & REG & 2-Butanone & 10 & $\mathrm{ug} / \mathrm{L}$ & $\mathrm{U}$ \\
\hline 835 & M01225 & $11 / 11 / 97$ & REG & 2-Hexanone & 10 & $\mathrm{ug} / \mathrm{L}$ & $U$ \\
\hline 835 & W00806 & $6 / 23 / 97$ & REG & 2-Hexanone & 10 & $\mathrm{ug} / \mathrm{L}$ & $\mathrm{U}$ \\
\hline 835 & M00041 & $12 / 3 / 96$ & REG & 2-Hexanone & 10 & ug/L & $U$ \\
\hline 835 & M00823 & $6 / 23 / 97$ & REG & 2-Hexanone & 10 & $\mathrm{ug} / \mathrm{L}$ & $\mathrm{U}$ \\
\hline 835 & S02553 & $2 / 24 / 95$ & REG & 2-Hexanone & 10 & $\mathrm{ug} / \mathrm{L}$ & $\mathrm{U}$ \\
\hline 835 & S03495 & $5 / 11 / 95$ & REG & 2-Hexanone & 5 & $\mathrm{ug} / \mathrm{L}$ & $\mathrm{U}$ \\
\hline 835 & S04714 & $7 / 19 / 95$ & REG & 2-Hexanone & 10 & $\mathrm{ug} / \mathrm{L}$ & $\mathrm{U}$ \\
\hline 835 & 835227REGUF & $2 / 27 / 91$ & REG & 2-Hexanone & 10 & $\mathrm{ug} / \mathrm{L}$ & $\mathrm{U}$ \\
\hline 835 & 83561REGUF & $6 / 1 / 90$ & REG & 2-Hexanone & 10 & $\mathrm{ug} / \mathrm{L}$ & U \\
\hline 835 & 8351113REGUF & $11 / 13 / 90$ & REG & 2-Hexanone & 10 & $\mathrm{ug} / \mathrm{L}$ & $\mathrm{U}$ \\
\hline 835 & 835130REGUF & $1 / 30 / 89$ & REG & 2-Hexanone & 10 & $\mathrm{ug} / \mathrm{L}$ & $\mathrm{U}$ \\
\hline 835 & 83558REGUF & $5 / 8 / 89$ & REG & 2-Hexanone & 10 & $\mathrm{ug} / \mathrm{L}$ & $\mathrm{U}$ \\
\hline 835 & 835722REGUF & $7 / 22 / 88$ & REG & 2-Hexanone & 10 & $\mathrm{ug} / \mathrm{L}$ & $\mathrm{U}$ \\
\hline 835 & 8351028REGUF & $10 / 28 / 88$ & REG & 2-Hexanone & 10 & $\mathrm{ug} / \mathrm{L}$ & $\mathrm{U}$ \\
\hline 835 & M01225 & $11 / 11 / 97$ & REG & 4-Methyl-2-pentanone & 10 & $\mathrm{ug} / \mathrm{L}$ & $\mathrm{U}$ \\
\hline 835 & W00806 & $6 / 23 / 97$ & REG & 4-Methyl-2-pentanone & 10 & $u g / L$ & $\mathrm{U}$ \\
\hline 835 & M00041 & $12 / 3 / 96$ & REG & 4-Methyl-2-pentanone & 10 & $\mathrm{ug} / \mathrm{L}$ & $\mathrm{U}$ \\
\hline 835 & M00823 & $6 / 23 / 97$ & REG & 4-Methyl-2-pentanone & 10 & $\mathrm{ug} / \mathrm{L}$ & $\mathrm{U}$ \\
\hline
\end{tabular}


B-6

\begin{tabular}{|c|c|c|c|c|c|c|c|}
\hline Station & Sample ID & Sample Date & Sample Type & Chemical & Results & Units & Qualifier \\
\hline 835 & S02553 & $2 / 24 / 95$ & REG & 4-Methyl-2-pentanone & 10 & $\mathrm{ug} / \mathrm{L}$ & $\mathrm{U}$ \\
\hline 835 & S03495 & $5 / 11 / 95$ & REG & 4-Methyl-2-pentanone & 5 & $\mathrm{ug} / \mathrm{L}$ & $\mathrm{U}$ \\
\hline 835 & S04714 & $7 / 19 / 95$ & REG & 4-Methyl-2-pentanone & 10 & $\mathrm{ug} / \mathrm{L}$ & $\mathrm{U}$ \\
\hline 835 & 835227REGUF & $2 / 27 / 91$ & REG & 4-Methyl-2-pentanone & 10 & $\mathrm{ug} / \mathrm{L}$ & $\mathrm{U}$ \\
\hline 835 & 83561REGUF & $6 / 1 / 90$ & REG & 4-Methyl-2-pentanone & 10 & $\mathrm{ug} / \mathrm{L}$ & $\mathrm{U}$ \\
\hline 835 & 8351113REGUF & $11 / 13 / 90$ & REG & 4-Methyl-2-pentanone & 10 & $\mathrm{ug} / \mathrm{L}$ & $\mathrm{U}$ \\
\hline 835 & 835130REGUF & $1 / 30 / 89$ & REG & 4-Methyl-2-pentanone & 10 & $\mathrm{ug} / \mathrm{L}$ & $\mathrm{U}$ \\
\hline 835 & 83558REGUF & $5 / 8 / 89$ & REG & 4-Methyl-2-pentanone & 10 & $u g / L$ & $\mathrm{U}$ \\
\hline 835 & 835722REGUF & $7 / 22 / 88$ & REG & 4-Methyl-2-pentanone & 10 & $\mathrm{ug} / \mathrm{L}$ & $\mathrm{U}$ \\
\hline 835 & 8351028REGUF & $10 / 28 / 88$ & REG & 4-Methyl-2-pentanone & 10 & $\mathrm{ug} / \mathrm{L}$ & $\mathrm{U}$ \\
\hline 835 & M01225 & $11 / 11 / 97$ & REG & Acetone & 3 & $\mathrm{ug} / \mathrm{L}$ & B \\
\hline 835 & W00806 & $6 / 23 / 97$ & REG & Acetone & 10 & $u g / L$ & $\mathrm{U}$ \\
\hline 835 & M00041 & $12 / 3 / 96$ & REG & Acetone & 10 & $\mathrm{ug} / \mathrm{L}$ & $\mathrm{U}$ \\
\hline 835 & M00823 & $6 / 23 / 97$ & REG & Acetone & 10 & $\mathrm{ug} / \mathrm{L}$ & $\mathrm{U}$ \\
\hline 835 & S02553 & $2 / 24 / 95$ & REG & Acetone & 10 & $\mathrm{ug} / \mathrm{L}$ & $\mathrm{U}$ \\
\hline 835 & S03495 & $5 / 11 / 95$ & REG & Acetone & 5 & $\mathrm{ug} / \mathrm{L}$ & $U$ \\
\hline 835 & S04714 & $7 / 19 / 95$ & REG & Acetone & 10 & $\mathrm{ug} / \mathrm{L}$ & $\mathrm{U}$ \\
\hline 835 & 835227REGUF & $2 / 27 / 91$ & REG & Acetone & 10 & $u g / L$ & $\mathrm{U}$ \\
\hline 835 & 83561REGUF & $6 / 1 / 90$ & REG & Acetone & 10 & $\mathrm{ug} / \mathrm{L}$ & $U$ \\
\hline 835 & 8351113REGUF & $11 / 13 / 90$ & REG & Acetone & 3 & ug/L & $\mathrm{J}$ \\
\hline 835 & 835130REGUF & $1 / 30 / 89$ & REG & Acetone & 10 & $\mathrm{ug} / \mathrm{L}$ & $\mathrm{U}$ \\
\hline 835 & 83558REGUF & $5 / 8 / 89$ & REG & Acetone & 3 & $\mathrm{ug} / \mathrm{L}$ & $\mathrm{J}$ \\
\hline 835 & 835722REGUF & $7 / 22 / 88$ & REG & Acetone & 10 & $\mathrm{ug} / \mathrm{L}$ & $\mathrm{U}$ \\
\hline 835 & 8351028REGUF & $10 / 28 / 88$ & REG & Acetone & 10 & $\mathrm{ug} / \mathrm{L}$ & $\mathrm{U}$ \\
\hline 835 & M01225 & $11 / 11 / 97$ & REG & Benzene & 5 & $\mathrm{ug} / \mathrm{L}$ & $\mathrm{U}$ \\
\hline 835 & W00806 & $6 / 23 / 97$ & REG & Benzene & 5 & $\mathrm{ug} / \mathrm{L}$ & $\mathrm{U}$ \\
\hline 835 & M00041 & $12 / 3 / 96$ & REG & Benzene & 5 & $\mathrm{ug} / \mathrm{L}$ & $\mathrm{U}$ \\
\hline 835 & M00823 & $6 / 23 / 97$ & REG & Benzene & 5 & $\mathrm{ug} / \mathrm{L}$ & $\mathrm{U}$ \\
\hline 835 & $\mathrm{~S} 02553$ & $2 / 24 / 95$ & REG & Benzene & 5 & ug/L & $\mathrm{U}$ \\
\hline 835 & S03495 & $5 / 11 / 95$ & REG & Benzene & 1 & $\mathrm{ug} / \mathrm{L}$ & $\mathrm{U}$ \\
\hline 835 & S04714 & $7 / 19 / 95$ & REG & Benzene & 5 & ug/L & $\mathrm{U}$ \\
\hline 835 & 835227REGUF & $2 / 27 / 91$ & REG & Benzene & 5 & $\mathrm{ug} / \mathrm{L}$ & $\mathrm{U}$ \\
\hline 835 & 83561REGUF & $6 / 1 / 90$ & REG & Benzene & 5 & $\mathrm{ug} / \mathrm{L}$ & $\mathrm{U}$ \\
\hline 835 & 8351113REGUF & $11 / 13 / 90$ & REG & Benzene & 5 & $u g / L$ & $\mathrm{U}$ \\
\hline 835 & 835130REGUF & $1 / 30 / 89$ & REG & Benzene & 3 & $\mathrm{ug} / \mathrm{L}$ & $\mathrm{J}$ \\
\hline 835 & 83558REGUF & $5 / 8 / 89$ & REG & Benzene & 2 & ug/L & J \\
\hline 835 & 835722REGUF & $7 / 22 / 88$ & REG & Benzene & 3 & $\mathrm{ug} / \mathrm{L}$ & $\mathrm{J}$ \\
\hline 835 & 8351028REGUF & $10 / 28 / 88$ & REG & Benzene & 2 & $\mathrm{ug} / \mathrm{L}$ & $\mathrm{J}$ \\
\hline 835 & S03495 & $5 / 11 / 95$ & REG & Bromochloromethane & 1 & $\mathrm{ug} / \mathrm{L}$ & $\mathrm{U}$ \\
\hline 835 & M01225 & $11 / 11 / 97$ & REG & Bromodichloromethane & 5 & $\mathrm{ug} / \mathrm{L}$ & $\mathrm{U}$ \\
\hline 835 & W00806 & $6 / 23 / 97$ & REG & Bromodichloromethane & 5 & $u g / L$ & $\mathrm{U}$ \\
\hline 835 & M00041 & $12 / 3 / 96$ & REG & Bromodichloromethane & 5 & $\mathrm{ug} / \mathrm{L}$ & $\mathrm{U}$ \\
\hline 835 & M00823 & $6 / 23 / 97$ & REG & Bromodichloromethane & 5 & $\mathrm{ug} / \mathrm{L}$ & $U$ \\
\hline 835 & S02553 & $2 / 24 / 95$ & REG & Bromodichloromethane & 5 & $\mathrm{ug} / \mathrm{L}$ & $\mathrm{U}$ \\
\hline 835 & S03495 & $5 / 11 / 95$ & REG & Bromodichloromethane & 1 & $\mathrm{ug} / \mathrm{L}$ & $\mathrm{U}$ \\
\hline 835 & S04714 & $7 / 19 / 95$ & REG & Bromodichloromethane & 5 & $\mathrm{ug} / \mathrm{L}$ & $\mathrm{U}$ \\
\hline 835 & 835227REGUF & $2 / 27 / 91$ & REG & Bromodichloromethane & 5 & $\mathrm{ug} / \mathrm{L}$ & $\mathrm{U}$ \\
\hline 835 & 8356lREGUF & $6 / 1 / 90$ & REG & Bromodichloromethane & 5 & $\mathrm{ug} / \mathrm{L}$ & $\mathrm{U}$ \\
\hline 835 & 8351113REGUF & $11 / 13 / 90$ & REG & Bromodichloromethane & 5 & $\mathrm{ug} / \mathrm{L}$ & $\mathrm{U}$ \\
\hline 835 & 835130REGUF & $1 / 30 / 89$ & REG & Bromodichloromethane & 5 & $\mathrm{ug} / \mathrm{L}$ & $\mathrm{U}$ \\
\hline
\end{tabular}


B-7

Station Sample ID Sample Date Sample Type Chemical

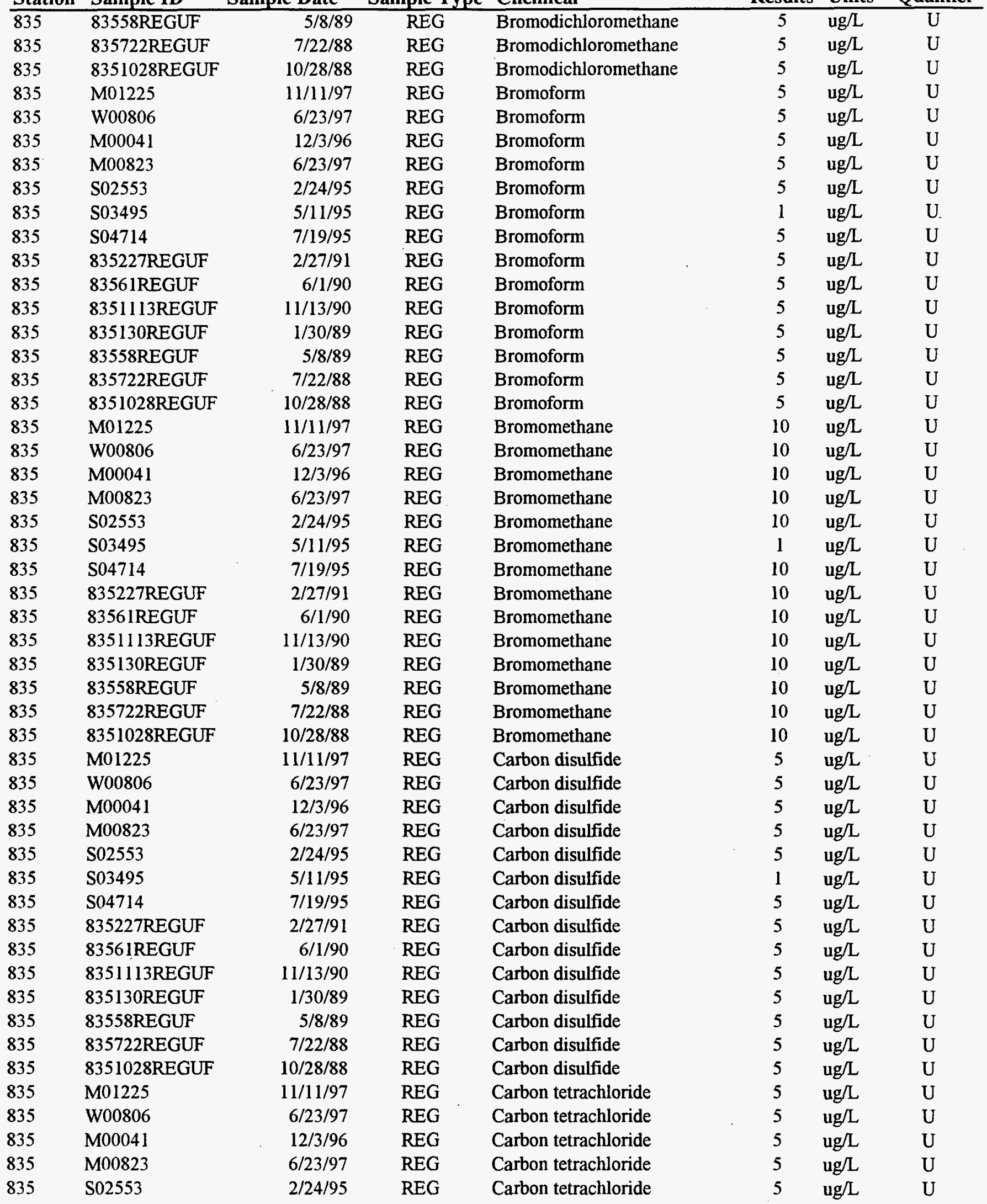


Station Sample ID Sample Date Sample Type Chemical

\begin{tabular}{|c|c|c|c|c|c|c|c|}
\hline Station & Sample ID & Sample Date & Sample Type & Chemical & Results & Units & Qualif \\
\hline 835 & S03495 & $5 / 11 / 95$ & REG & Carbon tetrachloride & 1 & $u g / L$ & $\overline{\mathrm{U}}$ \\
\hline 835 & S04714 & $7 / 19 / 95$ & REG & Carbon tetrachloride & 5 & $u g / L$ & $\mathrm{U}$ \\
\hline 835 & 835227REGUF & $2 / 27 / 91$ & REG & Carbon tetrachloride & 5 & $u g / L$ & $\mathrm{U}$ \\
\hline 835 & 8356IREGUF & $6 / 1 / 90$ & REG & Carbon tetrachloride & 5 & $\mathrm{ug} / \mathrm{L}$ & $\mathrm{U}$ \\
\hline 835 & 8351113REGUF & $11 / 13 / 90$ & REG & Carbon tetrachloride & 5 & $\mathrm{ug} / \mathrm{L}$ & $\mathrm{U}$ \\
\hline 835 & 835130REGUF & $1 / 30 / 89$ & REG & Carbon tetrachloride & 5 & $\mathrm{ug} / \mathrm{L}$ & $\mathrm{U}$ \\
\hline 835 & 83558REGUF & $5 / 8 / 89$ & REG & Carbon tetrachloride & 5 & $\mathrm{ug} / \mathrm{L}$ & $\mathrm{U}$ \\
\hline 835 & 835722REGUF & $7 / 22 / 88$ & REG & Carbon tetrachloride & 5 & $\mathrm{ug} / \mathrm{L}$ & $\mathrm{U}$ \\
\hline 835 & 8351028REGUF & $10 / 28 / 88$ & REG & Carbon tetrachloride & 5 & $u g / L$ & $\mathrm{U}$ \\
\hline 835 & M01225 & $11 / 11 / 97$ & REG & Chlorobenzene & 5 & $\mathrm{ug} / \mathrm{L}$ & $\mathrm{U}$ \\
\hline 835 & W00806 & $6 / 23 / 97$ & REG & Chlorobenzene & 5 & $\mathrm{ug} / \mathrm{L}$ & $\mathrm{U}$ \\
\hline 835 & $\mathrm{M} 00041$ & $12 / 3 / 96$ & REG & Chlorobenzene & 5 & $\mathrm{ug} / \mathrm{L}$ & $\mathrm{U}$ \\
\hline 835 & M00823 & $6 / 23 / 97$ & REG & Chlorobenzene & 5 & $\mathrm{ug} / \mathrm{L}$ & $\mathrm{U}$ \\
\hline 835 & $\mathrm{~S} 02553$ & $2 / 24 / 95$ & REG & Chlorobenzene & 5 & $\mathrm{ug} / \mathrm{L}$ & $\mathrm{U}$ \\
\hline 835 & S03495 & $5 / 11 / 95$ & REG & Chlorobenzene & 1 & $\mathrm{ug} / \mathrm{L}$ & $\mathrm{U}$ \\
\hline 835 & S04714 & $7 / 19 / 95$ & REG & Chlorobenzene & 5 & ug/L & $\mathrm{U}$ \\
\hline 835 & 835227REGUF & $2 / 27 / 91$ & REG & Chlorobenzene & 5 & $\mathrm{ug} / \mathrm{L}$ & $\mathrm{U}$ \\
\hline 835 & 83561REGUF & $6 / 1 / 90$ & REG & Chlorobenzene & 5 & $\mathrm{ug} / \mathrm{L}$ & $\mathrm{U}$ \\
\hline 835 & 8351113REGUF & $11 / 13 / 90$ & REG & Chlorobenzene & 5 & $u g / L$ & $\mathrm{U}$ \\
\hline 835 & 835130REGUF & $1 / 30 / 89$ & REG & Chlorobenzene & 5 & $\mathrm{ug} / \mathrm{L}$ & $\mathrm{U}$ \\
\hline 835 & 83558REGUF & $5 / 8 / 89$ & REG & Chlorobenzene & 5 & $u g / L$ & $\mathrm{U}$ \\
\hline 835 & 835722REGUF & $7 / 22 / 88$ & REG & Chlorobenzene & 5 & $u g / L$ & $\mathrm{U}$ \\
\hline 835 & 8351028REGUF & $10 / 28 / 88$ & REG & Chlorobenzene & 5 & $\mathrm{ug} / \mathrm{L}$ & $\mathrm{U}$ \\
\hline 835 & M01225 & $11 / 11 / 97$ & REG & Chloroethane & 10 & ug/L & $\mathbf{U}$ \\
\hline 835 & W00806 & $6 / 23 / 97$ & REG & Chloroethane & 10 & $\mathrm{ug} / \mathrm{L}$ & $\mathrm{U}$ \\
\hline 835 & M0004l & $12 / 3 / 96$ & REG & Chloroethane & 10 & $\mathrm{ug} / \mathrm{L}$ & $\mathrm{U}$ \\
\hline 835 & M00823 & $6 / 23 / 97$ & REG & Chloroethane & 10 & $\mathrm{ug} / \mathrm{L}$ & $\mathrm{U}$ \\
\hline 835 & S02553 & $2 / 24 / 95$ & REG & Chloroethane & 10 & $u g / L$ & $\mathrm{U}$ \\
\hline 835 & S03495 & $5 / 11 / 95$ & REG & Chloroethane & 1 & $\mathrm{ug} / \mathrm{L}$ & $\mathrm{U}$ \\
\hline 835 & S04714 & $7 / 19 / 95$ & REG & Chloroethane & 10 & $\mathrm{ug} / \mathrm{L}$ & $\mathrm{U}$ \\
\hline 835 & 835227REGUF & $2 / 27 / 91$ & REG & Chloroethane & 10 & $\mathrm{ug} / \mathrm{L}$ & $\mathrm{U}$ \\
\hline 835 & 83561REGUF & $6 / 1 / 90$ & REG & Chloroethane & 10 & ug $/ \mathrm{L}$ & $\mathbf{U}$ \\
\hline 835 & 8351113REGUF & $11 / 13 / 90$ & REG & Chloroethane & 10 & $\mathrm{ug} / \mathrm{L}$ & $\mathrm{U}$ \\
\hline 835 & 835130REGUF & $1 / 30 / 89$ & REG & Chloroethane & 10 & $\mathrm{ug} / \mathrm{L}$ & $\mathrm{U}$ \\
\hline 835 & 83558REGUF & $5 / 8 / 89$ & REG & Chloroethane & 10 & $\mathrm{ug} / \mathrm{L}$ & $\mathrm{U}$ \\
\hline 835 & 835722REGUF & $7 / 22 / 88$ & REG & Chloroethane & 10 & $u g / L$ & $\mathbf{U}$ \\
\hline 835 & 8351028REGUF & $10 / 28 / 88$ & REG & Chloroethane & 10 & $\mathrm{ug} / \mathrm{L}$ & $\mathrm{U}$ \\
\hline 835 & M01225 & $11 / 11 / 97$ & REG & Chloroform & 5 & $u g / L$ & $\mathrm{U}$ \\
\hline 835 & W00806 & $6 / 23 / 97$ & REG & Chloroform & 5 & $u g / L$ & $\mathrm{U}$ \\
\hline 835 & M00041 & $12 / 3 / 96$ & REG & Chloroform & 5 & ug $/ \mathrm{L}$ & $\mathrm{U}$ \\
\hline 835 & M00823 & $6 / 23 / 97$ & REG & Chloroform & 5 & $\mathrm{ug} / \mathrm{L}$ & $\mathrm{U}$ \\
\hline 835 & S02553 & $2 / 24 / 95$ & REG & Chloroform & 5 & $\mathrm{ug} / \mathrm{L}$ & $\mathbf{U}$ \\
\hline 835 & S03495 & $5 / 11 / 95$ & REG & Chloroform & 1 & $\mathrm{ug} / \mathrm{L}$ & $\mathrm{U}$ \\
\hline 835 & S04714 & $7 / 19 / 95$ & REG & Chloroform & 5 & ug/L & $\mathrm{U}$ \\
\hline 835 & 835227REGUF & $2 / 27 / 91$ & REG & Chloroform & 5 & $u g / L$ & $\mathrm{U}$ \\
\hline 835 & 83561REGUF & $6 / 1 / 90$ & REG & Chloroform & 5 & $\mathrm{ug} / \mathrm{L}$ & $\mathrm{U}$ \\
\hline 835 & 8351113REGUF & $11 / 13 / 90$ & REG & Chloroform & 5 & $u g / L$ & $\mathrm{U}$ \\
\hline 835 & 835130REGUF & $1 / 30 / 89$ & REG & Chloroform & 5 & $\mathrm{ug} / \mathrm{L}$ & $\mathrm{U}$ \\
\hline 835 & 83558REGUF & $5 / 8 / 89$ & REG & Chloroform & 5 & $\mathrm{ug} / \mathrm{L}$ & $\mathrm{U}$ \\
\hline 835 & 835722REGUF & $7 / 22 / 88$ & REG & Chloroform & 5 & $\mathrm{ug} / \mathrm{L}$ & $\mathrm{U}$ \\
\hline
\end{tabular}




\begin{tabular}{|c|c|c|c|c|c|c|c|}
\hline Station & Sample ID & Sample Date & Sample Type & Chemical & Results & Units & Qualifier \\
\hline 835 & 8351028REGUF & $10 / 28 / 88$ & REG & Chloroform & 5 & $\mathrm{ug} / \mathrm{L}$ & $\bar{U}$ \\
\hline 835 & M01225 & $11 / 11 / 97$ & REG & Chloromethane & 10 & $\mathrm{ug} / \mathrm{L}$ & $\mathrm{U}$ \\
\hline 835 & W00806 & $6 / 23 / 97$ & REG & Chloromethane & 10 & $\mathrm{ug} / \mathrm{L}$ & $\mathrm{U}$ \\
\hline 835 & M00041 & $12 / 3 / 96$ & REG & Chloromethane & 10 & $u g / L$ & $\mathrm{U}$ \\
\hline 835 & $\mathrm{M} 00823$ & $6 / 23 / 97$ & REG & Chloromethane & 10 & $\mathrm{ug} / \mathrm{L}$ & $\mathrm{U}$ \\
\hline 835 & S02553 & $2 / 24 / 95$ & REG & Chloromethane & 10 & $\mathrm{ug} / \mathrm{L}$ & $\mathrm{U}$ \\
\hline 835 & S03495 & $5 / 11 / 95$ & REG & Chloromethane & 1 & $\mathrm{ug} / \mathrm{L}$ & $\mathrm{U}$ \\
\hline 835 & S04714 & $7 / 19 / 95$ & REG & Chloromethane & 10 & $\mathrm{ug} / \mathrm{L}$ & $\mathrm{U}$ \\
\hline 835 & 835227REGUF & $2 / 27 / 91$ & REG & Chloromethane & 10 & $\mathrm{ug} / \mathrm{L}$ & $\mathrm{U}$ \\
\hline 835 & 83561REGUF & $6 / 1 / 90$ & REG & Chloromethane & 10 & $u g / L$ & $\mathrm{U}$ \\
\hline 835 & 8351113REGUF & $11 / 13 / 90$ & REG & Chloromethane & 10 & $\mathrm{ug} / \mathrm{L}$ & $\mathrm{U}$ \\
\hline 835 & 835130REGUF & $1 / 30 / 89$ & REG & Chloromethane & 10 & $u g / L$ & $\mathrm{U}$ \\
\hline 835 & 83558REGUF & $5 / 8 / 89$ & REG & Chloromethane & 10 & $\mathrm{ug} / \mathrm{L}$ & $\mathrm{U}$ \\
\hline 835 & 835722REGUF & $7 / 22 / 88$ & REG & Chloromethane & 10 & $\mathrm{ug} / \mathrm{L}$ & $\mathrm{U}$ \\
\hline 835 & 8351028REGUF & $10 / 28 / 88$ & REG & Chloromethane & 10 & $\mathrm{ug} / \mathrm{L}$ & $\mathrm{U}$ \\
\hline 835 & M01225 & $11 / 11 / 97$ & REG & cis-1,2-Dichloroethene & 5 & $\mathrm{ug} / \mathrm{L}$ & $\mathrm{U}$ \\
\hline 835 & M00041 & $12 / 3 / 96$ & REG & cis-1,2-Dichloroethene & 5 & $\mathrm{ug} / \mathrm{L}$ & U \\
\hline 835 & M00823 & $6 / 23 / 97$ & REG & cis-1,2-Dichloroethene & 5 & ug/L & $\mathrm{U}$ \\
\hline 835 & $\mathrm{~S} 02553$ & $2 / 24 / 95$ & REG & cis-1,2-Dichloroethene & 5 & $\mathrm{ug} / \mathrm{L}$ & $\mathrm{U}$ \\
\hline 835 & S03495 & $5 / 11 / 95$ & REG & cis-1,2-Dichloroethene & 1 & ug $/$ & $\mathrm{U}$ \\
\hline 835 & S04714 & $7 / 19 / 95$ & REG & cis-1,2-Dichloroethene & 5 & $\mathrm{ug} / \mathrm{L}$ & $\mathrm{U}$ \\
\hline 835 & M01225 & $11 / 11 / 97$ & REG & cis-1,3-Dichloropropen & 5 & $\mathrm{ug} / \mathrm{L}$ & $\mathrm{U}$ \\
\hline 835 & W00806 & $6 / 23 / 97$ & REG & cis-1,3-Dichloropropene & 5 & $\mathrm{ug} / \mathrm{L}$ & $\mathrm{U}$ \\
\hline 835 & M00041 & $12 / 3 / 96$ & REG & cis-1,3-Dichloropropene & 5 & $\mathrm{ug} / \mathrm{L}$ & $\mathrm{U}$ \\
\hline 835 & M00823 & $6 / 23 / 97$ & REG & cis-1,3-Dichloropropene & 5 & $\mathrm{ug} / \mathrm{L}$ & $\mathrm{U}$ \\
\hline 835 & $\mathrm{~S} 02553$ & $2 / 24 / 95$ & REG & cis-1,3-Dichloropropene & 5 & $\mathrm{ug} / \mathrm{L}$ & $\mathrm{U}$ \\
\hline 835 & S03495 & $5 / 11 / 95$ & REG & cis-1,3-Dichloropropene & 1 & $\mathrm{ug} / \mathrm{L}$ & $\mathrm{U}$ \\
\hline 835 & S04714 & $7 / 19 / 95$ & REG & cis-1,3-Dichloropropene & 5 & $\mathrm{ug} / \mathrm{L}$ & $\mathrm{U}$ \\
\hline 835 & 835227REGUF & $2 / 27 / 91$ & REG & cis-1,3-Dichloropropene & 5 & $\mathrm{ug} / \mathrm{L}$ & $\mathrm{U}$ \\
\hline 835 & 8356IREGUF & $6 / 1 / 90$ & REG & cis-1,3-Dichloropropene & 5 & $\mathrm{ug} / \mathrm{L}$ & $\mathrm{U}$ \\
\hline 835 & 8351113REGUF & $11 / 13 / 90$ & REG & cis-1,3-Dichloropropene & 5 & $\mathrm{ug} / \mathrm{L}$ & $\mathrm{U}$ \\
\hline 835 & 835130REGUF & $1 / 30 / 89$ & REG & cis-1,3-Dichloropropene & 5 & ug/L & $\mathrm{U}$ \\
\hline 835 & 83558REGUF & $5 / 8 / 89$ & REG & cis-1,3-Dichloropropene & 5 & $\mathrm{ug} / \mathrm{L}$ & $\mathrm{U}$ \\
\hline 835 & 835722REGUF & $7 / 22 / 88$ & REG & cis-1,3-Dichloropropene & 5 & $u g / L$ & $\mathrm{U}$ \\
\hline 835 & 8351028REGUF & $10 / 28 / 88$ & REG & cis-1,3-Dichloropropene & 5 & $\mathrm{ug} / \mathrm{L}$ & $\mathrm{U}$ \\
\hline 835 & M01225 & $11 / 11 / 97$ & REG & Dibromochloromethane & 5 & $\mathrm{ug} / \mathrm{L}$ & $\mathrm{U}$ \\
\hline 835 & W00806 & $6 / 23 / 97$ & REG & Dibromochloromethane & 5 & $\mathrm{ug} / \mathrm{L}$ & U \\
\hline 835 & M0004l & $12 / 3 / 96$ & REG & Dibromochloromethane & 5 & $\mathrm{ug} / \mathrm{L}$ & $\mathrm{U}$ \\
\hline 835 & M00823 & $6 / 23 / 97$ & REG & Dibromochloromethane & 5 & $\mathrm{ug} / \mathrm{L}$ & $\mathrm{U}$ \\
\hline 835 & S02553 & $2 / 24 / 95$ & REG & Dibromochloromethane & 5 & $\mathrm{ug} / \mathrm{L}$ & $\mathrm{U}$ \\
\hline 835 & S03495 & $5 / 11 / 95$ & REG & Dibromochloromethane & 1 & ug/L & $\mathrm{U}$ \\
\hline 835 & S04714 & $7 / 19 / 95$ & REG & Dibromochloromethane & 5 & $\mathrm{ug} / \mathrm{L}$ & $\mathrm{U}$ \\
\hline 835 & 835227REGUF & $2 / 27 / 91$ & REG & Dibromochloromethane & 5 & $\mathrm{ug} / \mathrm{L}$ & $\mathrm{U}$ \\
\hline 835 & 83561REGUF & $6 / 1 / 90$ & REG & Dibromochloromethane & 5 & ug/L & $\mathrm{U}$ \\
\hline 835 & 8351113REGUF & $11 / 13 / 90$ & REG & Dibromochloromethane & 5 & $\mathrm{ug} / \mathrm{L}$ & $\mathrm{U}$ \\
\hline 835 & 835130REGUF & $1 / 30 / 89$ & REG & Dibromochloromethane & 5 & $\mathrm{ug} / \mathrm{L}$ & $\mathrm{U}$ \\
\hline 835 & 83558REGUF & $5 / 8 / 89$ & REG & Dibromochloromethane & 5 & $\mathrm{ug} / \mathrm{L}$ & $U$ \\
\hline 835 & 835722REGUF & $7 / 22 / 88$ & REG & Dibromochloromethane & 5 & $\mathrm{ug} / \mathrm{L}$ & $\mathrm{U}$ \\
\hline 835 & 8351028REGUF & $10 / 28 / 88$ & REG & Dibromochloromethane & 5 & $\mathrm{ug} / \mathrm{L}$ & $\mathrm{U}$ \\
\hline 835 & M00041 & $12 / 3 / 96$ & REG & Dimethylbenzene & 5 & $\mathrm{ug} / \mathrm{L}$ & $\mathrm{U}$ \\
\hline
\end{tabular}


B-10

\begin{tabular}{|c|c|c|c|c|c|c|c|}
\hline Station & Sample ID & Sample Date & Sample Type & Chemical & Results & Units & Qualifier \\
\hline 835 & 835227REGUF & $2 / 27 / 91$ & REG & Dimethylbenzene & 5 & $\mathrm{ug} / \mathrm{L}$ & $\mathrm{U}$ \\
\hline 835 & 83561REGUF & $6 / 1 / 90$ & REG & Dimethylbenzene & 5 & $\mathrm{ug} / \mathrm{L}$ & $\mathrm{U}$ \\
\hline 835 & 8351113REGUF & $11 / 13 / 90$ & REG & Dimethylbenzene & 5 & ug/L & $\mathrm{U}$ \\
\hline 835 & 835130REGUF & $1 / 30 / 89$ & REG & Dimethylbenzene & 5 & $\mathrm{ug} / \mathrm{L}$ & $\mathrm{U}$ \\
\hline 835 & 83558REGUF & $5 / 8 / 89$ & REG & Dimethylbenzene & 5 & $\mathrm{ug} / \mathrm{L}$ & $\mathrm{U}$ \\
\hline 835 & 835722REGUF & $7 / 22 / 88$ & REG & Dimethylbenzene & 5 & $\mathrm{ug} / \mathrm{L}$ & $\mathrm{U}$ \\
\hline 835 & 8351028REGUF & $10 / 28 / 88$ & REG & Dimethylbenzene & 5 & ug/L & $\mathrm{U}$ \\
\hline 835 & M01225 & $11 / 11 / 97$ & REG & Ethylbenzene & 5 & $\mathrm{ug} / \mathrm{L}$ & $\mathrm{U}$ \\
\hline 835 & W00806 & $6 / 23 / 97$ & REG & Ethylbenzene & 5 & $\mathrm{ug} / \mathrm{L}$ & $\mathrm{U}$ \\
\hline 835 & M00041 & $12 / 3 / 96$ & REG & Ethylbenzene & 5 & $\mathrm{ug} / \mathrm{L}$ & $\mathrm{U}$ \\
\hline 835 & M00823 & $6 / 23 / 97$ & REG & Ethylbenzene & 5 & $u g / L$ & $\mathrm{U}$ \\
\hline 835 & $\mathrm{~S} 02553$ & $2 / 24 / 95$ & REG & Ethylbenzene & 5 & $\mathrm{ug} / \mathrm{L}$ & $\mathrm{U}$ \\
\hline 835 & S03495 & $5 / 11 / 95$ & REG & Ethylbenzene & 1 & $\mathrm{ug} / \mathrm{L}$ & $U$ \\
\hline 835 & S04714 & $7 / 19 / 95$ & REG & Ethylbenzene & 5 & $\mathrm{ug} / \mathrm{L}$ & $\mathrm{U}$ \\
\hline 835 & 835227REGUF & $2 / 27 / 91$ & REG & Ethylbenzene & 5 & $\mathrm{ug} / \mathrm{L}$ & $\mathrm{U}$ \\
\hline 835 & 83561REGUF & $6 / 1 / 90$ & REG & Ethylbenzene & 5 & $\mathrm{ug} / \mathrm{L}$ & $\mathrm{U}$ \\
\hline 835 & 8351113REGUF & $11 / 13 / 90$ & REG & Ethylbenzene & 5 & $\mathrm{ug} / \mathrm{L}$ & $\mathrm{U}$ \\
\hline 835 & 835130REGUF & $1 / 30 / 89$ & REG & Ethylbenzene & 5 & $\mathrm{ug} / \mathrm{L}$ & $\mathrm{U}$ \\
\hline 835 & 83558REGUF & $5 / 8 / 89$ & REG & Ethylbenzene & 5 & $\mathrm{ug} / \mathrm{L}$ & $\mathrm{U}$ \\
\hline 835 & 835722REGUF & $7 / 22 / 88$ & REG & Ethylbenzene & 5 & ug/L & $\mathrm{U}$ \\
\hline 835 & 8351028REGUF & $10 / 28 / 88$ & REG & Ethylbenzene & 5 & $\mathrm{ug} / \mathrm{L}$ & $\mathrm{U}$ \\
\hline 835 & M01225 & $11 / 11 / 97$ & REG & m,p-Xylene & 5 & $\mathrm{ug} / \mathrm{L}$ & $\mathrm{U}$ \\
\hline 835 & W00806 & $6 / 23 / 97$ & REG & m,p-Xylene & 5 & $\mathrm{ug} / \mathrm{L}$ & $\mathrm{U}$ \\
\hline 835 & M00041 & $12 / 3 / 96$ & REG & $\mathrm{m}, \mathrm{p}$-Xylene & 5 & ug/L & $\mathrm{U}$ \\
\hline 835 & M00823 & $6 / 23 / 97$ & REG & $\mathrm{m}, \mathrm{p}$-Xylene & 5 & $\mathrm{ug} / \mathrm{L}$ & $\mathrm{U}$ \\
\hline 835 & S02553 & $2 / 24 / 95$ & REG & $\mathrm{m}, \mathrm{p}$-Xylene & 5 & $\mathrm{ug} / \mathrm{L}$ & $\mathrm{U}$ \\
\hline 835 & S04714 & $7 / 19 / 95$ & REG & $\mathrm{m}, \mathrm{p}$-Xylene & 5 & ug/L & $\mathrm{U}$ \\
\hline 835 & M01225 & $11 / 11 / 97$ & REG & Methylene chloride & 5 & $\mathrm{ug} / \mathrm{L}$ & B \\
\hline 835 & W00806 & $6 / 23 / 97$ & REG & Methylene chloride & 9 & $\mathrm{ug} / \mathrm{L}$ & $=$ \\
\hline 835 & M0004l & $12 / 3 / 96$ & REG & Methylene chloride & 5 & $\mathrm{ug} / \mathrm{L}$ & U \\
\hline 835 & M00823 & $6 / 23 / 97$ & REG & Methylene chloride & 2 & $\mathrm{ug} / \mathrm{L}$ & $\mathrm{U}$ \\
\hline 835 & S02553 & $2 / 24 / 95$ & REG & Methylene chloride & 5 & $\mathrm{ug} / \mathrm{L}$ & $\mathbf{U}$ \\
\hline 835 & S03495 & $5 / 11 / 95$ & REG & Methylene chloride & 2 & $\mathrm{ug} / \mathrm{L}$ & $\mathrm{U}$ \\
\hline 835 & S04714 & $7 / 19 / 95$ & REG & Methylene chloride & 5 & $\mathrm{ug} / \mathrm{L}$ & $\mathrm{U}$ \\
\hline 835 & 835227REGUF & $2 / 27 / 91$ & REG & Methylene chloride & 5 & $\mathrm{ug} / \mathrm{L}$ & $\mathrm{U}$ \\
\hline 835 & 83561REGUF & $6 / 1 / 90$ & REG & Methylene chloride & 5 & $\mathrm{ug} / \mathrm{L}$ & $\mathrm{U}$ \\
\hline 835 & 8351113REGUF & $11 / 13 / 90$ & REG & Methylene chloride & 5 & $\mathrm{ug} / \mathrm{L}$ & $\mathrm{U}$ \\
\hline 835 & 835130REGUF & $1 / 30 / 89$ & REG & Methylene chloride & 0.8 & $\mathrm{ug} / \mathrm{L}$ & $\mathrm{J}$ \\
\hline 835 & 83558REGUF & $5 / 8 / 89$ & REG & Methylene chloride & 5 & $\mathrm{ug} / \mathrm{L}$ & $\mathrm{U}$ \\
\hline 835 & 835722REGUF & $7 / 22 / 88$ & REG & Methylene chloride & 1 & $\mathrm{ug} / \mathrm{L}$ & J \\
\hline 835 & 8351028REGUF & $10 / 28 / 88$ & REG & Methylene chloride & 3 & $\mathrm{ug} / \mathrm{L}$ & J \\
\hline 835 & M01225 & $11 / 11 / 97$ & REG & Styrene & 5 & $\mathrm{ug} / \mathrm{L}$ & $\mathrm{U}$ \\
\hline 835 & W00806 & $6 / 23 / 97$ & REG & Styrene & 5 & $\mathrm{ug} / \mathrm{L}$ & $\mathrm{U}$ \\
\hline 835 & M00041 & $12 / 3 / 96$ & REG & Styrene & 5 & $\mathrm{ug} / \mathrm{L}$ & U \\
\hline 835 & M00823 & $6 / 23 / 97$ & REG & Styrene & 5 & ug/L & U \\
\hline 835 & S02553 & $2 / 24 / 95$ & REG & Styrene & 5 & $\mathrm{ug} / \mathrm{L}$ & $\mathrm{U}$ \\
\hline 835 & S03495 & $5 / 11 / 95$ & REG & Styrene & 1 & ug/L & $\mathrm{U}$ \\
\hline 835 & S04714 & $7 / 19 / 95$ & REG & Styrene & 5 & $\mathrm{ug} / \mathrm{L}$ & $\mathrm{U}$ \\
\hline 835 & 835227REGUF & $2 / 27 / 91$ & REG & Styrene & 5 & $\mathrm{ug} / \mathrm{L}$ & $\mathrm{U}$ \\
\hline 835 & 83561REGUF & $6 / 1 / 90$ & REG & Styrene & 5 & $\mathrm{ug} / \mathrm{L}$ & $\mathrm{U}$ \\
\hline
\end{tabular}


B-11

Station Sample ID Sample Date Sample Type Chemical Results Units Qualifier

\begin{tabular}{|c|c|c|c|c|c|c|c|}
\hline 835 & 8351113REGUF & $11 / 13 / 90$ & REG & Styrene & 5 & $\mathrm{ug} / \mathrm{L}$ & $\mathrm{U}$ \\
\hline 835 & 835130REGUF & $1 / 30 / 89$ & REG & Styrene & 5 & $\mathrm{ug} / \mathrm{L}$ & $\mathrm{U}$ \\
\hline 835 & 83558REGUF & $5 / 8 / 89$ & REG & Styrene & 5 & $u g / L$ & $\mathrm{U}$ \\
\hline 835 & 835722REGUF & $7 / 22 / 88$ & REG & Styrene & 5 & $\mathrm{ug} / \mathrm{L}$ & $\mathrm{U}$ \\
\hline 835 & 8351028REGUF & $10 / 28 / 88$ & REG & Styrene & 5 & $\mathrm{ug} / \mathrm{L}$ & $\mathrm{U}$ \\
\hline 835 & M01225 & $11 / 11 / 97$ & REG & Tetrachloroethene & 5 & $\mathrm{ug} / \mathrm{L}$ & $\mathrm{U}$ \\
\hline 835 & W00806 & $6 / 23 / 97$ & REG & Tetrachloroethene & 5 & $\mathrm{ug} / \mathrm{L}$ & $\mathrm{U}$ \\
\hline 835 & M0004l & $12 / 3 / 96$ & REG & Tetrachloroethene & 5 & $\mathrm{ug} / \mathrm{L}$ & $\mathrm{U}$ \\
\hline 835 & M00823 & $6 / 23 / 97$ & REG & Tetrachloroethene & 5 & $\mathrm{ug} / \mathrm{L}$ & $\mathrm{U}$ \\
\hline 835 & S02553 & $2 / 24 / 95$ & REG & Tetrachloroethene & 5 & $\mathrm{ug} / \mathrm{L}$ & $\mathrm{U}$ \\
\hline 835 & S03495 & $5 / 11 / 95$ & REG & Tetrachloroethene & 1 & $\mathrm{ug} / \mathrm{L}$ & $\mathrm{U}$ \\
\hline 835 & S04714 & $7 / 19 / 95$ & REG & Tetrachloroethene & 5 & $\mathrm{ug} / \mathrm{L}$ & $\mathrm{U}$ \\
\hline 835 & 835227REGUF & $2 / 27 / 91$ & REG & Tetrachloroethene & 5 & $\mathrm{ug} / \mathrm{L}$ & $\mathrm{U}$ \\
\hline 835 & 8356IREGUF & $6 / 1 / 90$ & $\mathrm{REG}$ & Tetrachloroethene & 5 & $\mathrm{ug} / \mathrm{L}$ & $\mathrm{U}$ \\
\hline 835 & 8351113REGUF & $11 / 13 / 90$ & REG & Tetrachloroethene & 5 & $\mathrm{ug} / \mathrm{L}$ & $\mathrm{U}$ \\
\hline 835 & 835130REGUF & $1 / 30 / 89$ & REG & Tetrachloroethene & 5 & $\mathrm{ug} / \mathrm{L}$ & $\mathrm{U}$ \\
\hline 835 & 83558REGUF & $5 / 8 / 89$ & REG & Tetrachloroethene & 5 & ug/L & $\mathrm{U}$ \\
\hline 835 & 835722REGUF & $7 / 22 / 88$ & REG & Tetrachloroethene & 5 & $\mathrm{ug} / \mathrm{L}$ & $\mathrm{U}$ \\
\hline 835 & 8351028REGUF & $10 / 28 / 88$ & REG & Tetrachloroethene & 5 & $\mathrm{ug} / \mathrm{L}$ & $\mathrm{U}$ \\
\hline 835 & M01225 & $11 / 11 / 97$ & REG & Toluene & 5 & $\mathrm{ug} / \mathrm{L}$ & $\mathrm{U}$ \\
\hline 835 & W00806 & $6 / 23 / 97$ & REG & Toluene & 5 & $\mathrm{ug} / \mathrm{L}$ & $\mathrm{U}$ \\
\hline 835 & M00041 & $12 / 3 / 96$ & REG & Toluene & 5 & $\mathrm{ug} / \mathrm{L}$ & $\mathrm{U}$ \\
\hline 835 & M00823 & 6/23/97 & REG & Toluene & 5 & $\mathrm{ug} / \mathrm{L}$ & $\mathrm{U}$ \\
\hline 835 & S02553 & $2 / 24 / 95$ & REG & Toluene & 5 & $\mathrm{ug} / \mathrm{L}$ & $\mathrm{U}$ \\
\hline 835 & S03495 & $5 / 11 / 95$ & REG & Toluene & 1 & $\mathrm{ug} / \mathrm{L}$ & $\mathrm{U}$ \\
\hline 835 & S04714 & 7/19/95 & REG & Toluene & 5 & $\mathrm{ug} / \mathrm{L}$ & U \\
\hline 835 & 835227REGUF & $2 / 27 / 91$ & REG & Toluene & 5 & ug/L & $\mathrm{U}$ \\
\hline 835 & 83561REGUF & $6 / 1 / 90$ & REG & Toluene & 5 & $\mathrm{ug} / \mathrm{L}$ & $\mathrm{U}$ \\
\hline 835 & 8351113REGUF & $11 / 13 / 90$ & REG & Toluene & 5 & $\mathrm{ug} / \mathrm{L}$ & U \\
\hline 835 & 835130REGUF & $1 / 30 / 89$ & REG & Toluene & 5 & $\mathrm{ug} / \mathrm{L}$ & U \\
\hline 835 & 83558REGUF & $5 / 8 / 89$ & REG & Toluene & 2 & $\mathrm{ug} / \mathrm{L}$ & $\mathrm{J}$ \\
\hline 835 & 835722REGUF & $7 / 22 / 88$ & REG & Toluene & 5 & $\mathrm{ug} / \mathrm{L}$ & $\mathrm{U}$ \\
\hline 835 & 8351028REGUF & $10 / 28 / 88$ & REG & Toluene & 5 & $\mathrm{ug} / \mathrm{L}$ & $\mathrm{U}$ \\
\hline 835 & M01225 & $11 / 11 / 97$ & REG & trans-1,2-Dichloroethe & 5 & $\mathrm{ug} / \mathrm{L}$ & $\mathrm{U}$ \\
\hline 835 & M00041 & $12 / 3 / 96$ & REG & trans-1,2-Dichloroethene & 5 & $\mathrm{ug} / \mathrm{L}$ & $\mathrm{U}$ \\
\hline 835 & M00823 & $6 / 23 / 97$ & REG & trans-1,2-Dichloroethene & 5 & $\mathrm{ug} / \mathrm{L}$ & $\mathrm{U}$ \\
\hline 835 & S02553 & $2 / 24 / 95$ & REG & trans-1,2-Dichloroethene & 5 & $\mathrm{ug} / \mathrm{L}$ & $\mathrm{U}$ \\
\hline 835 & S03495 & $5 / 11 / 95$ & REG & trans-1,2-Dichloroethene & 1 & ug/L & $\mathrm{U}$ \\
\hline 835 & S04714 & $7 / 19 / 95$ & REG & trans-1,2-Dichloroethene & 5 & $\mathrm{ug} / \mathrm{L}$ & $\mathrm{U}$ \\
\hline 835 & M01225 & $11 / 11 / 97$ & REG & trans-1,3-Dichloroprop & 5 & $\mathrm{ug} / \mathrm{L}$ & $\mathrm{U}$ \\
\hline 835 & W00806 & $6 / 23 / 97$ & REG & trans-1,3-Dichloropropene & 5 & $\mathrm{ug} / \mathrm{L}$ & $\mathrm{U}$ \\
\hline 835 & M00041 & $12 / 3 / 96$ & REG & trans-1,3-Dichloropropene & 5 & $\mathrm{ug} / \mathrm{L}$ & $\mathrm{U}$ \\
\hline 835 & M00823 & $6 / 23 / 97$ & REG & trans-1,3-Dichloropropene & 5 & $\mathrm{ug} / \mathrm{L}$ & $\mathrm{U}$ \\
\hline 835 & S02553 & $2 / 24 / 95$ & REG & trans-1,3-Dichloropropene & 5 & ug/L & $\mathrm{U}$ \\
\hline 835 & S03495 & $5 / 11 / 95$ & REG & trans-1,3-Dichloropropene & 1 & ug/L & $\mathrm{U}$ \\
\hline 835 & S04714 & $7 / 19 / 95$ & REG & trans-1,3-Dichloropropene & 5 & $\mathrm{ug} / \mathrm{L}$ & $\mathrm{U}$ \\
\hline 835 & 835227REGUF & $2 / 27 / 91$ & REG & trans-1,3-Dichloropropene & 5 & $\mathrm{ug} / \mathrm{L}$ & $\mathbf{U}$ \\
\hline 835 & 83561REGUF & $6 / 1 / 90$ & REG & trans-1,3-Dichloropropene & 5 & $\mathrm{ug} / \mathrm{L}$ & $\mathrm{U}$ \\
\hline 835 & 8351113REGUF & $11 / 13 / 90$ & REG & trans-1,3-Dichloropropene & 5 & $\mathrm{ug} / \mathrm{L}$ & $\mathrm{U}$ \\
\hline 835 & 835130REGUF & $1 / 30 / 89$ & REG & trans-1,3-Dichloropropene & 5 & $\mathrm{ug} / \mathrm{L}$ & $\mathrm{U}$ \\
\hline
\end{tabular}


B-12

\begin{tabular}{|c|c|c|c|c|c|c|c|}
\hline Station & Sample ID & Sample Date & Sample Type & Chemical & Results & Units & Qualifier \\
\hline$\overline{835}$ & 83558REGUF & $5 / 8 / 89$ & REG & trans-1,3-Dichloropropene & 5 & $\mathrm{ug} / \mathrm{L}$ & $\mathrm{U}$ \\
\hline 835 & 835722REGUF & $7 / 22 / 88$ & REG & trans-1,3-Dichloropropene & 5 & $u g / L$ & U \\
\hline 835 & 8351028REGUF & $10 / 28 / 88$ & REG & trans-1,3-Dichloropropene & 5 & $\mathrm{ug} / \mathrm{L}$ & $\mathrm{U}$ \\
\hline 835 & M01225 & $11 / 11 / 97$ & REG & Trichloroethene & 5 & $\mathrm{ug} / \mathrm{L}$ & $U$ \\
\hline 835 & W00806 & $6 / 23 / 97$ & REG & Trichloroethene & 5 & $\mathrm{ug} / \mathrm{L}$ & $\mathrm{U}$ \\
\hline 835 & M00041 & $12 / 3 / 96$ & REG & Trichloroethene & 5 & $u g / L$ & $\mathrm{U}$ \\
\hline 835 & $\mathrm{M} 00823$ & $6 / 23 / 97$ & REG & Trichloroethene & 5 & $\mathrm{ug} / \mathrm{L}$ & $\mathrm{U}$ \\
\hline 835 & S02553 & $2 / 24 / 95$ & REG & Trichloroethene & 5 & $\mathrm{ug} / \mathrm{L}$ & $\mathrm{U}$ \\
\hline 835 & S03495 & $5 / 11 / 95$ & REG & Trichloroethene & 1 & $\mathrm{ug} / \mathrm{L}$ & $\mathrm{U}$ \\
\hline 835 & S04714 & $7 / 19 / 95$ & REG & Trichloroethene & 5 & $\mathrm{ug} / \mathrm{L}$ & $\mathrm{U}$ \\
\hline 835 & 835227REGUF & $2 / 27 / 91$ & REG & Trichloroethene & 5 & $\mathrm{ug} / \mathrm{L}$ & $\mathrm{U}$ \\
\hline 835 & 8356IREGUF & $6 / 1 / 90$ & REG & Trichloroethene & 5 & $\mathrm{ug} / \mathrm{L}$ & $\mathrm{U}$ \\
\hline 835 & 8351113REGUF & $11 / 13 / 90$ & REG & Trichloroethene & 5 & ug/L & $\mathrm{U}$ \\
\hline 835 & 835130REGUF & $1 / 30 / 89$ & REG & Trichloroethene & 5 & $\mathrm{ug} / \mathrm{L}$ & $\mathrm{U}$ \\
\hline 835 & 83558REGUF & $5 / 8 / 89$ & REG & Trichloroethene & 2 & $\mathrm{ug} / \mathrm{L}$ & $\mathrm{J}$ \\
\hline 835 & 835722REGUF & $7 / 22 / 88$ & REG & Trichloroethene & 5 & $\mathrm{ug} / \mathrm{L}$ & $\mathrm{U}$ \\
\hline 835 & 8351028REGUF & $10 / 28 / 88$ & REG & Trichloroethene & 5 & $u g / L$ & $\mathrm{U}$ \\
\hline 835 & M01225 & $11 / 11 / 97$ & REG & Vinyl acetate & 10 & $u g / L$ & $\mathrm{U}$ \\
\hline 835 & M00041 & $12 / 3 / 96$ & REG & Vinyl acetate & 10 & $\mathrm{ug} / \mathrm{L}$ & $\mathrm{U}$ \\
\hline 835 & M00823 & $6 / 23 / 97$ & REG & Vinyl acetate & 10 & $\mathrm{ug} / \mathrm{L}$ & $\mathrm{U}$ \\
\hline 835 & $\mathrm{~S} 02553$ & $2 / 24 / 95$ & REG & Vinyl acetate & 10 & $\mathrm{ug} / \mathrm{L}$ & $\mathrm{U}$ \\
\hline 835 & S04714 & $7 / 19 / 95$ & REG & Vinyl acetate & 10 & $\mathrm{ug} / \mathrm{L}$ & $\mathrm{U}$ \\
\hline 835 & 835227REGUF & $2 / 27 / 91$ & REG & Vinyl acetate & 10 & $\mathrm{ug} / \mathrm{L}$ & $\mathrm{U}$ \\
\hline 835 & 8356IREGUF & $6 / 1 / 90$ & REG & Vinyl acetate & 10 & $\mathrm{ug} / \mathrm{L}$ & $\mathrm{U}$ \\
\hline 835 & 8351113REGUF & $11 / 13 / 90$ & REG & Vinyl acetate & 10 & $\mathrm{ug} / \mathrm{L}$ & $\mathrm{U}$ \\
\hline 835 & 835130REGUF & $1 / 30 / 89$ & REG & Vinyl acetate & 10 & $\mathrm{ug} / \mathrm{L}$ & $\mathrm{U}$ \\
\hline 835 & 83558REGUF & $5 / 8 / 89$ & REG & Vinyl acetate & 10 & $\mathrm{ug} / \mathrm{L}$ & $\mathrm{U}$ \\
\hline 835 & 835722REGUF & $7 / 22 / 88$ & REG & Vinyl acetate & 10 & $\mathrm{ug} / \mathrm{L}$ & $\mathrm{U}$ \\
\hline 835 & 8351028REGUF & $10 / 28 / 88$ & REG & Vinyl acetate & 10 & $\mathrm{ug} / \mathrm{L}$ & U \\
\hline 835 & M01225 & $11 / 11 / 97$ & REG & Vinyl chloride & 10 & $\mathrm{ug} / \mathrm{L}$ & $\mathrm{U}$ \\
\hline 835 & W00806 & $6 / 23 / 97$ & REG & Vinyl chloride & 10 & $\mathrm{ug} / \mathrm{L}$ & $\mathrm{U}$ \\
\hline 835 & M00041 & $12 / 3 / 96$ & REG & Vinyl chloride & 10 & $\mathrm{ug} / \mathrm{L}$ & $\mathrm{U}$ \\
\hline 835 & M00823 & $6 / 23 / 97$ & REG & Vinyl chloride & 10 & ug/L & $\mathrm{U}$ \\
\hline 835 & S02553 & $2 / 24 / 95$ & REG & Vinyl chloride & 10 & $\mathrm{ug} / \mathrm{L}$ & $\mathrm{U}$ \\
\hline 835 & S03495 & $5 / 11 / 95$ & REG & Vinyl chloride & 1 & $\mathrm{ug} / \mathrm{L}$ & $\mathrm{U}$ \\
\hline 835 & S04714 & $7 / 19 / 95$ & REG & Vinyl chloride & 10 & $\mathrm{ug} / \mathrm{L}$ & $\mathrm{U}$ \\
\hline 835 & 835227REGUF & $2 / 27 / 91$ & REG & Vinyl chloride & 10 & $\mathrm{ug} / \mathrm{L}$ & U \\
\hline 835 & 83561REGUF & $6 / 1 / 90$ & REG & Vinyl chloride & 10 & $\mathrm{ug} / \mathrm{L}$ & $\mathrm{U}$ \\
\hline 835 & 8351113REGUF & $11 / 13 / 90$ & REG & Vinyl chloride & 10 & ug/L & $\mathrm{U}$ \\
\hline 835 & 835130REGUF & $1 / 30 / 89$ & REG & Vinyl chloride & 10 & $\mathrm{ug} / \mathrm{L}$ & $\mathrm{U}$ \\
\hline 835 & 83558REGUF & $5 / 8 / 89$ & REG & Vinyl chloride & 10 & $\mathrm{ug} / \mathrm{L}$ & $\mathrm{U}$ \\
\hline 835 & 835722REGUF & $7 / 22 / 88$ & REG & Vinyl chloride & 10 & $\mathrm{ug} / \mathrm{L}$ & $\mathrm{U}$ \\
\hline 835 & 8351028REGUF & $10 / 28 / 88$ & REG & Vinyl chloride & 10 & $\mathrm{ug} / \mathrm{L}$ & $\mathrm{U}$ \\
\hline 837 & M01229 & $11 / 10 / 97$ & REG & 1,1,1-Trichloroethane & 5 & $\mathrm{ug} / \mathrm{L}$ & $\mathrm{U}$ \\
\hline 837 & W00897 & $6 / 23 / 97$ & REG & 1,1,1-Trichloroethane & 5 & $\mathrm{ug} / \mathrm{L}$ & $\mathrm{U}$ \\
\hline 837 & M00047 & $11 / 13 / 96$ & REG & 1,1,1-Trichloroethane & 5 & $\mathrm{ug} / \mathrm{L}$ & $\mathrm{U}$ \\
\hline 837 & M01012 & $6 / 23 / 97$ & REG & 1,1,1-Trichloroethane & 5 & $\mathrm{ug} / \mathrm{L}$ & $\mathrm{U}$ \\
\hline 837 & S02571 & $2 / 14 / 95$ & REG & 1,1,1-Trichloroethane & 5 & $\mathrm{ug} / \mathrm{L}$ & $\mathrm{U}$ \\
\hline 837 & $\mathrm{~S} 03515$ & $5 / 11 / 95$ & REG & 1,1,1-Trichloroethane & 1 & ug/L & $\mathrm{U}$ \\
\hline 837 & S04732 & $8 / 11 / 95$ & REG & 1,1,1-Trichloroethane & 5 & $\mathrm{ug} / \mathrm{L}$ & $\mathrm{U}$ \\
\hline
\end{tabular}


B-13

Station Sample ID Sample Date Sample Type Chemical

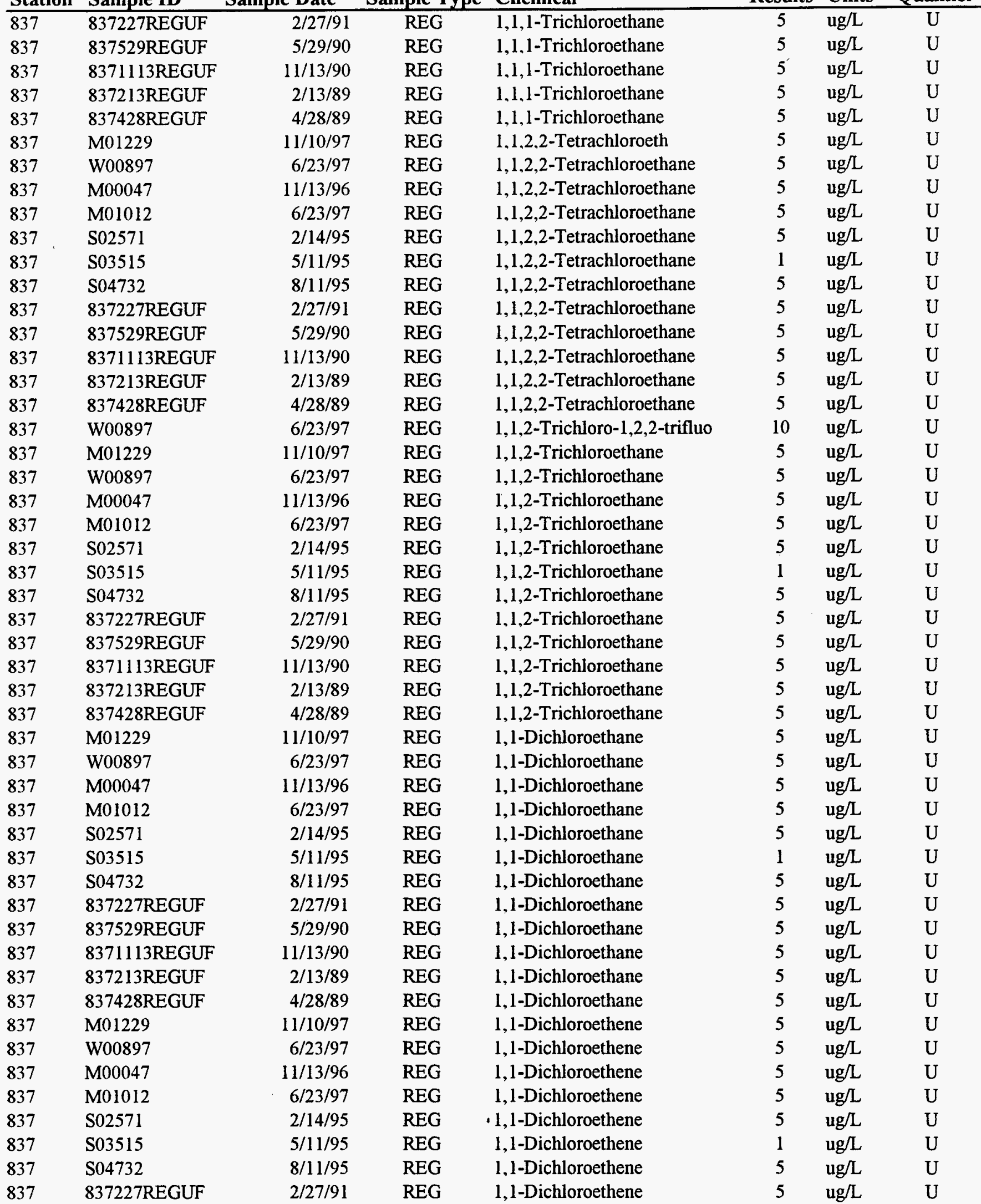


B-14

\begin{tabular}{|c|c|c|c|c|c|c|c|}
\hline Station & Sample ID & Sample Date & Sample Type & Chemical & Results & Units & Qualifier \\
\hline 837 & 837529REGUF & $5 / 29 / 90$ & REG & 1,1-Dichloroethene & 5 & $\mathrm{ug} / \mathrm{L}$ & $\mathrm{U}$ \\
\hline 837 & 8371113REGUF & $11 / 13 / 90$ & REG & 1,1-Dichloroethene & 5 & $\mathrm{ug} / \mathrm{L}$ & $\mathrm{U}$ \\
\hline 837 & 837213REGUF & $2 / 13 / 89$ & REG & 1.1-Dichloroethene & 5 & $\mathrm{ug} / \mathrm{L}$ & $\mathrm{U}$ \\
\hline 837 & 837428REGUF & $4 / 28 / 89$ & REG & 1,1-Dichloroethene & 5 & $\mathrm{ug} / \mathrm{L}$ & $\mathrm{U}$ \\
\hline 837 & S03515 & $5 / 11 / 95$ & REG & 1,2-Dibromo-3-chloropropane & 1 & $u g / L$ & $\mathrm{U}$ \\
\hline 837 & S03515 & $5 / 11 / 95$ & REG & 1,2-Dibromoethane & 1 & $u g / L$ & $\mathrm{U}$ \\
\hline 837 & M00047 & $11 / 13 / 96$ & REG & 1,2-Dichlorobenzene & 5 & ug/L & $\mathrm{U}$ \\
\hline 837 & M01229 & $11 / 10 / 97$ & REG & 1.2-Dichloroethane & 5 & $\mathrm{ug} / \mathrm{L}$ & $\mathrm{U}$ \\
\hline 837 & W00897 & $6 / 23 / 97$ & REG & 1,2-Dichloroethane & 5 & ug/L & $\mathrm{U}$ \\
\hline 837 & M00047 & $11 / 13 / 96$ & REG & 1,2-Dichloroethane & 5 & $\mathrm{ug} / \mathrm{L}$ & U \\
\hline 837 & M01012 & $6 / 23 / 97$ & REG & 1,2-Dichloroethane & 5 & $\mathrm{ug} / \mathrm{L}$ & $\mathrm{U}$ \\
\hline 837 & S02571 & $2 / 14 / 95$ & REG & 1,2-Dichloroethane & 5 & $\mathrm{ug} / \mathrm{L}$ & $\mathrm{U}$ \\
\hline 837 & S03515 & $5 / 11 / 95$ & REG & 1,2-Dichloroethane & 1 & $\mathrm{ug} / \mathrm{L}$ & $\mathrm{U}$ \\
\hline 837 & S04732 & $8 / 11 / 95$ & REG & 1,2-Dichloroethane & 5 & $u g / L$ & $\mathrm{U}$ \\
\hline 837 & 837227REGUF & $2 / 27 / 91$ & REG & 1,2-Dichloroethane & 5 & $\mathrm{ug} / \mathrm{L}$ & $\mathrm{U}$ \\
\hline 837 & 837529REGUF & $5 / 29 / 90$ & REG & 1,2-Dichloroethane & 5 & $u g / L$ & $\mathrm{U}$ \\
\hline 837 & 8371113REGUF & $11 / 13 / 90$ & REG & 1,2-Dichloroethane & 5 & $\mathrm{ug} / \mathrm{L}$ & $\mathrm{U}$ \\
\hline 837 & 837213REGUF & $2 / 13 / 89$ & REG & 1,2-Dichloroethane & 5 & $\mathrm{ug} / \mathrm{L}$ & $\mathrm{U}$ \\
\hline 837 & 837428REGUF & $4 / 28 / 89$ & REG & 1,2-Dichloroethane & 5 & $\mathrm{ug} / \mathrm{L}$ & $\mathrm{U}$ \\
\hline 837 & W00897 & $6 / 23 / 97$ & REG & 1,2-Dichloroethene & 5 & $u g / L$ & $\mathrm{U}$ \\
\hline 837 & M00047 & $11 / 13 / 96$ & REG & 1,2-Dichloroethene & 5 & $\mathrm{ug} / \mathrm{L}$ & $\mathrm{U}$ \\
\hline 837 & S02571 & $2 / 14 / 95$ & REG & 1,2-Dichloroethene & 5 & $\mathrm{ug} / \mathrm{L}$ & $\mathrm{U}$ \\
\hline 837 & S04732 & $8 / 11 / 95$ & REG & 1,2-Dichloroethene & 5 & $\mathrm{ug} / \mathrm{L}$ & $\mathrm{U}$ \\
\hline 837 & 837227REGUF & $2 / 27 / 91$ & REG & 1,2-Dichloroethene & 5 & $\mathrm{ug} / \mathrm{L}$ & $\mathrm{U}$ \\
\hline 837 & 837529REGUF & $5 / 29 / 90$ & REG & 1,2-Dichloroethene & 5 & $\mathrm{ug} / \mathrm{L}$ & $\mathrm{U}$ \\
\hline 837 & 8371113REGUF & $11 / 13 / 90$ & REG & 1,2-Dichloroethene & 5 & $\mathrm{ug} / \mathrm{L}$ & U \\
\hline 837 & 837213REGUF & $2 / 13 / 89$ & REG & 1,2-Dichloroethene & 5 & $\mathrm{ug} / \mathrm{L}$ & $\mathrm{U}$ \\
\hline 837 & 837428REGUF & $4 / 28 / 89$ & REG & 1,2-Dichloroethene & 5 & $\mathrm{ug} / \mathrm{L}$ & $\mathrm{U}$ \\
\hline 837 & M01229 & $11 / 10 / 97$ & REG & 1,2-Dichloropropane & 5 & $\mathrm{ug} / \mathrm{L}$ & $\mathrm{U}$ \\
\hline 837 & W00897 & $6 / 23 / 97$ & REG & 1,2-Dichloropropane & 5 & $\mathrm{ug} / \mathrm{L}$ & $\mathrm{U}$ \\
\hline 837 & M00047 & $11 / 13 / 96$ & REG & 1,2-Dichloropropane & 5 & $u g / L$ & $\mathrm{U}$ \\
\hline 837 & M01012 & $6 / 23 / 97$ & REG & 1,2-Dichloropropane & 5 & $\mathrm{ug} / \mathrm{L}$ & $\mathrm{U}$ \\
\hline 837 & S02571 & $2 / 14 / 95$ & REG & 1,2-Dichloropropane & 5 & $\mathrm{ug} / \mathrm{L}$ & $\mathrm{U}$ \\
\hline 837 & S03515 & $5 / 11 / 95$ & REG & 1,2-Dichloropropane & 1 & $\mathrm{ug} / \mathrm{L}$ & $\mathrm{U}$ \\
\hline 837 & S04732 & $8 / 11 / 95$ & REG & 1,2-Dichloropropane & 5 & $\mathrm{ug} / \mathrm{L}$ & $\mathrm{U}$ \\
\hline 837 & 837227REGUF & $2 / 27 / 91$ & REG & 1,2-Dichloropropane & 5 & $\mathrm{ug} / \mathrm{L}$ & $\mathrm{U}$ \\
\hline 837 & 837529REGUF & $5 / 29 / 90$ & REG & 1,2-Dichloropropane & 5 & $\mathrm{ug} / \mathrm{L}$ & $\mathrm{U}$ \\
\hline 837 & 8371113REGUF & $11 / 13 / 90$ & REG & 1,2-Dichloropropane & 5 & $\mathrm{ug} / \mathrm{L}$ & $\mathrm{U}$ \\
\hline 837 & 837213REGUF & $2 / 13 / 89$ & REG & 1,2-Dichloropropane & 5 & $\mathrm{ug} / \mathrm{L}$ & $\mathrm{U}$ \\
\hline 837 & 837428REGUF & $4 / 28 / 89$ & REG & 1,2-Dichloropropane & 5 & $\mathrm{ug} / \mathrm{L}$ & $\mathrm{U}$ \\
\hline 837 & M00047 & $11 / 13 / 96$ & REG & 1,2-Dimethylbenzene & 5 & $\mathrm{ug} / \mathrm{L}$ & $\mathrm{U}$ \\
\hline 837 & S02571 & $2 / 14 / 95$ & REG & 1,2-Dimethylbenzene & 5 & $\mathrm{ug} / \mathrm{L}$ & $\mathrm{U}$ \\
\hline 837 & S04732 & $8 / 11 / 95$ & REG & 1,2-Dimethylbenzene & 5 & $\mathrm{ug} / \mathrm{L}$ & $\mathrm{U}$ \\
\hline 837 & M00047 & $11 / 13 / 96$ & REG & 1,3-Dichlorobenzene & 5 & $\mathrm{ug} / \mathrm{L}$ & $\mathrm{U}$ \\
\hline 837 & M00047 & $11 / 13 / 96$ & REG & 1,4-Dichlorobenzene & 5 & $\mathrm{ug} / \mathrm{L}$ & $\mathrm{U}$ \\
\hline 837 & M01229 & $11 / 10 / 97$ & REG & 2-Butanone & 10 & $\mathrm{ug} / \mathrm{L}$ & $\mathrm{U}$ \\
\hline 837 & W00897 & $6 / 23 / 97$ & REG & 2+Butanone & 10 & $\mathrm{ug} / \mathrm{L}$ & $\mathrm{U}$ \\
\hline 837 & M00047 & $11 / 13 / 96$ & REG & 2-Butanone & 3 & $\mathrm{ug} / \mathrm{L}$ & $=$ \\
\hline 837 & M01012 & $6 / 23 / 97$ & REG & 2-Butanone & 23 & $\mathrm{ug} / \mathrm{L}$ & $=$ \\
\hline 837 & $\mathrm{~S} 02571$ & $2 / 14 / 95$ & REG & 2-Butanone & 5 & $\mathrm{ug} / \mathrm{L}$ & $\mathrm{J}$ \\
\hline
\end{tabular}


B-15

\begin{tabular}{|c|c|c|c|c|c|c|c|}
\hline Station & Sample ID & Sample Date & Sample Type & Chemical & Results & Units & Qualifier \\
\hline 837 & S03515 & $5 / 11 / 95$ & REG & 2-Butanone & 5 & $\mathrm{ug} / \mathrm{L}$ & $\mathrm{U}$ \\
\hline 837 & S04732 & $8 / 11 / 95$ & REG & 2-Butanone & 10 & $u g / L$ & $\mathrm{U}$ \\
\hline 837 & 837227REGUF & $2 / 27 / 91$ & REG & 2-Butanone & 10 & $\mathrm{ug} / \mathrm{L}$ & $\mathrm{U}$ \\
\hline 837 & 837529REGUF & $5 / 29 / 90$ & REG & 2-Butanone & 10 & $\mathrm{ug} / \mathrm{L}$ & $\mathrm{U}$ \\
\hline 837 & 8371113REGUF & $11 / 13 / 90$ & REG & 2-Butanone & 10 & $\mathrm{ug} / \mathrm{L}$ & $\mathrm{U}$ \\
\hline 837 & 837213REGUF & $2 / 13 / 89$ & REG & 2-Butanone & 10 & $\mathrm{ug} / \mathrm{L}$ & $\mathrm{U}$ \\
\hline 837 & 837428REGUF & $4 / 28 / 89$ & REG & 2-Butanone & 10 & $\mathrm{ug} / \mathrm{L}$ & $\mathrm{U}$ \\
\hline 837 & M01229 & $11 / 10 / 97$ & REG & 2-Hexanone & 10 & $\mathrm{ug} / \mathrm{L}$ & $\mathrm{U}$ \\
\hline 837 & W00897 & $6 / 23 / 97$ & REG & 2-Hexanone & 10 & $\mathrm{ug} / \mathrm{L}$ & $\mathrm{U}$ \\
\hline 837 & M00047 & $11 / 13 / 96$ & REG & 2-Hexanone & 10 & $\mathrm{ug} / \mathrm{L}$ & $\mathrm{U}$ \\
\hline 837 & M01012 & $6 / 23 / 97$ & REG & 2-Hexanone & 10 & $\mathrm{ug} / \mathrm{L}$ & $\mathrm{U}$ \\
\hline 837 & S02571 & $2 / 14 / 95$ & REG & 2-Hexanone & 10 & $\mathrm{ug} / \mathrm{L}$ & $\mathrm{U}$ \\
\hline 837 & S03515 & $5 / 11 / 95$ & REG & 2-Hexanone & 5 & $\mathrm{ug} / \mathrm{L}$ & $\mathrm{U}$ \\
\hline 837 & S04732 & $8 / 11 / 95$ & REG & 2-Hexanone & 10 & $\mathrm{ug} / \mathrm{L}$ & $\mathrm{U}$ \\
\hline 837 & 837227REGUF & $2 / 27 / 91$ & REG & 2-Hexanone & 10 & $\mathrm{ug} / \mathrm{L}$ & $\mathrm{U}$ \\
\hline 837 & 837529REGUF & $5 / 29 / 90$ & REG & 2-Hexanone & 10 & $\mathrm{ug} / \mathrm{L}$ & $\mathrm{U}$ \\
\hline 837 & 8371113REGUF & $11 / 13 / 90$ & REG & 2-Hexanone & 10 & $\mathrm{ug} / \mathrm{L}$ & $\mathrm{U}$ \\
\hline 837 & 837213REGUF & $2 / 13 / 89$ & REG & 2-Hexanone & 10 & $\mathrm{ug} / \mathrm{L}$ & $\mathrm{U}$ \\
\hline 837 & 837428REGUF & $4 / 28 / 89$ & REG & 2-Hexanone & 10 & $\mathrm{ug} / \mathrm{L}$ & $\mathrm{U}$ \\
\hline 837 & M01229 & $11 / 10 / 97$ & REG & 4-Methyl-2-pentanone & 10 & $\mathrm{ug} / \mathrm{L}$ & $\mathrm{U}$ \\
\hline 837 & W00897 & $6 / 23 / 97$ & REG & 4-Methyl-2-pentanone & 10 & $\mathrm{ug} / \mathrm{L}$ & $\mathrm{U}$ \\
\hline 837 & M00047 & $11 / 13 / 96$ & REG & 4-Methyl-2-pentanone & 10 & $\mathrm{ug} / \mathrm{L}$ & $\mathrm{U}$ \\
\hline 837 & M01012 & $6 / 23 / 97$ & REG & 4-Methyl-2-pentanone & 10 & $\mathrm{ug} / \mathrm{L}$ & $\mathrm{U}$ \\
\hline 837 & $\mathrm{~S} 02571$ & $2 / 14 / 95$ & REG & 4-Methyl-2-pentanone & 10 & $\mathrm{ug} / \mathrm{L}$ & $\mathrm{U}$ \\
\hline 837 & S03515 & $5 / 11 / 95$ & REG & 4-Methyl-2-pentanone & 5 & $\mathrm{ug} / \mathrm{L}$ & $\mathrm{U}$ \\
\hline 837 & S04732 & $8 / 11 / 95$ & REG & 4-Methyl-2-pentanone & 10 & $u g / L$ & $U$ \\
\hline 837 & 837227REGUF & $2 / 27 / 91$ & REG & 4-Methyl-2-pentanone & 10 & $\mathrm{ug} / \mathrm{L}$ & U \\
\hline 837 & 837529REGUF & $5 / 29 / 90$ & REG & 4-Methyl-2-pentanone & 10 & $\mathrm{ug} / \mathrm{L}$ & $\mathrm{U}$ \\
\hline 837 & 8371113REGUF & $11 / 13 / 90$ & REG & 4-Methyl-2-pentanone & 10 & $\mathrm{ug} / \mathrm{L}$ & $\mathrm{U}$ \\
\hline 837 & 837213REGUF & $2 / 13 / 89$ & REG & 4-Methyl-2-pentanone & 10 & $\mathrm{ug} / \mathrm{L}$ & $\mathrm{U}$ \\
\hline 837 & 837428REGUF & $4 / 28 / 89$ & REG & 4-Methyl-2-pentanone & 10 & $\mathrm{ug} / \mathrm{L}$ & $\mathrm{U}$ \\
\hline 837 & M01229 & $11 / 10 / 97$ & REG & Acetone & 5 & $\mathrm{ug} / \mathrm{L}$ & B \\
\hline 837 & W00897 & $6 / 23 / 97$ & REG & Acetone & 10 & $\mathrm{ug} / \mathrm{L}$ & $\mathrm{U}$ \\
\hline 837 & M00047 & $11 / 13 / 96$ & REG & Acetone & 10 & $\mathrm{ug} / \mathrm{L}$ & $\mathrm{U}$ \\
\hline 837 & M01012 & $6 / 23 / 97$ & REG & Acetone & 10 & $\mathrm{ug} / \mathrm{L}$ & $\mathrm{U}$ \\
\hline 837 & S02571 & $2 / 14 / 95$ & REG & Acetone & 10 & $\mathrm{ug} / \mathrm{L}$ & $\mathrm{U}$ \\
\hline 837 & S03515 & $5 / 11 / 95$ & REG & Acetone & 5 & $\mathrm{ug} / \mathrm{L}$ & $\mathrm{U}$ \\
\hline 837 & S04732 & $8 / 11 / 95$ & REG & Acetone & 10 & $\mathrm{ug} / \mathrm{L}$ & $\mathrm{U}$ \\
\hline 837 & 837227REGUF & $2 / 27 / 91$ & REG & Acetone & 10 & $\mathrm{ug} / \mathrm{L}$ & $\mathrm{U}$ \\
\hline 837 & 837529REGUF & $5 / 29 / 90$ & REG & Acetone & 10 & $\mathrm{ug} / \mathrm{L}$ & $\mathrm{U}$ \\
\hline 837 & 8371113REGUF & $11 / 13 / 90$ & REG & Acetone & 3 & $\mathrm{ug} / \mathrm{L}$ & $\mathrm{J}$ \\
\hline 837 & 837213REGUF & $2 / 13 / 89$ & REG & Acetone & 3 & $\mathrm{ug} / \mathrm{L}$ & $\mathrm{J}$ \\
\hline 837 & 837428REGUF & $4 / 28 / 89$ & REG & Acetone & 3 & $\mathrm{ug} / \mathrm{L}$ & $\mathrm{J}$ \\
\hline 837 & M01229 & $11 / 10 / 97$ & REG & Benzene & 5 & $\mathrm{ug} / \mathrm{L}$ & $\mathrm{U}$ \\
\hline 837 & W00897 & $6 / 23 / 97$ & REG & Benzene & 5 & $\mathrm{ug} / \mathrm{L}$ & $U$ \\
\hline 837 & M00047 & $11 / 13 / 96$ & REG & Benzene & 5 & $\mathrm{ug} / \mathrm{L}$ & $\mathrm{U}$ \\
\hline 837 & M01012 & $6 / 23 / 97$ & REG & Benzene & 5 & $\mathrm{ug} / \mathrm{L}$ & $\mathrm{U}$ \\
\hline 837 & S02571 & $2 / 14 / 95$ & REG & Benzene & 5 & $\mathrm{ug} / \mathrm{L}$ & $U$ \\
\hline 837 & S03515 & $5 / 11 / 95$ & REG & Benzene & 1 & $\mathrm{ug} / \mathrm{L}$ & $\mathrm{U}$ \\
\hline 837 & S04732 & $8 / 11 / 95$ & REG & Benzene & 5 & ug/L & $\mathrm{U}$ \\
\hline
\end{tabular}




\begin{tabular}{|c|c|c|c|c|c|c|c|}
\hline Station & Sample ID & Sample Date & Sample Type & Chemical & Results & Units & Qualifier \\
\hline$\overline{837}$ & 837227REGUF & $2 / 27 / 91$ & REG & Benzene & 5 & $\mathrm{ug} / \mathrm{L}$ & $\mathrm{U}$ \\
\hline 837 & 837529REGUF & $5 / 29 / 90$ & REG & Benzene & 5 & $\mathrm{ug} / \mathrm{L}$ & $\mathrm{U}$ \\
\hline 837 & 8371113REGUF & $11 / 13 / 90$ & REG & Benzene & 5 & $\mathrm{ug} / \mathrm{L}$ & $\mathrm{U}$ \\
\hline 837 & 837213REGUF & $2 / 13 / 89$ & REG & Benzene & 5 & $\mathrm{ug} / \mathrm{L}$ & $\mathrm{U}$ \\
\hline 837 & 837428REGUF & $4 / 28 / 89$ & REG & Benzene & 5 & $\mathrm{ug} / \mathrm{L}$ & $\mathrm{U}$ \\
\hline 837 & S03515 & $5 / 11 / 95$ & REG & Bromochloromethane & 1 & $\mathrm{ug} / \mathrm{L}$ & $\mathrm{U}$ \\
\hline 837 & M01229 & $11 / 10 / 97$ & REG & Bromodichloromethane & 5 & $\mathrm{ug} / \mathrm{L}$ & $\mathrm{U}$ \\
\hline 837 & W00897 & $6 / 23 / 97$ & REG & Bromodichloromethane & 5 & $\mathrm{ug} / \mathrm{L}$ & $\mathrm{U}$ \\
\hline 837 & M00047 & $11 / 13 / 96$ & REG & Bromodichloromethane & 5 & $\mathrm{ug} / \mathrm{L}$ & $\mathrm{U}$ \\
\hline 837 & $\mathrm{M} 01012$ & $6 / 23 / 97$ & REG & Bromodichloromethane & 5 & $\mathrm{ug} / \mathrm{L}$ & $\mathrm{U}$ \\
\hline 837 & $\mathrm{~S} 02571$ & $2 / 14 / 95$ & REG & Bromodichloromethane & 5 & $\mathrm{ug} / \mathrm{L}$ & $\mathrm{U}$ \\
\hline 837 & S03515 & $5 / 11 / 95$ & REG & Bromodichloromethane & 1 & $\mathrm{ug} / \mathrm{L}$ & $\mathrm{U}$ \\
\hline 837 & S04732 & $8 / 11 / 95$ & REG & Bromodichloromethane & 5 & $\mathrm{ug} / \mathrm{L}$ & $\mathrm{U}$ \\
\hline 837 & 837227REGUF & $2 / 27 / 91$ & REG & Bromodichloromethane & 5 & $\mathrm{ug} / \mathrm{L}$ & $\mathrm{U}$ \\
\hline 837 & 837529REGUF & $5 / 29 / 90$ & REG & Bromodichloromethane & 5 & ug/L & $\mathrm{U}$ \\
\hline 837 & 8371113REGUF & $11 / 13 / 90$ & REG & Bromodichloromethane & 5 & $\mathrm{ug} / \mathrm{L}$ & $\mathrm{U}$ \\
\hline 837 & 837213REGUF & $2 / 13 / 89$ & REG & Bromodichloromethane & 5 & $\mathrm{ug} / \mathrm{L}$ & $\mathrm{U}$ \\
\hline 837 & 837428REGUF & $4 / 28 / 89$ & REG & Bromodichloromethane & 5 & $\mathrm{ug} / \mathrm{L}$ & $\mathrm{U}$ \\
\hline 837 & M01229 & $11 / 10 / 97$ & REG & Bromoform & 5 & $\mathrm{ug} / \mathrm{L}$ & $\mathrm{U}$ \\
\hline 837 & W00897 & $6 / 23 / 97$ & REG & Bromoform & 5 & ug/L & $\mathrm{U}$ \\
\hline 837 & M00047 & $11 / 13 / 96$ & REG & Bromoform & 5 & $\mathrm{ug} / \mathrm{L}$ & $\mathrm{U}$ \\
\hline 837 & M01012 & $6 / 23 / 97$ & REG & Bromoform & 5 & $\mathrm{ug} / \mathrm{L}$ & $\mathrm{U}$ \\
\hline 837 & S02571 & $2 / 14 / 95$ & REG & Bromoform & 5 & $\mathrm{ug} / \mathrm{L}$ & $\mathrm{U}$ \\
\hline 837 & S03515 & $5 / 11 / 95$ & REG & Bromoform & 1 & $\mathrm{ug} / \mathrm{L}$ & $U$ \\
\hline 837 & S04732 & $8 / 11 / 95$ & REG & Bromoform & 5 & $\mathrm{ug} / \mathrm{L}$ & $\mathrm{U}$ \\
\hline 837 & 837227REGUF & $2 / 27 / 91$ & REG & Bromoform & 5 & $\mathrm{ug} / \mathrm{L}$ & $\mathrm{U}$ \\
\hline 837 & 837529REGUF & $5 / 29 / 90$ & REG & Bromoform & 5 & $\mathrm{ug} / \mathrm{L}$ & $\mathrm{U}$ \\
\hline 837 & 8371113REGUF & $11 / 13 / 90$ & REG & Bromoform & 5 & ug/L & $\mathrm{U}$ \\
\hline 837 & 837213REGUF & $2 / 13 / 89$ & REG & Bromoform & 5 & $\mathrm{ug} / \mathrm{L}$ & $\mathrm{U}$ \\
\hline 837 & 837428REGUF & $4 / 28 / 89$ & REG & Bromoform & 5 & $\mathrm{ug} / \mathrm{L}$ & $\mathrm{U}$ \\
\hline 837 & M01229 & $11 / 10 / 97$ & REG & Bromomethane & 10 & $\mathrm{ug} / \mathrm{L}$ & $\mathrm{U}$ \\
\hline 837 & W00897 & $6 / 23 / 97$ & REG & Bromomethane & 10 & $\mathrm{ug} / \mathrm{L}$ & U \\
\hline 837 & M00047 & $11 / 13 / 96$ & REG & Bromomethane & 10 & ug/L & $\mathrm{U}$ \\
\hline 837 & M01012 & $6 / 23 / 97$ & REG & Bromomethane & 10 & $\mathrm{ug} / \mathrm{L}$ & $\mathrm{U}$ \\
\hline 837 & S02571 & $2 / 14 / 95$ & REG & Bromomethane & 10 & $\mathrm{ug} / \mathrm{L}$ & $\mathrm{U}$ \\
\hline 837 & $\mathrm{~S} 03515$ & $5 / 11 / 95$ & REG & Bromomethane & 1 & $\mathrm{ug} / \mathrm{L}$ & $\mathrm{U}$ \\
\hline 837 & S04732 & $8 / 11 / 95$ & REG & Bromomethane & 10 & $\mathrm{ug} / \mathrm{L}$ & U \\
\hline 837 & 837227REGUF & $2 / 27 / 91$ & REG & Bromomethane & 10 & $\mathrm{ug} / \mathrm{L}$ & $\mathrm{U}$ \\
\hline 837 & 837529REGUF & $5 / 29 / 90$ & REG & Bromomethane & 10 & $\mathrm{ug} / \mathrm{L}$ & $\mathrm{U}$ \\
\hline 837 & 8371113REGUF & $11 / 13 / 90$ & REG & Bromomethane & 10 & $\mathrm{ug} / \mathrm{L}$ & $\mathrm{U}$ \\
\hline 837 & 837213REGUF & $2 / 13 / 89$ & REG & Bromomethane & 10 & $\mathrm{ug} / \mathrm{L}$ & U \\
\hline 837 & 837428REGUF & $4 / 28 / 89$ & REG & Bromomethane & 10 & $\mathrm{ug} / \mathrm{L}$ & $\mathrm{U}$ \\
\hline 837 & M01229 & $11 / 10 / 97$ & REG & Carbon disulfide & 5 & $\mathrm{ug} / \mathrm{L}$ & $\mathrm{U}$ \\
\hline 837 & W00897 & $6 / 23 / 97$ & REG & Carbon disulfide & 5 & $\mathrm{ug} / \mathrm{L}$ & $\mathrm{U}$ \\
\hline 837 & M00047 & $11 / 13 / 96$ & REG & Carbon disulfide & 5 & $\mathrm{ug} / \mathrm{L}$ & $\mathrm{U}$ \\
\hline 837 & M01012 & $6 / 23 / 97$ & REG & Carbon disulfide & 5 & $\mathrm{ug} / \mathrm{L}$ & $\mathrm{U}$ \\
\hline 837 & S02571 & $2 / 14 / 95$ & REG & Carbon disulfide & 5 & $\mathrm{ug} / \mathrm{L}$ & $\mathrm{U}$ \\
\hline 837 & S03515 & $5 / 11 / 95$ & REG & Carbon disulfide & 1 & $\mathrm{ug} / \mathrm{L}$ & $\mathrm{U}$ \\
\hline 837 & S04732 & $8 / 11 / 95$ & REG & Carbon disulfide & 5 & $\mathrm{ug} / \mathrm{L}$ & $\mathrm{U}$ \\
\hline 837 & 837227REGUF & $2 / 27 / 91$ & REG & Carbon disulfide & 5 & $\mathrm{ug} / \mathrm{L}$ & $U$ \\
\hline
\end{tabular}


Station Sample ID Sample Date Sample Type Chemical

\begin{tabular}{|c|c|c|c|c|c|c|c|}
\hline$\overline{837}$ & 837529REGUF & $5 / 29 / 90$ & REG & Carbon disulfide & 5 & $\mathrm{ug} / \mathrm{L}$ & $\mathrm{U}$ \\
\hline 837 & 8371113REGUF & $11 / 13 / 90$ & REG & Carbon disulfide & 2 & $\mathrm{ug} / \mathrm{L}$ & $\mathrm{J}$ \\
\hline 837 & 837213REGUF & $2 / 13 / 89$ & REG & Carbon disulfide & 5 & $\mathrm{ug} / \mathrm{L}$ & $\mathrm{U}$ \\
\hline 837 & 837428REGUF & $4 / 28 / 89$ & REG & Carbon disulfide & 5 & $\mathrm{ug} / \mathrm{L}$ & $\mathrm{U}$ \\
\hline 837 & M01229 & $11 / 10 / 97$ & REG & Carbon tetrachloride & 5 & $\mathrm{ug} / \mathrm{L}$ & $\mathrm{U}$ \\
\hline 837 & W00897 & $6 / 23 / 97$ & REG & Carbon tetrachloride & 5 & $\mathrm{ug} / \mathrm{L}$ & $\mathrm{U}$ \\
\hline 837 & M00047 & $11 / 13 / 96$ & REG & Carbon tetrachloride & 5 & $\mathrm{ug} / \mathrm{L}$ & $\mathrm{U}$ \\
\hline 837 & M01012 & $6 / 23 / 97$ & REG & Carbon tetrachloride & 5 & $\mathrm{ug} / \mathrm{L}$ & $\mathrm{U}$ \\
\hline 837 & $\mathrm{~S} 02571$ & $2 / 14 / 95$ & REG & Carbon tetrachloride & 5 & ug/L & $\mathrm{U}$ \\
\hline 837 & S03515 & $5 / 11 / 95$ & REG & Carbon tetrachloride & 1 & $\mathrm{ug} / \mathrm{L}$ & $\mathrm{U}$ \\
\hline 837 & S04732 & $8 / 11 / 95$ & REG & Carbon tetrachloride & 5 & $\mathrm{ug} / \mathrm{L}$ & $\mathrm{U}$ \\
\hline 837 & 837227REGUF & $2 / 27 / 91$ & REG & Carbon tetrachloride & 5 & $\mathrm{ug} / \mathrm{L}$ & $\mathrm{U}$ \\
\hline 837 & 837529REGUF & $5 / 29 / 90$ & REG & Carbon tetrachloride & 5 & $\mathrm{ug} / \mathrm{L}$ & $\mathrm{U}$ \\
\hline 837 & 8371113REGUF & $11 / 13 / 90$ & REG & Carbon tetrachloride & 5 & $\mathrm{ug} / \mathrm{L}$ & $\mathrm{U}$ \\
\hline 837 & 837213REGUF & $2 / 13 / 89$ & REG & Carbon tetrachloride & 5 & $\mathrm{ug} / \mathrm{L}$ & $\mathrm{U}$ \\
\hline 837 & 837428REGUF & $4 / 28 / 89$ & REG & Carbon tetrachloride & 5 & $\mathrm{ug} / \mathrm{L}$ & $\mathrm{U}$ \\
\hline 837 & M01229 & $11 / 10 / 97$ & REG & Chlorobenzene & 5 & $\mathrm{ug} / \mathrm{L}$ & $\mathrm{U}$ \\
\hline 837 & W00897 & $6 / 23 / 97$ & REG & Chlorobenzene & 5 & $\mathrm{ug} / \mathrm{L}$ & $\mathrm{U}$ \\
\hline 837 & M00047 & $11 / 13 / 96$ & REG & Chlorobenzene & 5 & $\mathrm{ug} / \mathrm{L}$ & $\mathrm{U}$ \\
\hline 837 & $\mathrm{M} 01012$ & $6 / 23 / 97$ & REG & Chlorobenzene & 5 & $\mathrm{ug} / \mathrm{L}$ & $\mathrm{U}$ \\
\hline 837 & $\mathrm{~S} 02571$ & $2 / 14 / 95$ & REG & Chlorobenzene & 5 & $\mathrm{ug} / \mathrm{L}$ & $\mathrm{U}$ \\
\hline 837 & $\mathrm{~S} 03515$ & $5 / 11 / 95$ & REG & Chlorobenzene & 1 & $\mathrm{ug} / \mathrm{L}$ & $\mathrm{U}$ \\
\hline 837 & S04732 & $8 / 11 / 95$ & REG & Chlorobenzene & 5 & $\mathrm{ug} / \mathrm{L}$ & $\mathrm{U}$ \\
\hline 837 & 837227REGUF & $2 / 27 / 91$ & REG & Chlorobenzene & 5 & $\mathrm{ug} / \mathrm{L}$ & $\mathrm{U}$ \\
\hline 837 & 837529REGUF & $5 / 29 / 90$ & REG & Chlorobenzene & 5 & $\mathrm{ug} / \mathrm{L}$ & $\mathrm{U}$ \\
\hline 837 & 8371113REGUF & $11 / 13 / 90$ & REG & Chlorobenzene & 5 & $\mathrm{ug} / \mathrm{L}$ & $\mathrm{U}$ \\
\hline 837 & 837213REGUF & $2 / 13 / 89$ & REG & Chlorobenzene & 5 & $\mathrm{ug} / \mathrm{L}$ & $\mathrm{U}$ \\
\hline 837 & 837428REGUF & $4 / 28 / 89$ & REG & Chlorobenzene & 5 & $\mathrm{ug} / \mathrm{L}$ & $\mathrm{U}$ \\
\hline 837 & M01229 & $11 / 10 / 97$ & REG & Chloroethane & 10 & $\mathrm{ug} / \mathrm{L}$ & $\mathrm{U}$ \\
\hline 837 & W00897 & $6 / 23 / 97$ & REG & Chloroethane & 10 & $\mathrm{ug} / \mathrm{L}$ & $\mathrm{U}$ \\
\hline 837 & M00047 & $11 / 13 / 96$ & REG & Chloroethane & 10 & ug/L & $\mathrm{U}$ \\
\hline 837 & M01012 & $6 / 23 / 97$ & REG & Chloroethane & 10 & ug/L & $\mathrm{U}$ \\
\hline 837 & S02571 & $2 / 14 / 95$ & REG & Chloroethane & 10 & $\mathrm{ug} / \mathrm{L}$ & $\mathrm{U}$ \\
\hline 837 & S03515 & $5 / 11 / 95$ & REG & Chloroethane & 1 & $\mathrm{ug} / \mathrm{L}$ & $\mathrm{U}$ \\
\hline 837 & S04732 & $8 / 11 / 95$ & REG & Chloroethane & 10 & $\mathrm{ug} / \mathrm{L}$ & $\mathrm{U}$ \\
\hline 837 & 837227REGUF & $2 / 27 / 91$ & $\mathrm{REG}$ & Chloroethane & 10 & $\mathrm{ug} / \mathrm{L}$ & $\mathrm{U}$ \\
\hline 837 & 837529REGUF & $5 / 29 / 90$ & REG & Chloroethane & 10 & $\mathrm{ug} / \mathrm{L}$ & $\mathrm{U}$ \\
\hline 837 & 8371113REGUF & $11 / 13 / 90$ & REG & Chloroethane & 10 & $\mathrm{ug} / \mathrm{L}$ & $\mathrm{U}$ \\
\hline 837 & 837213REGUF & $2 / 13 / 89$ & $\mathrm{REG}$ & Chloroethane & 10 & $\mathrm{ug} / \mathrm{L}$ & $\mathrm{U}$ \\
\hline 837 & 837428REGUF & $4 / 28 / 89$ & REG & Chloroethane & 10 & $\mathrm{ug} / \mathrm{L}$ & $\mathrm{U}$ \\
\hline 837 & M01229 & $11 / 10 / 97$ & $\mathrm{REG}$ & Chloroform & 5 & $\mathrm{ug} / \mathrm{L}$ & $\mathrm{U}$ \\
\hline 837 & W00897 & $6 / 23 / 97$ & REG & Chloroform & 5 & $\mathrm{ug} / \mathrm{L}$ & $\mathrm{U}$ \\
\hline 837 & M00047 & $11 / 13 / 96$ & REG & Chloroform & 5 & $\mathrm{ug} / \mathrm{L}$ & $\mathrm{U}$ \\
\hline 837 & M01012 & $6 / 23 / 97$ & REG & Chloroform & 5 & $\mathrm{ug} / \mathrm{L}$ & $\mathrm{U}$ \\
\hline 837 & S02571 & $2 / 14 / 95$ & REG & Chloroform & 5 & $\mathrm{ug} / \mathrm{L}$ & $\mathrm{U}$ \\
\hline 837 & S03515 & $5 / 11 / 95$ & REG & Chloroform & 1 & ug/L & $\mathrm{U}$ \\
\hline 837 & S04732 & $8 / 11 / 95$ & REG & Chloroform & 5 & $\mathrm{ug} / \mathrm{L}$ & $\mathrm{U}$ \\
\hline 837 & 837227REGUF & $2 / 27 / 91$ & REG & Chloroform & 5 & $\mathrm{ug} / \mathrm{L}$ & $\mathrm{U}$ \\
\hline 837 & 837529REGUF & $5 / 29 / 90$ & REG & Chloroform & 5 & $\mathrm{ug} / \mathrm{L}$ & $\mathrm{U}$ \\
\hline 837 & 8371113REGUF & $11 / 13 / 90$ & REG & Chloroform & 5 & $\mathrm{ug} / \mathrm{L}$ & $\mathrm{U}$ \\
\hline
\end{tabular}




\begin{tabular}{|c|c|c|c|c|c|c|c|}
\hline Station & Sample ID & Sample Date & Sample Type & Chemical & Results & Units & Qualifier \\
\hline 837 & 837213REGUF & $2 / 13 / 89$ & REG & Chloroform & 5 & $\mathrm{ug} / \mathrm{L}$ & $\mathrm{U}$ \\
\hline 837 & 837428REGUF & $4 / 28 / 89$ & REG & Chloroform & 5 & $\mathrm{ug} / \mathrm{L}$ & $\mathrm{U}$ \\
\hline 837 & M01229 & $11 / 10 / 97$ & REG & Chloromethane & 10 & $\mathrm{ug} / \mathrm{L}$ & $\mathrm{U}$ \\
\hline 837 & W00897 & $6 / 23 / 97$ & REG & Chloromethane & 10 & $\mathrm{ug} / \mathrm{L}$ & $\mathrm{U}$ \\
\hline 837 & M00047 & $11 / 13 / 96$ & REG & Chloromethane & 10 & $\mathrm{ug} / \mathrm{L}$ & $\mathrm{U}$ \\
\hline 837 & M01012 & $6 / 23 / 97$ & REG & Chloromethane & 10 & $\mathrm{ug} / \mathrm{L}$ & $\mathrm{U}$ \\
\hline 837 & S02571 & $2 / 14 / 95$ & REG & Chloromethane & 10 & $\mathrm{ug} / \mathrm{L}$ & $\mathrm{U}$ \\
\hline 837 & S03515 & $5 / 11 / 95$ & REG & Chloromethane & 1 & $\mathrm{ug} / \mathrm{L}$ & $\mathrm{U}$ \\
\hline 837 & S04732 & $8 / 11 / 95$ & REG & Chloromethane & 10 & $\mathrm{ug} / \mathrm{L}$ & $\mathrm{U}$ \\
\hline 837 & 837227REGUF & $2 / 27 / 91$ & REG & Chloromethane & 10 & $\mathrm{ug} / \mathrm{L}$ & $\mathrm{U}$ \\
\hline 837 & 837529REGUF & $5 / 29 / 90$ & REG & Chloromethane & 10 & $\mathrm{ug} / \mathrm{L}$ & $\mathrm{U}$ \\
\hline 837 & 8371113REGUF & $11 / 13 / 90$ & REG & Chloromethane & 10 & $\mathrm{ug} / \mathrm{L}$ & $\mathrm{U}$ \\
\hline 837 & 837213REGUF & $2 / 13 / 89$ & REG & Chloromethane & 10 & $\mathrm{ug} / \mathrm{L}$ & $\mathrm{U}$ \\
\hline 837 & 837428REGUF & $4 / 28 / 89$ & REG & Chloromethane & 10 & $\mathrm{ug} / \mathrm{L}$ & $\mathrm{U}$ \\
\hline 837 & M01229 & $11 / 10 / 97$ & REG & cis-1,2-Dichloroethene & 5 & $\mathrm{ug} / \mathrm{L}$ & $\mathrm{U}$ \\
\hline 837 & M00047 & $11 / 13 / 96$ & REG & cis-1,2-Dichloroethene & 5 & $\mathrm{ug} / \mathrm{L}$ & $\mathrm{U}$ \\
\hline 837 & M01012 & $6 / 23 / 97$ & REG & cis-1,2-Dichloroethene & 5 & $\mathrm{ug} / \mathrm{L}$ & $\mathrm{U}$ \\
\hline 837 & S02571 & $2 / 14 / 95$ & REG & cis-1,2-Dichloroethene & 5 & $\mathrm{ug} / \mathrm{L}$ & $\mathrm{U}$ \\
\hline 837 & S03515 & $5 / 11 / 95$ & REG & cis-1,2-Dichloroethene & 1 & $\mathrm{ug} / \mathrm{L}$ & $\mathrm{U}$ \\
\hline 837 & S04732 & $8 / 11 / 95$ & REG & cis-1,2-Dichloroethene & 5 & $\mathrm{ug} / \mathrm{L}$ & $\mathrm{U}$ \\
\hline 837 & M01229 & $11 / 10 / 97$ & REG & cis-1,3-Dichloropropen & 5 & $\mathrm{ug} / \mathrm{L}$ & $\mathrm{U}$ \\
\hline 837 & W00897 & $6 / 23 / 97$ & REG & cis-1,3-Dichloropropene & 5 & $\mathrm{ug} / \mathrm{L}$ & $\mathrm{U}$ \\
\hline 837 & M00047 & $11 / 13 / 96$ & REG & cis-1,3-Dichloropropene & 5 & $\mathrm{ug} / \mathrm{L}$ & $\mathrm{U}$ \\
\hline 837 & M01012 & $6 / 23 / 97$ & REG & cis-1,3-Dichloropropene & 5 & $\mathrm{ug} / \mathrm{L}$ & $\mathrm{U}$ \\
\hline 837 & $\mathrm{~S} 02571$ & $2 / 14 / 95$ & REG & cis-1,3-Dichloropropene & 5 & $\mathrm{ug} / \mathrm{L}$ & $\mathrm{U}$ \\
\hline 837 & $\mathrm{~S} 03515$ & $5 / 11 / 95$ & REG & cis-1,3-Dichloropropene & 1 & $\mathrm{ug} / \mathrm{L}$ & $\mathrm{U}$ \\
\hline 837 & S04732 & $8 / 11 / 95$ & REG & cis-1,3-Dichloropropene & 5 & $\mathrm{ug} / \mathrm{L}$ & $\mathbf{U}$ \\
\hline 837 & 837227REGUF & $2 / 27 / 91$ & REG & cis-1,3-Dichloropropene & 5 & $\mathrm{ug} / \mathrm{L}$ & $\mathrm{U}$ \\
\hline 837 & 837529REGUF & $5 / 29 / 90$ & REG & cis-1,3-Dichloropropene & 5 & ug/L & $\mathrm{U}$ \\
\hline 837 & 8371113REGUF & $11 / 13 / 90$ & REG & cis-1,3-Dichloropropene & 5 & $\mathrm{ug} / \mathrm{L}$ & $\mathrm{U}$ \\
\hline 837 & 837213REGUF & $2 / 13 / 89$ & REG & cis-1,3-Dichloropropene & 5 & $\mathrm{ug} / \mathrm{L}$ & $U$ \\
\hline 837 & 837428REGUF & $4 / 28 / 89$ & REG & cis-1,3-Dichloropropene & 5 & $\mathrm{ug} / \mathrm{L}$ & $\mathrm{U}$ \\
\hline 837 & M01229 & $11 / 10 / 97$ & REG & Dibromochloromethane & 5 & $\mathrm{ug} / \mathrm{L}$ & $\mathrm{U}$ \\
\hline 837 & W00897 & $6 / 23 / 97$ & REG & Dibromochloromethane & 5 & $\mathrm{ug} / \mathrm{L}$ & $\mathrm{U}$ \\
\hline 837 & M00047 & $11 / 13 / 96$ & REG & Dibromochloromethane & 5 & $\mathrm{ug} / \mathrm{L}$ & $\mathrm{U}$ \\
\hline 837 & M01012 & $6 / 23 / 97$ & REG & Dibromochloromethane & 5 & $\mathrm{ug} / \mathrm{L}$ & $\mathrm{U}$ \\
\hline 837 & S02571 & $2 / 14 / 95$ & REG & Dibromochloromethane & 5 & $\mathrm{ug} / \mathrm{L}$ & $\mathrm{U}$ \\
\hline 837 & S03515 & $5 / 11 / 95$ & REG & Dibromochloromethane & 1 & $\mathrm{ug} / \mathrm{L}$ & $\mathrm{U}$ \\
\hline 837 & S04732 & $8 / 11 / 95$ & REG & Dibromochloromethane & 5 & $\mathrm{ug} / \mathrm{L}$ & $\mathrm{U}$ \\
\hline 837 & 837227REGUF & $2 / 27 / 91$ & REG & Dibromochloromethane & 5 & $\mathrm{ug} / \mathrm{L}$ & $\mathrm{U}$ \\
\hline 837 & 837529REGUF & $5 / 29 / 90$ & REG & Dibromochloromethane & 5 & $\mathrm{ug} / \mathrm{L}$ & $\mathrm{U}$ \\
\hline 837 & 8371113REGUF & $11 / 13 / 90$ & REG & Dibromochloromethane & 5 & $\mathrm{ug} / \mathrm{L}$ & $\mathrm{U}$ \\
\hline 837 & 837213REGUF & $2 / 13 / 89$ & REG & Dibromochloromethane & 5 & $\mathrm{ug} / \mathrm{L}$ & $\mathrm{U}$ \\
\hline 837 & 837428REGUF & $4 / 28 / 89$ & REG & Dibromochloromethane & 5 & $\mathrm{ug} / \mathrm{L}$ & $\mathrm{U}$ \\
\hline 837 & M00047 & $11 / 13 / 96$ & REG & Dimethylbenzene & 5 & $u g / L$ & $\mathrm{U}$ \\
\hline 837 & 837227REGUF & $2 / 27 / 91$ & REG & Dimethylbenzene & 5 & $\mathrm{ug} / \mathrm{L}$ & $\mathrm{U}$ \\
\hline 837 & 837529REGUF & $5 / 29 / 90$ & REG & Dimethylbenzene & 5 & $\mathrm{ug} / \mathrm{L}$ & $\mathrm{U}$ \\
\hline 837 & 8371113REGUF & $11 / 13 / 90$ & REG & Dimethylbenzene & 5 & $\mathrm{ug} / \mathrm{L}$ & $\mathrm{U}$ \\
\hline 837 & 837213REGUF & $2 / 13 / 89$ & REG & Dimethylbenzene & 5 & $\mathrm{ug} / \mathrm{L}$ & $\mathrm{U}$ \\
\hline 837 & 837428REGUF & $4 / 28 / 89$ & REG & Dimethylbenzene & 5 & $\mathrm{ug} / \mathrm{L}$ & $\mathrm{U}$ \\
\hline
\end{tabular}


B-19

Station Sample ID Sample Date Sample Type Chemica

\begin{tabular}{|c|c|c|c|c|c|c|c|}
\hline 837 & M01229 & $11 / 10 / 97$ & REG & Ethylbenzene & 5 & $u g / L$ & $\overline{\mathrm{U}}$ \\
\hline 837 & W00897 & $6 / 23 / 97$ & REG & Ethylbenzene & 5 & $\mathrm{ug} / \mathrm{L}$ & $\mathrm{U}$ \\
\hline 837 & M00047 & $11 / 13 / 96$ & REG & Ethylbenzene & 5 & $\mathrm{ug} / \mathrm{L}$ & $\mathrm{U}$ \\
\hline 837 & M01012 & $6 / 23 / 97$ & REG & Ethylbenzene & 5 & $\mathrm{ug} / \mathrm{L}$ & $\mathrm{U}$ \\
\hline 837 & S02571 & $2 / 14 / 95$ & REG & Ethylbenzene & 5 & $\mathrm{ug} / \mathrm{L}$ & $\mathrm{U}$ \\
\hline 837 & S03515 & $5 / 11 / 95$ & REG & Ethylbenzene & 1 & $\mathrm{ug} / \mathrm{L}$ & $\mathrm{U}$ \\
\hline 837 & S04732 & $8 / 11 / 95$ & REG & Ethylbenzene & 5 & $\mathrm{ug} / \mathrm{L}$ & $\mathrm{U}$ \\
\hline 837 & 837227REGUF & $2 / 27 / 91$ & REG & Ethylbenzene & 5 & $\mathrm{ug} / \mathrm{L}$ & $\mathrm{U}$ \\
\hline 837 & 837529REGUF & $5 / 29 / 90$ & REG & Ethylbenzene & 5 & $\mathrm{ug} / \mathrm{L}$ & $\mathrm{U}$ \\
\hline 837 & 8371113REGUF & $11 / 13 / 90$ & REG & Ethylbenzene & 5 & $\mathrm{ug} / \mathrm{L}$ & $\mathbf{U}$ \\
\hline 837 & 837213REGUF & $2 / 13 / 89$ & REG & Ethylbenzene & 5 & $\mathrm{ug} / \mathrm{L}$ & $\mathrm{U}$ \\
\hline 837 & 837428REGUF & $4 / 28 / 89$ & REG & Ethylbenzene & 5 & $\mathrm{ug} / \mathrm{L}$ & $\mathrm{U}$ \\
\hline 837 & M01229 & $11 / 10 / 97$ & REG & m,p-Xylene & 5 & ug/L & $\mathrm{U}$ \\
\hline 837 & W00897 & $6 / 23 / 97$ & REG & m,p-Xylene & 5 & $\mathrm{ug} / \mathrm{L}$ & $\mathrm{U}$ \\
\hline 837 & M00047 & $11 / 13 / 96$ & REG & m,p-Xylene & 5 & $\mathrm{ug} / \mathrm{L}$ & $\mathrm{U}$ \\
\hline 837 & M01012 & $6 / 23 / 97$ & REG & m,p-Xylene & 5 & $\mathrm{ug} / \mathrm{L}$ & $\mathrm{U}$ \\
\hline 837 & S02571 & $2 / 14 / 95$ & REG & $\mathrm{m}, \mathrm{p}$-Xylene & 5 & $\mathrm{ug} / \mathrm{L}$ & $\mathrm{U}$ \\
\hline 837 & S04732 & $8 / 11 / 95$ & REG & m,p-Xylene & 5 & $\mathrm{ug} / \mathrm{L}$ & $\mathrm{U}$ \\
\hline 837 & M01229 & $11 / 10 / 97$ & REG & Methylene chloride & 4 & $\mathrm{ug} / \mathrm{L}$ & B \\
\hline 837 & W00897 & $6 / 23 / 97$ & REG & Methylene chloride & 10 & $u g / L$ & $=$ \\
\hline 837 & M00047 & $11 / 13 / 96$ & REG & Methylene chloride & 5 & $\mathrm{ug} / \mathrm{L}$ & $\mathrm{U}$ \\
\hline 837 & M01012 & $6 / 23 / 97$ & REG & Methylene chloride & 2 & $u g / L$ & $\mathrm{U}$ \\
\hline 837 & S02571 & $2 / 14 / 95$ & REG & Methylene chloride & 5 & $\mathrm{ug} / \mathrm{L}$ & $\mathrm{U}$ \\
\hline 837 & S03515 & $5 / 11 / 95$ & REG & Methylene chloride & 2 & $\mathrm{ug} / \mathrm{L}$ & $\mathrm{U}$ \\
\hline 837 & S04732 & $8 / 11 / 95$ & REG & Methylene chloride & 5 & $\mathrm{ug} / \mathrm{L}$ & $\mathrm{U}$ \\
\hline 837 & 837227REGUF & $2 / 27 / 91$ & REG & Methylene chloride & 5 & $\mathrm{ug} / \mathrm{L}$ & $\mathrm{U}$ \\
\hline 837 & 837529REGUF & $5 / 29 / 90$ & REG & Methylene chloride & 5 & $\mathrm{ug} / \mathrm{L}$ & $\mathrm{U}$ \\
\hline 837 & 8371113REGUF & $11 / 13 / 90$ & REG & Methylene chloride & 5 & $\mathrm{ug} / \mathrm{L}$ & $\mathrm{U}$ \\
\hline 837 & 837213REGUF & $2 / 13 / 89$ & REG & Methylene chloride & 1 & $\mathrm{ug} / \mathrm{L}$ & $\mathrm{J}$ \\
\hline 837 & 837428REGUF & $4 / 28 / 89$ & REG & Methylene chloride & 2 & $\mathrm{ug} / \mathrm{L}$ & $\mathbf{J}$ \\
\hline 837 & M01229 & $11 / 10 / 97$ & REG & Styrene & 5 & $\mathrm{ug} / \mathrm{L}$ & $\mathrm{U}$ \\
\hline 837 & W00897 & $6 / 23 / 97$ & REG & Styrene & 5 & $\mathrm{ug} / \mathrm{L}$ & $\mathrm{U}$ \\
\hline 837 & M00047 & $11 / 13 / 96$ & REG & Styrene & 5 & $\mathrm{ug} / \mathrm{L}$ & $\mathrm{U}$ \\
\hline 837 & M01012 & $6 / 23 / 97$ & REG & Styrene & 5 & $\mathrm{ug} / \mathrm{L}$ & $\mathrm{U}$ \\
\hline 837 & S02571 & $2 / 14 / 95$ & REG & Styrene & 5 & $\mathrm{ug} / \mathrm{L}$ & $\mathrm{U}$ \\
\hline 837 & S03515 & $5 / 11 / 95$ & REG & Styrene & 1 & $\mathrm{ug} / \mathrm{L}$ & $\mathrm{U}$ \\
\hline 837 & S04732 & $8 / 11 / 95$ & REG & Styrene & 5 & $\mathrm{ug} / \mathrm{L}$ & $\mathrm{U}$ \\
\hline 837 & 837227REGUF & $2 / 27 / 91$ & REG & Styrene & 5 & $\mathrm{ug} / \mathrm{L}$ & $\mathrm{U}$ \\
\hline 837 & 837529REGUF & $5 / 29 / 90$ & REG & Styrene & 5 & $\mathrm{ug} / \mathrm{L}$ & $\mathrm{U}$ \\
\hline 837 & 8371113REGUF & $11 / 13 / 90$ & REG & Styrene & 5 & $u g / L$ & $\mathrm{U}$ \\
\hline 837 & 837213REGUF & $2 / 13 / 89$ & REG & Styrene & 5 & ug/L & $\mathrm{U}$ \\
\hline 837 & 837428REGUF & $4 / 28 / 89$ & REG & Styrene & 5 & $\mathrm{ug} / \mathrm{L}$ & $\mathrm{U}$ \\
\hline 837 & M01229 & $11 / 10 / 97$ & REG & Tetrachloroethene & 5 & ug/L & $\mathrm{U}$ \\
\hline 837 & W00897 & $6 / 23 / 97$ & REG & Tetrachloroethene & 5 & $\mathrm{ug} / \mathrm{L}$ & $\mathrm{U}$ \\
\hline 837 & M00047 & $11 / 13 / 96$ & REG & Tetrachloroethene & 5 & $\mathrm{ug} / \mathrm{L}$ & $\mathrm{U}$ \\
\hline 837 & M01012 & $6 / 23 / 97$ & REG & Tetrachloroethene & 5 & $\mathrm{ug} / \mathrm{L}$ & $\mathrm{U}$ \\
\hline 837 & S02571 & $2 / 14 / 95$ & REG & Tetrachloroethene & 5 & $\mathrm{ug} / \mathrm{L}$ & $\mathbf{U}$ \\
\hline 837 & S03515 & $5 / 11 / 95$ & REG & Tetrachloroethene & 1 & $\mathrm{ug} / \mathrm{L}$ & $\mathrm{U}$ \\
\hline 837 & S04732 & $8 / 11 / 95$ & REG & Tetrachloroethene & 5 & $\mathrm{ug} / \mathrm{L}$ & $\mathrm{U}$ \\
\hline 837 & 837227REGUF & $2 / 27 / 91$ & REG & Tetrachloroethene & 5 & $\mathrm{ug} / \mathrm{L}$ & $\mathrm{U}$ \\
\hline
\end{tabular}




\begin{tabular}{|c|c|c|c|c|c|c|c|}
\hline Station & Sample ID & Sample Date & Sample Type & Chemical & Results & Units & Qualifier \\
\hline$\overline{837}$ & 837529REGUF & $5 / 29 / 90$ & REG & Tetrachloroethene & 5 & $u g / L$ & $\bar{U}$ \\
\hline 837 & 8371113REGUF & $11 / 13 / 90$ & REG & Tetrachloroethene & 5 & $\mathrm{ug} / \mathrm{L}$ & $\mathrm{U}$ \\
\hline 837 & 837213REGUF & $2 / 13 / 89$ & REG & Tetrachloroethene & 5 & ug/L & $\mathrm{U}$ \\
\hline 837 & 837428REGUF & $4 / 28 / 89$ & REG & Tetrachloroethene & 5 & $\mathrm{ug} / \mathrm{L}$ & $\mathrm{U}$ \\
\hline 837 & M01229 & $11 / 10 / 97$ & REG & Toluene & 5 & $\mathrm{ug} / \mathrm{L}$ & $\mathrm{U}$ \\
\hline 837 & W00897 & $6 / 23 / 97$ & REG & Toluene & 5 & ug/L & $\mathrm{U}$ \\
\hline 837 & M00047 & $11 / 13 / 96$ & REG & Toluene & 5 & ug/L & U \\
\hline 837 & M01012 & $6 / 23 / 97$ & REG & Toluene & 5 & $\mathrm{ug} / \mathrm{L}$ & $\mathrm{U}$ \\
\hline 837 & S02571 & $2 / 14 / 95$ & REG & Toluene & 5 & $\mathrm{ug} / \mathrm{L}$ & $\mathrm{U}$ \\
\hline 837 & S03515 & $5 / 11 / 95$ & REG & Toluene & 1 & $\mathrm{ug} / \mathrm{L}$ & $\mathrm{U}$ \\
\hline 837 & S04732 & $8 / 11 / 95$ & REG & Toluene & 5 & $u g / L$ & $\mathrm{U}$ \\
\hline 837 & 837227REGUF & $2 / 27 / 91$ & REG & Toluene & 5 & $\mathrm{ug} / \mathrm{L}$ & U \\
\hline 837 & 837529REGUF & $5 / 29 / 90$ & REG & Toluene & 5 & $\mathrm{ug} / \mathrm{L}$ & $\mathrm{U}$ \\
\hline 837 & 8371113REGUF & $11 / 13 / 90$ & REG & Toluene & 5 & ug/L & $\mathrm{U}$ \\
\hline 837 & 837213REGUF & $2 / 13 / 89$ & REG & Toluene & 5 & ug/L & $\mathrm{U}$ \\
\hline 837 & 837428REGUF & $4 / 28 / 89$ & REG & Toluene & 5 & $\mathrm{ug} / \mathrm{L}$ & $\mathrm{U}$ \\
\hline 837 & M01229 & $11 / 10 / 97$ & REG & trans-1,2-Dichloroethe & 5 & $\mathrm{ug} / \mathrm{L}$ & $\mathrm{U}$ \\
\hline 837 & M00047 & $11 / 13 / 96$ & REG & trans-1,2-Dichloroethene & 5 & $\mathrm{ug} / \mathrm{L}$ & U \\
\hline 837 & M01012 & $6 / 23 / 97$ & REG & trans-1,2-Dichloroethene & 5 & $\mathrm{ug} / \mathrm{L}$ & $\mathrm{U}$ \\
\hline 837 & S02571 & $2 / 14 / 95$ & REG & trans-1,2-Dichloroethene & 5 & $u g / L$ & $\mathrm{U}$ \\
\hline 837 & S03515 & $5 / 11 / 95$ & REG & trans-1,2-Dichloroethene & 1 & ug/L & $\mathrm{U}$ \\
\hline 837 & S04732 & $8 / 11 / 95$ & REG & trans-1,2-Dichloroethene & 5 & $\mathrm{ug} / \mathrm{L}$ & $\mathrm{U}$ \\
\hline 837 & M01229 & $11 / 10 / 97$ & REG & trans-1,3-Dichloroprop & 5 & ug/L & $\mathrm{U}$ \\
\hline 837 & W00897 & $6 / 23 / 97$ & REG & trans-1,3-Dichloropropene & 5 & $\mathrm{ug} / \mathrm{L}$ & $\mathrm{U}$ \\
\hline 837 & M00047 & $11 / 13 / 96$ & REG & trans-1,3-Dichloropropene & 5 & $\mathrm{ug} / \mathrm{L}$ & $\mathrm{U}$ \\
\hline 837 & M01012 & $6 / 23 / 97$ & REG & trans-1,3-Dichloropropene & 5 & $\mathrm{ug} / \mathrm{L}$ & $\mathrm{U}$ \\
\hline 837 & S02571 & $2 / 14 / 95$ & REG & trans-1,3-Dichloropropene & 5 & ug/L & $\mathrm{U}$ \\
\hline 837 & S03515 & $5 / 11 / 95$ & REG & trans-1,3-Dichloropropene & 1 & $\mathrm{ug} / \mathrm{L}$ & $\mathrm{U}$ \\
\hline 837 & S04732 & $8 / 11 / 95$ & REG & trans-1,3-Dichloropropene & 5 & $\mathrm{ug} / \mathrm{L}$ & $\mathrm{U}$ \\
\hline 837 & 837227REGUF & $2 / 27 / 91$ & REG & trans-1,3-Dichloropropene & 5 & $\mathrm{ug} / \mathrm{L}$ & $\mathrm{U}$ \\
\hline 837 & 837529REGUF & $5 / 29 / 90$ & REG & trans-1,3-Dichloropropene & 5 & $\mathrm{ug} / \mathrm{L}$ & $\mathrm{U}$ \\
\hline 837 & 8371113REGUF & $11 / 13 / 90$ & REG & trans-1,3-Dichloropropene & 5 & $\mathrm{ug} / \mathrm{L}$ & $\mathrm{U}$ \\
\hline 837 & 837213REGUF & $2 / 13 / 89$ & REG & trans-1,3-Dichloropropene & 5 & $\mathrm{ug} / \mathrm{L}$ & $\mathrm{U}$ \\
\hline 837 & 837428REGUF & $4 / 28 / 89$ & REG & trans-1,3-Dichloropropene & 5 & ug/L & $\mathrm{U}$ \\
\hline 837 & M01229 & $11 / 10 / 97$ & REG & Trichloroethene & 5 & $\mathrm{ug} / \mathrm{L}$ & U \\
\hline 837 & W00897 & $6 / 23 / 97$ & REG & Trichloroethene & 5 & $\mathrm{ug} / \mathrm{L}$ & $\mathrm{U}$ \\
\hline 837 & M00047 & $11 / 13 / 96$ & REG & Trichloroethene & 5 & $\mathrm{ug} / \mathrm{L}$ & $\mathrm{U}$ \\
\hline 837 & M01012 & $6 / 23 / 97$ & REG & Trichloroethene & 5 & $u g / L$ & $\mathrm{U}$ \\
\hline 837 & S02571 & $2 / 14 / 95$ & REG & Trichloroethene & 5 & $\mathrm{ug} / \mathrm{L}$ & $\mathrm{U}$ \\
\hline 837 & S03515 & $5 / 11 / 95$ & REG & Trichloroethene & 1 & $\mathrm{ug} / \mathrm{L}$ & U \\
\hline 837 & S04732 & $8 / 11 / 95$ & REG & Trichloroethene & 5 & $\mathrm{ug} / \mathrm{L}$ & $\mathrm{U}$ \\
\hline 837 & 837227REGUF & $2 / 27 / 91$ & REG & Trichloroethene & 5 & $\mathrm{ug} / \mathrm{L}$ & $\mathrm{U}$ \\
\hline 837 & 837529REGUF & $5 / 29 / 90$ & REG & Trichloroethene & 5 & $\mathrm{ug} / \mathrm{L}$ & $\mathrm{U}$ \\
\hline 837 & 8371113REGUF & $11 / 13 / 90$ & REG & Trichloroethene & 5 & ug/L & $\mathrm{U}$ \\
\hline 837 & 837213REGUF & $2 / 13 / 89$ & REG & Trichloroethene & 5 & $u g / L$ & $\mathrm{U}$ \\
\hline 837 & 837428REGUF & $4 / 28 / 89$ & REG & Trichloroethene & 5 & $\mathrm{ug} / \mathrm{L}$ & $\mathrm{U}$ \\
\hline 837 & M01229 & $11 / 10 / 97$ & REG & Vinyl acetate & 10 & $\mathrm{ug} / \mathrm{L}$ & $\mathrm{U}$ \\
\hline 837 & M00047 & $11 / 13 / 96$ & REG & Vinyl acetate & 10 & ug/L & $\mathrm{U}$ \\
\hline 837 & M01012 & $6 / 23 / 97$ & REG & Vinyl acetate & 10 & $\mathrm{ug} / \mathrm{L}$ & $\mathrm{U}$ \\
\hline 837 & S02571 & $2 / 14 / 95$ & REG & Vinyl acetate & 10 & $\mathrm{ug} / \mathrm{L}$ & U \\
\hline
\end{tabular}




\begin{tabular}{|c|c|c|c|c|c|c|c|}
\hline Station & Sample ID & Sample Date & Sample Type & Chemical & Results & Units & Qualifier \\
\hline 837 & S04732 & $8 / 11 / 95$ & REG & Vinyl acetate & 10 & $\mathrm{ug} / \mathrm{L}$ & $\mathrm{U}$ \\
\hline 837 & 837227REGUF & $2 / 27 / 91$ & REG & Vinyl acetate & 10 & $\mathrm{ug} / \mathrm{L}$ & $\mathrm{U}$ \\
\hline 837 & 837529REGUF & $5 / 29 / 90$ & REG & Vinyl acetate & 10 & $\mathrm{ug} / \mathrm{L}$ & $\mathrm{U}$ \\
\hline 837 & 8371113REGUF & $11 / 13 / 90$ & REG & Vinyl acetate & 10 & $\mathrm{ug} / \mathrm{L}$ & $\mathrm{U}$ \\
\hline 837 & 837213REGUF & $2 / 13 / 89$ & REG & Vinyl acetate & 10 & $\mathrm{ug} / \mathrm{L}$ & $\mathrm{U}$ \\
\hline 837 & 837428REGUF & $4 / 28 / 89$ & REG & Vinyl acetate & 10 & $\mathrm{ug} / \mathrm{L}$ & $\mathrm{U}$ \\
\hline 837 & M01229 & $11 / 10 / 97$ & REG & Vinyl chloride & 10 & $\mathrm{ug} / \mathrm{L}$ & $\mathrm{U}$ \\
\hline 837 & W00897 & $6 / 23 / 97$ & REG & Vinyl chloride & 10 & $\mathrm{ug} / \mathrm{L}$ & $\mathrm{U}$ \\
\hline 837 & M00047 & $11 / 13 / 96$ & REG & Vinyl chloride & 10 & $\mathrm{ug} / \mathrm{L}$ & $\mathrm{U}$ \\
\hline 837 & M01012 & $6 / 23 / 97$ & REG & Vinyl chloride & 10 & $\mathrm{ug} / \mathrm{L}$ & $\mathrm{U}$ \\
\hline 837 & $\mathrm{~S} 02571$ & $2 / 14 / 95$ & REG & Vinyl chloride & 10 & $\mathrm{ug} / \mathrm{L}$ & $\mathrm{U}$ \\
\hline 837 & S03515 & $5 / 11 / 95$ & REG & Vinyl chloride & 1 & $\mathrm{ug} / \mathrm{L}$ & $\mathrm{U}$ \\
\hline 837 & S04732 & $8 / 11 / 95$ & REG & Vinyl chloride & 10 & $\mathrm{ug} / \mathrm{L}$ & $\mathrm{U}$ \\
\hline 837 & 837227REGUF & $2 / 27 / 91$ & REG & Vinyl chloride & 10 & ug/L & $\mathrm{U}$ \\
\hline 837 & 837529REGUF & $5 / 29 / 90$ & REG & Vinyl chloride & 10 & $\mathrm{ug} / \mathrm{L}$ & $\mathrm{U}$ \\
\hline 837 & 8371113REGUF & $11 / 13 / 90$ & REG & Vinyl chloride & 10 & $\mathrm{ug} / \mathrm{L}$ & $\mathbf{U}$ \\
\hline 837 & 837213REGUF & $2 / 13 / 89$ & REG & Vinyl chloride & 10 & $\mathrm{ug} / \mathrm{L}$ & $\mathrm{U}$ \\
\hline 837 & 837428REGUF & $4 / 28 / 89$ & REG & Vinyl chloride & 10 & $\mathrm{ug} / \mathrm{L}$ & $\mathrm{U}$ \\
\hline 841 & M01244 & $11 / 12 / 97$ & REG & 1,1,1-Trichloroethane & 5 & $\mathrm{ug} / \mathrm{L}$ & $\mathrm{U}$ \\
\hline 841 & W00808 & $6 / 26 / 97$ & REG & $1,1,1$-Trichloroethane & 5 & $\mathrm{ug} / \mathrm{L}$ & $\mathrm{U}$ \\
\hline 841 & M00051 & $12 / 5 / 96$ & REG & 1,1,1-Trichloroethane & 5 & $\mathrm{ug} / \mathrm{L}$ & $\mathrm{U}$ \\
\hline 841 & M00836 & $6 / 26 / 97$ & REG & 1,1,1-Trichloroethane & 5 & $\mathrm{ug} / \mathrm{L}$ & $\mathrm{U}$ \\
\hline 841 & SMPL00280 & $5 / 27 / 94$ & REG & 1,1,1-Trichloroethane & 1 & $\mathrm{ug} / \mathrm{L}$ & $\mathrm{U}$ \\
\hline 841 & S00933 & $10 / 19 / 94$ & REG & 1,1,1-Trichloroethane & 5 & $\mathrm{ug} / \mathrm{L}$ & $U$ \\
\hline 841 & S02607 & $2 / 28 / 95$ & REG & 1,1,1-Trichloroethane & 5 & $\mathrm{ug} / \mathrm{L}$ & $\mathrm{U}$ \\
\hline 841 & S03565 & $5 / 16 / 95$ & REG & 1,1,1-Trichloroethane & 5 & $\mathrm{ug} / \mathrm{L}$ & $\mathrm{U}$ \\
\hline 841 & S04776 & $7 / 14 / 95$ & REG & $1,1,1$-Trichloroethane & 5 & $\mathrm{ug} / \mathrm{L}$ & U \\
\hline 841 & 841222REGUF & $2 / 22 / 91$ & REG & 1,1,1-Trichloroethane & 5 & $\mathrm{ug} / \mathrm{L}$ & $\mathrm{U}$ \\
\hline 841 & 841103REGUF & $10 / 3 / 91$ & REG & $1,1,1$-Trichloroethane & 5 & $\mathrm{ug} / \mathrm{L}$ & $\mathrm{U}$ \\
\hline 841 & 8411210REGUF & $12 / 10 / 91$ & REG & 1,1,1-Trichloroethane & 5 & $\mathrm{ug} / \mathrm{L}$ & $\mathrm{U}$ \\
\hline 841 & 841521REGUF & $5 / 21 / 90$ & REG & 1,1,1-Trichloroethane & 5 & $\mathrm{ug} / \mathrm{L}$ & $\mathrm{U}$ \\
\hline 841 & 841119REGUF & $11 / 9 / 90$ & REG & 1,1,1-Trichloroethane & 5 & $\mathrm{ug} / \mathrm{L}$ & $\mathrm{U}$ \\
\hline 841 & 841126REGUF & $1 / 26 / 89$ & REG & 1,1,1-Trichloroethane & 5 & $\mathrm{ug} / \mathrm{L}$ & $\mathrm{U}$ \\
\hline 841 & 841427REGUF & $4 / 27 / 89$ & REG & 1,1,1-Trichloroethane & 5 & $\mathrm{ug} / \mathrm{L}$ & $U$ \\
\hline 841 & 84178REGUF & $7 / 8 / 88$ & REG & 1,1,1-Trichloroethane & 5 & $\mathrm{ug} / \mathrm{L}$ & $\mathrm{U}$ \\
\hline 841 & 8411024 REGUF & $10 / 24 / 88$ & REG & 1,1,1-Trichloroethane & 5 & $\mathrm{ug} / \mathrm{L}$ & $\mathrm{U}$ \\
\hline 841 & M01244 & $11 / 12 / 97$ & REG & 1,1,2,2-Tetrachloroeth & 5 & ug/L & $\mathrm{U}$ \\
\hline 841 & W00808 & $6 / 26 / 97$ & REG & 1,1,2,2-Tetrachloroethane & 5 & $\mathrm{ug} / \mathrm{L}$ & $\mathrm{U}$ \\
\hline 841 & M00051 & $12 / 5 / 96$ & REG & 1,1,2,2-Tetrachloroethane & 5 & $\mathrm{ug} / \mathrm{L}$ & $\mathrm{U}$ \\
\hline 841 & M00836 & $6 / 26 / 97$ & REG & 1,1,2,2-Tetrachloroethane & 5 & $\mathrm{ug} / \mathrm{L}$ & $\mathrm{U}$ \\
\hline 841 & SMPL00280 & $5 / 27 / 94$ & REG & 1,1,2,2-Tetrachloroethane & 1 & $\mathrm{ug} / \mathrm{L}$ & $\mathrm{U}$ \\
\hline 841 & S00933 & $10 / 19 / 94$ & REG & $1,1,2,2$-Tetrachloroethane & 5 & $\mathrm{ug} / \mathrm{L}$ & $\mathrm{U}$ \\
\hline 841 & S02607 & $2 / 28 / 95$ & REG & $1,1,2,2$-Tetrachloroethane & 5 & ug/L & $\mathrm{U}$ \\
\hline 841 & S03565 & $5 / 16 / 95$ & REG & 1,1,2,2-Tetrachloroethane & 5 & $\mathrm{ug} / \mathrm{L}$ & $\mathrm{U}$ \\
\hline 841 & S04776 & $7 / 14 / 95$ & REG & 1,1.2,2-Tetrachloroethane & 5 & $\mathrm{ug} / \mathrm{L}$ & $\mathrm{U}$ \\
\hline 841 & 841222REGUF & $2 / 22 / 91$ & REG & 1,1,2,2-Tetrachloroethane & 5 & $\mathrm{ug} / \mathrm{L}$ & $\mathrm{U}$ \\
\hline 841 & 841103REGUF & $10 / 3 / 91$ & REG & 1,1,2,2-Tetrachloroethane & 5 & $\mathrm{ug} / \mathrm{L}$ & $\mathrm{U}$ \\
\hline 841 & 8411210 REGUF & $12 / 10 / 91$ & REG & 1,1,2,2-Tetrachloroethane & 5 & $\mathrm{ug} / \mathrm{L}$ & $\mathrm{U}$ \\
\hline 841 & 841521REGUF & $5 / 21 / 90$ & REG & 1,1,2,2-Tetrachloroethane & 5 & $\mathrm{ug} / \mathrm{L}$ & $\mathrm{U}$ \\
\hline 841 & 841119 REGUF & $11 / 9 / 90$ & REG & 1,1,2,2-Tetrachloroethane & 5 & $\mathrm{ug} / \mathrm{L}$ & $\mathrm{U}$ \\
\hline
\end{tabular}


B-22

\begin{tabular}{|c|c|c|c|c|c|c|c|}
\hline Station & Sample ID & Sample Date & Sample Type & Chemical & Results & Units & Qualifier \\
\hline 841 & 841126REGUF & $1 / 26 / 89$ & REG & 1,1,2,2-Tetrachloroethane & 5 & $\mathrm{ug} / \mathrm{L}$ & $\bar{U}$ \\
\hline 841 & 841427REGUF & $4 / 27 / 89$ & REG & 1,1,2,2-Tetrachloroethane & 5 & $\mathrm{ug} / \mathrm{L}$ & $\mathrm{U}$ \\
\hline 841 & 84178REGUF & $7 / 8 / 88$ & REG & 1,1,2,2-Tetrachloroethane & 5 & $\mathrm{ug} / \mathrm{L}$ & $\mathrm{U}$ \\
\hline 841 & 8411024 REGUF & $10 / 24 / 88$ & REG & 1,1,2,2-Tetrachloroethane & 5 & $\mathrm{ug} / \mathrm{L}$ & $\mathrm{U}$ \\
\hline 841 & W00808 & $6 / 26 / 97$ & REG & 1,1,2-Trichloro-1,2,2-trifluo & 10 & $\mathrm{ug} / \mathrm{L}$ & $\mathrm{U}$ \\
\hline 841 & $\mathrm{M} 01244$ & $11 / 12 / 97$ & REG & 1,1,2-Trichloroethane & 5 & $\mathrm{ug} / \mathrm{L}$ & $\mathrm{U}$ \\
\hline 841 & W00808 & $6 / 26 / 97$ & REG & 1,1,2-Trichloroethane & 5 & $\mathrm{ug} / \mathrm{L}$ & $\mathrm{U}$ \\
\hline 841 & M00051 & $12 / 5 / 96$ & REG & 1,1,2-Trichloroethane & 5 & ug/L & $\mathrm{U}$ \\
\hline 841 & M00836 & $6 / 26 / 97$ & REG & 1,1,2-Trichloroethane & 5 & $\mathrm{ug} / \mathrm{L}$ & $\mathrm{U}$ \\
\hline 841 & SMPL00280 & $5 / 27 / 94$ & REG & 1,1,2-Trichloroethane & 1 & $\mathrm{ug} / \mathrm{L}$ & $\mathrm{U}$ \\
\hline 841 & S00933 & $10 / 19 / 94$ & REG & 1,1,2-Trichloroethane & 5 & $\mathrm{ug} / \mathrm{L}$ & $\mathrm{U}$ \\
\hline 841 & S02607 & $2 / 28 / 95$ & REG & 1,1,2-Trichloroethane & 5 & $\mathrm{ug} / \mathrm{L}$ & $\mathrm{U}$ \\
\hline 841 & S03565 & $5 / 16 / 95$ & REG & 1,1.2-Trichloroethane & 5 & $\mathrm{ug} / \mathrm{L}$ & $\mathrm{U}$ \\
\hline 841 & S04776 & $7 / 14 / 95$ & REG & 1,1,2-Trichloroethane & 5 & $\mathrm{ug} / \mathrm{L}$ & $\mathrm{U}$ \\
\hline 841 & 841222REGUF & $2 / 22 / 91$ & REG & 1,1,2-Trichloroethane & 5 & $\mathrm{ug} / \mathrm{L}$ & $U$ \\
\hline 841 & 841103REGUF & $10 / 3 / 91$ & REG & 1,1,2-Trichloroethane & 5 & $\mathrm{ug} / \mathrm{L}$ & $\mathrm{U}$ \\
\hline 841 & 8411210REGUF & $12 / 10 / 91$ & REG & 1,1,2-Trichloroethane & 5 & $\mathrm{ug} / \mathrm{L}$ & $\mathrm{U}$ \\
\hline 841 & 84152 IREGUF & $5 / 21 / 90$ & REG & 1,1,2-Trichloroethane & 5 & $\mathrm{ug} / \mathrm{L}$ & $\mathrm{U}$ \\
\hline 841 & 841119REGUF & $11 / 9 / 90$ & REG & 1,1,2-Trichloroethane & 5 & $\mathrm{ug} / \mathrm{L}$ & $\mathrm{U}$ \\
\hline 841 & 841126REGUF & $1 / 26 / 89$ & REG & 1,1,2-Trichloroethane & 5 & $\mathrm{ug} / \mathrm{L}$ & $\mathrm{U}$ \\
\hline 841 & 841427REGUF & $4 / 27 / 89$ & REG & 1,1,2-Trichloroethane & 5 & $\mathrm{ug} / \mathrm{L}$ & $\mathrm{U}$ \\
\hline 841 & 84178REGUF & $7 / 8 / 88$ & REG & 1,1,2-Trichloroethane & 5 & $\mathrm{ug} / \mathrm{L}$ & $\mathrm{U}$ \\
\hline 841 & 8411024REGUF & $10 / 24 / 88$ & REG & 1,1,2-Trichloroethane & 5 & $\mathrm{ug} / \mathrm{L}$ & $\mathrm{U}$ \\
\hline 841 & M01244 & $11 / 12 / 97$ & REG & 1,1-Dichloroethane & 5 & $\mathrm{ug} / \mathrm{L}$ & $\mathrm{U}$ \\
\hline 841 & W00808 & $6 / 26 / 97$ & REG & 1,1-Dichloroethane & 5 & $\mathrm{ug} / \mathrm{L}$ & $\mathrm{U}$ \\
\hline 841 & M00051 & $12 / 5 / 96$ & REG & 1,1-Dichloroethane & 5 & $\mathrm{ug} / \mathrm{L}$ & $\mathrm{U}$ \\
\hline 841 & M00836 & $6 / 26 / 97$ & REG & 1,1-Dichloroethane & 5 & $\mathrm{ug} / \mathrm{L}$ & $\mathrm{U}$ \\
\hline 841 & SMPL00280 & $5 / 27 / 94$ & REG & 1,1-Dichloroethane & 1 & $\mathrm{ug} / \mathrm{L}$ & $\mathrm{U}$ \\
\hline 841 & S00933 & $10 / 19 / 94$ & REG & 1,1-Dichloroethane & 5 & $\mathrm{ug} / \mathrm{L}$ & $\mathrm{U}$ \\
\hline 841 & S02607 & $2 / 28 / 95$ & REG & 1,1-Dichloroethane & 5 & $\mathrm{ug} / \mathrm{L}$ & $\mathrm{U}$ \\
\hline 841 & S03565 & $5 / 16 / 95$ & REG & 1,1-Dichloroethane & 5 & $\mathrm{ug} / \mathrm{L}$ & $\mathbf{U}$ \\
\hline 841 & S04776 & $7 / 14 / 95$ & REG & 1,1-Dichloroethane & 5 & $\mathrm{ug} / \mathrm{L}$ & $\mathrm{U}$ \\
\hline 841 & 841222REGUF & $2 / 22 / 91$ & REG & 1,1-Dichloroethane & 5 & $\mathrm{ug} / \mathrm{L}$ & $\mathrm{U}$ \\
\hline 841 & 841103 REGUF & $10 / 3 / 91$ & REG & 1,1-Dichloroethane & 5 & $\mathrm{ug} / \mathrm{L}$ & $\mathrm{U}$ \\
\hline 841 & 8411210REGUF & $12 / 10 / 91$ & REG & 1,1-Dichloroethane & 5 & $\mathrm{ug} / \mathrm{L}$ & $\mathrm{U}$ \\
\hline 841 & 841521REGUF & $5 / 21 / 90$ & REG & 1,1-Dichloroethane & 5 & $\mathrm{ug} / \mathrm{L}$ & $\mathrm{U}$ \\
\hline 841 & 841119REGUF & $11 / 9 / 90$ & REG & 1,1-Dichloroethane & 5 & $\mathrm{ug} / \mathrm{L}$ & $\mathrm{U}$ \\
\hline 841 & 841126REGUF & $1 / 26 / 89$ & REG & 1,1-Dichloroethane & 5 & $u g / L$ & $\mathrm{U}$ \\
\hline 841 & 841427REGUF & $4 / 27 / 89$ & REG & 1,1-Dichloroethane & 5 & $\mathrm{ug} / \mathrm{L}$ & $\mathbf{U}$ \\
\hline 841 & 84178REGUF & $7 / 8 / 88$ & REG & 1,1-Dichloroethane & 5 & $\mathrm{ug} / \mathrm{L}$ & $\mathbf{U}$ \\
\hline 841 & 8411024REGUF & $10 / 24 / 88$ & REG & 1,1-Dichloroethane & 5 & $\mathrm{ug} / \mathrm{L}$ & $\mathbf{U}$ \\
\hline 841 & M01244 & $11 / 12 / 97$ & REG & 1,1-Dichloroethene & 5 & $u g / L$ & $\mathrm{U}$ \\
\hline 841 & W00808 & $6 / 26 / 97$ & REG & 1,1-Dichloroethene & 5 & $\mathrm{ug} / \mathrm{L}$ & $\mathrm{U}$ \\
\hline 841 & M00051 & $12 / 5 / 96$ & REG & 1,1-Dichloroethene & 5 & $\mathrm{ug} / \mathrm{L}$ & $\mathrm{U}$ \\
\hline 841 & M00836 & $6 / 26 / 97$ & REG & 1,1-Dichloroethene & 5 & $\mathrm{ug} / \mathrm{L}$ & U \\
\hline 841 & SMPL00280 & $5 / 27 / 94$ & REG & 1,1-Dichloroethene & 1 & $\mathrm{ug} / \mathrm{L}$ & $\mathrm{U}$ \\
\hline 841 & S00933 & $10 / 19 / 94$ & REG & 1,1-Dichloroethene & 5 & $\mathrm{ug} / \mathrm{L}$ & $\mathrm{U}$ \\
\hline 841 & S02607 & $2 / 28 / 95$ & REG & 1,1-Dichloroethene & 5 & $u g / L$ & $\mathrm{U}$ \\
\hline 841 & S03565 & $5 / 16 / 95$ & REG & 1,1-Dichloroethene & 5 & $\mathrm{ug} / \mathrm{L}$ & $\mathrm{U}$ \\
\hline 841 & S04776 & $7 / 14 / 95$ & REG & 1,1-Dichloroethene & 5 & $\mathrm{ug} / \mathrm{L}$ & $\mathrm{U}$ \\
\hline
\end{tabular}




\begin{tabular}{|c|c|c|c|c|c|c|c|}
\hline Station & Sample ID & Sample Date & Sample Type & Chemical & Results & Units & Qualifier \\
\hline 841 & 841222REGUF & $2 / 22 / 91$ & REG & 1,1-Dichloroethene & 5 & $\mathrm{ug} / \mathrm{L}$ & $\mathrm{U}$ \\
\hline 841 & 841103 REGUF & $10 / 3 / 91$ & REG & 1.1-Dichloroethene & 5 & ug/L & $\mathrm{U}$ \\
\hline 841 & 8411210 REGUF & $12 / 10 / 91$ & REG & 1,1-Dichloroethene & 5 & $u g / L$ & $\mathrm{U}$ \\
\hline 841 & 841521REGUF & $5 / 21 / 90$ & REG & 1.1-Dichloroethene & 5 & $u g / L$ & $\mathrm{U}$. \\
\hline 841 & 841119REGUF & $11 / 9 / 90$ & REG & 1,1-Dichloroethene & 5 & $\mathrm{ug} / \mathrm{L}$ & $\mathrm{U}$ \\
\hline 841 & 841126REGUF & $1 / 26 / 89$ & REG & 1,1-Dichloroethene & 5 & $\mathrm{ug} / \mathrm{L}$ & $\mathrm{U}$ \\
\hline 841 & 841427REGUF & $4 / 27 / 89$ & REG & 1,1-Dichloroethene & 5 & $\mathrm{ug} / \mathrm{L}$ & $\mathrm{U}$ \\
\hline 841 & 84178REGUF & $7 / 8 / 88$ & REG & 1,1-Dichloroethene & 5 & $\mathrm{ug} / \mathrm{L}$ & $\mathrm{U}$ \\
\hline 841 & 8411024REGUF & $10 / 24 / 88$ & REG & 1.1-Dichloroethene & 5 & ug/L & $\mathrm{U}$ \\
\hline 841 & SMPL00280 & $5 / 27 / 94$ & REG & 1,2-Dibromo-3-chloropropane & 1 & ug/L & $U$ \\
\hline 841 & SMPL00280 & $5 / 27 / 94$ & REG & 1,2-Dibromoethane & 1 & $\mathrm{ug} / \mathrm{L}$ & $\mathrm{U}$ \\
\hline 841 & M00051 & $12 / 5 / 96$ & REG & 1,2-Dichlorobenzene & 5 & $\mathrm{ug} / \mathrm{L}$ & $\mathrm{U}$ \\
\hline 841 & SMPL00280 & $5 / 27 / 94$ & REG & 1,2-Dichlorobenzene & 1 & $\mathrm{ug} / \mathrm{L}$ & $\mathrm{U}$ \\
\hline 841 & M01244 & $11 / 12 / 97$ & REG & 1,2-Dichloroethane & 5 & ug/L & $\mathrm{U}$ \\
\hline 841 & W00808 & $6 / 26 / 97$ & REG & 1,2-Dichloroethane & 5 & $\mathrm{ug} / \mathrm{L}$ & $\mathrm{U}$ \\
\hline 841 & M00051 & $12 / 5 / 96$ & REG & 1,2-Dichloroethane & 1 & $\mathrm{ug} / \mathrm{L}$ & $=$ \\
\hline 841 & M00836 & $6 / 26 / 97$ & REG & 1,2-Dichloroethane & 5 & $\mathrm{ug} / \mathrm{L}$ & $\mathrm{U}$ \\
\hline 841 & SMPL00280 & $5 / 27 / 94$ & REG & 1,2-Dichloroethane & 1 & $\mathrm{ug} / \mathrm{L}$ & $\mathrm{U}$ \\
\hline 841 & S00933 & $10 / 19 / 94$ & REG & 1,2-Dichloroethane & 5 & $\mathrm{ug} / \mathrm{L}$ & $\mathrm{U}$ \\
\hline 841 & S02607 & $2 / 28 / 95$ & REG & 1,2-Dichloroethane & 1 & $\mathrm{ug} / \mathrm{L}$ & $J$ \\
\hline 841 & S03565 & $5 / 16 / 95$ & REG & 1,2-Dichloroethane & 1 & ug/L & $\mathrm{J}$ \\
\hline 841 & S04776 & $7 / 14 / 95$ & REG & 1,2-Dichloroethane & 5 & ug/L & $\mathrm{U}$ \\
\hline 841 & 841222REGUF & $2 / 22 / 91$ & REG & 1,2-Dichloroethane & 5 & $\mathrm{ug} / \mathrm{L}$ & $\mathrm{U}$ \\
\hline 841 & $841103 R E G U F$ & $10 / 3 / 91$ & REG & 1,2-Dichloroethane & 5 & ug/L & $\mathrm{U}$ \\
\hline 841 & 8411210 REGUF & $12 / 10 / 91$ & REG & 1,2-Dichloroethane & 5 & $\mathrm{ug} / \mathrm{L}$ & $\mathrm{U}$ \\
\hline 841 & 84152 IREGUF & $5 / 21 / 90$ & REG & 1,2-Dichloroethane & 5 & $\mathrm{ug} / \mathrm{L}$ & $\mathrm{U}$ \\
\hline 841 & 841119REGUF & $11 / 9 / 90$ & REG & 1,2-Dichloroethane & 5 & $\mathrm{ug} / \mathrm{L}$ & $\mathrm{U}$ \\
\hline 841 & 841126REGUF & $1 / 26 / 89$ & REG & 1,2-Dichloroethane & 5 & $\mathrm{ug} / \mathrm{L}$ & $\mathrm{U}$ \\
\hline 841 & 841427REGUF & $4 / 27 / 89$ & REG & 1,2-Dichloroethane & 5 & $\mathrm{ug} / \mathrm{L}$ & $\mathbf{U}$ \\
\hline 841 & 84178REGUF & $7 / 8 / 88$ & REG & 1,2-Dichloroethane & 5 & $\mathrm{ug} / \mathrm{L}$ & $\mathrm{U}$ \\
\hline 841 & 8411024REGUF & $10 / 24 / 88$ & REG & 1,2-Dichloroethane & 5 & $\mathrm{ug} / \mathrm{L}$ & $\mathrm{U}$ \\
\hline 841 & W00808 & $6 / 26 / 97$ & REG & 1,2-Dichloroethene & 5 & $\mathrm{ug} / \mathrm{L}$ & $\mathrm{U}$ \\
\hline 841 & M00051 & $12 / 5 / 96$ & REG & 1,2-Dichloroethene & 1 & $\mathrm{ug} / \mathrm{L}$ & $=$ \\
\hline 841 & S00933 & $10 / 19 / 94$ & REG & 1,2-Dichloroethene & 5 & $\mathrm{ug} / \mathrm{L}$ & $U$ \\
\hline 841 & S02607 & $2 / 28 / 95$ & REG & 1,2-Dichloroethene & 5 & ug/L & $\mathrm{U}$ \\
\hline 841 & S03565 & $5 / 16 / 95$ & REG & 1,2-Dichloroethene & 5 & $\mathrm{ug} / \mathrm{L}$ & $\dot{U}$ \\
\hline 841 & S04776 & $7 / 14 / 95$ & REG & 1,2-Dichloroethene & 5 & $\mathrm{ug} / \mathrm{L}$ & $\mathrm{U}$ \\
\hline 841 & 841222REGUF & $2 / 22 / 91$ & REG & 1,2-Dichloroethene & 5 & $\mathrm{ug} / \mathrm{L}$ & $\mathrm{U}$ \\
\hline 841 & 841103REGUF & $10 / 3 / 91$ & REG & 1,2-Dichloroethene & 5 & ug/L & $\mathrm{U}$ \\
\hline 841 & 8411210 REGUF & $12 / 10 / 91$ & REG & 1,2-Dichloroethene & 5 & $\mathrm{ug} / \mathrm{L}$ & U \\
\hline 841 & 841521REGUF & $5 / 21 / 90$ & REG & 1,2-Dichloroethene & 5 & $\mathrm{ug} / \mathrm{L}$ & $\mathrm{U}$ \\
\hline 841 & 841119REGUF & $11 / 9 / 90$ & REG & 1,2-Dichloroethene & 5 & $\mathrm{ug} / \mathrm{L}$ & $\mathrm{U}$ \\
\hline 841 & 841126REGUF & $1 / 26 / 89$ & REG & 1,2-Dichloroethene & 5 & $u g / L$ & $\mathrm{U}$ \\
\hline 841 & 841427REGUF & $4 / 27 / 89$ & REG & 1,2-Dichloroethene & 5 & $\mathrm{ug} / \mathrm{L}$ & $U$ \\
\hline 841 & 84178REGUF & $7 / 8 / 88$ & REG & 1,2-Dichloroethene & 5 & ug/L & $\mathrm{U}$ \\
\hline 841 & 8411024REGUF & $10 / 24 / 88$ & REG & 1,2-Dichloroethene & 18 & ug/L & $=$ \\
\hline 841 & M01244 & $11 / 12 / 97$ & REG & 1,2-Dichloropropane & 5 & $\mathrm{ug} / \mathrm{L}$ & $\mathrm{U}$ \\
\hline 841 & W00808 & $6 / 26 / 97$ & REG & 1,2-Dichloropropane & 5 & ug/L & $\mathrm{U}$ \\
\hline 841 & M00051 & $12 / 5 / 96$ & REG & 1,2-Dichloropropane & 5 & $\mathrm{ug} / \mathrm{L}$ & $\mathrm{U}$ \\
\hline 841 & M00836 & $6 / 26 / 97$ & REG & 1,2-Dichloropropane & 5 & $\mathrm{ug} / \mathrm{L}$ & $\mathrm{U}$ \\
\hline
\end{tabular}


B-24

Station Sample ID Sample Date Sample Type Chemical

\begin{tabular}{|c|c|c|c|c|c|c|c|}
\hline Station & Sample ID & Sample Date & Sample Type & Chemical & Results & Units & Qualifier \\
\hline$\overline{841}$ & $\overrightarrow{\text { SMPL00280 }}$ & $5 / 27 / 94$ & REG & 1,2-Dichloropropane & 1 & $\mathrm{ug} / \mathrm{L}$ & $\mathrm{U}$ \\
\hline 841 & S00933 & $10 / 19 / 94$ & REG & 1.2-Dichloropropane & 5 & $\mathrm{ug} / \mathrm{L}$ & $\mathrm{U}$ \\
\hline 841 & S02607 & $2 / 28 / 95$ & REG & 1,2-Dichloropropane & 5 & $\mathrm{ug} / \mathrm{L}$ & $\mathrm{U}$ \\
\hline 841 & S03565 & $5 / 16 / 95$ & REG & 1,2-Dichloropropane & 5 & ug/L & $\mathrm{U}$ \\
\hline 841 & S04776 & $7 / 14 / 95$ & REG & 1,2-Dichloropropane & 5 & $\mathrm{ug} / \mathrm{L}$ & $\mathrm{U}$ \\
\hline 841 & 841222REGUF & $2 / 22 / 91$ & REG & 1.2-Dichloropropane & 5 & $\mathrm{ug} / \mathrm{L}$ & $\mathrm{U}$ \\
\hline 841 & 841103REGUF & $10 / 3 / 91$ & REG & 1,2-Dichloropropane & 5 & ug/L & $\mathrm{U}$ \\
\hline 841 & 8411210REGUF & $12 / 10 / 91$ & REG & 1,2-Dichloropropane & 5 & $\mathrm{ug} / \mathrm{L}$ & $\mathrm{U}$ \\
\hline $84 I$ & 841521REGUF & $5 / 21 / 90$ & REG & 1,2-Dichloropropane & 5 & $\mathrm{ug} / \mathrm{L}$ & $\mathrm{U}$ \\
\hline 841 & 841119REGUF & $11 / 9 / 90$ & REG & 1,2-Dichloropropane & 5 & $\mathrm{ug} / \mathrm{L}$ & $\mathrm{U}$ \\
\hline 841 & 841126REGUF & $1 / 26 / 89$ & REG & 1,2-Dichloropropane & 5 & $\mathrm{ug} / \mathrm{L}$ & $\mathrm{U}$ \\
\hline 841 & 841427REGUF & $4 / 27 / 89$ & REG & 1,2-Dichloropropane & 5 & ug/L & $\mathrm{U}$ \\
\hline 841 & 84178REGUF & $7 / 8 / 88$ & REG & 1,2-Dichloropropane & 5 & $\mathrm{ug} / \mathrm{L}$ & $\mathrm{U}$ \\
\hline 841 & 8411024REGUF & $10 / 24 / 88$ & REG & 1,2-Dichloropropane & 5 & $u g / L$ & $\mathrm{U}$ \\
\hline 841 & M00051 & $12 / 5 / 96$ & REG & 1,2-Dimethylbenzene & 5 & $\mathrm{ug} / \mathrm{L}$ & $\mathrm{U}$ \\
\hline 841 & S02607 & $2 / 28 / 95$ & REG & 1.2-Dimethylbenzene & 5 & $\mathrm{ug} / \mathrm{L}$ & $U$ \\
\hline 841 & S03565 & $5 / 16 / 95$ & REG & 1,2-Dimethylbenzene & 5 & $u g / L$ & $\mathrm{U}$ \\
\hline 841 & S04776 & $7 / 14 / 95$ & REG & 1,2-Dimethylbenzene & 5 & ug/L & $\mathrm{U}$ \\
\hline 841 & M00051 & $12 / 5 / 96$ & REG & 1,3-Dichlorobenzene & 5 & $\mathrm{ug} / \mathrm{L}$ & $\mathrm{U}$ \\
\hline 841 & SMPL00280 & $5 / 27 / 94$ & REG & 1,3-Dichlorobenzene & 1 & $u g / L$ & $\mathrm{U}$ \\
\hline 841 & M00051 & $12 / 5 / 96$ & REG & 1,4-Dichlorobenzene & 5 & $\mathrm{ug} / \mathrm{L}$ & $\mathrm{U}$ \\
\hline 841 & SMPL00280 & $5 / 27 / 94$ & REG & 1,4-Dichlorobenzene & 1 & $\mathrm{ug} / \mathrm{L}$ & $\mathrm{U}$ \\
\hline 841 & Mo1244 & $11 / 12 / 97$ & REG & 2-Butanone & 10 & ug/L & $\mathrm{U}$ \\
\hline 841 & W00808 & $6 / 26 / 97$ & REG & 2-Butanone & 10 & $\mathrm{ug} / \mathrm{L}$ & $\mathrm{U}$ \\
\hline 841 & M00051 & $12 / 5 / 96$ & REG & 2-Butanone & 2 & $\mathrm{ug} / \mathrm{L}$ & $=$ \\
\hline 841 & M00836 & $6 / 26 / 97$ & REG & 2-Butanone & 10 & $\mathrm{ug} / \mathrm{L}$ & $\mathrm{U}$ \\
\hline 841 & SMPL00280 & $5 / 27 / 94$ & REG & 2-Butanone & 5 & $\mathrm{ug} / \mathrm{L}$ & $\mathrm{U}$ \\
\hline 841 & S00933 & $10 / 19 / 94$ & REG & 2-Butanone & 3.5 & $\mathrm{ug} / \mathrm{L}$ & $\mathrm{J}$ \\
\hline 841 & S02607 & $2 / 28 / 95$ & REG & 2-Butanone & 4 & $\mathrm{ug} / \mathrm{L}$ & $J$ \\
\hline 841 & S03565 & $5 / 16 / 95$ & REG & 2-Butanone & 2 & $\mathrm{ug} / \mathrm{L}$ & $\mathrm{J}$ \\
\hline 841 & S04776 & $7 / 14 / 95$ & REG & 2-Butanone & 10 & $\mathrm{ug} / \mathrm{L}$ & $\mathrm{U}$ \\
\hline 841 & 841222REGUF & $2 / 22 / 91$ & REG & 2-Butanone & 10 & $\mathrm{ug} / \mathrm{L}$ & $\mathrm{U}$ \\
\hline 841 & 841103REGUF & $10 / 3 / 91$ & REG & 2-Butanone & 10 & $\mathrm{ug} / \mathrm{L}$ & $\mathrm{U}$ \\
\hline 841 & 8411210REGUF & $12 / 10 / 91$ & REG & 2-Butanone & 10 & ug/L & $\mathrm{U}$ \\
\hline 841 & 841521REGUF & $5 / 21 / 90$ & REG & 2-Butanone & 10 & $\mathrm{ug} / \mathrm{L}$ & $U$ \\
\hline 841 & 841119REGUF & $11 / 9 / 90$ & REG & 2-Butanone & 10 & $\mathrm{ug} / \mathrm{L}$ & $\mathrm{U}$ \\
\hline 841 & 841126REGUF & $1 / 26 / 89$ & REG & 2-Butanone & 10 & $\mathrm{ug} / \mathrm{L}$ & $\mathrm{U}$ \\
\hline 841 & 841427REGUF & $4 / 27 / 89$ & REG & 2-Butanone & 10 & $\mathrm{ug} / \mathrm{L}$ & $\mathrm{U}$ \\
\hline 841 & 84178REGUF & $7 / 8 / 88$ & REG & 2-Butanone & 10 & ug/L & $\mathrm{U}$ \\
\hline 841 & 8411024REGUF & $10 / 24 / 88$ & REG & 2-Butanone & 10 & ug/L & U \\
\hline 841 & M01244 & $11 / 12 / 97$ & REG & 2-Hexanone & 10 & ug/L & $\mathrm{U}$ \\
\hline 841 & W00808 & $6 / 26 / 97$ & REG & 2-Hexanone & 10 & ug/L & $\mathrm{U}$ \\
\hline 841 & M00051 & $12 / 5 / 96$ & REG & 2-Hexanone & 10 & ug/L & $\mathrm{U}$ \\
\hline 841 & M00836 & $6 / 26 / 97$ & REG & 2-Hexanone & 10 & $\mathrm{ug} / \mathrm{L}$ & $\mathrm{U}$ \\
\hline 841 & SMPL00280 & $5 / 27 / 94$ & REG & 2-Hexanone & 5 & $\mathrm{ug} / \mathrm{L}$ & $\mathrm{U}$ \\
\hline 841 & S00933 & $10 / 19 / 94$ & REG & 2-Hexanone & 10 & $\mathrm{ug} / \mathrm{L}$ & $\mathrm{U}$ \\
\hline 841 & S02607 & $2 / 28 / 95$ & REG & 2-Hexanone & 10 & $\mathrm{ug} / \mathrm{L}$ & $\mathrm{U}$ \\
\hline 841 & S03565 & $5 / 16 / 95$ & REG & 2-Hexanone & 10 & ug/L & $\mathrm{U}$ \\
\hline 841 & S04776 & $7 / 14 / 95$ & REG & 2-Hexanone & 10 & $\mathrm{ug} / \mathrm{L}$ & $\mathrm{U}$ \\
\hline 841 & 841222REGUF & $2 / 22 / 91$ & REG & 2-Hexanone & 10 & $\mathrm{ug} / \mathrm{L}$ & $\mathrm{U}$ \\
\hline
\end{tabular}




\section{B-25}

Station Sample ID Sample Date Sample Type Chemical

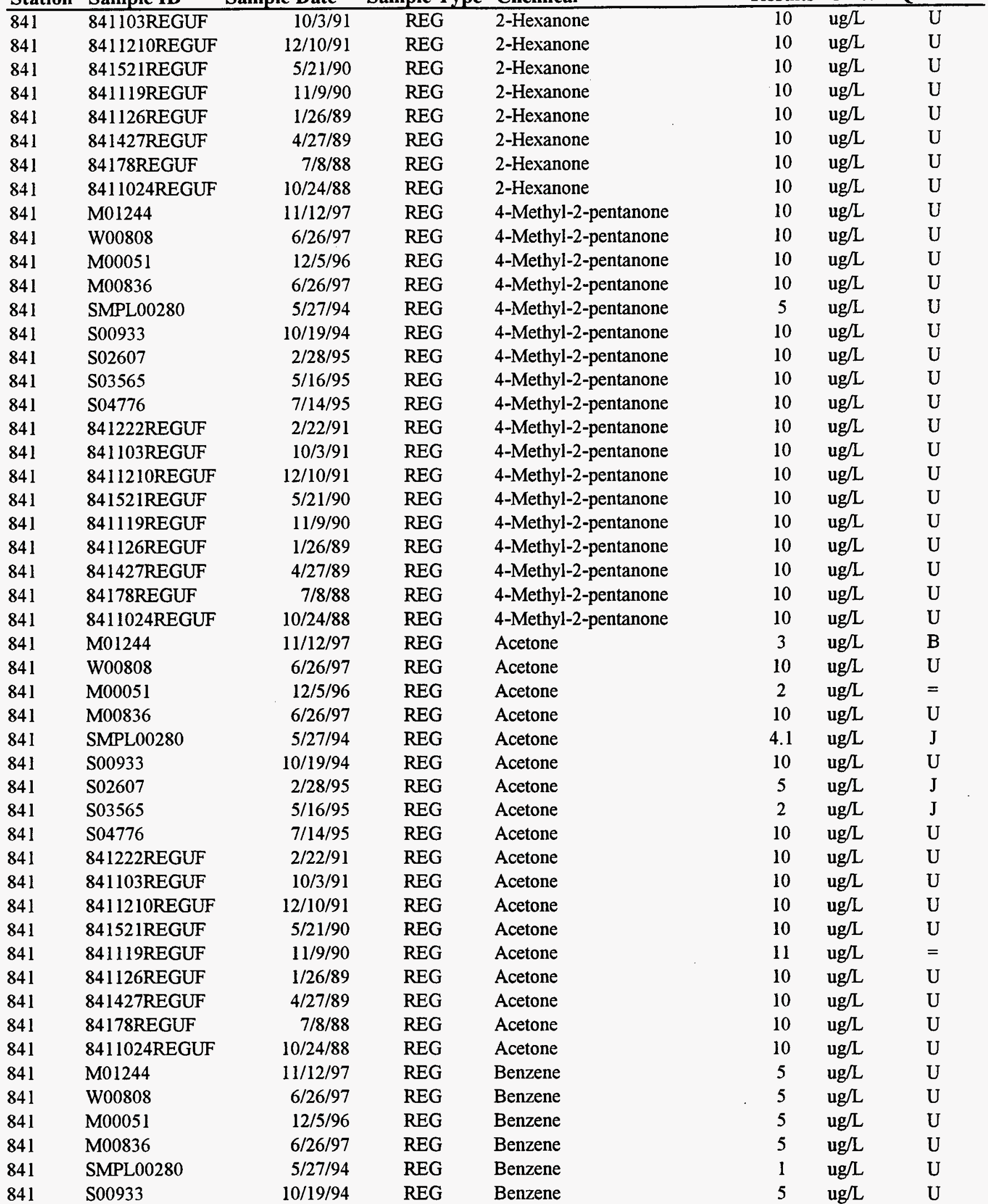




\begin{tabular}{|c|c|c|c|c|c|c|c|}
\hline Station & Sample ID & Sample Date & Sample Type & Chemical & Results & Units & Qualifier \\
\hline$\overline{841}$ & S02607 & $2 / 28 / 95$ & REG & Benzene & 5 & $\mathrm{ug} / \mathrm{L}$ & $\bar{U}$ \\
\hline 841 & S03565 & $5 / 16 / 95$ & REG & Benzene & 5 & $\mathrm{ug} / \mathrm{L}$ & $\mathrm{U}$ \\
\hline 841 & S04776 & $7 / 14 / 95$ & REG & Benzene & 5 & $\mathrm{ug} / \mathrm{L}$ & $\mathrm{U}$ \\
\hline 841 & 841222REGUF & $2 / 22 / 91$ & REG & Benzene & 5 & ug $/ \mathrm{L}$ & $\mathrm{U}$ \\
\hline 841 & 841103REGUF & $10 / 3 / 91$ & REG & Benzene & 5 & $\mathrm{ug} / \mathrm{L}$ & $\mathrm{U}$ \\
\hline 841 & 8411210REGUF & $12 / 10 / 91$ & REG & Benzene & 5 & ug/L & $\mathrm{U}$ \\
\hline 841 & 841521REGUF & $5 / 21 / 90$ & REG & Benzene & 5 & ug/L & $\mathrm{U}$ \\
\hline 841 & 841119REGUF & $11 / 9 / 90$ & REG & Benzene & 5 & ug/L & $\mathrm{U}$ \\
\hline 841 & 841126REGUF & $1 / 26 / 89$ & REG & Benzene & 5 & $\mathrm{ug} / \mathrm{L}$ & U \\
\hline 841 & 841427REGUF & $4 / 27 / 89$ & REG & Benzene & 5 & ug/L & U \\
\hline 841 & 84178REGUF & $7 / 8 / 88$ & REG & Benzene & 5 & ug $/ \mathrm{L}$ & $\mathrm{U}$ \\
\hline 841 & 8411024REGUF & $10 / 24 / 88$ & REG & Benzene & 5 & ug $/ \mathrm{L}$ & $\mathrm{U}$ \\
\hline 841 & SMPL00280 & $5 / 27 / 94$ & REG & Bromochloromethane & 1 & ug/L & $\mathrm{U}$ \\
\hline 841 & M01244 & $11 / 12 / 97$ & REG & Bromodichloromethane & 5 & ug/L & $\mathrm{U}$ \\
\hline 841 & W00808 & $6 / 26 / 97$ & REG & Bromodichloromethane & 5 & ug/L & $\mathrm{U}$ \\
\hline 841 & M00051 & $12 / 5 / 96$ & REG & Bromodichloromethane & 5 & ug/L & $\mathrm{U}$ \\
\hline 841 & M00836 & $6 / 26 / 97$ & REG & Bromodichloromethane & 5 & ug/L & U \\
\hline 841 & SMPL00280 & $5 / 27 / 94$ & REG & Bromodichloromethane & 1 & ug/L & $\mathrm{U}$ \\
\hline 841 & S00933 & $10 / 19 / 94$ & REG & Bromodichloromethane & 5 & ug/L & $\mathrm{U}$ \\
\hline 841 & S02607 & $2 / 28 / 95$ & REG & Bromodichloromethane & 5 & ug/L & $\mathrm{U}$ \\
\hline 841 & S03565 & $5 / 16 / 95$ & REG & Bromodichloromethane & 5 & ug $/ \mathrm{L}$ & $\mathrm{U}$ \\
\hline 841 & S04776 & $7 / 14 / 95$ & REG & Bromodichloromethane & 5 & ug/L & $\mathrm{U}$ \\
\hline 841 & 841222REGUF & $2 / 22 / 91$ & REG & Bromodichloromethane & 5 & ug/L & $\mathrm{U}$ \\
\hline 841 & 841103REGUF & $10 / 3 / 91$ & REG & Bromodichloromethane & 5 & ug/L & $\mathrm{U}$ \\
\hline 841 & 8411210 REGUF & $12 / 10 / 91$ & REG & Bromodichloromethane & 5 & ug/L & $\mathrm{U}$ \\
\hline 841 & 841521REGUF & $5 / 21 / 90$ & REG & Bromodichloromethane & 5 & ug/L & $\mathrm{U}$ \\
\hline 841 & 841119REGUF & $11 / 9 / 90$ & REG & Bromodichloromethane & 5 & ug/L & U \\
\hline 841 & 841126REGUF & $1 / 26 / 89$ & REG & Bromodichloromethane & 5 & $\mathrm{ug} / \mathrm{L}$ & $\mathrm{U}$ \\
\hline 841 & 841427REGUF & $4 / 27 / 89$ & REG & Bromodichloromethane & 5 & ug/L & $\mathrm{U}$ \\
\hline 841 & 84178REGUF & $7 / 8 / 88$ & REG & Bromodichloromethane & 5 & ug/L & $\mathrm{U}$ \\
\hline 841 & 8411024REGUF & $10 / 24 / 88$ & REG & Bromodichloromethane & 5 & ug/L & $\mathrm{U}$ \\
\hline 841 & M01244 & $11 / 12 / 97$ & REG & Bromoform & 5 & $u g / L$ & U \\
\hline 841 & W00808 & $6 / 26 / 97$ & REG & Bromoform & 5 & ug/L & $\mathrm{U}$ \\
\hline 841 & M0005I & $12 / 5 / 96$ & REG & Bromoform & 5 & ug/L & $\mathrm{U}$ \\
\hline 841 & M00836 & $6 / 26 / 97$ & REG & Bromoform & 5 & ug/L & $\mathrm{U}$ \\
\hline 841 & SMPL00280 & $5 / 27 / 94$ & REG & Bromoform & 1 & $\mathrm{ug} / \mathrm{L}$ & $\mathrm{U}$ \\
\hline 841 & S00933 & $10 / 19 / 94$ & REG & Bromoform & 5 & $\mathrm{ug} / \mathrm{L}$ & $\mathrm{U}$ \\
\hline 841 & S02607 & $2 / 28 / 95$ & REG & Bromoform & 5 & $\mathrm{ug} / \mathrm{L}$ & $\mathrm{U}$ \\
\hline 841 & S03565 & $5 / 16 / 95$ & REG & Bromoform & 5 & $\mathrm{ug} / \mathrm{L}$ & $\mathrm{U}$ \\
\hline 841 & S04776 & $7 / 14 / 95$ & REG & Bromoform & 5 & $\mathrm{ug} / \mathrm{L}$ & $\mathrm{U}$ \\
\hline 841 & 841222REGUF & $2 / 22 / 91$ & REG & Bromoform & 5 & $\mathrm{ug} / \mathrm{L}$ & $\mathrm{U}$ \\
\hline 841 & 841103REGUF & $10 / 3 / 91$ & REG & Bromoform & 5 & $\mathrm{ug} / \mathrm{L}$ & $\mathrm{U}$ \\
\hline 841 & 8411210REGUF & $12 / 10 / 91$ & REG & Bromoform & 5 & ug/L & $\mathrm{U}$ \\
\hline 841 & 84152 IREGUF & $5 / 21 / 90$ & REG & Bromoform & 5 & $\mathrm{ug} / \mathrm{L}$ & $\mathrm{U}$ \\
\hline 841 & 841119 REGUF & $11 / 9 / 90$ & REG & Bromoform & 5 & $u g / L$ & $\mathrm{U}$ \\
\hline 841 & 841126REGUF & $1 / 26 / 89$ & REG & Bromoform & 5 & ug/L & $\mathrm{U}$ \\
\hline 841 & 841427REGUF & $4 / 27 / 89$ & REG & Bromoform & 5 & $\mathrm{ug} / \mathrm{L}$ & $\mathrm{U}$ \\
\hline 841 & 84178REGUF & $7 / 8 / 88$ & REG & Bromoform & 5 & $\mathrm{ug} / \mathrm{L}$ & $\mathrm{U}$ \\
\hline 841 & 841 1024REGUF & $10 / 24 / 88$ & REG & Bromoform & 5 & $\mathrm{ug} / \mathrm{L}$ & $\mathrm{U}$ \\
\hline 841 & M01244 & $11 / 12 / 97$ & REG & Bromomethane & 10 & ug/L & $\mathrm{U}$ \\
\hline
\end{tabular}


Station Sample ID Sample Date Sample Type Chemical

\begin{tabular}{|c|c|c|c|c|c|c|c|}
\hline & & & & & & & \\
\hline 841 & W00808 & $6 / 26 / 97$ & REG & Bromomethane & 10 & $\mathrm{ug} / \mathrm{L}$ & $\mathrm{U}$ \\
\hline 841 & M00051 & $12 / 5 / 96$ & REG & Bromomethane & 10 & $\mathrm{ug} / \mathrm{L}$ & $\mathrm{U}$ \\
\hline 841 & M00836 & $6 / 26 / 97$ & REG & Bromomethane & 10 & $\mathrm{ug} / \mathrm{L}$ & $\mathrm{U}$ \\
\hline 841 & SMPL00280 & $5 / 27 / 94$ & REG & Bromomethane & 1 & $\mathrm{ug} / \mathrm{L}$ & $\mathrm{U}$ \\
\hline 841 & S00933 & $10 / 19 / 94$ & REG & Bromomethane & 10 & $\mathrm{ug} / \mathrm{L}$ & $\mathrm{U}$ \\
\hline 841 & S02607 & $2 / 28 / 95$ & REG & Bromomethane & 10 & $\mathrm{ug} / \mathrm{L}$ & $\mathrm{U}$ \\
\hline 841 & S03565 & $5 / 16 / 95$ & REG & Bromomethane & 10 & $\mathrm{ug} / \mathrm{L}$ & $\mathrm{U}$ \\
\hline 841 & S04776 & $7 / 14 / 95$ & REG & Bromomethane & 10 & $\mathrm{ug} / \mathrm{L}$ & $\mathrm{U}$ \\
\hline 841 & 841222REGUF & $2 / 22 / 91$ & REG & Bromomethane & 10 & $\mathrm{ug} / \mathrm{L}$ & $\mathrm{U}$ \\
\hline 841 & 841103REGUF & $10 / 3 / 91$ & REG & Bromomethane & 10 & $\mathrm{ug} / \mathrm{L}$ & $\mathrm{U}$ \\
\hline 841 & 8411210 REGUF & $12 / 10 / 91$ & REG & Bromomethane & 10 & $\mathrm{ug} / \mathrm{L}$ & $\mathrm{U}$ \\
\hline 841 & 84152 lREGUF & $5 / 21 / 90$ & REG & Bromomethane & 10 & $\mathrm{ug} / \mathrm{L}$ & $\mathrm{U}$ \\
\hline 841 & 841119REGUF & $11 / 9 / 90$ & REG & Bromomethane & 10 & ug/L & $\mathrm{U}$ \\
\hline 841 & 841126REGUF & $1 / 26 / 89$ & REG & Bromomethane & 10 & $u g / L$ & $\mathrm{U}$ \\
\hline 841 & 841427REGUF & $4 / 27 / 89$ & REG & Bromomethane & 10 & $\mathrm{ug} / \mathrm{L}$ & $\mathrm{U}$ \\
\hline 841 & 84178REGUF & $7 / 8 / 88$ & REG & Bromomethane & 10 & $\mathrm{ug} / \mathrm{L}$ & $\mathrm{U}$ \\
\hline 841 & 8411024REGUF & $10 / 24 / 88$ & REG & Bromomethane & 10 & $\mathrm{ug} / \mathrm{L}$ & $\mathrm{U}$ \\
\hline 841 & M01244 & $11 / 12 / 97$ & REG & Carbon disulfide & 5 & $u g / L$ & $\mathrm{U}$ \\
\hline 841 & W00808 & $6 / 26 / 97$ & REG & Carbon disulfide & 5 & $\mathrm{ug} / \mathrm{L}$ & $\mathrm{U}$ \\
\hline 841 & M00051 & $12 / 5 / 96$ & REG & Carbon disulfide & 5 & $\mathrm{ug} / \mathrm{L}$ & $\mathrm{U}$ \\
\hline 841 & M00836 & $6 / 26 / 97$ & REG & Carbon disulfide & 5 & $\mathrm{ug} / \mathrm{L}$ & $\mathrm{U}$ \\
\hline 841 & SMPL00280 & $5 / 27 / 94$ & REG & Carbon disulfide & 1 & ug/L & $\mathrm{U}$ \\
\hline 841 & S00933 & $10 / 19 / 94$ & REG & Carbon disulfide & 5 & $\mathrm{ug} / \mathrm{L}$ & $\mathrm{U}$ \\
\hline 841 & S02607 & $2 / 28 / 95$ & REG & Carbon disulfide & 5 & ug/L & $\mathrm{U}$ \\
\hline 841 & S03565 & $5 / 16 / 95$ & REG & Carbon disulfide & 5 & $\mathrm{ug} / \mathrm{L}$ & $\mathrm{U}$ \\
\hline 841 & S04776 & $7 / 14 / 95$ & REG & Carbon disulfide & 5 & $\mathrm{ug} / \mathrm{L}$ & $\mathrm{U}$ \\
\hline 841 & 841222REGUF & $2 / 22 / 91$ & REG & Carbon disulfide & 5 & $\mathrm{ug} / \mathrm{L}$ & $\mathrm{U}$ \\
\hline 841 & 841103REGUF & $10 / 3 / 91$ & REG & Carbon disulfide & 160 & $u g / L$ & $=$ \\
\hline 841 & 8411210REGUF & $12 / 10 / 91$ & REG & Carbon disulfide & 5 & $\mathrm{ug} / \mathrm{L}$ & $\mathrm{U}$ \\
\hline 841 & 841521REGUF & $5 / 21 / 90$ & REG & Carbon disulfide & 5 & $\mathrm{ug} / \mathrm{L}$ & $\mathrm{U}$ \\
\hline 841 & 841119REGUF & $11 / 9 / 90$ & REG & Carbon disulfide & 1 & $\mathrm{ug} / \mathrm{L}$ & $\mathbf{J}$ \\
\hline 841 & 841126REGUF & $1 / 26 / 89$ & REG & Carbon disulfide & 5 & $\mathrm{ug} / \mathrm{L}$ & $\mathrm{U}$ \\
\hline 841 & 841427REGUF & $4 / 27 / 89$ & REG & Carbon disulfide & 5 & $\mathrm{ug} / \mathrm{L}$ & $\mathrm{U}$ \\
\hline 841 & 84178REGUF & $7 / 8 / 88$ & REG & Carbon disulfide & 5 & $\mathrm{ug} / \mathrm{L}$ & $\mathrm{U}$ \\
\hline 841 & 8411024REGUF & $10 / 24 / 88$ & REG & Carbon disulfide & 5 & $\mathrm{ug} / \mathrm{L}$ & $\mathbf{U}$ \\
\hline 841 & M01244 & $11 / 12 / 97$ & REG & Carbon tetrachloride & 5 & $\mathrm{ug} / \mathrm{L}$ & $\mathrm{U}$ \\
\hline 841 & W00808 & $6 / 26 / 97$ & REG & Carbon tetrachloride & 5 & $\mathrm{ug} / \mathrm{L}$ & $\mathrm{U}$ \\
\hline 841 & M00051 & $12 / 5 / 96$ & REG & Carbon tetrachloride & 5 & $\mathrm{ug} / \mathrm{L}$ & $\mathrm{U}$ \\
\hline 841 & M00836 & $6 / 26 / 97$ & REG & Carbon tetrachloride & 5 & $\mathrm{ug} / \mathrm{L}$ & $\mathrm{U}$ \\
\hline 841 & SMPL00280 & $5 / 27 / 94$ & REG & Carbon tetrachloride & 1 & $\mathrm{ug} / \mathrm{L}$ & $\mathrm{U}$ \\
\hline 841 & S00933 & $10 / 19 / 94$ & REG & Carbon tetrachloride & 5 & $\mathrm{ug} / \mathrm{L}$ & $\mathbf{U}$ \\
\hline 841 & S02607 & $2 / 28 / 95$ & REG & Carbon tetrachloride & 5 & $\mathrm{ug} / \mathrm{L}$ & $\mathrm{U}$ \\
\hline 841 & S03565 & $5 / 16 / 95$ & REG & Carbon tetrachloride & 5 & $\mathrm{ug} / \mathrm{L}$ & $\mathrm{U}$ \\
\hline 841 & S04776 & $7 / 14 / 95$ & REG & Carbon tetrachloride & 5 & $\mathrm{ug} / \mathrm{L}$ & $\mathrm{U}$ \\
\hline 841 & 841222REGUF & $2 / 22 / 91$ & REG & Carbon tetrachloride & 5 & $\mathrm{ug} / \mathrm{L}$ & $\mathrm{U}$ \\
\hline 841 & 841103REGUF & $10 / 3 / 91$ & REG & Carbon tetrachloride & 5 & $\mathrm{ug} / \mathrm{L}$ & $\mathrm{U}$ \\
\hline 841 & 8411210REGUF & $12 / 10 / 91$ & REG & Carbon tetrachloride & 5 & $\mathrm{ug} / \mathrm{L}$ & $\mathrm{U}$ \\
\hline 841 & 84152IREGUF & $5 / 21 / 90$ & REG & Carbon tetrachloride & 5 & $\mathrm{ug} / \mathrm{L}$ & $\mathrm{U}$ \\
\hline 841 & 841119 REGUF & $11 / 9 / 90$ & REG & Carbon tetrachloride & 5 & $\mathrm{ug} / \mathrm{L}$ & $\mathrm{U}$ \\
\hline 841 & 841126REGUF & $1 / 26 / 89$ & REG & Carbon tetrachloride & 5 & $\mathrm{ug} / \mathrm{L}$ & $\mathrm{U}$ \\
\hline
\end{tabular}


B-28

Station Sample ID Sample Date Sample Type Chemical

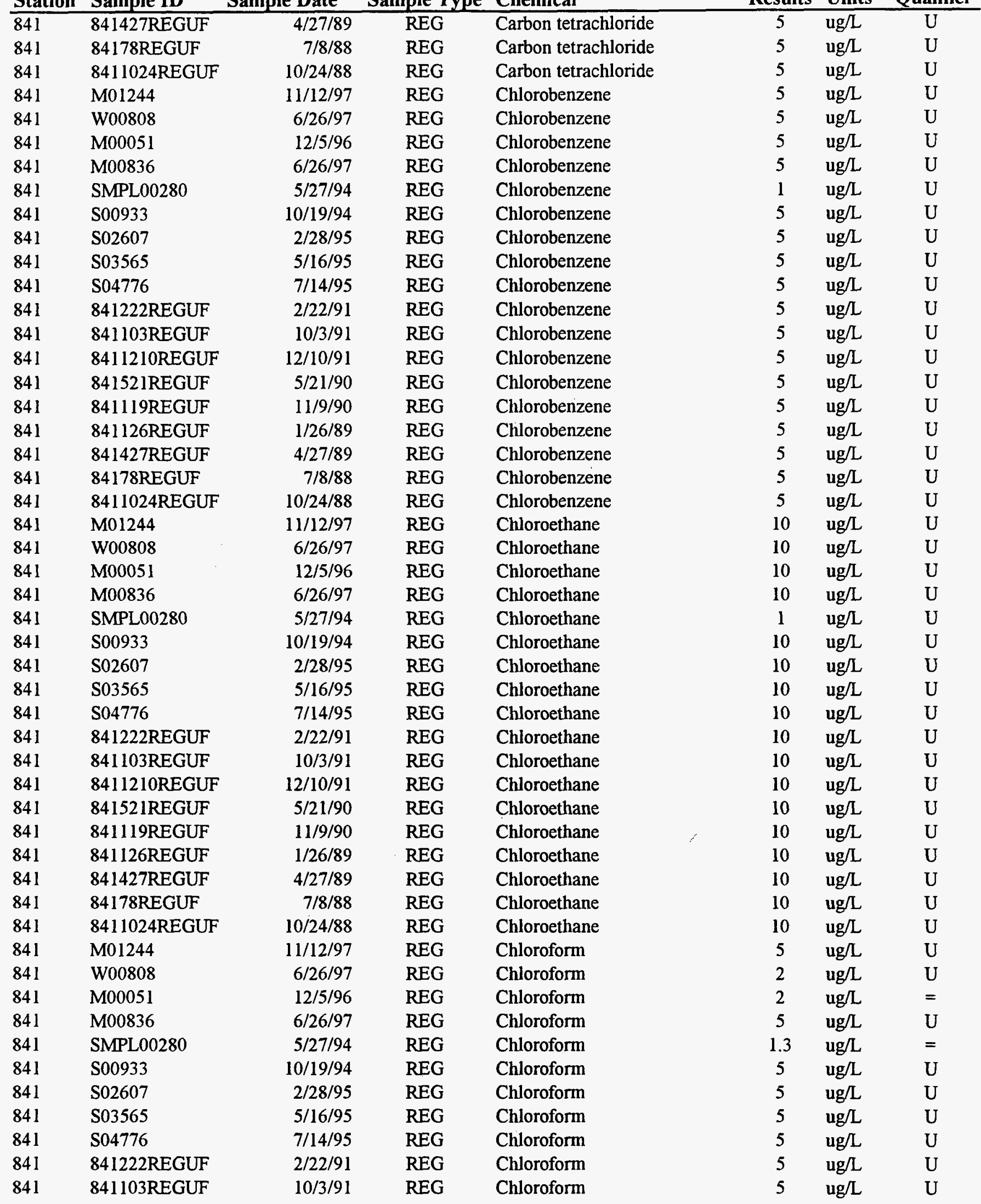




\begin{tabular}{|c|c|c|c|c|c|c|c|}
\hline Station & Sample ID & Sample Date & Sample Type & Chemical & Results & Units & Qualifier \\
\hline 841 & 8411210REGUF & $12 / 10 / 91$ & REG & Chloroform & 5 & $\mathrm{ug} / \mathrm{L}$ & $\mathrm{U}$ \\
\hline 841 & 841521REGUF & $5 / 21 / 90$ & REG & Chloroform & 5 & $\mathrm{ug} / \mathrm{L}$ & $\mathrm{U}$ \\
\hline 841 & 841119REGUF & $11 / 9 / 90$ & REG & Chloroform & 5 & $\mathrm{ug} / \mathrm{L}$ & $\mathrm{U}$ \\
\hline 841 & 841126REGUF & $1 / 26 / 89$ & REG & Chloroform & 5 & $\mathrm{ug} / \mathrm{L}$ & $\mathrm{U}$ \\
\hline 841 & 841427REGUF & $4 / 27 / 89$ & REG & Chloroform & 5 & $\mathrm{ug} / \mathrm{L}$ & U \\
\hline 841 & 84178REGUF & $7 / 8 / 88$ & REG & Chloroform & 5 & $\mathrm{ug} / \mathrm{L}$ & $\mathrm{U}$ \\
\hline 841 & 8411024REGUF & $10 / 24 / 88$ & REG & Chloroform & 5 & $\mathrm{ug} / \mathrm{L}$ & $\mathrm{U}$ \\
\hline 841 & M01244 & $11 / 12 / 97$ & REG & Chloromethane & 2 & $\mathrm{ug} / \mathrm{L}$ & B \\
\hline 841 & W00808 & $6 / 26 / 97$ & REG & Chloromethane & 10 & $\mathrm{ug} / \mathrm{L}$ & $\mathrm{U}$ \\
\hline 841 & M0005I & $12 / 5 / 96$ & REG & Chloromethane & 10 & $\mathrm{ug} / \mathrm{L}$ & $\mathrm{U}$ \\
\hline 841 & M00836 & $6 / 26 / 97$ & REG & Chloromethane & 10 & $\mathrm{ug} / \mathrm{L}$ & $\mathrm{U}$ \\
\hline 841 & SMPL00280 & $5 / 27 / 94$ & REG & Chloromethane & 1 & $\mathrm{ug} / \mathrm{L}$ & $\mathrm{U}$ \\
\hline 841 & S00933 & $10 / 19 / 94$ & REG & Chloromethane & 10 & $\mathrm{ug} / \mathrm{L}$ & $\mathrm{U}$ \\
\hline 841 & S02607 & $2 / 28 / 95$ & REG & Chloromethane & 10 & $\mathrm{ug} / \mathrm{L}$ & $\mathrm{U}$ \\
\hline 841 & S03565 & $5 / 16 / 95$ & REG & Chloromethane & 10 & $\mathrm{ug} / \mathrm{L}$ & $\mathrm{U}$ \\
\hline 841 & S04776 & $7 / 14 / 95$ & REG & Chloromethane & 10 & $\mathrm{ug} / \mathrm{L}$ & $\mathrm{U}$ \\
\hline 841 & 841222REGUF & $2 / 22 / 91$ & REG & Chloromethane & 10 & $\mathrm{ug} / \mathrm{L}$ & $\mathrm{U}$ \\
\hline 841 & 841103REGUF & $10 / 3 / 91$ & REG & Chloromethane & 10 & $u g / L$ & $\mathrm{U}$ \\
\hline 841 & 8411210 REGUF & $12 / 10 / 91$ & REG & Chloromethane & 10 & $\mathrm{ug} / \mathrm{L}$ & $\mathrm{U}$ \\
\hline 841 & 841521REGUF & $5 / 21 / 90$ & REG & Chloromethane & 10 & $\mathrm{ug} / \mathrm{L}$ & $\mathrm{U}$ \\
\hline 841 & 841119REGUF & $11 / 9 / 90$ & REG & Chloromethane & 10 & $\mathrm{ug} / \mathrm{L}$ & $\mathrm{U}$ \\
\hline 841 & 841126REGUF & $1 / 26 / 89$ & REG & Chloromethane & 10 & $\mathrm{ug} / \mathrm{L}$ & $\mathrm{U}$ \\
\hline 841 & 841427REGUF & $4 / 27 / 89$ & REG & Chloromethane & 10 & $u g / L$ & $\mathrm{U}$ \\
\hline 841 & 84178REGUF & $7 / 8 / 88$ & REG & Chloromethane & 10 & $\mathrm{ug} / \mathrm{L}$ & $\mathrm{U}$ \\
\hline 841 & 8411024REGUF & $10 / 24 / 88$ & REG & Chloromethane & 10 & $\mathrm{ug} / \mathrm{L}$ & U \\
\hline 841 & M01244 & $11 / 12 / 97$ & REG & cis-1,2-Dichloroethene & 5 & $\mathrm{ug} / \mathrm{L}$ & $\mathrm{U}$ \\
\hline 841 & M00051 & $12 / 5 / 96$ & REG & cis-1,2-Dichloroethene & 1 & $\mathrm{ug} / \mathrm{L}$ & $=$ \\
\hline 841 & M00836 & $6 / 26 / 97$ & REG & cis-1,2-Dichloroethene & 5 & $\mathrm{ug} / \mathrm{L}$ & $\mathrm{U}$ \\
\hline 841 & SMPL00280 & $5 / 27 / 94$ & REG & cis-1,2-Dichloroethene & 1 & $\mathrm{ug} / \mathrm{L}$ & $\mathrm{U}$ \\
\hline 841 & S00933 & $10 / 19 / 94$ & REG & cis-1,2-Dichloroethene & 5 & $\mathrm{ug} / \mathrm{L}$ & $\mathrm{U}$ \\
\hline 841 & S02607 & $2 / 28 / 95$ & REG & cis-1,2-Dichloroethene & 5 & $\mathrm{ug} / \mathrm{L}$ & $\mathrm{U}$ \\
\hline 841 & S03565 & $5 / 16 / 95$ & REG & cis-1,2-Dichloroethene & 5 & $\mathrm{ug} / \mathrm{L}$ & $\mathrm{U}$ \\
\hline 841 & S04776 & $7 / 14 / 95$ & REG & cis-1,2-Dichloroethene & 5 & $\mathrm{ug} / \mathrm{L}$ & $\mathrm{U}$ \\
\hline 841 & M01244 & $11 / 12 / 97$ & REG & cis-1,3-Dichloropropen & 5 & $\mathrm{ug} / \mathrm{L}$ & U \\
\hline 841 & W00808 & $6 / 26 / 97$ & REG & cis-1,3-Dichloropropene & 5 & $\mathrm{ug} / \mathrm{L}$ & $\mathrm{U}$ \\
\hline 841 & M00051 & $12 / 5 / 96$ & REG & cis-1,3-Dichloropropene & 5 & $\mathrm{ug} / \mathrm{L}$ & $\mathrm{U}$ \\
\hline 841 & M00836 & $6 / 26 / 97$ & REG & cis-1,3-Dichloropropene & 5 & $u g / L$ & $\mathrm{U}$ \\
\hline 841 & SMPL00280 & $5 / 27 / 94$ & REG & cis-1,3-Dichloropropene & 1 & $\mathrm{ug} / \mathrm{L}$ & $\mathrm{U}$ \\
\hline 841 & S00933 & $10 / 19 / 94$ & REG & cis-1,3-Dichloropropene & 5 & ug/L & $\mathrm{U}$ \\
\hline 841 & S02607 & $2 / 28 / 95$ & REG & cis-1,3-Dichloropropene & 5 & ug/L & $\mathrm{U}$ \\
\hline 841 & S03565 & $5 / 16 / 95$ & REG & cis-1,3-Dichloropropene & 5 & $\mathrm{ug} / \mathrm{L}$ & $\mathrm{U}$ \\
\hline 841 & S04776 & $7 / 14 / 95$ & REG & cis-1,3-Dichloropropene & 5 & $\mathrm{ug} / \mathrm{L}$ & $\mathrm{U}$ \\
\hline 841 & 841222REGUF & $2 / 22 / 91$ & REG & cis-1,3-Dichloropropene & 5 & $\mathrm{ug} / \mathrm{L}$ & $\mathrm{U}$ \\
\hline 841 & 841103REGUF & $10 / 3 / 91$ & REG & cis-1,3-Dichloropropene & 5 & $\mathrm{ug} / \mathrm{L}$ & $\mathrm{U}$ \\
\hline 841 & 8411210REGUF & $12 / 10 / 91$ & REG & cis-1,3-Dichloropropene & 5 & $\mathrm{ug} / \mathrm{L}$ & $\mathbf{U}$ \\
\hline 841 & 841521REGUF & $5 / 21 / 90$ & REG & cis-1,3-Dichloropropene & 5 & $\mathrm{ug} / \mathrm{L}$ & $\mathrm{U}$ \\
\hline 841 & 841119REGUF & $11 / 9 / 90$ & REG & cis-1,3-Dichloropropene & 5 & $\mathrm{ug} / \mathrm{L}$ & $\mathrm{U}$ \\
\hline 841 & 841126REGUF & $1 / 26 / 89$ & REG & cis-1,3-Dichloropropene & 5 & $\mathrm{ug} / \mathrm{L}$ & $\mathrm{U}$ \\
\hline 841 & 841427REGUF & $4 / 27 / 89$ & REG & cis-1,3-Dichloropropene & 5 & $\mathrm{ug} / \mathrm{L}$ & $\mathrm{U}$ \\
\hline 841 & 84178REGUF & $7 / 8 / 88$ & REG & cis-1,3-Dichloropropene & 5 & $\mathrm{ug} / \mathrm{L}$ & $\mathrm{U}$ \\
\hline
\end{tabular}




\begin{tabular}{|c|c|c|c|c|c|c|c|}
\hline Station & Sample ID & Sample Date & Sample Type & Chemical & Results & Units & Qualifier \\
\hline 841 & 8411024REGUF & $10 / 24 / 88$ & $\mathrm{REG}$ & cis-1,3-Dichloropropene & 5 & $\mathrm{ug} / \mathrm{L}$ & $\mathrm{U}$ \\
\hline 841 & M01244 & $11 / 12 / 97$ & REG & Dibromochloromethane & 5 & $\mathrm{ug} / \mathrm{L}$ & $\mathrm{U}$ \\
\hline 841 & W00808 & $6 / 26 / 97$ & REG & Dibromochloromethane & 5 & $\mathrm{ug} / \mathrm{L}$ & $\mathrm{U}$ \\
\hline 841 & M00051 & $12 / 5 / 96$ & REG & Dibromochloromethane & 5 & $\mathrm{ug} / \mathrm{L}$ & $\mathrm{U}$ \\
\hline 841 & M00836 & $6 / 26 / 97$ & REG & Dibromochloromethane & 5 & $\mathrm{ug} / \mathrm{L}$ & $\mathrm{U}$ \\
\hline 841 & SMPL00280 & $5 / 27 / 94$ & REG & Dibromochloromethane & 1 & $u g / L$ & $\mathrm{U}$ \\
\hline 841 & S00933 & $10 / 19 / 94$ & REG & Dibromochloromethane & 5 & $u g / L$ & $\mathrm{U}$ \\
\hline 841 & S02607 & $2 / 28 / 95$ & REG & Dibromochloromethane & 5 & $\mathrm{ug} / \mathrm{L}$ & $\mathrm{U}$ \\
\hline 841 & S03565 & $5 / 16 / 95$ & REG & Dibromochloromethane & 5 & $u g / L$ & $\mathrm{U}$ \\
\hline 841 & S04776 & $7 / 14 / 95$ & REG & Dibromochloromethane & 5 & $\mathrm{ug} / \mathrm{L}$ & $\mathrm{U}$ \\
\hline 841 & 841222REGUF & $2 / 22 / 91$ & REG & Dibromochloromethane & 5 & $\mathrm{ug} / \mathrm{L}$ & $\mathrm{U}$ \\
\hline 841 & 841103REGUF & $10 / 3 / 91$ & REG & Dibromochloromethane & 5 & $\mathrm{ug} / \mathrm{L}$ & $\mathrm{U}$ \\
\hline $84 \mathrm{I}$ & 8411210REGUF & $12 / 10 / 91$ & REG & Dibromochloromethane & 5 & $\mathrm{ug} / \mathrm{L}$ & $\mathrm{U}$ \\
\hline 841 & 841521REGUF & $5 / 21 / 90$ & REG & Dibromochloromethane & 5 & $\mathrm{ug} / \mathrm{L}$ & $\mathrm{U}$ \\
\hline 841 & 841119REGUF & $11 / 9 / 90$ & REG & Dibromochloromethane & 5 & ug/L & $\mathrm{U}$ \\
\hline 841 & 841126REGUF & $1 / 26 / 89$ & REG & Dibromochloromethane & 5 & $\mathrm{ug} / \mathrm{L}$ & $\mathrm{U}$ \\
\hline 841 & 841427REGUF & $4 / 27 / 89$ & REG & Dibromochloromethane & 5 & $u g / L$ & $\mathrm{U}$ \\
\hline 841 & 84178REGUF & $7 / 8 / 88$ & REG & Dibromochloromethane & 5 & $\mathrm{ug} / \mathrm{L}$ & $\mathrm{U}$ \\
\hline 841 & 8411024REGUF & $10 / 24 / 88$ & REG & Dibromochloromethane & 5 & $\mathrm{ug} / \mathrm{L}$ & $\mathrm{U}$ \\
\hline 841 & M00051 & $12 / 5 / 96$ & REG & Dimethylbenzene & 5 & $\mathrm{ug} / \mathrm{L}$ & $\mathrm{U}$ \\
\hline 841 & SMPL00280 & $5 / 27 / 94$ & REG & Dimethylbenzene & 1 & $\mathrm{ug} / \mathrm{L}$ & $\mathrm{U}$ \\
\hline 841 & 841222REGUF & $2 / 22 / 91$ & REG & Dimethylbenzene & 5 & $\mathrm{ug} / \mathrm{L}$ & $\mathrm{U}$ \\
\hline 841 & $841103 R E G U F$ & $10 / 3 / 91$ & REG & Dimethylbenzene & 5 & $\mathrm{ug} / \mathrm{L}$ & $\mathrm{U}$ \\
\hline 841 & 84I1210REGUF & $12 / 10 / 91$ & REG & Dimethylbenzene & 5 & $\mathrm{ug} / \mathrm{L}$ & $\mathrm{U}$ \\
\hline 841 & 841521REGUF & $5 / 21 / 90$ & REG & Dimethylbenzene & 5 & $\mathrm{ug} / \mathrm{L}$ & $\mathrm{U}$ \\
\hline 841 & 841119REGUF & $11 / 9 / 90$ & REG & Dimethylbenzene & 5 & $\mathrm{ug} / \mathrm{L}$ & $\mathrm{U}$ \\
\hline 841 & 841126REGUF & $1 / 26 / 89$ & REG & Dimethylbenzene & 5 & $\mathrm{ug} / \mathrm{L}$ & $\mathrm{U}$ \\
\hline 841 & 841427REGUF & $4 / 27 / 89$ & REG & Dimethylbenzene & 5 & $u g / L$ & $\mathrm{U}$ \\
\hline 841 & 84178REGUF & $7 / 8 / 88$ & REG & Dimethylbenzene & 5 & $\mathrm{ug} / \mathrm{L}$ & U \\
\hline 841 & 8411024REGUF & $10 / 24 / 88$ & REG & Dimethylbenzene & 5 & $\mathrm{ug} / \mathrm{L}$ & $\mathrm{U}$ \\
\hline 841 & M01244 & $11 / 12 / 97$ & REG & Ethylbenzene & 5 & $\mathrm{ug} / \mathrm{L}$ & $\mathrm{U}$ \\
\hline 841 & W00808 & $6 / 26 / 97$ & REG & Ethylbenzene & 5 & $\mathrm{ug} / \mathrm{L}$ & $\mathrm{U}$ \\
\hline 841 & M00051 & $12 / 5 / 96$ & REG & Ethylbenzene & 5 & $\mathrm{ug} / \mathrm{L}$ & $\mathrm{U}$ \\
\hline 841 & M00836 & $6 / 26 / 97$ & REG & Ethylbenzene & 5 & $\mathrm{ug} / \mathrm{L}$ & $\mathrm{U}$ \\
\hline 841 & SMPL00280 & $5 / 27 / 94$ & REG & Ethylbenzene & 1 & $\mathrm{ug} / \mathrm{L}$ & $\mathrm{U}$ \\
\hline 841 & S00933 & $10 / 19 / 94$ & REG & Ethylbenzene & 5 & $\mathrm{ug} / \mathrm{L}$ & $\mathrm{U}$ \\
\hline 841 & S02607 & $2 / 28 / 95$ & REG & Ethylbenzene & 5 & ug/L & $\mathrm{U}$ \\
\hline 841 & S03565 & $5 / 16 / 95$ & REG & Ethylbenzene & 5 & $\mathrm{ug} / \mathrm{L}$ & U \\
\hline 841 & S04776 & $7 / 14 / 95$ & REG & Ethylbenzene & 5 & $\mathrm{ug} / \mathrm{L}$ & $\mathrm{U}$ \\
\hline 841 & 841222REGUF & $2 / 22 / 91$ & REG & Ethylbenzene & 5 & $\mathrm{ug} / \mathrm{L}$ & $\mathrm{U}$ \\
\hline 841 & 841103REGUF & $10 / 3 / 91$ & REG & Ethylbenzene & 5 & $\mathrm{ug} / \mathrm{L}$ & $\mathrm{U}$ \\
\hline 841 & 8411210REGUF & $12 / 10 / 91$ & REG & Ethylbenzene & 5 & ug/L & $\mathrm{U}$ \\
\hline 841 & 841521REGUF & $5 / 21 / 90$ & REG & Ethylbenzene & 5 & $\mathrm{ug} / \mathrm{L}$ & $U$ \\
\hline 841 & 841119REGUF & $11 / 9 / 90$ & REG & Ethylbenzene & 5 & $\mathrm{ug} / \mathrm{L}$ & $\mathrm{U}$ \\
\hline 841 & $841126 \mathrm{REGUF}$ & $1 / 26 / 89$ & REG & Ethylbenzene & 5 & $\mathrm{ug} / \mathrm{L}$ & $\mathrm{U}$ \\
\hline 841 & 841427REGUF & $4 / 27 / 89$ & REG & Ethylbenzene & 5 & $\mathrm{ug} / \mathrm{L}$ & $\mathrm{U}$ \\
\hline 841 & 84178REGUF & $7 / 8 / 88$ & REG & Ethylbenzene & 5 & $\mathrm{ug} / \mathrm{L}$ & $\mathrm{U}$ \\
\hline 841 & 8411024REGUF & $10 / 24 / 88$ & REG & Ethylbenzene & 5 & $\mathrm{ug} / \mathrm{L}$ & $\mathrm{U}$ \\
\hline 841 & M01244 & $11 / 12 / 97$ & REG & m,p-Xylene & 5 & $\mathrm{ug} / \mathrm{L}$ & $\mathrm{U}$ \\
\hline 841 & W00808 & $6 / 26 / 97$ & REG & m,p-Xylene & 5 & ug/ & $\mathrm{U}$ \\
\hline
\end{tabular}


B-31

Station Sample ID Sample Date Sample Type Chemical

Results Units Qualifier

\begin{tabular}{|c|c|c|c|c|c|c|c|}
\hline 841 & M00051 & $12 / 5 / 96$ & REG & $\mathrm{m}, \mathrm{p}$-Xylene & 5 & ug/L & $\bar{U}$ \\
\hline 841 & M00836 & $6 / 26 / 97$ & REG & m,p-Xylene & 5 & $\mathrm{ug} / \mathrm{L}$ & $\mathrm{U}$ \\
\hline 841 & S02607 & $2 / 28 / 95$ & REG & $\mathrm{m}, \mathrm{p}$-Xylene & 5 & $\mathrm{ug} / \mathrm{L}$ & $\mathrm{U}$ \\
\hline 841 & S03565 & $5 / 16 / 95$ & REG & m,p-Xylene & 5 & ug/L & $\mathrm{U}$ \\
\hline 841 & S04776 & $7 / 14 / 95$ & REG & m,p-Xylene & 5 & ug/L & $\mathrm{U}$ \\
\hline 841 & M01244 & $11 / 12 / 97$ & REG & Methylene chloride & 4 & ug/L & B \\
\hline 841 & W00808 & $6 / 26 / 97$ & REG & Methylene chloride & 10 & $\mathrm{ug} / \mathrm{L}$ & $=$ \\
\hline 841 & M00051 & $12 / 5 / 96$ & REG & Methylene chloride & 5 & $u g / L$ & $\mathrm{U}$ \\
\hline 841 & M00836 & $6 / 26 / 97$ & REG & Methylene chloride & 2 & ug $/ \mathrm{L}$ & $\mathrm{U}$ \\
\hline 841 & SMPL00280 & $5 / 27 / 94$ & REG & Methylene chloride & 2 & ug/L & $\mathrm{U}$ \\
\hline 841 & S00933 & $10 / 19 / 94$ & REG & Methylene chloride & 5 & ug/L & $\mathrm{U}$ \\
\hline 841 & S02607 & $2 / 28 / 95$ & REG & Methylene chloride & 5 & $\mathrm{ug} / \mathrm{L}$ & $\mathrm{U}$ \\
\hline 841 & S03565 & $5 / 16 / 95$ & REG & Methylene chloride & 5 & $\mathrm{ug} / \mathrm{L}$ & $\mathrm{U}$ \\
\hline 841 & S04776 & $7 / 14 / 95$ & REG & Methylene chloride & 5 & $\mathrm{ug} / \mathrm{L}$ & $\mathrm{U}$ \\
\hline 841 & 841222REGUF & $2 / 22 / 91$ & REG & Methylene chloride & 5 & $\mathrm{ug} / \mathrm{L}$ & $\mathrm{U}$ \\
\hline 841 & $841103 R E G U F$ & $10 / 3 / 91$ & REG & Methylene chloride & 5 & $\mathrm{ug} / \mathrm{L}$ & $\mathrm{U}$ \\
\hline 841 & 8411210REGUF & $12 / 10 / 91$ & REG & Methylene chloride & 5 & $\mathrm{ug} / \mathrm{L}$ & $\mathrm{U}$ \\
\hline 841 & 841521REGUF & $5 / 21 / 90$ & REG & Methylene chloride & 1 & $\mathrm{ug} / \mathrm{L}$ & $\mathrm{J}$ \\
\hline 841 & 841119REGUF & $11 / 9 / 90$ & REG & Methylene chloride & 5 & $\mathrm{ug} / \mathrm{L}$ & $\mathrm{U}$ \\
\hline 841 & 841126REGUF & $1 / 26 / 89$ & REG & Methylene chloride & 1 & $\mathrm{ug} / \mathrm{L}$ & $\mathrm{J}$ \\
\hline 841 & 841427REGUF & $4 / 27 / 89$ & REG & Methylene chloride & 0.9 & $\mathrm{ug} / \mathrm{L}$ & $\mathrm{J}$ \\
\hline 841 & 84178REGUF & $7 / 8 / 88$ & REG & Methylene chloride & 1 & $\mathrm{ug} / \mathrm{L}$ & $\mathrm{J}$ \\
\hline 841 & 8411024REGUF & $10 / 24 / 88$ & REG & Methylene chloride & 7 & $\mathrm{ug} / \mathrm{L}$ & $=$ \\
\hline 841 & M01244 & $11 / 12 / 97$ & REG & Styrene & 5 & $\mathrm{ug} / \mathrm{L}$ & $\mathrm{U}$ \\
\hline 841 & W00808 & $6 / 26 / 97$ & REG & Styrene & 5 & $\mathrm{ug} / \mathrm{L}$ & $\mathrm{U}$ \\
\hline 841 & M00051 & $12 / 5 / 96$ & REG & Styrene & 5 & $\mathrm{ug} / \mathrm{L}$ & $\mathrm{U}$ \\
\hline 841 & M00836 & $6 / 26 / 97$ & REG & Styrene & 5 & $\mathrm{ug} / \mathrm{L}$ & $\mathrm{U}$ \\
\hline 841 & SMPL00280 & $5 / 27 / 94$ & REG & Styrene & 1 & $\mathrm{ug} / \mathrm{L}$ & $\mathrm{U}$ \\
\hline 841 & S00933 & $10 / 19 / 94$ & REG & Styrene & 5 & $\mathrm{ug} / \mathrm{L}$ & $\mathrm{U}$ \\
\hline 841 & S02607 & $2 / 28 / 95$ & REG & Styrene & 5 & $\mathrm{ug} / \mathrm{L}$ & $\mathrm{U}$ \\
\hline 841 & S03565 & $5 / 16 / 95$ & REG & Styrene & 5 & $\mathrm{ug} / \mathrm{L}$ & $\mathrm{U}$ \\
\hline 841 & S04776 & $7 / 14 / 95$ & REG & Styrene & 5 & $\mathrm{ug} / \mathrm{L}$ & $\mathrm{U}$ \\
\hline 841 & 841222REGUF & $2 / 22 / 91$ & REG & Styrene & 5 & $\mathrm{ug} / \mathrm{L}$ & $\mathrm{U}$ \\
\hline 841 & 841103REGUF & $10 / 3 / 91$ & REG & Styrene & 5 & $\mathrm{ug} / \mathrm{L}$ & $\mathrm{U}$ \\
\hline 841 & 8411210 REGUF & $12 / 10 / 91$ & REG & Styrene & 5 & $\mathrm{ug} / \mathrm{L}$ & $\mathrm{U}$ \\
\hline 841 & 841521REGUF & $5 / 21 / 90$ & REG & Styrene & 5 & $\mathrm{ug} / \mathrm{L}$ & $\mathrm{U}$ \\
\hline 841 & 841119REGUF & $11 / 9 / 90$ & REG & Styrene & 5 & $\mathrm{ug} / \mathrm{L}$ & $\mathrm{U}$ \\
\hline 841 & 841126REGUF & $1 / 26 / 89$ & REG & Styrene & 5 & ug/L & $\mathrm{U}$ \\
\hline 841 & 841427REGUF & $4 / 27 / 89$ & REG & Styrene & 5 & $\mathrm{ug} / \mathrm{L}$ & $\mathrm{U}$ \\
\hline 841 & 84178REGUF & $7 / 8 / 88$ & REG & Styrene & 5 & ug/L & $\mathrm{U}$ \\
\hline 841 & 8411024REGUF & $10 / 24 / 88$ & REG & Styrene & 5 & $\mathrm{ug} / \mathrm{L}$ & $\mathrm{U}$ \\
\hline 841 & M01244 & $11 / 12 / 97$ & REG & Tetrachloroethene & 5 & $\mathrm{ug} / \mathrm{L}$ & $\mathrm{U}$ \\
\hline 841 & W00808 & $6 / 26 / 97$ & REG & Tetrachloroethene & 5 & $\mathrm{ug} / \mathrm{L}$ & $\mathrm{U}$ \\
\hline 841 & M00051 & $12 / 5 / 96$ & REG & Tetrachloroethene & 5 & $u g / L$ & $\mathrm{U}$ \\
\hline 841 & M00836 & $6 / 26 / 97$ & REG & Tetrachloroethene & 5 & $\mathrm{ug} / \mathrm{L}$ & $\mathrm{U}$ \\
\hline 841 & SMPL00280 & $5 / 27 / 94$ & REG & Tetrachloroethene & 1 & $\mathrm{ug} / \mathrm{L}$ & $\mathrm{U}$ \\
\hline 841 & S00933 & $10 / 19 / 94$ & REG & Tetrachloroethene & 5 & $\mathrm{ug} / \mathrm{L}$ & $\mathrm{U}$ \\
\hline 841 & S02607 & $2 / 28 / 95$ & REG & Tetrachloroethene & 5 & $\mathrm{ug} / \mathrm{L}$ & $\mathrm{U}$ \\
\hline 841 & S03565 & $5 / 16 / 95$ & REG & Tetrachloroethene & 5 & $\mathrm{ug} / \mathrm{L}$ & $\mathrm{U}$ \\
\hline 841 & S04776 & $7 / 14 / 95$ & REG & Tetrachloroethene & 5 & $\mathrm{ug} / \mathrm{L}$ & $\mathrm{U}$ \\
\hline
\end{tabular}

Page 29 


\begin{tabular}{|c|c|c|c|c|c|c|c|}
\hline Station & Sample ID & Sample Date & Sample Type & Chemical & Results & Units & Qualifier \\
\hline 841 & 841222REGUF & $2 / 22 / 91$ & REG & Tetrachloroethene & 5 & $\mathrm{ug} / \mathrm{L}$ & $\mathrm{U}$ \\
\hline 841 & 841103REGUF & $10 / 3 / 91$ & REG & Tetrachloroethene & 5 & $u g / L$ & $\mathrm{U}$ \\
\hline 841 & 8411210 REGUF & $12 / 10 / 91$ & REG & Tetrachloroethene & 5 & $\mathrm{ug} / \mathrm{L}$ & $\mathrm{U}$ \\
\hline 841 & 841521REGUF & $5 / 21 / 90$ & REG & Tetrachloroethene & 5 & $u g / L$ & $\mathrm{U}$ \\
\hline 841 & 841119REGUF & $11 / 9 / 90$ & REG & Tetrachloroethene & 3 & $\mathrm{ug} / \mathrm{L}$ & $J$ \\
\hline 841 & 841126REGUF & $1 / 26 / 89$ & REG & Tetrachloroethene & 5 & $\mathrm{ug} / \mathrm{L}$ & $\mathrm{U}$ \\
\hline 841 & 841427REGUF & $4 / 27 / 89$ & REG & Tetrachloroethene & 5 & $\mathrm{ug} / \mathrm{L}$ & $\mathrm{U}$ \\
\hline 841 & 84178REGUF & $7 / 8 / 88$ & REG & Tetrachloroethene & 5 & $\mathrm{ug} / \mathrm{L}$ & $\mathrm{U}$ \\
\hline 841 & 8411024REGUF & $10 / 24 / 88$ & REG & Tetrachloroethene & 16 & $\mathrm{ug} / \mathrm{L}$ & $=$ \\
\hline 841 & M01244 & $11 / 12 / 97$ & REG & Toluene & 5 & $\mathrm{ug} / \mathrm{L}$ & $\mathrm{U}$ \\
\hline 841 & W00808 & $6 / 26 / 97$ & REG & Toluene & 5 & $\mathrm{ug} / \mathrm{L}$ & $\mathrm{U}$ \\
\hline 841 & M00051 & $12 / 5 / 96$ & REG & Toluene & 5 & $\mathrm{ug} / \mathrm{L}$ & $\mathrm{U}$ \\
\hline 841 & M00836 & $6 / 26 / 97$ & REG & Toluene & 5 & $\mathrm{ug} / \mathrm{L}$ & $\mathrm{U}$ \\
\hline 841 & SMPL00280 & $5 / 27 / 94$ & REG & Toluene & 1 & $\mathrm{ug} / \mathrm{L}$ & $\mathrm{U}$ \\
\hline 841 & S00933 & $10 / 19 / 94$ & REG & Toluene & 5 & $\mathrm{ug} / \mathrm{L}$ & $\mathrm{U}$ \\
\hline 841 & S02607 & $2 / 28 / 95$ & REG & Toluene & 5 & $\mathrm{ug} / \mathrm{L}$ & $\mathrm{U}$ \\
\hline 841 & S03565 & $5 / 16 / 95$ & REG & Toluene & 5 & $\mathrm{ug} / \mathrm{L}$ & $\mathrm{U}$ \\
\hline 841 & S04776 & $7 / 14 / 95$ & REG & Toluene & 5 & $\mathrm{ug} / \mathrm{L}$ & $\mathrm{U}$ \\
\hline 841 & 841222REGUF & $2 / 22 / 91$ & REG & Toluene & 5 & $\mathrm{ug} / \mathrm{L}$ & $\mathrm{U}$ \\
\hline 841 & 841103REGUF & $10 / 3 / 91$ & REG & Toluene & 5 & $\mathrm{ug} / \mathrm{L}$ & $\mathrm{U}$ \\
\hline 841 & 8411210REGUF & $12 / 10 / 91$ & REG & Toluene & 5 & $\mathrm{ug} / \mathrm{L}$ & $\mathrm{U}$ \\
\hline 841 & 841521REGUF & $5 / 21 / 90$ & REG & Toluene & 1 & $\mathrm{ug} / \mathrm{L}$ & $\mathrm{J}$ \\
\hline 841 & 841119REGUF & $11 / 9 / 90$ & REG & Toluene & 5 & $\mathrm{ug} / \mathrm{L}$ & $\mathrm{U}$ \\
\hline 841 & 841126REGUF & $1 / 26 / 89$ & REG & Toluene & 5 & $\mathrm{ug} / \mathrm{L}$ & $\mathrm{U}$ \\
\hline 841 & 841427REGUF & $4 / 27 / 89$ & REG & Toluene & 5 & $\mathrm{ug} / \mathrm{L}$ & $\mathrm{U}$ \\
\hline 841 & 84178REGUF & $7 / 8 / 88$ & REG & Toluene & 2 & $\mathrm{ug} / \mathrm{L}$ & $\mathrm{J}$ \\
\hline 841 & 8411024REGUF & $10 / 24 / 88$ & REG & Toluene & 5 & $\mathrm{ug} / \mathrm{L}$ & $\mathrm{U}$ \\
\hline 841 & M01244 & $11 / 12 / 97$ & REG & trans-1,2-Dichloroethe & 5 & $\mathrm{ug} / \mathrm{L}$ & U \\
\hline 841 & $\mathrm{M} 00051$ & $12 / 5 / 96$ & REG & trans-1,2-Dichloroethene & 5 & $\mathrm{ug} / \mathrm{L}$ & $\mathrm{U}$ \\
\hline 841 & M00836 & $6 / 26 / 97$ & REG & trans-1,2-Dichloroethene & 5 & $\mathrm{ug} / \mathrm{L}$ & $U$ \\
\hline 841 & SMPL00280 & $5 / 27 / 94$ & REG & trans-1,2-Dichloroethene & 1 & $\mathrm{ug} / \mathrm{L}$ & $\mathrm{U}$ \\
\hline 841 & S00933 & $10 / 19 / 94$ & REG & trans-1,2-Dichloroethene & 5 & $\mathrm{ug} / \mathrm{L}$ & $\mathrm{U}$ \\
\hline 841 & S02607 & $2 / 28 / 95$ & REG & trans-1,2-Dichloroethene & 5 & $\mathrm{ug} / \mathrm{L}$ & $\mathrm{U}$ \\
\hline 841 & S03565 & $5 / 16 / 95$ & REG & trans-1,2-Dichloroethene & 5 & $\mathrm{ug} / \mathrm{L}$ & $\mathrm{U}$ \\
\hline 841 & S04776 & $7 / 14 / 95$ & REG & trans-1,2-Dichloroethene & 5 & $\mathrm{ug} / \mathrm{L}$ & $\mathrm{U}$ \\
\hline 841 & M01244 & $11 / 12 / 97$ & REG & trans-1,3-Dichloroprop & 5 & $\mathrm{ug} / \mathrm{L}$ & $\mathrm{U}$ \\
\hline 841 & W00808 & $6 / 26 / 97$ & REG & trans-1,3-Dichloropropene & 5 & $\mathrm{ug} / \mathrm{L}$ & $\mathrm{U}$ \\
\hline 841 & M00051 & $12 / 5 / 96$ & REG & trans-1,3-Dichloropropene & 5 & $\mathrm{ug} / \mathrm{L}$ & $\mathrm{U}$ \\
\hline 841 & M00836 & $6 / 26 / 97$ & REG & trans-1,3-Dichloropropene & 5 & $\mathrm{ug} / \mathrm{L}$ & $\mathrm{U}$ \\
\hline 841 & SMPL00280 & $5 / 27 / 94$ & REG & trans-1,3-Dichloropropene & 1 & ug/L & $U$ \\
\hline 841 & S00933 & $10 / 19 / 94$ & REG & trans-1,3-Dichloropropene & 5 & $\mathrm{ug} / \mathrm{L}$ & $\mathrm{U}$ \\
\hline 841 & S02607 & $2 / 28 / 95$ & REG & trans-1,3-Dichloropropene & 5 & $\mathrm{ug} / \mathrm{L}$ & $\mathrm{U}$ \\
\hline 841 & S03565 & $5 / 16 / 95$ & REG & trans-1,3-Dichloropropene & 5 & $\mathrm{ug} / \mathrm{L}$ & $\mathrm{U}$ \\
\hline 841 & S04776 & $7 / 14 / 95$ & REG & trans-1,3-Dichloropropene & 5 & ug/L & $\mathrm{U}$ \\
\hline 841 & 841222REGUF & $2 / 22 / 91$ & REG & trans-1,3-Dichloropropene & 5 & ug/L & $\mathrm{U}$ \\
\hline 841 & 841103REGUF & $10 / 3 / 91$ & REG & trans-1,3-Dichloropropene & 5 & $\mathrm{ug} / \mathrm{L}$ & $\mathrm{U}$ \\
\hline 841 & 8411210REGUF & $12 / 10 / 91$ & REG & trans-1,3-Dichloropropene & 5 & ug/L & $\mathrm{U}$ \\
\hline 841 & 841521REGUF & $5 / 21 / 90$ & REG & trans-1,3-Dichloropropene & 5 & ug/L & $\mathrm{U}$ \\
\hline 841 & 841119REGUF & $11 / 9 / 90$ & REG & trans-1,3-Dichloropropene & 5 & $\mathrm{ug} / \mathrm{L}$ & $\mathrm{U}$ \\
\hline 841 & 841126REGUF & $1 / 26 / 89$ & REG & trans-1,3-Dichloropropene & 5 & $u g / L$ & $\mathrm{U}$ \\
\hline
\end{tabular}


B-33

\begin{tabular}{|c|c|c|c|c|c|c|c|}
\hline Station & Sample ID & Sample Date & Sample Type & Chemical & Results & Units & Qualifier \\
\hline 841 & 84 1427REGUF & $4 / 27 / 89$ & REG & trans-1,3-Dichloropropene & 5 & $\mathrm{ug} / \mathrm{L}$ & $\bar{U}$ \\
\hline 841 & 84178REGUF & $7 / 8 / 88$ & REG & trans-1,3-Dichloropropene & 5 & $\mathrm{ug} / \mathrm{L}$ & $\mathrm{U}$ \\
\hline 841 & 8411024REGUF & $10 / 24 / 88$ & REG & trans-1,3-Dichloropropene & 5 & $\mathrm{ug} / \mathrm{L}$ & $\mathrm{U}$ \\
\hline 841 & M01244 & $11 / 12 / 97$ & REG & Trichloroethene & 7 & $\mathrm{ug} / \mathrm{L}$ & $=$ \\
\hline 841 & W00808 & $6 / 26 / 97$ & REG & Trichloroethene & 10 & $\mathrm{ug} / \mathrm{L}$ & $=$ \\
\hline 841 & M00051 & $12 / 5 / 96$ & REG & Trichloroethene & 14 & $\mathrm{ug} / \mathrm{L}$ & $=$ \\
\hline 841 & M00836 & $6 / 26 / 97$ & REG & Trichloroethene & 8 & ug/L & $=$ \\
\hline 841 & SMPL00280 & $5 / 27 / 94$ & REG & Trichloroethene & 6.1 & $\mathrm{ug} / \mathrm{L}$ & $=$ \\
\hline 841 & S00933 & $10 / 19 / 94$ & REG & Trichloroethene & 3.4 & $\mathrm{ug} / \mathrm{L}$ & $\mathrm{J}$ \\
\hline 841 & S02607 & $2 / 28 / 95$ & REG & Trichloroethene & 5 & $\mathrm{ug} / \mathrm{L}$ & $\mathrm{J}$ \\
\hline 841 & S03565 & $5 / 16 / 95$ & REG & Trichloroethene & 7 & $\mathrm{ug} / \mathrm{L}$ & $=$ \\
\hline 841 & S04776 & $7 / 14 / 95$ & REG & Trichloroethene & 5 & $\mathrm{ug} / \mathrm{L}$ & $=$ \\
\hline 841 & 841222REGUF & $2 / 22 / 91$ & REG & Trichloroethene & 4 & $\mathrm{ug} / \mathrm{L}$ & $\mathrm{J}$ \\
\hline 841 & 841 103REGUF & $10 / 3 / 91$ & REG & Trichloroethene & 5 & $\mathrm{ug} / \mathrm{L}$ & $\mathrm{U}$ \\
\hline 841 & 8411210REGUF & $12 / 10 / 91$ & REG & Trichloroethene & 7 & $\mathrm{ug} / \mathrm{L}$ & $=$ \\
\hline 841 & 841521REGUF & $5 / 21 / 90$ & REG & Trichloroethene & 5 & $\mathrm{ug} / \mathrm{L}$ & $=$ \\
\hline 841 & 841119REGUF & $11 / 9 / 90$ & REG & Trichloroethene & 3 & $\mathrm{ug} / \mathrm{L}$ & J \\
\hline 841 & $841126 \mathrm{REGUF}$ & $1 / 26 / 89$ & REG & Trichloroethene & 9 & $\mathrm{ug} / \mathrm{L}$ & $=$ \\
\hline 841 & 841427REGUF & $4 / 27 / 89$ & REG & Trichloroethene & 5 & $\mathrm{ug} / \mathrm{L}$ & $\mathbf{J}$ \\
\hline 841 & 84178REGUF & $7 / 8 / 88$ & REG & Trichloroethene & 7 & $\mathrm{ug} / \mathrm{L}$ & $=$ \\
\hline 841 & 8411024REGUF & $10 / 24 / 88$ & REG & Trichloroethene & 19 & $\mathrm{ug} / \mathrm{L}$ & $=$ \\
\hline 841 & M01244 & $11 / 12 / 97$ & REG & Vinyl acetate & 10 & $\mathrm{ug} / \mathrm{L}$ & $U$ \\
\hline 841 & M00051 & $12 / 5 / 96$ & REG & Vinyl acetate & 10 & $\mathrm{ug} / \mathrm{L}$ & $\mathrm{U}$ \\
\hline 841 & M00836 & $6 / 26 / 97$ & REG & Vinyl acetate & 10 & $\mathrm{ug} / \mathrm{L}$ & $\mathrm{U}$ \\
\hline 841 & S00933 & $10 / 19 / 94$ & REG & Vinyl acetate & 10 & $\mathrm{ug} / \mathrm{L}$ & $\mathrm{U}$ \\
\hline 841 & S02607 & $2 / 28 / 95$ & REG & Vinyl acetate & 10 & ug/L & $\mathrm{U}$ \\
\hline 841 & S03565 & $5 / 16 / 95$ & REG & Vinyl acetate & 10 & $\mathrm{ug} / \mathrm{L}$ & $\mathrm{U}$ \\
\hline 841 & S04776 & $7 / 14 / 95$ & REG & Vinyl acetate & 10 & $\mathrm{ug} / \mathrm{L}$ & $\mathrm{U}$ \\
\hline 841 & 841222REGUF & $2 / 22 / 91$ & REG & Vinyl acetate & 10 & ug/L & $\mathrm{U}$ \\
\hline 841 & 841103REGUF & $10 / 3 / 91$ & REG & Vinyl acetate & 10 & $\mathrm{ug} / \mathrm{L}$ & $\mathrm{U}$ \\
\hline 841 & 8411210 REGUF & $12 / 10 / 91$ & REG & Vinyl acetate & 10 & $\mathrm{ug} / \mathrm{L}$ & $\mathrm{U}$ \\
\hline 841 & 841521REGUF & $5 / 21 / 90$ & REG & Vinyl acetate & 10 & $\mathrm{ug} / \mathrm{L}$ & $\mathrm{U}$ \\
\hline 841 & 841119REGUF & $11 / 9 / 90$ & REG & Vinyl acetate & 10 & $\mathrm{ug} / \mathrm{L}$ & $\mathrm{U}$ \\
\hline 841 & 841126REGUF & $1 / 26 / 89$ & REG & Vinyl acetate & 10 & $\mathrm{ug} / \mathrm{L}$ & $U$ \\
\hline 841 & 841427REGUF & $4 / 27 / 89$ & REG & Vinyl acetate & 10 & $\mathrm{ug} / \mathrm{L}$ & $\mathrm{U}$ \\
\hline 841 & 84178REGUF & $7 / 8 / 88$ & REG & Vinyl acetate & 10 & $\mathrm{ug} / \mathrm{L}$ & $\mathrm{U}$ \\
\hline 841 & 8411024 REGUF & $10 / 24 / 88$ & REG & Vinyl acetate & 10 & $\mathrm{ug} / \mathrm{L}$ & $\mathrm{U}$ \\
\hline 841 & M01244 & $11 / 12 / 97$ & REG & Vinyl chloride & 10 & $\mathrm{ug} / \mathrm{L}$ & $\mathrm{U}$ \\
\hline 841 & W00808 & $6 / 26 / 97$ & REG & Vinyl chloride & 10 & $\mathrm{ug} / \mathrm{L}$ & $\mathrm{U}$ \\
\hline 841 & M00051 & $12 / 5 / 96$ & REG & Vinyl chloride & 10 & $\mathrm{ug} / \mathrm{L}$ & $\mathrm{U}$ \\
\hline 841 & M00836 & $6 / 26 / 97$ & REG & Vinyl chloride & 10 & $\mathrm{ug} / \mathrm{L}$ & $\mathrm{U}$ \\
\hline 841 & SMPL00280 & $5 / 27 / 94$ & REG & Vinyl chloride & 1 & $\mathrm{ug} / \mathrm{L}$ & $\mathrm{U}$ \\
\hline 841 & S00933 & $10 / 19 / 94$ & REG & Vinyl chloride & 10 & $u g / L$ & $\mathrm{U}$ \\
\hline 841 & S02607 & $2 / 28 / 95$ & REG & Vinyl chloride & 10 & $\mathrm{ug} / \mathrm{L}$ & $\mathrm{U}$ \\
\hline 841 & S03565 & $5 / 16 / 95$ & REG & Vinyl chloride & 10 & $\mathrm{ug} / \mathrm{L}$ & $\mathrm{U}$ \\
\hline 841 & S04776 & $7 / 14 / 95$ & REG & Vinyl chloride & 10 & $\mathrm{ug} / \mathrm{L}$ & $\mathrm{U}$ \\
\hline 841 & 841222REGUF & $2 / 22 / 91$ & REG & Vinyl chloride & 10 & $\mathrm{ug} / \mathrm{L}$ & $\mathrm{U}$ \\
\hline 841 & 841103 REGUF & $10 / 3 / 91$ & REG & Vinyl chloride & 10 & $\mathrm{ug} / \mathrm{L}$ & $\mathrm{U}$ \\
\hline 841 & 8411210REGUF & $12 / 10 / 91$ & REG & Vinyl chloride & 10 & $\mathrm{ug} / \mathrm{L}$ & $\mathrm{U}$ \\
\hline 841 & 841521REGUF & $5 / 21 / 90$ & REG & Vinyl chloride & 10 & $\mathrm{ug} / \mathrm{L}$ & $\mathrm{U}$ \\
\hline
\end{tabular}


Station Sample ID Sample Date Sample Type Chemical

\begin{tabular}{|c|c|c|c|c|c|c|c|}
\hline 841 & 841119REGUF & $11 / 9 / 90$ & REG & Vinyl chloride & 10 & $\mathrm{ug} / \mathrm{L}$ & $\mathrm{U}$ \\
\hline 841 & 841126REGUF & $1 / 26 / 89$ & REG & Vinyl chloride & 10 & $\mathrm{ug} / \mathrm{L}$ & $\mathrm{U}$ \\
\hline 841 & 841427REGUF & $4 / 27 / 89$ & REG & Vinyl chloride & 10 & $\mathrm{ug} / \mathrm{L}$ & $\mathrm{U}$ \\
\hline 841 & 84178REGUF & $7 / 8 / 88$ & REG & Vinyl chloride & 10 & $u g / L$ & $\mathrm{U}$ \\
\hline 841 & 8411024REGUF & $10 / 24 / 88$ & REG & Vinyl chloride & 10 & $\mathrm{ug} / \mathrm{L}$ & $\mathrm{U}$ \\
\hline 842 & M01250 & $11 / 11 / 97$ & REG & 1,1,1-Trichloroethane & 5 & $\mathrm{ug} / \mathrm{L}$ & $\mathrm{U}$ \\
\hline 842 & W00809 & $6 / 18 / 97$ & REG & 1.1,1-Trichloroethane & 5 & ug/L & $\mathrm{U}$ \\
\hline 842 & M00055 & $12 / 3 / 96$ & REG & $1,1,1$-Trichloroethane & 5 & $\mathrm{ug} / \mathrm{L}$ & $\mathrm{U}$ \\
\hline 842 & M00843 & $6 / 18 / 97$ & REG & 1,1,1-Trichloroethane & 5 & ug/L & $\mathrm{U}$ \\
\hline 842 & SMPL00266 & $5 / 25 / 94$ & REG & 1,1,1-Trichloroethane & 10 & $\mathrm{ug} / \mathrm{L}$ & $\mathrm{U}$ \\
\hline 842 & S00957 & $10 / 12 / 94$ & REG & 1,1,1-Trichloroethane & 5 & $\mathrm{ug} / \mathrm{L}$ & $\mathrm{U}$ \\
\hline 842 & S02616 & $2 / 28 / 95$ & REG & $1,1,1$-Trichloroethane & 5 & $\mathrm{ug} / \mathrm{L}$ & $\mathrm{U}$ \\
\hline 842 & S03576 & $5 / 8 / 95$ & REG & $1,1,1$-Trichloroethane & 5 & $\mathrm{ug} / \mathrm{L}$ & $\mathrm{U}$ \\
\hline 842 & S04793 & $7 / 14 / 95$ & REG & $1,1,1-T r i c h l o r o e t h a n e$ & 5 & $\mathrm{ug} / \mathrm{L}$ & $\mathrm{U}$ \\
\hline 842 & 842222REGUF & $2 / 22 / 91$ & REG & 1,1,1-Trichloroethane & 5 & $\mathrm{ug} / \mathrm{L}$ & $\mathrm{U}$ \\
\hline 842 & 842104REGUF & $10 / 4 / 91$ & REG & 1,1,1-Trichloroethane & 5 & $\mathrm{ug} / \mathrm{L}$ & $\mathrm{U}$ \\
\hline 842 & 8421210REGUF & $12 / 10 / 91$ & REG & 1,1,1-Trichloroethane & 5 & $\mathrm{ug} / \mathrm{L}$ & $\mathrm{U}$ \\
\hline 842 & 84252 IREGUF & $5 / 21 / 90$ & REG & 1,1,1-Trichloroethane & 5 & ug/L & $\mathrm{U}$ \\
\hline 842 & 842119REGUF & $11 / 9 / 90$ & REG & 1,1,1-Trichloroethane & 5 & ug/L & $\mathrm{U}$ \\
\hline 842 & 842126REGUF & $1 / 26 / 89$ & REG & 1,1,1-Trichloroethane & 5 & $\mathrm{ug} / \mathrm{L}$ & $\mathrm{U}$ \\
\hline 842 & 842427REGUF & $4 / 27 / 89$ & REG & 1,1,1-Trichloroethane & 5 & ug/L & $\mathrm{U}$ \\
\hline 842 & M01250 & $11 / 11 / 97$ & REG & 1,1,2,2-Tetrachloroeth & 5 & $\mathrm{ug} / \mathrm{L}$ & $\mathrm{U}$ \\
\hline 842 & W00809 & $6 / 18 / 97$ & REG & 1,1,2,2-Tetrachloroethane & 5 & ug/L & $\mathrm{U}$ \\
\hline 842 & M00055 & $12 / 3 / 96$ & REG & 1,1,2,2-Tetrachloroethane & 5 & $\mathrm{ug} / \mathrm{L}$ & $\mathrm{U}$ \\
\hline 842 & M00843 & $6 / 18 / 97$ & REG & 1,1,2,2-Tetrachloroethane & 5 & $\mathrm{ug} / \mathrm{L}$ & U \\
\hline 842 & SMPL00266 & $5 / 25 / 94$ & REG & $1,1,2,2$-Tetrachloroethane & 10 & $\mathrm{ug} / \mathrm{L}$ & $\mathrm{U}$ \\
\hline 842 & S00957 & $10 / 12 / 94$ & REG & 1,1,2,2-Tetrachloroethane & 5 & ug/L & $\mathrm{U}$ \\
\hline 842 & $\mathrm{~S} 02616$ & $2 / 28 / 95$ & REG & 1,1,2,2-Tetrachloroethane & 5 & $\mathrm{ug} / \mathrm{L}$ & $\mathrm{U}$ \\
\hline 842 & S03576 & $5 / 8 / 95$ & REG & 1,1,2,2-Tetrachloroethane & 5 & $\mathrm{ug} / \mathrm{L}$ & $\mathrm{U}$ \\
\hline 842 & S04793 & $7 / 14 / 95$ & REG & 1,1,2,2-Tetrachloroethane & 5 & ug $/ \mathrm{L}$ & $\mathrm{U}$ \\
\hline 842 & 842222REGUF & $2 / 22 / 91$ & REG & 1,1,2,2-Tetrachloroethane & 5 & ug/L & $\mathrm{U}$ \\
\hline 842 & 842104REGUF & $10 / 4 / 91$ & REG & $1,1,2,2$-Tetrachloroethane & 5 & $\mathrm{ug} / \mathrm{L}$ & $\mathrm{U}$ \\
\hline 842 & 8421210REGUF & $12 / 10 / 91$ & REG & 1,1,2,2-Tetrachloroethane & 5 & $\mathrm{ug} / \mathrm{L}$ & $\mathrm{U}$ \\
\hline 842 & 842521REGUF & $5 / 21 / 90$ & REG & 1,1,2,2-Tetrachloroethane & 5 & $\mathrm{ug} / \mathrm{L}$ & $U$ \\
\hline 842 & 842119REGUF & $11 / 9 / 90$ & REG & $1,1,2,2$-Tetrachloroethane & 5 & $\mathrm{ug} / \mathrm{L}$ & $\mathrm{U}$ \\
\hline 842 & 842126REGUF & $1 / 26 / 89$ & REG & 1,1,2,2-Tetrachloroethane & 5 & $\mathrm{ug} / \mathrm{L}$ & $\mathrm{U}$ \\
\hline 842 & 842427REGUF & $4 / 27 / 89$ & REG & 1,1,2,2-Tetrachloroethane & 5 & $\mathrm{ug} / \mathrm{L}$ & $\mathrm{U}$ \\
\hline 842 & W00809 & $6 / 18 / 97$ & REG & 1,1,2-Trichloro-1,2,2-trifluo & 9 & $\mathrm{ug} / \mathrm{L}$ & $\mathrm{U}$ \\
\hline 842 & M01250 & $11 / 11 / 97$ & REG & 1,1,2-Trichloroethane & 5 & $u g / L$ & $\mathrm{U}$ \\
\hline 842 & W00809 & $6 / 18 / 97$ & REG & 1,1,2-Trichloroethane & 5 & $\mathrm{ug} / \mathrm{L}$ & $\mathrm{U}$ \\
\hline 842 & M00055 & $12 / 3 / 96$ & REG & 1,1,2-Trichloroethane & 5 & ug/L & $\mathrm{U}$ \\
\hline 842 & M00843 & $6 / 18 / 97$ & REG & 1,1,2-Trichloroethane & 5 & $\mathrm{ug} / \mathrm{L}$ & $\mathrm{U}$ \\
\hline 842 & SMPL00266 & $5 / 25 / 94$ & REG & 1,1,2-Trichloroethane & 10 & $u g / L$ & $\mathrm{U}$ \\
\hline 842 & S00957 & $10 / 12 / 94$ & REG & 1,1,2-Trichloroethane & 5 & ug/L & $\mathrm{U}$ \\
\hline 842 & S02616 & $2 / 28 / 95$ & REG & 1,1,2-Trichloroethane & 5 & $\mathrm{ug} / \mathrm{L}$ & $\mathrm{U}$ \\
\hline 842 & S03576 & $5 / 8 / 95$ & REG & 1,1,2-Trichloroethane & 5 & $\mathrm{ug} / \mathrm{L}$ & $\mathrm{U}$ \\
\hline 842 & S04793 & $7 / 14 / 95$ & REG & 1,1,2-Trichloroethane & 5 & $\mathrm{ug} / \mathrm{L}$ & $\mathrm{U}$ \\
\hline 842 & 842222REGUF & $2 / 22 / 91$ & REG & 1,1,2-Trichloroethane & 5 & $\mathrm{ug} / \mathrm{L}$ & $\mathrm{U}$ \\
\hline 842 & 842104REGUF & $10 / 4 / 91$ & REG & 1,1,2-Trichloroethane & 5 & $\mathrm{ug} / \mathrm{L}$ & $\mathrm{U}$ \\
\hline 842 & 8421210REGUF & $12 / 10 / 91$ & REG & 1,1,2-Trichloroethane & 5 & $\mathrm{ug} / \mathrm{L}$ & $\mathrm{U}$ \\
\hline
\end{tabular}




\begin{tabular}{|c|c|c|c|c|c|c|c|}
\hline Station & Sample ID & Sample Date & Sample Type & Chemical & Results & Units & Qualifier \\
\hline 842 & 842521REGUF & $5 / 21 / 90$ & REG & 1,1,2-Trichloroethane & 5 & $\mathrm{ug} / \mathrm{L}$ & $\mathrm{U}$ \\
\hline 842 & 842119REGUF & $11 / 9 / 90$ & REG & 1,1,2-Trichloroethane & 5 & $\mathrm{ug} / \mathrm{L}$ & $\mathrm{U}$ \\
\hline 842 & 842126REGUF & $1 / 26 / 89$ & REG & 1,1,2-Trichloroethane & 5 & $\mathrm{ug} / \mathrm{L}$ & U \\
\hline 842 & 842427REGUF & $4 / 27 / 89$ & REG & 1,1,2-Trichloroethane & 5 & $\mathrm{ug} / \mathrm{L}$ & $\mathrm{U}$ \\
\hline 842 & M01250 & $11 / 11 / 97$ & REG & 1,1-Dichloroethane & 2 & $\mathrm{ug} / \mathrm{L}$ & $\mathrm{J}$ \\
\hline 842 & W00809 & $6 / 18 / 97$ & REG & 1,1-Dichloroethane & 5 & $\mathrm{ug} / \mathrm{L}$ & U \\
\hline 842 & M00055 & $12 / 3 / 96$ & REG & 1,1-Dichloroethane & 1 & $\mathrm{ug} / \mathrm{L}$ & $=$ \\
\hline 842 & M00843 & $6 / 18 / 97$ & REG & 1,1-Dichloroethane & 1 & $\mathrm{ug} / \mathrm{L}$ & $\mathrm{U}$ \\
\hline 842 & SMPL00266 & $5 / 25 / 94$ & REG & 1,1-Dichloroethane & 10 & $\mathrm{ug} / \mathrm{L}$ & $\mathrm{U}$ \\
\hline 842 & S00957 & $10 / 12 / 94$ & REG & 1,1-Dichloroethane & 2.5 & $\mathrm{ug} / \mathrm{L}$ & $J$ \\
\hline 842 & S02616 & $2 / 28 / 95$ & REG & 1,1-Dichloroethane & 5 & $\mathrm{ug} / \mathrm{L}$ & $\mathrm{U}$ \\
\hline 842 & S03576 & $5 / 8 / 95$ & REG & 1,1-Dichloroethane & 2 & $\mathrm{ug} / \mathrm{L}$ & $\mathrm{J}$ \\
\hline 842 & S04793 & $7 / 14 / 95$ & REG & 1,1-Dichloroethane & 2 & $\mathrm{ug} / \mathrm{L}$ & J \\
\hline 842 & 842222REGUF & $2 / 22 / 91$ & REG & 1,1-Dichloroethane & 5 & $\mathrm{ug} / \mathrm{L}$ & $\mathrm{U}$ \\
\hline 842 & 842104REGUF & $10 / 4 / 91$ & REG & 1,1-Dichloroethane & 5 & ug/L & $\mathrm{U}$ \\
\hline 842 & 8421210REGUF & $12 / 10 / 91$ & REG & 1,1-Dichloroethane & 2 & ug/L & $\mathrm{J}$ \\
\hline 842 & 842521REGUF & $5 / 21 / 90$ & REG & 1,1-Dichloroethane & 3 & $\mathrm{ug} / \mathrm{L}$ & $J$ \\
\hline 842 & 842119REGUF & $11 / 9 / 90$ & REG & 1,1-Dichloroethane & 3 & $\mathrm{ug} / \mathrm{L}$ & $\mathbf{J}$ \\
\hline 842 & 842126REGUF & $1 / 26 / 89$ & REG & 1,1-Dichloroethane & 3 & ug/L & J \\
\hline 842 & 842427REGUF & $4 / 27 / 89$ & REG & 1,1-Dichloroethane & 9 & ug/L & $=$ \\
\hline 842 & M01250 & $11 / 11 / 97$ & REG & 1,1-Dichloroethene & 5 & $\mathrm{ug} / \mathrm{L}$ & $\mathrm{U}$ \\
\hline 842 & W00809 & $6 / 18 / 97$ & REG & 1,1-Dichloroethene & 5 & ug $/ \mathrm{L}$ & U \\
\hline 842 & M00055 & $12 / 3 / 96$ & REG & 1,1-Dichloroethene & 5 & $\mathrm{ug} / \mathrm{L}$ & $\mathrm{U}$ \\
\hline 842 & M00843 & $6 / 18 / 97$ & REG & 1,1-Dichloroethene & 5 & ug/L & $\mathrm{U}$ \\
\hline 842 & SMPL00266 & $5 / 25 / 94$ & REG & 1,1-Dichloroethene & 10 & ug/L & $\mathbf{U}$ \\
\hline 842 & S00957 & $10 / 12 / 94$ & REG & 1,1-Dichloroethene & 5 & $\mathrm{ug} / \mathrm{L}$ & $\mathrm{U}$ \\
\hline 842 & S02616 & $2 / 28 / 95$ & REG & 1,1-Dichloroethene & 5 & $\mathrm{ug} / \mathrm{L}$ & $\mathrm{U}$ \\
\hline 842 & $\mathrm{~S} 03576$ & $5 / 8 / 95$ & REG & 1,1-Dichloroethene & 5 & $\mathrm{ug} / \mathrm{L}$ & $\mathrm{U}$ \\
\hline 842 & S04793 & $7 / 14 / 95$ & REG & 1,1-Dichloroethene & 5 & $\mathrm{ug} / \mathrm{L}$ & $\mathrm{U}$ \\
\hline 842 & 842222REGUF & $2 / 22 / 91$ & REG & 1,1-Dichloroethene & 5 & $\mathrm{ug} / \mathrm{L}$ & $\mathrm{U}$ \\
\hline 842 & 842104REGUF & $10 / 4 / 91$ & REG & 1,1-Dichloroethene & 5 & $\mathrm{ug} / \mathrm{L}$ & $\mathrm{U}$ \\
\hline 842 & 8421210REGUF & $12 / 10 / 91$ & REG & 1,1-Dichloroethene & 5 & $\mathrm{ug} / \mathrm{L}$ & $\mathrm{U}$ \\
\hline 842 & 842521REGUF & $5 / 21 / 90$ & REG & 1,1-Dichloroethene & 5 & $\mathrm{ug} / \mathrm{L}$ & $\mathrm{U}$ \\
\hline 842 & 842119REGUF & $11 / 9 / 90$ & REG & 1,1-Dichloroethene & 1 & $\mathrm{ug} / \mathrm{L}$ & $\mathrm{J}$ \\
\hline 842 & 842126REGUF & $1 / 26 / 89$ & REG & 1,1-Dichloroethene & 5 & $u g / L$ & $\mathrm{U}$ \\
\hline 842 & 842427REGUF & $4 / 27 / 89$ & REG & 1,1-Dichloroethene & 5 & $\mathrm{ug} / \mathrm{L}$ & $\mathrm{U}$ \\
\hline 842 & SMPL00266 & $5 / 25 / 94$ & REG & 1,2-Dibromo-3-chloropropane & 10 & $\mathrm{ug} / \mathrm{L}$ & $\mathrm{U}$ \\
\hline 842 & SMPL00266 & $5 / 25 / 94$ & REG & 1,2-Dibromoethane & 10 & $\mathrm{ug} / \mathrm{L}$ & $\mathrm{U}$ \\
\hline 842 & M00055 & $12 / 3 / 96$ & REG & 1,2-Dichlorobenzene & 5 & $\mathrm{ug} / \mathrm{L}$ & $\mathrm{U}$ \\
\hline 842 & SMPL00266 & $5 / 25 / 94$ & REG & 1,2-Dichlorobenzene & 10 & $\mathrm{ug} / \mathrm{L}$ & $\mathrm{U}$ \\
\hline 842 & M01250 & $11 / 11 / 97$ & REG & 1,2-Dichloroethane & 10 & $\mathrm{ug} / \mathrm{L}$ & $=$ \\
\hline 842 & W00809 & $6 / 18 / 97$ & REG & 1,2-Dichloroethane & 11 & $\mathrm{ug} / \mathrm{L}$ & $=$ \\
\hline 842 & M00055 & $12 / 3 / 96$ & REG & 1,2-Dichloroethane & 7 & $\mathrm{ug} / \mathrm{L}$ & $=$ \\
\hline 842 & M00843 & $6 / 18 / 97$ & REG & 1,2-Dichloroethane & 8 & $\mathrm{ug} / \mathrm{L}$ & $=$ \\
\hline 842 & S00957 & $10 / 12 / 94$ & REG & 1,2-Dichloroethane & 12 & ug/L & $=$ \\
\hline 842 & S02616 & $2 / 28 / 95$ & REG & 1,2-Dichloroethane & 10 & $\mathrm{ug} / \mathrm{L}$ & $=$ \\
\hline 842 & S03576 & $5 / 8 / 95$ & REG & 1,2-Dichloroethane & 11 & $\mathrm{ug} / \mathrm{L}$ & $=$ \\
\hline 842 & S04793 & $7 / 14 / 95$ & REG & 1,2-Dichloroethane & 11 & $\mathrm{ug} / \mathrm{L}$ & $=$ \\
\hline 842 & 842222REGUF & $2 / 22 / 91$ & REG & 1,2-Dichloroethane & 19 & $\mathrm{ug} / \mathrm{L}$ & $=$ \\
\hline 842 & 842 104REGUF & $10 / 4 / 91$ & REG & 1,2-Dichloroethane & 15 & $\mathrm{ug} / \mathrm{L}$ & $=$ \\
\hline
\end{tabular}


B-36

Station Sample ID Sample Date Sample Type Chemical

\begin{tabular}{|c|c|c|c|c|}
\hline 842 & 8421210REGUF & $12 / 10 / 91$ & REG & 1,2-Dichloroethane \\
\hline 842 & 842521REGUF & $5 / 21 / 90$ & REG & 1.2-Dichloroethane \\
\hline 842 & 842119REGUF & $11 / 9 / 90$ & REG & 1.2-Dichloroethane \\
\hline 842 & 842126REGUF & $1 / 26 / 89$ & REG & 1,2-Dichloroethane \\
\hline 842 & 842427REGUF & $4 / 27 / 89$ & REG & 1.2-Dichloroethane \\
\hline 842 & W00809 & $6 / 18 / 97$ & REG & 1,2-Dichloroethene \\
\hline 842 & M00055 & $12 / 3 / 96$ & REG & 1,2-Dichloroethene \\
\hline 842 & S00957 & $10 / 12 / 94$ & REG & 1,2-Dichloroethene \\
\hline 842 & S02616 & $2 / 28 / 95$ & REG & 1,2-Dichloroethene \\
\hline 842 & S03576 & $5 / 8 / 95$ & REG & 1,2-Dichloroethene \\
\hline 842 & S04793 & $7 / 14 / 95$ & REG & 1,2-Dichloroethene \\
\hline 842 & 842222REGUF & $2 / 22 / 91$ & REG & 1,2-Dichloroethene \\
\hline 842 & 842104REGUF & $10 / 4 / 91$ & REG & 1,2-Dichloroethene \\
\hline 842 & 8421210 REGUF & $12 / 10 / 91$ & REG & 1,2-Dichloroethene \\
\hline 842 & 842521REGUF & $5 / 21 / 90$ & REG & 1,2-Dichloroethene \\
\hline 842 & 842119REGUF & $11 / 9 / 90$ & REG & 1,2-Dichloroethene \\
\hline 842 & 842126REGUF & $1 / 26 / 89$ & REG & 1,2-Dichloroethene \\
\hline 842 & 842427REGUF & $4 / 27 / 89$ & REG & 1,2-Dichloroethene \\
\hline 842 & M01250 & $11 / 11 / 97$ & REG & 1,2-Dichloropropane \\
\hline 842 & W00809 & $6 / 18 / 97$ & REG & 1,2-Dichloropropane \\
\hline 842 & M00055 & $12 / 3 / 96$ & REG & 1,2-Dichloropropane \\
\hline 842 & M00843 & $6 / 18 / 97$ & REG & 1,2-Dichloropropane \\
\hline 842 & SMPL00266 & $5 / 25 / 94$ & REG & 1,2-Dichloropropane \\
\hline 842 & S00957 & $10 / 12 / 94$ & REG & 1,2-Dichloropropane \\
\hline 842 & S02616 & $2 / 28 / 95$ & REG & 1,2-Dichloropropane \\
\hline 842 & S03576 & $5 / 8 / 95$ & REG & 1,2-Dichloropropane \\
\hline 842 & S04793 & $7 / 14 / 95$ & REG & 1,2-Dichloropropane \\
\hline 842 & 842222REGUF & $2 / 22 / 91$ & REG & 1,2-Dichloropropane \\
\hline 842 & 842104REGUF & $10 / 4 / 91$ & REG & 1,2-Dichloropropane \\
\hline 842 & 8421210REGUF & $12 / 10 / 91$ & REG & 1,2-Dichloropropane \\
\hline 842 & 842521REGUF & $5 / 21 / 90$ & REG & 1,2-Dichloropropane \\
\hline 842 & 842119REGUF & $11 / 9 / 90$ & REG & 1,2-Dichloropropane \\
\hline 842 & 842126REGUF & $1 / 26 / 89$ & REG & 1,2-Dichloropropane \\
\hline 842 & 842427REGUF & $4 / 27 / 89$ & REG & 1,2-Dichloropropane \\
\hline 842 & M00055 & $12 / 3 / 96$ & REG & 1,2-Dimethylbenzene \\
\hline 842 & S02616 & $2 / 28 / 95$ & REG & 1,2-Dimethylbenzene \\
\hline 842 & S03576 & $5 / 8 / 95$ & REG & 1,2-Dimethylbenzene \\
\hline 842 & S04793 & $7 / 14 / 95$ & REG & 1,2-Dimethylbenzene \\
\hline 842 & M00055 & $12 / 3 / 96$ & REG & 1,3-Dichlorobenzene \\
\hline 842 & SMPL00266 & $5 / 25 / 94$ & REG & 1,3-Dichlorobenzene \\
\hline 842 & M00055 & $12 / 3 / 96$ & REG & 1,4-Dichlorobenzene \\
\hline 842 & SMPL00266 & $5 / 25 / 94$ & REG & 1,4-Dichlorobenzene \\
\hline 842 & M01250 & $11 / 11 / 97$ & REG & 2-Butanone \\
\hline 842 & W00809 & $6 / 18 / 97$ & REG & 2-Butanone \\
\hline 842 & M00055 & $12 / 3 / 96$ & REG & 2-Butanone \\
\hline 842 & M00843 & $6 / 18 / 97$ & REG & 2-Butanone \\
\hline 842 & SMPL00266 & $5 / 25 / 94$ & REG & 2-Butanone \\
\hline 842 & S00957 & $10 / 12 / 94$ & REG & 2-Butanone \\
\hline 842 & S02616 & $2 / 28 / 95$ & REG & 2-Butanone \\
\hline 842 & S03576 & $5 / 8 / 95$ & REG & 2-Butanone \\
\hline
\end{tabular}

Results Units Qualifier

\begin{tabular}{|c|c|c|}
\hline 11 & $\mathrm{ug} / \mathrm{L}$ & \\
\hline 5 & $\mathrm{ug} / \mathrm{L}$ & $\mathrm{U}$ \\
\hline 15 & $\mathrm{ug} / \mathrm{L}$ & $=$ \\
\hline 19 & $\mathrm{ug} / \mathrm{L}$ & $=$ \\
\hline 44 & $\mathrm{ug} / \mathrm{L}$ & $=$ \\
\hline 12 & $\mathrm{ug} / \mathrm{L}$ & $=$ \\
\hline 6 & $u g / L$ & $=$ \\
\hline 10 & $\mathrm{ug} / \mathrm{L}$ & $=$ \\
\hline 5 & $\mathrm{ug} / \mathrm{L}$ & $\mathrm{U}$ \\
\hline 9 & $\mathrm{ug} / \mathrm{L}$ & $=$ \\
\hline 10 & $u g / L$ & $=$ \\
\hline 7 & $\mathrm{ug} / \mathrm{L}$ & $=$ \\
\hline 10 & $\mathrm{ug} / \mathrm{L}$ & $=$ \\
\hline 7 & $\mathrm{ug} / \mathrm{L}$ & $=$ \\
\hline 9 & $\mathrm{ug} / \mathrm{L}$ & $=$ \\
\hline 1 & $\mathrm{ug} / \mathrm{L}$ & $\mathrm{J}$ \\
\hline 8 & $\mathrm{ug} / \mathrm{L}$ & $=$ \\
\hline 19 & $\mathrm{ug} / \mathrm{L}$ & $=$ \\
\hline 5 & $\mathrm{ug} / \mathrm{L}$ & $\mathrm{U}$ \\
\hline 5 & ug $/ \mathrm{L}$ & $\mathrm{U}$ \\
\hline 5 & $\mathrm{ug} / \mathrm{L}$ & $\mathrm{U}$ \\
\hline 5 & $\mathrm{ug} / \mathrm{L}$ & $\mathrm{U}$ \\
\hline 10 & $\mathrm{ug} / \mathrm{L}$ & $\mathrm{U}$ \\
\hline 5 & $\mathrm{ug} / \mathrm{L}$ & $\mathrm{U}$ \\
\hline 5 & $\mathrm{ug} / \mathrm{L}$ & $\mathrm{U}$ \\
\hline 5 & $\mathrm{ug} / \mathrm{L}$ & $\mathrm{U}$ \\
\hline 5 & $\mathrm{ug} / \mathrm{L}$ & $\mathrm{U}$ \\
\hline 5 & $\mathrm{ug} / \mathrm{L}$ & $\mathrm{U}$ \\
\hline 5 & $\mathrm{ug} / \mathrm{L}$ & $\mathrm{U}$ \\
\hline 5 & $\mathrm{ug} / \mathrm{h}$ & $\mathrm{U}$ \\
\hline 5 & $\mathrm{ug} / \mathrm{L}$ & $\mathrm{U}$ \\
\hline 5 & $\mathrm{ug} / \mathrm{L}$ & $\mathrm{U}$ \\
\hline 5 & ug/L & $\mathrm{U}$ \\
\hline 5 & $\mathrm{ug} / \mathrm{L}$ & $\mathrm{U}$ \\
\hline 5 & $\mathrm{ug} / \mathrm{L}$ & $\mathrm{U}$ \\
\hline 5 & $\mathrm{ug} / \mathrm{L}$ & $\mathrm{U}$ \\
\hline 5 & $\mathrm{ug} / \mathrm{L}$ & $\mathrm{U}$ \\
\hline 5 & $\mathrm{ug} / \mathrm{L}$ & $\mathrm{U}$ \\
\hline 5 & $\mathrm{ug} / \mathrm{L}$ & $\mathrm{U}$ \\
\hline 10 & $\mathrm{ug} / \mathrm{L}$ & $\mathrm{U}$ \\
\hline 5 & $\mathrm{ug} / \mathrm{L}$ & $\mathrm{U}$ \\
\hline 10 & $\mathrm{ug} / \mathrm{L}$ & $\mathrm{U}$ \\
\hline 1 & $\mathrm{ug} / \mathrm{L}$ & $\mathrm{J}$ \\
\hline 10 & $\mathrm{ug} / \mathrm{L}$ & $\mathrm{U}$ \\
\hline 10 & $\mathrm{ug} / \mathrm{L}$ & $\mathrm{U}$ \\
\hline 10 & $\mathrm{ug} / \mathrm{L}$ & $\mathrm{U}$ \\
\hline 50 & $\mathrm{ug} / \mathrm{L}$ & $\mathrm{U}$ \\
\hline 6.6 & $\mathrm{ug} / \mathrm{L}$ & $\mathrm{J}$ \\
\hline 4 & $u g / L$ & $\mathrm{~J}$ \\
\hline 2 & $\mathrm{ug} / \mathrm{L}$ & $\mathrm{J}$ \\
\hline
\end{tabular}


Station Sample ID Sample Date Sample Type Chemical

\begin{tabular}{|c|c|c|c|c|c|c|c|}
\hline Station & Sample ID & Sample Date & Sample Type & Chemical & Results & Units & Qualifier \\
\hline 842 & S04793 & $7 / 14 / 95$ & REG & 2-Butanone & 10 & $\mathrm{ug} / \mathrm{L}$ & $\mathrm{U}$ \\
\hline 842 & 842222REGUF & $2 / 22 / 91$ & REG & 2-Butanone & 10 & $\mathrm{ug} / \mathrm{L}$ & $\mathrm{U}$ \\
\hline 842 & 842104REGUF & $10 / 4 / 91$ & REG & 2-Butanone & 10 & $\mathrm{ug} / \mathrm{L}$ & $\mathrm{U}$ \\
\hline 842 & 8421210REGUF & $12 / 10 / 91$ & REG & 2-Butanone & 10 & $\mathrm{ug} / \mathrm{L}$ & $U$ \\
\hline 842 & 842521REGUF & $5 / 21 / 90$ & REG & 2-Butanone & 10 & $\mathrm{ug} / \mathrm{L}$ & $\mathrm{U}$ \\
\hline 842 & 842119REGUF & $11 / 9 / 90$ & REG & 2-Butanone & 10 & $\mathrm{ug} / \mathrm{L}$ & $U$ \\
\hline 842 & 842126REGUF & $1 / 26 / 89$ & REG & 2-Butanone & 10 & $\mathrm{ug} / \mathrm{L}$ & $\mathrm{U}$ \\
\hline 842 & 842427REGUF & $4 / 27 / 89$ & REG & 2-Butanone & 10 & $\mathrm{ug} / \mathrm{L}$ & $\mathrm{U}$ \\
\hline 842 & M01250 & $11 / 11 / 97$ & REG & 2-Hexanone & 10 & ug/L & $\mathrm{U}$ \\
\hline 842 & W00809 & $6 / 18 / 97$ & REG & 2-Hexanone & 10 & $\mathrm{ug} / \mathrm{L}$ & $\mathrm{U}$ \\
\hline 842 & M00055 & $12 / 3 / 96$ & REG & 2-Hexanone & 10 & $\mathrm{ug} / \mathrm{L}$ & $\mathrm{U}$ \\
\hline 842 & M00843 & $6 / 18 / 97$ & REG & 2-Hexanone & 10 & $\mathrm{ug} / \mathrm{L}$ & $\mathrm{U}$ \\
\hline 842 & SMPL00266 & $5 / 25 / 94$ & REG & 2-Hexanone & 50 & $\mathrm{ug} / \mathrm{L}$ & $\mathrm{U}$ \\
\hline 842 & S00957 & $10 / 12 / 94$ & REG & 2-Hexanone & 10 & $\mathrm{ug} / \mathrm{L}$ & $\mathrm{U}$ \\
\hline 842 & S02616 & $2 / 28 / 95$ & REG & 2-Hexanone & 10 & $\mathrm{ug} / \mathrm{L}$ & $\mathrm{U}$ \\
\hline 842 & S03576 & $5 / 8 / 95$ & REG & 2-Hexanone & 10 & $\mathrm{ug} / \mathrm{L}$ & $\mathrm{U}$ \\
\hline 842 & S04793 & $7 / 14 / 95$ & REG & 2-Hexanone & 10 & $\mathrm{ug} / \mathrm{L}$ & $\mathrm{U}$ \\
\hline 842 & 842222REGUF & $2 / 22 / 91$ & REG & 2-Hexanone & 10 & $\mathrm{ug} / \mathrm{L}$ & $\mathrm{U}$ \\
\hline 842 & 842104REGUF & $10 / 4 / 91$ & REG & 2-Hexanone & 10 & $\mathrm{ug} / \mathrm{L}$ & $\mathrm{U}$ \\
\hline 842 & 8421210REGUF & $12 / 10 / 91$ & REG & 2-Hexanone & 10 & $\mathrm{ug} / \mathrm{L}$ & $\mathrm{U}$ \\
\hline 842 & 842521REGUF & $5 / 21 / 90$ & REG & 2-Hexanone & 10 & $\mathrm{ug} / \mathrm{L}$ & $\mathrm{U}$ \\
\hline 842 & 842119REGUF & $11 / 9 / 90$ & REG & 2-Hexanone & 10 & $\mathrm{ug} / \mathrm{L}$ & $\mathrm{U}$ \\
\hline 842 & 842126REGUF & $1 / 26 / 89$ & REG & 2-Hexanone & 10 & $\mathrm{ug} / \mathrm{L}$ & $\mathrm{U}$ \\
\hline 842 & 842427REGUF & $4 / 27 / 89$ & REG & 2-Hexanone & 10 & $\mathrm{ug} / \mathrm{L}$ & $\mathrm{U}$ \\
\hline 842 & M01250 & $11 / 11 / 97$ & REG & 4-Methyl-2-pentanone & 10 & $\mathrm{ug} / \mathrm{L}$ & $\mathrm{U}$ \\
\hline 842 & W00809 & $6 / 18 / 97$ & REG & 4-Methyl-2-pentanone & 10 & $\mathrm{ug} / \mathrm{L}$ & $\mathrm{U}$ \\
\hline 842 & M00055 & $12 / 3 / 96$ & REG & 4-Methyl-2-pentanone & 10 & ug/L & $\mathrm{U}$ \\
\hline 842 & M00843 & $6 / 18 / 97$ & REG & 4-Methyl-2-pentanone & 10 & $\mathrm{ug} / \mathrm{L}$ & $\mathrm{U}$ \\
\hline 842 & SMPL00266 & $5 / 25 / 94$ & REG & 4-Methyl-2-pentanone & 50 & $\mathrm{ug} / \mathrm{L}$ & $\mathbf{U}$ \\
\hline 842 & S00957 & $10 / 12 / 94$ & REG & 4-Methyl-2-pentanone & 10 & $\mathrm{ug} / \mathrm{L}$ & $\mathrm{U}$ \\
\hline 842 & S02616 & $2 / 28 / 95$ & REG & 4-Methyl-2-pentanone & 10 & $\mathrm{ug} / \mathrm{L}$ & $\mathrm{U}$ \\
\hline 842 & S03576 & $5 / 8 / 95$ & REG & 4-Methyl-2-pentanone & 10 & $\mathrm{ug} / \mathrm{L}$ & $\mathrm{U}$ \\
\hline 842 & S04793 & $7 / 14 / 95$ & REG & 4-Methyl-2-pentanone & 10 & $\mathrm{ug} / \mathrm{L}$ & $\mathrm{U}$ \\
\hline 842 & 842222REGUF & $2 / 22 / 91$ & REG & 4-Methyl-2-pentanone & 10 & $\mathrm{ug} / \mathrm{L}$ & $\mathrm{U}$ \\
\hline 842 & 842104REGUF & $10 / 4 / 91$ & REG & 4-Methyl-2-pentanone & 10 & $\mathrm{ug} / \mathrm{L}$ & $\mathrm{U}$ \\
\hline 842 & 8421210REGUF & $12 / 10 / 91$ & REG & 4-Methyl-2-pentanone & 10 & $\mathrm{ug} / \mathrm{L}$ & $\mathrm{U}$ \\
\hline 842 & 842521REGUF & $5 / 21 / 90$ & REG & 4-Methyl-2-pentanone & 10 & $\mathrm{ug} / \mathrm{L}$ & $\mathrm{U}$ \\
\hline 842 & 842119REGUF & $11 / 9 / 90$ & REG & 4-Methyl-2-pentanone & 10 & $\mathrm{ug} / \mathrm{L}$ & $\mathrm{U}$ \\
\hline 842 & 842126REGUF & $1 / 26 / 89$ & REG & 4-Methyl-2-pentanone & 10 & $\mathrm{ug} / \mathrm{L}$ & $\mathrm{U}$ \\
\hline 842 & 842427REGUF & $4 / 27 / 89$ & REG & 4-Methyl-2-pentanone & 10 & $\mathrm{ug} / \mathrm{L}$ & $\mathrm{U}$ \\
\hline 842 & M01250 & $11 / 11 / 97$ & REG & Acetone & 6 & $\mathrm{ug} / \mathrm{L}$ & B \\
\hline 842 & W00809 & $6 / 18 / 97$ & REG & Acetone & 10 & $\mathrm{ug} / \mathrm{L}$ & $\mathrm{U}$ \\
\hline 842 & M00055 & $12 / 3 / 96$ & REG & Acetone & 10 & $\mathrm{ug} / \mathrm{L}$ & $\mathrm{U}$ \\
\hline 842 & M00843 & $6 / 18 / 97$ & REG & Acetone & 4 & $\mathrm{ug} / \mathrm{L}$ & $\mathrm{U}$ \\
\hline 842 & S00957 & $10 / 12 / 94$ & REG & Acetone & 6.3 & $\mathrm{ug} / \mathrm{L}$ & $\mathbf{J}$ \\
\hline 842 & S02616 & $2 / 28 / 95$ & REG & Acetone & 10 & $\mathrm{ug} / \mathrm{L}$ & $\mathrm{U}$ \\
\hline 842 & S03576 & $5 / 8 / 95$ & REG & Acetone & 2 & $\mathrm{ug} / \mathrm{L}$ & $\mathrm{J}$ \\
\hline 842 & S04793 & $7 / 14 / 95$ & REG & Acetone & 10 & $\mathrm{ug} / \mathrm{L}$ & $\mathrm{U}$ \\
\hline 842 & 842222REGUF & $2 / 22 / 91$ & REG & Acetone & 10 & $\mathrm{ug} / \mathrm{L}$ & $\mathrm{U}$ \\
\hline 842 & 842104REGUF & $10 / 4 / 91$ & REG & Acetone & 10 & $\mathrm{ug} / \mathrm{L}$ & $\mathrm{U}$ \\
\hline
\end{tabular}




\begin{tabular}{|c|c|c|c|c|c|c|c|}
\hline Station & Sample ID & Sample Date & Sample Type & Chemical & Results & Units & Qualifier \\
\hline$\overline{842}$ & 8421210REGUF & $12 / 10 / 91$ & REG & Acetone & 10 & $\mathrm{ug} / \mathrm{L}$ & $\mathrm{U}$ \\
\hline 842 & 842521REGUF & $5 / 21 / 90$ & REG & Acetone & 10 & $\mathrm{ug} / \mathrm{L}$ & $\mathrm{U}$ \\
\hline 842 & 842119REGUF & $11 / 9 / 90$ & REG & Acetone & 7 & $\mathrm{ug} / \mathrm{L}$ & J \\
\hline 842 & 842126REGUF & $1 / 26 / 89$ & REG & Acetone & 10 & $\mathrm{ug} / \mathrm{L}$ & $\mathrm{U}$ \\
\hline 842 & 842427REGUF & $4 / 27 / 89$ & REG & Acetone & 10 & $\mathrm{ug} / \mathrm{L}$ & $\mathrm{U}$ \\
\hline 842 & M01250 & $11 / 11 / 97$ & REG & Benzene & 5 & $u g / L$ & $\mathrm{U}$ \\
\hline 842 & W00809 & $6 / 18 / 97$ & REG & Benzene & 5 & $\mathrm{ug} / \mathrm{L}$ & $\mathrm{U}$ \\
\hline 842 & M00055 & $12 / 3 / 96$ & REG & Benzene & 5 & $\mathrm{ug} / \mathrm{L}$ & $\mathrm{U}$ \\
\hline 842 & M00843 & $6 / 18 / 97$ & REG & Benzene & 5 & $u g / L$ & $U$ \\
\hline 842 & SMPL00266 & $5 / 25 / 94$ & REG & Benzene & 10 & $\mathrm{ug} / \mathrm{L}$ & $\mathrm{U}$ \\
\hline 842 & S00957 & $10 / 12 / 94$ & REG & Benzene & 5 & $\mathrm{ug} / \mathrm{L}$ & $\mathrm{U}$ \\
\hline 842 & S02616 & $2 / 28 / 95$ & REG & Benzene & 5 & $\mathrm{ug} / \mathrm{L}$ & $\mathrm{U}$ \\
\hline 842 & S03576 & $5 / 8 / 95$ & REG & Benzene & 5 & ug/L & $\mathrm{U}$ \\
\hline 842 & S04793 & $7 / 14 / 95$ & REG & Benzene & 5 & $\mathrm{ug} / \mathrm{L}$ & $\mathrm{U}$ \\
\hline 842 & 842222REGUF & $2 / 22 / 91$ & REG & Benzene & 5 & $\mathrm{ug} / \mathrm{L}$ & $\mathrm{U}$ \\
\hline 842 & 842104REGUF & $10 / 4 / 91$ & REG & Benzene & 5 & $\mathrm{ug} / \mathrm{L}$ & $\mathrm{U}$ \\
\hline 842 & 8421210REGUF & $12 / 10 / 91$ & REG & Benzene & 5 & $\mathrm{ug} / \mathrm{L}$ & $\mathrm{U}$ \\
\hline 842 & 842521REGUF & $5 / 21 / 90$ & REG & Benzene & 5 & $\mathrm{ug} / \mathrm{L}$ & $\mathrm{U}$ \\
\hline 842 & 842119REGUF & $11 / 9 / 90$ & REG & Benzene & 5 & $\mathrm{ug} / \mathrm{L}$ & $\mathrm{U}$ \\
\hline 842 & 842126REGUF & $1 / 26 / 89$ & REG & Benzene & 5 & $\mathrm{ug} / \mathrm{L}$ & $\mathrm{U}$ \\
\hline 842 & 842427REGUF & $4 / 27 / 89$ & REG & Benzene & 5 & $\mathrm{ug} / \mathrm{L}$ & U \\
\hline 842 & SMPL00266 & $5 / 25 / 94$ & REG & Bromochloromethane & 10 & $\mathrm{ug} / \mathrm{L}$ & $\mathrm{U}$ \\
\hline 842 & M01250 & $11 / 11 / 97$ & REG & Bromodichloromethane & 5 & $\mathrm{ug} / \mathrm{L}$ & $\mathrm{U}$ \\
\hline 842 & W00809 & $6 / 18 / 97$ & REG & Bromodichloromethane & 5 & $u g / L$ & $\mathrm{U}$ \\
\hline 842 & M00055 & $12 / 3 / 96$ & REG & Bromodichloromethane & 5 & $u g / L$ & $\mathrm{U}$ \\
\hline 842 & M00843 & $6 / 18 / 97$ & REG & Bromodichloromethane & 5 & $\mathrm{ug} / \mathrm{L}$ & $\mathrm{U}$ \\
\hline 842 & SMPL00266 & $5 / 25 / 94$ & REG & Bromodichloromethane & 10 & $u g / L$ & $\mathbf{U}$ \\
\hline 842 & S00957 & $10 / 12 / 94$ & REG & Bromodichloromethane & 5 & $\mathrm{ug} / \mathrm{L}$ & $\mathrm{U}$ \\
\hline 842 & S02616 & $2 / 28 / 95$ & REG & Bromodichloromethane & 5 & $\mathrm{ug} / \mathrm{L}$ & $\mathrm{U}$ \\
\hline 842 & S03576 & $5 / 8 / 95$ & REG & Bromodichloromethane & 5 & $\mathrm{ug} / \mathrm{L}$ & $\mathrm{U}$ \\
\hline 842 & S04793 & $7 / 14 / 95$ & REG & Bromodichloromethane & 5 & $u g / L$ & $\mathrm{U}$ \\
\hline 842 & 842222REGUF & $2 / 22 / 91$ & REG & Bromodichloromethane & 5 & ug/L & $\mathrm{U}$ \\
\hline 842 & 842104REGUF & $10 / 4 / 91$ & REG & Bromodichloromethane & 5 & $\mathrm{ug} / \mathrm{L}$ & $\mathrm{U}$ \\
\hline 842 & 8421210REGUF & $12 / 10 / 91$ & REG & Bromodichloromethane & 5 & $\mathrm{ug} / \mathrm{L}$ & $\mathrm{U}$ \\
\hline 842 & 842521REGUF & $5 / 21 / 90$ & REG & Bromodichloromethane & 5 & ug/L & $\mathrm{U}$ \\
\hline 842 & 842119REGUF & $11 / 9 / 90$ & REG & Bromodichloromethane & 5 & $\mathrm{ug} / \mathrm{L}$ & $\mathrm{U}$ \\
\hline 842 & 842126REGUF & $1 / 26 / 89$ & REG & Bromodichloromethane & 5 & $\mathrm{ug} / \mathrm{L}$ & $\mathrm{U}$ \\
\hline 842 & 842427REGUF & $4 / 27 / 89$ & REG & Bromodichloromethane & 5 & $\mathrm{ug} / \mathrm{L}$ & $\mathrm{U}$ \\
\hline 842 & M01250 & $11 / 11 / 97$ & REG & Bromoform & 5 & $\mathrm{ug} / \mathrm{L}$ & $\mathrm{U}$ \\
\hline 842 & W00809 & $6 / 18 / 97$ & REG & Bromoform & 5 & $\mathrm{ug} / \mathrm{L}$ & $\mathrm{U}$ \\
\hline 842 & M00055 & $12 / 3 / 96$ & REG & Bromoform & 5 & $\mathrm{ug} / \mathrm{L}$ & $\mathrm{U}$ \\
\hline 842 & M00843 & $6 / 18 / 97$ & REG & Bromoform & 5 & $\mathrm{ug} / \mathrm{L}$ & $\mathrm{U}$ \\
\hline 842 & SMPL00266 & $5 / 25 / 94$ & REG & Bromoform & 10 & $\mathrm{ug} / \mathrm{L}$ & $\mathrm{U}$ \\
\hline 842 & S00957 & $10 / 12 / 94$ & REG & Bromoform & 5 & $u g / L$ & $\mathrm{U}$ \\
\hline 842 & S02616 & $2 / 28 / 95$ & REG & Bromoform & 5 & $\mathrm{ug} / \mathrm{L}$ & $\mathrm{U}$ \\
\hline 842 & S03576 & $5 / 8 / 95$ & REG & Bromoform & 5 & $\mathrm{ug} / \mathrm{L}$ & $\mathrm{U}$ \\
\hline 842 & S04793 & $7 / 14 / 95$ & REG & Bromoform & 5 & $\mathrm{ug} / \mathrm{L}$ & $\mathrm{U}$ \\
\hline 842 & 842222REGUF & $2 / 22 / 91$ & REG & Bromoform & 5 & $\mathrm{ug} / \mathrm{L}$ & $\mathrm{U}$ \\
\hline 842 & 842104REGUF & $10 / 4 / 91$ & REG & Bromoform & 5 & $\mathrm{ug} / \mathrm{L}$ & $\mathrm{U}$ \\
\hline 842 & 8421210REGUF & $12 / 10 / 91$ & REG & Bromoform & 5 & $\mathrm{ug} / \mathrm{L}$ & $\mathrm{U}$ \\
\hline
\end{tabular}




\begin{tabular}{|c|c|c|c|c|c|c|c|}
\hline Station & Sample ID & Sample Date & Sample Type & Chemical & Results & Units & Qualifier \\
\hline$\overline{842}$ & 842521REGUF & $5 / 21 / 90$ & REG & Bromoform & 5 & $\mathrm{ug} / \mathrm{L}$ & $\bar{U}$ \\
\hline 842 & 842119REGUF & $11 / 9 / 90$ & REG & Bromoform & 5 & $\mathrm{ug} / \mathrm{L}$ & $\mathrm{U}$ \\
\hline 842 & 842126REGUF & $1 / 26 / 89$ & REG & Bromoform & 5 & $\mathrm{ug} / \mathrm{L}$ & $\mathrm{U}$ \\
\hline 842 & 842427REGUF & $4 / 27 / 89$ & REG & Bromoform & 5 & $\mathrm{ug} / \mathrm{L}$ & $\mathrm{U}$ \\
\hline 842 & M01250 & $11 / 11 / 97$ & REG & Bromomethane & 10 & $\mathrm{ug} / \mathrm{L}$ & $\mathrm{U}$ \\
\hline 842 & W00809 & $6 / 18 / 97$ & REG & Bromomethane & 10 & $\mathrm{ug} / \mathrm{L}$ & $\mathrm{U}$ \\
\hline 842 & M00055 & $12 / 3 / 96$ & REG & Bromomethane & 10 & $\mathrm{ug} / \mathrm{L}$ & $\mathrm{U}$ \\
\hline 842 & M00843 & $6 / 18 / 97$ & REG & Bromomethane & 10 & $\mathrm{ug} / \mathrm{L}$ & $\mathrm{U}$ \\
\hline 842 & SMPL00266 & $5 / 25 / 94$ & REG & Bromomethane & 10 & $u g / L$ & $\mathrm{U}$ \\
\hline 842 & S00957 & $10 / 12 / 94$ & REG & Bromomethane & 10 & ug/L & $\mathrm{U}$ \\
\hline 842 & S02616 & $2 / 28 / 95$ & REG & Bromomethane & 10 & $\mathrm{ug} / \mathrm{L}$ & U \\
\hline 842 & S03576 & $5 / 8 / 95$ & REG & Bromomethane & 10 & $\mathrm{ug} / \mathrm{L}$ & $\mathrm{U}$ \\
\hline 842 & S04793 & $7 / 14 / 95$ & REG & Bromomethane & 10 & $\mathrm{ug} / \mathrm{L}$ & $U$ \\
\hline 842 & 842222REGUF & $2 / 22 / 91$ & REG & Bromomethane & 10 & $\mathrm{ug} / \mathrm{L}$ & $\mathrm{U}$ \\
\hline 842 & 842104REGUF & $10 / 4 / 91$ & REG & Bromomethane & 10 & $\mathrm{ug} / \mathrm{L}$ & $\mathrm{U}$ \\
\hline 842 & 8421210REGUF & $12 / 10 / 91$ & REG & Bromomethane & 10 & $\mathrm{ug} / \mathrm{L}$ & $\mathrm{U}$ \\
\hline 842 & 842521REGUF & $5 / 21 / 90$ & REG & Bromomethane & 10 & $\mathrm{ug} / \mathrm{L}$ & $\mathrm{U}$ \\
\hline 842 & 842119REGUF & $11 / 9 / 90$ & REG & Bromomethane & 10 & ug $/ \mathrm{L}$ & $\mathrm{U}$ \\
\hline 842 & 842126REGUF & $1 / 26 / 89$ & REG & Bromomethane & 10 & $\mathrm{ug} / \mathrm{L}$ & U \\
\hline 842 & 842427REGUF & $4 / 27 / 89$ & REG & Bromomethane & 10 & $\mathrm{ug} / \mathrm{L}$ & $\mathrm{U}$ \\
\hline 842 & M01250 & $11 / 11 / 97$ & REG & Carbon disulfide & 5 & $\mathrm{ug} / \mathrm{L}$ & $\mathrm{U}$ \\
\hline 842 & W00809 & $6 / 18 / 97$ & REG & Carbon disulfide & 5 & $\mathrm{ug} / \mathrm{L}$ & $\mathrm{U}$ \\
\hline 842 & M00055 & $12 / 3 / 96$ & REG & Carbon disulfide & 5 & $\mathrm{ug} / \mathrm{L}$ & $\mathrm{U}$ \\
\hline 842 & M00843 & $6 / 18 / 97$ & REG & Carbon disulfide & 5 & $\mathrm{ug} / \mathrm{L}$ & $\mathrm{U}$ \\
\hline 842 & SMPL00266 & $5 / 25 / 94$ & REG & Carbon disulfide & 10 & $\mathrm{ug} / \mathrm{L}$ & $U$ \\
\hline 842 & S00957 & $10 / 12 / 94$ & REG & Carbon disulfide & 5 & $\mathrm{ug} / \mathrm{L}$ & $\mathrm{U}$ \\
\hline 842 & S02616 & $2 / 28 / 95$ & REG & Carbon disulfide & 5 & $\mathrm{ug} / \mathrm{L}$ & $\mathrm{U}$ \\
\hline 842 & S03576 & $5 / 8 / 95$ & REG & Carbon disulfide & 5 & $\mathrm{ug} / \mathrm{L}$ & $\mathrm{U}$ \\
\hline 842 & S04793 & $7 / 14 / 95$ & REG & Carbon disulfide & 5 & ug/L & $\mathrm{U}$ \\
\hline 842 & 842222REGUF & $2 / 22 / 91$ & REG & Carbon disulfide & 5 & $\mathrm{ug} / \mathrm{L}$ & $\mathrm{U}$ \\
\hline 842 & 842104REGUF & $10 / 4 / 91$ & REG & Carbon disulfide & 5 & $\mathrm{ug} / \mathrm{L}$ & $\mathrm{U}$ \\
\hline 842 & 8421210REGUF & $12 / 10 / 91$ & REG & Carbon disulfide & 5 & ug/L & $\mathrm{U}$ \\
\hline 842 & 842521REGUF & $5 / 21 / 90$ & REG & Carbon disulfide & 5 & $\mathrm{ug} / \mathrm{L}$ & $\mathrm{U}$ \\
\hline 842 & 842119REGUF & $11 / 9 / 90$ & REG & Carbon disulfide & 5 & $\mathrm{ug} / \mathrm{L}$ & $\mathrm{U}$ \\
\hline 842 & 842126REGUF & $1 / 26 / 89$ & REG & Carbon disulfide & 5 & $\mathrm{ug} / \mathrm{L}$ & $\mathrm{U}$ \\
\hline 842 & 842427REGUF & $4 / 27 / 89$ & REG & Carbon disulfide & 5 & $\mathrm{ug} / \mathrm{L}$ & $\mathrm{U}$ \\
\hline 842 & M01250 & $11 / 11 / 97$ & REG & Carbon tetrachloride & 29 & $\mathrm{ug} / \mathrm{L}$ & $=$ \\
\hline 842 & W00809 & $6 / 18 / 97$ & REG & Carbon tetrachloride & 20 & $\mathrm{ug} / \mathrm{L}$ & $=$ \\
\hline 842 & M00055 & $12 / 3 / 96$ & REG & Carbon tetrachloride & 27 & $\mathrm{ug} / \mathrm{L}$ & $=$ \\
\hline 842 & M00843 & $6 / 18 / 97$ & REG & Carbon tetrachloride & 28 & $\mathrm{ug} / \mathrm{L}$ & $=$ \\
\hline 842 & S00957 & $10 / 12 / 94$ & REG & Carbon tetrachloride & 34 & $\mathrm{ug} / \mathrm{L}$ & $=$ \\
\hline 842 & S02616 & $2 / 28 / 95$ & REG & Carbon tetrachloride & 34 & $\mathrm{ug} / \mathrm{L}$ & $=$ \\
\hline 842 & S03576 & $5 / 8 / 95$ & REG & Carbon tetrachloride & 34 & ug/L & $=$ \\
\hline 842 & S04793 & $7 / 14 / 95$ & REG & Carbon tetrachloride & 32 & $\mathrm{ug} / \mathrm{L}$ & $=$ \\
\hline 842 & 842222REGUF & $2 / 22 / 91$ & REG & Carbon tetrachloride & 65 & ug/L & $=$ \\
\hline 842 & 842104REGUF & $10 / 4 / 91$ & REG & Carbon tetrachloride & 51 & $\mathrm{ug} / \mathrm{L}$ & $=$ \\
\hline 842 & 8421210REGUF & $12 / 10 / 91$ & REG & Carbon tetrachloride & 40 & $\mathrm{ug} / \mathrm{L}$ & $=$ \\
\hline 842 & 842521REGUF & $5 / 21 / 90$ & REG & Carbon tetrachloride & 73 & $\mathrm{ug} / \mathrm{L}$ & $=$ \\
\hline 842 & 842119REGUF & $11 / 9 / 90$ & REG & Carbon tetrachloride & 33 & $\mathrm{ug} / \mathrm{L}$ & $=$ \\
\hline 842 & 842126REGUF & $1 / 26 / 89$ & REG & Carbon tetrachloride & 86 & $\mathrm{ug} / \mathrm{L}$ & $=$ \\
\hline
\end{tabular}


Station Sample ID Sample Date Sample Type Chemical

\begin{tabular}{|c|c|c|c|c|c|c|c|}
\hline 842 & 842427REGUF & $4 / 27 / 89$ & REG & Carbon tetrachloride & 82 & $\mathrm{ug} / \mathrm{L}$ & $=$ \\
\hline 842 & M01250 & $11 / 11 / 97$ & REG & Chlorobenzene & 5 & $\mathrm{ug} / \mathrm{L}$ & $\mathrm{U}$ \\
\hline 842 & W00809 & $6 / 18 / 97$ & REG & Chlorobenzene & 5 & $\mathrm{ug} / \mathrm{L}$ & $\mathrm{U}$ \\
\hline 842 & M00055 & $12 / 3 / 96$ & REG & Chlorobenzene & 5 & $\mathrm{ug} / \mathrm{L}$ & $U$ \\
\hline 842 & M00843 & $6 / 18 / 97$ & REG & Chlorobenzene & 5 & $\mathrm{ug} / \mathrm{L}$ & $\mathrm{U}$ \\
\hline 842 & SMPL00266 & $5 / 25 / 94$ & REG & Chlorobenzene & 10 & $\mathrm{ug} / \mathrm{L}$ & $\mathrm{U}$ \\
\hline 842 & S00957 & $10 / 12 / 94$ & REG & Chlorobenzene & 5 & $\mathrm{ug} / \mathrm{L}$ & $\mathrm{U}$ \\
\hline 842 & S02616 & $2 / 28 / 95$ & REG & Chlorobenzene & 5 & ug/L & $\mathrm{U}$ \\
\hline 842 & S03576 & $5 / 8 / 95$ & REG & Chlorobenzene & 5 & $\mathrm{ug} / \mathrm{L}$ & $\mathrm{U}$ \\
\hline 842 & S04793 & $7 / 14 / 95$ & REG & Chlorobenzene & 5 & $\mathrm{ug} / \mathrm{L}$ & $\mathrm{U}$ \\
\hline 842 & 842222REGUF & $2 / 22 / 91$ & REG & Chlorobenzene & 5 & $\mathrm{ug} / \mathrm{L}$ & $\mathrm{U}$ \\
\hline 842 & 842104REGUF & $10 / 4 / 91$ & REG & Chlorobenzene & 5 & $\mathrm{ug} / \mathrm{L}$ & $\mathrm{U}$ \\
\hline 842 & 8421210REGUF & $12 / 10 / 91$ & REG & Chlorobenzene & 5 & $\mathrm{ug} / \mathrm{L}$ & $\mathrm{U}$ \\
\hline 842 & 842521REGUF & $5 / 21 / 90$ & REG & Chlorobenzene & 5 & $u g / L$ & U \\
\hline 842 & 842119REGUF & $11 / 9 / 90$ & REG & Chlorobenzene & 5 & $\mathrm{ug} / \mathrm{L}$ & $\mathrm{U}$ \\
\hline 842 & 842126REGUF & $1 / 26 / 89$ & REG & Chlorobenzene & 5 & $u g / L$ & $\mathrm{U}$ \\
\hline 842 & 842427REGUF & $4 / 27 / 89$ & REG & Chlorobenzene & 5 & $\mathrm{ug} / \mathrm{L}$ & $\mathrm{U}$ \\
\hline 842 & $\mathrm{M} 01250$ & $11 / 11 / 97$ & REG & Chloroethane & 10 & $\mathrm{ug} / \mathrm{L}$ & $\mathrm{U}$ \\
\hline 842 & W00809 & $6 / 18 / 97$ & REG & Chloroethane & 10 & $\mathrm{ug} / \mathrm{L}$ & $\mathrm{U}$ \\
\hline 842 & M00055 & $12 / 3 / 96$ & REG & Chloroethane & 10 & $u g / L$ & $\mathrm{U}$ \\
\hline 842 & M00843 & $6 / 18 / 97$ & REG & Chloroethane & 10 & $\mathrm{ug} / \mathrm{L}$ & $\mathrm{U}$ \\
\hline 842 & SMPL00266 & $5 / 25 / 94$ & REG & Chloroethane & 10 & $u g / L$ & $U$ \\
\hline 842 & S00957 & $10 / 12 / 94$ & REG & Chloroethane & 10 & $\mathrm{ug} / \mathrm{L}$ & $\mathrm{U}$ \\
\hline 842 & S02616 & $2 / 28 / 95$ & REG & Chloroethane & 10 & $\mathrm{ug} / \mathrm{L}$ & $\mathrm{U}$ \\
\hline 842 & S03576 & $5 / 8 / 95$ & REG & Chloroethane & 10 & $\mathrm{ug} / \mathrm{L}$ & $\mathrm{U}$ \\
\hline 842 & S04793 & $7 / 14 / 95$ & REG & Chloroethane & 10 & $\mathrm{ug} / \mathrm{L}$ & $\mathrm{U}$ \\
\hline 842 & 842222REGUF & $2 / 22 / 91$ & REG & Chloroethane & 10 & $\mathrm{ug} / \mathrm{L}$ & $\mathrm{U}$ \\
\hline 842 & 842104REGUF & $10 / 4 / 91$ & REG & Chloroethane & 10 & $u g / L$ & $\mathrm{U}$ \\
\hline 842 & 8421210REGUF & $12 / 10 / 91$ & REG & Chloroethane & 10 & $\mathrm{ug} / \mathrm{L}$ & $\mathrm{U}$ \\
\hline 842 & 842521REGUF & $5 / 21 / 90$ & REG & Chloroethane & 10 & $u g / L$ & $\mathrm{U}$ \\
\hline 842 & 842119REGUF & $11 / 9 / 90$ & REG & Chloroethane & 10 & $u g / L$ & $\mathrm{U}$ \\
\hline 842 & 842126 REGUF & $1 / 26 / 89$ & REG & Chloroethane & 10 & ug/L & $\mathrm{U}$ \\
\hline 842 & 842427REGUF & $4 / 27 / 89$ & REG & Chloroethane & 10 & $u g / L$ & U \\
\hline 842 & M01250 & $11 / 11 / 97$ & REG & Chloroform & 29 & $\mathrm{ug} / \mathrm{L}$ & $=$ \\
\hline 842 & W00809 & $6 / 18 / 97$ & REG & Chloroform & 34 & $\mathrm{ug} / \mathrm{L}$ & $=$ \\
\hline 842 & M00055 & $12 / 3 / 96$ & REG & Chloroform & 30 & $\mathrm{ug} / \mathrm{L}$ & $=$ \\
\hline 842 & M00843 & $6 / 18 / 97$ & REG & Chloroform & 30 & $\mathrm{ug} / \mathrm{L}$ & $=$ \\
\hline 842 & S00957 & $10 / 12 / 94$ & REG & Chloroform & 40 & $\mathrm{ug} / \mathrm{L}$ & $=$ \\
\hline 842 & S02616 & $2 / 28 / 95$ & REG & Chloroform & 37 & $\mathrm{ug} / \mathrm{L}$ & $=$ \\
\hline 842 & S03576 & $5 / 8 / 95$ & REG & Chloroform & 40 & $u g / L$ & $=$ \\
\hline 842 & S04793 & $7 / 14 / 95$ & REG & Chloroform & 35 & $\mathrm{ug} / \mathrm{L}$ & $=$ \\
\hline 842 & 842222REGUF & $2 / 22 / 91$ & REG & Chloroform & 60 & $u g / L$ & $=$ \\
\hline 842 & 842104REGUF & $10 / 4 / 91$ & REG & Chloroform & 50 & $\mathrm{ug} / \mathrm{L}$ & $=$ \\
\hline 842 & 8421210 REGUF & $12 / 10 / 91$ & REG & Chloroform & 46 & $u g / L$ & $=$ \\
\hline 842 & 842521REGUF & $5 / 21 / 90$ & REG & Chloroform & 61 & $\mathrm{ug} / \mathrm{L}$ & $=$ \\
\hline 842 & 842119REGUF & $11 / 9 / 90$ & REG & Chloroform & 63 & $\mathrm{ug} / \mathrm{L}$ & $=$ \\
\hline 842 & 842126REGUF & $1 / 26 / 89$ & REG & Chloroform & 62 & $u g / L$ & $=$ \\
\hline 842 & 842427REGUF & $4 / 27 / 89$ & REG & Chloroform & 97 & $\mathrm{ug} / \mathrm{L}$ & $=$ \\
\hline 842 & M01250 & $11 / 11 / 97$ & REG & Chloromethane & 10 & $\mathrm{ug} / \mathrm{L}$ & $\mathrm{U}$ \\
\hline 842 & W00809 & $6 / 18 / 97$ & REG & Chloromethane & 10 & $\mathrm{ug} / \mathrm{L}$ & $\mathrm{U}$ \\
\hline
\end{tabular}




\begin{tabular}{|c|c|c|c|c|c|c|c|}
\hline Station & Sample ID & Sample Date & Sample Type & Chemical & Results & Units & Qualifier \\
\hline$\overline{842}$ & M00055 & $12 / 3 / 96$ & REG & Chloromethane & 10 & $\mathrm{ug} / \mathrm{L}$ & $\bar{U}$ \\
\hline 842 & M00843 & $6 / 18 / 97$ & REG & Chloromethane & 10 & $\mathrm{ug} / \mathrm{L}$ & $\mathrm{U}$ \\
\hline 842 & SMPL00266 & $5 / 25 / 94$ & REG & Chloromethane & 10 & $\mathrm{ug} / \mathrm{L}$ & $\mathrm{U}$ \\
\hline 842 & S00957 & $10 / 12 / 94$ & REG & Chloromethane & 10 & $u g / L$ & U \\
\hline 842 & S02616 & $2 / 28 / 95$ & REG & Chloromethane & 10 & ug/L & $\mathrm{U}$ \\
\hline 842 & S03576 & $5 / 8 / 95$ & REG & Chloromethane & 10 & $\mathrm{ug} / \mathrm{L}$ & $\mathrm{U}$ \\
\hline 842 & S04793 & $7 / 14 / 95$ & REG & Chloromethane & 10 & ug/L & $\mathrm{U}$ \\
\hline 842 & 842222REGUF & $2 / 22 / 91$ & REG & Chloromethane & 10 & ug/L & $\mathrm{U}$ \\
\hline 842 & 842104REGUF & $10 / 4 / 91$ & REG & Chloromethane & 10 & $\mathrm{ug} / \mathrm{L}$ & $\mathrm{U}$ \\
\hline 842 & 8421210REGUF & $12 / 10 / 91$ & REG & Chloromethane & 10 & ug/L & $\mathrm{U}$ \\
\hline 842 & 842521REGUF & $5 / 21 / 90$ & REG & Chloromethane & 10 & ug $/ \mathrm{L}$ & $\mathrm{U}$ \\
\hline 842 & 842119REGUF & $11 / 9 / 90$ & REG & Chloromethane & 10 & $\mathrm{ug} / \mathrm{L}$ & $\mathrm{U}$ \\
\hline 842 & 842126REGUF & $1 / 26 / 89$ & REG & Chloromethane & 10 & $\mathrm{ug} / \mathrm{L}$ & $\mathrm{U}$ \\
\hline 842 & 842427REGUF & $4 / 27 / 89$ & REG & Chloromethane & 10 & $\mathrm{ug} / \mathrm{L}$ & $\mathrm{U}$ \\
\hline 842 & M01250 & $11 / 11 / 97$ & REG & cis-1,2-Dichloroethene & 10 & $\mathrm{ug} / \mathrm{L}$ & $=$ \\
\hline 842 & M00055 & $12 / 3 / 96$ & REG & cis-1,2-Dichloroethene & 6 & ug/L & $=$ \\
\hline 842 & M00843 & $6 / 18 / 97$ & REG & cis-1,2-Dichloroethene & 7 & $\mathrm{ug} / \mathrm{L}$ & $=$ \\
\hline 842 & SMPL00266 & $5 / 25 / 94$ & REG & cis-1,2-Dichloroethene & 9.9 & $\mathrm{ug} / \mathrm{L}$ & $\mathrm{J}$ \\
\hline 842 & S00957 & $10 / 12 / 94$ & REG & cis-1,2-Dichloroethene & 10 & $u g / L$ & $=$ \\
\hline 842 & S02616 & $2 / 28 / 95$ & REG & cis-1,2-Dichloroethene & 8 & $\mathrm{ug} / \mathrm{L}$ & $=$ \\
\hline 842 & S03576 & $5 / 8 / 95$ & REG & cis-1,2-Dichloroethene & 9 & $\mathrm{ug} / \mathrm{L}$ & $=$ \\
\hline 842 & S04793 & $7 / 14 / 95$ & REG & cis-1,2-Dichloroethene & 10 & $\mathrm{ug} / \mathrm{L}$ & $=$ \\
\hline 842 & $\mathrm{M} 01250$ & $11 / 11 / 97$ & REG & cis-1,3-Dichloropropen & 5 & $\mathrm{ug} / \mathrm{L}$ & $\mathrm{U}$ \\
\hline 842 & W00809 & $6 / 18 / 97$ & REG & cis-1,3-Dichloropropene & 5 & $\mathrm{ug} / \mathrm{L}$ & $\mathrm{U}$ \\
\hline 842 & M00055 & $12 / 3 / 96$ & REG & cis-1,3-Dichloropropene & 5 & $\mathrm{ug} / \mathrm{L}$ & $\mathrm{U}$ \\
\hline 842 & M00843 & $6 / 18 / 97$ & REG & cis-1,3-Dichloropropene & 5 & $\mathrm{ug} / \mathrm{L}$ & $\mathrm{U}$ \\
\hline 842 & SMPL00266 & $5 / 25 / 94$ & REG & cis-1,3-Dichloropropene & 10 & $\mathrm{ug} / \mathrm{L}$ & $\mathrm{U}$ \\
\hline 842 & S00957 & $10 / 12 / 94$ & REG & cis-1,3-Dichloropropene & 5 & $\mathrm{ug} / \mathrm{L}$ & $\mathrm{U}$ \\
\hline 842 & S02616 & $2 / 28 / 95$ & REG & cis-1,3-Dichloropropene & 5 & $\mathrm{ug} / \mathrm{L}$ & $\mathrm{U}$ \\
\hline 842 & S03576 & $5 / 8 / 95$ & REG & cis-1,3-Dichloropropene & 5 & $\mathrm{ug} / \mathrm{L}$ & $\mathrm{U}$ \\
\hline 842 & S04793 & $7 / 14 / 95$ & REG & cis-1,3-Dichloropropene & 5 & $\mathrm{ug} / \mathrm{L}$ & $\mathrm{U}$ \\
\hline 842 & 842222REGUF & $2 / 22 / 91$ & REG & cis-1,3-Dichloropropene & 5 & $\mathrm{ug} / \mathrm{L}$ & $\mathrm{U}$ \\
\hline 842 & 842104REGUF & $10 / 4 / 91$ & REG & cis-1,3-Dichloropropene & 5 & $\mathrm{ug} / \mathrm{L}$ & $\mathrm{U}$ \\
\hline 842 & 8421210REGUF & $12 / 10 / 91$ & REG & cis-1,3-Dichloropropene & 5 & $\mathrm{ug} / \mathrm{L}$ & U \\
\hline 842 & 842521REGUF & $5 / 21 / 90$ & REG & cis-1,3-Dichloropropene & 5 & ug/L & U \\
\hline 842 & 842119REGUF & $11 / 9 / 90$ & REG & cis-1,3-Dichloropropene & 5 & ug/L & $\mathrm{U}$ \\
\hline 842 & 842126REGUF & $1 / 26 / 89$ & REG & cis-1,3-Dichloropropene & 5 & ug/L & $\mathrm{U}$ \\
\hline 842 & 842427REGUF & $4 / 27 / 89$ & REG & cis-1,3-Dichloropropene & 5 & ug/L & $\mathrm{U}$ \\
\hline 842 & $\mathrm{M} 01250$ & $11 / 11 / 97$ & REG & Dibromochloromethane & 5 & $\mathrm{ug} / \mathrm{L}$ & $\mathrm{U}$ \\
\hline 842 & W00809 & $6 / 18 / 97$ & REG & Dibromochloromethane & 5 & $\mathrm{ug} / \mathrm{L}$ & $\mathrm{U}$ \\
\hline 842 & M00055 & $12 / 3 / 96$ & REG & Dibromochloromethane & 5 & $\mathrm{ug} / \mathrm{L}$ & $\mathrm{U}$ \\
\hline 842 & M00843 & $6 / 18 / 97$ & REG & Dibromochloromethane & 5 & ug/L & $\mathrm{U}$ \\
\hline 842 & SMPL00266 & $5 / 25 / 94$ & REG & Dibromochloromethane & 10 & ug $/ \mathrm{L}$ & $\mathrm{U}$ \\
\hline 842 & S00957 & $10 / 12 / 94$ & REG & Dibromochloromethane & 5 & $\mathrm{ug} / \mathrm{L}$ & $\mathrm{U}$ \\
\hline 842 & S02616 & $2 / 28 / 95$ & REG & Dibromochloromethane & 5 & $\mathrm{ug} / \mathrm{L}$ & $\mathrm{U}$ \\
\hline 842 & S03576 & $5 / 8 / 95$ & REG & Dibromochloromethane & 5 & $\mathrm{ug} / \mathrm{L}$ & $\mathrm{U}$ \\
\hline 842 & S04793 & $7 / 14 / 95$ & REG & Dibromochloromethane & 5 & $\mathrm{ug} / \mathrm{L}$ & $\mathrm{U}$ \\
\hline 842 & 842222REGUF & $2 / 22 / 91$ & REG & Dibromochloromethane & 5 & $\mathrm{ug} / \mathrm{L}$ & $\mathrm{U}$ \\
\hline 842 & 842 104REGUF & $10 / 4 / 91$ & REG & Dibromochloromethane & 5 & $\mathrm{ug} / \mathrm{L}$ & $\mathrm{U}$ \\
\hline 842 & 8421210REGUF & $12 / 10 / 91$ & REG & Dibromochloromethane & 5 & ug/L & $\mathrm{U}$ \\
\hline
\end{tabular}


Station Sample ID Sample Date Sample Type Chemical

\begin{tabular}{|c|c|c|c|c|c|c|c|}
\hline 842 & 842521REGUF & $5 / 21 / 90$ & REG & Dibromochloromethane & 5 & $u g / L$ & $\mathrm{U}$ \\
\hline 842 & 842119REGUF & $11 / 9 / 90$ & REG & Dibromochloromethane & 5 & $\mathrm{ug} / \mathrm{L}$ & $\mathrm{U}$ \\
\hline 842 & 842126REGUF & $1 / 26 / 89$ & REG & Dibromochloromethane & 5 & $\mathrm{ug} / \mathrm{L}$ & $\mathrm{U}$ \\
\hline 842 & 842427REGUF & $4 / 27 / 89$ & REG & Dibromochloromethane & 5 & $\mathrm{ug} / \mathrm{L}$ & $\mathrm{U}$ \\
\hline 842 & M00055 & $12 / 3 / 96$ & REG & Dimethylbenzene & 5 & $\mathrm{ug} / \mathrm{L}$ & $\mathrm{U}$ \\
\hline 842 & SMPL00266 & $5 / 25 / 94$ & REG & Dimethylbenzene & 10 & $\mathrm{ug} / \mathrm{L}$ & $\mathrm{U}$ \\
\hline 842 & 842222REGUF & $2 / 22 / 91$ & REG & Dimethylbenzene & 5 & $\mathrm{ug} / \mathrm{L}$ & $\mathrm{U}$ \\
\hline 842 & 842104REGUF & $10 / 4 / 91$ & REG & Dimethylbenzene & 5 & $\mathrm{ug} / \mathrm{L}$ & $\mathrm{U}$ \\
\hline 842 & 8421210REGUF & $12 / 10 / 91$ & REG & Dimethylbenzene & 5 & $\mathrm{ug} / \mathrm{L}$ & $\mathrm{U}$ \\
\hline 842 & 842521REGUF & $5 / 21 / 90$ & REG & Dimethylbenzene & 5 & $\mathrm{ug} / \mathrm{L}$ & $\mathrm{U}$ \\
\hline 842 & 842119REGUF & $11 / 9 / 90$ & REG & Dimethylbenzene & 5 & $\mathrm{ug} / \mathrm{L}$ & $\mathrm{U}$ \\
\hline 842 & 842126REGUF & $1 / 26 / 89$ & REG & Dimethylbenzene & 5 & $\mathrm{ug} / \mathrm{L}$ & $\mathrm{U}$ \\
\hline 842 & 842427REGUF & $4 / 27 / 89$ & REG & Dimethylbenzene & 5 & $\mathrm{ug} / \mathrm{L}$ & $\mathrm{U}$ \\
\hline 842 & M01250 & $11 / 11 / 97$ & REG & Ethylbenzene & 5 & $\mathrm{ug} / \mathrm{L}$ & $\mathrm{U}$ \\
\hline 842 & W00809 & $6 / 18 / 97$ & REG & Ethylbenzene & 5 & $\mathrm{ug} / \mathrm{L}$ & $\mathrm{U}$ \\
\hline 842 & M00055 & $12 / 3 / 96$ & REG & Ethylbenzene & 5 & $\mathrm{ug} / \mathrm{L}$ & $\mathrm{U}$ \\
\hline 842 & M00843 & $6 / 18 / 97$ & REG & Ethylbenzene & 5 & $\mathrm{ug} / \mathrm{L}$ & $\mathrm{U}$ \\
\hline 842 & SMPL00266 & $5 / 25 / 94$ & REG & Ethylbenzene & 10 & $\mathrm{ug} / \mathrm{L}$ & $\mathrm{U}$ \\
\hline 842 & S00957 & $10 / 12 / 94$ & REG & Ethylbenzene & 5 & $\mathrm{ug} / \mathrm{L}$ & $\mathrm{U}$ \\
\hline 842 & S02616 & $2 / 28 / 95$ & REG & Ethylbenzene & 5 & $\mathrm{ug} / \mathrm{L}$ & $U$ \\
\hline 842 & S03576 & $5 / 8 / 95$ & REG & Ethylbenzene & 5 & $\mathrm{ug} / \mathrm{L}$ & $\mathrm{U}$ \\
\hline 842 & S04793 & $7 / 14 / 95$ & REG & Ethylbenzene & 5 & $\mathrm{ug} / \mathrm{L}$ & $\mathrm{U}$ \\
\hline 842 & 842222REGUF & $2 / 22 / 91$ & REG & Ethylbenzene & 5 & $\mathrm{ug} / \mathrm{L}$ & $\mathrm{U}$ \\
\hline 842 & 842104REGUF & $10 / 4 / 91$ & REG & Ethylbenzene & 5 & $\mathrm{ug} / \mathrm{L}$ & $\mathrm{U}$ \\
\hline 842 & 8421210REGUF & $12 / 10 / 91$ & REG & Ethylbenzene & 5 & $\mathrm{ug} / \mathrm{L}$ & $\mathbf{U}$ \\
\hline 842 & 842521REGUF & $5 / 21 / 90$ & REG & Ethylbenzene & 5 & $\mathrm{ug} / \mathrm{L}$ & $\mathrm{U}$ \\
\hline 842 & 842119REGUF & $11 / 9 / 90$ & REG & Ethylbenzene & 5 & $\mathrm{ug} / \mathrm{L}$ & $\mathrm{U}$ \\
\hline 842 & 842126REGUF & $1 / 26 / 89$ & REG & Ethylbenzene & 5 & $\mathrm{ug} / \mathrm{L}$ & $\mathrm{U}$ \\
\hline 842 & 842427REGUF & $4 / 27 / 89$ & REG & Ethylbenzene & 5 & $\mathrm{ug} / \mathrm{L}$ & $\mathrm{U}$ \\
\hline 842 & M01250 & $11 / 11 / 97$ & REG & m,p-Xylene & 5 & $\mathrm{ug} / \mathrm{L}$ & $\mathrm{U}$ \\
\hline 842 & W00809 & $6 / 18 / 97$ & REG & m,p-Xylene & 5 & $\mathrm{ug} / \mathrm{L}$ & $\mathrm{U}$ \\
\hline 842 & M00055 & $12 / 3 / 96$ & REG & m,p-Xylene & 5 & $\mathrm{ug} / \mathrm{L}$ & $\mathrm{U}$ \\
\hline 842 & M00843 & $6 / 18 / 97$ & REG & m,p-Xylene & 5 & $\mathrm{ug} / \mathrm{L}$ & $\mathrm{U}$ \\
\hline 842 & $\mathrm{~S} 02616$ & $2 / 28 / 95$ & REG & m,p-Xylene & 5 & $\mathrm{ug} / \mathrm{L}$ & $\mathrm{U}$ \\
\hline 842 & S03576 & $5 / 8 / 95$ & REG & m,p-Xylene & 5 & $\mathrm{ug} / \mathrm{L}$ & $\mathrm{U}$ \\
\hline 842 & S04793 & $7 / 14 / 95$ & REG & m,p-Xylene & 5 & $\mathrm{ug} / \mathrm{L}$ & $\mathrm{U}$ \\
\hline 842 & M01250 & $11 / 11 / 97$ & REG & Methylene chloride & 2 & $\mathrm{ug} / \mathrm{L}$ & B \\
\hline 842 & W00809 & $6 / 18 / 97$ & REG & Methylene chloride & 5 & $\mathrm{ug} / \mathrm{L}$ & $\mathrm{U}$ \\
\hline 842 & $\mathrm{M} 00055$ & $12 / 3 / 96$ & REG & Methylene chloride & 5 & $\mathrm{ug} / \mathrm{L}$ & $\mathrm{U}$ \\
\hline 842 & M00843 & $6 / 18 / 97$ & REG & Methylene chloride & 2 & $\mathrm{ug} / \mathrm{L}$ & $\mathrm{U}$ \\
\hline 842 & S00957 & $10 / 12 / 94$ & REG & Methylene chloride & 5 & $\mathrm{ug} / \mathrm{L}$ & $\mathrm{U}$ \\
\hline 842 & S02616 & $2 / 28 / 95$ & REG & Methylene chloride & 5 & $\mathrm{ug} / \mathrm{L}$ & $\mathrm{U}$ \\
\hline 842 & S03576 & $5 / 8 / 95$ & REG & Methylene chloride & 5 & $\mathrm{ug} / \mathrm{L}$ & $\mathrm{U}$ \\
\hline 842 & S04793 & $7 / 14 / 95$ & REG & Methylene chloride & 5 & ug/L & $\mathrm{U}$ \\
\hline 842 & 842222REGUF & $2 / 22 / 91$ & REG & Methylene chloride & 5 & $\mathrm{ug} / \mathrm{L}$ & $\mathrm{U}$ \\
\hline 842 & 842104REGUF & $10 / 4 / 91$ & REG & Methylene chloride & 5 & $\mathrm{ug} / \mathrm{L}$ & $\mathrm{U}$ \\
\hline 842 & 8421210REGUF & $12 / 10 / 91$ & REG & Methylene chloride & 5 & $\mathrm{ug} / \mathrm{L}$ & $\mathrm{U}$ \\
\hline 842 & 842521REGUF & $5 / 21 / 90$ & REG & Methylene chloride & 1 & $\mathrm{ug} / \mathrm{L}$ & J \\
\hline 842 & 842119REGUF & $11 / 9 / 90$ & REG & Methylene chloride & 5 & $\mathrm{ug} / \mathrm{L}$ & $\mathrm{U}$ \\
\hline 842 & 842126REGUF & $1 / 26 / 89$ & REG & Methylene chloride & 1 & $\mathrm{ug} / \mathrm{L}$ & J \\
\hline
\end{tabular}


Station Sample ID Sample Date Sample Type Chemical

\begin{tabular}{|c|c|c|c|c|c|c|c|}
\hline Station & sampic io & Sample Date & & & & & \\
\hline 842 & 842427REGUF & $4 / 27 / 89$ & REG & Methylene chloride & 2 & $\mathrm{ug} / \mathrm{L}$ & $\mathrm{J}$ \\
\hline 842 & M01250 & $11 / 11 / 97$ & REG & Styrene & 5 & $u g / L$ & $\mathrm{U}$ \\
\hline 842 & W00809 & $6 / 18 / 97$ & REG & Styrene & 5 & $u g / L$ & $\mathrm{U}$ \\
\hline 842 & M00055 & $12 / 3 / 96$ & REG & Styrene & 5 & ug/L & $\mathrm{U}$ \\
\hline 842 & M00843 & $6 / 18 / 97$ & REG & Styrene & 5 & $\mathrm{ug} / \mathrm{L}$ & $\mathrm{U}$ \\
\hline 842 & SMPL00266 & $5 / 25 / 94$ & REG & Styrene & 10 & $u g / L$ & $\mathrm{U}$ \\
\hline 842 & S00957 & $10 / 12 / 94$ & REG & Styrene & 5 & $\mathrm{ug} / \mathrm{L}$ & $\mathrm{U}$ \\
\hline 842 & S02616 & $2 / 28 / 95$ & REG & Styrene & 5 & $\mathrm{ug} / \mathrm{L}$ & $\mathrm{U}$ \\
\hline 842 & S03576 & $5 / 8 / 95$ & REG & Styrene & 5 & $\mathrm{ug} / \mathrm{L}$ & $\mathrm{U}$ \\
\hline 842 & S04793 & $7 / 14 / 95$ & REG & Styrene & 5 & $\mathrm{ug} / \mathrm{L}$ & $\mathrm{U}$ \\
\hline 842 & 842222REGUF & $2 / 22 / 91$ & REG & Styrene & 5 & $\mathrm{ug} / \mathrm{L}$ & $\mathrm{U}$ \\
\hline 842 & 842104REGUF & $10 / 4 / 91$ & REG & Styrene & 5 & $\mathrm{ug} / \mathrm{L}$ & $\mathrm{U}$ \\
\hline 842 & 8421210REGUF & $12 / 10 / 91$ & REG & Styrene & 5 & $\mathrm{ug} / \mathrm{L}$ & $\mathrm{U}$ \\
\hline 842 & 842521 REGUF & $5 / 21 / 90$ & REG & Styrene & 5 & $\mathrm{ug} / \mathrm{L}$ & $\mathrm{U}$ \\
\hline 842 & 842119REGUF & $11 / 9 / 90$ & REG & Styrene & 5 & $\mathrm{ug} / \mathrm{L}$ & $\mathrm{U}$ \\
\hline 842 & 842126REGUF & $1 / 26 / 89$ & REG & Styrene & 5 & $\mathrm{ug} / \mathrm{L}$ & $\mathrm{U}$ \\
\hline 842 & 842427REGUF & $4 / 27 / 89$ & REG & Styrene & 5 & $\mathrm{ug} / \mathrm{L}$ & $\mathrm{U}$ \\
\hline 842 & M01250 & $11 / 11 / 97$ & REG & Tetrachloroethene & 5 & ug/L & $\mathrm{U}$ \\
\hline 842 & W00809 & $6 / 18 / 97$ & REG & Tetrachloroethene & 5 & ug/L & $\mathrm{U}$ \\
\hline 842 & M00055 & $12 / 3 / 96$ & REG & Tetrachloroethene & 5 & ug/L & $\mathrm{U}$ \\
\hline 842 & M00843 & $6 / 18 / 97$ & REG & Tetrachloroethene & 5 & $\mathrm{ug} / \mathrm{L}$ & $\mathrm{U}$ \\
\hline 842 & SMPL00266 & $5 / 25 / 94$ & REG & Tetrachloroethene & 10 & $\mathrm{ug} / \mathrm{L}$. & $\mathrm{U}$ \\
\hline 842 & S00957 & $10 / 12 / 94$ & REG & Tetrachloroethene & 5 & $\mathrm{ug} / \mathrm{L}$ & $\mathrm{U}$ \\
\hline 842 & S02616 & $2 / 28 / 95$ & REG & Tetrachloroethene & 5 & $\mathrm{ug} / \mathrm{L}$ & $\mathrm{U}$ \\
\hline 842 & S03576 & $5 / 8 / 95$ & REG & Tetrachloroethene & 5 & $\mathrm{ug} / \mathrm{L}$ & $\mathrm{U}$ \\
\hline 842 & S04793 & $7 / 14 / 95$ & REG & Tetrachloroethene & 1 & $\mathrm{ug} / \mathrm{L}$ & J \\
\hline 842 & 842222REGUF & $2 / 22 / 91$ & REG & Tetrachloroethene & 5 & $u g / L$ & $\mathrm{U}$ \\
\hline 842 & 842104REGUF & $10 / 4 / 91$ & REG & Tetrachloroethene & 5 & $\mathrm{ug} / \mathrm{L}$ & $\mathrm{U}$ \\
\hline 842 & 8421210 REGUF & $12 / 10 / 91$ & REG & Tetrachloroethene & 5 & $u g / L$ & $\mathrm{U}$ \\
\hline 842 & 842521REGUF & $5 / 21 / 90$ & REG & Tetrachloroethene & 5 & $\mathrm{ug} / \mathrm{L}$ & $\mathrm{U}$ \\
\hline 842 & 842119REGUF & $11 / 9 / 90$ & REG & Tetrachloroethene & 1 & $\mathrm{ug} / \mathrm{L}$ & $\mathrm{J}$ \\
\hline 842 & 842126REGUF & $1 / 26 / 89$ & REG & Tetrachloroethene & 5 & $\mathrm{ug} / \mathrm{L}$ & $\mathrm{U}$ \\
\hline 842 & 842427REGUF & $4 / 27 / 89$ & REG & Tetrachloroethene & 2 & $\mathrm{ug} / \mathrm{L}$ & $\mathrm{J}$ \\
\hline 842 & M01250 & $11 / 11 / 97$ & REG & Toluene & 5 & $\mathrm{ug} / \mathrm{L}$ & $\mathrm{U}$ \\
\hline 842 & W00809 & $6 / 18 / 97$ & REG & Toluene & 5 & ug/L & $\mathrm{U}$ \\
\hline 842 & M00055 & $12 / 3 / 96$ & REG & Toluene & 5 & $\mathrm{ug} / \mathrm{L}$ & $\mathrm{U}$ \\
\hline 842 & $\mathrm{M} 00843$ & $6 / 18 / 97$ & REG & Toluene & 5 & $u g / L$ & $\mathrm{U}$ \\
\hline 842 & SMPL00266 & $5 / 25 / 94$ & REG & Toluene & 10 & $\mathrm{ug} / \mathrm{L}$ & $\mathrm{U}$ \\
\hline 842 & S00957 & $10 / 12 / 94$ & REG & Toluene & 5 & $\mathrm{ug} / \mathrm{L}$ & $\mathrm{U}$ \\
\hline 842 & S02616 & $2 / 28 / 95$ & REG & Toluene & 5 & $\mathrm{ug} / \mathrm{L}$ & $\mathrm{U}$ \\
\hline 842 & S03576 & $5 / 8 / 95$ & REG & Toluene & 5 & $\mathrm{ug} / \mathrm{L}$ & $\mathrm{U}$ \\
\hline 842 & S04793 & $7 / 14 / 95$ & REG & Toluene & 5 & $\mathrm{ug} / \mathrm{L}$ & $\mathrm{U}$ \\
\hline 842 & 842222REGUF & $2 / 22 / 91$ & REG & Toluene & 5 & $\mathrm{ug} / \mathrm{L}$ & $\mathrm{U}$ \\
\hline 842 & 842104REGUF & $10 / 4 / 91$ & REG & Toluene & 5 & $\mathrm{ug} / \mathrm{L}$ & $\mathrm{U}$ \\
\hline 842 & 8421210REGUF & $12 / 10 / 91$ & REG & Toluene & 5 & $\mathrm{ug} / \mathrm{L}$ & $U$ \\
\hline 842 & 842521REGUF & $5 / 21 / 90$ & REG & Toluene & 1 & $\mathrm{ug} / \mathrm{L}$ & $\mathrm{J}$ \\
\hline 842 & 842119REGUF & $11 / 9 / 90$ & REG & Toluene & 5 & $\operatorname{ug} / \mathrm{L}$ & $\mathrm{U}$ \\
\hline 842 & 842126REGUF & $1 / 26 / 89$ & REG & Toluene & 5 & ug/L & $\mathrm{U}$ \\
\hline 842 & 842427REGUF & $4 / 27 / 89$ & REG & Toluene & 5 & $\mathrm{ug} / \mathrm{L}$ & $\mathrm{U}$ \\
\hline 842 & M01250 & $11 / 11 / 97$ & REG & trans-1,2-Dichloroethe & 5 & $\mathrm{ug} / \mathrm{L}$ & $\mathrm{U}$ \\
\hline
\end{tabular}




\begin{tabular}{|c|c|c|c|c|c|c|c|}
\hline Station & Sample ID & Sample Date & Sample Type & Chemical & Results & Units & Qualifier \\
\hline$\overline{842}$ & M00055 & $12 / 3 / 96$ & REG & trans-1,2-Dichloroethene & 5 & $u g / L$ & $\bar{U}$ \\
\hline 842 & M00843 & $6 / 18 / 97$ & REG & trans-1,2-Dichloroethene & 5 & $\mathrm{ug} / \mathrm{L}$ & $U$ \\
\hline 842 & SMPL00266 & $5 / 25 / 94$ & REG & trans-1,2-Dichloroethene & 10 & $\mathrm{ug} / \mathrm{L}$ & $\mathrm{U}$ \\
\hline 842 & S00957 & $10 / 12 / 94$ & REG & trans-1,2-Dichloroethene & 5 & $\mathrm{ug} / \mathrm{L}$ & $\mathrm{U}$ \\
\hline 842 & S02616 & $2 / 28 / 95$ & REG & trans-1,2-Dichloroethene & 5 & $\mathrm{ug} / \mathrm{L}$ & $\mathrm{U}$ \\
\hline 842 & S03576 & $5 / 8 / 95$ & REG & trans-1,2-Dichloroethene & 5 & $\mathrm{ug} / \mathrm{L}$ & $\mathrm{U}$ \\
\hline 842 & S04793 & $7 / 14 / 95$ & REG & trans-1,2-Dichloroethene & 5 & $\mathrm{ug} / \mathrm{L}$ & $\mathrm{U}$ \\
\hline 842 & M01250 & $11 / 11 / 97$ & REG & trans-1,3-Dichloroprop & 5 & $\mathrm{ug} / \mathrm{L}$ & $\mathrm{U}$ \\
\hline 842 & W00809 & $6 / 18 / 97$ & REG & trans-1,3-Dichloropropene & 5 & $\mathrm{ug} / \mathrm{L}$ & $\mathrm{U}$ \\
\hline 842 & M00055 & $12 / 3 / 96$ & REG & trans-1,3-Dichloropropene & 5 & $\mathrm{ug} / \mathrm{L}$ & $\mathrm{U}$ \\
\hline 842 & $\mathrm{M} 00843$ & $6 / 18 / 97$ & REG & trans-1,3-Dichloropropene & 5 & $\mathrm{ug} / \mathrm{L}$ & $\mathrm{U}$ \\
\hline 842 & SMPL00266 & $5 / 25 / 94$ & REG & trans-1,3-Dichloropropene & 10 & $\mathrm{ug} / \mathrm{L}$ & $\mathrm{U}$ \\
\hline 842 & S00957 & $10 / 12 / 94$ & REG & trans-1.3-Dichloropropene & 5 & $\mathrm{ug} / \mathrm{L}$ & $\mathrm{U}$ \\
\hline 842 & S02616 & $2 / 28 / 95$ & REG & trans-1,3-Dichloropropene & 5 & $\mathrm{ug} / \mathrm{L}$ & $\mathrm{U}$ \\
\hline 842 & S03576 & $5 / 8 / 95$ & REG & trans-1,3-Dichloropropene & 5 & $\mathrm{ug} / \mathrm{L}$ & $\mathrm{U}$ \\
\hline 842 & S04793 & $7 / 14 / 95$ & REG & trans-1,3-Dichloropropene & 5 & $\mathrm{ug} / \mathrm{L}$ & $\mathrm{U}$ \\
\hline 842 & 842222REGUF & $2 / 22 / 91$ & REG & trans-1,3-Dichloropropene & 5 & ug/L & $\mathrm{U}$ \\
\hline 842 & 842104REGUF & $10 / 4 / 91$ & REG & trans-1,3-Dichloropropene & 5 & ug/L & $\mathrm{U}$ \\
\hline 842 & 8421210REGUF & $12 / 10 / 91$ & REG & trans-1,3-Dichloropropene & 5 & $u g / L$ & $\mathrm{U}$ \\
\hline 842 & 842521REGUF & $5 / 21 / 90$ & REG & trans-1,3-Dichloropropene & 5 & $\mathrm{ug} / \mathrm{L}$ & $\mathrm{U}$ \\
\hline 842 & 842119REGUF & $11 / 9 / 90$ & REG & trans-1,3-Dichloropropene & 5 & ug/L & $\mathrm{U}$ \\
\hline 842 & 842126REGUF & $1 / 26 / 89$ & REG & trans-1,3-Dichloropropene & 5 & $\mathrm{ug} / \mathrm{L}$ & $\mathrm{U}$ \\
\hline 842 & 842427REGUF & $4 / 27 / 89$ & REG & trans-1,3-Dichloropropene & 5 & $\mathrm{ug} / \mathrm{L}$ & $\mathrm{U}$ \\
\hline 842 & M01250 & $11 / 11 / 97$ & REG & Trichloroethene & 150 & $\mathrm{ug} / \mathrm{L}$ & $=$ \\
\hline 842 & W00809 & $6 / 18 / 97$ & REG & Trichloroethene & 140 & $\mathrm{ug} / \mathrm{L}$ & $=$ \\
\hline 842 & M00055 & $12 / 3 / 96$ & REG & Trichloroethene & 110 & $\mathrm{ug} / \mathrm{L}$ & $=$ \\
\hline 842 & M00843 & $6 / 18 / 97$ & REG & Trichloroethene & 110 & $\mathrm{ug} / \mathrm{L}$ & $=$ \\
\hline 842 & S00957 & $10 / 12 / 94$ & REG & Trichloroethene & 180 & $\mathrm{ug} / \mathrm{L}$ & $=$ \\
\hline 842 & S02616 & $2 / 28 / 95$ & REG & Trichloroethene & 170 & $\mathrm{ug} / \mathrm{L}$ & $=$ \\
\hline 842 & S03576 & $5 / 8 / 95$ & REG & Trichloroethene & 180 & $u g / L$ & $=$ \\
\hline 842 & S04793 & $7 / 14 / 95$ & REG & Trichloroethene & 200 & $\mathrm{ug} / \mathrm{L}$ & $=$ \\
\hline 842 & 842222REGUF & $2 / 22 / 91$ & REG & Trichloroethene & 330 & $\mathrm{ug} / \mathrm{L}$ & $=$ \\
\hline 842 & 842104REGUF & $10 / 4 / 91$ & REG & Trichloroethene & 280 & $\mathrm{ug} / \mathrm{L}$ & $=$ \\
\hline 842 & 8421210REGUF & $12 / 10 / 91$ & REG & Trichloroethene & 220 & $\mathrm{ug} / \mathrm{L}$ & $=$ \\
\hline 842 & 842521REGUF & $5 / 21 / 90$ & REG & Trichloroethene & 320 & $\mathrm{ug} / \mathrm{L}$ & $=$ \\
\hline 842 & 842119REGUF & $11 / 9 / 90$ & REG & Trichloroethene & 310 & $\mathrm{ug} / \mathrm{L}$ & $=$ \\
\hline 842 & 842126REGUF & $1 / 26 / 89$ & REG & Trichloroethene & 360 & $\mathrm{ug} / \mathrm{L}$ & $=$ \\
\hline 842 & 842427REGUF & $4 / 27 / 89$ & REG & Trichloroethene & 490 & $\mathrm{ug} / \mathrm{L}$ & $=$ \\
\hline 842 & M01250 & $11 / 11 / 97$ & REG & Vinyl acetate & 10 & $\mathrm{ug} / \mathrm{L}$ & $\mathrm{U}$ \\
\hline 842 & M00055 & $12 / 3 / 96$ & REG & Vinyl acetate & 10 & $\mathrm{ug} / \mathrm{L}$ & $\mathrm{U}$ \\
\hline 842 & M00843 & $6 / 18 / 97$ & REG & Vinyl acetate & 10 & $u g / L$ & $\mathrm{U}$ \\
\hline 842 & S00957 & $10 / 12 / 94$ & REG & Vinyl acetate & 10 & $\mathrm{ug} / \mathrm{L}$ & $U$ \\
\hline 842 & S02616 & $2 / 28 / 95$ & REG & Vinyl acetate & 10 & ug/L & $\mathrm{U}$ \\
\hline 842 & S03576 & $5 / 8 / 95$ & REG & Vinyl acetate & 10 & $\mathrm{ug} / \mathrm{L}$ & $\mathrm{U}$ \\
\hline 842 & S04793 & $7 / 14 / 95$ & REG & Vinyl acetate & 10 & $\mathrm{ug} / \mathrm{L}$ & $\mathrm{U}$ \\
\hline 842 & 842222REGUF & $2 / 22 / 91$ & REG & Vinyl acetate & 10 & $u g / L$ & $\mathrm{U}$ \\
\hline 842 & 842104REGUF & $10 / 4 / 91$ & REG & Vinyl acetate & 10 & $\mathrm{ug} / \mathrm{L}$ & $\mathrm{U}$ \\
\hline 842 & 8421210REGUF & $12 / 10 / 91$ & REG & Vinyl acetate & 10 & ug/L & U \\
\hline 842 & 84252IREGUF & $5 / 21 / 90$ & REG & Vinyl acetate & 10 & $\mathrm{ug} / \mathrm{L}$ & U \\
\hline 842 & 842119REGUF & $11 / 9 / 90$ & REG & Vinyl acetate & 10 & ug/L & $\mathrm{U}$ \\
\hline
\end{tabular}


Station Sample ID Sample Date Sample Type Chemical

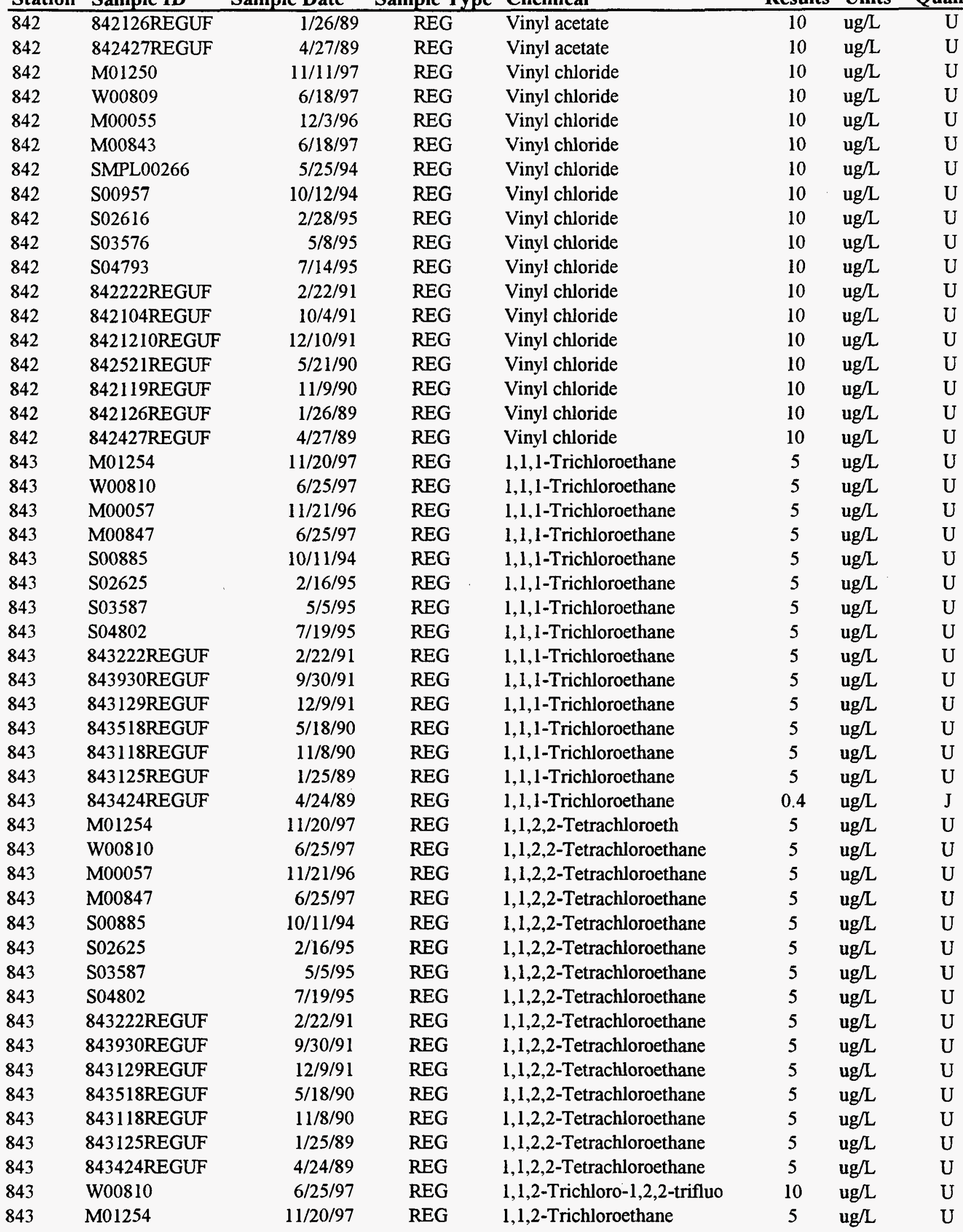


Station Sample ID Sample Date Sample Type Chemical

\begin{tabular}{|c|c|c|c|c|}
\hline$\overline{843}$ & W00810 & $6 / 25 / 97$ & REG & 1,1,2-Trichloroethane \\
\hline 843 & M00057 & $11 / 21 / 96$ & REG & 1,1,2-Trichloroethan \\
\hline 843 & M00847 & $6 / 25 / 97$ & REG & 1,1,2-Trichloroetha \\
\hline 843 & S00885 & $10 / 11 / 94$ & REG & 1,1,2-Trichloroetha \\
\hline 843 & S02625 & $2 / 16 / 95$ & REG & 1,1,2-Trichloroetha \\
\hline 843 & S03587 & $5 / 5 / 95$ & REG & 1,1,2-Trichloroetha \\
\hline 843 & $\mathrm{~S} 04802$ & $7 / 19 / 95$ & REG & 1,1,2-Trichloroetl \\
\hline 843 & 843222REGUF & $2 / 22 / 91$ & REG & 1,1,2-Trichloroetha \\
\hline 843 & 843930REGUF & $9 / 30 / 91$ & REG & 1,1,2-Trichloroetha \\
\hline 843 & 843129REGUF & $12 / 9 / 91$ & REG & 1,1,2-Trichloroetha \\
\hline 843 & 843518REGUF & $5 / 18 / 90$ & REG & 1,1,2-Trichloroetha \\
\hline 843 & 843118REGUF & $11 / 8 / 90$ & REG & 1,1,2-Trichloroeth \\
\hline 843 & 843125REGUF & $1 / 25 / 89$ & REG & 1,1,2-Trichloroetha \\
\hline 843 & 843424REGUF & $4 / 24 / 89$ & REG & 1,1,2-Trichloroetha \\
\hline 843 & M01254 & $11 / 20 / 97$ & REG & 1,1-Dichloroethane \\
\hline 843 & W00810 & $6 / 25 / 97$ & REG & 1,1-Dichloroethane \\
\hline 843 & M00057 & $11 / 21 / 96$ & REG & 1,1-Dichloroethane \\
\hline 843 & M00847 & $6 / 25 / 97$ & REG & 1,1-Dichloroethane \\
\hline 843 & S00885 & $10 / 11 / 94$ & REG & 1,1-Dichloroethane \\
\hline 843 & $\mathrm{~S} 02625$ & $2 / 16 / 95$ & REG & 1,1-Dichloroethane \\
\hline 843 & S03587 & $5 / 5 / 95$ & REG & 1,1-Dichloroethane \\
\hline 843 & S04802 & $7 / 19 / 95$ & REG & 1,1-Dichloroethane \\
\hline 843 & 843222REGUF & $2 / 22 / 91$ & REG & 1,1-Dichloroethane \\
\hline 843 & 843930REGUF & $9 / 30 / 91$ & REG & 1,1-Dichloroethane \\
\hline 843 & 843129REGUF & $12 / 9 / 91$ & REG & 1,1-Dichloroethane \\
\hline 843 & 843518REGUF & $5 / 18 / 90$ & REG & 1,1-Dichloroethane \\
\hline 843 & 843118REGUF & $11 / 8 / 90$ & REG & 1,1-Dichloroethane \\
\hline 843 & 843125REGUF & $1 / 25 / 89$ & REG & 1,1-Dichloroethane \\
\hline 843 & 843424REGUF & $4 / 24 / 89$ & REG & 1,1-Dichloroethane \\
\hline 843 & M01254 & $11 / 20 / 97$ & REG & 1,1-Dichloroethene \\
\hline 843 & W00810 & $6 / 25 / 97$ & REG & 1,1-Dichloroethene \\
\hline 843 & M00057 & $11 / 21 / 96$ & REG & 1,1-Dichloroethene \\
\hline 843 & M00847 & $6 / 25 / 97$ & REG & 1,1-Dichloroethene \\
\hline 843 & S00885 & $10 / 11 / 94$ & REG & 1,1-Dichloroethene \\
\hline 843 & S02625 & $2 / 16 / 95$ & REG & 1,1-Dichloroethene \\
\hline 843 & S03587 & $5 / 5 / 95$ & REG & 1,1-Dichloroethene \\
\hline 843 & S04802 & $7 / 19 / 95$ & REG & 1,1-Dichloroethene \\
\hline 843 & 843222REGUF & $2 / 22 / 91$ & REG & 1,1-Dichloroethene \\
\hline 843 & 843930REGUF & $9 / 30 / 91$ & REG & 1,1-Dichloroethene \\
\hline 843 & 843129REGUF & $12 / 9 / 91$ & REG & 1,1-Dichloroethene \\
\hline 843 & 843518REGUF & $5 / 18 / 90$ & REG & 1,1-Dichloroethene \\
\hline 843 & 843118REGUF & $11 / 8 / 90$ & REG & 1,1-Dichloroethene \\
\hline 843 & 843125REGUF & $1 / 25 / 89$ & REG & 1,1-Dichloroethene \\
\hline 843 & 843424REGUF & $4 / 24 / 89$ & REG & 1,1-Dichloroethene \\
\hline 843 & M00057 & $11 / 21 / 96$ & REG & 1,2-Dichlorobenzene \\
\hline 843 & M01254 & $11 / 20 / 97$ & REG & 1,2-Dichloroethane \\
\hline 843 & W00810 & $6 / 25 / 97$ & REG & 1,2-Dichloroethane \\
\hline 843 & M00057 & $11 / 21 / 96$ & REG & 1,2-Dichloroethane \\
\hline 843 & M00847 & $6 / 25 / 97$ & REG & 1,2-Dichloroethane \\
\hline 843 & S00885 & $10 / 11 / 94$ & REG & 1,2-Dichloroethane \\
\hline
\end{tabular}

Results Units Qualifier

$\begin{array}{lll}5 & \mathrm{ug} / \mathrm{L} & \mathrm{U} \\ 5 & \mathrm{ug} / \mathrm{L} & \mathrm{U} \\ 5 & \mathrm{ug} / \mathrm{L} & \mathrm{U}\end{array}$

$5 \quad \mathrm{ug} / \mathrm{L} \quad \mathrm{U}$

5 ug/L $\quad \mathrm{U}$

5 ug/L U

5 ug/L U

5 ug/L U

5 ug/L U

$5 \mathrm{ug} / \mathrm{L} \quad \mathrm{U}$

5 ug/L U

$5 \quad \mathrm{ug} / \mathrm{L} \quad \mathrm{U}$

5 ug/L U

5 ug/L U

$5 \mathrm{ug} / \mathrm{L} \quad \mathrm{U}$

5 ug/L U

$5 \quad u g / L \quad U$

5 ug/L U

$5 \quad u g / L \quad U$

5 ug/L U

5 ug/ $\quad U$

5 ug/ $\quad \mathrm{U}$

5 ug/L U

5 ug/ $\quad U$

$5 \quad \mathrm{ug} / \mathrm{L} \quad \mathrm{U}$

5 ug/ . U

5 ug/ $\quad \mathrm{U}$

5 ug/ $\quad \mathrm{U}$

$5 \quad u g / \mathrm{L} \quad \mathrm{U}$

$5 \quad u g / L \quad U$

5 ug/ $/$ U

$5 \mathrm{ug} / \mathrm{L} \quad \mathrm{U}$

$5 \quad u g / L \quad U$

$5 \quad \mathrm{ug} / \mathrm{L} \quad \mathrm{U}$

$5 \quad u g / L \quad U$

5 ug/L U

$5 \quad u g / L \quad U$

5 ug/L U

5 ug/ $\quad \mathrm{U}$

5 ug/L U

$5 \quad u g / L \quad U$

5 ug/ $\quad U$

$5 \quad$ ug/L U

5 ug/L U

5 ug/L U

5 ug/L U

$\begin{array}{lll}5 & u g / L & u g / L\end{array}$

5 ug/L U

5 ug/L U

$5 \quad u g / L \quad U$ 
Station Sample ID Sample Date Sample Type Chemical

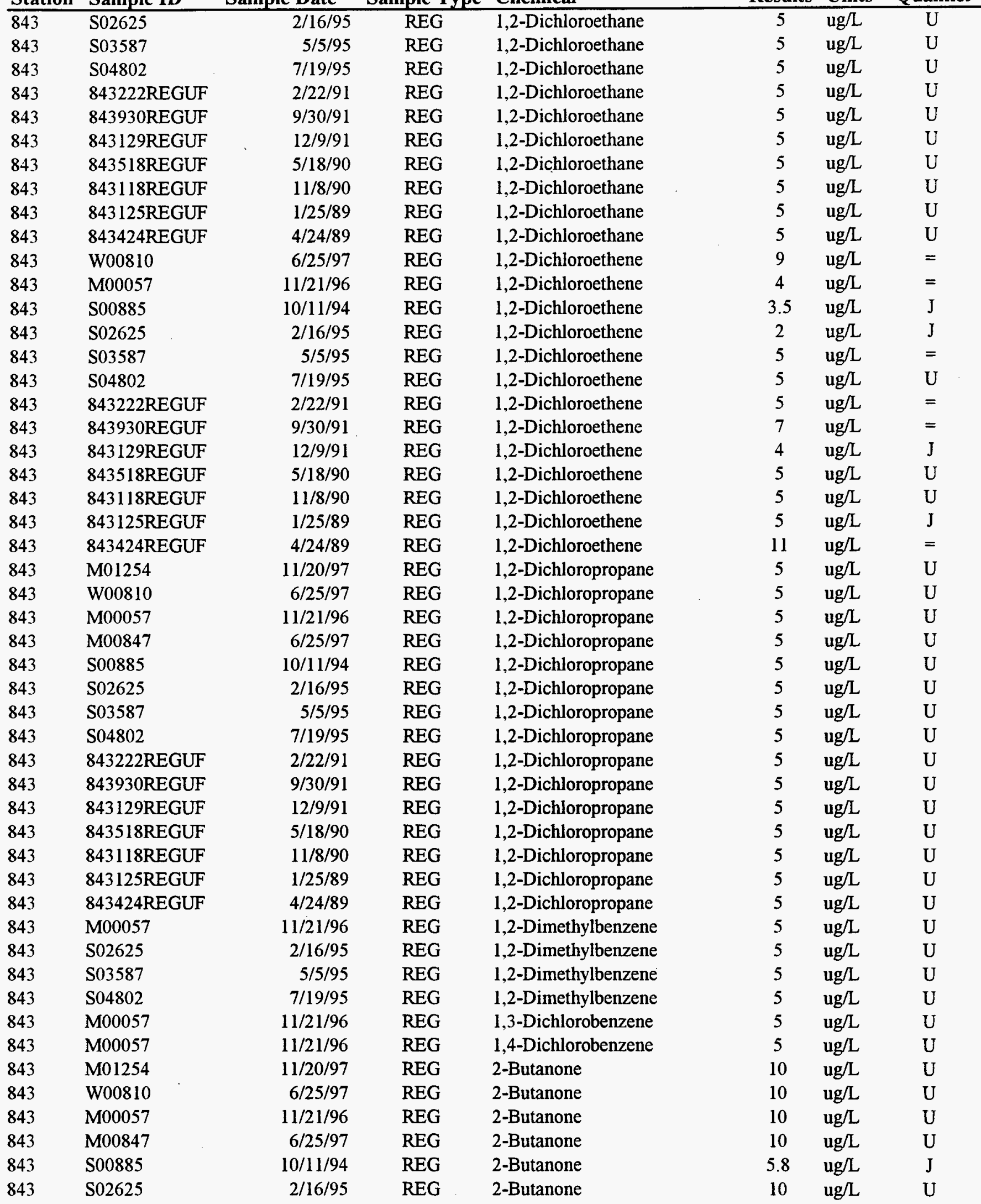




\begin{tabular}{|c|c|c|c|c|c|c|c|}
\hline Station & Sample ID & Sample Date & Sample Type & Chemical & Results & Units & Qualifier \\
\hline$\overline{843}$ & S03587 & $5 / 5 / 95$ & REG & 2-Butanone & 3 & $\mathrm{ug} / \mathrm{L}$ & $\mathrm{J}$ \\
\hline 843 & S04802 & $7 / 19 / 95$ & REG & 2-Butanone & 10 & $\mathrm{ug} / \mathrm{L}$ & U \\
\hline 843 & 843222REGUF & $2 / 22 / 91$ & REG & 2-Butanone & 10 & $\mathrm{ug} / \mathrm{L}$ & $\mathrm{U}$ \\
\hline 843 & 843930REGUF & $9 / 30 / 91$ & REG & 2-Butanone & 10 & $\mathrm{ug} / \mathrm{L}$ & $\mathrm{U}$ \\
\hline 843 & 843129REGUF & $12 / 9 / 91$ & REG & 2-Butanone & 10 & $u g / L$ & $\mathrm{U}$ \\
\hline 843 & 843518REGUF & $5 / 18 / 90$ & REG & 2-Butanone & 10 & $\mathrm{ug} / \mathrm{L}$ & $\mathrm{U}$ \\
\hline 843 & 843118REGUF & $11 / 8 / 90$ & REG & 2-Butanone & 10 & $\mathrm{ug} / \mathrm{L}$ & $\mathrm{U}$ \\
\hline 843 & 843125REGUF & $1 / 25 / 89$ & REG & 2-Butanone & 10 & $\mathrm{ug} / \mathrm{L}$ & U \\
\hline 843 & 843424REGUF & $4 / 24 / 89$ & REG & 2-Butanone & 10 & $\mathrm{ug} / \mathrm{L}$ & $\mathrm{U}$ \\
\hline 843 & M01254 & $11 / 20 / 97$ & REG & 2-Hexanone & 10 & $\mathrm{ug} / \mathrm{L}$ & U \\
\hline 843 & W00810 & $6 / 25 / 97$ & REG & 2-Hexanone & 10 & $\mathrm{ug} / \mathrm{L}$ & $\mathrm{U}$ \\
\hline 843 & M00057 & $11 / 21 / 96$ & REG & 2-Hexanone & 10 & $\mathrm{ug} / \mathrm{L}$ & $\mathrm{U}$ \\
\hline 843 & M00847 & $6 / 25 / 97$ & REG & 2-Hexanone & 10 & $u g / L$ & $\mathrm{U}$ \\
\hline 843 & S00885 & $10 / 11 / 94$ & REG & 2-Hexanone & 10 & $u g / L$ & $\mathrm{U}$ \\
\hline 843 & S02625 & $2 / 16 / 95$ & REG & 2-Hexanone & 10 & $\mathrm{ug} / \mathrm{L}$ & $\mathrm{U}$ \\
\hline 843 & S03587 & $5 / 5 / 95$ & REG & 2-Hexanone & 10 & $\mathrm{ug} / \mathrm{L}$ & $\mathrm{U}$ \\
\hline 843 & S04802 & $7 / 19 / 95$ & REG & 2-Hexanone & 10 & $\mathrm{ug} / \mathrm{L}$ & $\mathrm{U}$ \\
\hline 843 & 843222REGUF & $2 / 22 / 91$ & REG & 2-Hexanone & 10 & $\mathrm{ug} / \mathrm{L}$ & $\mathrm{U}$ \\
\hline 843 & 843930REGUF & $9 / 30 / 91$ & REG & 2-Hexanone & 10 & $\mathrm{ug} / \mathrm{L}$ & $\mathrm{U}$ \\
\hline 843 & 843129REGUF & $12 / 9 / 91$ & REG & 2-Hexanone & 10 & $\mathrm{ug} / \mathrm{L}$ & $\mathrm{U}$ \\
\hline 843 & 843518REGUF & $5 / 18 / 90$ & REG & 2-Hexanone & 10 & $\mathrm{ug} / \mathrm{L}$ & $\mathrm{U}$ \\
\hline 843 & 843118REGUF & $11 / 8 / 90$ & REG & 2-Hexanone & 10 & ug/L & U \\
\hline 843 & 843125REGUF & $1 / 25 / 89$ & REG & 2-Hexanone & 10 & ug/L & $\mathrm{U}$ \\
\hline 843 & 843424REGUF & $4 / 24 / 89$ & REG & 2-Hexanone & 10 & ug/L & $\mathrm{U}$ \\
\hline 843 & M01254 & $11 / 20 / 97$ & REG & 4-Methyl-2-pentanone & 10 & ug/L & $\mathrm{U}$ \\
\hline 843 & W00810 & $6 / 25 / 97$ & REG & 4-Methyl-2-pentanone & 10 & $\mathrm{ug} / \mathrm{L}$ & $\mathrm{U}$ \\
\hline 843 & M00057 & $11 / 21 / 96$ & REG & 4-Methyl-2-pentanone & 10 & $\mathrm{ug} / \mathrm{L}$ & $\mathrm{U}$ \\
\hline 843 & M00847 & $6 / 25 / 97$ & REG & 4-Methyl-2-pentanone & 10 & $\mathrm{ug} / \mathrm{L}$ & $\mathrm{U}$ \\
\hline 843 & S00885 & $10 / 11 / 94$ & REG & 4-Methyl-2-pentanone & 10 & ug/L & $U$ \\
\hline 843 & $\mathrm{~S} 02625$ & $2 / 16 / 95$ & REG & 4-Methyl-2-pentanone & 10 & $\mathrm{ug} / \mathrm{L}$ & $\mathrm{U}$ \\
\hline 843 & S03587 & $5 / 5 / 95$ & REG & 4-Methyl-2-pentanone & 10 & ug/L & $\mathrm{U}$ \\
\hline 843 & S04802 & $7 / 19 / 95$ & REG & 4-Methyl-2-pentanone & 10 & ug/L & $\mathrm{U}$ \\
\hline 843 & 843222REGUF & $2 / 22 / 91$ & REG & 4-Methyl-2-pentanone & 10 & $\mathrm{ug} / \mathrm{L}$ & $\mathrm{U}$ \\
\hline 843 & 843930REGUF & $9 / 30 / 91$ & REG & 4-Methyl-2-pentanone & 10 & $\mathrm{ug} / \mathrm{L}$ & $\mathrm{U}$ \\
\hline 843 & 843129REGUF & $12 / 9 / 91$ & REG & 4-Methyl-2-pentanone & 10 & $\mathrm{ug} / \mathrm{L}$ & $\mathrm{U}$ \\
\hline 843 & 843518REGUF & $5 / 18 / 90$ & REG & 4-Methyl-2-pentanone & 10 & $u g / L$ & U \\
\hline 843 & 843118REGUF & $11 / 8 / 90$ & REG & 4-Methyl-2-pentanone & 10 & $\mathrm{ug} / \mathrm{L}$ & $\mathrm{U}$ \\
\hline 843 & 843125REGUF & $1 / 25 / 89$ & REG & 4-Methyl-2-pentanone & 10 & $\mathrm{ug} / \mathrm{L}$ & $\mathrm{U}$ \\
\hline 843 & 843424REGUF & $4 / 24 / 89$ & REG & 4-Methyl-2-pentanone & 10 & ug/L & $\mathrm{U}$ \\
\hline 843 & M01254 & $11 / 20 / 97$ & REG & Acetone & 6 & $u g / L$ & $\mathbf{B}$ \\
\hline 843 & W00810 & $6 / 25 / 97$ & REG & Acetone & 10 & ug/L & $\mathrm{U}$ \\
\hline 843 & M00057 & $11 / 21 / 96$ & REG & Acetone & 10 & $\mathrm{ug} / \mathrm{L}$ & $\mathrm{U}$ \\
\hline 843 & M00847 & $6 / 25 / 97$ & REG & Acetone & 10 & $\mathrm{ug} / \mathrm{L}$ & $\mathrm{U}$ \\
\hline 843 & S00885 & $10 / 11 / 94$ & REG & Acetone & 10 & $u g / L$ & $\mathrm{U}$ \\
\hline 843 & $\mathrm{~S} 02625$ & $2 / 16 / 95$ & REG & Acetone & 10 & ug/L & $\mathrm{U}$ \\
\hline 843 & S03587 & $5 / 5 / 95$ & REG & Acetone & 1 & $\mathrm{ug} / \mathrm{L}$ & J \\
\hline 843 & S04802 & $7 / 19 / 95$ & REG & Acetone & 10 & $\mathrm{ug} / \mathrm{L}$ & $\mathrm{U}$ \\
\hline 843 & 843222REGUF & $2 / 22 / 91$ & REG & Acetone & 10 & $\mathrm{ug} / \mathrm{L}$ & $\mathrm{U}$ \\
\hline 843 & 843930REGUF & $9 / 30 / 91$ & REG & Acetone & 10 & $\mathrm{ug} / \mathrm{L}$ & $\mathrm{U}$ \\
\hline 843 & 843129REGUF & $12 / 9 / 91$ & REG & Acetone & 3 & $\mathrm{ug} / \mathrm{L}$ & J \\
\hline
\end{tabular}


Station Sample ID

\begin{tabular}{ll}
\hline 843 & 843518REGUF \\
843 & 843118REGUF \\
843 & 843125REGUF \\
843 & 843424REGUF \\
843 & M01254
\end{tabular}

843 W00810

$843 \quad$ M00057

$843 \quad$ M00847

$843 \quad$ S00885

843 S02625

$843 \quad \mathrm{~S} 03587$

$843 \quad \mathrm{~S} 04802$

843 843222REGUF

843 843930REGUF

843 843129REGUF

843 843518REGUF

843 843118REGUF

843 843125REGUF

843 843424REGUF

843 M01254

843 W00810

$843 \quad$ M00057

843 M00847

$843 \quad$ S00885

843 S02625

$843 \quad$ S03587

$843 \quad$ S04802

843 843222REGUF

843 843930REGUF

843 843129REGUF

843 843518REGUF

843 843118REGUF

843 843125REGUF

843 843424REGUF

843 M01254

843 W00810

$843 \quad$ M00057

$843 \quad$ M00847

$843 \quad$ S00885

$843 \quad$ S02625

$843 \quad$ S03587

$843 \quad \mathrm{~S} 04802$

843 843222REGUF

843 843930REGUF

843 843129REGUF

843 843518REGUF

843 843118REGUF

843

843

843
Sample Date

$5 / 18 / 90$

$11 / 8 / 90$

$1 / 25 / 89$

$4 / 24 / 89$

$11 / 20 / 97$

$6 / 25 / 97$

$11 / 21 / 96$

$6 / 25 / 97$

$10 / 11 / 94$

$2 / 16 / 95$

$5 / 5 / 95$

$7 / 19 / 95$

2/22/91

9/30/91

$12 / 9 / 91$

$5 / 18 / 90$

$11 / 8 / 90$

$1 / 25 / 89$

$4 / 24 / 89$

$11 / 20 / 97$

$6 / 25 / 97$

$11 / 21 / 96$

$6 / 25 / 97$

$10 / 11 / 94$

2/16/95

$5 / 5 / 95$

$7 / 19 / 95$

2/22/91

9/30/91

$12 / 9 / 91$

$5 / 18 / 90$

$11 / 8 / 90$

1/25/89

$4 / 24 / 89$

$11 / 20 / 97$

$6 / 25 / 97$

$11 / 21 / 96$

$6 / 25 / 97$

$10 / 11 / 94$

$2 / 16 / 95$

$5 / 5 / 95$

$7 / 19 / 95$

2/22/91

$9 / 30 / 91$

12/9/91

$5 / 18 / 90$

$11 / 8 / 90$

$1 / 25 / 89$

4/24/89

$11 / 20 / 97$
Sample Type Chemical

REG

REG

REG

Acetone

Acetone

REG

Acetone

REG

Acetone

REG

Benzene

REG

REG

REG

REG

REG

REG

REG

REG

REG

REG

REG

REG

REG

REG

REG

REG

REG

REG

REG

REG

REG

REG

REG

REG

REG

REG

REG

REG

REG

REG

REG

REG

REG

REG

REG

REG

REG

REG

REG

REG

REG

REG

REG

REG
Benzene

Benzene

Benzene

Benzene

Benzene

Benzene

Benzene

Benzene

Benzene

Benzene

Benzene

Benzene

Benzene

Benzene

Bromodichloromethane

Bromodichloromethane

Bromodichloromethane

Bromodichloromethane

Bromodichloromethane

Bromodichloromethane

Bromodichloromethane

Bromodichloromethane

Bromodichloromethane

Bromodichloromethane

Bromodichloromethane

Bromodichloromethane

Bromodichloromethane

Bromodichloromethane

Bromodichloromethane

Bromoform

Bromoform

Bromoform

Bromoform

Bromoform

Bromoform

Bromoform

Bromoform

Bromoform

Bromoform

Bromoform

Bromoform

Bromoform

Bromoform

Bromoform

Bromomethane
Results Units Qualifier

$10 \mathrm{ug} / \mathrm{L} \quad \mathrm{U}$

$10 \mathrm{ug} / \mathrm{L} \quad \cdot \mathrm{U}$

$10 \quad \mathrm{ug} / \mathrm{L} \quad \mathrm{U}$

$3 \mathrm{ug} / \mathrm{L} \quad \mathrm{J}$

5 ug/L U

$5 \mathrm{ug} / \mathrm{L} \quad \mathrm{U}$

$5 \quad \mathrm{ug} / \mathrm{L} \quad \mathrm{U}$

$5 \quad u g / L \quad U$

$5 \quad u g / L \quad U$

$5 \mathrm{ug} / \mathrm{L} \quad \mathrm{U}$

$5 \quad \mathrm{ug} / \mathrm{L} \quad \mathrm{U}$

$5 \quad u g / L \quad U$

$5 \mathrm{ug} / \mathrm{L} \quad \mathrm{U}$

$5 \quad u g / L \quad U$

$5 \quad u g / L \quad U$

$5 \quad u g / L \quad U$

$5 \quad u g / L \quad U$

$5 \mathrm{ug} / \mathrm{L} \quad \mathrm{U}$

5 ug/L U

$5 \quad u g / L \quad U$

$5 \mathrm{ug} / \mathrm{L} \quad \mathrm{U}$

$5 \mathrm{ug} / \mathrm{L} \quad \mathrm{U}$

5 ug/L U

5 ug/ $\quad \mathrm{U}$

$5 \mathrm{ug} / \mathrm{L} \quad \mathrm{U}$

$5 \mathrm{ug} / \mathrm{L} \quad \mathrm{U}$

$5 \mathrm{ug} / \mathrm{L} \quad \mathrm{U}$

$5 \quad u g / L \quad U$

5 ug/L U

$5 \mathrm{ug} / \mathrm{L} \quad \mathrm{U}$

5 ug/ $\quad \mathrm{U}$

$5 \quad \mathrm{ug} / \mathrm{L} \quad \mathrm{U}$

5 ug/ $\quad U$

$5 \mathrm{ug} / \mathrm{L} \quad \mathrm{U}$

$5 \mathrm{ug} / \mathrm{L} \quad \mathrm{U}$

$5 \mathrm{ug} / \mathrm{L} \quad \mathrm{U}$

5 ug/L U

$5 \quad u g / L \quad U$

$5 \mathrm{ug} / \mathrm{L} \quad \mathrm{U}$

$5 \quad \mathrm{ug} / \mathrm{L} \quad \mathrm{U}$

$5 \quad u g / L \quad U$

$5 \quad u g / L \quad U$

$5 \mathrm{ug} / \mathrm{L} \quad \mathrm{U}$

5 ug/L U

$5 \mathrm{ug} / \mathrm{L} \quad \mathrm{U}$

$5 \mathrm{ug} / \mathrm{L} \quad \mathrm{U}$

$5 \quad u g / L \quad U$

$5 \mathrm{ug} / \mathrm{L} \quad \mathrm{U}$

5 ug/L U

$10 \mathrm{ug} / \mathrm{L} \quad \mathrm{U}$ 
Station Sample ID Sample Date Sample Type Chemical

\begin{tabular}{|c|c|c|c|c|c|c|c|}
\hline 843 & W00810 & $6 / 25 / 97$ & REG & Bromomethane & 10 & $\mathrm{ug} / \mathrm{L}$ & $\mathrm{U}$ \\
\hline 843 & M00057 & $11 / 21 / 96$ & REG & Bromomethane & 10 & $\mathrm{ug} / \mathrm{L}$ & $\mathrm{U}$ \\
\hline 843 & M00847 & $6 / 25 / 97$ & REG & Bromomethane & 10 & $\mathrm{ug} / \mathrm{L}$ & $\mathrm{U}$ \\
\hline 843 & S00885 & $10 / 11 / 94$ & REG & Bromomethane & 10 & ug/L & $\mathrm{U}$ \\
\hline 843 & S02625 & $2 / 16 / 95$ & REG & Bromomethane & 10 & ug $/ \mathrm{L}$ & $\mathrm{U}$ \\
\hline 843 & S03587 & $5 / 5 / 95$ & REG & Bromomethane & 10 & $\mathrm{ug} / \mathrm{L}$ & $\mathrm{U}$ \\
\hline 843 & S04802 & $7 / 19 / 95$ & REG & Bromomethane & 10 & $\mathrm{ug} / \mathrm{L}$ & $\mathrm{U}$ \\
\hline 843 & 843222REGUF & $2 / 22 / 91$ & REG & Bromomethane & 10 & $\mathrm{ug} / \mathrm{L}$ & $\mathrm{U}$ \\
\hline 843 & 843930REGUF & $9 / 30 / 91$ & REG & Bromomethane & 10 & $\mathrm{ug} / \mathrm{L}$ & $\mathrm{U}$ \\
\hline 843 & 843129REGUF & $12 / 9 / 91$ & REG & Bromomethane & 10 & $\mathrm{ug} / \mathrm{L}$ & $\mathrm{U}$ \\
\hline 843 & 843518REGUF & $5 / 18 / 90$ & REG & Bromomethane & 10 & ug/L & $\mathrm{U}$ \\
\hline 843 & 843118REGUJ & $11 / 8 / 90$ & REG & Bromomethane & 10 & $\mathrm{ug} / \mathrm{L}$ & $\mathrm{U}$ \\
\hline 843 & 843125REGUF & $1 / 25 / 89$ & REG & Bromomethane & 10 & $\mathrm{ug} / \mathrm{L}$ & $\mathrm{U}$ \\
\hline 843 & 843424REGUF & $4 / 24 / 89$ & REG & Bromomethane & 10 & ug/L & $\mathrm{U}$ \\
\hline 843 & M01254 & $11 / 20 / 97$ & REG & Carbon disulfide & 5 & $\mathrm{ug} / \mathrm{L}$ & $\mathrm{U}$ \\
\hline 843 & W00810 & $6 / 25 / 97$ & REG & Carbon disulfide & 5 & $\mathrm{ug} / \mathrm{L}$ & $\mathrm{U}$ \\
\hline 843 & M00057 & $11 / 21 / 96$ & REG & Carbon disulfide & 5 & $\mathrm{ug} / \mathrm{L}$ & $\mathrm{U}$ \\
\hline 843 & M00847 & $6 / 25 / 97$ & REG & Carbon disulfide & 5 & $\mathrm{ug} / \mathrm{L}$ & $\mathrm{U}$ \\
\hline 843 & S00885 & $10 / 11 / 94$ & REG & Carbon disulfide & 5 & $\mathrm{ug} / \mathrm{L}$ & $\mathrm{U}$ \\
\hline 843 & S02625 & $2 / 16 / 95$ & REG & Carbon disulfide & 5 & $\mathrm{ug} / \mathrm{L}$ & $\mathrm{U}$ \\
\hline 843 & S03587 & $5 / 5 / 95$ & REG & Carbon disulfide & 5 & $\mathrm{ug} / \mathrm{L}$ & $\mathrm{U}$ \\
\hline 843 & S04802 & $7 / 19 / 95$ & REG & Carbon disulfide & 5 & $\mathrm{ug} / \mathrm{L}$ & $\mathrm{U}$ \\
\hline 843 & 843222REGUF & $2 / 22 / 91$ & REG & Carbon disulfide & 5 & $\mathrm{ug} / \mathrm{L}$ & $\mathrm{U}$ \\
\hline 843 & 843930REGUF & $9 / 30 / 91$ & REG & Carbon disulfide & 5 & $\mathrm{ug} / \mathrm{L}$ & $\mathrm{U}$ \\
\hline 843 & 843129REGUF & $12 / 9 / 91$ & REG & Carbon disulfide & 5 & $\mathrm{ug} / \mathrm{L}$ & $\mathrm{U}$ \\
\hline 843 & 843518REGUF & $5 / 18 / 90$ & REG & Carbon disulfide & 5 & ug/L & $\mathrm{U}$ \\
\hline 843 & 843118REGUF & $11 / 8 / 90$ & REG & Carbon disulfide & 5 & $\mathrm{ug} / \mathrm{L}$ & $\mathrm{U}$ \\
\hline 843 & 843125REGUF & $1 / 25 / 89$ & REG & Carbon disulfide & 5 & $\mathrm{ug} / \mathrm{L}$ & $\mathrm{U}$ \\
\hline 843 & 843424REGUF & $4 / 24 / 89$ & REG & Carbon disulfide & 5 & $\mathrm{ug} / \mathrm{L}$ & $\mathrm{U}$ \\
\hline 843 & M01254 & $11 / 20 / 97$ & REG & Carbon tetrachloride & 5 & $\mathrm{ug} / \mathrm{L}$ & $\mathrm{U}$ \\
\hline 843 & W00810 & $6 / 25 / 97$ & REG & Carbon tetrachloride & 5 & $\mathrm{ug} / \mathrm{L}$ & $\mathrm{U}$ \\
\hline 843 & M00057 & $11 / 21 / 96$ & REG & Carbon tetrachloride & 5 & $\mathrm{ug} / \mathrm{L}$ & $\mathrm{U}$ \\
\hline 843 & M00847 & $6 / 25 / 97$ & REG & Carbon tetrachloride & 5 & $\mathrm{ug} / \mathrm{L}$ & $\mathrm{U}$ \\
\hline 843 & S00885 & $10 / 11 / 94$ & REG & Carbon tetrachloride & 5 & $\mathrm{ug} / \mathrm{L}$ & $U$ \\
\hline 843 & S02625 & $2 / 16 / 95$ & REG & Carbon tetrachloride & 5 & $\mathrm{ug} / \mathrm{L}$ & $\mathrm{U}$ \\
\hline 843 & S03587 & $5 / 5 / 95$ & REG & Carbon tetrachloride & 5 & $\mathrm{ug} / \mathrm{L}$ & $\mathrm{U}$ \\
\hline 843 & S04802 & $7 / 19 / 95$ & REG & Carbon tetrachloride & 5 & $\mathrm{ug} / \mathrm{L}$ & $\mathrm{U}$ \\
\hline 843 & 843222REGUF & $2 / 22 / 91$ & REG & Carbon tetrachloride & 5 & $\mathrm{ug} / \mathrm{L}$ & $\mathrm{U}$ \\
\hline 843 & 843930REGUF & $9 / 30 / 91$ & REG & Carbon tetrachloride & 5 & ug $/ \mathrm{L}$ & $\mathrm{U}$ \\
\hline 843 & 843129REGUF & $12 / 9 / 91$ & REG & Carbon tetrachloride & 5 & $\mathrm{ug} / \mathrm{L}$ & $\mathrm{U}$ \\
\hline 843 & 843518REGUF & $5 / 18 / 90$ & REG & Carbon tetrachloride & 5 & $\mathrm{ug} / \mathrm{L}$ & $\mathrm{U}$ \\
\hline 843 & 843118REGUF & $11 / 8 / 90$ & REG & Carbon tetrachloride & 5 & $\mathrm{ug} / \mathrm{L}$ & $\mathrm{U}$ \\
\hline 843 & 843125REGUF & $1 / 25 / 89$ & REG & Carbon tetrachloride & 5 & $\mathrm{ug} / \mathrm{L}$ & $\mathrm{U}$ \\
\hline 843 & 843424REGUF & $4 / 24 / 89$ & REG & Carbon tetrachloride & 0.5 & $\mathrm{ug} / \mathrm{L}$ & $\mathrm{J}$ \\
\hline 843 & M01254 & $11 / 20 / 97$ & REG & Chlorobenzene & 5 & $\mathrm{ug} / \mathrm{L}$ & $\mathrm{U}$ \\
\hline 843 & W00810 & $6 / 25 / 97$ & REG. & Chlorobenzene & 5 & $\mathrm{ug} / \mathrm{L}$ & $\mathrm{U}$ \\
\hline 843 & M00057 & $11 / 21 / 96$ & REG & Chlorobenzene & 5 & $\mathrm{ug} / \mathrm{L}$ & $\mathrm{U}$ \\
\hline 843 & M00847 & $6 / 25 / 97$ & REG & Chlorobenzene & 5 & $\mathrm{ug} / \mathrm{L}$ & $\mathrm{U}$ \\
\hline 843 & S00885 & $10 / 11 / 94$ & REG & Chlorobenzene & 5 & $\mathrm{ug} / \mathrm{L}$ & $\mathrm{U}$ \\
\hline 843 & S02625 & $2 / 16 / 95$ & REG & Chlorobenzene & 5 & $\mathrm{ug} / \mathrm{L}$ & $\mathrm{U}$ \\
\hline
\end{tabular}


Station Sample ID Sample Date Sample Type Chemical

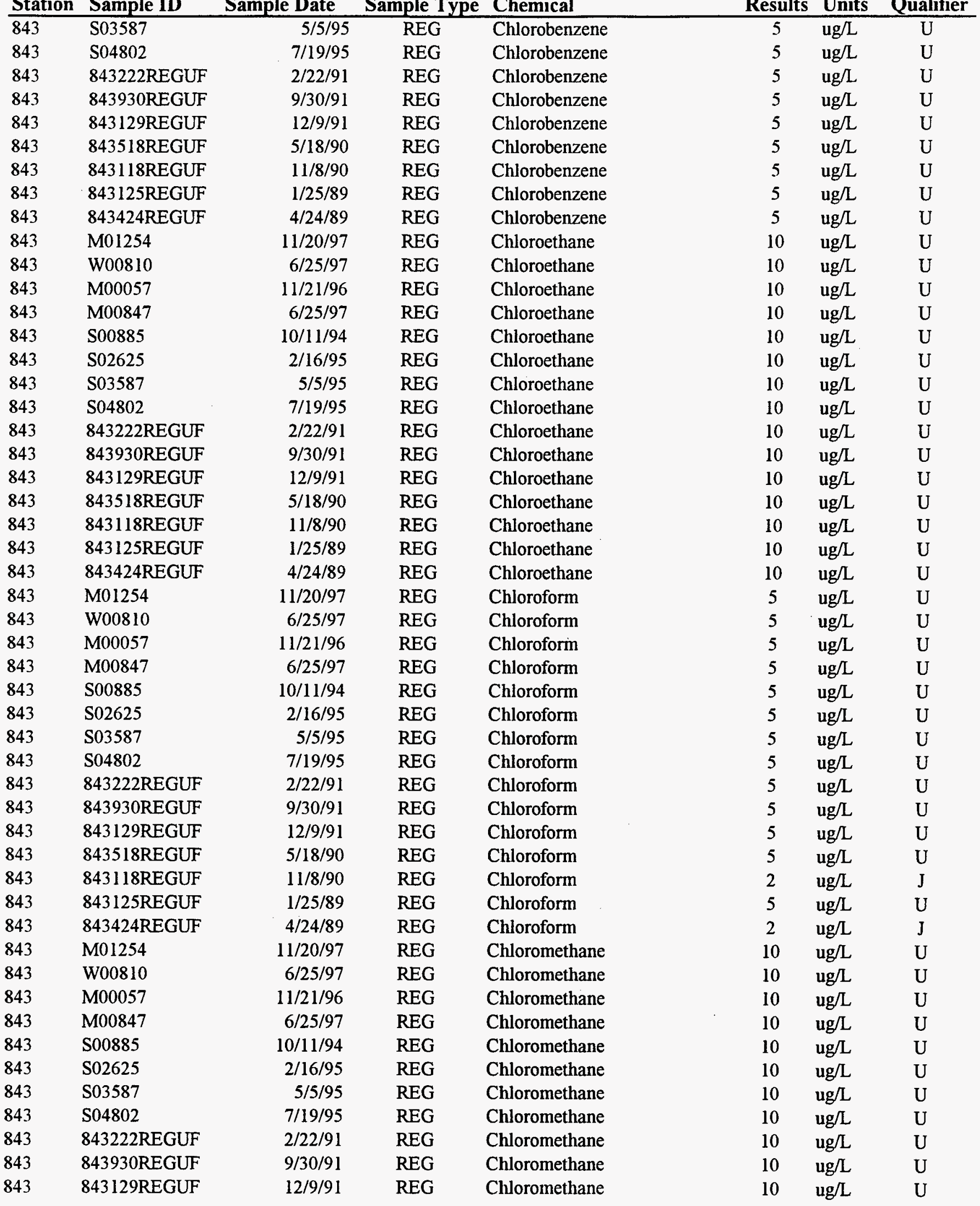




\begin{tabular}{|c|c|c|c|c|c|c|c|}
\hline Station & Sample ID & Sample Date & Sample Type & Chemical & Results & Units & Qualifier \\
\hline 843 & 843518REGUF & $5 / 18 / 90$ & REG & Chloromethane & 10 & $\mathrm{ug} / \mathrm{L}$ & $\mathrm{U}$ \\
\hline 843 & 843118REGUF & $11 / 8 / 90$ & REG & Chloromethane & 10 & $\mathrm{ug} / \mathrm{L}$ & $\mathrm{U}$ \\
\hline 843 & 843125REGUF & $1 / 25 / 89$ & REG & Chloromethane & 10 & $\mathrm{ug} / \mathrm{L}$ & $\mathrm{U}$ \\
\hline 843 & 843424REGUF & $4 / 24 / 89$ & REG & Chloromethane & 4 & $\mathrm{ug} / \mathrm{L}$ & $\mathrm{J}$ \\
\hline 843 & M01254 & $11 / 20 / 97$ & REG & cis-1,2-Dichloroethene & 6 & $u g / L$ & $=$ \\
\hline 843 & M00057 & $11 / 21 / 96$ & REG & cis-1,2-Dichloroethene & 4 & $\mathrm{ug} / \mathrm{L}$ & $=$ \\
\hline 843 & M00847 & $6 / 25 / 97$ & REG & cis-1,2-Dichloroethene & 13 & $\mathrm{ug} / \mathrm{L}$ & $=$ \\
\hline 843 & S00885 & $10 / 11 / 94$ & REG & cis-1,2-Dichloroethene & 3.5 & $\mathrm{ug} / \mathrm{L}$ & $\mathrm{J}$ \\
\hline 843 & S02625 & $2 / 16 / 95$ & REG & cis-1,2-Dichloroethene & 2 & $u g / L$ & $\mathrm{~J}$ \\
\hline 843 & S03587 & $5 / 5 / 95$ & REG & cis-1,2-Dichloroethene & 5 & $\mathrm{ug} / \mathrm{L}$ & $=$ \\
\hline 843 & S04802 & $7 / 19 / 95$ & REG & cis-1,2-Dichloroethene & 4 & $u g / L$ & $\mathrm{~J}$ \\
\hline 843 & M01254 & $11 / 20 / 97$ & REG & cis-1,3-Dichloropropen & 5 & $\mathrm{ug} / \mathrm{L}$ & $\mathrm{U}$ \\
\hline 843 & W00810 & $6 / 25 / 97$ & REG & cis-1,3-Dichloropropene & 5 & $\mathrm{ug} / \mathrm{L}$ & $\mathrm{U}$ \\
\hline 843 & M00057 & $11 / 21 / 96$ & REG & cis-1,3-Dichloropropene & 5 & $\mathrm{ug} / \mathrm{L}$ & $\mathrm{U}$ \\
\hline 843 & M00847 & $6 / 25 / 97$ & REG & cis-1,3-Dichloropropene & 5 & $\mathrm{ug} / \mathrm{L}$ & $\mathrm{U}$ \\
\hline 843 & S00885 & $10 / 11 / 94$ & REG & cis-1,3-Dichloropropene & 5 & $\mathrm{ug} / \mathrm{L}$ & $\mathrm{U}$ \\
\hline 843 & S02625 & $2 / 16 / 95$ & REG & cis-1,3-Dichloropropene & 5 & $\mathrm{ug} / \mathrm{L}$ & $\mathrm{U}$ \\
\hline 843 & S03587 & $5 / 5 / 95$ & REG & cis-1,3-Dichloropropene & 5 & $\mathrm{ug} / \mathrm{L}$ & $\mathrm{U}$ \\
\hline 843 & S04802 & $7 / 19 / 95$ & REG & cis-1,3-Dichloropropene & 5 & $\mathrm{ug} / \mathrm{L}$ & $\mathrm{U}$ \\
\hline 843 & 843222REGUF & $2 / 22 / 91$ & REG & cis-1,3-Dichloropropene & 5 & $\mathrm{ug} / \mathrm{L}$ & $\mathbf{U}$ \\
\hline 843 & 843930REGUF & $9 / 30 / 91$ & REG & cis-1,3-Dichloropropene & 5 & $\mathrm{ug} / \mathrm{L}$ & U \\
\hline 843 & 843129REGUF & $12 / 9 / 91$ & REG & cis-1,3-Dichloropropene & 5 & $\mathrm{ug} / \mathrm{L}$ & $\mathrm{U}$ \\
\hline 843 & 843518REGUF & $5 / 18 / 90$ & REG & cis-1,3-Dichloropropene & 5 & $\mathrm{ug} / \mathrm{L}$ & $\mathrm{U}$ \\
\hline 843 & 843118REGUF & $11 / 8 / 90$ & REG & cis-1,3-Dichloropropene & 5 & $u g / L$ & $\mathrm{U}$ \\
\hline 843 & 843125REGUF & $1 / 25 / 89$ & REG & cis-1,3-Dichloropropene & 5 & $\mathrm{ug} / \mathrm{L}$ & $\mathrm{U}$ \\
\hline 843 & 843424REGUF & $4 / 24 / 89$ & REG & cis-1,3-Dichloropropene & 5 & $\mathrm{ug} / \mathrm{L}$ & $\mathrm{U}$ \\
\hline 843 & M01254 & $11 / 20 / 97$ & REG & Dibromochloromethane & 5 & $\mathrm{ug} / \mathrm{L}$ & $\mathrm{U}$ \\
\hline 843 & W00810 & $6 / 25 / 97$ & REG & Dibromochloromethane & 5 & $u g / L$ & $\mathrm{U}$ \\
\hline 843 & M00057 & $11 / 21 / 96$ & REG & Dibromochloromethane & 5 & $\mathrm{ug} / \mathrm{L}$ & $\mathrm{U}$ \\
\hline 843 & M00847 & $6 / 25 / 97$ & REG & Dibromochloromethane & 5 & $\mathrm{ug} / \mathrm{L}$ & $\mathrm{U}$ \\
\hline 843 & S00885 & $10 / 11 / 94$ & REG & Dibromochloromethane & 5 & $\mathrm{ug} / \mathrm{L}$ & $\mathrm{U}$ \\
\hline 843 & S02625 & $2 / 16 / 95$ & REG & Dibromochloromethane & 5 & $\mathrm{ug} / \mathrm{L}$ & $\mathrm{U}$ \\
\hline 843 & S03587 & $5 / 5 / 95$ & REG & Dibromochloromethane & 5 & $\mathrm{ug} / \mathrm{L}$ & $\mathrm{U}$ \\
\hline 843 & S04802 & $7 / 19 / 95$ & REG & Dibromochloromethane & 5 & ug/L & $\mathrm{U}$ \\
\hline 843 & 843222REGUF & $2 / 22 / 91$ & REG & Dibromochloromethane & 5 & $\mathrm{ug} / \mathrm{L}$ & $\mathrm{U}$ \\
\hline 843 & 843930REGUF & $9 / 30 / 91$ & REG & Dibromochloromethane & 5 & $\mathrm{ug} / \mathrm{L}$ & $\mathrm{U}$ \\
\hline 843 & 843129REGUF & $12 / 9 / 91$ & REG & Dibromochloromethane & 5 & $\mathrm{ug} / \mathrm{L}$ & $\mathbf{U}$ \\
\hline 843 & 843518REGUF & $5 / 18 / 90$ & REG & Dibromochloromethane & 5 & $\mathrm{ug} / \mathrm{L}$ & $\mathrm{U}$ \\
\hline 843 & 843118REGUF & $11 / 8 / 90$ & REG & Dibromochloromethane & 5 & $\mathrm{ug} / \mathrm{L}$ & U \\
\hline 843 & 843125REGUF & $1 / 25 / 89$ & REG & Dibromochloromethane & 5 & $\mathrm{ug} / \mathrm{L}$ & $\mathrm{U}$ \\
\hline 843 & 843424REGUF & $4 / 24 / 89$ & REG & Dibromochloromethane & 5 & $\mathrm{ug} / \mathrm{L}$ & $\mathrm{U}$ \\
\hline 843 & M00057 & $11 / 21 / 96$ & REG & Dimethylbenzene & 5 & $u g / L$ & $\mathrm{U}$ \\
\hline 843 & 843222REGUF & $2 / 22 / 91$ & REG & Dimethylbenzene & 5 & $\mathrm{ug} / \mathrm{L}$ & $\mathrm{U}$ \\
\hline 843 & 843930REGUF & $9 / 30 / 91$ & REG & Dimethylbenzene & 5 & $\mathrm{ug} / \mathrm{L}$ & $\mathbf{U}$ \\
\hline 843 & 843129REGUF & $12 / 9 / 91$ & REG & Dimethylbenzene & 5 & $\mathrm{ug} / \mathrm{L}$ & $\mathrm{U}$ \\
\hline 843 & 843518REGUF & $5 / 18 / 90$ & REG & Dimethylbenzene & 5 & $\mathrm{ug} / \mathrm{L}$ & $\mathrm{U}$ \\
\hline 843 & 843118REGUF & $11 / 8 / 90$ & REG & Dimethylbenzene & 5 & $\mathrm{ug} / \mathrm{L}$ & $\mathrm{U}$ \\
\hline 843 & 843125REGUF & $1 / 25 / 89$ & REG & Dimethylbenzene & 5 & $\mathrm{ug} / \mathrm{L}$ & U \\
\hline 843 & 843424REGUF & $4 / 24 / 89$ & REG & Dimethylbenzene & 5 & $\mathrm{ug} / \mathrm{L}$ & $\mathrm{U}$ \\
\hline 843 & M01254 & $11 / 20 / 97$ & REG & Ethylbenzene & 5 & ug/L & $\mathrm{U}$ \\
\hline
\end{tabular}


Station Sample ID Sample Date Sample Type Chemical

\begin{tabular}{|c|c|c|}
\hline 4317 & S01876 & $12 / 15 / 94$ \\
\hline+.317 & $\mathrm{~S} 02345$ & $1 / 25 / 95$ \\
\hline 4317 & S03456 & $4 / 13 / 95$ \\
\hline $4: 17$ & M01304 & $11 / 19 / 97$ \\
\hline$+\$ 17$ & W00820 & $6 / 17 / 97$ \\
\hline 4317 & M00091 & $11 / 20 / 96$ \\
\hline+317 & M00896 & $6 / 17 / 97$ \\
\hline+317 & SMPL00816 & $9 / 12 / 94$ \\
\hline+317 & S01876 & $12 / 15 / 94$ \\
\hline+317 & S02345 & $1 / 25 / 95$ \\
\hline+317 & S03456 & $4 / 13 / 95$ \\
\hline $4 \$ 17$ & M01304 & $11 / 19 / 97$ \\
\hline 4.117 & M00091 & $11 / 20 / 96$ \\
\hline 4.317 & M00896 & $6 / 17 / 97$ \\
\hline 4317 & SMPL00816 & $9 / 12 / 94$ \\
\hline 4317 & S01876 & $12 / 15 / 94$ \\
\hline 4317 & S02345 & $1 / 25 / 95$ \\
\hline 4317 & S03456 & $4 / 13 / 95$ \\
\hline 4317 & M01304 & $11 / 19 / 97$ \\
\hline+317 & W00820 & $6 / 17 / 97$ \\
\hline+317 & M00091 & $11 / 20 / 96$ \\
\hline 4317 & M00896 & $6 / 17 / 97$ \\
\hline+317 & SMPL00816 & $9 / 12 / 94$ \\
\hline+317 & S01876 & $12 / 15 / 94$ \\
\hline+317 & S02345 & $1 / 25 / 95$ \\
\hline+317 & S03456 & $4 / 13 / 95$ \\
\hline $4: 17$ & M01304 & $11 / 19 / 97$ \\
\hline 4317 & W00820 & $6 / 17 / 97$ \\
\hline 4.317 & M00091 & $11 / 20 / 96$ \\
\hline 4317 & M00896 & $6 / 17 / 97$ \\
\hline 4317 & SMPL00816 & $9 / 12 / 94$ \\
\hline 4.317 & S01876 & $12 / 15 / 94$ \\
\hline $4: 17$ & $\mathrm{~S} 02345$ & 1/25/95 \\
\hline 4317 & S03456 & $4 / 13 / 95$ \\
\hline $4: 17$ & M01304 & $11 / 19 / 97$ \\
\hline 4317 & M00091 & $11 / 20 / 96$ \\
\hline 4317 & M00896 & $6 / 17 / 97$ \\
\hline 4317 & SMPL00816 & $9 / 12 / 94$ \\
\hline+317 & M01304 & $11 / 19 / 97$ \\
\hline $4: 17$ & W00820 & $6 / 17 / 97$ \\
\hline 4317 & M00091 & $11 / 20 / 96$ \\
\hline 4317 & M00896 & $6 / 17 / 97$ \\
\hline+317 & SMPL00816 & $9 / 12 / 94$ \\
\hline+317 & S01876 & $12 / 15 / 94$ \\
\hline 4317 & S02345 & $1 / 25 / 95$ \\
\hline+317 & S03456 & $4 / 13 / 95$ \\
\hline
\end{tabular}

Tetrachloroethene

Tetrachloroethene

Tetrachloroethene

Toluene

Toluene

Toluene

Toluene

Toluene

Toluene

Toluene

Toluene

trans-1.2-Dichloroethe

trans-1.2-Dichloroethene

trans-1,2-Dichloroethene

trans-1,2-Dichloroethene

trans-1,2-Dichloroethene

trans-1,2-Dichloroethene

urans-1,2-Dichloroethene

trans-1,3-Dichloroprop

trans-1.3-Dichloropropene

trans-1,3-Dichloropropene

trans-1,3-Dichloropropene

trans-1,3-Dichloropropene

trans-1,3-Dichloropropene

trans-1,3-Dichloropropene

trans-1.3-Dichloropropene

Trichloroethene

Trichloroethene

Trichloroethene

Trichloroethene

Trichloroethene

Trichloroethene

Trichloroethene

Trichloroethene

Vinyl acetate

Vinyl acetate

Vinyl acetate

Vinyl acetate

Vinyl chloride

Vinyl chloride

Vinyl chloride

Vinyl chloride

Vinyl chloride

Vinyl chloride

Vinyl chloride

Vinyl chloride
Results Units Qualifier

$\begin{array}{lll}\text { I } & \text { ug/L } & U \\ \text { I } & u g / L & U\end{array}$

$\begin{array}{lll}1 & u g / L & U\end{array}$

$5 \mathrm{ug} / \mathrm{L} \quad \mathrm{U}$

$5 \mathrm{ug} / \mathrm{L} \quad \mathrm{U}$

$5 \quad u g / L \quad U$

$5 \quad u g / L \quad U$

5 ug/L U

$1 \mathrm{ug} / \mathrm{L} \quad \mathrm{U}$

1 ug/L U

1 ug/L U

5 ug/L U

$5 \mathrm{ug} / \mathrm{L} \quad \mathrm{U}$

5 ug/L U

$5 \quad u g / L \quad U$

1 ug/

1 ug/

1 ug/L

5 ug/L U

$5 \quad u g / L \quad U$

5 ug/L U

$5 \mathrm{ug} / \mathrm{L} \quad \mathrm{U}$

$5 \quad u g / L$

1 ug/L

1 ug/L

1 ug/L

5 ug/L U

5 ug/L $\quad$ U

$\begin{array}{lll}5 & u g / L & U \\ 5 & u g / L & U\end{array}$

$5 \quad u g / L \quad U$

1 ug/L U

$1 \mathrm{ug} / \mathrm{L}, \mathrm{U}$

$1 \mathrm{ug} / \mathrm{L} U$

$10 \mathrm{ug} / \mathrm{L} \quad \mathrm{U}$

$10 \mathrm{ug} / \mathrm{L} \quad \mathrm{U}$

$10 \mathrm{ug} / \mathrm{L} \quad \mathrm{U}$

$10 \mathrm{ug} / \mathrm{L} \quad \mathrm{U}$

$10 \mathrm{ug} / \mathrm{L} U$

$10 \mathrm{ug} / \mathrm{L} \quad \mathrm{U}$

$10 \mathrm{ug} / \mathrm{L} \quad \mathrm{U}$

$10 \mathrm{ug} / \mathrm{L} \quad \mathrm{U}$

$10 \mathrm{ug} / \mathrm{L} \quad \mathrm{U}$

$1 \mathrm{ug} / \mathrm{L} \quad \mathrm{U}$

1 ug/L U

1 ug/L U 
B-54

\begin{tabular}{|c|c|c|c|c|c|c|c|}
\hline Station & Sample ID & Sample Date & Sample Type & Chemical & Results & Units & Qualifier \\
\hline$\overline{843}$ & W00810 & $6 / 25 / 97$ & REG & Ethỵlbenzene & 5 & $\mathrm{ug} / \mathrm{L}$ & $\mathrm{U}$ \\
\hline 843 & M00057 & $11 / 21 / 96$ & REG & Ethylbenzene & 5 & $\mathrm{ug} / \mathrm{L}$ & U \\
\hline 843 & $\mathrm{MO}(1) 847$ & $6 / 25 / 97$ & REG & Ethylbenzene & 5 & ug $\Omega$ & U \\
\hline 843 & $S 00885$ & $10 / 11 / 94$ & REG & Ethylbenzene & 5 & ug $/$ & $\mathrm{U}$ \\
\hline 843 & $S 02625$ & $2 / 16 / 95$ & REG & Ethilbenzene & 5 & ug $/$ L & U \\
\hline 843 & $S 0.3 .587$ & $5 / 5 / 95$ & REG & Ethylbenzene & 5 & ug/L & $\mathrm{U}$ \\
\hline 843 & S $0+802$ & $7 / 19 / 95$ & REG & Ethỵlbenzene & 5 & ug/L & $\mathrm{U}$ \\
\hline 843 & 843222REGUF & $2 / 22 / 91$ & REG & Ethyllbenzene & 5 & $\mathrm{ug} / \mathrm{L}$ & $\mathrm{U}$ \\
\hline 843 & 843930REGUF & $9 / 30 / 91$ & REG & Ethylbenzene & 5 & ug/L & $\mathrm{U}$ \\
\hline 843 & 843129REGUF & $12 / 9 / 91$ & REG & Ethylbenzene & 5 & $\mathrm{ug} / \mathrm{L}$ & $U$ \\
\hline 843 & 843518REGUF & $5 / 18 / 90$ & REG & Ethylbenzene & 5 & ug $/$ & $\mathrm{U}$ \\
\hline 843 & 8+3118REGUF & $11 / 8 / 90$ & REG & Ethylbenzene & 5 & ug/L & $\mathrm{U}$ \\
\hline 843 & 843125REGUF & $1 / 25 / 89$ & REG & Ethylbenzene & 5 & $\mathrm{ug} / \mathrm{L}$ & $\mathrm{U}$ \\
\hline 843 & 843424REGUF & $4 / 24 / 89$ & REG & Ethỵlbenzene & 5 & $\mathrm{ug} / \mathrm{L}$ & U \\
\hline 843 & M01254 & $11 / 20 / 97$ & REG & m.p-Xylene & 5 & $\mathrm{ug} / \mathrm{L}$ & $\mathrm{U}$ \\
\hline 843 & W00810 & $6 / 25 / 97$ & REG & m.p-Xylene & 5 & $\mathrm{ug} / \mathrm{L}$ & $\mathrm{U}$ \\
\hline 843 & M00057 & $11 / 21 / 96$ & REG & m,p-Xylene & 5 & $\mathrm{ug} / \mathrm{L}$ & U \\
\hline 843 & M00847 & $6 / 25 / 97$ & REG & m.p-Xylene & 5 & $\mathrm{ug} / \mathrm{L}$ & $\mathrm{U}$ \\
\hline 843 & S02625 & $2 / 16 / 95$ & REG & m.p-Xylene & 5 & $\mathrm{ug} / \mathrm{L}$ & U \\
\hline 843 & S03587 & $5 / 5 / 95$ & REG & m.p-Xylene & 5 & $\mathrm{ug} / \mathrm{L}$ & $\mathrm{U}$ \\
\hline 843 & $\mathrm{~S} 04802$ & $7 / 19 / 95$ & REG & m.p-Xylene & 5 & $\mathrm{ug} / \mathrm{L}$ & $\mathrm{U}$ \\
\hline 843 & M01254 & $11 / 20 / 97$ & REG & Methylene chloride & 2 & ug $/ \mathrm{L}$ & B \\
\hline 843 & W00810 & $6 / 25 / 97$ & REG & Methylene chloride & 9 & ug $/ \mathrm{L}$ & $=$ \\
\hline 843 & M00057 & $11 / 21 / 96$ & REG & Methylene chloride & 5 & ug $/ \mathrm{L}$ & U \\
\hline 843 & M00847 & $6 / 25 / 97$ & REG & Methylene chloride & 2 & ug/L & $\mathrm{U}$ \\
\hline 843 & S00885 & $10 / 11 / 94$ & REG & Methylene chloride & 5 & $u g / L$ & U \\
\hline 843 & S02625 & $2 / 16 / 95$ & REG & Methy lene chloride & 5 & $\mathrm{ug} / \mathrm{L}$ & $\mathrm{U}$ \\
\hline 843 & S03587 & $5 / 5 / 95$ & REG & Methylene chloride & 5 & ug $/ \mathrm{L}$ & $\mathrm{U}$ \\
\hline 843 & S04802 & $7 / 19 / 95$ & REG & Methylene chloride & 5 & $\mathrm{ug} / \mathrm{L}$ & $\mathrm{U}$ \\
\hline 843 & 843222REGUF & $2 / 22 / 91$ & REG & Methylene chloride & 5 & $\mathrm{ug} / \mathrm{L}$ & $\mathrm{U}$ \\
\hline 843 & 843930REGUF & $9 / 30 / 91$ & REG & Methylene chloride & 5 & $\mathrm{ug} / \mathrm{L}$ & $\mathrm{U}$ \\
\hline 843 & 843129REGUF & $12 / 9 / 91$ & REG & Methỵlene chloride & 5 & $\mathrm{ug} / \mathrm{L}$ & $\mathrm{U}$ \\
\hline 843 & 843518REGUF & $5 / 18 / 90$ & REG & Methylene chloride & 2 & ug/L & J \\
\hline 843 & $8+3118$ REGUF & $11 / 8 / 90$ & REG & Methylene chloride & 5 & $\mathrm{ug} / \mathrm{L}$ & $U$ \\
\hline 843 & 843125 REGUF & $1 / 25 / 89$ & REG & Methylene chloride & 1 & ug/L & $\mathrm{J}$ \\
\hline 843 & 843424REGUF & $4 / 24 / 89$ & REG & Methylene chloride & 1 & ug/L & J \\
\hline 843 & M01254 & $11 / 20 / 97$ & REG & Styrene & 5 & ug/L & $\mathrm{U}$ \\
\hline 843 & W00810 & $6 / 25 / 97$ & REG & Styrene & 5 & $\mathrm{ug} / \mathrm{L}$ & $\mathrm{U}$ \\
\hline 843 & M00057 & $11 / 21 / 96$ & REG & Styrene & 5 & $\mathrm{ug} / \mathrm{L}$ & $\mathrm{U}$ \\
\hline 843 & M00847 & $6 / 25 / 97$ & REG & Styrene & 5 & $\mathrm{ug} / \mathrm{L}$ & $\mathrm{U}$ \\
\hline 843 & S00885 & $10 / 11 / 94$ & REG & Styrene & 5 & $\mathrm{ug} / \mathrm{L}$ & $\mathrm{U}$ \\
\hline 843 & S02625 & $2 / 16 / 95$ & REG & Styrene & 5 & ug/L & $U$ \\
\hline 843 & S03587 & $5 / 5 / 95$ & REG & Styrene & 5 & ug $/ \mathrm{L}$ & $\mathrm{U}$ \\
\hline $8+3$ & S0 4802 & $7 / 19 / 95$ & REG & Styrene & 5 & ug/L & $\mathrm{U}$ \\
\hline 843 & 843222REGUF & $2 / 22 / 91$ & REG & Styrene & 5 & ug $/$ & U \\
\hline 843 & 843930REGUF & $9 / 30 / 91$ & REG & Styrene & 5 & $\mathrm{ug} / \mathrm{L}$ & $\mathrm{U}$ \\
\hline 843 & 843129REGUF & $12 / 9 / 91$ & REG & Styrene & 5 & ug/L & $\mathrm{U}$ \\
\hline 843 & 843518REGUF & $5 / 18 / 90$ & REG & Styrene & 5 & $\mathrm{ug} / \mathrm{L}$ & $\mathrm{U}$ \\
\hline 843 & 843118 REGUF & $11 / 8 / 90$ & REG & Styrene & 5 & $\mathrm{ug} / \mathrm{L}$ & $\mathrm{U}$ \\
\hline 843 & 843125 REGUF & $1 / 25 / 89$ & REG & Styrene & 5 & $\mathrm{ug} / \mathrm{L}$ & $\mathrm{U}$ \\
\hline
\end{tabular}




\begin{tabular}{|c|c|c|c|c|c|c|c|}
\hline Station & Sample ID & Sample Date & Sample Type & Chemical & Results & Units & Qualifier \\
\hline $8+3$ & 843424REGUF & $4 / 24 / 89$ & REG & Styrene & 5 & $\mathrm{ug} / \mathrm{L}$ & $\mathrm{U}$ \\
\hline $8+3$ & M01254 & $11 / 20 / 97$ & REG & Tetrachloroethene & 5 & ug/L & $\mathrm{U}$ \\
\hline 843 & W00810 & $6 / 25 / 97$ & REG & Tetrachloroethene & 5 & $\mathrm{ug} / \mathrm{L}$ & U \\
\hline 843 & M00057 & $11 / 21 / 96$ & REG & Tetrachloroethene & 5 & $\mathrm{ug} / \mathrm{L}$ & $\mathrm{U}$ \\
\hline $8+3$ & M00847 & $6 / 25 / 97$ & REG & Tetrachloroethene & 5 & $\mathrm{ug} / \mathrm{L}$ & U \\
\hline $8+3$ & S00885 & $10 / 11 / 94$ & REG & Tetrachloroethene & 5 & $\mathrm{ug} / \mathrm{L}$ & U \\
\hline $8+3$ & S02625 & $2 / 16 / 95$ & REG & Tetrachloroethene & 5 & $\mathrm{ug} / \mathrm{L}$ & U \\
\hline 843 & S03587 & $5 / 5 / 95$ & REG & Tetrachloroethene & 5 & ug/L & U \\
\hline 843 & S04802 & $7 / 19 / 95$ & REG & Tetrachloroethene & 5 & ug/L & $\mathrm{U}$ \\
\hline 843 & 843222REGUF & $2 / 22 / 91$ & REG & Tetrachloroethene & 5 & ug/L & $\mathrm{U}$ \\
\hline 843 & 843930REGUF & 9/30/91 & REG & Tetrachloroethene & 5 & $\mathrm{ug} / \mathrm{L}$ & U \\
\hline 843 & 843129REGUF & $12 / 9 / 91$ & REG & Tetrachloroethene & 5 & $\mathrm{ug} / \mathrm{L}$ & U \\
\hline 843 & 843518REGUF & $5 / 18 / 90$ & REG & Tetrachioroethene & 5 & $\mathrm{ug} / \mathrm{L}$ & $\mathrm{U}$ \\
\hline 843 & 843118REGUF & $11 / 8 / 90$ & REG & Tetrachloroethene & 5 & $\mathrm{ug} / \mathrm{L}$ & $\mathrm{U}$ \\
\hline 843 & 843125REGUF & $1 / 25 / 89$ & REG & Tetrachloroethene & 5 & $\mathrm{ug} / \mathrm{L}$ & U \\
\hline 843 & 843424REGUF & $4 / 24 / 89$ & REG & Tetrachloroethene & 5 & ug/L & $\mathrm{U}$ \\
\hline 843 & $\mathrm{M} 01254$ & $11 / 20 / 97$ & REG & Toluene & 5 & $\mathrm{ug} / \mathrm{L}$ & $\mathrm{U}$ \\
\hline 843 & W00810 & $6 / 25 / 97$ & REG & Toluene & 5 & $\mathrm{ug} / \mathrm{L}$ & $\mathrm{U}$ \\
\hline 843 & M00057 & $11 / 21 / 96$ & REG & Toluene & 5 & $\mathrm{ug} / \mathrm{L}$ & $\mathrm{U}$ \\
\hline 843 & M00847 & $6 / 25 / 97$ & REG & Toluene & 5 & $\mathrm{ug} / \mathrm{L}$ & $\mathrm{U}$ \\
\hline $8+3$ & S00885 & $10 / 11 / 94$ & REG & Toluene & 5 & $\mathrm{ug} / \mathrm{L}$ & $\mathrm{U}$ \\
\hline 843 & S02625 & $2 / 16 / 95$ & REG & Toluene & 5 & $\mathrm{ug} / \mathrm{L}$ & $\mathrm{U}$ \\
\hline 843 & S03587 & $5 / 5 / 95$ & REG & Toluene & 5 & $\mathrm{ug} / \mathrm{L}$ & U \\
\hline $8+3$ & S04802 & $7 / 19 / 95$ & REG & Toluene & 5 & $\mathrm{ug} / \mathrm{L}$ & $\mathrm{U}$ \\
\hline 843 & 843222REGUF & $2 / 22 / 91$ & REG & Toluene & 5 & $\mathrm{ug} / \mathrm{L}$ & $\mathrm{U}$ \\
\hline 843 & 843930REGUF & $9 / 30 / 91$ & REG & Toluene & 5 & $\mathrm{ug} / \mathrm{L}$ & $\mathrm{U}$ \\
\hline 843 & 843129REGUF & $12 / 9 / 91$ & REG & Toluene & 5 & ug/L & $\mathrm{U}$ \\
\hline 843 & 843518REGUF & $5 / 18 / 90$ & REG & Toluene & 2 & $\mathrm{ug} / \mathrm{L}$ & J \\
\hline 843 & 843118REGUF & $11 / 8 / 90$ & REG & Toluene & 5 & $\mathrm{ug} / \mathrm{L}$ & $\mathrm{U}$ \\
\hline 843 & 843125REGUF & $1 / 25 / 89$ & REG & Toluene & 5 & $\mathrm{ug} / \mathrm{L}$ & $\mathrm{U}$ \\
\hline 843 & 843424REGUF & $4 / 24 / 89$ & REG & Toluene & 5 & $\mathrm{ug} / \mathrm{L}$ & $\mathrm{U}$ \\
\hline 843 & M01254 & $11 / 20 / 97$ & REG & trans-1.2-Dichloroethe & 5 & $\mathrm{ug} / L$ & $\mathrm{U}$ \\
\hline 843 & M00057 & $11 / 21 / 96$ & REG & trans-1,2-Dichloroethene & 5 & $\mathrm{ug} / \mathrm{L}$ & $\mathrm{U}$ \\
\hline 843 & M00847 & $6 / 25 / 97$ & REG & trans-1.2-Dichloroethene & 5 & $\mathrm{ug} / \mathrm{L}$ & U \\
\hline 843 & S00885 & $10 / 11 / 94$ & REG & trans-1.2-Dichloroethene & 5 & $\mathrm{ug} / \mathrm{L}$ & $\mathrm{U}$ \\
\hline 843 & S02625 & $2 / 16 / 95$ & REG & trans-1.2-Dichloroethene & 5 & $\mathrm{ug} / \mathrm{L}$ & $\mathrm{U}$ \\
\hline 843 & S03587 & $5 / 5 / 95$ & REG & trans-1.2-Dichloroethene & 5 & $\mathrm{ug} / \mathrm{L}$ & $U$ \\
\hline 843 & S04802 & $7 / 19 / 95$ & REG & trans-1.2-Dichloroethene & 5 & $\mathrm{ug} / \mathrm{L}$ & $\mathrm{U}$ \\
\hline 843 & M01254 & $11 / 20 / 97$ & REG & trans-1.3-Dichloroprop & 5 & $\mathrm{ug} / \mathrm{L}$ & $\mathrm{U}$ \\
\hline 843 & W00810 & $6 / 25 / 97$ & REG & trans-1.3-Dichloropropene & 5 & $\mathrm{ug} / \mathrm{L}$ & $\mathrm{U}$ \\
\hline $8+3$ & M00057 & $11 / 21 / 96$ & REG & trans-1.3-Dichloropropene & 5 & $\mathrm{ug} / \mathrm{L}$ & $\mathrm{U}$ \\
\hline 843 & M00847 & $6 / 25 / 97$ & REG & trans-1.3-Dichloropropene & 5 & $\mathrm{ug} / \mathrm{L}$ & $\mathrm{U}$ \\
\hline $8+3$ & S00885 & $10 / 11 / 94$ & REG & trans-1,3-Dichloropropene & 5 & $\mathrm{ug} / \mathrm{L}$ & $\mathrm{U}$ \\
\hline $8+3$ & S02625 & $2 / 16 / 95$ & REG & trans-1,3-Dichloropropene & 5 & $\mathrm{ug} / \mathrm{L}$ & $\mathrm{U}$ \\
\hline 843 & S03587 & $5 / 5 / 95$ & REG & trans-1,3-Dichloropropene & 5 & $\mathrm{ug} / \mathrm{L}$ & U \\
\hline 843 & S04802 & $7 / 19 / 95$ & REG & trans-1,3-Dichloropropene & 5 & $\mathrm{ug} / \mathrm{L}$ & $\mathrm{U}$ \\
\hline 843 & 843222REGUF & $2 / 22 / 91$ & REG & trans-1.3-Dichloropropene & 5 & $\mathrm{ug} / \mathrm{L}$ & $\mathrm{U}$ \\
\hline 843 & 843930REGUF & $9 / 30 / 91$ & REG & trans-1,3-Dichloropropene & 5 & ug/L & $\mathrm{U}$ \\
\hline $8+3$ & 843129REGUF & $12 / 9 / 91$ & REG & trans-1.3-Dichloropropene & 5 & $\mathrm{ug} / \mathrm{L}$ & $\mathrm{U}$ \\
\hline 843 & 843518REGUF & $5 / 18 / 90$ & REG & trans-1.3-Dichloropropene & 5 & $\mathrm{ug} / \mathrm{L}$ & $\mathrm{U}$ \\
\hline
\end{tabular}




\begin{tabular}{|c|c|c|c|c|c|c|c|}
\hline Station & Sample ID & Sample Date & Sample Type & Chemical & Results & Units & Qualifier \\
\hline$\overline{8+3}$ & 843118REGUF & $11 / 8 / 90$ & REG & trans-1.3-Dichloropropene & 5 & $\mathrm{ug} / \mathrm{L}$ & $\mathrm{U}$ \\
\hline 843 & 843125REGUF & $1 / 25 / 89$ & REG & trans-1.3-Dichloropropene & 5 & $\mathrm{ug} / \mathrm{L}$ & $\mathrm{U}$ \\
\hline $8+3$ & 843424REGUF & $4 / 24 / 89$ & REG & trans-1.3-Dichloropropene & 5 & $u g / L$ & $\mathrm{U}$ \\
\hline $8+3$ & $\mathrm{M} 01254$ & $11 / 20 / 97$ & REG & Trichloroethene & 5 & $\mathrm{ug} / \mathrm{L}$ & $\mathrm{U}$ \\
\hline $8+3$ & W00810 & $6 / 25 / 97$ & REG & Trichloroethene & 2 & $\mathrm{ug} / \mathrm{L}$ & $\mathrm{U}$ \\
\hline 843 & M00057 & $11 / 21 / 96$ & REG & Trichloroethene & 5 & $\mathrm{ug} / \mathrm{L}$ & $\mathrm{U}$ \\
\hline 843 & M00847 & $6 / 25 / 97$ & REG & Trichloroethene & 2 & $\mathrm{ug} / \mathrm{L}$ & $\mathrm{U}$ \\
\hline 843 & S00885 & $10 / 11 / 94$ & REG & Trichloroethene & 5 & $\mathrm{ug} / \mathrm{L}$ & $\mathrm{U}$ \\
\hline 843 & S02625 & $2 / 16 / 95$ & REG & Trichloroethene & 5 & $\mathrm{ug} / \mathrm{L}$ & $\mathrm{U}$ \\
\hline 843 & S03587 & $5 / 5 / 95$ & REG & Trichloroethene & 5 & $\mathrm{ug} / \mathrm{L}$ & $\mathrm{U}$ \\
\hline 843 & S04802 & $7 / 19 / 95$ & REG & Trichloroethene & 5 & $\mathrm{ug} / \mathrm{L}$ & $\mathrm{U}$ \\
\hline 843 & 843222REGUF & $2 / 22 / 91$ & REG & Trichloroethene & 5 & $\mathrm{ug} / \mathrm{L}$ & U \\
\hline 843 & 843930REGUF & $9 / 30 / 91$ & REG & Trichloroethene & 5 & $\mathrm{ug} / \mathrm{L}$ & $\mathrm{U}$ \\
\hline 843 & 843129REGUF & $12 / 9 / 91$ & REG & Trichloroethene & 5 & ug $/$ & $U$ \\
\hline 843 & 843518REGUF & $5 / 18 / 90$ & REG & Trichloroethene & 5 & ug/L & $\mathrm{U}$ \\
\hline 843 & 843118REGUF & $11 / 8 / 90$ & REG & Trichloroethene & 5 & $u g / L$ & $\mathrm{U}$ \\
\hline 843 & 843125REGUF & $1 / 25 / 89$ & REG & Trichloroethene & 5 & $u g / L$ & $\mathrm{U}$ \\
\hline 843 & 843424REGUF & $4 / 24 / 89$ & REG & Trichloroethene & 1 & $\mathrm{ug} / \mathrm{L}$ & $\mathrm{J}$ \\
\hline 843 & M01254 & $11 / 20 / 97$ & REG & Vinyl acetate & 10 & $\mathrm{ug} / \mathrm{L}$ & $\mathrm{U}$ \\
\hline 843 & M00057 & $11 / 21 / 96$ & REG & Vinyl acetate & 10 & ug/L & $\mathrm{U}$ \\
\hline 843 & $\mathrm{M} 00847$ & $6 / 25 / 97$ & REG & Vinyl acetate & 10 & $\mathrm{ug} / \mathrm{L}$ & $\mathrm{U}$ \\
\hline 843 & S00885 & $10 / 11 / 94$ & REG & Vinyl acetate & 10 & $u g / L$ & $\mathrm{U}$ \\
\hline 843 & S02625 & $2 / 16 / 95$ & REG & Vinyl acetate & 10 & ug $/ \mathrm{L}$ & $\mathrm{U}$ \\
\hline 843 & S03587 & $5 / 5 / 95$ & REG & Vinyl acetate & 10 & ug $/ \mathrm{L}$ & $\mathrm{U}$ \\
\hline 843 & S04802 & $7 / 19 / 95$ & REG & Vinyl acetate & 10 & ug/L & $\mathrm{U}$ \\
\hline 843 & 843222REGUF & $2 / 22 / 91$ & REG & Vinyl acetate & 10 & ug/L & $\mathrm{U}$ \\
\hline 843 & 843930REGUF & 9/30/91 & REG & Vinyl acetate & 10 & ug/L & $\mathrm{U}$ \\
\hline 843 & 843129REGUF & $12 / 9 / 91$ & REG & Vinvil acetate & 10 & $u g / L$ & $\mathbf{U}$ \\
\hline 843 & 843518REGUF & $5 / 18 / 90$ & REG & Vinyl acetate & 10 & $\mathrm{ug} / \mathrm{L}$ & $\mathrm{U}$ \\
\hline 843 & 843118REGUF & $11 / 8 / 90$ & REG & Vinyl acetate & 10 & $\mathrm{ug} / \mathrm{L}$ & $\mathrm{U}$ \\
\hline 843 & 843125REGUF & $1 / 25 / 89$ & REG & Vinyl acetate & 10 & ug/ & $\mathrm{U}$ \\
\hline 843 & 843424REGUF & $4 / 24 / 89$ & REG & Vinyl acetate & 10 & $\mathrm{ug} / \mathrm{L}$ & $\mathrm{U}$ \\
\hline 843 & M01254 & $11 / 20 / 97$ & REG & Vinyll chloride & 10 & ug/L & $\mathrm{U}$ \\
\hline 843 & W00810 & $6 / 25 / 97$ & REG & Vinyl chloride & 10 & $\mathrm{ug} / \mathrm{L}$ & $\mathrm{U}$ \\
\hline 843 & M00057 & $11 / 21 / 96$ & REG & Vinyl chloride & 10 & ug/L & $\mathrm{U}$ \\
\hline 843 & M00847 & $6 / 25 / 97$ & REG & Vinyl chloride & 10 & $\mathrm{ug} / \mathrm{L}$ & $\mathbf{U}$ \\
\hline 843 & S00885 & $10 / 11 / 94$ & REG & Vinyl chloride & 10 & $\mathrm{ug} / \mathrm{L}$ & $\mathrm{U}$ \\
\hline 843 & S02625 & $2 / 16 / 95$ & REG & Vinyl chloride & 10 & ug/ & $\mathrm{U}$ \\
\hline 843 & S03587 & $5 / 5 / 95$ & REG & Vinyll chloride & 10 & $\mathrm{ug} / \mathrm{L}$ & $\mathrm{U}$ \\
\hline 843 & S04802 & $7 / 19 / 95$ & REG & Vinyl chloride & 10 & $\mathrm{ug} / \mathrm{L}$ & $U$ \\
\hline 843 & 843222REGUF & $2 / 22 / 91$ & REG & Vinỵl chloride & 10 & $\mathrm{ug} / \mathrm{L}$ & $\mathrm{U}$ \\
\hline 843 & 843930REGUF & $9 / 30 / 91$ & REG & Vinyl chloride & 10 & $\mathrm{ug} / \mathrm{L}$ & $\mathrm{U}$ \\
\hline $8+3$ & 843129REGUF & $12 / 9 / 91$ & REG & Vinyl chloride & 10 & $\mathrm{ug} / \mathrm{L}$ & $\mathrm{U}$ \\
\hline 843 & 843518REGUF & $5 / 18 / 90$ & REG & Vinyl chloride & 10 & ug/L & $\mathrm{U}$ \\
\hline $8+3$ & 843118REGUF & $11 / 8 / 90$ & REG & Vinyl chloride & 10 & $\mathrm{ug} / \mathrm{L}$ & $\mathrm{U}$ \\
\hline 843 & 843125REGUF & $1 / 25 / 89$ & REG & Vinyl chloride & 10 & $\mathrm{ug} / \mathrm{L}$ & $\mathrm{U}$ \\
\hline 843 & 843424REGUF & $4 / 24 / 89$ & REG & Vinyl chloride & 10 & $\mathrm{ug} / \mathrm{L}$ & $\mathrm{U}$ \\
\hline 844 & M01267 & $11 / 20 / 97$ & REG & 1.1.1-Trichloroethane & 5 & $\mathrm{ug} / \mathrm{L}$ & $\mathrm{U}$ \\
\hline 844 & W00811 & $6 / 25 / 97$ & REG & 1.1.1-Trichloroethane & 5 & $\mathrm{ug} / \mathrm{L}$ & $\mathrm{U}$ \\
\hline 844 & M00066 & $11 / 26 / 96$ & REG & 1.1.1-Trichloroethane & 5 & $\mathrm{ug} / \mathrm{L}$ & $\mathrm{U}$ \\
\hline
\end{tabular}


B-57

\begin{tabular}{|c|c|c|c|c|c|c|c|}
\hline Station & Sample ID & Sample Date & Sample Type & Chemical & Results & Units & Qualifier \\
\hline $8+4$ & $M(0) 858$ & $6 / 25 / 97$ & REG & 1.1.1-Trichloroethane & 5 & ug/L & $\mathrm{U}$ \\
\hline 844 & So090)9 & $10 / 12 / 94$ & REG & 1.1.1-Trichloroethane & 5 & ug $/ \mathrm{L}$ & $\mathrm{U}$ \\
\hline 844 & S02634 & $2 / 7 / 95$ & REG & 1.1.1-Trichloroethane & 5 & $\mathrm{ug} / \mathrm{L}$ & $\mathrm{U}$ \\
\hline 844 & S03598 & $5 / 17 / 95$ & REG & 1.1.1-Trichloroethane & 5 & $\mathrm{ug} / \mathrm{L}$ & $\mathrm{U}$ \\
\hline 844 & $\mathrm{~S} 04811$ & $7 / 19 / 95$ & REG & 1.1.1-Trichloroethane & 5 & $\mathrm{ug} / \mathrm{L}$ & U \\
\hline 844 & $84+220$ REGUF & $2 / 20 / 91$ & REG & 1.1.1-Trichloroethane & 5 & ug/L & $\mathrm{U}$ \\
\hline 844 & $8+410$ IREGUF & $10 / 1 / 91$ & REG & 1.1.1-Trichloroethane & 5 & ug/L & $\mathrm{U}$ \\
\hline 844 & 8+4129REGUF & $12 / 9 / 91$ & REG & 1.1.1-Trichloroethane & 1 & ug/L & J \\
\hline 844 & 844518REGUF & $5 / 18 / 90$ & REG & 1.1.1-Trichloroethane & 5 & $u g / L$ & $\mathrm{U}$ \\
\hline 844 & 844118REGUF & $11 / 8 / 90$ & REG & 1.1.1-Trichloroethane & 5 & $\mathrm{ug} / \mathrm{L}$ & $\mathrm{U}$ \\
\hline 844 & 844125REGUF & $1 / 25 / 89$ & REG & 1.1.1-Trichloroethane & 5 & $\mathrm{ug} / \mathrm{L}$ & $\mathrm{U}$ \\
\hline 844 & 8444IIREGUF & $4 / 11 / 89$ & REG & 1.1.1-Trichloroethane & 5 & $\mathrm{ug} / \mathrm{L}$ & $U$ \\
\hline $8+4$ & 84476REGUF & $7 / 6 / 88$ & REG & 1.1.1-Trichloroethane & 5 & $\mathrm{ug} / \mathrm{L}$ & $\mathrm{U}$ \\
\hline 844 & 844 102 IREGUF & $10 / 21 / 88$ & REG & 1.1.1-Trichloroethane & 5 & $\mathrm{ug} / \mathrm{L}$ & $\mathrm{U}$ \\
\hline 844 & M01267 & $11 / 20 / 97$ & REG & 1.1.2.2-Tetrachloroeth & 5 & $\mathrm{ug} / \mathrm{L}$ & $\mathrm{U}$ \\
\hline 844 & W00811 & $6 / 25 / 97$ & REG & 1.1.2,2-Tetrachloroethane & 5 & $\mathrm{ug} / \mathrm{L}$ & $\mathrm{U}$ \\
\hline 844 & M00066 & $11 / 26 / 96$ & REG & 1.1.2.2-Tetrachloroethane & 5 & $\mathrm{ug} / \mathrm{L}$ & $\mathrm{U}$ \\
\hline 844 & M00858 & $6 / 25 / 97$ & REG & 1.1.2,2-Tetrachloroethane & 5 & $\mathrm{ug} / \mathrm{L}$ & $\mathrm{U}$ \\
\hline 844 & S00909 & $10 / 12 / 94$ & REG & 1.1.2.2-Tetrachloroethane & 5 & $\mathrm{ug} / \mathrm{L}$ & $\mathrm{U}$ \\
\hline 844 & S02634 & $2 / 7 / 95$ & REG & 1.1.2.2-Tetrachloroethane & 5 & $\mathrm{ug} / \mathrm{L}$ & $\mathrm{U}$ \\
\hline 844 & S03598 & $5 / 17 / 95$ & REG & 1.1.2.2-Tetrachloroethane & 5 & $\mathrm{ug} / \mathrm{L}$ & $\mathrm{U}$ \\
\hline 844 & S04811 & $7 / 19 / 95$ & REG & 1.1,2.2-Tetrachloroethane & 5 & $\mathrm{ug} / \mathrm{L}$ & $\mathrm{U}$ \\
\hline 844 & 844220REGUF & $2 / 20 / 91$ & REG & 1.1.2.2-Tetrachloroethane & 5 & $\mathrm{ug} / \mathrm{L}$ & $\mathrm{U}$ \\
\hline 844 & 844101REGUF & $10 / 1 / 91$ & REG & 1.1.2.2-Tetrachloroethane & 5 & ug/L & $\mathrm{U}$ \\
\hline $8+4$ & 844129REGUF & $12 / 9 / 91$ & REG & 1.1.2.2-Tetrachloroethane & 5 & $\mathrm{ug} / \mathrm{L}$ & $\mathrm{U}$ \\
\hline 844 & 844518REGUF & $5 / 18 / 90$ & REG & 1.1.2.2-Tetrachloroethane & 5 & $\mathrm{ug} / \mathrm{L}$ & $\mathrm{U}$ \\
\hline 844 & 844118REGUF & $11 / 8 / 90$ & REG & 1.1.2.2-Tetrachloroethane & 5 & $\mathrm{ug} / \mathrm{L}$ & $U$ \\
\hline 844 & $8+4125$ REGUF & $1 / 25 / 89$ & REG & 1.1.2.2-Tetrachloroethane & 5 & $\mathrm{ug} / \mathrm{L}$ & $\mathrm{U}$ \\
\hline 844 & 8444111REGUF & $4 / 11 / 89$ & REG & 1.1.2.2-Tetrachloroethane & 5 & $\mathrm{ug} / \mathrm{L}$ & $\mathrm{U}$ \\
\hline 844 & 84476REGUF & $7 / 6 / 88$ & REG & 1.1.2.2-Tetrachloroethane & 5 & $\mathrm{ug} / \mathrm{L}$ & $\mathrm{U}$ \\
\hline 844 & 8441021REGUF & $10 / 21 / 88$ & REG & 1.1.2.2-Tetrachloroethane & 5 & $\mathrm{ug} / \mathrm{L}$ & $\mathrm{U}$ \\
\hline 844 & w00811 & $6 / 25 / 97$ & REG & 1.1.2-Trichloro-1.2.2-trifluo & 10 & $\mathrm{ug} / \mathrm{L}$ & $\mathrm{U}$ \\
\hline 844 & M01267 & $11 / 20 / 97$ & REG & 1.1.2-Trichloroethane & 5 & $\mathrm{ug} / \mathrm{L}$ & $\mathrm{U}$ \\
\hline 844 & W00811 & $6 / 25 / 97$ & REG & 1.1.2-Trichloroethane & 5 & $\mathrm{ug} / \mathrm{L}$ & $\mathrm{U}$ \\
\hline $8+4$ & $\mathrm{M} \cap 0066$ & $11 / 26 / 96$ & REG & 1.1.2-Trichloroethane & 5 & $\mathrm{ug} / \mathrm{L}$ & L' \\
\hline 844 & M00858 & $6 / 25 / 97$ & REG & 1.1.2-Trichloroethane & 5 & $\mathrm{ug} / \mathrm{L}$ & $\mathrm{U}$ \\
\hline $8+4$ & S00909 & $10 / 12 / 94$ & REG & 1.1.2-Trichloroethane & 5 & $\mathrm{ug} / \mathrm{L}$ & $\mathrm{U}$ \\
\hline $8+4$ & S02634 & $2 / 7 / 95$ & REG & 1.1.2-Trichloroethane & 5 & $\mathrm{ug} / \mathrm{L}$ & $\mathrm{U}$ \\
\hline 844 & S03598 & $5 / 17 / 95$ & REG & 1.1.2-Trichloroethane & 5 & $\mathrm{ug} / \mathrm{L}$ & $\mathrm{U}$ \\
\hline 844 & S04811 & $7 / 19 / 95$ & REG & 1.1.2-Trichloroethane & 5 & $\mathrm{ug} / \mathrm{L}$ & $\mathrm{U}$ \\
\hline 844 & 84+220REGUF & $2 / 20 / 91$ & REG & 1.1.2-Trichloroethane & 5 & $\mathrm{ug} / \mathrm{L}$ & $\mathrm{U}$ \\
\hline 844 & 844101REGUF & $10 / 1 / 91$ & REG & 1.1.2-Trichloroethane & 5 & ug/L & $\mathrm{U}$ \\
\hline 844 & 844129REGUF & $12 / 9 / 91$ & REG & 1.1.2-Trichloroethane & 5 & $\mathrm{ug} / \mathrm{L}$ & $\mathrm{U}$ \\
\hline 844 & $844518 R E G U F$ & $5 / 18 / 90$ & REG & 1.1.2-Trichloroethane & 5 & $\mathrm{ug} / \mathrm{L}$ & $\mathrm{U}$ \\
\hline 844 & 844118REGUF & $11 / 8 / 90$ & REG & 1.1.2-Trichloroethane & 5 & $\mathrm{ug} / \mathrm{L}$ & $\mathbf{U}$ \\
\hline 844 & 844125REGUF & $1 / 25 / 89$ & REG & 1.1.2-Trichloroethane & 5 & $\mathrm{ug} / \mathrm{L}$ & $\mathrm{U}$ \\
\hline $8+4$ & 844411REGUF & $4 / 11 / 89$ & REG & 1.1.2-Trichloroethane & 5 & $\mathrm{ug} / \mathrm{L}$ & $\mathrm{U}$ \\
\hline $8+4$ & 84476REGUF & $7 / 6 / 88$ & REG & 1.1.2-Trichloroethane & 5 & $\mathrm{ug} / \mathrm{L}$ & $\mathrm{U}$ \\
\hline 844 & $84+1021$ REGUF & $10 / 21 / 88$ & REG & 1.1.2-Trichloroethane & 5 & ug/L & $\mathrm{U}$ \\
\hline 844 & $\mathrm{M} 01267$ & $11 / 20 / 97$ & REG & 1.1-Dichloroethane & 5 & $\mathrm{ug} / \mathrm{L}$ & U \\
\hline
\end{tabular}


B-58

\begin{tabular}{|c|c|c|c|c|c|c|c|}
\hline Station & Sample ID & Sample Date & Sample Type & Chemical & Results & Units & Qualifier \\
\hline $8+4$ & W00811 & $6 / 25 / 97$ & REG & 1.1-Dichloroethane & 5 & $\mathrm{ug} / \mathrm{L}$ & $\bar{U}$ \\
\hline 844 & M00066 & $11 / 26 / 96$ & REG & 1.1-Dichloroethane & 5 & $\mathrm{ug} / \mathrm{L}$ & $\mathrm{U}$ \\
\hline $8+4$ & M00858 & $6 / 25 / 97$ & REG & 1.1-Dichloroethane & 5 & $\mathrm{ug} / \mathrm{L}$ & $\mathrm{U}$ \\
\hline $8+4$ & S00909 & $10 / 12 / 94$ & REG & 1.1-Dichlorocthane & 5 & $u g / L$ & U \\
\hline $8+4$ & S02634 & $2 / 7 / 95$ & REG & 1.1-Dichloroethane & 5 & ug/L & $\mathrm{U}$ \\
\hline 844 & S03598 & $5 / 17 / 95$ & REG & 1.1-Dichloroethane & 5 & ug/ $/$ & $\mathrm{U}$ \\
\hline $8+4$ & S04811 & $7 / 19 / 95$ & REG & 1.1-Dichloroethane & 5 & $\mathrm{ug} / \mathrm{L}$ & $\mathrm{U}$ \\
\hline 844 & 844220REGUF & $2 / 20 / 91$ & REG & 1.1-Dichloroethane & 5 & $\mathrm{ug} / \mathrm{L}$ & $\mathrm{U}$ \\
\hline 844 & 844101REGUF & $10 / 1 / 91$ & REG & 1,1-Dichloroethane & 5 & ug/L & $\mathrm{U}$ \\
\hline 844 & 844129REGUF & $12 / 9 / 91$ & REG & 1,1-Dichloroethane & 5 & ug/L & $\mathrm{U}$ \\
\hline 844 & 844518REGUF & $5 / 18 / 90$ & REG & 1.1-Dichloroethane & 5 & $\mathrm{ug} / \mathrm{L}$ & $\mathrm{U}$ \\
\hline 844 & 844118REGUF & $11 / 8 / 90$ & REG & 1.1-Dichloroethane & 5 & ug/L & $\mathrm{U}$ \\
\hline 844 & $844125 R E G U F$ & $1 / 25 / 89$ & REG & 1.1-Dichloroethane & 5 & $\mathrm{ug} / \mathrm{L}$ & $\mathrm{U}$ \\
\hline 844 & 84441 IREGUF & $4 / 11 / 89$ & REG & 1,1-Dichloroethane & 5 & $\mathrm{ug} / \mathrm{L}$ & $\mathrm{U}$ \\
\hline 844 & 84476REGUF & $7 / 6 / 88$ & REG & 1.1-Dichloroethane & 5 & $\mathrm{ug} / \mathrm{L}$ & $\mathrm{U}$ \\
\hline 844 & 844102 IREGUF & $10 / 21 / 88$ & REG & 1.1-Dichloroethane & 5 & $\mathrm{ug} / \mathrm{L}$ & $\mathrm{U}$ \\
\hline 844 & M01267 & $11 / 20 / 97$ & REG & 1.1-Dichloroethene & 5 & $\mathrm{ug} / \mathrm{L}$ & $\mathrm{U}$ \\
\hline 844 & W00811 & $6 / 25 / 97$ & REG & 1,1-Dichloroethene & 5 & $\mathrm{ug} / \mathrm{L}$ & $\mathrm{U}$ \\
\hline 844 & M00066 & $11 / 26 / 96$ & REG & 1.1-Dichloroethene & 5 & $\mathrm{ug} / \mathrm{L}$ & $\mathrm{U}$ \\
\hline 844 & M00858 & $6 / 25 / 97$ & REG & 1.1-Dichloroethene & 5 & ug/L & $\mathrm{U}$ \\
\hline 844 & S00909 & $10 / 12 / 94$ & REG & 1.1-Dichloroethene & 5 & ug/L & $\mathrm{U}$ \\
\hline 844 & S02634 & $2 / 7 / 95$ & REG & 1.1-Dichloroethene & 5 & $\mathrm{ug} / \mathrm{L}$ & $\mathrm{U}$ \\
\hline 844 & S03598 & $5 / 17 / 95$ & REG & 1,1-Dichloroethene & 5 & ug/L & $\mathrm{U}$ \\
\hline 844 & S04811 & $7 / 19 / 95$ & REG & 1,1-Dichloroethene & 5 & $\mathrm{ug} / \mathrm{L}$ & $\mathrm{U}$ \\
\hline 844 & 844220REGUF & $2 / 20 / 91$ & REG & 1,1-Dichloroethene & 5 & $\mathrm{ug} / \mathrm{L}$ & $\mathrm{U}$ \\
\hline 844 & 844101REGUF & $10 / 1 / 91$ & REG & 1,1-Dichloroethene & 5 & ug/L & $\mathrm{U}$ \\
\hline 844 & 844129REGUF & $12 / 9 / 91$ & REG & 1.1-Dichloroethene & 5 & $\mathrm{ug} / \mathrm{L}$ & $\mathrm{U}$ \\
\hline 844 & 844518REGUF & $5 / 18 / 90$ & REG & 1.1-Dichloroethene & 5 & $\mathrm{ug} / \mathrm{L}$ & $\mathrm{U}$ \\
\hline 844 & 844118REGUF & $11 / 8 / 90$ & REG & 1,1-Dichloroethene & 5 & $\mathrm{ug} / \mathrm{L}$ & $\mathrm{U}$ \\
\hline 844 & 844125REGUF & $1 / 25 / 89$ & REG & 1,1-Dichloroethene & 5 & ug/L & $\mathrm{U}$ \\
\hline 844 & 844411REGUF & $4 / 11 / 89$ & REG & 1,1-Dichloroethene & 5 & $\mathrm{ug} / \mathrm{L}$ & $\mathrm{U}$ \\
\hline 844 & 84476REGUF & $7 / 6 / 88$ & REG & 1.1-Dichloroethene & 5 & ug/L & $\mathrm{U}$ \\
\hline 844 & 8441021 REGUF & $10 / 21 / 88$ & REG & 1,1-Dichloroethene & 5 & ug/L & $\mathrm{U}$ \\
\hline 844 & M00066 & $11 / 26 / 96$ & REG & 1,2-Dichlorobenzene & 5 & ug/ & $\mathrm{U}$ \\
\hline 844 & $\mathrm{M} 01267$ & $11 / 20 / 97$ & REG & 1.2-Dichloroethane & 5 & $\mathrm{ug} / \mathrm{L}$ & $U$ \\
\hline 844 & W00811 & $6 / 25 / 97$ & REG & 1.2-Dichloroethane & 5 & $\mathrm{ug} / \mathrm{L}$ & $\mathrm{U}$ \\
\hline $8+4$ & M00066 & $11 / 26 / 96$ & REG & 1.2-Dichloroethane & 5 & ug/L & $\mathrm{U}$ \\
\hline 844 & M00858 & $6 / 25 / 97$ & REG & 1.2-Dichloroethane & 5 & ug/L & $\mathrm{U}$ \\
\hline 844 & S00909 & $10 / 12 / 94$ & REG & 1.2-Dichloroethane & 5 & ug/L & $\mathrm{U}$ \\
\hline 844 & S02634 & $2 / 7 / 95$ & REG & 1.2-Dichloroethane & 5 & $\mathrm{ug} / \mathrm{L}$ & $\mathrm{U}$ \\
\hline 844 & S03598 & $5 / 17 / 95$ & REG & 1.2-Dichloroethane & 5 & ug $/ \mathrm{L}$ & $\mathrm{U}$ \\
\hline 844 & S04811 & $7 / 19 / 95$ & REG & 1,2-Dichloroethane & 5 & $\mathrm{ug} / \mathrm{L}$ & $\mathrm{U}$ \\
\hline 844 & 844220REGUF & $2 / 20 / 91$ & REG & 1.2-Dichloroethane & 5 & $\mathrm{ug} / \mathrm{L}$ & $\mathrm{U}$ \\
\hline 844 & 844101REGUF & $10 / 1 / 91$ & REG & 1.2-Dichloroethane & 5 & $\mathrm{ug} / \mathrm{L}$ & U \\
\hline $8+4$ & 844129REGUF & $12 / 9 / 91$ & REG & 1.2-Dichloroethane & 5 & $\mathrm{ug} / \mathrm{L}$ & $\mathrm{U}$ \\
\hline 844 & 844518REGUF & $5 / 18 / 90$ & REG & 1.2-Dichloroethane & 5 & $\mathrm{ug} / \mathrm{L}$ & U \\
\hline 844 & 844118REGUF & $11 / 8 / 90$ & REG & 1,2-Dichloroethane & 5 & ug/L & $\mathrm{U}$ \\
\hline 844 & 844125REGUF & $1 / 25 / 89$ & REG & 1.2-Dichloroethane & 5 & $\mathrm{ug} / \mathrm{L}$ & $\mathrm{U}$ \\
\hline 844 & 84441 IREGUF & $4 / 11 / 89$ & REG & 1.2-Dichloroethane & 5 & $\mathrm{ug} / \mathrm{L}$ & $\mathrm{U}$ \\
\hline 844 & 84476REGUF & $7 / 6 / 88$ & REG & 1.2-Dichloroethane & 5 & $\mathrm{ug} / \mathrm{L}$ & $\mathrm{U}$ \\
\hline
\end{tabular}




\begin{tabular}{|c|c|c|c|c|c|c|c|}
\hline Station & Sample ID & Sample Date & Sample Type & Chemical & Results & Units & Qualifier \\
\hline $8+4$ & $8+4102$ IREGUF & $10 / 21 / 88$ & REG & 1.2-Dichloroethane & 5 & $\mathrm{ug} / \mathrm{L}$ & $\mathrm{U}$ \\
\hline 844 & W00811 & $6 / 25 / 97$ & REG & 1.2-Dichloroethene & 5 & $\mathrm{ug} / \mathrm{L}$ & U \\
\hline 844 & M00066 & $11 / 26 / 96$ & REG & 1.2-Dichloroethene & 5 & $\mathrm{ug} / \mathrm{L}$ & $\mathrm{U}$ \\
\hline 844 & S00909 & $10 / 12 / 94$ & REG & 1.2-Dichloroethene & 5 & $\mathrm{ug} / \mathrm{L}$ & $\mathrm{U}$ \\
\hline $84 t$ & S02634 & $2 / 7 / 95$ & REG & 1.2-Dichloroethene & 5 & $\mathrm{ug} / \mathrm{L}$ & $\mathrm{U}$ \\
\hline 844 & S03598 & $5 / 17 / 95$ & REG & 1.2-Dichloroethene & 5 & ug/L & $\mathrm{U}$ \\
\hline $8+4$ & S04811 & $7 / 19 / 95$ & REG & 1.2-Dichloroethene & 5 & ug $/ \mathrm{L}$ & $\mathrm{U}$ \\
\hline $8+4$ & 844220REGUF & $2 / 20 / 91$ & REG & 1.2-Dichloroethene & 5 & ug $\Omega$ & $\mathrm{U}$ \\
\hline $8+4$ & 844101REGUF & $10 / 1 / 91$ & REG & 1,2-Dichloroethene & 5 & $\mathrm{ug} / \mathrm{L}$ & $\mathrm{U}$ \\
\hline 844 & 844129REGUF & $12 / 9 / 91$ & REG & 1.2-Dichloroethene & 5 & $u g / L$ & $\mathrm{U}$ \\
\hline 844 & 844518REGUF & $5 / 18 / 90$ & REG & 1.2-Dichloroethene & 8 & $\mathrm{ug} / \mathrm{L}$ & $=$ \\
\hline 844 & 844118REGUF & $11 / 8 / 90$ & REG & 1.2-Dichloroethene & 5 & $\mathrm{ug} /$ & $\mathrm{U}$ \\
\hline 844 & 844125REGUF & $1 / 25 / 89$ & REG & 1,2-Dichloroethene & 5 & ug/L & $\mathrm{U}$ \\
\hline 844 & 844411 REGUF & $4 / 11 / 89$ & REG & 1,2-Dichloroethene & 5 & $\mathrm{ug} / \mathrm{L}$ & $\mathrm{U}$ \\
\hline 844 & 84476REGUF & $7 / 6 / 88$ & REG & 1,2-Dichloroethene & 5 & $\mathrm{ug} / \mathrm{L}$ & $\mathrm{U}$ \\
\hline 844 & 8441021 REGUF & $10 / 21 / 88$ & REG & 1.2-Dichloroethene & 5 & ug/L & $\mathrm{U}$ \\
\hline 844 & M01267 & $11 / 20 / 97$ & REG & 1,2-Dichloropropane & 5 & $u g / L$ & U \\
\hline 844 & W00811 & $6 / 25 / 97$ & REG & 1.2-Dichloropropane & 5 & $\mathrm{ug} / \mathrm{L}$ & $\mathrm{U}$ \\
\hline 844 & M00066 & $11 / 26 / 96$ & REG & 1.2-Dichloropropane & 5 & $\mathrm{ug} / \mathrm{L}$ & $\mathrm{U}$ \\
\hline 844 & M00858 & $6 / 25 / 97$ & REG & 1,2-Dichloropropane & 5 & $\mathrm{ug} / \mathrm{L}$ & $\mathrm{U}$ \\
\hline 844 & S00909 & $10 / 12 / 94$ & REG & 1.2-Dichloropropane & 5 & $\mathrm{ug} / \mathrm{L}$ & $\mathrm{U}$ \\
\hline 844 & S02634 & $2 / 7 / 95$ & REG & 1.2-Dichloropropane & 5 & $\mathrm{ug} / \mathrm{L}$ & $\mathrm{U}$ \\
\hline $8+4$ & S03598 & $5 / 17 / 95$ & REG & 1.2-Dichloropropane & 5 & $\mathrm{ug} / \mathrm{L}$ & $\mathrm{U}$ \\
\hline 844 & $S 04811$ & $7 / 19 / 95$ & REG & 1.2-Dichloropropane & 5 & $\mathrm{ug} / \mathrm{L}$ & $\mathrm{U}$ \\
\hline $8+4$ & 844220REGUF & $2 / 20 / 91$ & REG & 1,2-Dichloropropane & 5 & $\mathrm{ug} / \mathrm{L}$ & U \\
\hline 844 & 84410IREGUF & $10 / 1 / 91$ & REG & 1,2-Dichloropropane & 5 & $\mathrm{ug} / \mathrm{L}$ & $\mathrm{U}$ \\
\hline $8+4$ & 844129REGUF & $12 / 9 / 91$ & REG & 1,2-Dichloropropane & 5 & $\mathrm{ug} / \mathrm{L}$ & $\mathrm{U}$ \\
\hline 844 & 844518REGUF & $5 / 18 / 90$ & REG & 1.2-Dichloropropane & 5 & $u g / L$ & $\mathrm{U}$ \\
\hline 844 & 844118REGUF & $11 / 8 / 90$ & REG & 1,2-Dichloropropane & 5 & $\mathrm{ug} / \mathrm{L}$ & $U$ \\
\hline 844 & 844125REGUF & $1 / 25 / 89$ & REG & 1,2-Dichloropropane & 5 & $u g / L$ & $\mathrm{U}$ \\
\hline 844 & 84+411REGUF & $4 / 11 / 89$ & REG & 1.2-Dichloropropane & 5 & $\mathrm{ug} / \mathrm{L}$ & $\mathrm{U}$ \\
\hline 844 & 84476REGUF & $7 / 6 / 88$ & REG & 1.2-Dichloropropane & 5 & ug/L & $\mathrm{U}$ \\
\hline $8+4$ & $8+41021$ REGUF & $10 / 21 / 88$ & REG & 1.2-Dichloropropane & 5 & $\mathrm{ug} / \mathrm{L}$ & $\mathrm{U}$ \\
\hline $8+4$ & M00066 & $11 / 26 / 96$ & REG & 1.2-Dimethylbenzene & 5 & ug/L & $\mathrm{U}$ \\
\hline 844 & Sn2634 & $2 / 7 / 95$ & REG & 1.2-Dimethylbenzene & 5 & $\mathrm{ug} / \mathrm{L}$ & $\mathrm{U}$ \\
\hline $8+4$ & S03598 & $5 / 17 / 95$ & REG & 1.2-Dimethylbenzene & 5 & $\mathrm{ug} / \mathrm{L}$ & $\mathrm{U}$ \\
\hline 844 & S04811 & $7 / 19 / 95$ & REG & 1.2-Dimethylbenzene & 5 & $\mathrm{ug} / \mathrm{L}$ & $\mathrm{U}$ \\
\hline 844 & M00066 & $11 / 26 / 96$ & REG & 1.3-Dichlorobenzene & 5 & $\mathrm{ug} / \mathrm{L}$ & $\mathrm{U}$ \\
\hline 844 & M00066 & $11 / 26 / 96$ & REG & 1.4-Dichlorobenzene & 5 & $\mathrm{ug} / \mathrm{L}$ & $\mathrm{U}$ \\
\hline 844 & M01267 & $11 / 20 / 97$ & REG & 2-Butanone & 10 & $\mathrm{ug} / \mathrm{L}$ & $\mathrm{U}$ \\
\hline $8+4$ & W00811 & $6 / 25 / 97$ & REG & 2-Butanone & 10 & $\mathrm{ug} / \mathrm{L}$ & $\mathrm{U}$ \\
\hline 844 & M00066 & $11 / 26 / 96$ & REG & 2-Butanone & 10 & ug/L & $\mathrm{U}$ \\
\hline 844 & M00858 & $6 / 25 / 97$ & REG & 2-Butanone & 10 & $\mathrm{ug} / \mathrm{L}$ & $\mathrm{U}$ \\
\hline 844 & S00909 & $10 / 12 / 94$ & REG & 2-Butanone & 6.4 & $\mathrm{ug} / \mathrm{L}$ & $\mathrm{J}$ \\
\hline 844 & S02634 & $2 / 7 / 95$ & REG & 2-Butanone & 4 & $\mathrm{ug} / \mathrm{L}$ & $\mathrm{J}$ \\
\hline $8+4$ & S03598 & $5 / 17 / 95$ & REG & 2-Butanone & 4 & $\mathrm{ug} / \mathrm{L}$ & $\mathrm{J}$ \\
\hline 844 & S04811 & $7 / 19 / 95$ & REG & 2-Butanone & 10 & $\mathrm{ug} / \mathrm{L}$ & $\mathbf{U}$ \\
\hline 844 & 844220REGUF & $2 / 20 / 91$ & REG & 2-Butanone & 10 & ug/L & $\mathrm{U}$ \\
\hline 844 & 84+101REGUF & $10 / 1 / 91$ & REG & 2-Butanone & 10 & ug/L & $\mathrm{U}$ \\
\hline $8+4$ & 844129REGUF & $12 / 9 / 91$ & REG & 2-Butanone & 10 & ug $/$ & U \\
\hline
\end{tabular}




\begin{tabular}{|c|c|c|c|c|c|c|c|}
\hline Station & Sample ID & Sample Date & Sample Type & Chemical & Results & Units & Qualifier \\
\hline $8+4$ & 844518REGUF & $5 / 18 / 90$ & REG & 2-Butanone & 10 & $\mathrm{ug} / \mathrm{L}$ & $\mathrm{U}$ \\
\hline $8+4$ & 844118REGUF & $11 / 8 / 90$ & REG & 2-Butanone & 10 & $\mathrm{ug} / \mathrm{L}$ & $\mathrm{U}$ \\
\hline $8+4$ & $84+125$ REGUF & $1 / 25 / 89$ & REG & 2-Butanone & 10 & $\mathrm{ug} / \mathrm{L}$ & $\mathrm{U}$ \\
\hline $8+4$ & $8+4+11$ REGUF & $4 / 11 / 89$ & REG & 2-Butanone & 10 & $\mathrm{ug} / \mathrm{L}$ & U \\
\hline $8+4$ & $84+76$ REGUF & $7 / 6 / 88$ & REG & 2-Butanone & 10 & $\mathrm{ug} / \mathrm{L}$ & $\mathrm{U}$ \\
\hline 844 & $8+41021$ REGUF & $10 / 21 / 88$ & REG & 2-Butanone & 10 & $\mathrm{ug} / \mathrm{L}$ & $\mathrm{U}$ \\
\hline 844 & Mo1267 & $11 / 20 / 97$ & REG & 2-Hexanone & 10 & $\mathrm{ug} / \mathrm{L}$ & $\mathrm{U}$ \\
\hline 844 & W00811 & $6 / 25 / 97$ & REG & 2-Hexanone & 10 & $\mathrm{ug} / \mathrm{L}$ & $\mathrm{U}$ \\
\hline 844 & M00066 & $11 / 26 / 96$ & REG & 2-Hexanone & 10 & $\mathrm{ug} / \mathrm{L}$ & U \\
\hline 844 & M00858 & $6 / 25 / 97$ & REG & 2-Hexanone & 10 & $\mathrm{ug} / \mathrm{L}$ & $\mathrm{U}$ \\
\hline 844 & S00909 & $10 / 12 / 94$ & REG & 2-Hexanone & 10 & $\mathrm{ug} / \mathrm{L}$ & $\mathrm{U}$ \\
\hline $8+4$ & S02634 & $2 / 7 / 95$ & REG & 2-Hexanone & 10 & $\mathrm{ug} / \mathrm{L}$ & $U$ \\
\hline $8+4$ & S03598 & $5 / 17 / 95$ & REG & 2-Hexanone & 10 & $\mathrm{ug} / \mathrm{L}$ & $\mathrm{U}$ \\
\hline 844 & S04811 & $7 / 19 / 95$ & REG & 2-Hexanone & 10 & ug/L & $U$ \\
\hline 844 & 844220REGUF & $2 / 20 / 91$ & REG & 2-Hexanone & 10 & $\mathrm{ug} / \mathrm{L}$ & $\mathrm{U}$ \\
\hline 844 & 844101REGUF & $10 / 1 / 91$ & REG & 2-Hexanone & 10 & ug/L & $\mathrm{U}$ \\
\hline 844 & 844129REGUF & $12 / 9 / 91$ & REG & 2-Hexanone & 10 & $\mathrm{ug} / \mathrm{L}$ & $\mathrm{U}$ \\
\hline 844 & 844518REGUF & $5 / 18 / 90$ & REG & 2-Hexanone & 10 & $\mathrm{ug} / \mathrm{L}$ & $\mathrm{U}$ \\
\hline 844 & 844118REGUF & $11 / 8 / 90$ & REG & 2-Hexanone & 10 & $\mathrm{ug} / \mathrm{L}$ & $\mathrm{U}$ \\
\hline 844 & 844125REGUF & $1 / 25 / 89$ & REG & 2-Hexanone & 10 & $\mathrm{ug} / \mathrm{L}$ & $\mathrm{U}$ \\
\hline 844 & 844411REGUF & $4 / 11 / 89$ & REG & 2-Hexanone & 10 & $\mathrm{ug} / \mathrm{L}$ & $\mathrm{U}$ \\
\hline 844 & 84476REGUF & $7 / 6 / 88$ & REG & 2-Hexanone & 10 & $\mathrm{ug} / \mathrm{L}$ & $\mathrm{U}$ \\
\hline 844 & 8441021REGUF & $10 / 21 / 88$ & REG & 2-Hexanone & 10 & $\mathrm{ug} / \mathrm{L}$ & $\mathrm{U}$ \\
\hline 844 & M01267 & $11 / 20 / 97$ & REG & 4-Methyyl-2-pentanone & 10 & $\mathrm{ug} / \mathrm{L}$ & $\mathrm{U}$ \\
\hline $8+4$ & W00811 & $6 / 25 / 97$ & REG & 4-Methyl-2-pentanone & 10 & $\mathrm{ug} / \mathrm{L}$ & $\mathrm{U}$ \\
\hline 844 & M00066 & $11 / 26 / 96$ & REG & 4-Methyl-2-pentanone & 10 & $\mathrm{ug} / \mathrm{L}$ & $\mathrm{U}$ \\
\hline 844 & M00858 & $6 / 25 / 97$ & REG & 4-Methyl-2-pentanone & 10 & $\mathrm{ug} / \mathrm{L}$ & $\mathrm{U}$ \\
\hline $8+4$ & S00909 & $10 / 12 / 94$ & REG & 4-Methyll-2-pentanone & 10 & $\mathrm{ug} / \mathrm{L}$ & U \\
\hline 844 & S02634 & $2 / 7 / 95$ & REG & 4-Methyl-2-pentanone & 10 & $\mathrm{ug} / \mathrm{L}$ & U \\
\hline 844 & S03598 & $5 / 17 / 95$ & REG & 4-Methyl-2-pentanone & 10 & $\mathrm{ug} / \mathrm{L}$ & $\mathrm{U}$ \\
\hline 844 & S04811 & $7 / 19 / 95$ & REG & 4-Methyl-2-pentanone & 10 & $\mathrm{ug} / \mathrm{L}$ & $\mathrm{U}$ \\
\hline $8+4$ & 84+220REGUF & $2 / 20 / 91$ & REG & 4-Methyl-2-pentanone & 10 & $\mathrm{ug} / \mathrm{L}$ & $\mathrm{U}$ \\
\hline 844 & 84+101REGUF & $10 / 1 / 91$ & REG & 4-Methyl-2-pentanone & 10 & $\mathrm{ug} / \mathrm{L}$ & $\mathrm{U}$ \\
\hline $8+4$ & 844129REGUF & $12 / 9 / 91$ & REG & 4-Methỵ1-2-pentanone & 10 & $\mathrm{ug} / \mathrm{L}$ & U \\
\hline 844 & 844518REGUF & $5 / 18 / 90$ & REG & 4-Methili-2-pentanone & 10 & $\mathrm{ug} / \mathrm{L}$ & $\mathrm{U}$ \\
\hline 844 & 84+118REGUF & $11 / 8 / 90$ & REG & 4-Methyll-2-pentanone & 10 & $\mathrm{ug} / \mathrm{L}$ & U \\
\hline 844 & $84+125$ REGUF & $1 / 25 / 89$ & REG & 4-Methyl-2-pentanone & 10 & $\mathrm{ug} / \mathrm{L}$ & U \\
\hline 844 & 84+411REGUF & $4 / 11 / 89$ & REG & 4-Methyl-2-pentanone & 10 & $\mathrm{ug} / \mathrm{L}$ & $\mathrm{U}$ \\
\hline 844 & 84476REGUF & $7 / 6 / 88$ & REG & 4-Methỵl-2-pentanone & 10 & $\mathrm{ug} / \mathrm{L}$ & U \\
\hline 844 & 8441021 REGUF & $10 / 21 / 88$ & REG & 4-Methyl-2-pentanone & 10 & $\mathrm{ug} / \mathrm{L}$ & $\mathrm{U}$ \\
\hline 844 & M01267 & $11 / 20 / 97$ & REG & Acetone & 3 & $\mathrm{ug} / \mathrm{L}$ & B \\
\hline 844 & W00811 & $6 / 25 / 97$ & REG & Acetone & 10 & $\mathrm{ug} / \mathrm{L}$ & $\mathrm{U}$ \\
\hline 844 & M00066 & $11 / 26 / 96$ & REG & Acetone & 10 & $\mathrm{ug} / \mathrm{L}$ & $\mathrm{U}$ \\
\hline 844 & M00858 & $6 / 25 / 97$ & REG & Acetone & 10 & $\mathrm{ug} / \mathrm{L}$ & U \\
\hline 844 & S00909 & $10 / 12 / 94$ & REG & Acetone & 10 & $\mathrm{ug} / \mathrm{L}$ & $\mathrm{U}$ \\
\hline 844 & S0263t & $2 / 7 / 95$ & REG & Acetone & 10 & $u g / L$ & $\mathrm{U}$ \\
\hline 844 & S03598 & $5 / 17 / 95$ & REG & Acetone & 6 & ug/L & J \\
\hline 844 & S04811 & $7 / 19 / 95$ & REG & Acetone & 10 & ug $\Omega$ & $\mathrm{U}$ \\
\hline $8+4$ & $84+220$ REGUF & $2 / 20 / 91$ & REG & Acetone & 10 & $\mathrm{ug} / \mathrm{L}$ & $\mathrm{U}$ \\
\hline $8+4$ & 84+10IREGUF & $10 / 1 / 91$ & REG & Acetone & 5 & $\mathrm{ug} / \mathrm{L}$ & $\mathrm{J}$ \\
\hline
\end{tabular}




\begin{tabular}{|c|c|c|c|c|c|c|c|}
\hline Station & Sample ID & Sample Date & Sample Type & Chemical & Results & Units & Qualifier \\
\hline $8+4$ & $84+129$ REGUF & $12 / 9 / 9 \mid$ & REG & Acetone & 4 & ug/L & $\mathrm{J}$ \\
\hline 844 & $8+4518$ REGUF & $5 / 18 / 90$ & REG & Acetone & 10 & $\mathrm{ug} / \mathrm{L}$ & $U$ \\
\hline 844 & $8+4118$ REGUF & $11 / 8 / 90$ & REG & Acetone & 4 & $\mathrm{ug} / \mathrm{L}$ & J \\
\hline 844 & $84+125$ REGUF & $1 / 25 / 89$ & REG & Acetone & 10 & $\mathrm{ug} / \mathrm{L}$ & U \\
\hline 844 & $8+4+11$ IREGUF & $4 / 11 / 89$ & REG & Acetone & 3 & $\mathrm{ug} / \mathrm{L}$ & J \\
\hline 844 & 84476REGUF & $7 / 6 / 88$ & REG & Acetone & 1 & $\mathrm{ug} / \mathrm{L}$ & $\mathrm{J}$ \\
\hline 844 & $8+41021$ REGUF & $10 / 21 / 88$ & REG & Acetone & 10 & $\mathrm{ug} / \mathrm{L}$ & $\mathrm{U}$ \\
\hline 844 & M01267 & $11 / 20 / 97$ & REG & Benzene & 5 & $\mathrm{ug} / \mathrm{L}$ & $\mathrm{U}$ \\
\hline 844 & W00811 & $6 / 25 / 97$ & REG & Benzene & 5 & $\mathrm{ug} / \mathrm{L}$ & $\mathrm{U}$ \\
\hline 844 & M00066 & $11 / 26 / 96$ & REG & Benzene & 5 & $\mathrm{ug} / \mathrm{L}$ & $\mathrm{U}$ \\
\hline 844 & M00858 & $6 / 25 / 97$ & REG & Benzene & 5 & $\mathrm{ug} / \mathrm{L}$ & $U$ \\
\hline 844 & S00909 & $10 / 12 / 94$ & REG & Benzene & 5 & $\mathrm{ug} / \mathrm{L}$ & $\mathrm{U}$ \\
\hline 844 & S02634 & $2 / 7 / 95$ & REG & Benzene & 5 & $\mathrm{ug} / \mathrm{L}$ & $\mathrm{U}$ \\
\hline 844 & S03598 & $5 / 17 / 95$ & REG & Benzene & 5 & $\mathrm{ug} / \mathrm{L}$ & $\mathrm{U}$ \\
\hline 844 & S04811 & $7 / 19 / 95$ & REG & Benzene & 5 & $\mathrm{ug} / \mathrm{L}$ & $\mathrm{U}$ \\
\hline 844 & 844220REGUF & $2 / 20 / 91$ & REG & Benzene & 5 & $\mathrm{ug} / \mathrm{L}$ & $\mathrm{U}$ \\
\hline 844 & 844101REGUF & $10 / 1 / 91$ & REG & Benzene & 5 & $\mathrm{ug} / \mathrm{L}$ & $\mathrm{U}$ \\
\hline 844 & 844129REGUF & $12 / 9 / 91$ & REG & Benzene & 3 & $\mathrm{ug} / \mathrm{L}$ & $\mathbf{J}$ \\
\hline 844 & 844518REGUF & $5 / 18 / 90$ & REG & Benzene & 5 & $u g / L$ & $\mathrm{U}$ \\
\hline 844 & 844118 REGUF & $11 / 8 / 90$ & REG & Benzene & 5 & $\mathrm{ug} / \mathrm{L}$ & $\mathrm{U}$ \\
\hline 844 & 844125REGUF & $1 / 25 / 89$ & REG & Benzene & 5 & $\mathrm{ug} / \mathrm{L}$ & $\mathrm{U}$ \\
\hline 844 & 84441 IREGUF & $4 / 11 / 89$ & REG & Benzene & 5 & $\mathrm{ug} / \mathrm{L}$ & $\mathrm{U}$ \\
\hline 844 & 84476REGUF & $7 / 6 / 88$ & REG & Benzene & 5 & $\mathrm{ug} / \mathrm{L}$ & $\mathrm{U}$ \\
\hline 844 & 8441021 REGUF & $10 / 21 / 88$ & REG & Benzene & 5 & $\mathrm{ug} / \mathrm{L}$ & $\mathrm{U}$ \\
\hline 844 & Mo1267 & $11 / 20 / 97$ & REG & Bromodichloromethane & 5 & $\mathrm{ug} / \mathrm{L}$ & $\mathrm{U}$ \\
\hline 844 & W00811 & $6 / 25 / 97$ & REG & Bromodichloromethane & 5 & $\mathrm{ug} / \mathrm{L}$ & $\mathrm{U}$ \\
\hline 844 & M00066 & $11 / 26 / 96$ & REG & Bromodichloromethane & 5 & $\mathrm{ug} / \mathrm{L}$ & $\mathrm{U}$ \\
\hline 844 & M00858 & $6 / 25 / 97$ & REG & Bromodichloromethane & 5 & $\mathrm{ug} / \mathrm{L}$ & $U$ \\
\hline 844 & S00909 & $10 / 12 / 94$ & REG & Bromodichloromethane & 5 & $\mathrm{ug} / \mathrm{L}$ & $\mathrm{U}$ \\
\hline 844 & S02634 & $2 / 7 / 95$ & REG & Bromodichloromethane & 5 & $\mathrm{ug} / \mathrm{L}$ & $\mathrm{U}$ \\
\hline 844 & S03598 & $5 / 17 / 95$ & REG & Bromodichloromethane & 5 & $\mathrm{ug} / \mathrm{L}$ & $\mathrm{U}$ \\
\hline 844 & S04811 & $7 / 19 / 95$ & REG & Bromodichloromethane & 5 & $\mathrm{ug} / \mathrm{L}$ & $\mathrm{U}$ \\
\hline 844 & 844220REGUF & $2 / 20 / 91$ & REG & Bromodichloromethane & 5 & $\mathrm{ug} / \mathrm{L}$ & $\mathrm{U}$ \\
\hline 844 & $84+101$ REGUF & $10 / 1 / 91$ & REG & Bromodichloromethane & 5 & $\mathrm{ug} / \mathrm{L}$ & $\mathrm{U}$ \\
\hline 844 & 844129REGUF & $12 / 9 / 91$ & REG & Bromodichloromethane & 5 & $\mathrm{ug} / \mathrm{L}$ & $\mathrm{U}$ \\
\hline 844 & 844518REGUF & $5 / 18 / 90$ & REG & Bromodichloromethane & 5 & $\mathrm{ug} / \mathrm{L}$ & $\mathrm{U}$ \\
\hline 844 & 84+118REGUF & $11 / 8 / 90$ & REG & Bromodichloromethane & 5 & $\mathrm{ug} / \mathrm{L}$ & $\mathrm{U}$ \\
\hline 844 & $84+125$ REGUF & $1 / 25 / 89$ & REG & Bromodichloromethane & 5 & ug/L & $\mathrm{U}$ \\
\hline 844 & $8+4+1$ IREGUF & $4 / 11 / 89$ & REG & Bromodichloromethane & 5 & $\mathrm{ug} / \mathrm{L}$ & $\mathrm{U}$ \\
\hline 844 & 84476REGUF & $7 / 6 / 88$ & REG & Bromodichloromethane & 5 & $\mathrm{ug} / \mathrm{L}$ & $\mathrm{U}$ \\
\hline 844 & 8441021REGUF & $10 / 21 / 88$ & REG & Bromodichloromethane & 5 & $\mathrm{ug} / \mathrm{L}$ & $\mathrm{U}$ \\
\hline 844 & M01267 & $11 / 20 / 97$ & REG & Bromoform & 5 & $\mathrm{ug} / \mathrm{L}$ & $\mathrm{U}$ \\
\hline 844 & W00811 & $6 / 25 / 97$ & REG & Bromoform & 5 & $u g / L$ & $\mathrm{U}$ \\
\hline 844 & M00066 & $11 / 26 / 96$ & REG & Bromoform & 5 & ug/L & $\mathrm{U}$ \\
\hline 844 & M00858 & $6 / 25 / 97$ & REG & Bromoform & 5 & $\mathrm{ug} / \mathrm{L}$ & $\mathrm{U}$ \\
\hline 844 & S00909 & $10 / 12 / 94$ & REG & Bromoform & 5 & $\mathrm{ug} / \mathrm{L}$ & $\mathrm{U}$ \\
\hline 844 & S02634 & $2 / 7 / 95$ & REG & Bromoform & 5 & $\mathrm{ug} / \mathrm{L}$ & $\mathrm{U}$ \\
\hline 844 & S03598 & $5 / 17 / 95$ & REG & Bromoform & 5 & $\mathrm{ug} / \mathrm{L}$ & $\mathrm{U}$ \\
\hline 844 & S04811 & $7 / 19 / 95$ & REG & Bromoform & 5 & $\mathrm{ug} / \mathrm{L}$ & $\mathrm{U}$ \\
\hline 844 & 844220REGUF & $2 / 20 / 91$ & REG & Bromoform & 5 & $\mathrm{ug} / \mathrm{L}$ & $U$ \\
\hline
\end{tabular}




\begin{tabular}{|c|c|c|c|c|c|c|c|}
\hline Station & Sample ID & Sample Date & Sample Type & Chemical & Results & Units & Qualifier \\
\hline $8+4$ & 844101 REGUF & $10 / 1 / 91$ & REG & Bromoform & 5 & $\mathrm{ug} / \mathrm{L}$ & $\overline{\mathrm{U}}$ \\
\hline 844 & 844129REGUF & $12 / 9 / 91$ & REG & Bromoform & 5 & $\mathrm{ug} / \mathrm{L}$ & $\mathrm{U}$ \\
\hline $8+4$ & 844518REGUF & $5 / 18 / 90$ & REG & Bromoform & 5 & $\mathrm{ug} / \mathrm{L}$ & $\mathrm{U}$ \\
\hline $8+4$ & 844118REGUF & $11 / 8 / 90$ & REG & Bromoform & 5 & $\mathrm{ug} / \mathrm{L}$ & $\mathrm{U}$ \\
\hline 844 & 844125 REGUF & $1 / 25 / 89$ & REG & Bromoform & 5 & $\mathrm{ug} / \mathrm{L}$ & U \\
\hline 844 & 844411REGUF & $4 / 11 / 89$ & REG & Bromoform & 5 & ug/L & $\mathrm{U}$ \\
\hline $8+4$ & 84476REGUF & $7 / 6 / 88$ & REG & Bromoform & 5 & ug/L & $\mathrm{U}$ \\
\hline 844 & 844102IREGUF & $10 / 21 / 88$ & REG & Bromoform & 5 & ug $/ \mathrm{L}$ & $\mathrm{U}$ \\
\hline 844 & M01267 & $11 / 20 / 97$ & REG & Bromomethane & 10 & ug/L & U \\
\hline 844 & W00811 & $6 / 25 / 97$ & REG & Bromomethane & 10 & $\mathrm{ug} / \mathrm{L}$ & $\mathrm{U}$ \\
\hline 844 & M00066 & $11 / 26 / 96$ & REG & Bromomethane & 10 & $\mathrm{ug} / \mathrm{L}$ & $\mathrm{U}$ \\
\hline 844 & M00858 & $6 / 25 / 97$ & REG & Bromomethane & 10 & $\mathrm{ug} / \mathrm{L}$ & U \\
\hline 844 & S00909 & $10 / 12 / 94$ & REG & Bromomethane & 10 & ug $/ \mathrm{L}$ & U \\
\hline 844 & S02634 & $2 / 7 / 95$ & REG & Bromomethane & 10 & $\mathrm{ug} / \mathrm{L}$ & U \\
\hline 844 & S03598 & $5 / 17 / 95$ & REG & Bromomethane & 10 & ug/L & U \\
\hline 844 & S04811 & $7 / 19 / 95$ & REG & Bromomethane & 10 & $\mathrm{ug} / \mathrm{L}$ & $\mathrm{U}$ \\
\hline 844 & 844220REGUF & $2 / 20 / 91$ & REG & Bromomethane & 10 & ug/ & $\mathrm{U}$ \\
\hline 844 & 844101REGUF & $10 / 1 / 91$ & REG & Bromomethane & 10 & ug/L & $\mathrm{U}$ \\
\hline 844 & 844129REGUF & $12 / 9 / 91$ & REG & Bromomethane & 10 & $\mathrm{ug} / \mathrm{L}$ & $\mathrm{U}$ \\
\hline 844 & 844518REGUF & $5 / 18 / 90$ & REG & Bromomethane & 10 & $\mathrm{ug} / \mathrm{L}$ & $\mathrm{U}$ \\
\hline $8+4$ & 844118REGUF & $11 / 8 / 90$ & REG & Bromomethane & 10 & $\mathrm{ug} / \mathrm{L}$ & $\mathrm{U}$ \\
\hline $8+4$ & 844125REGUF & $1 / 25 / 89$ & REG & Bromomethane & 10 & ug $/ \mathrm{L}$ & $\mathrm{U}$ \\
\hline 844 & 84441 IREGUF & $4 / 11 / 89$ & REG & Bromomethane & 10 & ug/L & $\mathrm{U}$ \\
\hline 844 & 84476REGUF & $7 / 6 / 88$ & REG & Bromomethane & 10 & ug/L & $\mathrm{U}$ \\
\hline $8+4$ & 8441021REGUF & $10 / 21 / 88$ & REG & Bromomethane & 10 & $\mathrm{ug} / \mathrm{L}$ & $\mathrm{U}$ \\
\hline 844 & M01267 & $11 / 20 / 97$ & REG & Carbon disulfide & 5 & ug $/ \mathrm{L}$ & $\mathrm{U}$ \\
\hline $8+4$ & W00811 & $6 / 25 / 97$ & REG & Carbon disulfide & 5 & ug $/ \mathrm{L}$ & $\mathrm{U}$ \\
\hline $8+4$ & M00066 & $11 / 26 / 96$ & REG & Carbon disulfide & 5 & $\mathrm{ug} / \mathrm{L}$ & $\mathrm{U}$ \\
\hline 844 & M00858 & $6 / 25 / 97$ & REG & Carbon disulfide & 5 & $u g / L$ & $\mathrm{U}$ \\
\hline 844 & S00909 & $10 / 12 / 94$ & REG & Carbon disulfide & 5 & ug/L & $\mathrm{U}$ \\
\hline 844 & S02634 & $2 / 7 / 95$ & REG & Carbon disulfide & 5 & $\mathrm{ug} / \mathrm{L}$ & $\mathrm{U}$ \\
\hline 844 & S03598 & $5 / 17 / 95$ & REG & Carbon disulfide & 4 & ug $/ \mathrm{L}$ & $\mathrm{J}$ \\
\hline $8+4$ & S04811 & $7 / 19 / 95$ & REG & Carbon disulfide & 5 & $\mathrm{ug} / \mathrm{L}$ & $\mathrm{U}$ \\
\hline 844 & 844220REGUF & $2 / 20 / 91$ & REG & Carbon disulfide & 5 & ug $/ \mathrm{L}$ & $\mathrm{U}$ \\
\hline 844 & 84+10IREGUF & $10 / 1 / 91$ & REG & Carbon disulfide & 5 & ug, $I$ & $\mathrm{U}$ \\
\hline 844 & 844129REGUF & $12 / 9 / 91$ & REG & Carbon disulfide & 33 & ug/h & $=$ \\
\hline 844 & 8445 18REGUF & $5 / 18 / 90$ & REG & Carbon disulfide & 5 & $\mathrm{ug} / \mathrm{L}$ & $\mathrm{U}$ \\
\hline 844 & 8441 18REGUF & $11 / 8 / 90$ & REG & Carbon disulfide & 5 & ug/L & $\mathrm{U}$ \\
\hline 844 & 844125REGUF & $1 / 25 / 89$ & REG & Carbon disulfide & 5 & $\mathrm{ug} / \mathrm{L}$ & $\mathrm{U}$ \\
\hline 844 & 84441 IREGUF & $4 / 11 / 89$ & REG & Carbon disulfide & 5 & ug/L & $\mathrm{U}$ \\
\hline $8+4$ & 84476REGUF & $7 / 6 / 88$ & REG & Carbon disulfide & 5 & ug $/ \mathrm{L}$ & $\mathrm{U}$ \\
\hline 844 & 8441021REGUF & $10 / 21 / 88$ & REG & Carbon disulfide & 5 & ug/L & $\mathrm{U}$ \\
\hline 844 & M01267 & $11 / 20 / 97$ & REG & Carbon tetrachloride & 5 & ug/L & $\mathrm{U}$ \\
\hline $8+4$ & W00811 & $6 / 25 / 97$ & REG & Carbon tetrachloride & 5 & $\mathrm{ug} / \mathrm{L}$ & $\mathrm{U}$ \\
\hline 844 & M00066 & $11 / 26 / 96$ & REG & Carbon tetrachloride & 5 & ug/L & $\mathrm{U}$ \\
\hline 844 & M00858 & $6 / 25 / 97$ & REG & Carbon tetrachloride & 5 & ug/L & $\mathrm{U}$ \\
\hline $8+4$ & 500909 & $10 / 12 / 94$ & REG & Carbon tetrachloride & 5 & $\mathrm{ug} / \mathrm{L}$ & U \\
\hline $8+4$ & S02634 & $2 / 7 / 95$ & REG & Carbon tetrachloride & 5 & $u g / L$ & $\mathrm{U}$ \\
\hline $8+4$ & S03598 & $5 / 17 / 95$ & REG & Carbon tetrachloride & 5 & $\mathrm{ug} / \mathrm{L}$ & $\mathrm{U}$ \\
\hline $8+4$ & S04811 & $7 / 19 / 95$ & REG & Carbon tetrachloride & 5 & $\mathrm{ug} / \mathrm{L}$ & $\mathrm{U}$ \\
\hline
\end{tabular}




\begin{tabular}{|c|c|c|c|c|c|c|c|}
\hline Station & Sample ID & Sample Date & Sample Type & Chemical & Results & Units & Qualifier \\
\hline $8+4$ & 844220 REGUF & $2 / 20 / 91$ & REG & Carbon tetrachloride & 5 & $\mathrm{ug} / \mathrm{L}$ & $\mathrm{U}$ \\
\hline 844 & 84410 IREGUF & $10 / 1 / 91$ & REG & Carbon tetrachloride & 5 & $\mathrm{ug} / \mathrm{L}$ & U \\
\hline 844 & 84+129REGUF & $12 / 9 / 91$ & REG & Carbon tetrachloride & 5 & ug/L & $\mathrm{U}$ \\
\hline 844 & 8445 18REGUF & $5 / 18 / 90$ & REG & Carbon tetrachloride & 5 & ug/L & $\mathrm{U}$ \\
\hline 844 & 844118 REGUF & $11 / 8 / 90$ & REG & Carbon tetrachloride & 5 & ug/L & $\mathrm{U}$ \\
\hline 844 & 844125REGUF & $1 / 25 / 89$ & REG & Carbon tetrachloride & 5 & ug/L & $\mathrm{U}$ \\
\hline 844 & 844411REGUF & $4 / 11 / 89$ & REG & Carbon tetrachloride & 5 & ug/L & $\mathrm{U}$ \\
\hline 844 & 84476REGUF & $7 / 6 / 88$ & REG & Carbon tetrachloride & 5 & $\mathrm{ug} / \mathrm{L}$ & $\mathrm{U}$ \\
\hline 844 & 8441021REGUF & $10 / 21 / 88$ & REG & Carbon tetrachloride & 5 & ug/L & U \\
\hline 844 & M01267 & $11 / 20 / 97$ & REG & Chlorobenzene & 5 & $u g / L$ & $\mathrm{U}$ \\
\hline 844 & W00811 & $6 / 25 / 97$ & REG & Chlorobenzene & 5 & $\mathrm{ug} / \mathrm{L}$ & U \\
\hline 844 & M00066 & $11 / 26 / 96$ & REG & Chlorobenzene & 5 & $\mathrm{ug} / \mathrm{L}$ & U \\
\hline 844 & M00858 & $6 / 25 / 97$ & REG & Chlorobenzene & 5 & $\mathrm{ug} / \mathrm{L}$ & $U$ \\
\hline 844 & S00909 & $10 / 12 / 94$ & REG & Chlorobenzene & 5 & ug $/ \mathrm{L}$ & $\mathrm{U}$ \\
\hline 844 & S02634 & $2 / 7 / 95$ & REG & Chlorobenzene & 5 & $\mathrm{ug} / \mathrm{L}$ & $\mathrm{U}$ \\
\hline 844 & S03598 & $5 / 17 / 95$ & REG & Chlorobenzene & 5 & $\mathrm{ug} / \mathrm{L}$ & $\mathrm{U}$ \\
\hline 844 & S04811 & $7 / 19 / 95$ & REG & Chlorobenzene & 5 & $\mathrm{ug} / \mathrm{L}$ & $\mathrm{U}$ \\
\hline 844 & 844220REGUF & $2 / 20 / 91$ & REG & Chlorobenzene & 5 & $\mathrm{ug} / \mathrm{L}$ & $\mathrm{U}$ \\
\hline 844 & 844101REGUF & $10 / 1 / 91$ & REG & Chlorobenzene & 5 & $\mathrm{ug} / \mathrm{L}$ & $\mathrm{U}$ \\
\hline 844 & 844 129REGUF & $12 / 9 / 91$ & REG & Chlorobenzene & 5 & $\mathrm{ug} / \mathrm{L}$ & $\mathrm{U}$ \\
\hline 844 & 844518REGUF & $5 / 18 / 90$ & REG & Chlorobenzene & 5 & $\mathrm{ug} / \mathrm{L}$ & $U$ \\
\hline 844 & 844118REGUF & $11 / 8 / 90$ & REG & Chlorobenzene & 5 & $\mathrm{ug} / \mathrm{L}$ & $\mathrm{U}$ \\
\hline 844 & 844125REGUF & $1 / 25 / 89$ & REG & Chlorobenzene & 5 & $\mathrm{ug} / \mathrm{L}$ & $U$ \\
\hline 844 & 84441 IREGUF & $4 / 11 / 89$ & REG & Chlorobenzene & 5 & $\mathrm{ug} / \mathrm{L}$ & $\mathrm{U}$ \\
\hline 844 & 84476REGUF & $7 / 6 / 88$ & REG & Chlorobenzene & 5 & ug/L & $\mathrm{U}$ \\
\hline 844 & 8441021REGUF & $10 / 21 / 88$ & REG & Chlorobenzene & 5 & ug/L & $\mathbf{U}$ \\
\hline 844 & M01267 & $11 / 20 / 97$ & REG & Chloroethane & 10 & ug/L & $\mathrm{U}$ \\
\hline $8+4$ & W00811 & $6 / 25 / 97$ & REG & Chloroethane & 10 & ug/L & $\mathrm{U}$ \\
\hline 844 & M00066 & $11 / 26 / 96$ & REG & Chloroethane & 10 & $\mathrm{ug} / \mathrm{L}$ & $\mathrm{U}$ \\
\hline 844 & M00858 & $6 / 25 / 97$ & REG & Chloroethane & 10 & $\mathrm{ug} / \mathrm{L}$ & $\mathrm{U}$ \\
\hline 844 & S00909 & $10 / 12 / 94$ & REG & Chloroethane & 10 & $\mathrm{ug} / \mathrm{L}$ & $\mathrm{U}$ \\
\hline 844 & S0263t & $2 / 7 / 95$ & REG & Chloroethane & 10 & $\mathrm{ug} / \mathrm{L}$ & $\mathrm{U}$ \\
\hline 844 & S03598 & $5 / 17 / 95$ & REG & Chloroethane & 10 & $\mathrm{ug} / \mathrm{L}$ & $\mathrm{U}$ \\
\hline $8+4$ & S04811 & $7 / 19 / 95$ & REG & Chloroethane & 10 & $\mathrm{ug} / \mathrm{L}$ & $\mathrm{U}$ \\
\hline 844 & 84+220REGUF & $2 / 20 / 91$ & REG & Chloroethane & 10 & $\mathrm{ug} / \mathrm{L}$ & $\mathrm{U}$ \\
\hline 844 & 844101REGUF & $10 / 1 / 91$ & REG & Chloroethane & 10 & ug $/$ & $\mathrm{U}$ \\
\hline 844 & 844129REGUF & $12 / 9 / 91$ & REG & Chloroethane & 10 & ug/L & U \\
\hline 844 & 844518REGUF & $5 / 18 / 90$ & REG & Chloroethane & 10 & ug/L & $\mathrm{U}$ \\
\hline 844 & $8+4118$ REGUF & $11 / 8 / 90$ & REG & Chloroethane & 10 & ug/L & $\mathrm{U}$ \\
\hline 844 & 844125REGUF & $1 / 25 / 89$ & REG & Chloroethane & 10 & $\mathrm{ug} / \mathrm{L}$ & $\mathrm{U}$ \\
\hline 844 & 84+411REGUF & $4 / 11 / 89$ & REG & Chloroethane & 10 & $\mathrm{ug} / \mathrm{L}$ & $\mathrm{U}$ \\
\hline 844 & 84476REGUF & $7 / 6 / 88$ & REG & Chloroethane & 10 & $\mathrm{ug} / \mathrm{L}$ & $\mathrm{U}$ \\
\hline 844 & 8441021REGUF & $10 / 21 / 88$ & REG & Chloroethane & 10 & ug/L & $\mathrm{U}$ \\
\hline 844 & M01267 & $11 / 20 / 97$ & REG & Chloroform & 5 & ug $/ \mathrm{L}$ & $\mathrm{U}$ \\
\hline 844 & W00811 & $6 / 25 / 97$ & REG & Chloroform & 5 & ug/L & $\mathrm{U}$ \\
\hline 844 & M00066 & $11 / 26 / 96$ & REG & Chloroform & 5 & $\mathrm{ug} / \mathrm{L}$ & $\mathrm{U}$ \\
\hline 844 & M00858 & $6 / 25 / 97$ & REG & Chloroform & 5 & $\mathrm{ug} / \mathrm{L}$ & $\mathrm{U}$ \\
\hline 844 & S00909 & $10 / 12 / 94$ & REG & Chloroform & 5 & ug/L & $\mathrm{U}$ \\
\hline 844 & S02634 & $2 / 7 / 95$ & REG & Chloroform & 5 & $\mathrm{ug} / \mathrm{L}$ & $\mathrm{U}$ \\
\hline 844 & S03598 & $5 / 17 / 95$ & REG & Chloroform & 5 & ug/L & $\mathrm{U}$ \\
\hline
\end{tabular}


B-64

\begin{tabular}{|c|c|c|c|c|c|c|c|}
\hline Station & Sample ID & Sample Date & Sample Type & Chemical & Results & Units & Qualifier \\
\hline 844 & S04811 & $7 / 19 / 95$ & REG & Chloroform & 5 & ug/L & $\bar{U}$ \\
\hline 844 & 844220REGUF & $2 / 20 / 91$ & REG & Chloroform & 5 & ug $/ \mathrm{L}$ & $\mathrm{U}$ \\
\hline 844 & 844101REGUF & $10 / 1 / 91$ & REG & Chloroform & 5 & ug/ & $\mathrm{U}$ \\
\hline 844 & 844129REGUF & $12 / 9 / 91$ & REG & Chloroform & 5 & $\mathrm{ug} / \mathrm{L}$ & $\mathrm{U}$ \\
\hline 844 & 844518REGUF & $5 / 18 / 90$ & REG & Chloroform & 2 & $\mathrm{ug} / \mathrm{L}$ & J \\
\hline 844 & 844118REGUF & $11 / 8 / 90$ & REG & Chloroform & 5 & $u g / L$ & $\mathrm{U}$ \\
\hline 844 & 844125REGUF & $1 / 25 / 89$ & REG & Chloroform & 5 & $\mathrm{ug} / \mathrm{L}$ & $\mathrm{U}$ \\
\hline 844 & 844411 REGUF & $4 / 11 / 89$ & REG & Chloroform & 5 & $\mathrm{ug} / \mathrm{L}$ & U \\
\hline 844 & 84476REGUF & $7 / 6 / 88$ & REG & Chloroform & 5 & ug/L & U \\
\hline 844 & 8441021REGUF & $10 / 21 / 88$ & REG & Chloroform & 5 & $\mathrm{ug} / \mathrm{L}$ & $\mathrm{U}$ \\
\hline 844 & M01267 & $11 / 20 / 97$ & REG & Chloromethane & 10 & $\mathrm{ug} / \mathrm{L}$ & $\mathrm{U}$ \\
\hline 844 & W00811 & $6 / 25 / 97$ & REG & Chloromethane & 10 & $\mathrm{ug} / \mathrm{L}$ & $U$ \\
\hline 844 & M00066 & $11 / 26 / 96$ & REG & Chloromethane & 10 & ug/L & U \\
\hline 844 & M00858 & $6 / 25 / 97$ & REG & Chloromethane & 10 & $u g / L$ & $\mathrm{U}$ \\
\hline 844 & S00909 & $10 / 12 / 94$ & REG & Chloromethane & 10 & $\mathrm{ug} / \mathrm{L}$ & $\mathrm{U}$ \\
\hline 844 & S02634 & $2 / 7 / 95$ & REG & Chloromethane & 10 & $\mathrm{ug} / \mathrm{L}$ & $\mathrm{U}$ \\
\hline 844 & S03598 & $5 / 17 / 95$ & REG & Chloromethane & 10 & $\mathrm{ug} / \mathrm{L}$ & U \\
\hline 844 & S04811 & $7 / 19 / 95$ & REG & Chloromethane & 10 & $\mathrm{ug} / \mathrm{L}$ & U \\
\hline 844 & 844220REGUF & $2 / 20 / 91$ & REG & Chloromethane & 10 & $\mathrm{ug} / \mathrm{L}$ & $\mathrm{U}$ \\
\hline 844 & 844101REGUF & $10 / 1 / 91$ & REG & Chloromethane & 10 & $\mathrm{ug} / \mathrm{L}$ & $\mathrm{U}$ \\
\hline 844 & 844129REGUF & $12 / 9 / 91$ & REG & Chloromethane & 10 & $\mathrm{ug} / \mathrm{L}$ & U \\
\hline 844 & 844518REGUF & $5 / 18 / 90$ & REG & Chloromethane & 10 & $u g / L$ & $\mathrm{U}$ \\
\hline 844 & 844118REGUF & $11 / 8 / 90$ & REG & Chloromethane & 10 & $\mathrm{ug} / \mathrm{L}$ & $U$ \\
\hline 844 & 844125REGUF & $1 / 25 / 89$ & REG & Chloromethane & 10 & $\mathrm{ug} / \mathrm{L}$ & U \\
\hline 844 & 84441IREGUF & $4 / 11 / 89$ & REG & Chloromethane & 10 & ug/ & $\mathrm{U}$ \\
\hline 844 & 84476REGUF & $7 / 6 / 88$ & REG & Chloromethane & 10 & $u g / L$ & $\mathrm{U}$ \\
\hline 844 & 8441021REGUF & $10 / 21 / 88$ & REG & Chloromethane & 10 & ug/L & $\mathrm{U}$ \\
\hline 844 & M01267 & $11 / 20 / 97$ & REG & cis-1.2-Dichloroethene & 5 & $u g / L$ & $\mathrm{U}$ \\
\hline 844 & M00066 & $11 / 26 / 96$ & REG & cis-1,2-Dichloroethene & 5 & $u g / L$ & $\mathrm{U}$ \\
\hline 844 & M00858 & $6 / 25 / 97$ & REG & cis-1,2-Dichloroethene & 5 & ug/L & $\mathrm{U}$ \\
\hline 844 & S00909 & $10 / 12 / 94$ & REG & cis-1.2-Dichloroethene & 5 & $\mathrm{ug} / \mathrm{L}$ & U \\
\hline 844 & S02634 & $2 / 7 / 95$ & REG & cis-1.2-Dichloroethene & 5 & ug $/ \mathrm{L}$ & $\mathrm{U}$ \\
\hline 844 & S03598 & $5 / 17 / 95$ & REG & cis-1.2-Dichloroethene & 5 & ug/L & $\mathrm{U}$ \\
\hline 844 & S04811 & $7 / 19 / 95$ & REG & cis-1,2-Dichloroethene & 5 & $\mathrm{ug} / \mathrm{L}$ & $\mathrm{U}$ \\
\hline $8+4$ & M01267 & $11 / 20 / 97$ & REG & cis-1.3-Dichloropropen & 5 & $u g / L$ & $\mathrm{U}$ \\
\hline 844 & W00811 & $6 / 25 / 97$ & REG & cis-1.3-Dichloropropene & 5 & $\mathrm{ug} / \mathrm{L}$ & $\mathrm{U}$ \\
\hline 844 & M00066 & $11 / 26 / 96$ & REG & cis-1.3-Dichloropropene & 5 & $u g / \mathrm{L}$ & $\mathrm{U}$ \\
\hline 844 & M00858 & $6 / 25 / 97$ & REG & cis-1.3-Dichloropropene & 5 & ug/L & $\mathrm{U}$ \\
\hline 844 & S00909 & $10 / 12 / 94$ & REG & cis-1.3-Dichloropropene & 5 & ug/L & $\mathrm{U}$ \\
\hline 844 & S02634 & $2 / 7 / 95$ & REG & cis-1.3-Dichloropropene & 5 & $\mathrm{ug} / \mathrm{L}$ & $\mathrm{U}$ \\
\hline 844 & S03598 & $5 / 17 / 95$ & REG & cis-1.3-Dichloropropene & 5 & $\mathrm{ug} / \mathrm{L}$ & $U$ \\
\hline 844 & S04811 & $7 / 19 / 95$ & REG & cis-1.3-Dichloropropene & 5 & $\mathrm{ug} / \mathrm{L}$ & U \\
\hline 844 & 844220REGUF & $2 / 20 / 91$ & REG & cis-1.3-Dichloropropene & 5 & $\mathrm{ug} / \mathrm{L}$ & $\mathrm{U}$ \\
\hline $8+4$ & 84410IREGUF & $10 / 1 / 91$ & REG & cis-1,3-Dichloropropene & 5 & $u g / L$ & U \\
\hline 844 & 844129REGUF & $12 / 9 / 91$ & REG & cis-1.3-Dichloropropene & 5 & $\mathrm{ug} / \mathrm{L}$ & $\mathrm{U}$ \\
\hline 844 & 844518REGUF & $5 / 18 / 90$ & REG & cis-1.3-Dichloropropene & 5 & $\mathrm{ug} / \mathrm{L}$ & $\mathrm{U}$ \\
\hline 844 & 844118REGUF & $11 / 8 / 90$ & REG & cis-1.3-Dichloropropene & 5 & $\mathrm{ug} / \mathrm{L}$ & $\mathrm{U}$ \\
\hline 844 & 844125REGUF & $1 / 25 / 89$ & REG & cis-1,3-Dichloropropene & 5 & $\mathrm{ug} / \mathrm{L}$ & $\mathrm{U}$ \\
\hline 844 & 8444IIIREGUF & $4 / 11 / 89$ & REG & cis-1.3-Dichloropropene & 5 & ug/L & $\mathrm{U}$ \\
\hline 844 & 84476REGUF & $7 / 6 / 88$ & REG & cis-1.3-Dichloropropene & 5 & $\mathrm{ug} / \mathrm{L}$ & $\mathrm{U}$ \\
\hline
\end{tabular}




\begin{tabular}{|c|c|c|c|c|c|c|c|}
\hline Station & Sample ID & Sample Date & Sample Type & Chemical & Results & Units & Qualifier \\
\hline $8+4$ & S03598 & $5 / 17 / 95$ & REG & m.p-Xylene & 5 & $\mathrm{ug} / \mathrm{L}$ & $\mathrm{U}$ \\
\hline 844 & S04811 & $7 / 19 / 95$ & REG & m.p-Xylene & 5 & $\mathrm{ug} / \mathrm{L}$ & $\mathrm{U}$ \\
\hline $8+4$ & Mo1267 & $11 / 20 / 97$ & REG & Methylene chloride & 6 & ug $/ \mathrm{L}$ & B \\
\hline 844 & W00811 & $6 / 25 / 97$ & REG & Methylene chloride & 10 & $\mathrm{ug} \Omega$ & $=$ \\
\hline 844 & M00066 & $11 / 26 / 96$ & REG & Methylene chloride & 5 & ug/L & $\mathrm{U}$ \\
\hline 844 & M00858 & $6 / 25 / 97$ & REG & Methylene chloride & 2 & $\mathrm{ug} / \mathrm{L}$ & $\mathrm{U}$ \\
\hline 844 & S00909 & $10 / 12 / 94$ & REG & Methylene chloride & 5 & $\mathrm{ug} / \mathrm{L}$ & $\mathrm{U}$ \\
\hline 844 & S02634 & $2 / 7 / 95$ & REG & Methylene chloride & 5 & ug/L & $\mathrm{U}$ \\
\hline 844 & S03598 & $5 / 17 / 95$ & REG & Methylene chloride & 5 & $\mathrm{ug} / \mathrm{L}$ & $\mathrm{U}$ \\
\hline 844 & S04811 & $7 / 19 / 95$ & REG & Methylene chloride & 5 & $\mathrm{ug} / \mathrm{L}$ & $U$ \\
\hline 844 & 844220REGUF & $2 / 20 / 91$ & REG & Methylene chloride & 5 & $\mathrm{ug} / \mathrm{L}$ & $\mathrm{U}$ \\
\hline 844 & $84+10$ IREGUF & $10 / 1 / 91$ & REG & Methylene chloride & 5 & $\mathrm{ug} / \mathrm{L}$ & $\mathrm{U}$ \\
\hline 844 & 844129REGUF & $12 / 9 / 91$ & REG & Methylene chloride & 5 & $\mathrm{ug} / \mathrm{L}$ & $\mathrm{U}$ \\
\hline 844 & 844518REGUF & $5 / 18 / 90$ & REG & Methylene chloride & 2 & $\mathrm{ug} / \mathrm{L}$ & $\mathbf{J}$ \\
\hline 844 & 844118REGUF & $11 / 8 / 90$ & REG & Methylene chloride & 5 & $\mathrm{ug} / \mathrm{L}$ & $\mathrm{U}$ \\
\hline 844 & 844125REGUF & $1 / 25 / 89$ & REG & Methylene chloride & 1 & $\mathrm{ug} / \mathrm{L}$ & $J$ \\
\hline 844 & 84441 IREGUF & $4 / 11 / 89$ & REG & Methylene chloride & 1 & $u g / L$ & $\mathbf{J}$ \\
\hline 844 & 84476REGUF & $7 / 6 / 88$ & REG & Methylene chloride & 2 & $u g / L$ & J \\
\hline 844 & 8441021REGUF & $10 / 21 / 88$ & REG & Methylene chloride & 7 & $\mathrm{ug} / \mathrm{L}$ & $=$ \\
\hline 844 & M01267 & $11 / 20 / 97$ & REG & Styrene & 5 & $\mathrm{ug} / \mathrm{L}$ & $\mathrm{U}$ \\
\hline 844 & W00811 & $6 / 25 / 97$ & REG & Styrene & 5 & $\mathrm{ug} / \mathrm{L}$ & $\mathrm{U}$ \\
\hline $8+4$ & M00066 & $11 / 26 / 96$ & REG & Styrene & 5 & $\mathrm{ug} / \mathrm{L}$ & $\mathrm{U}$ \\
\hline $8+4$ & M00858 & $6 / 25 / 97$ & REG & Styrene & 5 & $\mathrm{ug} / \mathrm{L}$ & $\mathrm{U}$ \\
\hline 844 & S00909 & $10 / 12 / 94$ & REG & Styrene & 5 & $\mathrm{ug} / \mathrm{L}$ & U \\
\hline 844 & S02634 & $2 / 7 / 95$ & REG & Styrene & 5 & $\mathrm{ug} / \mathrm{L}$ & $\mathrm{U}$ \\
\hline 844 & S03598 & $5 / 17 / 95$ & REG & Styrene & 5 & $\mathrm{ug} / \mathrm{L}$ & $\mathrm{U}$ \\
\hline 844 & S04811 & $7 / 19 / 95$ & REG & Styrene & 5 & $\mathrm{ug} / \mathrm{L}$ & $\mathrm{U}$ \\
\hline 844 & 844220REGUF & $2 / 20 / 91$ & REG & Styrene & 5 & $\mathrm{ug} / \mathrm{L}$ & $\mathrm{U}$ \\
\hline 844 & 844101REGUF & $10 / 1 / 91$ & REG & Styrene & 5 & ug/L & $\mathrm{U}$ \\
\hline 844 & 844129REGUF & $12 / 9 / 91$ & REG & Styrene & 1 & $\mathrm{ug} / \mathrm{L}$ & $\mathrm{J}$ \\
\hline 844 & 844518REGUF & $5 / 18 / 90$ & REG & Styrene & 5 & $\mathrm{ug} / \mathrm{L}$ & $\mathrm{U}$ \\
\hline 844 & 844118REGUF & $11 / 8 / 90$ & REG & Styrene & 5 & ug/L & $\mathrm{U}$ \\
\hline 844 & 844125REGUF & $1 / 25 / 89$ & REG & Styrrene & 5 & $\mathrm{ug} / \mathrm{L}$ & $U$ \\
\hline $8+4$ & 8444IIREGUF & $4 / 11 / 89$ & REG & Styrene & 5 & ug $/ \mathrm{L}$ & $\mathrm{U}$ \\
\hline $8+4$ & 84476REGUF & $7 / 6 / 88$ & REG & Styrene & 5 & ug/L & $\mathrm{U}$ \\
\hline 844 & 8441021 REGUF & $10 / 21 / 88$ & REG & Styrene & 5 & ug $/ \mathrm{L}$ & $\mathrm{U}$ \\
\hline 844 & M01267 & $11 / 20 / 97$ & REG & Tetrachloroethene & 5 & $\mathrm{ug} / \mathrm{L}$ & $\mathrm{U}$ \\
\hline $8+4$ & W00811 & $6 / 25 / 97$ & REG & Tetrachloroethene & 5 & $\mathrm{ug} / \mathrm{L}$ & $\mathrm{U}$ \\
\hline 844 & M00066 & $11 / 26 / 96$ & REG & Tetrachloroethene & 5 & $\mathrm{ug} / \mathrm{L}$ & $\mathrm{U}$ \\
\hline 844 & M00858 & $6 / 25 / 97$ & REG & Tetrachloroethene & 5 & $\mathrm{ug} / \mathrm{L}$ & $\mathrm{U}$ \\
\hline $8+4$ & S00909 & $10 / 12 / 94$ & REG & Tetrachloroethene & 5 & $\mathrm{ug} / \mathrm{L}$ & $U$ \\
\hline 844 & S02634 & $2 / 7 / 95$ & REG & Tetrachloroethene & 5 & $\mathrm{ug} / \mathrm{L}$ & $\mathrm{U}$ \\
\hline 844 & S03598 & $5 / 17 / 95$ & REG & Tetrachloroethene & 5 & $\mathrm{ug} / \mathrm{L}$ & $\mathrm{U}$ \\
\hline 844 & S04811 & $7 / 19 / 95$ & REG & Tetrachloroethene & 5 & $\mathrm{ug} / \mathrm{L}$ & $\mathrm{U}$ \\
\hline 844 & 844220REGUF & $2 / 20 / 91$ & REG & Tetrachloroethene & 5 & $\mathrm{ug} / \mathrm{L}$ & $\mathrm{U}$ \\
\hline 844 & 84410IREGUF & $10 / 1 / 91$ & REG & Tetrachloroethene & 5 & ug/L & $\mathrm{U}$ \\
\hline $8+4$ & 844129REGUF & $12 / 9 / 91$ & REG & Tetrachloroethene & 5 & $u g / L$ & $\mathrm{U}$ \\
\hline 844 & 8445 I 8REGUF & $5 / 18 / 90$ & REG & Tetrachloroethene & 5 & $\mathrm{ug} / \mathrm{L}$ & $\mathrm{U}$ \\
\hline 844 & 84+118REGUF & $11 / 8 / 90$ & REG & Tetrachloroethene & 3 & $\mathrm{ug} / \mathrm{L}$ & $\mathrm{J}$ \\
\hline 844 & 844125 REGUF & $1 / 25 / 89$ & REG & Tetrachloroethene & 5 & $\mathrm{ug} / \mathrm{L}$ & U \\
\hline
\end{tabular}




\begin{tabular}{|c|c|c|c|c|c|c|c|}
\hline Station & Sample ID & Sample Date & Sample Type & Chemical & Results & Units & Qualifier \\
\hline $8+4$ & S03598 & $5 / 17 / 95$ & REG & Trichloroethene & 5 & ug/L & $\mathrm{U}$ \\
\hline $8+4$ & $S 04811$ & $7 / 19 / 95$ & REG & Trichloroethene & 5 & $\mathrm{ug} / \mathrm{L}$ & $\mathrm{U}$ \\
\hline $8+4$ & $84+220$ REGUF & $2 / 20 / 91$ & REG & Trichloroethene & 5 & $\mathrm{ug} / \mathrm{L}$ & $\mathrm{U}$ \\
\hline $8+4$ & $8+4101$ REGUF & $10 / 1 / 91$ & REG & Trichloroethene & 5 & ug/L & U \\
\hline $8+4$ & $84+129$ REGUF & $12 / 9 / 91$ & REG & Trichloroethene & 1 & $\mathrm{ug} / \mathrm{L}$ & $J$ \\
\hline $8+4$ & 844ミ18REGUF & $5 / 18 / 90$ & REG & Trichloroethene & 2 & $\mathrm{ug} / \mathrm{L}$ & $J$ \\
\hline 844 & 844118REGUF & $11 / 8 / 90$ & REG & Trichloroethene & 5 & $\mathrm{ug} / \mathrm{L}$ & U \\
\hline 844 & 844125REGUF & $1 / 25 / 89$ & REG & Trichloroethene & 5 & $\mathrm{ug} / \mathrm{L}$ & $\mathrm{U}$ \\
\hline 844 & 844+1 IREGUF & $4 / 11 / 89$ & REG & Trichloroethene & 5 & $\mathrm{ug} / \mathrm{L}$ & $\mathrm{U}$ \\
\hline 844 & 84476REGUF & $7 / 6 / 88$ & REG & Trichloroethene & 5 & $\mathrm{ug} / \mathrm{L}$ & $\mathrm{U}$ \\
\hline 844 & 844102 IREGUF & $10 / 21 / 88$ & REG & Trichloroethene & 5 & $\mathrm{ug} / \mathrm{L}$ & $\mathrm{U}$ \\
\hline 844 & M01267 & $11 / 20 / 97$ & REG & Vinyl acetate & 10 & $\mathrm{ug} / \mathrm{L}$ & $\mathrm{U}$ \\
\hline 844 & M00066 & $11 / 26 / 96$ & REG & Vinyl acetate & 10 & $\mathrm{ug} / \mathrm{L}$ & $\mathrm{U}$ \\
\hline 844 & M00858 & $6 / 25 / 97$ & REG & Vinyl acetate & 10 & $\mathrm{ug} / \mathrm{L}$ & $\mathrm{U}$ \\
\hline 844 & S00909 & $10 / 12 / 94$ & REG & Vinyl acetate & 10 & $u g / L$ & $\mathrm{U}$ \\
\hline 844 & S02634 & $2 / 7 / 95$ & REG & Vinyl acetate & 10 & ug/ & U \\
\hline 844 & S03598 & $5 / 17 / 95$ & REG & Vinyl acetate & 10 & $\mathrm{ug} / \mathrm{L}$ & $\mathrm{U}$ \\
\hline 844 & S04811 & $7 / 19 / 95$ & REG & Vinyl acetate & 10 & $\mathrm{ug} / \mathrm{L}$ & $\mathrm{U}$ \\
\hline 844 & 844220REGUF & $2 / 20 / 91$ & REG & Vinyl acetate & 10 & $u g / L$ & U \\
\hline 844 & 844101 REGUF & $10 / 1 / 91$ & REG & Vinyl acetate & 10 & ug $/ \mathrm{L}$ & $\mathrm{U}$ \\
\hline 844 & 844129REGUF & $12 / 9 / 91$ & REG & Vinyl acetate & 10 & $\mathrm{ug} / \mathrm{L}$ & $\mathrm{U}$ \\
\hline 844 & 844518REGUF & $5 / 18 / 90$ & REG & Vinyl acetate & 10 & $\mathrm{ug} / \mathrm{L}$ & U \\
\hline 844 & $84+118$ REGUF & $11 / 8 / 90$ & REG & Vinyl acetate & 10 & $\mathrm{ug} / \mathrm{L}$ & $\mathrm{U}$ \\
\hline 844 & 844125REGUF & $1 / 25 / 89$ & REG & Vinyl acetate & 10 & $\mathrm{ug} / \mathrm{L}$ & U \\
\hline 844 & 8444 IIREGUF & $4 / 11 / 89$ & REG & Vinyl acetate & 10 & $\mathrm{ug} / \mathrm{L}$ & $\mathrm{U}$ \\
\hline 844 & 84476 REGUF & $7 / 6 / 88$ & REG & Vinyl acetate & 10 & $\mathrm{ug} / \mathrm{L}$ & $\mathrm{U}$ \\
\hline 844 & $84+1021$ REGUF & $10 / 21 / 88$ & REG & Vinyl acetate & 10 & $\mathrm{ug} / \mathrm{L}$ & $\mathrm{U}$ \\
\hline 844 & M01267 & $11 / 20 / 97$ & REG & Vinyl chloride & 10 & $\mathrm{ug} / \mathrm{L}$ & $\mathrm{U}$ \\
\hline $8+4$ & W00811 & $6 / 25 / 97$ & REG & Vinyl chloride & 10 & ug/L & $\mathrm{U}$ \\
\hline 844 & M00066 & $11 / 26 / 96$ & REG & Vinyl chloride & 10 & $\mathrm{ug} / \mathrm{L}$ & $\mathrm{U}$ \\
\hline 844 & M00858 & $6 / 25 / 97$ & REG & Vinyl chloride & 10 & $\mathrm{ug} / \mathrm{L}$ & $\mathrm{U}$ \\
\hline 844 & S00909 & $10 / 12 / 94$ & REG & Vinyl chloride & 10 & $\mathrm{ug} / \mathrm{L}$ & U \\
\hline 844 & S02634 & $2 / 7 / 95$ & REG & Vinyl chloride & 10 & $\mathrm{ug} / \mathrm{L}$ & $\mathrm{U}$ \\
\hline $8+4$ & S03598 & $5 / 17 / 95$ & REG & Vinyl chloride & 10 & ug $/$ & $\mathrm{U}$ \\
\hline $8+4$ & S04811 & $7 / 19 / 95$ & REG & Vinyl chloride & 10 & ug $\Omega$ & U \\
\hline 844 & $8+4220$ REGUF & $2 / 20 / 91$ & REG & Vinyl chloride & 10 & ug $/$. & $\mathrm{U}$ \\
\hline 844 & $84+101$ REGUF & $10 / 1 / 91$ & REG & Vinyl chloride & 10 & $\mathrm{ug} / \mathrm{L}$ & $\mathrm{U}$ \\
\hline 844 & 844129REGUF & $12 / 9 / 91$ & REG & Vinyl chloride & 10 & $\mathrm{ug} / \mathrm{L}$ & $\mathrm{U}$ \\
\hline 844 & 844518REGUF & $5 / 18 / 90$ & REG & Vinyl chloride & 10 & $\mathrm{ug} / \mathrm{L}$ & $\mathrm{U}$ \\
\hline 844 & 844118 REGUF & $11 / 8 / 90$ & REG & Vinyl chloride & 10 & $\mathrm{ug} / \mathrm{L}$ & $\mathrm{U}$ \\
\hline $8+4$ & 844125 REGUF & $1 / 25 / 89$ & REG & Vinyl chloride & 10 & ug $/$ & $\mathrm{U}$ \\
\hline 844 & 8444 11REGUF & $4 / 11 / 89$ & REG & Vinyl chloride & 10 & $\mathrm{ug} / \mathrm{L}$ & $\mathrm{U}$ \\
\hline 844 & 84476REGUF & $7 / 6 / 88$ & REG & Vinyl chloride & 10 & $\mathrm{ug} / \mathrm{L}$ & U \\
\hline 844 & $84+102$ IREGUF & $10 / 21 / 88$ & REG & Vinyl chloride & 10 & ug/ & $\mathrm{U}$ \\
\hline 846 & M01273 & $11 / 19 / 97$ & REG & 1,1,1-Trichloroethane & 5 & $\mathrm{ug} / \mathrm{L}$ & $\mathrm{U}$ \\
\hline 846 & W00899 & $6 / 24 / 97$ & REG & 1.1,1-Trichloroethane & 5 & ug $/$ & $\mathrm{U}$ \\
\hline 846 & M00070 & $12 / 4 / 96$ & REG & 1.1,1-Trichloroethane & 5 & $\mathrm{ug} / \mathrm{L}$ & U \\
\hline 846 & M01018 & $6 / 24 / 97$ & REG & 1.1.1-Trichloroethane & 5 & $\mathrm{ug} / \mathrm{L}$ & $\mathrm{U}$ \\
\hline 846 & S01116 & $12 / 12 / 94$ & REG & 1.1.1-Trichloroethane & 1 & $\mathrm{ug} / \mathrm{L}$ & $\mathrm{U}$ \\
\hline $8+6$ & 502736 & $2 / 27 / 95$ & REG & 1.1.1-Trichloroethane & 5 & ug $/ \mathrm{L}$ & $\mathrm{U}$ \\
\hline
\end{tabular}


B-67

\begin{tabular}{|c|c|c|c|c|c|c|c|}
\hline Station & Sample ID & Sample Date & Sample Type & Chemical & Results & Units & Qualifier \\
\hline$\overline{846}$ & S04829 & $8 / 24 / 95$ & REG & 1,1,1-Trichloroethane & 5 & ug/L & $\bar{U}$ \\
\hline 846 & 846215REGUF & $2 / 15 / 91$ & REG & 1.1.1-Trichloroethane & 5 & ug/L & $\mathrm{U}$ \\
\hline 846 & 8465I6REGUF & $5 / 16 / 90$ & REG & 1.1.1-Trichloroethane & 5 & $\mathrm{ug} / \mathrm{L}$ & $\mathrm{U}$ \\
\hline 846 & 8461030REGUF & $10 / 30 / 90$ & REG & 1.1.1-Trichloroethane & 5 & $\mathrm{ug} / \mathrm{L}$ & $\mathrm{U}$ \\
\hline 846 & 846111REGUF & $1 / 11 / 89$ & REG & 1.1.I-Trichloroethane & 5 & ug/L & $\mathrm{U}$ \\
\hline 846 & 846417REGUF & $4 / 17 / 89$ & REG & 1.1.1-Trichloroethane & 5 & $\mathrm{ug} / \mathrm{L}$ & $\mathrm{U}$ \\
\hline 846 & 84665REGUF & $6 / 5 / 88$ & REG & 1,1.1-Trichloroethane & 5 & $\mathrm{ug} / \mathrm{L}$ & $\mathrm{U}$ \\
\hline 846 & 846105REGUF & $10 / 5 / 88$ & REG & 1,1,1-Trichloroethane & 5 & ug/L & $\mathrm{U}$ \\
\hline 846 & M01273 & $11 / 19 / 97$ & REG & 1,1,2,2-Tetrachloroeth & 5 & $\mathrm{ug} / \mathrm{L}$ & $\mathbf{U}$ \\
\hline 846 & W00899 & $6 / 24 / 97$ & REG & 1,1,2,2-Tetrachloroethane & 5 & ug/L & $\mathrm{U}$ \\
\hline 846 & M00070 & $12 / 4 / 96$ & REG & 1,1,2,2-Tetrachloroethane & 5 & ug/L & $\mathrm{U}$ \\
\hline 846 & M01018 & $6 / 24 / 97$ & REG & 1.1.2,2-Tetrachloroethane & 5 & $\mathrm{ug} / \mathrm{L}$ & $\mathrm{U}$ \\
\hline 846 & S01116 & $12 / 12 / 94$ & REG & 1.1,2,2-Tetrachloroethane & 1 & ug $/ \mathrm{L}$ & U \\
\hline 846 & S02736 & $2 / 27 / 95$ & REG & 1.1.2,2-Tetrachloroethane & 5 & $\mathrm{ug} / \mathrm{L}$ & $\mathrm{U}$ \\
\hline 846 & S04829 & $8 / 24 / 95$ & REG & 1.1,2,2-Tetrachloroethane & 5 & ug/L & $\mathrm{U}$ \\
\hline 846 & 846215REGUF & $2 / 15 / 91$ & REG & 1,1,2,2-Tetrachloroethane & 5 & ug/L & $\mathrm{U}$ \\
\hline 846 & 846516REGUF & $5 / 16 / 90$ & REG & 1,1,2,2-Tetrachloroethane & 5 & ug/L & $\mathrm{U}$ \\
\hline 846 & 8461030REGUF & $10 / 30 / 90$ & REG & 1,1.2.2-Tetrachloroethane & 5 & $\mathrm{ug} / \mathrm{L}$ & U \\
\hline 846 & 846111 REGUF & $1 / 11 / 89$ & REG & 1,1.2,2-Tetrachloroethane & 5 & ug/L & $\mathrm{U}$ \\
\hline 846 & 846417REGUF & $4 / 17 / 89$ & REG & 1,1,2,2-Tetrachloroethane & 5 & ug $/ \mathrm{L}$ & $\mathrm{U}$ \\
\hline 846 & 84665REGUF & $6 / 5 / 88$ & REG & $1,1,2,2$-Tetrachloroethane & 5 & ug/L & $\mathrm{U}$ \\
\hline 846 & 846105REGUF & $10 / 5 / 88$ & REG & 1,1,2,2-Tetrachloroethane & 5 & $\mathrm{ug} / \mathrm{L}$ & $\mathrm{U}$ \\
\hline $8+6$ & W00899 & $6 / 24 / 97$ & REG & 1,1,2-Trichloro-1,2.2-trifluo & 10 & $u g / L$ & $\mathrm{U}$ \\
\hline 846 & M01273 & $11 / 19 / 97$ & REG & 1,1,2-Trichloroethane & 5 & ug/L & $\mathrm{U}$ \\
\hline 846 & W00899 & $6 / 24 / 97$ & REG & 1,1,2-Trichlorœthane & 5 & ug $/ \mathrm{L}$ & U \\
\hline 846 & M00070 & $12 / 4 / 96$ & REG & 1,1.2-Trichloroethane & 5 & $\mathrm{ug} / \mathrm{L}$ & $\mathrm{U}$ \\
\hline 846 & M01018 & $6 / 24 / 97$ & REG & 1,1.2-Trichloroethane & 5 & ug/L & $\mathrm{U}$ \\
\hline 846 & S01116 & $12 / 12 / 94$ & REG & 1,1.2-Trichloroethane & 1 & ug/L & $\mathrm{U}$ \\
\hline 846 & S02736 & $2 / 27 / 95$ & REG & 1,1,2-Trichloroethane & 5 & ug/L & $\mathrm{U}$ \\
\hline 846 & S04829 & $8 / 24 / 95$ & REG & 1,1,2-Trichloroethane & 5 & $\mathrm{ug} / \mathrm{L}$ & $\mathrm{U}$ \\
\hline 846 & 846215REGUF & $2 / 15 / 91$ & REG & 1,1,2-Trichloroethane & 5 & $\mathrm{ug} / \mathrm{L}$ & $\mathrm{U}$ \\
\hline 846 & 846516REGUF & $5 / 16 / 90$ & REG & 1,1,2-Trichlorothane & 5 & $\mathrm{ug} / \mathrm{L}$ & $\mathrm{U}$ \\
\hline 846 & 846 1030REGUF & $10 / 30 / 90$ & REG & 1.1.2-Trichloroethane & 5 & $\mathrm{ug} / \mathrm{L}$ & $\mathrm{U}$ \\
\hline 846 & 846111 REGUF & $1 / 11 / 89$ & REG & 1,1.2-Trichloroethane & 5 & ug/L & $\mathrm{U}$ \\
\hline 846 & 846+17REGUF & $4 / 17 / 89$ & REG & 1.1.2-Trichloroethane & 5 & $\mathrm{ug} / \mathrm{L}$ & $\mathrm{U}$ \\
\hline 846 & 84665REGUF & $6 / 5 / 88$ & REG & 1.1.2-Trichloroethane & 5 & $\mathrm{ug} / \mathrm{L}$ & $\mathrm{U}$ \\
\hline 846 & 846105REGUF & $10 / 5 / 88$ & REG & 1.1.2-Trichloroethane & 5 & $u g / L$ & $\mathrm{U}$ \\
\hline $8+6$ & M01273 & $11 / 19 / 97$ & REG & 1.1-Dichloroethane & 5 & $\mathrm{ug} / \mathrm{L}$ & $\mathrm{U}$ \\
\hline $8+6$ & W00899 & $6 / 24 / 97$ & REG & 1.1-Dichloroethane & 5 & $\mathrm{ug} / \mathrm{L}$ & $\mathrm{U}$ \\
\hline 846 & M00070 & $12 / 4 / 96$ & REG & 1.1-Dichloroethane & 5 & ug/L & $\mathrm{U}$ \\
\hline $8+6$ & M01018 & $6 / 24 / 97$ & REG & 1.1-Dichloroethane & 5 & ug/L & $\mathrm{U}$ \\
\hline 846 & So1116 & $12 / 12 / 94$ & REG & 1,1-Dichloroethane & 1 & $\mathrm{ug} / \mathrm{L}$ & $\mathrm{U}$ \\
\hline 846 & S02736 & $2 / 27 / 95$ & REG & 1.1-Dichloroethane & 5 & $\mathrm{ug} / \mathrm{L}$ & $\mathrm{U}$ \\
\hline 846 & S04829 & $8 / 24 / 95$ & REG & 1,1-Dichloroethane & 5 & $\mathrm{ug} / \mathrm{L}$ & $\mathrm{U}$ \\
\hline 846 & 846215REGUF & $2 / 15 / 91$ & REG & 1.1-Dichloroethane & 5 & $\mathrm{ug} / \mathrm{L}$ & $\mathrm{U}$ \\
\hline 846 & 846516REGUF & $5 / 16 / 90$ & REG & 1,1-Dichloroethane & 5 & $\mathrm{ug} / \mathrm{L}$ & $\mathrm{U}$ \\
\hline 846 & 8461030REGUF & $10 / 30 / 90$ & REG & 1.1-Dichloroethane & 5 & $\mathrm{ug} / \mathrm{L}$ & $\mathrm{U}$ \\
\hline 846 & 846111REGUF & $1 / 11 / 89$ & REG & 1.1-Dichloroethane & 5 & $\mathrm{ug} / \mathrm{L}$ & $\mathrm{U}$ \\
\hline 846 & 846417REGUF & $4 / 17 / 89$ & REG & 1.1-Dichloroethane & 5 & ug/L & $\mathrm{U}$ \\
\hline 846 & 84665REGUF & $6 / 5 / 88$ & REG & 1,1-Dichloroethane & 5 & $\mathrm{ug} / \mathrm{L}$ & $\mathrm{U}$ \\
\hline
\end{tabular}




\begin{tabular}{|c|c|c|c|c|c|c|c|}
\hline Station & Sample ID & Sample Date & Sample Type & Chemical & Results & Units & Qualifier \\
\hline $8+6$ & 846105 REGUF & $10 / 5 / 88$ & REG & 1.1-Dichloroethane & 5 & ug/L & $\bar{U}$ \\
\hline $8+6$ & M01273 & $11 / 19 / 97$ & REG & 1.1-Dichloroethene & 5 & ug $/$ & $\mathrm{U}$ \\
\hline $8+6$ & W00899 & $6 / 24 / 97$ & REG & 1.1-Dichloroethene & 5 & $\mathrm{ug} / \mathrm{L}$ & $\mathrm{U}$ \\
\hline 846 & M00070 & $12 / 4 / 96$ & REG & 1.1-Dichloroethene & 5 & ug $/ \mathrm{L}$ & $\mathrm{U}$ \\
\hline 846 & M01018 & $6 / 24 / 97$ & REG & 1.1-Dichloroethene & 5 & ug/L & $\mathrm{U}$ \\
\hline 846 & S01116 & $12 / 12 / 94$ & REG & 1.1-Dichloroethene & 1 & $\mathrm{ug} / \mathrm{L}$ & U \\
\hline 846 & S02736 & $2 / 27 / 95$ & REG & 1.1-Dichloroethene & 5 & $\mathrm{ug} / \mathrm{L}$ & $\mathrm{U}$ \\
\hline 846 & S04829 & $8 / 24 / 95$ & REG & 1.1-Dichloroethene & 5 & ug/L & U \\
\hline 846 & 846215REGUF & $2 / 15 / 91$ & REG & 1,1-Dichloroethene & 5 & ug/L & U \\
\hline 846 & 846516REGUF & $5 / 16 / 90$ & REG & 1,I-Dichloroethene & 5 & $u g / L$ & $\mathrm{U}$ \\
\hline 846 & 8461030REGUF & $10 / 30 / 90$ & REG & 1.1-Dichloroethene & 5 & $\mathrm{ug} / \mathrm{L}$ & $\mathrm{U}$ \\
\hline 846 & 846I11REGUF & $1 / 11 / 89$ & REG & 1,1-Dichloroethene & 5 & $\mathrm{ug} / \mathrm{L}$ & $\mathrm{U}$ \\
\hline 846 & 846417REGUF & $4 / 17 / 89$ & REG & 1,1-Dichloroethene & 5 & $u g / L$ & U \\
\hline 846 & 84665REGUF & $6 / 5 / 88$ & REG & 1,1-Dichloroethene & 5 & ug/L & $\mathrm{U}$ \\
\hline 846 & 846 I05REGUF & $10 / 5 / 88$ & REG & 1,1-Dichloroethene & 5 & $\mathrm{ug} / \mathrm{L}$ & $\mathrm{U}$ \\
\hline 846 & S01116 & $12 / 12 / 94$ & REG & 1,2-Dibromo-3-chloropropane & 1 & $\mathrm{ug} / \mathrm{L}$ & $\mathrm{U}$ \\
\hline 846 & S01116 & $12 / 12 / 94$ & REG & 1,2-Dibromoethane & 1 & ug/L & $\mathrm{U}$ \\
\hline 846 & M00070 & $12 / 4 / 96$ & REG & 1,2-Dichlorobenzene & 5 & ug/L & $\mathrm{U}$ \\
\hline 846 & M01273 & $11 / 19 / 97$ & REG & 1,2-Dichloroethane & 5 & $\mathrm{ug} / \mathrm{L}$ & $\mathrm{U}$ \\
\hline 846 & W00899 & $6 / 24 / 97$ & REG & 1.2-Dichloroethane & 5 & ug/L & $\mathrm{U}$ \\
\hline 846 & M00070 & $12 / 4 / 96$ & REG & 1.2-Dichloroethane & 5 & $\mathrm{ug} / \mathrm{L}$ & $\mathrm{U}$ \\
\hline 846 & M01018 & $6 / 24 / 97$ & REG & 1.2-Dichloroethane & 5 & ug/ & $\mathrm{U}$ \\
\hline 846 & S01116. & $12 / 12 / 94$ & REG & 1.2-Dichloroethane & 1 & ug/L & $\mathrm{U}$ \\
\hline 846 & S02736 & $2 / 27 / 95$ & REG & 1.2-Dichloroethane & 5 & $\mathrm{ug} / \mathrm{L}$ & U \\
\hline 846 & S04829 & $8 / 24 / 95$ & REG & 1,2-Dichloroethane & 5 & ug $\Omega$ & $\mathrm{U}$ \\
\hline 846 & 84621 RREGUF & $2 / 15 / 91$ & REG & 1.2-Dichloroethane & 5 & ug/ & U \\
\hline $8+6$ & 8465I6REGUF & $5 / 16 / 90$ & REG & 1.2-Dichloroethane & 5 & ug/ & U \\
\hline 846 & 8461030REGUF & $10 / 30 / 90$ & REG & 1.2-Dichloroethane & 5 & ug $/ \mathrm{L}$ & $\mathrm{U}$ \\
\hline 846 & 84611 IREGUF & $1 / 11 / 89$ & REG & 1,2-Dichloroethane & 5 & ug/L & $\mathrm{U}$ \\
\hline 846 & 846417REGUF & $4 / 17 / 89$ & REG & 1.2-Dichloroethane & 5 & ug $/$ & $\mathrm{U}$ \\
\hline 846 & 84665REGUF & $6 / 5 / 88$ & REG & 1,2-Dichloroethane & 5 & ug $/ \mathrm{L}$ & $\mathrm{U}$ \\
\hline 846 & 84610SREGUF & $10 / 5 / 88$ & REG & 1.2-Dichloroethane & 5 & ug $/ \mathrm{L}$ & $\mathrm{U}$ \\
\hline 846 & W00899 & $6 / 24 / 97$ & REG & 1.2-Dichloroethene & 5 & $\mathrm{ug} / \mathrm{L}$ & $\mathrm{U}$ \\
\hline 846 & M00070 & $12 / 4 / 96$ & REG & 1,2-Dichloroethene & 5 & ug/L & $\mathrm{U}$ \\
\hline 846 & S02736 & $2 / 27 / 95$ & REG & 1,2-Dichloroethene & 5 & ug $/ \mathrm{L}$ & U \\
\hline 846 & S04829 & $8 / 24 / 95$ & REG & 1.2-Dichloroethene & 5 & $\mathrm{ug} / \mathrm{L}$ & U \\
\hline 846 & 846215REGUF & $2 / 15 / 91$ & REG & 1.2-Dichloroethene & 5 & ug $/ \mathrm{L}$ & U \\
\hline 846 & 846516REGUF & $5 / 16 / 90$ & REG & 1.2-Dichloroethene & 5 & ug/L & $\mathrm{U}$ \\
\hline 846 & 8461030REGUF & $10 / 30 / 90$ & REG & 1.2-Dichloroethene & 5 & ug/L & $\mathrm{U}$ \\
\hline $8+6$ & 8+6111REGUF & $1 / 11 / 89$ & REG & 1.2-Dichloroethene & 5 & ug $/ \mathrm{L}$ & U \\
\hline $8+6$ & $8+6+17$ REGUF & $4 / 17 / 89$ & REG & 1.2-Dichloroethene & 5 & ugl & U \\
\hline 846 & 84665REGUF & $6 / 5 / 88$ & REG & 1.2-Dichloroethene & 5 & ug $/ \mathrm{L}$ & $\mathrm{U}$ \\
\hline 846 & 846105REGUF & $10 / 5 / 88$ & REG & 1.2-Dichloroethene & 5 & $\mathrm{ug} / \mathrm{L}$ & U \\
\hline 846 & M01273 & $11 / 19 / 97$ & REG & 1,2-Dichloropropane & 5 & ug $/ \mathrm{L}$ & $\mathrm{U}$ \\
\hline 846 & W00899 & $6 / 24 / 97$ & REG & 1.2-Dichloropropane & 5 & ug $/ \mathrm{L}$ & U \\
\hline 846 & M00070 & $12 / 4 / 96$ & REG & 1.2-Dichloropropane & 5 & ug/ & U \\
\hline 846 & M01018 & $6 / 24 / 97$ & REG & 1,2-Dichloropropane & 5 & ug/L & $\mathrm{U}$ \\
\hline 846 & So1116 & $12 / 12 / 94$ & REG & 1.2-Dichloropropane & 1 & ug/L & $\mathrm{U}$ \\
\hline 846 & S02736 & $2 / 27 / 95$ & REG & 1.2-Dichloropropane & 5 & ug $/ \mathrm{L}$ & $\mathrm{U}$ \\
\hline 846 & S04829 & $8 / 24 / 95$ & REG & 1.2-Dichloropropane & 5 & ug $/ \mathrm{L}$ & $\mathrm{U}$ \\
\hline
\end{tabular}


B-69

\begin{tabular}{|c|c|c|c|c|c|c|c|}
\hline Station & Sample ID & Sample Date & Sample Type & Chemical & Results & Units & Qualifier \\
\hline$\overline{846}$ & 846215REGUF & $2 / 15 / 91$ & REG & 1.2-Dichloropropane & 5 & $\mathrm{ug} / \mathrm{L}$ & $\mathrm{U}$ \\
\hline 846 & 846516REGUF & $5 / 16 / 90$ & REG & 1.2-Dichloropropane & 5 & ug/L & U \\
\hline 846 & 8461030 REGUF & $10 / 30 / 90$ & REG & 1.2-Dichloropropane & 5 & ug $/ \mathrm{L}$ & $\mathrm{U}$ \\
\hline $8+6$ & 84611IREGUF & $1 / 11 / 89$ & REG & 1.2-Dichloropropane & 5 & $\mathrm{ug} / \mathrm{L}$ & $\mathrm{U}$ \\
\hline 846 & 846417REGUF & $4 / 17 / 89$ & REG & 1.2-Dichloropropane & 5 & $\mathrm{ug} / \mathrm{L}$ & $\mathrm{U}$ \\
\hline 846 & 84665REGUF & $6 / 5 / 88$ & REG & 1.2-Dichloropropane & 5 & $\mathrm{ug} / \mathrm{L}$ & $\mathrm{U}$ \\
\hline 846 & 846105REGUF & $10 / 5 / 88$ & REG & 1.2-Dichloropropane & 5 & $\mathrm{ug} / \mathrm{L}$ & $\mathrm{U}$ \\
\hline 846 & M00070 & $12 / 4 / 96$ & REG & 1.2-Dimethylbenzene & 5 & $\mathrm{ug} / \mathrm{L}$ & $U$ \\
\hline 846 & S02736 & $2 / 27 / 95$ & REG & 1.2-Dimethyllbenzene & 5 & ug $/ \mathrm{L}$ & $\mathrm{U}$ \\
\hline 846 & S04829 & $8 / 24 / 95$ & REG & 1.2-Dimethylbenzene & 5 & $\mathrm{ug} /$ & $\mathrm{U}$ \\
\hline 846 & M00070 & $12 / 4 / 96$ & REG & 1.3-Dichlorobenzene & 5 & $\mathrm{ug} / \mathrm{L}$ & $\mathrm{U}$ \\
\hline 846 & M00070 & $12 / 4 / 96$ & REG & 1.4-Dichlorobenzene & 5 & $\mathrm{ug} / \mathrm{L}$ & $\mathrm{U}$ \\
\hline 846 & M01273 & $11 / 19 / 97$ & REG & 2-Butanone & 10 & $\mathrm{ug} / \mathrm{L}$ & $\mathrm{U}$ \\
\hline 846 & W00899 & $6 / 24 / 97$ & REG & 2-Butanone & 10 & $\mathrm{ug} / \mathrm{L}$ & $U$ \\
\hline 846 & M00070 & $12 / 4 / 96$ & REG & 2-Butanone & 3 & ug $/ \mathrm{L}$ & $=$ \\
\hline 846 & M01018 & $6 / 24 / 97$ & REG & 2-Butanone & 10 & $\mathrm{ug} / \mathrm{L}$ & $U$ \\
\hline 846 & S01116 & $12 / 12 / 94$ & REG & 2-Butanone & 5 & $\mathrm{ug} / \mathrm{L}$ & $\mathrm{U}$ \\
\hline 846 & S02736 & $2 / 27 / 95$ & REG & 2-Butanone & 4 & ug/L & $\mathrm{J}$ \\
\hline 846 & S04829 & $8 / 24 / 95$ & REG & 2-Butanone & 3 & $u g / L$ & $\mathrm{~J}$ \\
\hline 846 & 846215REGUF & $2 / 15 / 91$ & REG & 2-Butanone & 10 & $u g / L$ & $\mathrm{U}$ \\
\hline 846 & 846516REGUF & $5 / 16 / 90$ & REG & 2-Butanone & 10 & $\mathrm{ug} / \mathrm{L}$ & $\mathrm{U}$ \\
\hline 846 & 8461030REGUF & $10 / 30 / 90$ & REG & 2-Butanone & 10 & $u g / L$ & $\mathrm{U}$ \\
\hline 846 & 846111REGUF & $1 / 11 / 89$ & REG & 2-Butanone & 10 & $u g / L$ & $\mathrm{U}$ \\
\hline 846 & 846417REGUF & $4 / 17 / 89$ & REG & 2-Butanone & 10 & $u g / L$ & $\mathbf{U}$ \\
\hline 846 & 84665REGUF & $6 / 5 / 88$ & REG & 2-Butanone & 10 & ug $/ \mathrm{L}$ & $\mathrm{U}$ \\
\hline 846 & 846105REGUF & $10 / 5 / 88$ & REG & 2-Butanone & 10 & ug $/ \mathrm{L}$ & $\mathrm{U}$ \\
\hline 846 & M01273 & $11 / 19 / 97$ & REG & 2-Hexanone & 10 & ug $/ \mathrm{L}$ & $\mathrm{U}$ \\
\hline 846 & W00899 & $6 / 24 / 97$ & REG & 2-Hexanone & 10 & ug $/ \mathrm{L}$ & $\mathrm{U}$ \\
\hline 846 & M00070 & $12 / 4 / 96$ & REG & 2-Hexanone & 10 & $\mathrm{ug} / \mathrm{L}$ & $\mathrm{U}$ \\
\hline 846 & M01018 & $6 / 24 / 97$ & REG & 2-Hexanone & 10 & ug/L & $\mathrm{U}$ \\
\hline 846 & S01116 & $12 / 12 / 94$ & REG & 2-Hexanone & 5 & $\mathrm{ug} / \mathrm{L}$ & $\mathbf{U}$ \\
\hline 846 & S02736 & $2 / 27 / 95$ & REG & 2-Hexanone & 10 & $\mathrm{ug} / \mathrm{L}$ & $\mathrm{U}$ \\
\hline 846 & S04829 & $8 / 24 / 95$ & REG & 2-Hexanone & 10 & $\mathrm{ug} / \mathrm{L}$ & $\mathrm{U}$ \\
\hline 846 & 846215REGUF & $2 / 15 / 91$ & REG & 2-Hexanone & 10 & ug $/ \mathrm{L}$ & $\mathrm{U}$ \\
\hline $8+6$ & 846516REGUF & $5 / 16 / 90$ & REG & 2-Hexanone & 10 & $\mathrm{ug} / \mathrm{L}$ & $\mathrm{U}$ \\
\hline 846 & 8461030REGUF & $10 / 30 / 90$ & REG & 2-Hexanone & 10 & $\mathrm{ug} / \mathrm{L}$ & $\mathrm{U}$ \\
\hline 846 & 846111REGUF & $1 / 11 / 89$ & REG & 2-Hexanone & 10 & $\mathrm{ug} / \mathrm{L}$ & $\mathrm{U}$ \\
\hline 846 & 846417REGUF & $4 / 17 / 89$ & REG & 2-Hexanone & 10 & $\mathrm{ug} / \mathrm{L}$ & $\mathrm{U}$ \\
\hline 846 & 84665REGUF & $6 / 5 / 88$ & REG & 2-Hexanone & 10 & ug/L & $\mathrm{U}$ \\
\hline 846 & 846105REGUF & $10 / 5 / 88$ & REG & 2-Hexanone & 10 & $\mathrm{ug} / \mathrm{L}$ & $\mathrm{U}$ \\
\hline 846 & M01273 & $11 / 19 / 97$ & REG & 4-Methỵl-2-pentanone & 10 & $\mathrm{ug} / \mathrm{L}$ & $\mathrm{U}$ \\
\hline 846 & W00899 & $6 / 24 / 97$ & REG & 4-Methỵ1-2-pentanone & 10 & $\mathrm{ug} / \mathrm{L}$ & $\mathrm{U}$ \\
\hline 846 & M00070 & $12 / 4 / 96$ & REG & 4-Methy̧1-2-pentanone & 10 & $\mathrm{ug} / \mathrm{L}$ & $\mathrm{U}$ \\
\hline 846 & M01018 & $6 / 24 / 97$ & REG & 4-Methyl-2-pentanone & 10 & $\mathrm{ug} / \mathrm{L}$ & $\mathrm{U}$ \\
\hline 846 & S01116 & $12 / 12 / 94$ & REG & 4-Methyl-2-pentanone & 5 & $\mathrm{ug} / \mathrm{L}$ & $\mathrm{U}$ \\
\hline 846 & $\mathrm{~S} 02736$ & $2 / 27 / 95$ & REG & 4-Methỵ1-2-pentanone & 10 & $\mathrm{ug} / \mathrm{L}$ & $\mathrm{U}$ \\
\hline 846 & S04829 & $8 / 24 / 95$ & REG & 4-Methyl-2-pentanone & 10 & $\mathrm{ug} / \mathrm{L}$ & $\mathrm{U}$ \\
\hline 846 & 846215REGUF & $2 / 15 / 91$ & REG & 4-Methỵl-2-pentanone & 10 & $\mathrm{ug} / \mathrm{L}$ & $\mathrm{U}$ \\
\hline $8+6$ & 846516REGUF & $5 / 16 / 90$ & REG & 4-Methỵl-2-pentanone & 10 & $\mathrm{ug} / \mathrm{L}$ & $\mathrm{U}$ \\
\hline 846 & 8461030REGUF & $10 / 30 / 90$ & REG & 4-Methis-2-pentanone & 10 & $\mathrm{ug} / \mathrm{L}$ & $\mathrm{U}$ \\
\hline
\end{tabular}




\begin{tabular}{|c|c|c|c|c|c|c|c|}
\hline Station & Sample ID & Sample Date & Sample Type & Chemical & Results & Units & Qualifier \\
\hline 846 & 846111 REGUF & $1 / 11 / 89$ & $\overline{R E G}$ & 4-Methỵ1-2-pentanone & 10 & $\mathrm{ug} / \mathrm{L}$ & $U$ \\
\hline 846 & 846+17REGUF & $4 / 17 / 89$ & REG & 4-Methyl-2-pentanone & 2 & $\mathrm{ug} / \mathrm{L}$ & J \\
\hline $8+6$ & 84665REGUF & $6 / 5 / 88$ & REG & 4-Methỵl-2-pentanone & 10 & $u g /$ & $\mathrm{U}$ \\
\hline 846 & 846105REGUF & $10 / 5 / 88$ & REG & t-Methỵl-2-pentanone & 10 & $\mathrm{ug} / \mathrm{L}$ & $\mathrm{U}$ \\
\hline 846 & M01273 & $11 / 19 / 97$ & REG & Acetone & 10 & $\mathrm{ug} /$ & $\mathrm{U}$ \\
\hline 846 & W00899 & $6 / 24 / 97$ & REG & Acetone & 10 & $\mathrm{ug} / \mathrm{L}$ & $\mathrm{U}$ \\
\hline 846 & M00070 & $12 / 4 / 96$ & REG & Acetone & 2 & $\mathrm{ug} / \mathrm{L}$ & $=$ \\
\hline 846 & M01018 & $6 / 24 / 97$ & REG & Acetone & 10 & $\mathrm{ug} / \mathrm{L}$ & $\mathrm{U}$ \\
\hline 846 & S01116 & $12 / 12 / 94$ & REG & Acetone & 4.5 & $\mathrm{ug} / \mathrm{L}$ & $J$ \\
\hline 846 & S02736 & $2 / 27 / 95$ & REG & Acetone & 10 & ug/L & $\mathrm{U}$ \\
\hline 846 & S04829 & $8 / 24 / 95$ & REG & Acetone & 10 & $\mathrm{ug} / \mathrm{L}$ & $\mathrm{U}$ \\
\hline 846 & 846215REGUF & $2 / 15 / 91$ & REG & Acetone & 10 & $\mathrm{ug} / \mathrm{L}$ & $U$ \\
\hline 846 & 846516REGUF & $5 / 16 / 90$ & REG & Acetone & 10 & $\mathrm{ug} / \mathrm{L}$ & $\mathrm{U}$ \\
\hline 846 & 8461030REGUF & $10 / 30 / 90$ & REG & Acetone & 10 & $\mathrm{ug} / \mathrm{L}$ & $\mathrm{U}$ \\
\hline 846 & 846111REGUF & $1 / 11 / 89$ & REG & Acetone & 10 & $\mathrm{ug} / \mathrm{L}$ & $\mathrm{U}$ \\
\hline 846 & 8464l7REGUF & $4 / 17 / 89$ & REG & Acetone & 3 & ug/ & $\mathrm{J}$ \\
\hline 846 & 84665REGUF & $6 / 5 / 88$ & REG & Acetone & 10 & $\mathrm{ug} / \mathrm{L}$ & $\mathrm{U}$ \\
\hline 846 & 846105REGUF & $10 / 5 / 88$ & REG & Acetone & 4 & $\mathrm{ug} / \mathrm{L}$ & $\mathrm{J}$ \\
\hline 846 & M01273 & $11 / 19 / 97$ & REG & Benzene & 5 & $\mathrm{ug} / \mathrm{L}$ & $U$ \\
\hline 846 & W00899 & 6/24/97 & REG & Benzene & 5 & ug/L & $\mathrm{U}$ \\
\hline 846 & M00070 & $12 / 4 / 96$ & REG & Benzene & 5 & ug/L & $\mathrm{U}$ \\
\hline 846 & M01018 & $6 / 24 / 97$ & REG & Benzene & 5 & ug/L & $\mathrm{U}$ \\
\hline 846 & S01116 & $12 / 12 / 94$ & REG & Benzene & 1 & ug $/ \mathrm{L}$ & $\mathrm{U}$ \\
\hline 846 & S02736 & $2 / 27 / 95$ & REG & Benzene & 5 & $\mathrm{ug} / \mathrm{L}$ & $\mathrm{U}$ \\
\hline 846 & S04829 & $8 / 24 / 95$ & REG & Benzene & 5 & ug $\lambda$ & $\mathrm{U}$ \\
\hline 846 & 846215REGUF & $2 / 15 / 91$ & REG & Benzene & 5 & $\mathrm{ug} / \mathrm{L}$ & $\mathrm{U}$ \\
\hline 846 & 846516REGUF & $5 / 16 / 90$ & REG & Benzene & 5 & $\mathrm{ug} / \mathrm{L}$ & $\mathrm{U}$ \\
\hline 846 & 8461030REGUF & $10 / 30 / 90$ & REG & Benzene & 5 & ug $/ \mathrm{L}$ & $\mathrm{U}$ \\
\hline 846 & 846111REGUF & $1 / 11 / 89$ & REG & Benzene & 5 & ug $/$ & $\mathrm{U}$ \\
\hline 846 & 846417REGUF & $4 / 17 / 89$ & REG & Benzene & 5 & $u g / L$ & $\mathrm{U}$ \\
\hline 846 & 84665REGUF & $6 / 5 / 88$ & REG & Benzene & 5 & $\mathrm{ug} / \mathrm{L}$ & $\mathrm{U}$ \\
\hline 846 & 846105REGUF & $10 / 5 / 88$ & REG & Benzene & 5 & $\mathrm{ug} / \mathrm{L}$ & $\mathrm{U}$ \\
\hline 846 & S01116 & $12 / 12 / 94$ & REG & Bromochloromethane & 1 & $\mathrm{ug} / \mathrm{L}$ & $\mathrm{U}$ \\
\hline 846 & M01273 & $11 / 19 / 97$ & REG & Bromodichloromethane & 5 & ug $/$ & $\mathrm{U}$ \\
\hline $8+6$ & W00899 & $6 / 24 / 97$ & REG & Bromodichloromethane & 5 & $\mathrm{ug} / \mathrm{L}$ & $\mathrm{U}$ \\
\hline 846 & M00070 & $12 / 4 / 96$ & REG & Bromodichloromethane & 5 & $\mathrm{ug} / \mathrm{L}$ & $\mathrm{U}$ \\
\hline 846 & M01018 & $6 / 24 / 97$ & REG & Bromodichloromethane & 5 & $\mathrm{ug} / \mathrm{L}$ & $\mathrm{U}$ \\
\hline 846 & S01116 & $12 / 12 / 94$ & REG & Bromodichloromethane & 1 & $\mathrm{ug} / \mathrm{L}$ & $\mathrm{U}$ \\
\hline 846 & S02736 & $2 / 27 / 95$ & REG & Bromodichloromethane & 5 & ug $\Omega$ & $\mathrm{U}$ \\
\hline 846 & S04829 & $8 / 24 / 95$ & REG & Bromodichloromethane & 5 & ug/ & $\mathrm{U}$ \\
\hline 846 & 846215REGUF & $2 / 15 / 91$ & REG & Bromodichloromethane & 5 & ug/L & $\mathrm{U}$ \\
\hline 846 & 846516REGUF & $5 / 16 / 90$ & REG & Bromodichloromethane & 5 & $\mathrm{ug} / \mathrm{L}$ & $\mathrm{U}$ \\
\hline 846 & 8461030REGUF & $10 / 30 / 90$ & REG & Bromodichloromethane & 5 & ug $/$ & U \\
\hline 846 & 846111REGUF & $1 / 11 / 89$ & REG & Bromodichloromethane & 5 & ug/L & U \\
\hline 846 & 846417REGUF & $4 / 17 / 89$ & REG & Bromodichloromethane & 5 & $\mathrm{ug} / \mathrm{L}$ & $\mathrm{U}$ \\
\hline 846 & 84665REGUF & $6 / 5 / 88$ & REG & Bromodichloromethane & 5 & ug/L & $\mathrm{U}$ \\
\hline 846 & 846 I05REGUF & $10 / 5 / 88$ & REG & Bromodichloromethane & 5 & ug/L & $\mathrm{U}$ \\
\hline 846 & M01273 & $11 / 19 / 97$ & REG & Bromoform & 5 & ugl & $\mathrm{U}$ \\
\hline $8+6$ & W00899 & $6 / 24 / 97$ & REG & Bromoform & 5 & $\mathrm{ug} / \mathrm{L}$ & $\mathrm{U}$ \\
\hline $8+6$ & M00070 & $12 / 4 / 96$ & REG & Bromoform & 5 & $\mathrm{ug} / \mathrm{L}$ & $\mathrm{U}$ \\
\hline
\end{tabular}


B-71

\begin{tabular}{|c|c|c|c|c|c|c|c|}
\hline Station & Sample ID & Sample Date & Sample Type & Chemical & Results & Units & Qualifier \\
\hline$\overline{8+6}$ & 846417REGUF & $4 / 17 / 89$ & REG & Carbon tetrachloride & 5 & $\mathrm{ug} / \mathrm{L}$ & $\mathrm{U}$ \\
\hline 846 & 84665REGUF & $6 / 5 / 88$ & REG & Carbon tetrachloride & 5 & $\mathrm{ug} / \mathrm{L}$ & U \\
\hline $8+6$ & 846IOSREGUF & $10 / 5 / 88$ & REG & Carbon tetrachloride & 5 & $\mathrm{ug} / \mathrm{L}$ & U \\
\hline 846 & M01273 & $11 / 19 / 97$ & REG & Chlorobenzene & 5 & $\mathrm{ug} / \mathrm{L}$ & $\mathrm{U}$ \\
\hline 846 & W00899 & $6 / 24 / 97$ & REG & Chlorobenzene & 5 & ug/L & U \\
\hline 846 & M00070 & $12 / 4 / 96$ & REG & Chlorobenzene & 5 & ug/L & $\mathrm{U}$ \\
\hline 846 & M01018 & $6 / 24 / 97$ & REG & Chlorobenzene & 5 & ug $/$ & $\mathrm{U}$ \\
\hline 846 & S01116 & $12 / 12 / 94$ & REG & Chlorobenzene & 1 & $\mathrm{ug} / \mathrm{L}$ & U \\
\hline 846 & S02736 & $2 / 27 / 95$ & REG & Chlorobenzene & 5 & $\mathrm{ug} / \mathrm{L}$ & U \\
\hline 846 & S04829 & $8 / 24 / 95$ & REG & Chlorobenzene & 5 & ug/L & $\mathrm{U}$ \\
\hline 846 & 846215REGUF & $2 / 15 / 91$ & REG & Chlorobenzene & 5 & ug $/ \mathrm{L}$ & $\mathrm{U}$ \\
\hline 846 & 8465 l6REGUF & $5 / 16 / 90$ & REG & Chlorobenzene & 5 & $\mathrm{ug} / \mathrm{L}$ & $\mathrm{U}$ \\
\hline 846 & 8461030REGUF & $10 / 30 / 90$ & REG & Chlorobenzene & 5 & $\mathrm{ug} / \mathrm{L}$ & $\mathrm{U}$ \\
\hline 846 & 846111REGUF & $1 / 11 / 89$ & REG & Chlorobenzene & 5 & $\mathrm{ug} / \mathrm{L}$ & $\mathrm{U}$ \\
\hline 846 & 8464 17REGUF & $4 / 17 / 89$ & REG & Chlorobenzene & 5 & $\mathrm{ug} / \mathrm{L}$ & $\mathrm{U}$ \\
\hline 846 & 84665REGUF & $6 / 5 / 88$ & REG & Chlorobenzene & 5 & $\mathrm{ug} / \mathrm{L}$ & $\mathrm{U}$ \\
\hline 846 & 846105REGUF & $10 / 5 / 88$ & REG & Chlorobenzene & 5 & ug $/ \mathrm{L}$ & $\mathbf{U}$ \\
\hline 846 & M01273 & $11 / 19 / 97$ & REG & Chloroethane & 10 & $\mathrm{ug} / \mathrm{L}$ & $\mathrm{U}$ \\
\hline 846 & W00899 & $6 / 24 / 97$ & REG & Chloroethane & 10 & $\mathrm{ug} / \mathrm{L}$ & $\mathrm{U}$ \\
\hline 846 & M00070 & $12 / 4 / 96$ & REG & Chloroethane & 10 & $\mathrm{ug} / \mathrm{L}$ & $\mathrm{U}$ \\
\hline 846 & M01018 & $6 / 24 / 97$ & REG & Chloroethane & 10 & $\mathrm{ug} / \mathrm{L}$ & $\mathrm{U}$ \\
\hline 846 & S01116 & $12 / 12 / 94$ & REG & Chloroethane & 1 & ug $/ \mathrm{L}$ & $\mathrm{U}$ \\
\hline 846 & S02736 & $2 / 27 / 95$ & REG & Chloroethane & 10 & $\mathrm{ug} / \mathrm{L}$ & $\mathrm{U}$ \\
\hline 846 & S04829 & $8 / 24 / 95$ & REG & Chloroethane & 10 & ug/L & U \\
\hline 846 & 846215REGUF & $2 / 15 / 91$ & REG & Chloroethane & 10 & ug/L & $\mathrm{U}$ \\
\hline 846 & 846516REGUF & $5 / 16 / 90$ & REG & Chloroethane & 10 & $\mathrm{ug} / \mathrm{L}$ & $\mathrm{U}$ \\
\hline 846 & 8461030REGUF & $10 / 30 / 90$ & REG & Chloroethane & 10 & ug $/ \mathrm{L}$ & $\mathrm{U}$ \\
\hline $8+6$ & 84611IREGUF & $1 / 11 / 89$ & REG & Chloroethane & 10 & $\mathrm{ug} / \mathrm{L}$ & $\mathrm{U}$ \\
\hline 846 & 846417REGUF & $4 / 17 / 89$ & REG & Chloroethane & 10 & $\mathrm{ug} / \mathrm{L}$ & $\mathrm{U}$ \\
\hline 846 & 84665REGUF & $6 / 5 / 88$ & REG & Chloroethane & 10 & $\mathrm{ug} / \mathrm{L}$ & $\mathrm{U}$ \\
\hline 846 & 846105REGUF & $10 / 5 / 88$ & REG & Chloroethane & 10 & ug/ & $\mathrm{U}$ \\
\hline $8+6$ & M01273 & $11 / 19 / 97$ & REG & Chloroform & 5 & $\mathrm{ug} / \mathrm{L}$ & $\mathrm{U}$ \\
\hline 846 & W00899 & $6 / 24 / 97$ & REG & Chloroform & 5 & $\mathrm{ug} / \mathrm{L}$ & $\mathrm{U}$ \\
\hline $8+6$ & M00070 & $12 / 4 / 96$ & REG & Chloroform & 5 & ug/L & $\mathrm{U}$ \\
\hline $8+6$ & Mo1018 & $6 / 24 / 97$ & REG & Chloroform & 5 & $\mathrm{ug} / \mathrm{L}$ & $\mathrm{U}^{\mathrm{T}}$ \\
\hline $8+6$ & S01116 & $12 / 12 / 94$ & REG & Chloroform & 1 & $\mathrm{ug} / \mathrm{L}$ & $\mathrm{U}$ \\
\hline 846 & S02736 & $2 / 27 / 95$ & REG & Chloroform & 5 & $\mathrm{ug} / \mathrm{L}$ & $\mathrm{U}$ \\
\hline 846 & S04829 & $8 / 24 / 95$ & REG & Chloroform & 5 & ug/L & $\mathrm{U}$ \\
\hline 846 & 846215REGUF & $2 / 15 / 91$ & REG & Chloroform & 5 & $\operatorname{ug} / \mathrm{L}$ & $\mathrm{U}$ \\
\hline 846 & 846516REGUF & $5 / 16 / 90$ & REG & Chloroform & 5 & $\mathrm{ug} / \mathrm{L}$ & $\mathrm{U}$ \\
\hline 846 & 8461030 REGUF & $10 / 30 / 90$ & REG & Chloroform & 5 & $\mathrm{ug} / \mathrm{L}$ & $\mathrm{U}$ \\
\hline 846 & 846111REGUF & $1 / 11 / 89$ & REG & Chloroform & 5 & $\mathrm{ug} / \mathrm{L}$ & $\mathrm{U}$ \\
\hline 846 & 846417REGUF & $4 / 17 / 89$ & REG & Chloroform & 5 & ug/ & $\tilde{U}$ \\
\hline 846 & 84665REGUF & $6 / 5 / 88$ & REG & Chloroform & 5 & ug/L & $\mathrm{U}$ \\
\hline 846 & 846105REGUF & $10 / 5 / 88$ & REG & Chloroform & 5 & $\mathrm{ug} / \mathrm{L}$ & $\mathrm{U}$ \\
\hline 846 & $\mathrm{M} 01273$ & $11 / 19 / 97$ & REG & Chloromethane & 10 & ug/L & $\mathrm{U}$ \\
\hline 846 & W00899 & $6 / 24 / 97$ & REG & Chloromethane & 10 & $\mathrm{ug} / \mathrm{L}$ & $\mathrm{U}$ \\
\hline 846 & M00070 & $12 / 4 / 96$ & REG & Chloromethane & 10 & ug $/$ & $\mathrm{U}$ \\
\hline 846 & M01018 & $6 / 24 / 97$ & REG & Chloromethane & 10 & $\mathrm{ug} / \mathrm{L}$ & $\mathrm{U}$ \\
\hline $8+6$ & S01116 & $12 / 12 / 94$ & REG & Chloromethane & 1 & $\mathrm{ug} / \mathrm{L}$ & $\mathrm{U}$ \\
\hline
\end{tabular}


B-72

Station Sample ID Sample Date Sample Type Chemical

\begin{tabular}{|c|c|c|c|c|c|c|c|}
\hline 846 & $S 02736$ & $2 / 27 / 95$ & REG & Chloromethane & 10 & $\mathrm{ug} / \mathrm{L}$ & $\mathrm{U}$ \\
\hline 846 & $50+829$ & $8 / 24 / 95$ & REG & Chloromethane & 10 & ug/L & $\mathrm{U}$ \\
\hline 846 & $846215 R E G U F$ & $2 / 15 / 91$ & REG & Chloromethane & 10 & $u g / L$ & $\mathrm{U}$ \\
\hline 846 & 846516 REGUF & $5 / 16 / 90)$ & REG & Chloromethane & 10 & $\mathrm{ug} / \mathrm{L}$ & $\mathrm{U}$ \\
\hline 846 & 8461030REGUF & $10 / 30 / 90$ & REG & Chloromethane & 10 & $u g / \mathrm{L}$ & $\mathrm{U}$ \\
\hline $8+6$ & 846111REGUF & $1 / 11 / 89$ & REG & Chloromethane & 10 & $\mathrm{ug} / \mathrm{L}$ & $\mathrm{U}$ \\
\hline 846 & 846417REGUF & $4 / 17 / 89$ & REG & Chloromethane & 10 & $\mathrm{ug} / \mathrm{L}$ & $\mathrm{U}$ \\
\hline 846 & 84665REGUF & $6 / 5 / 88$ & REG & Chloromethane & 10 & $u g / L$ & $\mathrm{U}$ \\
\hline 846 & 846105REGUF & $10 / 5 / 88$ & REG & Chloromethane & 10 & $\mathrm{ug} / \mathrm{L}$ & $\mathrm{U}$ \\
\hline 846 & M01273 & $11 / 19 / 97$ & REG & cis-1.2-Dichloroethene & 5 & $\mathrm{ug} / \mathrm{L}$ & $\mathrm{U}$ \\
\hline 846 & M00070 & $12 / 4 / 96$ & REG & cis-1.2-Dichloroethene & 5 & $\mathrm{ug} / \mathrm{L}$ & $\mathbf{U}$ \\
\hline 846 & M01018 & $6 / 24 / 97$ & REG & cis-1.2-Dichloroethene & 5 & $\mathrm{ug} / \mathrm{L}$ & $\mathrm{U}$ \\
\hline 846 & S01116 & $12 / 12 / 94$ & REG & cis-1.2-Dichloroethene & 1 & $u g / L$ & $\mathrm{U}$ \\
\hline 846 & S02736 & $2 / 27 / 95$ & REG & cis-1.2-Dichloroethene & 5 & $\mathrm{ug} / \mathrm{L}$ & $\mathrm{U}$ \\
\hline 846 & SO4829 & $8 / 24 / 95$ & REG & cis-1.2-Dichloroethene & 5 & $\mathrm{ug} / \mathrm{L}$ & $\mathrm{U}$ \\
\hline 846 & $\mathrm{M} 01273$ & $11 / 19 / 97$ & REG & cis-1.3-Dichloropropen & 5 & $u g / L$ & $\mathrm{U}$ \\
\hline 846 & W00899 & $6 / 24 / 97$ & REG & cis-1.3-Dichloropropene & 5 & $\mathrm{ug} / \mathrm{L}$ & $\mathrm{U}$ \\
\hline 846 & M00070 & $12 / 4 / 96$ & REG & cis-1.3-Dichloropropene & 5 & ug $/ \mathrm{L}$ & $\mathrm{U}$ \\
\hline 846 & M01018 & $6 / 24 / 97$ & REG & cis-1.3-Dichloropropene & 5 & $\mathrm{ug} / \mathrm{L}$ & $\mathrm{U}$ \\
\hline 846 & S01116 & $12 / 12 / 94$ & REG & cis-1.3-Dichloropropene & 1 & $u g / L$ & $\mathrm{U}$ \\
\hline 846 & S02736 & $2 / 27 / 95$ & REG & cis-1.3-Dichloropropene & 5 & $\mathrm{ug} / \mathrm{L}$ & U \\
\hline 846 & S04829 & $8 / 24 / 95$ & REG & cis-1.3-Dichloropropene & 5 & $u g / L$ & $\mathrm{U}$ \\
\hline 846 & $8+6215 R E G U F$ & $2 / 15 / 91$ & REG & cis-1.3-Dichloropropene & 5 & ug/ & $\mathrm{U}$ \\
\hline 846 & 846516REGUF & $5 / 16 / 90$ & REG & cis-1.3-Dichloropropene & 5 & $u g / L$ & $\mathrm{U}$ \\
\hline 846 & 8461030REGUF & $10 / 30 / 90$ & REG & cis-1.3-Dichloropropene & 5 & ug/L & $\mathrm{U}$ \\
\hline 846 & 84611IREGUF & $1 / 11 / 89$ & REG & cis-1.3-Dichloropropene & 5 & $\mathrm{ug} / \mathrm{L}$ & $\mathrm{U}$ \\
\hline 846 & $8+6+17$ REGUF & $4 / 17 / 89$ & REG & cis-1.3-Dichloropropene & 5 & $u g / L$ & $\mathrm{U}$ \\
\hline 846 & 8+665REGUF & $6 / 5 / 88$ & REG & cis-1.3-Dichloropropene & 5 & $\mathrm{ug} / \mathrm{L}$ & $\mathrm{U}$ \\
\hline 846 & 846105REGUF & $10 / 5 / 88$ & REG & cis-1.3-Dichloropropene & 5 & ug/ $/$ & $\mathrm{U}$ \\
\hline 846 & M01273 & $11 / 19 / 97$ & REG & Dibromochloromethane & 5 & $\mathrm{ug} / \mathrm{L}$ & $\mathrm{U}$ \\
\hline 846 & W00899 & $6 / 24 / 97$ & REG & Dibromochloromethane & 5 & $\mathrm{ug} / \mathrm{L}$ & $\mathrm{U}$ \\
\hline 846 & $\mathrm{M} 00070$ & $12 / 4 / 96$ & REG & Dibromochloromethane & 5 & $\mathrm{ug} / \mathrm{L}$ & $\mathrm{U}$ \\
\hline 846 & M01018 & $6 / 24 / 97$ & REG & Dibromochloromethane & 5 & $u g / L$ & $\mathbf{U}$ \\
\hline 846 & S01116 & $12 / 12 / 94$ & REG & Dibromochloromethane & 1 & $u g / L$ & $\mathbf{U}$ \\
\hline $8+6$ & S02736 & $2 / 27: 95$ & REG & Dibromochloromethane & 5 & ug/L & $\mathrm{U}$ \\
\hline 846 & S04829 & $8 / 24 / 95$ & REG & Dibromochloromethane & 5 & ug $\mathrm{L}$ & $\mathbf{U}$ \\
\hline 846 & $8+6215 R E G U F$ & $2 / 15 / 91$ & REG & Dibromochloromethane & 5 & $u g /$ & $\mathrm{U}$ \\
\hline 846 & $8+6516$ REGUF & $5 / 16 / 90$ & REG & Dibromochloromethane & 5 & ug/L & $\mathrm{U}$ \\
\hline $8+6$ & 8461030REGUF & $10 / 30 / 90$ & REG & Dibromochloromethane & 5 & $\mathrm{ug} / \mathrm{L}$ & $\mathrm{U}$ \\
\hline 846 & 84611 IREGUF & $1 / 11 / 89$ & REG & Dibromochloromethane & 5 & $\mathrm{ug} / \mathrm{L}$ & $\mathrm{U}$ \\
\hline 846 & $8+6+17$ REGUF & $4 / 17 / 89$ & REG & Dibromochloromethane & 5 & $\mathrm{ug} / \mathrm{L}$ & $\mathrm{U}$ \\
\hline 846 & 84665REGUF & $6 / 5 / 88$ & REG & Dibromochloromethane & 5 & $\mathrm{ug} / \mathrm{L}$ & $\mathrm{U}$ \\
\hline 846 & 846105REGUF & $10 / 5 / 88$ & REG & Dibromochloromethane & 5 & $\mathrm{ug} / \mathrm{L}$ & $\mathrm{U}$ \\
\hline $8+6$ & $\mathbf{M} 00070$ & $12 / 4 / 96$ & REG & Dimethylbenzene & 5 & ug $/ \mathrm{L}$ & $\mathrm{U}$ \\
\hline 846 & 846215REGUF & $2 / 15 / 91$ & REG & Dimethylbenzene & 5 & $\mathrm{ug} / \mathrm{L}$ & $\mathrm{U}$ \\
\hline 846 & 846516REGUF & $5 / 16 / 90$ & REG & Dimethylbenzene & 5 & ug $/ \mathrm{L}$ & $\mathrm{U}$ \\
\hline 846 & 8461030REGUF & $10 / 30 / 90$ & REG & Dimethylbenzene & 5 & ug/ $/$ & $\mathrm{U}$ \\
\hline 846 & 846111REGUF & $1 / 11 / 89$ & REG & Dimethylbenzene & 5 & ug/ & $\mathrm{U}$ \\
\hline $8+6$ & $846+17$ REGUF & $4 / 17 / 89$ & REG & Dimethylbenzene & 5 & ug/L & $\mathrm{U}$ \\
\hline $8+6$ & 84665REGUF & $6 / 5 / 88$ & REG & Dimethylbenzene & 5 & ug $/ \mathrm{L}$ & $\mathrm{U}$ \\
\hline
\end{tabular}


B-73

\begin{tabular}{|c|c|c|c|c|c|c|c|}
\hline Station & Sample ID & Sample Date & Sample Type & Chemical & Results & Units & Qualifier \\
\hline $8+6$ & 846105 REGUF & $10 / 5 / 88$ & REG & Dimellyylbenzene & 5 & $\mathrm{ug} / \mathrm{L}$ & $\bar{U}$ \\
\hline 846 & M01273 & $11 / 19 / 97$ & REG & Ethỵlbenzene & 5 & $\mathrm{ug} /$ & $\mathrm{U}$ \\
\hline 846 & W00899 & $6 / 24 / 97$ & REG & Ethylbenzene & 5 & $\mathrm{ug} / \mathrm{L}$ & U \\
\hline 846 & $\mathrm{M} 00070$ & 12/4/96 & REG & Ethylbenzene & 5 & $u g / L$ & $\mathrm{U}$ \\
\hline 846 & Mol018 & $6 / 24 / 97$ & REG & Ethylbenzene & 5 & $\mathrm{ug} / \mathrm{L}$ & U \\
\hline 846 & S01116 & $12 / 12 / 94$ & REG & Ethylbenzene & 1 & $\mathrm{ug} / \mathrm{L}$ & $\mathrm{U}$ \\
\hline 846 & S02736 & $2 / 27 / 95$ & REG & Ethylbenzene & 5 & $\mathrm{ug} / \mathrm{L}$ & U \\
\hline 846 & S04829 & $8 / 24 / 95$ & REG & Ethylbenzene & 5 & ug/ & U \\
\hline 846 & 846215REGUF & $2 / 15 / 91$ & REG & Ethylbenzene & 5 & $\mathrm{ug} / \mathrm{L}$ & U \\
\hline 846 & 846516REGUF & $5 / 16 / 90$ & REG & Ethylbenzene & 5 & $\mathrm{ug} / \mathrm{L}$ & U \\
\hline 846 & 8461030REGUF & $10 / 30 / 90$ & REG & Ethylbenzene & 5 & $\mathrm{ug} / \mathrm{L}$ & $\mathrm{U}$ \\
\hline 846 & 846IIIREGUF & $1 / 11 / 89$ & REG & Ethylbenzene & 5 & $\mathrm{ug} / \mathrm{L}$ & $\mathrm{U}$ \\
\hline 846 & 846417REGUF & $4 / 17 / 89$ & REG & Ethylbenzene & 5 & $\mathrm{ug} / \mathrm{L}$ & U \\
\hline 846 & 84665REGUF & $6 / 5 / 88$ & REG & Ethylbenzene & 2 & $\mathrm{ug} / \mathrm{L}$ & J \\
\hline 846 & 846105REGUF & $10 / 5 / 88$ & REG & Ethylbenzene & 5 & $\mathrm{ug} / \mathrm{L}$ & $\mathrm{U}$ \\
\hline 846 & M01273 & $11 / 19 / 97$ & REG & m,p-Xylene & 5 & $\mathrm{ug} / \mathrm{L}$ & $\mathrm{U}$ \\
\hline 846 & W00899 & $6 / 24 / 97$ & REG & m,p-Xylene & 5 & ug/L & $\mathrm{U}$ \\
\hline 846 & M00070 & $12 / 4 / 96$ & REG & $\mathrm{m}, \mathrm{p}$-Xylene & 5 & $\mathrm{ug} / \mathrm{L}$ & $\mathrm{U}$ \\
\hline 846 & M01018 & $6 / 24 / 97$ & REG & m,p-Xylene & 5 & $\mathrm{ug} / \mathrm{L}$ & $\mathrm{U}$ \\
\hline 846 & S02736 & $2 / 27 / 95$ & REG & m,p-Xylene & 5 & $\mathrm{ug} / \mathrm{L}$ & $\mathrm{U}$ \\
\hline 846 & S04829 & $8 / 24 / 95$ & REG & $\mathrm{m}, \mathrm{p}-\mathrm{Xylene}$ & 5 & $\mathrm{ug} / \mathrm{L}$ & $\mathrm{U}$ \\
\hline 846 & M01273 & $11 / 19 / 97$ & REG & Methylene chloride & 12 & $\mathrm{ug} / \mathrm{L}$ & B \\
\hline 846 & W00899 & $6 / 24 / 97$ & REG & Methylene chloride & 10 & $\mathrm{ug} / \mathrm{L}$ & $=$ \\
\hline 846 & M00070 & $12 / 4 / 96$ & REG & Methylene chloride & 5 & $\mathrm{ug} / \mathrm{L}$ & $\mathrm{U}$ \\
\hline 846 & M01018 & $6 / 24 / 97$ & REG & Methylene chloride & 6 & $\mathrm{ug} / \mathrm{L}$ & $=$ \\
\hline 846 & S01116 & $12 / 12 / 94$ & REG & Methylene chloride & 2 & ug/L & $\mathrm{U}$ \\
\hline 846 & S02736 & $2 / 27 / 95$ & REG & Methylene chloride & 5 & $\mathrm{ug} / \mathrm{L}$ & $\mathrm{U}$ \\
\hline 846 & S04829 & $8 / 24 / 95$ & REG & Methylene chloride & 5 & $\mathrm{ug} / \mathrm{L}$ & $\mathrm{U}$ \\
\hline 846 & 846215REGUF & $2 / 15 / 91$ & REG & Methylene chloride & 5 & ug $\Omega$ & $\mathrm{U}$ \\
\hline 846 & 846516REGUF & $5 / 16 / 90$ & REG & Methylene chloride & 5 & $\mathrm{ug} / \mathrm{L}$ & $\mathrm{U}$ \\
\hline 846 & 8461030 REGUF & $10 / 30 / 90$ & REG & Methylene chloride & 5 & ug/L & $\mathrm{U}$ \\
\hline 846 & 846111REGUF & $1 / 11 / 89$ & REG & Methylene chloride & 2 & $\mathrm{ug} / \mathrm{L}$ & $\mathrm{J}$ \\
\hline 846 & $846+17$ REGUF & $+/ 17 / 89$ & REG & Methylene chloride & 1 & $\mathrm{ug} / \mathrm{L}$ & $\mathrm{J}$ \\
\hline $8+6$ & 84665REGUF & $6 / 5 / 88$ & REG & Methylene chloride & 5 & $\mathrm{ug} / \mathrm{L}$ & U \\
\hline 846 & 846105REGUF & $10 / 5 / 88$ & REG & Methylene chloride & 1 & $\mathrm{ug} / \mathrm{L}$ & $\mathrm{J}$ \\
\hline 846 & M01273 & $11 / 19 / 97$ & REG & Styrene & 5 & ug $/$ & $U$ \\
\hline 846 & W00899 & $6 / 24 / 97$ & REG & Styrene & 5 & ug $/$ & U \\
\hline 846 & M00070 & $12 / 4 / 96$ & REG & Styrene & 5 & $\mathrm{ug} / \mathrm{L}$ & $\mathrm{U}$ \\
\hline 846 & M01018 & $6 / 24 / 97$ & REG & Styrene & 5 & $\mathrm{ug} / \mathrm{L}$ & $\mathrm{U}$ \\
\hline 846 & S01116 & $12 / 12 / 94$ & REG & Styrene & 1 & $\mathrm{ug} / \mathrm{L}$ & $\mathrm{U}$ \\
\hline $8+6$ & S02736 & $2 / 27 / 95$ & REG & Styrene & 5 & ug $/ \mathrm{L}$ & $\mathrm{U}$ \\
\hline 846 & S04829 & $8 / 24 / 95$ & REG & Styrene & 5 & $\mathrm{ug} / \mathrm{L}$ & $\mathrm{U}$ \\
\hline 846 & 846215REGUF & $2 / 15 / 91$ & REG & Styrene & 5 & ug $/ \mathrm{L}$ & $\mathrm{U}$ \\
\hline 846 & 846516REGUF & $5 / 16 / 90$ & REG & Styrene & 5 & $\mathrm{ug} / \mathrm{L}$ & $U$ \\
\hline 846 & 8461030REGUF & $10 / 30 / 90$ & REG & Styrene & 5 & $\mathrm{ug} / \mathrm{L}$ & $\mathrm{U}$ \\
\hline 846 & 846111REGUF & $1 / 11 / 89$ & REG & Styrene & 5 & $\mathrm{ug} / \mathrm{L}$ & $\mathrm{U}$ \\
\hline 846 & $846+17$ REGUF & $4 / 17 / 89$ & REG & Styrene & 5 & $\mathrm{ug} / \mathrm{L}$ & $\mathrm{U}$ \\
\hline 846 & 84665REGUF & $6 / 5 / 88$ & REG & Styrene & 5 & $\mathrm{ug} / \mathrm{L}$ & $\mathrm{U}$ \\
\hline 846 & 846105 REGUF & $10 / 5 / 88$ & REG & Styrene & 5 & $\mathrm{ug} / \mathrm{L}$ & $\mathrm{U}$ \\
\hline 846 & $\mathrm{M} 01273$ & $11 / 19 / 97$ & REG & Tetrachloroethene & 5 & $\mathrm{ug} / \mathrm{L}$ & $\mathrm{U}$ \\
\hline
\end{tabular}


B-74

Station Sample ID Sample Date Sample Type Chemical

\begin{tabular}{|c|c|c|c|c|c|c|c|}
\hline 846 & W00899 & $6 / 24 / 97$ & REG & Tetrachloroethene & 5 & $\mathrm{ug} / \mathrm{h}$ & $\mathrm{U}$ \\
\hline 846 & M00070 & $12 / 4 / 96$ & REG & Tetrachloroethene & 5 & $\mathrm{ug} / \mathrm{L}$ & $\mathrm{U}$ \\
\hline 846 & M01018 & $6 / 24 / 97$ & REG & Tetrachloroethene & 5 & $u g / \mathrm{L}$ & $\mathrm{U}$ \\
\hline 846 & S01116 & $12 / 12 / 94$ & REG & Tetrachloroethene & 1 & ug $/ \mathrm{L}$ & $\mathrm{U}$ \\
\hline 846 & S02736 & $2 / 27 / 95$ & REG & Tetrachloroethene & 5 & ug $/ \mathrm{L}$ & $\mathbf{U}$ \\
\hline 846 & S04829 & $8 / 24 / 95$ & REG & Tetrachloroethene & 5 & $\mathrm{ug} / \Omega$ & $\mathrm{U}$ \\
\hline 846 & 846215REGUF & $2 / 15 / 91$ & REG & Tetrachloroethene & 5 & ug $/$ & $\mathrm{U}$ \\
\hline 846 & 846516REGUF & $5 / 16 / 90$ & REG & Tetrachloroethene & 5 & $u g / L$ & $\mathrm{U}$ \\
\hline 846 & 8461030REGUF & $10 / 30 / 90$ & REG & Tetrachloroethene & 5 & $u g /$ & $\mathrm{U}$ \\
\hline 846 & 846111REGUF & $1 / 11 / 89$ & REG & Tetrachloroethene & 5 & ug $/ \mathrm{L}$ & $\mathrm{U}$ \\
\hline 846 & 846417REGUF & $4 / 17 / 89$ & REG & Tetrachloroethene & 5 & ug $/ \mathrm{L}$ & $\mathrm{U}$ \\
\hline $8+6$ & 84665REGUF & $6 / 5 / 88$ & REG & Tetrachloroethene & 5 & ug/ $/$ & $\mathrm{U}$ \\
\hline 846 & 846105REGUF & $10 / 5 / 88$ & REG & Tetrachloroethene & 5 & ug/L & $\mathbf{U}$ \\
\hline 846 & M01273 & $11 / 19 / 97$ & REG & Toluene & 5 & ug/L & $\mathrm{U}$ \\
\hline 846 & W00899 & $6 / 24 / 97$ & REG & Toluene & 5 & ug/L & $\mathbf{U}$ \\
\hline 846 & M00070 & $12 / 4 / 96$ & REG & Toluene & 5 & $\mathrm{ug} / \mathrm{L}$ & $\mathbf{U}$ \\
\hline 846 & M01018 & $6 / 24 / 97$ & REG & Toluene & 5 & $\mathrm{ug} / \mathrm{L}$ & U \\
\hline 846 & S01116 & $12 / 12 / 94$ & REG & Toluene & 1 & ug $/$ & $\mathrm{U}$ \\
\hline 846 & S02736 & $2 / 27 / 95$ & REG & Toluene & 5 & ug $/$ & $\mathrm{U}$ \\
\hline 846 & S04829 & $8 / 24 / 95$ & REG & Toluene & 5 & $\mathrm{ug} / \mathrm{L}$ & $\mathrm{U}$ \\
\hline 846 & 846215REGUF & $2 / 15 / 91$ & REG & Toluene & 5 & ug $/ \mathrm{L}$ & $\mathrm{U}$ \\
\hline $846^{\circ}$ & 846516REGUF & $5 / 16 / 90$ & REG & Toluene & 1 & ug $/ \mathrm{L}$ & $\mathrm{J}$ \\
\hline 846 & 8461030REGUF & $10 / 30 / 90$ & REG & Toluene & 5 & $\mathrm{ug} / \mathrm{L}$ & $\mathrm{U}$ \\
\hline 846 & 846111 REGUF & $1 / 11 / 89$ & REG & Toluene & 5 & ug/ & $\mathrm{U}$ \\
\hline 846 & 846417REGUF & $4 / 17 / 89$ & REG & Toluene & 5 & ug/L & $\mathbf{U}$ \\
\hline 846 & 84665REGUF & $6 / 5 / 88$ & REG & Toluene & 5 & ug $\Omega$ & $\mathrm{U}$ \\
\hline $8+6$ & 846105REGUF & $10 / 5 / 88$ & REG & Toluene & 5 & ug/L & U \\
\hline 846 & M01273 & $11 / 19 / 97$ & REG & trans-1.2-Dichloroethe & 5 & ug $/ \mathrm{L}$ & $\mathrm{U}$ \\
\hline 846 & M00070 & $12 / 4 / 96$ & REG & trans-1.2-Dichloroethene & 5 & ug $/$ & $\mathrm{U}$ \\
\hline 846 & M01018 & $6 / 24 / 97$ & REG & trans-1,2-Dichloroethene & 5 & ug/L & $\mathrm{U}$ \\
\hline 846 & S01116 & $12 / 12 / 94$ & REG & trans-1.2-Dichloroethene & 1 & $\mathrm{ug} / \mathrm{L}$ & $\mathrm{U}$ \\
\hline 846 & S02736 & $2 / 27 / 95$ & REG & trans-1.2-Dichloroethene & 5 & ug/ & U \\
\hline $8+6$ & S04829 & $8 / 24 / 95$ & REG & trans-1.2-Dichloroethene & 5 & ug $/ \mathrm{L}$ & $\mathrm{U}$ \\
\hline 846 & M01273 & $11 / 19 / 97$ & REG & trans-1.3-Dichloroprop & 5 & ug/ & $\mathrm{U}$ \\
\hline $8+6$ & W00899 & $6 / 24 / 97$ & REG & trans-1.3-Dichloropropene & 5 & ug $/ \mathrm{L}$ & $\mathrm{U}$ \\
\hline 846 & M00070 & $12 / 4 / 96$ & REG & trans-1.3-Dichloropropene & 5 & ug $/ \mathrm{L}$ & $\mathrm{U}$ \\
\hline 846 & M01018 & $6 / 24 / 97$ & REG & trans-1.3-Dichloropropene & 5 & ug $/$ & $\mathrm{U}$ \\
\hline 846 & S01116 & $12 / 12 / 94$ & REG & trans-1,3-Dichloropropene & 1 & $u g / L$ & $\mathrm{U}$ \\
\hline 846 & S02736 & $2 / 27 / 95$ & REG & trans-1.3-Dichloropropene & 5 & $\mathrm{ug} / \mathrm{L}$ & U \\
\hline 846 & S04829 & $8 / 24 / 95$ & REG & trans-1.3-Dichloropropene & 5 & $\mathrm{ug} / \mathrm{L}$ & $\mathrm{U}$ \\
\hline $8+6$ & $8+6215$ REGUF & $2 / 15 / 91$ & REG & trans-1.3-Dichloropropene & 5 & ug $/ \mathrm{L}$ & $\mathrm{U}$ \\
\hline 846 & 8465 I6REGUF & $5 / 16 / 90$ & REG & trans-1.3-Dichloropropene & 5 & ug $/$ & $\mathrm{U}$ \\
\hline 846 & 8461030REGUF & $10 / 30 / 90$ & REG & trans-1,3-Dichloropropene & 5 & ug $/ \mathrm{L}$ & $\mathrm{U}$ \\
\hline 846 & 84611IREGUF & $1 / 11 / 89$ & REG & trans-1,3-Dichloropropene & 5 & ug/L & $\mathrm{U}$ \\
\hline 846 & 846417REGUF & $4 / 17 / 89$ & REG & trans-1,3-Dichloropropene & 5 & $\mathrm{ug} / \mathrm{L}$ & $\mathrm{U}$ \\
\hline 846 & 84665REGUF & $6 / 5 / 88$ & REG & trans-1,3-Dichloropropene & 5 & $\mathrm{ug} / \mathrm{L}$ & $\mathrm{U}$ \\
\hline 846 & 84610SREGUF & $10 / 5 / 88$ & REG & trans-1.3-Dichloropropene & 5 & ug/L & $\mathrm{U}$ \\
\hline 846 & M01273 & $11 / 19 / 97$ & REG & Trichloroethene & 5 & $\mathrm{ug} / \mathrm{L}$ & $\mathrm{U}$ \\
\hline 846 & W00899 & $6 / 24 / 97$ & REG & Trichloroethene & 5 & ug/L & $\mathrm{U}$ \\
\hline $8+6$ & M00070 & $12 / 4 / 96$ & REG & Trichloroethene & 5 & $\mathrm{ug} / \mathrm{L}$ & $\mathrm{U}$ \\
\hline
\end{tabular}


B-75

\begin{tabular}{|c|c|c|c|c|c|c|c|}
\hline Station & Sample ID & Sample Date & Sample Type & Chemical & Results & Units & Qualifier \\
\hline 846 & $\mathrm{M} 01018$ & $6 / 24 / 97$ & REG & Trichloroethene & 5 & $u g / L$ & $\bar{U}$ \\
\hline 846 & S01116 & $12 / 12 / 94$ & REG & Trichloroethene & 1 & $u g / L$ & U \\
\hline 846 & S02736 & $2 / 27 / 95$ & REG & Trichloroethene & 5 & ug/L & $\mathrm{U}$ \\
\hline 846 & S0 +829 & $8 / 24 / 95$ & REG & Trichloroethene & 5 & ug $\mathcal{L}$ & $\mathrm{U}$ \\
\hline $8+6$ & 846215REGUF & $2 / 15 / 91$ & REG & Trichloroethene & 5 & ug/L & $\mathrm{U}$ \\
\hline $8+6$ & 846516REGUF & $5 / 16 / 90$ & REG & Trichloroethene & 5 & $\mathrm{ug} / \mathrm{L}$ & $\mathrm{U}$ \\
\hline 846 & 8461030REGUF & $10 / 30 / 90$ & REG & Trichloroethene & 5 & $\mathrm{ug} / \mathrm{L}$ & $\mathrm{U}$ \\
\hline 846 & 84611IREGUF & $1 / 11 / 89$ & REG & Trichloroethene & 5 & $\mathrm{ug} / \mathrm{L}$ & U \\
\hline 846 & 846417REGUF & $4 / 17 / 89$ & REG & Trichloroethene & 5 & ug/L & $\mathrm{U}$ \\
\hline 846 & 84665REGUF & $6 / 5 / 88$ & REG & Trichloroethene & 5 & $\mathrm{ug} / \mathrm{L}$ & $\mathrm{U}$ \\
\hline 846 & 846 I0SREGUF & $10 / 5 / 88$ & REG & Trichloroethene & 5 & ug/L & U \\
\hline 846 & M01273 & $11 / 19 / 97$ & REG & Vinyl acetate & 10 & $\mathrm{ug} / \mathrm{L}$ & $\mathrm{U}$ \\
\hline 846 & M00070 & $12 / 4 / 96$ & REG & Vinyl acetate & 10 & $\mathrm{ug} / \mathrm{L}$ & $\mathrm{U}$ \\
\hline 846 & M01018 & $6 / 24 / 97$ & REG & Vinyl acetate & 10 & $\mathrm{ug} / \mathrm{L}$ & $\mathrm{U}$ \\
\hline 846 & S02736 & $2 / 27 / 95$ & REG & Vinyl acetate & 10 & $\mathrm{ug} / \mathrm{L}$ & $\mathrm{U}$ \\
\hline 846 & S04829 & $8 / 24 / 95$ & REG & Vinyl acetate & 10 & $u g / L$ & $\mathbf{U}$ \\
\hline 846 & 846215REGUF & $2 / 15 / 91$ & REG & Vinyl acetate & 10 & $\mathrm{ug} / \mathrm{L}^{\prime}$ & $\mathrm{U}$ \\
\hline 846 & 846516REGUF & $5 / 16 / 90$ & REG & Vinyl acetate & 10 & $\mathrm{ug} / \mathrm{L}$ & $\mathrm{U}$ \\
\hline 846 & 8461030REGUF & $10 / 30 / 90$ & REG & Vinyl acetate & 10 & $\mathrm{ug} / \mathrm{L}$ & $\mathbf{U}$ \\
\hline 846 & 846111REGUF & $1 / 11 / 89$ & REG & Vinyl acetate & 10 & $\mathrm{ug} / \mathrm{L}$ & $\mathrm{U}$ \\
\hline 846 & 846417REGUF & $4 / 17 / 89$ & REG & Vinyl acetate & 10 & $\mathrm{ug} / \mathrm{L}$ & $\mathrm{U}$ \\
\hline 846 & 84665REGUF & $6 / 5 / 88$ & REG & Vinyl acetate & 10 & $\mathrm{ug} / \mathrm{L}$ & $\mathrm{U}$ \\
\hline $8+6$ & 846105REGUF & $10 / 5 / 88$ & REG & Vinvl acetate & 10 & $\mathrm{ug} / \mathrm{L}$ & $\mathrm{U}$ \\
\hline 846 & M01273 & $11 / 19 / 97$ & REG & Vinyl chloride & 10 & $\mathrm{ug} / \mathrm{L}$ & $\mathrm{U}$ \\
\hline 846 & W00899 & $6 / 24 / 97$ & REG & Vinyl chloride & 10 & $\mathrm{ug} / \mathrm{L}$ & $\mathrm{U}$ \\
\hline 846 & M00070 & $12 / 4 / 96$ & REG & Vinyl chloride & 10 & ug/ & $\mathrm{U}$ \\
\hline 846 & M01018 & $6 / 24 / 97$ & REG & Vinyl chloride & 10 & $\mathrm{ug} / \mathrm{L}$ & $\mathrm{U}$ \\
\hline 846 & S01116 & $12 / 12 / 94$ & REG & Vinyl chloride & 1 & ug/L & $\mathrm{U}$ \\
\hline 846 & S02736 & $2 / 27 / 95$ & REG & Vinyl chloride & 10 & ug $/$. & $\mathrm{U}$ \\
\hline 846 & S04829 & $8 / 24 / 95$ & REG & Vinyl chloride & 10 & ug/ & $\mathbf{U}$ \\
\hline 846 & 846215REGUF & $2 / 15 / 91$ & REG & Vinyl chloride & 10 & $\mathrm{ug} / \mathrm{L}$ & $\mathrm{U}$ \\
\hline $8+6$ & 846516REGUF & $5 / 16 / 90$ & REG & Vinyl chloride & 10 & $\mathrm{ug} / \mathrm{L}$ & $\mathrm{U}$ \\
\hline 846 & 8461030REGUF & $10 / 30 / 90$ & REG & Vinyl chloride & 10 & ug $/ \mathrm{L}$ & $\mathrm{U}$ \\
\hline 846 & 846111REGUF & $1 / 11 / 89$ & REG & Vinyl chloride & 10 & ug/L & $\mathbf{U}$ \\
\hline $8+6$ & $846+17$ REGUF & $4 / 17 / 89$ & REG & Vinyl chloride & 10 & $\mathrm{ug} / \mathrm{h}$ & $\mathrm{U}$ \\
\hline 846 & 84665REGUF & $6 / 5 / 88$ & REG & Vinyl chloride & 10 & ug $/$ & $\mathbf{U}$ \\
\hline 846 & 846105REGUF & $10 / 5 / 88$ & REG & Vinyl chloride & 10 & ug/L & $\mathrm{U}$ \\
\hline 857 & M01279 & $11 / 13 / 97$ & REG & 1.1,1-Trichloroethane & 5 & ug $/ \mathrm{L}$ & $\mathrm{U}$ \\
\hline 857 & W00814 & $6 / 17 / 97$ & REG & 1.1,1-Trichloroethane & 5 & $\mathrm{ug} / \mathrm{L}$ & $\mathrm{U}$ \\
\hline 857 & $\mathrm{M} 00870$ & $6 / 17 / 97$ & REG & 1.1.1-Trichloroethane & 5 & $\mathrm{ug} / \mathrm{L}$ & $\mathrm{U}$ \\
\hline 857 & S01222 & $11 / 1 / 94$ & REG & 1.1.1-Trichloroethane & 1 & ug $/ \mathrm{L}$ & $\mathrm{U}$ \\
\hline 857 & S02769 & $3 / 6 / 95$ & REG & 1.1,1-Trichloroethane & 5 & $u g / L$ & $\mathrm{U}$ \\
\hline 857 & S04927 & $8 / 3 / 95$ & REG & 1.1.1-Trichloroethane & 5 & $\mathrm{ug} / \mathrm{L}$ & U \\
\hline 857 & 85721 SREGUF & $2 / 15 / 91$ & REG & 1.1.1-Trichloroethane & 5 & $\mathrm{ug} / \mathrm{L}$ & $\mathrm{U}$ \\
\hline 857 & 857516REGUF & $5 / 16 / 90$ & REG & 1.1.1-Trichloroethane & 5 & ug $/ \mathrm{L}$ & $\mathrm{U}$ \\
\hline 857 & 857112REGUF & $11 / 2 / 90$ & REG & 1,1,1-Trichloroethane & 5 & $\operatorname{ug} / \mathrm{L}$ & $\mathrm{U}$ \\
\hline 857 & 85729REGUF & $2 / 9 / 89$ & REG & 1.1.1-Trichloroethane & 5 & ug $/ \mathrm{L}$ & U \\
\hline 857 & 857418REGUF & $4 / 18 / 89$ & REG & 1.1.1-Trichloroethane & 5 & ug $/ \mathrm{L}$ & U \\
\hline 857 & $85761+$ REGUF & $6 / 14 / 88$ & REG & 1.1.1-Trichloroethane & 5 & $\mathrm{ug} / \mathrm{L}$ & U \\
\hline 857 & 8571010REGUF & $10 / 10 / 88$ & REG & 1.1.1-Trichloroethane & 5 & ug/L & U \\
\hline
\end{tabular}




\begin{tabular}{|c|c|c|c|c|c|c|c|}
\hline Station & Sample ID & Sample Date & Sample Type & Chemical & Results & Units & Qualifier \\
\hline 857 & M01279 & $11 / 13 / 97$ & REG & 1.1.2.2-Tetrachloroeth & 5 & ug/L & $\mathrm{U}$ \\
\hline 857 & W00817 & $6 / 17 / 97$ & REG & 1.1.2.2-Tetrachloroethane & 5 & ug $/ \mathrm{L}$ & $\mathrm{U}$ \\
\hline 8.57 & M0n870 & $6 / 17 / 97$ & REG & 1.1.2.2-Tetrachloroethane & 5 & ug/L & U \\
\hline 857 & $\mathrm{~S} 01222$ & $11 / 1 / 94$ & REG & 1.1.2.2-Tetrachloroethane & 1 & ug/L & $\mathrm{U}$ \\
\hline 857 & S02769 & $3 / 6 / 95$ & REG & 1.1.2.2-Tetrachloroethane & 5 & $\mathrm{ug} / \mathrm{L}$ & $\mathrm{U}$ \\
\hline 857 & S04927 & $8 / 3 / 95$ & REG & 1.1.2.2-Tetrachloroethane & 5 & $\mathrm{ug} / \mathrm{L}$ & $\mathrm{U}$ \\
\hline 857 & 857215REGUF & $2 / 15 / 91$ & REG & 1,1.2.2-Tetrachloroethane & 5 & $\mathrm{ug} / \mathrm{L}$ & $\mathrm{U}$ \\
\hline 857 & 857516REGUF & $5 / 16 / 90$ & REG & 1,1,2,2-Tetrachloroethane & 5 & $\mathrm{ug} / \mathrm{L}$ & $\mathrm{U}$ \\
\hline 857 & 857112REGUF & $11 / 2 / 90$ & REG & 1,1,2,2-Tetrachloroethane & 5 & $\mathrm{ug} / \mathrm{L}$ & $\mathrm{U}$ \\
\hline 857 & 85729REGUF & $2 / 9 / 89$ & REG & 1.1,2,2-Tetrachloroethane & 5 & ug/ & $\mathrm{U}$ \\
\hline 857 & 857418REGUF & $4 / 18 / 89$ & REG & 1,1,2.2-Tetrachloroethane & 5 & $\mathrm{ug} / \mathrm{L}$ & $\mathrm{U}$ \\
\hline 857 & 857614REGUF & $6 / 14 / 88$ & REG & 1,1.2,2-Tetrachloroethane & 5 & $\mathrm{ug} / \mathrm{L}$ & $\mathrm{U}$ \\
\hline 857 & 8571010REGUF & $10 / 10 / 88$ & REG & 1.1.2,2-Tetrachloroethane & 5 & $\mathrm{ug} / \mathrm{L}$ & $\mathrm{U}$ \\
\hline 857 & W00814 & $6 / 17 / 97$ & REG & 1,1,2-Trichloro-1.2.2-trifluo & 10 & ug $/ \mathrm{h}$ & $\mathrm{U}$ \\
\hline 857 & M01279 & $11 / 13 / 97$ & REG & 1,1.2-Trichloroethane & 5 & $\mathrm{ug} / \mathrm{L}$ & $\mathrm{U}$ \\
\hline 857 & W00814 & $6 / 17 / 97$ & REG & 1,1.2-Trichloroethane & 5 & $\mathrm{ug} / \mathrm{L}$ & $\mathrm{U}$ \\
\hline 857 & M00870 & $6 / 17 / 97$ & REG & 1,1,2-Trichloroethane & 5 & $\mathrm{ug} / \mathrm{L}$ & $\mathrm{U}$ \\
\hline 857 & S01222 & $11 / 1 / 94$ & REG & 1.1.2-Trichloroethane & 1 & $\mathrm{ug} / \mathrm{L}$ & $\mathrm{U}$ \\
\hline 857 & S02769 & $3 / 6 / 95$ & REG & 1,1,2-Trichloroethane & 5 & ug/ & $\mathrm{U}$ \\
\hline 857 & S04927 & $8 / 3 / 95$ & REG & 1.1.2-Trichloroethane & 5 & $\mathrm{ug} / \mathrm{L}$ & $\mathrm{U}$ \\
\hline 857 & 857215REGUF & $2 / 15 / 91$ & REG & 1,1.2-Trichloroethane & 5 & $\mathrm{ug} /$ & $\mathrm{U}$ \\
\hline 857 & 857516REGUF & $5 / 16 / 90$ & REG & 1,1,2-Trichloroethane & 5 & ug/ & $\mathrm{U}$ \\
\hline 857 & 857112REGUF & $11 / 2 / 90$ & REG & 1,1,2-Trichloroethane & 5 & ug/ & $\mathbf{U}$ \\
\hline 857 & 85729REGUF & $2 / 9 / 89$ & REG & 1.1,2-Trichloroethane & 5 & $\mathrm{ug} / \mathrm{L}$ & $\mathrm{U}$ \\
\hline 857 & 857418REGUF & $4 / 18 / 89$ & REG & 1,1,2-Trichloroethane & 5 & ug/L & $\mathrm{U}$ \\
\hline 857 & 857614REGUF & $6 / 14 / 88$ & REG & 1.1.2-Trichloroethane & 5 & $\mathrm{ug} / \mathrm{L}$ & $\mathrm{U}$ \\
\hline 857 & 8571010REGUF & $10 / 10 / 88$ & REG & 1.1.2-Trichloroethane & 5 & $\mathrm{ug} / \mathrm{L}$ & $\mathrm{U}$ \\
\hline 857 & Mo1279 & $11 / 13 / 97$ & REG & 1.1-Dichloroethane & 5 & $\mathrm{ug} / \mathrm{L}$ & $\mathrm{U}$ \\
\hline 857 & W00814 & $6 / 17 / 97$ & REG & 1,1-Dichloroethane & 5 & ug $\Omega$ & $\mathrm{U}$ \\
\hline 857 & M00870 & $6 / 17 / 97$ & REG & 1.1-Dichloroethane & 5 & ug/ & $\mathrm{U}$ \\
\hline 857 & S01222 & $11 / 1 / 94$ & REG & 1,1-Dichloroethane & 1 & $\mathrm{ug} / \mathrm{L}$ & $\mathrm{U}$ \\
\hline 857 & S02769 & $3 / 6 / 95$ & REG & 1.1-Dichloroethane & 5 & ug/ & $\mathrm{U}$ \\
\hline 857 & S04927 & $8 / 3 / 95$ & REG & 1,1-Dichloroethane & 5 & $\mathrm{ug} / \mathrm{L}$ & $\mathrm{U}$ \\
\hline 857 & 857215REGUF & $2 / 15 / 91$ & REG & 1.1-Dichloroethane & 5 & $\mathrm{ug} / \mathrm{L}$ & $\mathrm{U}$ \\
\hline 857 & 857516REGUF & $5 / 16 / 90$ & REG & 1.1-Dichloroethane & 5 & ug/L & $\mathrm{U}$ \\
\hline 857 & 857112 REGUF & $11 / 2 / 90$ & REG & 1,1-Dichloroethane & 5 & $\mathrm{ug} / \mathrm{L}$ & $\mathrm{U}$ \\
\hline 857 & 85729REGUF & $2 / 9 / 89$ & REG & 1.1-Dichloroethane & 5 & $\mathrm{ug} / \mathrm{L}$ & $\mathrm{U}$ \\
\hline 857 & 857418REGUF & $4 / 18 / 89$ & REG & 1.1-Dichloroethane & 5 & $u g / L$ & $\mathrm{U}$ \\
\hline 857 & 857614REGUF & $6 / 14 / 88$ & REG & 1,1-Dichloroethane & 5 & $\mathrm{ug} / \mathrm{L}$ & $\mathrm{U}$ \\
\hline 857 & 8571010REGUF & $10 / 10 / 88$ & REG & 1.1-Dichloroethane & 5 & $\mathrm{ug} / \mathrm{L}$ & U \\
\hline 857 & M01279 & $11 / 13 / 97$ & REG & 1,1-Dichloroethene & $j$ & $\mathrm{ug} / \mathrm{L}$ & $\mathrm{U}$ \\
\hline 857 & W00814 & $6 / 17 / 97$ & REG & 1.1-Dichloroethene & 5 & $\mathrm{ug} / \mathrm{L}$ & $\mathrm{U}$ \\
\hline 857 & M00870 & $6 / 17 / 97$ & REG & 1.1-Dichloroethene & 5 & $\mathrm{ug} / \mathrm{L}$ & $\mathrm{U}$ \\
\hline 857 & S01222 & $11 / 1 / 94$ & REG & 1,1-Dichloroethene & 1 & $\mathrm{ug} / \mathrm{L}$ & U \\
\hline 857 & S02769 & $3 / 6 / 95$ & REG & 1,1-Dichloroethene & 5 & ug $/ \mathrm{L}$ & $\mathrm{U}$ \\
\hline 857 & S04927 & $8 / 3 / 95$ & REG & 1,1-Dichloroethene & 5 & ug/L & $\mathrm{U}$ \\
\hline 857 & 857215REGUF & $2 / 15 / 91$ & REG & 1.1-Dichloroethene & 5 & ug/ & $\mathrm{U}$ \\
\hline 857 & 857516REGUF & $5 / 16 / 90$ & REG & 1.1-Dichloroethene & 5 & ug/L & $\mathrm{U}$ \\
\hline 857 & 857112 REGUF & $11 / 2 / 90$ & REG & 1.1-Dichloroethene & 5 & $\mathrm{ug} / \mathrm{L}$ & $\mathrm{U}$ \\
\hline 857 & 85729REGUF & $2 / 9 / 89$ & REG & 1.1-Dichloroethene & 5 & $\operatorname{ug} \Omega$ & $\mathrm{U}$ \\
\hline
\end{tabular}




\begin{tabular}{|c|c|c|c|c|c|c|c|}
\hline Station & Sample ID & Sample Date & Sample Type & Chemical & Results & Units & Qualifier \\
\hline 8.57 & 857+18REGUF & $4 / 18 / 89$ & REG & 1.1-Dichloroethene & 5 & $\mathrm{ug} / \mathrm{L}$ & $\overline{\mathrm{U}}$ \\
\hline 857 & 857614REGUF & $6 / 14 / 88$ & REG & 1.1-Dichloroethene & 5 & $\mathrm{ug} / \mathrm{L}$ & $\mathrm{U}$ \\
\hline 857 & 8571010REGUF & $10 / 10 / 88$ & REG & 1.1-Dichloroethene & 5 & $\mathrm{ug} / \mathrm{L}$ & $\mathrm{U}$ \\
\hline 857 & S01222 & $11 / 1 / 94$ & REG & 1.2-Dibromo-3-chloropropane & 1 & $\mathrm{ug} / \mathrm{L}$ & U \\
\hline 857 & S01222 & $11 / 1 / 94$ & REG & 1.2-Dibromoethane & 1 & $\mathrm{ug} / \mathrm{L}$ & U \\
\hline 857 & M01279 & $11 / 13 / 97$ & REG & 1.2-Dichloroethane & 5 & $\mathrm{ug} / \mathrm{L}$ & $\mathrm{U}$ \\
\hline 857 & W00814 & $6 / 17 / 97$ & REG & 1.2-Dichloroethane & 5 & ug/L & $\mathrm{U}$ \\
\hline 857 & M00870 & $6 / 17 / 97$ & REG & 1.2-Dichloroethane & 5 & $\mathrm{ug} / \mathrm{L}$ & $\mathrm{U}$ \\
\hline 857 & S01222 & $11 / 1 / 94$ & REG & 1,2-Dichloroethane & 1 & $\mathrm{ug} / \mathrm{L}$ & $\mathrm{U}$ \\
\hline 857 & S02769 & $3 / 6 / 95$ & REG & 1,2-Dichloroethane & 5 & $\mathrm{ug} / \mathrm{L}$ & $\mathrm{U}$ \\
\hline 857 & S04927 & $8 / 3 / 95$ & REG & 1.2-Dichloroethane & 5 & $\mathrm{ug} / \mathrm{L}$ & $\mathrm{U}$ \\
\hline 857 & 857215REGUF & $2 / 15 / 91$ & REG & 1.2-Dichloroethane & 5 & $\mathrm{ug} / \mathrm{L}$ & $\mathrm{U}$ \\
\hline 857 & 857516REGUF & $5 / 16 / 90$ & REG & 1,2-Dichloroethane & 5 & $\mathrm{ug} / \mathrm{L}$ & $\mathrm{U}$ \\
\hline 857 & 857112REGUF & $11 / 2 / 90$ & REG & 1,2-Dichloroethane & 5 & $\mathrm{ug} / \mathrm{L}$ & U \\
\hline 857 & 85729REGUF & $2 / 9 / 89$ & REG & 1,2-Dichloroethane & 5 & $\mathrm{ug} / \mathrm{L}$ & $\mathrm{U}$ \\
\hline 857 & 857418REGUF & $4 / 18 / 89$ & REG & 1.2-Dichloroethane & 5 & $\mathrm{ug} / \mathrm{L}$ & $\mathrm{U}$ \\
\hline 857 & 857614REGUF & $6 / 14 / 88$ & REG & 1,2-Dichloroethane & 5 & ug $/$ & $\mathrm{U}$ \\
\hline 857 & 8571010REGUF & $10 / 10 / 88$ & REG & 1,2-Dichloroethane & 5 & $u g / L$ & $\mathrm{U}$ \\
\hline 857 & W00814 & $6 / 17 / 97$ & REG & 1,2-Dichloroethene & 5 & $\mathrm{ug} / \mathrm{L}$ & U \\
\hline 857 & S02769 & $3 / 6 / 95$ & REG & 1.2-Dichloroethene & 5 & $\mathrm{ug} / \mathrm{L}$ & $\mathrm{U}$ \\
\hline 857 & S04927 & $8 / 3 / 95$ & REG & 1.2-Dichloroethene & 5 & $\mathrm{ug} / \mathrm{L}$ & $\mathrm{U}$ \\
\hline 857 & 857215REGUF & $2 / 15 / 91$ & REG & 1.2-Dichloroethene & 5 & ug/L & $\mathbf{U}$ \\
\hline 857 & 857516REGUF & $5 / 16 / 90$ & REG & 1.2-Dichloroethene & 5 & ug/L & $\mathrm{U}$ \\
\hline 857 & 857112REGUF & $11 / 2 / 90$ & REG & 1.2-Dichloroethene & 5 & $\mathrm{ug} / \mathrm{L}$ & $\mathrm{U}$ \\
\hline 857 & 85729REGUF & $2 / 9 / 89$ & REG & 1.2-Dichloroethene & 5 & $u g / L$ & $\mathrm{U}$ \\
\hline 857 & 857418REGUF & $4 / 18 / 89$ & REG & 1.2-Dichloroethene & 5 & $\mathrm{ug} / \mathrm{L}$ & $\mathrm{U}$ \\
\hline 857 & 85761+REGUF & $6 / 14 / 88$ & REG & 1.2-Dichloroethene & 5 & $\mathrm{ug} / \mathrm{L}$ & $\mathrm{U}$ \\
\hline 857 & 8571010REGUF & $10 / 10 / 88$ & REG & 1.2-Dichloroethene & 5 & $u g / L$ & $\mathrm{U}$ \\
\hline 857 & M01279 & $11 / 13 / 97$ & REG & 1,2-Dichloropropane & 5 & $\mathrm{ug} / \mathrm{L}$ & $\mathrm{U}$ \\
\hline 857 & W00814 & $6 / 17 / 97$ & REG & 1.2-Dichloropropane & 5 & $\mathrm{ug} / \mathrm{L}$ & $\mathrm{U}$ \\
\hline 857 & M00870 & $6 / 17 / 97$ & REG & 1.2-Dichloropropane & 5 & $u g / L$ & $\mathrm{U}$ \\
\hline 857 & S01222 & $11 / 1 / 94$ & REG & 1.2-Dichloropropane & 1 & $\mathrm{ug} / \mathrm{L}$ & $\mathrm{U}$ \\
\hline 857 & S02769 & $3 / 6 / 95$ & REG & 1.2-Dichloropropane & 5 & ug/L & $\mathrm{U}$ \\
\hline 857 & S04927 & $8 / 3 / 95$ & REG & 1.2-Dichloropropane & 5 & $\mathrm{ug} / \mathrm{L}$ & $\mathrm{U}$ \\
\hline 857 & 857215REGUF & $2 / 15 / 91$ & REG & 1.2-Dishloropropane & 5 & $\mathrm{ug} / \mathrm{L}$ & $\mathrm{U}$ \\
\hline 857 & 857516REGUF & $5 / 16 / 90$ & REG & 1.2-Dichloropropane & 5 & $\mathrm{ug} / \mathrm{L}$ & $\mathrm{U}$ \\
\hline 857 & 857112REGUF & $11 / 2 / 90$ & REG & 1.2-Dichloropropane & 5 & $\mathrm{ug} / \mathrm{L}$ & $\mathrm{U}$ \\
\hline 857 & 85729REGUF & $2 / 9 / 89$ & REG & 1.2-Dichloropropane & 5 & ug/L & $\mathrm{U}$ \\
\hline 857 & 857418REGUF & $4 / 18 / 89$ & REG & 1.2-Dichloropropane & 5 & ug/L & $\mathrm{U}$ \\
\hline 857 & 857614REGUF & $6 / 14 / 88$ & REG & 1.2-Dichloropropane & 5 & ug $/$ & U \\
\hline 857 & 8571010REGUF & $10 / 10 / 88$ & REG & 1.2-Dichloropropane & 5 & $\mathrm{ug} / \mathrm{L}$ & $\mathrm{U}$ \\
\hline 857 & S02769 & $3 / 6 / 95$ & REG & 1.2-Dimethylbenzene & 5 & $\mathrm{ug} / \mathrm{L}$ & $\mathrm{U}$ \\
\hline 857 & S04927 & $8 / 3 / 95$ & REG & 1.2-Dimethylbenzene & 5 & ug $/ \mathrm{L}$ & $\mathrm{U}$ \\
\hline 857 & M01279 & $11 / 13 / 97$ & REG & 2-Butanone & 10 & $\mathrm{ug} / \mathrm{L}$ & $\mathrm{U}$ \\
\hline 857 & W00814 & $6 / 17 / 97$ & REG & 2-Butanone & 10 & $\mathrm{ug} / \mathrm{L}$ & $\mathrm{U}$ \\
\hline 857 & M00870 & $6 / 17 / 97$ & REG & 2-Butanone & 10 & $\mathrm{ug} / \mathrm{L}$ & $\mathrm{U}$ \\
\hline 857 & S01222 & $11 / 1 / 94$ & REG & 2-Butanone & 5 & $\mathrm{ug} / \mathrm{L}$ & $\mathbf{U}$ \\
\hline 857 & S02769 & $3 / 6 / 95$ & REG & 2-Butanone & 2 & ug/L & J \\
\hline 857 & S04927 & $8 / 3 / 95$ & REG & 2-Butanone & 10 & ug $/$ & $\mathrm{U}$ \\
\hline 857 & 857215REGUF & $2 / 15 / 91$ & REG & 2-Butanone & 10 & ug/ $/$ & $\mathrm{U}$ \\
\hline
\end{tabular}




\begin{tabular}{|c|c|c|c|c|c|c|c|}
\hline Station & Sample ID & Sample Date & Sample Type & Chemical & Results & Units & Qualifier \\
\hline 857 & 857516REGUF & $5 / 16 / 90$ & REG & 2-Butanone & 10 & ug/ & $\bar{U}$ \\
\hline 857 & 857112REGUF & $11 / 2 / 90$ & REG & 2-Butanone & 10 & ug/L & $\mathrm{U}$ \\
\hline 857 & 85729REGUF & $2 / 9 / 89$ & REG & 2-Butanone & 10 & $\mathrm{ug} / \mathrm{L}$ & $\mathrm{U}$ \\
\hline 857 & 857418 REGUF & $4 / 18 / 89$ & REG & 2-Butanone & 10 & $\mathrm{ug} / \mathrm{L}$ & U \\
\hline 857 & 857614REGUF & $6 / 14 / 88$ & REG & 2-Butanone & 10 & ug/L & $\mathrm{U}$ \\
\hline 857 & 8571010REGUF & $10 / 10 / 88$ & REG & 2-Butanone & 10 & $\mathrm{ug} / \mathrm{L}$ & $\mathrm{U}$ \\
\hline 857 & M01279 & $11 / 13 / 97$ & REG & 2-Hexanone & 10 & ug/L & $\mathrm{U}$ \\
\hline 857 & W00814 & $6 / 17 / 97$ & REG & 2-Hexanone & 10 & $\mathrm{ug} / \mathrm{L}$ & $\mathrm{U}$ \\
\hline 857 & M00870 & $6 / 17 / 97$ & REG & 2-Hexanone & 10 & ug/L & $\mathrm{U}$ \\
\hline 857 & S01222 & $11 / 1 / 94$ & REG & 2-Hexanone & 5 & $\mathrm{ug} / \mathrm{L}$ & $\mathrm{U}$ \\
\hline 857 & S02769 & $3 / 6 / 95$ & REG & 2-Hexanone & 10 & $\mathrm{ug} / \mathrm{L}$ & $\mathrm{U}$ \\
\hline 857 & S04927 & $8 / 3 / 95$ & REG & 2-Hexanone & 10 & $u g / L$ & $\mathrm{U}$ \\
\hline 857 & 857215REGUF & $2 / 15 / 91$ & REG & 2-Hexanone & 10 & $\mathrm{ug} / \mathrm{L}$ & $\mathrm{U}$ \\
\hline 857 & 857516REGUF & $5 / 16 / 90$ & REG & 2-Hexanone & 10 & $\mathrm{ug} / \mathrm{L}$ & $\mathrm{U}$ \\
\hline 857 & 857112REGUF & $11 / 2 / 90$ & REG & 2-Hexanone & 10 & $u g / L$ & $\mathbf{U}$ \\
\hline 857 & 85729REGUF & $2 / 9 / 89$ & REG & 2-Hexanone & 10 & $u g / L$ & $\mathrm{U}$ \\
\hline 857 & 857418REGUF & $4 / 18 / 89$ & REG & 2-Hexanone & 10 & $\mathrm{ug} / \mathrm{L}$ & $\mathrm{U}$ \\
\hline 857 & 857614REGUF & $6 / 14 / 88$ & REG & 2-Hexanone & 10 & $\mathrm{ug} / \mathrm{L}$ & $\mathrm{U}$ \\
\hline 857 & 8571010REGUF & $10 / 10 / 88$ & REG & 2-Hexanone & 10 & ug $/ \mathrm{L}$ & $\mathrm{U}$ \\
\hline 857 & M01279 & $11 / 13 / 97$ & REG & 4-Methỵl-2-pentanone & 10 & $\mathrm{ug} / \mathrm{L}$ & $\mathrm{U}$ \\
\hline 857 & W00814 & $6 / 17 / 97$ & REG & 4-Methyl-2-pentanone & 10 & $u g / L$ & $\mathrm{U}$ \\
\hline 857 & M00870 & $6 / 17 / 97$ & REG & 4-Methyl-2-pentanone & 10 & $\mathrm{ug} / \mathrm{L}$ & $\mathrm{U}$ \\
\hline 857 & S01222 & $11 / 1 / 94$ & REG & t-Methyl-2-pentanone & 5 & ug $/ \mathrm{L}$ & $\mathrm{U}$ \\
\hline 857 & S02769 & $3 / 6 / 95$ & REG & 4-Methỵl-2-pentanone & 10 & $\mathrm{ug} / \mathrm{L}$ & $\mathrm{U}$ \\
\hline 857 & S04927 & $8 / 3 / 95$ & REG & 4-Methyl-2-pentanone & 10 & $\mathrm{ug} / \mathrm{L}$ & $\mathrm{U}$ \\
\hline 857 & 857215REGUF & $2 / 15 / 91$ & REG & 4-Methyl-2-pentanone & 10 & ug/L & $\mathrm{U}$ \\
\hline 857 & 857516REGUF & $5 / 16 / 90$ & REG & 4-Methyll-2-pentanone & 10 & $\mathrm{ug} / \mathrm{L}$ & $\mathrm{U}$ \\
\hline 857 & 857112REGUF & $11 / 2 / 90$ & REG & 4-Methyl-2-pentanone & 10 & ug/L & U \\
\hline 857 & 85729REGUF & $2 / 9 / 89$ & REG & 4-Methyl-2-pentanone & 10 & ug $/ \mathrm{L}$ & $\mathrm{U}$ \\
\hline 857 & 857418REGUF & $4 / 18 / 89$ & REG & 4-Methyl-2-pentanone & 10 & ug/ & $\mathrm{U}$ \\
\hline 857 & 857614REGUF & $6 / 14 / 88$ & REG & 4-Methyl-2-pentanone & 10 & ug/L & $\mathrm{U}$ \\
\hline 857 & 8571010REGUF & $10 / 10 / 88$ & REG & 4-Methyl-2-pentanone & 10 & $\mathrm{ug} / \mathrm{L}$ & U \\
\hline 857 & M01279 & $11 / 13 / 97$ & REG & Acetone & 7 & ug/L & B \\
\hline 857 & W00814 & $6 / 17 / 97$ & REG & Acetone & 10 & ug/L & $\mathrm{U}$ \\
\hline 857 & M00870 & $6 / 17 / 97$ & REG & Acetone & 4 & ug/L & $\mathrm{U}$ \\
\hline 857 & S01222 & $11 / 1 / 94$ & REG & Acetone & 1 & ug/L & $\mathrm{U}$ \\
\hline 857 & S02769 & $3 / 6 / 95$ & REG & Acetone & 10 & ug/ & $\mathrm{U}$ \\
\hline 857 & S04927 & $8 / 3 / 95$ & REG & Acetone & 10 & ug/L & $\mathrm{U}$ \\
\hline 857 & 857215REGUF & $2 / 15 / 91$ & REG & Acetone & 10 & $\mathrm{ug} / \mathrm{L}$ & U \\
\hline 857 & 857516REGUF & $5 / 16 / 90$ & REG & Acetone & 10 & ug/L & U \\
\hline 857 & 857112REGUF & $11 / 2 / 90$ & REG & Acetone & 10 & ug/L & U \\
\hline 857 & 85729REGUF & $2 / 9 / 89$ & REG & Acetone & 4 & ug $/ \mathrm{L}$ & $\mathbf{J}$ \\
\hline 857 & 857418REGUF & $4 / 18 / 89$ & REG & Acetone & 93 & ug/ & $=$ \\
\hline 857 & 85761+REGUF & $6 / 14 / 88$ & REG & Acetone & 11 & ug/L & $=$ \\
\hline 857 & 8571010REGUF & $10 / 10 / 88$ & REG & Acetone & 1 & ug $/$ & $\mathrm{J}$ \\
\hline 857 & M01279 & $11 / 13 / 97$ & REG & Benzene & 5 & ug/ & $\mathrm{U}$ \\
\hline 857 & W00814 & $6 / 17 / 97$ & REG & Benzene & 5 & ug/L & $\mathrm{U}$ \\
\hline 857 & M00870 & $6 / 17 / 97$ & REG & Benzene & 5 & $\mathrm{ug} / \mathrm{L}$ & $\mathrm{U}$ \\
\hline 857 & S01222 & $11 / 1 / 94$ & REG & Benzene & 1 & $\mathrm{ug} / \mathrm{L}$ & $\mathrm{U}$ \\
\hline 857 & S02769 & $3 / 6 / 95$ & REG & Benzene & 5 & $\mathrm{ug} / \mathrm{L}$ & $\mathrm{U}$ \\
\hline
\end{tabular}


B-79

\begin{tabular}{|c|c|c|c|c|c|c|c|}
\hline Station & Sample ID & Sample Date & Sample Type & Chemical & Results & Units & Qualifier \\
\hline 857 & S04927 & $8 / 3 / 95$ & REG & Benzene & 5 & ug $/ \mathrm{L}$ & $\bar{U}$ \\
\hline 8.57 & 857215REGUF & $2 / 15 / 91$ & REG & Benzene & 5 & $\mathrm{ug} / \mathrm{L}$ & $\mathrm{U}$ \\
\hline 857 & 857516REGUF & $5 / 16 / 90$ & REG & Benzene & 5 & $\mathrm{ug} / \mathrm{L}$ & $\mathrm{U}$ \\
\hline 857 & 857112REGUF & $11 / 2 / 90$ & REG & Benzene & 5 & $\mathrm{ug} / \mathrm{L}$ & $U$ \\
\hline 857 & 85729REGUF & $2 / 9 / 89$ & REG & Benzene & 5 & $u g / L$ & $U$ \\
\hline 857 & $857+18$ REGUF & $4 / 18 / 89$ & REG & Benzene & 5 & $\mathrm{ug} / \mathrm{L}$ & $\mathrm{U}$ \\
\hline 857 & 857614REGUF & $6 / 14 / 88$ & REG & Benzene & 5 & ug/ & $\mathrm{U}$ \\
\hline 857 & 8571010REGUF & $10 / 10 / 88$ & REG & Benzene & 5 & $\mathrm{ug} / \mathrm{L}$ & $\mathrm{U}$ \\
\hline 857 & S01222 & $11 / 1 / 94$ & REG & Bromochloromethane & 1 & $\mathrm{ug} / \mathrm{L}$ & $\mathrm{U}$ \\
\hline 857 & M01279 & $11 / 13 / 97$ & REG & Bromodichloromethane & 5 & ug $/ \mathrm{L}$ & $\mathrm{U}$ \\
\hline 857 & W00814 & $6 / 17 / 97$ & REG & Bromodichloromethane & 5 & $\mathrm{ug} / \mathrm{L}$ & $\mathrm{U}$ \\
\hline 857 & M00870 & $6 / 17 / 97$ & REG & Bromodichloromethane & 5 & $\operatorname{ug} \Omega$ & $\mathrm{U}$ \\
\hline 857 & $\mathrm{~S} 01222$ & $11 / 1 / 94$ & REG & Bromodichloromethane & 1 & ug/L & $\mathrm{U}$ \\
\hline 857 & S02769 & $3 / 6 / 95$ & REG & Bromodichloromethane & 5 & $\mathrm{ug} / \mathrm{L}$ & $\mathrm{U}$ \\
\hline 857 & S04927 & $8 / 3 / 95$ & REG & Bromodichloromethane & 5 & $\mathrm{ug} / \mathrm{L}$ & $\mathrm{U}$ \\
\hline 857 & 857215REGUF & $2 / 15 / 91$ & REG & Bromodichloromethane & 5 & $\mathrm{ug} / \mathrm{L}$ & $\mathrm{U}$ \\
\hline 857 & 857516REGUF & $5 / 16 / 90$ & REG & Bromodichloromethane & 5 & $\mathrm{ug} / \mathrm{L}$ & U \\
\hline 857 & 857112REGUF & $11 / 2 / 90$ & REG & Bromodichloromethane & 5 & $\mathrm{ug} / \mathrm{L}$ & $\mathrm{U}$ \\
\hline 857 & 85729REGUF & $2 / 9 / 89$ & REG & Bromodichloromethane & 5 & $\mathrm{ug} / \mathrm{L}$ & $\mathrm{U}$ \\
\hline 857 & 857418REGUF & $4 / 18 / 89$ & REG & Bromodichloromethane & 5 & $u g / L$ & $U$ \\
\hline 857 & 857614REGUF & $6 / 14 / 88$ & REG & Bromodichloromethane & 5 & ug/ & $\mathrm{U}$ \\
\hline 857 & 8571010REGUF & $10 / 10 / 88$ & REG & Bromodichloromethane & 5 & ug/ & $\mathrm{U}$ \\
\hline 857 & M01279 & $11 / 13 / 97$ & REG & Bromoform & 5 & ug/L & U \\
\hline 857 & W00814 & $6 / 17 / 97$ & REG & Bromoform & 5 & ug $/ \mathrm{L}$ & $\mathrm{U}$ \\
\hline 857 & M00870 & $6 / 17 / 97$ & REG & Bromoform & 5 & ug/L & $\mathrm{U}$ \\
\hline 857 & S01222 & $11 / 1 / 94$ & REG & Bromoform & 1 & ug $/ \mathrm{L}$ & $\mathrm{U}$ \\
\hline 857 & S02769 & $3 / 6 / 95$ & REG & Bromoform & 5 & ug $/$ & $U$ \\
\hline 857 & S04927 & $8 / 3 / 95$ & REG & Bromoform & 5 & ug/L & $\mathrm{U}$ \\
\hline 857 & 857215REGUF & $2 / 15 / 91$ & REG & Bromoform & 5 & ug $\Omega$ & $\mathrm{U}$ \\
\hline 857 & 857516REGUF & $5 / 16 / 90$ & REG & Bromoform & 5 & ug/L & $\mathrm{U}$ \\
\hline 857 & 857112REGUF & $11 / 2 / 90$ & REG & Bromoform & 5 & $\mathrm{ug} / \mathrm{L}$ & $\mathrm{U}$ \\
\hline 857 & 85729REGUF & $2 / 9 / 89$ & REG & Bromoform & 5 & $\mathrm{ug} / \mathrm{L}$ & $\mathrm{U}$ \\
\hline 857 & 857418REGUF & $4 / 18 / 89$ & REG & Bromoform & 5 & $\mathrm{ug} / \mathrm{L}$ & $U$ \\
\hline 857 & 857614REGUF & $6 / 14 / 88$ & REG & Bromoform & 5 & ug/L & $\mathrm{U}$ \\
\hline 8.57 & 8571010 REGUF & $10 / 10 / 88$ & REG & Bromoform & 5 & ug $/$ & $\mathrm{U}$ \\
\hline 857 & M01279 & $11 / 13 / 97$ & REG & Bromomethane & 10 & ug $/$ & $\mathrm{U}$ \\
\hline 857 & W00814 & $6 / 17 / 97$ & REG & Bromomethane & 10 & ug $/ \mathrm{L}$ & $U$ \\
\hline 857 & M00870 & $6 / 17 / 97$ & REG & Bromomethane & 10 & $\mathrm{ug} / \mathrm{L}$ & $\mathrm{U}$ \\
\hline 857 & S01222 & $11 / 1 / 94$ & REG & Bromomethane & 1 & $\mathrm{ug} / \mathrm{L}$ & $\mathrm{U}$ \\
\hline 857 & S02769 & $3 / 6 / 95$ & REG & Bromomethane & 10 & $\mathrm{ug} / \mathrm{L}$ & $\mathrm{U}$ \\
\hline 857 & S04927 & $8 / 3 / 95$ & REG & Bromomethane & 10 & $\mathrm{ug} / \mathrm{L}$ & $\mathrm{U}$ \\
\hline 857 & 857215REGUF & $2 / 15 / 91$ & REG & Bromomethane & 10 & $\mathrm{ug} / \mathrm{L}$ & $\mathrm{U}$ \\
\hline 857 & 857516REGUF & $5 / 16 / 90$ & REG & Bromomethane & 10 & $\mathrm{ug} / \mathrm{L}$ & $\mathrm{U}$ \\
\hline 857 & 857112REGUF & $11 / 2 / 90$ & REG & Bromomethane & 10 & ug/L & $\mathrm{U}$ \\
\hline 857 & 85729REGUF & $2 / 9 / 89$ & REG & Bromomethane & 10 & $\operatorname{ug} / \mathrm{L}$ & $\mathrm{U}$ \\
\hline 857 & 857418REGUF & $4 / 18 / 89$ & REG & Bromomethane & 10 & ug/L & $\mathrm{U}$ \\
\hline 857 & 857614REGUF & $6 / 14 / 88$ & REG & Bromomethane & 10 & $\mathrm{ug} / \mathrm{L}$ & $\mathrm{U}$ \\
\hline 857 & 8571010REGUF & $10 / 10 / 88$ & REG & Bromomethane & 10 & ug/L & $\mathrm{U}$ \\
\hline 857 & M01279 & $11 / 13 / 97$ & REG & Carbon disulfide & 5 & ug $\Omega$ & $\mathrm{U}$ \\
\hline 857 & W00814 & $6 / 17 / 97$ & REG & Carbon disulfide & 5 & ug/L & $\mathrm{U}$ \\
\hline
\end{tabular}


Station Sample ID Sample Date Sample Type Chemical

\begin{tabular}{|c|c|c|c|c|c|c|c|}
\hline Station & Sampic Is & Sample & sample 1уре & & & & \\
\hline 857 & $\mathrm{M} 00870$ & $6 / 17 / 97$ & REG & Carbon disulfide & 5 & $\mathrm{ug} / \mathrm{L}$ & $\bar{U}$ \\
\hline 857 & S01222 & $11 / 1 / 94$ & REG & Carbon disulfide & 1 & $\mathrm{ug} / \mathrm{L}$ & $U$ \\
\hline 857 & S02769 & $3 / 6 / 95$ & REG & Carbon disulfide & 5 & $\mathrm{ug} / \mathrm{L}$ & U \\
\hline 857 & S04927 & $8 / 3 / 95$ & REG & Carbon disulfide & 5 & $u g / L$ & U \\
\hline 857 & 857215REGUF & $2 / 15 / 91$ & REG & Carbon disulfide & 5 & ug/L & $\mathrm{U}$ \\
\hline 857 & 857516REGUF & $5 / 16 / 90$ & REG & Carbon disulfide & 5 & ug/L & $\mathrm{U}$ \\
\hline 857 & 857112 REGUF & $11 / 2 / 90$ & REG & Carbon disulfide & 5 & $\mathrm{ug} / \mathrm{L}$ & $\mathrm{U}$ \\
\hline 857 & 85729REGUF & $2 / 9 / 89$ & REG & Carbon disulfide & 5 & ug $/ \mathrm{L}$ & $\mathrm{U}$ \\
\hline 857 & 857418REGUF & $4 / 18 / 89$ & REG & Carbon disulfide & 5 & $\mathrm{ug} / \mathrm{L}$ & $\mathrm{U}$ \\
\hline 857 & 857614REGUF & $6 / 14 / 88$ & REG & Carton disulfide & 5 & ug/L & $\mathrm{U}$ \\
\hline 857 & 8571010REGUF & $10 / 10 / 88$ & REG & Carbon disulfide & 5 & $\mathrm{ug} / \mathrm{L}$ & U \\
\hline 857 & M01279 & $11 / 13 / 97$ & REG & Carbon tetrachloride & 5 & $\mathrm{ug} / \mathrm{L}$ & $\mathrm{U}$ \\
\hline 857 & W00814 & $6 / 17 / 97$ & REG & Carbon tetrachioride & 5 & ug/L & $\mathrm{U}$ \\
\hline 857 & M00870 & $6 / 17 / 97$ & REG & Carbon tetrachloride & 5 & ug/L & $\mathrm{U}$ \\
\hline 857 & S01222 & $11 / 1 / 94$ & REG & Carbon tetrachloride & 1 & ug $/$ & $\mathrm{U}$ \\
\hline 857 & S02769 & $3 / 6 / 95$ & REG & Carbon tetrachloride & 5 & $\mathrm{ug} / \mathrm{L}$ & $\mathrm{U}$ \\
\hline 857 & S04927 & $8 / 3 / 95$ & REG & Carbon tetrachloride & 5 & ug/L & $\mathrm{U}$ \\
\hline 857 & 857215REGUF & $2 / 15 / 91$ & REG & Carbon tetrachioride & 5 & ug/L & $\mathrm{U}$ \\
\hline 857 & 8575I6REGUF & $5 / 16 / 90$ & REG & Carbon tetrachloride & 5 & ug $/$ & $\mathrm{U}$ \\
\hline 857 & 857112REGUF & $11 / 2 / 90$ & REG & Carbon tetrachloride & 5 & $\mathrm{ug} / \mathrm{L}$ & $\mathrm{U}$ \\
\hline 857 & 85729REGUF & $2 / 9 / 89$ & REG & Carbon tetrachloride & 5 & $\mathrm{ug} / \mathrm{L}$ & $\mathrm{U}$ \\
\hline 857 & 857418REGUF & $4 / 18 / 89$ & REG & Carbon tetrachloride & 5 & $\mathrm{ug} / \mathrm{L}$ & $\mathrm{U}$ \\
\hline 857 & 857614REGUF & $6 / 14 / 88$ & REG & Carbon tetrachloride & 5 & ug $\Omega$ & $\mathrm{U}$ \\
\hline 857 & 8571010REGUF & $10 / 10 / 88$ & REG & Carbon tetrachloride & 5 & $\mathrm{ug} / \mathrm{L}$ & $\mathrm{U}$ \\
\hline 857 & M01279 & $11 / 13 / 97$ & REG & Chlorobenzene & 5 & $\mathrm{ug} / \mathrm{L}$ & $\mathbf{U}$ \\
\hline 857 & W00814 & $6 / 17 / 97$ & REG & Chlorobenzene & 5 & ug/L & U \\
\hline 857 & M00870 & $6 / 17 / 97$ & REG & Chlorobenzene & 5 & ug/L & $\mathrm{U}$ \\
\hline 857 & S01222 & $11 / 1 / 94$ & REG & Chlorobenzene & 1 & $u g / L$ & U \\
\hline 857 & S02769 & $3 / 6 / 95$ & REG & Chlorobenzene & 5 & $\mathrm{ug} / \mathrm{L}$ & $\mathrm{U}$ \\
\hline 857 & S04927 & $8 / 3 / 95$ & REG & Chlorobenzene & 5 & ug/L & $\mathrm{U}$ \\
\hline 857 & 857215REGUF & $2 / 15 / 91$ & REG & Chlorobenzene & 5 & $\mathrm{ug} / \mathrm{L}$ & $\mathrm{U}$ \\
\hline 857 & 857516REGUF & $5 / 16 / 90$ & REG & Chlorobenzene & 5 & $\mathrm{ug} / \mathrm{L}$ & $\mathrm{U}$ \\
\hline 857 & 857112REGUF & $11 / 2 / 90$ & REG & Chlorobenzene & 5 & $\mathrm{ug} / \mathrm{L}$ & $\mathrm{U}$ \\
\hline 857 & 85729REGUF & $2 / 9 / 89$ & REG & Chlorobenzene & 5 & ug/L & $\mathrm{U}$ \\
\hline 857 & $857+18$ REGUF & $4 / 18 / 89$ & REG & Chlorobenzene & 5 & ug/L & $\mathrm{U}$ \\
\hline 857 & 857614REGUF & $6 / 14 / 88$ & REG & Chlorobenzene & 5 & ug/L & $\mathrm{U}$ \\
\hline 857 & 8571010REGUF & $10 / 10 / 88$ & REG & Chlorobenzene & 5 & $\mathrm{ug} / \mathrm{L}$ & $\mathrm{U}$ \\
\hline 857 & M01279 & $11 / 13 / 97$ & REG & Chloroethane & 10 & ug/L & U \\
\hline 857 & W00814 & $6 / 17 / 97$ & REG & Chloroethane & 10 & $\mathrm{ug} / \mathrm{L}$ & $\mathrm{U}$ \\
\hline 857 & M00870 & $6 / 17 / 97$ & REG & Chloroethane & 10 & ug $/ \mathrm{L}$ & $\mathrm{U}$ \\
\hline 857 & $\mathrm{~S} 01222$ & $11 / 1 / 94$ & REG & Chloroethane & 1 & $\mathrm{ug} / \mathrm{L}$ & $\mathrm{U}$ \\
\hline 857 & S02769 & $3 / 6 / 95$ & REG & Chloroethane & 10 & ug/L & U \\
\hline 857 & S04927 & $8 / 3 / 95$ & REG & Chloroethane & 10 & $\mathrm{ug} / \mathrm{L}$ & $\mathrm{U}$ \\
\hline 857 & 857215 REGUF & $2 / 15 / 91$ & REG & Chloroethane & 10 & $\mathrm{ug} / \mathrm{L}$ & $\mathrm{U}$ \\
\hline 857 & 857516REGUF & $5 / 16 / 90$ & REG & Chloroethane & 10 & $\mathrm{ug} / \mathrm{L}$ & $\mathrm{U}$ \\
\hline 857 & 857112REGUF & $11 / 2 / 90$ & REG & Chloroethane & 10 & $\mathrm{ug} / \mathrm{L}$ & $\mathrm{U}$ \\
\hline 857 & 85729REGUF & $2 / 9 / 89$ & REG & Chloroethane & 10 & $\mathrm{ug} / \mathrm{L}$ & $\mathrm{U}$ \\
\hline 857 & 857418REGUF & $4 / 18 / 89$ & REG & Chloroethane & 10 & $\mathrm{ug} / \mathrm{L}$ & $\mathrm{U}$ \\
\hline 857 & 857614REGUF & $6 / 14 / 88$ & REG & Chloroethane & 10 & $\mathrm{ug} / \mathrm{L}$ & $\mathrm{U}$ \\
\hline 857 & 8571010 REGUF & $10 / 10 / 88$ & REG & Chloroethane & 10 & $\mathrm{ug} / \mathrm{L}$ & $\mathrm{U}$ \\
\hline
\end{tabular}


Station Sample ID Sample Date Sample Type Chemical

\begin{tabular}{|c|c|c|c|c|c|c|c|}
\hline & & & & & & & \\
\hline 857 & $\mathrm{M} 01279$ & $11 / 13 / 97$ & REG & Chloroform & 5 & $\mathrm{ug} / \mathrm{L}$ & $\bar{U}$ \\
\hline 857 & W00814 & $6 / 17 / 97$ & REG & Chloroform & 5 & $\mathrm{ug} / \mathrm{L}$ & U \\
\hline 857 & $M 00870$ & $6 / 17 / 97$ & REG & Chloroform & 5 & $\mathrm{ug} / \mathrm{L}$ & $\mathrm{U}$ \\
\hline 857 & So1 1222 & $11 / 1 / 94$ & REG & Chloroform & 1 & ug/L & $\mathrm{U}$ \\
\hline 857 & S02769 & $3 / 6 / 95$ & REG & Chloroform & 5 & $\mathrm{ug} / \mathrm{L}$ & $\mathrm{U}$ \\
\hline 857 & S0+927 & $8 / 3 / 95$ & REG & Chloroform & 5 & ug/L & $\mathrm{U}$ \\
\hline 857 & 857215REGUF & $2 / 15 / 91$ & REG & Chloroform & 5 & $\mathrm{ug} / \mathrm{L}$ & $\mathrm{U}$ \\
\hline 857 & 857516REGUF & $5 / 16 / 90$ & REG & Chloroform & 5 & ug/L & $\mathrm{U}$ \\
\hline 857 & 857112REGUF & $11 / 2 / 90$ & REG & Chloroform & 5 & ug $/ \mathrm{L}$ & $\mathrm{U}$ \\
\hline 857 & 85729REGUF & $2 / 9 / 89$ & REG & Chloroform & 5 & ug $/$ & $\mathrm{U}$ \\
\hline 857 & 857418REGUF & $4 / 18 / 89$ & REG & Chloroform & 5 & $\mathrm{ug} / \mathrm{L}$ & $\mathrm{U}$ \\
\hline 857 & 857614REGUF & $6 / 14 / 88$ & REG & Chloroform & 5 & $\mathrm{ug} / \mathrm{L}$ & $\mathrm{U}$ \\
\hline 857 & 8571010REGUF & $10 / 10 / 88$ & REG & Chloroform & 5 & $\mathrm{ug} / \mathrm{L}$ & U \\
\hline 857 & M01279 & $11 / 13 / 97$ & REG & Chloromethane & 10 & ug/L & $\mathrm{U}$ \\
\hline 857 & W00814 & $6 / 17 / 97$ & REG & Chloromethane & 10 & $\mathrm{ug} / \mathrm{L}$ & $\mathrm{U}$ \\
\hline 857 & M00870 & $6 / 17 / 97$ & REG & Chloromethane & 10 & ug $/$ & $\mathrm{U}$ \\
\hline 857 & S01222 & $11 / 1 / 94$ & REG & Chloromethane & 1 & $\mathrm{ug} / \mathrm{L}$ & U \\
\hline 857 & S02769 & $3 / 6 / 95$ & REG & Chloromethane & 10 & $\mathrm{ug} / \mathrm{L}$ & $\mathbf{U}$ \\
\hline 857 & S04927 & $8 / 3 / 95$ & REG & Chloromethane & 10 & ug/L & $\mathrm{U}$ \\
\hline 857 & 857215REGUF & $2 / 15 / 91$ & REG & Chloromethane & 10 & $\mathrm{ug} / \mathrm{L}$ & $\mathrm{U}$ \\
\hline 857 & 857516REGUF & $5 / 16 / 90$ & REG & Chloromethane & 10 & $\mathrm{ug} / \mathrm{L}$ & U \\
\hline 857 & 857112REGUF & $11 / 2 / 90$ & REG & Chloromethane & 10 & $\mathrm{ug} / \mathrm{L}$ & $\mathrm{U}$ \\
\hline 857 & 85729REGUF & $2 / 9 / 89$ & REG & Chloromethane & 10 & $\mathrm{ug} / \mathrm{L}$ & U \\
\hline 857 & 857418REGUF & $4 / 18 / 89$ & REG & Chloromethane & 10 & $\mathrm{ug} / \mathrm{L}$ & $\mathrm{U}$ \\
\hline 857 & 857614REGUF & $6 / 14 / 88$ & REG & Chloromethane & 10 & $\mathrm{ug} / \mathrm{L}$ & $\mathrm{U}$ \\
\hline 857 & 8571010REGUF & $10 / 10 / 88$ & REG & Chloromethane & 10 & $\mathrm{ug} / \mathrm{L}$ & $\mathrm{U}$ \\
\hline 857 & M01279 & $11 / 13 / 97$ & REG & cis-1.2-Dichloroethene & 5 & $\mathrm{ug} / \mathrm{L}$ & $\mathrm{U}$ \\
\hline 857 & M00870 & $6 / 17 / 97$ & REG & cis-1,2-Dichloroethene & 5 & $\mathrm{ug} / \mathrm{L}$ & $\mathrm{U}$ \\
\hline 857 & S01222 & $11 / 1 / 94$ & REG & cis-1.2-Dichloroethene & 1 & $\mathrm{ug} / \mathrm{L}$ & $\mathrm{U}$ \\
\hline 857 & S02769 & $3 / 6 / 95$ & REG & cis-1,2-Dichloroethene & 5 & ug/L & $\mathrm{U}$ \\
\hline 857 & S04927 & $8 / 3 / 95$ & REG & cis-1.2-Dichloroethene & 5 & ug $/ \mathrm{L}$ & $\mathrm{U}$ \\
\hline 857 & M01279 & $11 / 13 / 97$ & REG & cis-1.3-Dichloropropen & 5 & $\mathrm{ug} / \mathrm{L}$ & $\mathrm{U}$ \\
\hline 857 & W00814 & $6 / 17 / 97$ & REG & cis-1.3-Dichloropropene & 5 & $\mathrm{ug} / \mathrm{L}$ & $\mathrm{U}$ \\
\hline 857 & M00870 & $6 / 17 / 97$ & REG & cis-1.3-Dichloropropene & 5 & $\mathrm{ug} / \mathrm{L}$ & $\mathrm{U}$ \\
\hline 857 & S01222 & $11 / 1 / 94$ & REG & cis-1.3-Dichloropropene & 1 & ug/L & $\mathrm{U}$ \\
\hline 857 & S02769 & $3 / 6 / 95$ & REG & cis-1.3-Dichloropropene & 5 & $\mathrm{ug} / \mathrm{L}$ & $\mathrm{U}$ \\
\hline 857 & S04927 & $8 / 3 / 95$ & REG & cis-1.3-Dichloropropene & 5 & ug/L & $\mathrm{U}$ \\
\hline 857 & 857215REGUF & $2 / 15 / 91$ & REG & cis-1.3-Dichloropropene & 5 & $\mathrm{ug} / \mathrm{L}$ & $\mathrm{U}$ \\
\hline 857 & 857516REGUF & $5 / 16 / 90$ & REG & cis-1.3-Dichloropropene & 5 & $\mathrm{ug} / \mathrm{L}$ & $\mathrm{U}$ \\
\hline 857 & 857112REGUF & $11 / 2 / 90$ & REG & cis-1.3-Dichloropropene & 5 & ug $/ \mathrm{L}$ & $\mathrm{U}$ \\
\hline 857 & 85729REGUF & $2 / 9 / 89$ & REG & cis-1,3-Dichloropropene & 5 & ug/ $\mathrm{L}$ & $\mathrm{U}$ \\
\hline 857 & 857418REGUF & $4 / 18 / 89$ & REG & cis-1.3-Dichloropropene & 5 & ug/L & $\mathrm{U}$ \\
\hline 857 & 85761+REGUF & $6 / 14 / 88$ & REG & cis-1,3-Dichloropropene & 5 & ug/L & U \\
\hline 857 & 8571010REGUF & $10 / 10 / 88$ & REG & cis-1,3-Dichloropropene & 5 & ug/L & $\mathrm{U}$ \\
\hline 857 & M01279 & $11 / 13 / 97$ & REG & Dibromochloromethane & 5 & ug/L & $\mathrm{U}$ \\
\hline 857 & W00814 & $6 / 17 / 97$ & REG & Dibromochloromethane & 5 & ug/L & $\mathrm{U}$ \\
\hline 857 & M00870 & $6 / 17 / 97$ & REG & Dibromochloromethane & 5 & ug/L & $\mathrm{U}$ \\
\hline 857 & S01222 & $11 / 1 / 94$ & REG & Dibromochloromethane & 1 & ug/L & $\mathrm{U}$ \\
\hline 857 & S02769 & $3 / 6 / 95$ & REG & Dibromochloromethane & 5 & $\mathrm{ug} / \mathrm{L}$ & $\mathrm{U}$ \\
\hline 857 & S04927 & $8 / 3 / 95$ & REG & Dibromochloromethane & 5 & ug $/$ & $\mathrm{U}$ \\
\hline
\end{tabular}




\begin{tabular}{|c|c|c|c|c|c|c|c|}
\hline Station & Sample ID & Sample Date & Sample Type & Chemical & Results & Units & Qualifier \\
\hline 857 & 857215REGUF & $2 / 15 / 91$ & REG & Dibromochloromethane & 5 & $u g / \mathrm{L}$ & $\mathrm{U}$ \\
\hline 857 & 857516REGUF & $5 / 16 / 90$ & REG & Dibromochloromethane & 5 & $\mathrm{ug} / \mathrm{L}$ & U \\
\hline 857 & 857112REGUF & $11 / 2 / 90$ & REG & Dibromochloromethane & 5 & ug/L & U \\
\hline 857 & 85729REGUF & $2 / 9 / 89$ & REG & Dibromochloromethane & 5 & ug/L & $\mathrm{U}$ \\
\hline 857 & 857418REGUF & $4 / 18 / 89$ & REG & Dibromochloromethane & 5 & $\mathrm{ug} / \mathrm{L}$ & $\mathrm{U}$ \\
\hline 857 & 857614REGUF & $6 / 14 / 88$ & REG & Dibromochloromethane & 5 & $u g / L$ & U \\
\hline 857 & 8571010REGUF & $10 / 10 / 88$ & REG & Dibromochloromethane & 5 & $\mathrm{ug} / \mathrm{L}$ & $\mathrm{U}$ \\
\hline 857 & 857215REGUF & $2 / 15 / 91$ & REG & Dimethỵlbenzene & 5 & ug/L & $\mathrm{U}$ \\
\hline 857 & 857516REGUF & $5 / 16 / 90$ & REG & Dimethylbenzene & 5 & ug/L & $\mathrm{U}$ \\
\hline 857 & 857112REGUF & $11 / 2 / 90$ & REG & Dimethylbenzene & 5 & ug/L & $\mathrm{U}$ \\
\hline 857 & 85729REGUF & $2 / 9 / 89$ & REG & Dimethylbenzene & 5 & $\mathrm{ug} / \mathrm{L}$ & $U$ \\
\hline 857 & 857418REGUF & $4 / 18 / 89$ & REG & Dimethylbenzene & 5 & $\mathrm{ug} / \mathrm{L}$ & $\mathrm{U}$ \\
\hline 857 & 857614REGUF & $6 / 14 / 88$ & REG & Dimethylbenzene & 5 & $u g / L$ & $\mathrm{U}$ \\
\hline 857 & 8571010REGUF & $10 / 10 / 88$ & REG & Dimethylbenzene & 5 & $\mathrm{ug} / \mathrm{L}$ & $U$ \\
\hline 857 & M01279 & $11 / 13 / 97$ & REG & Ethylbenzene & 5 & ug/L & $\mathrm{U}$ \\
\hline 857 & W00814 & $6 / 17 / 97$ & REG & Ethylbenzene & 5 & $\mathrm{ug} / \mathrm{L}$ & $\mathrm{U}$ \\
\hline 857 & M00870 & $6 / 17 / 97$ & REG & Ethylbenzene & 5 & ug $/ \mathrm{L}$ & $\mathrm{U}$ \\
\hline 857 & S01222 & $11 / 1 / 94$ & REG & Ethylbenzene & 1 & $u g / L$ & $\mathrm{U}$ \\
\hline 857 & S02769 & $3 / 6 / 95$ & REG & Ethylbenzene & 5 & $\mathrm{ug} / \mathrm{L}$ & $\mathrm{U}$ \\
\hline 857 & S04927 & $8 / 3 / 95$ & REG & Ethylbenzene & 5 & $\mathrm{ug} / \mathrm{L}$ & $\mathrm{U}$ \\
\hline 857 & 857215REGUF & $2 / 15 / 91$ & REG & Ethylbenzene & 5 & $\mathrm{ug} / \mathrm{L}$ & $\mathrm{U}$ \\
\hline 857 & 857516REGUF & $5 / 16 / 90$ & REG & Ethylbenzene & 5 & $\mathrm{ug} / \mathrm{L}$ & $\mathrm{U}$ \\
\hline 857 & 857112REGUF & $11 / 2 / 90$ & REG & Ethylbenzene & 5 & $\mathrm{ug} / \mathrm{L}$ & $\mathrm{U}$ \\
\hline 857 & 85729REGUF & $2 / 9 / 89$ & REG & Ethylbenzene & 5 & $\mathrm{ug} / \mathrm{L}$ & $\mathrm{U}$ \\
\hline 857 & 857418REGUF & $4 / 18 / 89$ & REG & Ethylbenzene & 5 & $\mathrm{ug} / \mathrm{L}$ & U \\
\hline 857 & 857614REGUF & $6 / 14 / 88$ & REG & Ethylbenzene & 2 & $\mathrm{ug} / \mathrm{L}$ & $\mathrm{J}$ \\
\hline 857 & 8571010 REGUF & $10 / 10 / 88$ & REG & Ethylbenzene & 5 & $\mathrm{ug} / \mathrm{L}$ & $\mathrm{U}$ \\
\hline 857 & M01279 & $11 / 13 / 97$ & REG & m,p-Xylene & 5 & $u g / L$ & $\mathrm{U}$ \\
\hline 857 & W00814 & $6 / 17 / 97$ & REG & $\mathrm{m}, \mathrm{p}$-Xylene & 5 & $\mathrm{ug} / \mathrm{L}$ & $\mathrm{U}$ \\
\hline 857 & M00870 & $6 / 17 / 97$ & REG & m,p-Xylene & 5 & $\mathrm{ug} / \mathrm{L}$ & $\mathrm{U}$ \\
\hline 857 & S02769 & $3 / 6 / 95$ & REG & m,p-Xylene & 5 & $u g / L$ & $\mathrm{U}$ \\
\hline 857 & S04927 & $8 / 3 / 95$ & REG & m.p-Xylene & 5 & ug/L & $U$ \\
\hline 857 & M01279 & $11 / 13 / 97$ & REG & Methylene chloride & 7 & $\mathrm{ug} / \mathrm{L}$ & B \\
\hline 857 & W00814 & $6 / 17 / 97$ & REG & Methylene chloride & 8 & $\mathrm{ug} / \mathrm{L}$ & $=$ \\
\hline 857 & M00870 & $6 / 17 / 97$ & REG & Methylene chloride & 7 & $u g / L$ & $=$ \\
\hline 857 & S01222 & $11 / 1 / 94$ & REG & Methỵlene chloride & 2 & $\mathrm{ug} / \mathrm{L}$ & UJ \\
\hline 857 & S02769 & $3 / 6 / 95$ & REG & Methylene chloride & 5 & $\mathrm{ug} / \mathrm{L}$ & $\mathrm{U}$ \\
\hline 857 & S04927 & $8 / 3 / 95$ & REG & Methylene chloride & 5 & $\mathrm{ug} / \mathrm{L}$ & $\mathrm{U}$ \\
\hline 857 & 857215REGUF & $2 / 15 / 91$ & REG & Methylene chloride & 5 & ug/ & $\mathrm{U}$ \\
\hline 857 & 857516REGUF & $5 / 16 / 90$ & REG & Methylene chloride & 5 & ug $/$ & $\mathrm{U}$ \\
\hline 857 & 857112REGUF & $11 / 2 / 90$ & REG & Methylene chloride & 5 & $\mathrm{ug} / \mathrm{L}$ & $\mathrm{U}$ \\
\hline 857 & 85729REGUF & $2 / 9 / 89$ & REG & Methylene chloride & 0.6 & ug/L & J \\
\hline 857 & 857418REGUF & $4 / 18 / 89$ & REG & Methylene chloride & 1 & $\mathrm{ug} / \mathrm{L}$ & J \\
\hline 857 & 85761+REGUF & $6 / 1+/ 88$ & REG & Methylene chloride & 5 & ug/ & $\mathrm{U}$ \\
\hline 857 & 8571010REGUF & $10 / 10 / 88$ & REG & Methylene chloride & 1 & $u g / L$ & J \\
\hline 857 & M01279 & $11 / 13 / 97$ & REG & Styrene & 5 & $\mathrm{ug} / \mathrm{L}$ & $\mathrm{U}$ \\
\hline 857 & W00814 & $6 / 17 / 97$ & REG & Styrene & 5 & ug/L & $\mathrm{U}$ \\
\hline 857 & M00870 & $6 / 17 / 97$ & REG & Styrene & 5 & ug/L & $\mathrm{U}$ \\
\hline 857 & S01222 & $11 / 1 / 94$ & REG & Styrene & 1 & $\mathrm{ug} / \mathrm{L}$ & $U$ \\
\hline 857 & S02769 & $3 / 6 / 95$ & REG & Styrene & 5 & $\mathrm{ug} / \mathrm{L}$ & $\mathrm{U}$ \\
\hline
\end{tabular}


B-83

\begin{tabular}{|c|c|c|c|c|c|c|c|}
\hline Station & Sample ID & Sample Date & Sample Type & Chemical & Results & Units & Qualifier \\
\hline 857 & S04927 & $8 / 3 / 95$ & REG & Styrene & 5 & $u g / L$ & $U$ \\
\hline 857 & 857215REGUF & $2 / 15 / 91$ & REG & Styrene & 5 & ug $/ \mathrm{L}$ & $U$ \\
\hline 857 & 857516REGUF & $5 / 16 / 90$ & REG & Sty̆rene & 5 & $\mathrm{ug} / \mathrm{L}$ & $\mathrm{U}$ \\
\hline 857 & 857112REGUF & $11 / 2 / 90$ & REG & Styrene & 5 & $\mathrm{ug} / \mathrm{L}$ & U \\
\hline 857 & 85729REGUF & $2 / 9 / 89$ & REG & Styrene & 5 & $\mathrm{ug} / \mathrm{L}$ & $\mathrm{U}$ \\
\hline 857 & 857418REGUF & $4 / 18 / 89$ & REG & Styrene & 5 & $\mathrm{ug} / \mathrm{L}$ & $\mathrm{U}$ \\
\hline 857 & 857614REGUF & $6 / 14 / 88$ & REG & Styrene & 5 & $u g / L$ & $\mathrm{U}$ \\
\hline 857 & 8571010REGUF & $10 / 10 / 88$ & REG & Styrene & 5 & $\mathrm{ug} / \mathrm{L}$ & $U$ \\
\hline 857 & M01279 & $11 / 13 / 97$ & REG & Tetrachloroethene & 5 & $u g / L$ & $\mathrm{U}$ \\
\hline 857 & W00814 & $6 / 17 / 97$ & REG & Tetrachloroethene & 5 & $\mathrm{ug} / \mathrm{L}$ & U \\
\hline 857 & M00870 & $6 / 17 / 97$ & REG & Tetrachloroethene & 5 & $\mathrm{ug} / \mathrm{L}$ & $\mathrm{U}$ \\
\hline 857 & S01222 & $11 / 1 / 94$ & REG & Tetrachloroethene & 1 & $\mathrm{ug} / \mathrm{L}$ & U \\
\hline 857 & S02769 & $3 / 6 / 95$ & REG & Tetrachloroethene & 5 & ug/L & $\mathrm{U}$ \\
\hline 857 & S04927 & $8 / 3 / 95$ & REG & Tetrachloroethene & 5 & ug $\Lambda$ & $\mathrm{U}$ \\
\hline 857 & 857215REGUF & $2 / 15 / 91$ & REG & Tetrachloroethene & 5 & $\mathrm{ug} / \mathrm{L}$ & U \\
\hline 857 & 857516REGUF & $5 / 16 / 90$ & REG & Tetrachloroethene & 5 & ug $/ L$ & U \\
\hline 857 & 857112REGUF & $11 / 2 / 90$ & REG & Tetrachloroethene & 5 & $\mathrm{ug} / \mathrm{L}$ & $=$ \\
\hline 857 & 85729REGUF & $2 / 9 / 89$ & REG & Tetrachloroethene & 5 & $\mathrm{ug} / \mathrm{L}$ & $\mathrm{U}$ \\
\hline 857 & 857418REGUF & $4 / 18 / 89$ & REG & Tetrachloroethene & 5 & ug/ & $\mathrm{U}$ \\
\hline 857 & 857614REGUF & $6 / 14 / 88$ & REG & Tetrachloroethene & 5 & ug/L & $\mathrm{U}$ \\
\hline 857 & 8571010REGUF & $10 / 10 / 88$ & REG & Tetrachloroethene & 5 & $\mathrm{ug} / \mathrm{L}$ & $\mathrm{U}$ \\
\hline 857 & M01279 & $11 / 13 / 97$ & REG & Toluene & 5 & ug/L & $\mathrm{U}$ \\
\hline 857 & W008I4 & $6 / 17 / 97$ & REG & Toluene & 5 & ug/L & $\mathrm{U}$ \\
\hline 857 & M00870 & $6 / 17 / 97$ & REG & Toluene & 5 & $\mathrm{ug} / \mathrm{L}$ & U \\
\hline 857 & So1222 & $11 / 1 / 94$ & REG & Toluene & 1 & ug/L & $\mathrm{U}$ \\
\hline 857 & S02769 & $3 / 6 / 95$ & REG & Toluene & 5 & ug $/$ & $U$ \\
\hline 857 & S04927 & $8 / 3 / 95$ & REG & Toluene & 5 & ug/L & $\mathrm{U}$ \\
\hline 857 & 857215REGUF & $2 / 15 / 91$ & REG & Toluene & 5 & $\mathrm{ug} / \mathrm{L}$ & $\mathrm{U}$ \\
\hline 857 & 857516REGUF & $5 / 16 / 90$ & REG & Toluene & 1 & $\mathrm{ug} / \mathrm{L}$ & $\mathrm{J}$ \\
\hline 857 & 857112REGUF & $11 / 2 / 90$ & REG & Toluene & 5 & $u g / L$ & U \\
\hline 857 & 85729REGUF & 2/9/89 & REG & Toluene & 5 & ug $/ \mathrm{L}$ & $\mathrm{U}$ \\
\hline 857 & 857418REGUF & $4 / 18 / 89$ & REG & Toluene & 5 & $\mathrm{ug} / \mathrm{L}$ & $\mathrm{U}$ \\
\hline 857 & 857614REGUF & $6 / 14 / 88$ & REG & Toluene & 5 & $u g / L$ & $\mathrm{U}$ \\
\hline 857 & 8571010REGUF & $10 / 10 / 88$ & REG & Toluene & 5 & ug $/ \mathrm{L}$ & $\mathrm{U}$ \\
\hline 857 & M01279 & $11 / 13 / 97$ & REG & trans-1.2-Dichloroethe & 5 & ug $/ \mathrm{L}$ & $\mathrm{U}$ \\
\hline 857 & M00870 & $6 / 17 / 97$ & REG & trans-1,2-Dichloroethene & 5 & $u g / L$ & $\mathrm{U}$ \\
\hline 857 & S01222 & $11 / 1 / 94$ & REG & trans-1.2-Dichloroethene & 1 & $\mathrm{ug} / \mathrm{L}$ & $\mathrm{U}$ \\
\hline 857 & S02769 & $3 / 6 / 95$ & REG & trans-1,2-Dichloroethene & 5 & ug $/ \mathrm{L}$ & $\mathrm{U}$ \\
\hline 857 & S04927 & $8 / 3 / 95$ & REG & trans-1.2-Dichloroethene & 5 & ug $/ \mathrm{L}$ & $\mathrm{U}$ \\
\hline 857 & M01279 & $11 / 13 / 97$ & REG & trans-1.3-Dichloroprop & 5 & $\mathrm{ug} / \mathrm{L}$ & $U$ \\
\hline 857 & W00814 & $6 / 17 / 97$ & REG & trans-1.3-Dichloropropene & 5 & $\operatorname{ug} / \mathcal{L}$ & $\mathrm{U}$ \\
\hline 857 & M00870 & $6 / 17 / 97$ & REG & trans-1,3-Dichloropropene & 5 & ug $/ \mathrm{L}$ & $\mathrm{U}$ \\
\hline 857 & S01222 & $11 / 1 / 94$ & REG & trans-1,3-Dichloropropene & 1 & ug $/ \mathrm{L}$ & $\mathrm{U}$ \\
\hline 857 & S02769 & $3 / 6 / 95$ & REG & trans-1,3-Dichloropropene & 5 & ug/L & $\mathrm{U}$ \\
\hline 857 & S04927 & $8 / 3 / 95$ & REG & trans-1,3-Dichloropropene & 5 & ug $/ \mathrm{L}$ & $\mathrm{U}$ \\
\hline 857 & 857215REGUF & $2 / 15 / 91$ & REG & trans-1,3-Dichloropropene & 5 & $\mathrm{ug} / \mathrm{L}$ & $\mathrm{U}$ \\
\hline 857 & 857516REGUF & $5 / 16 / 90$ & REG & trans-1,3-Dichloropropene & 5 & ug/L & $\mathrm{U}$ \\
\hline 857 & 857112REGUF & $11 / 2 / 90$ & REG & trans-1,3-Dichloropropene & 5 & ug $/$ & $U$ \\
\hline 857 & 85729REGUF & $2 / 9 / 89$ & REG & trans-1.3-Dichloropropene & 5 & ug/L & $\mathrm{U}$ \\
\hline 857 & 857418REGUF & $4 / 18 / 89$ & REG & trans-1.3-Dichloropropene & 5 & $\mathrm{ug} / \mathrm{L}$ & $\mathrm{U}$ \\
\hline
\end{tabular}




\begin{tabular}{|c|c|c|c|c|c|c|c|}
\hline Station & Sample ID & Sample Date & Sample Type & Chemical & Results & Units & Qualifier \\
\hline 8.57 & 8576I4REGUF & $6 / 14 / 88$ & REG & trans-1.3-Dichloropropene & 5 & $\mathrm{ug} / \mathrm{L}$ & $\bar{U}$ \\
\hline 857 & 8571010 REGUF & $10 / 10 / 88$ & REG & trans-1,3-Dichloropropene & 5 & $\mathrm{ug} / \mathrm{L}$ & $\mathrm{U}$ \\
\hline 857 & M01279 & $11 / 13 / 97$ & REG & Trichloroethene & 5 & ug $\Omega$ & $\mathrm{U}$ \\
\hline 857 & W00814 & $6 / 17 / 97$ & REG & Trichloroethene & 5 & ug $\Omega$ & $\mathrm{U}$ \\
\hline 8.57 & M00870 & $6 / 17 / 97$ & REG & Trichloroethene & 5 & ug $/ \mathrm{L}$ & U \\
\hline 857 & S01222 & $11 / 1 / 94$ & REG & Trichloroethene & 1 & $\mathrm{ug} / \mathrm{L}$ & $\mathrm{U}$ \\
\hline 857 & S02769 & $3 / 6 / 95$ & REG & Trichloroethene & 5 & ug $/ \mathrm{L}$ & $\mathrm{U}$ \\
\hline 857 & S04927 & $8 / 3 / 95$ & REG & Trichloroethene & 5 & ug $/$ & $\mathrm{U}$ \\
\hline 857 & 857215REGUF & $2 / 15 / 91$ & REG & Trichloroethene & 5 & ug/L & $\mathrm{U}$ \\
\hline 857 & 857516REGUF & $5 / 16 / 90$ & REG & Trichloroethene & 5 & ug/L & $\mathrm{U}$ \\
\hline 857 & 857112REGUF & $11 / 2 / 90$ & REG & Trichloroethene & 5 & $\mathrm{ug} / \mathrm{L}$ & $\mathrm{U}$ \\
\hline 857 & 85729REGUF & $2 / 9 / 89$ & REG & Trichloroethene & 5 & $\mathrm{ug} / \mathrm{L}$ & $U$ \\
\hline 857 & 8574 I8REGUF & $4 / 18 / 89$ & REG & Trichloroethene & 5 & $\mathrm{ug} / \mathrm{L}$ & $U$ \\
\hline 857 & 857614REGUF & $6 / 14 / 88$ & REG & Trichloroethene & 5 & ug/L & $\mathrm{U}$ \\
\hline 857 & 8571010REGUF & $10 / 10 / 88$ & REG & Trichloroethene & 5 & ug/L & U \\
\hline 857 & M01279 & $11 / 13 / 97$ & REG & Vinyl acetate & 10 & ug/L & $\mathrm{U}$ \\
\hline 857 & M00870 & $6 / 17 / 97$ & REG & Vinyl acetate & 10 & ug/L & $\mathrm{U}$ \\
\hline 857 & S02769 & $3 / 6 / 95$ & REG & Vinyl acetate & 10 & ug/L & $\mathrm{U}$ \\
\hline 857 & S04927 & $8 / 3 / 95$ & REG & Vinyl acetate & 10 & ug/L & U \\
\hline 857 & 857215REGUF & $2 / 15 / 91$ & REG & Vinyl acetate & 10 & ug $/ \mathrm{L}$ & $\mathrm{U}$ \\
\hline 857 & 857516REGUF & $5 / 16 / 90$ & REG & Vinyl acetate & 10 & ug $/$ L & U \\
\hline 857 & 857112REGUF & $11 / 2 / 90$ & REG & Vinyl acetate & 10 & ug/L & U \\
\hline 857 & 85729REGUF & $2 / 9 / 89$ & REG & Vinyl acetate & 10 & ug/L & $U$ \\
\hline 857 & 857418REGUF & $4 / 18 / 89$ & REG & Vinyl acetate & 10 & ug/L & $\mathrm{U}$ \\
\hline 857 & 857614REGUF & $6 / 14 / 88$ & REG & Vinyl acetate & 10 & ug/L & $\mathrm{U}$ \\
\hline 857 & 8571010REGUF & $10 / 10 / 88$ & REG & Vinyl acetate & 10 & ug/L & $\mathrm{U}$ \\
\hline 857 & M01279 & $11 / 13 / 97$ & REG & Vinyl chloride & 10 & ug $/ \mathrm{L}$ & $\mathrm{U}$ \\
\hline 857 & W00814 & $6 / 17 / 97$ & REG & Vinyl chloride & 10 & ug/ & $\mathrm{U}$ \\
\hline 857 & M00870 & $6 / 17 / 97$ & REG & Vinyl chloride & 10 & ug $/$ & $\mathrm{U}$ \\
\hline 857 & S01222 & $11 / 1 / 94$ & REG & Vinyl chloride & 1 & ug/L & U \\
\hline 857 & S02769 & $3 / 6 / 95$ & REG & Vinyl chloride & 10 & ug/L & U \\
\hline 857 & S04927 & $8 / 3 / 95$ & REG & Vinyl chloride & 10 & $\mathrm{ug} / \mathrm{L}$ & U \\
\hline 857 & 857215REGUF & $2 / 15 / 91$ & REG & Vinyl chloride & 10 & ug/L & $\mathrm{U}$ \\
\hline 857 & 857516REGUF & $5 / 16 / 90$ & REG & Vinyl chloride & 10 & $\mathrm{ug} / \mathrm{L}$ & $\mathrm{U}$ \\
\hline 857 & 857112REGUF & $11 / 2 / 90$ & REG & Vinyl chloride & 10 & ug $/$ & $\mathrm{U}$ \\
\hline 857 & 85729REGUF & $2 / 9 / 89$ & REG & Vinyl chloride & 10 & ug/L & $\mathrm{U}$ \\
\hline 857 & 857418REGUF & $4 / 18 / 89$ & REG & Vinyl chloride & 10 & ug $/ \mathrm{L}$ & $\mathrm{U}$ \\
\hline 857 & 857614REGUF & $6 / 14 / 88$ & REG & Vinyl chloride & 10 & ug/L & $\mathrm{U}$ \\
\hline 857 & 8571010REGUF & $10 / 10 / 88$ & REG & Vinyl chloride & 10 & ug $/ \mathrm{L}$ & $\mathrm{U}$ \\
\hline 858 & M01285 & $11 / 17 / 97$ & REG & 1.1.1-Trichloroethane & 5 & $\mathrm{ug} / \mathrm{L}$ & $\mathrm{U}$ \\
\hline 858 & W00815 & $6 / 16 / 97$ & REG & I.1,1-Trichloroethane & 5 & $\mathrm{ug} / \mathrm{L}$ & $\mathrm{U}$ \\
\hline 858 & M00078 & $11 / 14 / 96$ & REG & I,I,1-Trichloroethane & 5 & ug $/ \mathrm{L}$ & $\mathrm{U}$ \\
\hline 858 & M00877 & $6 / 16 / 97$ & REG & 1.1.1-Trichloroethane & 5 & $\mathrm{ug} / \mathrm{L}$ & U \\
\hline 858 & $\mathrm{~S} 02772$ & $3 / 6 / 95$ & REG & 1,1,1-Trichloroethane & 5 & $\mathrm{ug} / \mathrm{L}$ & U \\
\hline 858 & S04936 & $8 / 3 / 95$ & REG & 1,1,1-Trichloroethane & 5 & ug/L & $\mathrm{U}$ \\
\hline 858 & 858215REGUF & $2 / 15 / 91$ & REG & 1,1,1-Trichloroethane & 5 & ug $/ \mathrm{L}$ & U \\
\hline 858 & 858516REGUF & $5 / 16 / 90$ & REG & 1.1.1-Trichloroethane & 5 & ug $/ \mathrm{L}$ & $\mathrm{U}$ \\
\hline 858 & 858111 REGUF & $11 / 1 / 90$ & REG & 1.1,1-Trichloroethane & 5 & $\mathrm{ug} / \mathrm{L}$ & $\mathrm{U}$ \\
\hline 858 & 858116REGUF & $1 / 16 / 89$ & REG & 1.1,1-Trichloroethane & 5 & $\mathrm{ug} / \mathrm{L}$ & $\mathrm{U}$ \\
\hline 858 & 858425REGUF & $4 / 25 / 89$ & REG & 1,1,1-Trichloroethane & 5 & ug $\Omega$ & $\mathrm{U}$ \\
\hline
\end{tabular}


Station Sample ID Sample Date Sample Type Chemical

\begin{tabular}{|c|c|c|c|c|c|c|}
\hline$\overline{858}$ & M01285 & $11 / 17 / 97$ & REG & 1.1,2.2-Tetrachloroeth & 5 & $\mathrm{ug} / \mathrm{L}$ \\
\hline 858 & W00815 & $6 / 16 / 97$ & REG & 1.1.2.2-Tetrachloroethane & 5 & $\mathrm{ug} / \mathrm{L}$ \\
\hline 858 & $\mathrm{M} 00078$ & $11 / 1+/ 96$ & REG & 1.1.2.2-Tetrachloroethane & 5 & $\mathrm{ug} / \mathrm{L}$ \\
\hline 858 & M00877 & $6 / 16 / 97$ & REG & 1.1.2.2-Tetrachloroethane & 5 & $\mathrm{ug} / \mathrm{L}$ \\
\hline 858 & $\mathrm{~S} 02772$ & $3 / 6 / 95$ & REG & 1.1,2,2-Tetrachloroethane & 5 & $\mathrm{ug} / \mathrm{L}$ \\
\hline 858 & S04936 & $8 / 3 / 95$ & REG & 1.1,2.2-Tetrachloroethane & 5 & ug $/ \mathrm{L}$ \\
\hline 858 & 858215REGUF & $2 / 15 / 91$ & REG & 1,1,2,2-Tetrachloroethane & 5 & $\mathrm{ug} / \mathrm{L}$ \\
\hline 858 & 858516REGUF & $5 / 16 / 90$ & REG & 1,1,2,2-Tetrachloroethane & 5 & ug/L \\
\hline 858 & 858111REGUF & $11 / 1 / 90$ & REG & 1.1,2,2-Tetrachloroethane & 5 & ug/L \\
\hline 858 & 858116REGUF & $1 / 16 / 89$ & REG & 1,1,2,2-Tetrachloroethane & 5 & $\mathrm{ug} / \mathrm{L}$ \\
\hline 858 & 858425REGUF & $4 / 25 / 89$ & REG & 1.1,2.2-Tetrachloroethane & 5 & $\mathrm{ug} / \mathrm{L}$ \\
\hline 858 & W00815 & $6 / 16 / 97$ & REG & 1,1,2-Trichloro-1,2,2-trifluo & 10 & $\mathrm{ug} / \mathrm{L}$ \\
\hline 858 & M01285 & $11 / 17 / 97$ & REG & 1.1,2-Trichloroethane & 5 & ug/L \\
\hline 858 & W00815 & $6 / 16 / 97$ & REG & 1,1,2-Trichloroethane & 5 & ug/L \\
\hline 858 & M00078 & $11 / 14 / 96$ & REG & 1.1,2-Trichloroethane & 5 & ug/L \\
\hline 858 & M00877 & $6 / 16 / 97$ & REG & 1,1,2-Trichloroethane & 5 & ug/L \\
\hline 858 & S02772 & $3 / 6 / 95$ & REG & 1,1,2-Trichloroethane & 5 & $\mathrm{ug} / \mathrm{L}$ \\
\hline 858 & S04936 & $8 / 3 / 95$ & REG & 1.1,2-Trichloroethane & 5 & ug/L \\
\hline 858 & 858215REGUF & $2 / 15 / 91$ & REG & 1,1,2-Trichloroethane & 5 & ug/L \\
\hline 858 & 858516REGUF & $5 / 16 / 90$ & REG & 1,1,2-Trichloroethane & 5 & $\mathrm{ug} / \mathrm{L}$ \\
\hline 858 & 858111 REGUF & $11 / 1 / 90$ & REG & 1.1.2-Trichloroethane & 5 & ug $/ \mathrm{L}$ \\
\hline 858 & 858116REGUF & $1 / 16 / 89$ & REG & 1,1,2-Trichloroethane & 5 & ug $/ \mathrm{L}$ \\
\hline 858 & $858+25$ REGUF & $4 / 25 / 89$ & REG & 1.1,2-Trichloroethane & 5 & ug/L \\
\hline 858 & M01285 & $11 / 17 / 97$ & REG & 1.1-Dichloroethane & 5 & ug/L \\
\hline 858 & W00815 & $6 / 16 / 97$ & REG & 1,1-Dichloroethane & 5 & ug/L \\
\hline 858 & M00078 & $11 / 14 / 96$ & REG & 1,1-Dichloroethane & 5 & $\mathrm{ug} / \mathrm{L}$ \\
\hline 858 & M00877 & $6 / 16 / 97$ & REG & 1.1-Dichloroethane & 5 & $\mathrm{ug} / \mathrm{L}$ \\
\hline 858 & S02772 & $3 / 6 / 95$ & REG & 1.1-Dichloroethane & 5 & $\mathrm{ug} / \mathrm{L}$ \\
\hline 858 & S04936 & $8 / 3 / 95$ & REG & 1.1-Dichloroethane & 5 & $\mathrm{ug} / \mathrm{L}$ \\
\hline 858 & 858215REGUF & $2 / 15 / 91$ & REG & 1,1-Dichloroethane & 5 & ug/L \\
\hline 858 & 858516REGUF & $5 / 16 / 90$ & REG & 1,1-Dichloroethane & 5 & $\mathrm{ug} / \mathrm{L}$ \\
\hline 858 & 858111REGUF & $11 / 1 / 90$ & REG & 1.1-Dichloroethane & 5 & $\mathrm{ug} / \mathrm{L}$ \\
\hline 858 & 858116REGUF & $1 / 16 / 89$ & REG & 1,1-Dichloroethane & 5 & $\mathrm{ug} / \mathrm{L}$ \\
\hline 858 & 858425REGUF & $4 / 25 / 89$ & REG & 1.1-Dichloroethane & 5 & $\mathrm{ug} / \mathrm{L}$ \\
\hline 858 & M01285 & $11 / 17 / 97$ & REG & 1,1-Dichloroethene & 5 & $\mathrm{ug} / \mathrm{L}$ \\
\hline 858 & W00815 & $6 / 16 / 97$ & REG & 1.1-Dichloroethene & 5 & $\mathrm{ug} / \mathrm{L}$ \\
\hline 858 & M00078 & $11 / 14 / 96$ & REG & 1.1-Dichloroethene & 5 & ug/L \\
\hline 858 & M00877 & $6 / 16 / 97$ & REG & 1.1-Dichloroethene & 5 & ug/L \\
\hline 858 & $\mathrm{~S} 02772$ & $3 / 6 / 95$ & REG & 1.1-Dichloroethene & 5 & $\mathrm{ug} / \mathrm{L}$ \\
\hline 858 & S04936 & $8 / 3 / 95$ & REG & 1.1-Dichloroethene & 5 & $\mathrm{ug} / \mathrm{L}$ \\
\hline 858 & 858215 REGUF & $2 / 15 / 91$ & REG & 1.1-Dichloroethene & 5 & ug/L \\
\hline 858 & 858516REGUF & $5 / 16 / 90$ & REG & 1,1-Dichloroethene & 5 & $\mathrm{ug} / \mathrm{L}$ \\
\hline 858 & 858111 REGUF & $11 / 1 / 90$ & REG & 1.1-Dichloroethene & 5 & $\mathrm{ug} / \mathrm{L}$ \\
\hline 858 & 858116 REGUF & $1 / 16 / 89$ & REG & 1.1-Dichloroethene & 5 & $\mathrm{ug} / \mathrm{L}$ \\
\hline 858 & 858425REGUF & $4 / 25 / 89$ & REG & 1.1-Dichloroethene & 5 & $\mathrm{ug} / \mathrm{L}$ \\
\hline 858 & M00078 & $11 / 14 / 96$ & REG & 1.2-Dichlorobenzene & 5 & $\mathrm{ug} / \mathrm{L}$ \\
\hline 858 & M01285 & $11 / 17 / 97$ & REG & 1.2-Dichloroethane & 5 & ug $/ \mathrm{L}$ \\
\hline 858 & W00815 & $6 / 16 / 97$ & REG & 1.2-Dichloroethane & 5 & $\mathrm{ug} / \mathrm{L}$ \\
\hline 858 & M00078 & $11 / 14 / 96$ & REG & 1.2-Dichloroethane & 5 & $\mathrm{ug} / \mathrm{L}$ \\
\hline 858 & M00877 & $6 / 16 / 97$ & REG & 1.2-Dichloroethane & 5 & $\mathrm{ug} / \mathrm{L}$ \\
\hline
\end{tabular}




\begin{tabular}{|c|c|c|c|c|c|c|c|}
\hline Station & Sample ID & Sample Date & Sample Type & Chemical & Results & Units & Qualifier \\
\hline 8.58 & S02772 & $3 / 6 / 95$ & REG & 1.2-Dichloroethane & 5 & $\mathrm{ug} / \mathrm{L}$ & $\bar{U}$ \\
\hline 858 & S04936 & $8 / 3 / 95$ & REG & 1.2-Dichloroethane & 5 & $\mathrm{ug} / \mathrm{L}$ & $\mathrm{U}$ \\
\hline 858 & 858215 REGUF & $2 / 15 / 91$ & REG & 1.2-Dichloroethane & 5 & $\mathrm{ug} / \mathrm{L}$ & U \\
\hline 858 & 858516REGUF & $5 / 16 / 90$ & REG & 1.2-Dichloroethane & 5 & $\mathrm{ug} / \mathrm{L}$ & $\mathrm{U}$ \\
\hline 858 & 858111REGUF & $11 / 1 / 90$ & REG & 1.2-Dichloroethane & 5 & $\mathrm{ug} / \mathrm{L}$ & U \\
\hline 858 & 858116REGUF & $1 / 16 / 89$ & REG & 1.2-Dichloroethane & 5 & ug/L & $\mathrm{U}$ \\
\hline 858 & 858425REGUF & $4 / 25 / 89$ & REG & 1.2-Dichloroethane & 5 & $\mathrm{ug} / \mathrm{L}$ & U \\
\hline 858 & W00815 & $6 / 16 / 97$ & REG & 1.2-Dichloroethene & 5 & ug/L & $\mathrm{U}$ \\
\hline 858 & M00078 & $11 / 14 / 96$ & REG & 1,2-Dichloroethene & 5 & ug $\Omega$ & $U$ \\
\hline 858 & S02772 & $3 / 6 / 95$ & REG & 1,2-Dichloroethene & 5 & $\mathrm{ug} / \mathrm{L}$ & $\mathrm{U}$ \\
\hline 858 & S04936 & $8 / 3 / 95$ & REG & 1,2-Dichloroethene & 5 & $u g / L$ & $\mathrm{U}$ \\
\hline 858 & 858215REGUF & $2 / 15 / 91$ & REG & 1,2-Dichloroethene & 5 & $\mathrm{ug} / \mathrm{L}$ & $\mathrm{U}$ \\
\hline 858 & 858516REGUF & $5 / 16 / 90$ & REG & 1.2-Dichloroethene & 5 & $\mathrm{ug} / \mathrm{L}$ & $\mathrm{U}$ \\
\hline 858 & 858111REGUF & $11 / 1 / 90$ & REG & 1,2-Dichloroethene & 5 & $u g / L$ & U \\
\hline 858 & 858116REGUF & $1 / 16 / 89$ & REG & 1,2-Dichloroethene & 5 & ug $/ \mathrm{L}$ & $\mathrm{U}$ \\
\hline 858 & 858425REGUF & $4 / 25 / 89$ & REG & 1,2-Dichloroethene & 5 & $u g / L$ & $\mathrm{U}$ \\
\hline 858 & M01285 & $11 / 17 / 97$ & REG & 1.2-Dichloropropane & 5 & $\mathrm{ug} / \mathrm{L}$ & $\mathrm{U}$ \\
\hline 858 & W00815 & $6 / 16 / 97$ & REG & 1,2-Dichloropropane & 5 & ug/L & $\mathrm{U}$ \\
\hline 858 & M00078 & $11 / 14 / 96$ & REG & 1,2-Dichloropropane & 5 & $u g / L$ & U \\
\hline 858 & M00877 & $6 / 16 / 97$ & REG & 1,2-Dichloropropane & 5 & ug $/ \mathrm{L}$ & $\mathrm{U}$ \\
\hline 858 & $\mathrm{~S} 02772$ & $3 / 6 / 95$ & REG & 1,2-Dichloropropane & 5 & $u g / L$ & $\mathrm{U}$ \\
\hline 858 & S04936 & $8 / 3 / 95$ & REG & 1,2-Dichloropropane & 5 & $\mathrm{ug} / \mathrm{L}$ & $\mathrm{U}$ \\
\hline 858 & 858215REGUF & $2 / 15 / 91$ & REG & 1,2-Dichloropropane & 5 & $\mathrm{ug} / \mathrm{L}$ & $\mathrm{U}$ \\
\hline 858 & 858516REGUF & $5 / 16 / 90$ & REG & 1.2-Dichloropropane & 5 & ug/L & $\mathrm{U}$ \\
\hline 858 & 858111 REGUF & $11 / 1 / 90$ & REG & 1,2-Dichloropropane & 5 & ug $/ \mathrm{L}$ & $\mathrm{U}$ \\
\hline 858 & 858116REGUF & $1 / 16 / 89$ & REG & 1.2-Dichloropropane & 5 & $\mathrm{ug} / \mathrm{L}$ & $\mathrm{U}$ \\
\hline 858 & 858425 REGUF & $4 / 25 / 89$ & REG & 1.2-Dichloropropane & 5 & $\mathrm{ug} / \mathrm{L}$ & $\mathrm{U}$ \\
\hline 858 & M00078 & $11 / 14 / 96$ & REG & 1.2-Dimethylbenzene & 5 & $\mathrm{ug} / \mathrm{L}$ & U \\
\hline 858 & S02772 & $3 / 6 / 95$ & REG & 1,2-Dimethylbenzene & 5 & ug $/$ & $\mathrm{U}$ \\
\hline 858 & S04936 & $8 / 3 / 95$ & REG & 1,2-Dimethylbenzene & 5 & $\mathrm{ug} / \mathrm{L}$ & $\mathrm{U}$ \\
\hline 858 & M00078 & $11 / 14 / 96$ & REG & 1,3-Dichlorobenzene & 5 & $\mathrm{ug} / \mathrm{L}$ & $\mathrm{U}$ \\
\hline 858 & M00078 & $11 / 14 / 96$ & REG & 1,4-Dichlorobenzene & 5 & $u g /$ & $\mathrm{U}$ \\
\hline 858 & M01285 & $11 / 17 / 97$ & REG & 2-Butanone & 10 & ug $/ \mathrm{L}$ & U \\
\hline 858 & W00815 & $6 / 16 / 97$ & REG & 2-Butanone & 10 & $\mathrm{ug} / \mathrm{L}$ & $\mathrm{U}$ \\
\hline 858 & M00078 & $11 / 14 / 96$ & REG & 2-Butanone & 3 & ug $/ \mathrm{L}$ & $=$ \\
\hline 858 & M00877 & $6 / 16 / 97$ & REG & 2-Butanone & 10 & $\mathrm{ug} / \mathrm{L}$ & U \\
\hline 858 & S02772 & $3 / 6 / 95$ & REG & 2-Butanone & 2 & $\mathrm{ug} / \mathrm{L}$ & $\mathbf{J}$ \\
\hline 858 & S04936 & $8 / 3 / 95$ & REG & 2-Butanone & 10 & $\mathrm{ug} / \mathrm{L}$ & $\mathrm{U}$ \\
\hline 858 & $858215 R E G U F$ & $2 / 15 / 91$ & REG & 2-Butanone & 10 & $\mathrm{ug} / \mathrm{L}$ & $\mathrm{U}$ \\
\hline 858 & 858516 REGUF & $5 / 16 / 90$ & REG & 2-Butanone & 10 & $\mathrm{ug} / \mathrm{L}$ & $\mathrm{U}$ \\
\hline 858 & 858111REGUF & $11 / 1 / 90$ & REG & 2-Butanone & 10 & $\mathrm{ug} / \mathrm{L}$ & U \\
\hline 858 & 858116 REGUF & $1 / 16 / 89$ & REG & 2-Butanone & 10 & $\mathrm{ug} / \mathrm{L}$ & $\mathrm{U}$ \\
\hline 858 & 858425REGUF & $4 / 25 / 89$ & REG & 2-Butanone & 10 & $u g / L$ & $\mathrm{U}$ \\
\hline 858 & M01285 & $11 / 17 / 97$ & REG & 2-Hexanone & 10 & $u g / L$ & $\mathrm{U}$ \\
\hline 858 & W00815 & $6 / 16 / 97$ & REG & 2-Hexanone & 10 & $\mathrm{ug} / \mathrm{L}$ & $\mathbf{U}$ \\
\hline 858 & M00078 & $11 / 14 / 96$ & REG & 2-Hexanone & 10 & $\mathrm{ug} / \mathrm{L}$ & $\mathrm{U}$ \\
\hline 858 & M00877 & $6 / 16 / 97$ & REG & 2-Hexanone & 10 & $\mathrm{ug} / \mathrm{L}$ & U \\
\hline 858 & S02772 & $3 / 6 / 95$ & REG & 2-Hexanone & 10 & $\mathrm{ug} / \mathrm{L}$ & $\mathrm{U}$ \\
\hline 8.58 & S04936 & $8 / 3 / 95$ & REG & 2-Hexanone & 10 & $\mathrm{ug} / \mathrm{L}$ & $\mathrm{U}$ \\
\hline 858 & 858215 REGUF & $2 / 15 / 91$ & REG & 2-Hexanone & 10 & $\mathrm{ug} / \mathrm{L}$ & $\mathrm{U}$ \\
\hline
\end{tabular}




\begin{tabular}{|c|c|c|c|c|c|c|c|}
\hline Station & Sample ID & Sample Date & Sample Type & Chemical & Results & Units & Qualifier \\
\hline$\overline{858}$ & 858516REGUF & $5 / 16 / 90$ & REG & 2-Hexanone & 10 & ug/L & $\bar{U}$ \\
\hline 858 & 858111 REGUF & $11 / 1 / 90$ & REG & 2-Hexanone & 10 & ug $/ \mathrm{L}$ & U \\
\hline 858 & 858116REGUF & $1 / 16 / 89$ & REG & 2-Hexanone & 10 & $\operatorname{ug} / \mathrm{L}$ & $\mathrm{U}$ \\
\hline 858 & $858+25 R E G U F$ & $4 / 25 / 89$ & REG & 2-Hexanone & 10 & ug $/ \mathcal{L}$ & $\mathrm{U}$ \\
\hline 858 & M01285 & $11 / 17 / 97$ & REG & t-Methỵl-2-pentanone & 10 & ug/L & $\mathrm{U}$ \\
\hline 858 & W00815 & $6 / 16 / 97$ & REG & 4-Methyl-2-pentanone & 10 & ug $/ \mathrm{L}$ & $\mathrm{U}$ \\
\hline 858 & M00078 & $11 / 14 / 96$ & REG & 4-Methyl-2-pentanone & 10 & $\mathrm{ug} / \mathrm{L}$ & U \\
\hline 858 & M00877 & $6 / 16 / 97$ & REG & t-Methyl-2-pentanone & 10 & $\mathrm{ug} / \mathrm{L}$ & U \\
\hline 858 & $\mathrm{~S} 02772$ & $3 / 6 / 95$ & REG & 4-Methyl-2-pentanone & 10 & $u g / L$ & U \\
\hline 858 & S04936 & $8 / 3 / 95$ & REG & 4-Methyl-2-pentanone & 10 & ug/L & $\mathrm{U}$ \\
\hline 858 & 858215REGUF & $2 / 15 / 91$ & REG & 4-Methyl-2-pentanone & 10 & ug/L & $\mathrm{U}$ \\
\hline 858 & 858516REGUF & $5 / 16 / 90$ & REG & 4-Methyl-2-pentanone & 10 & $\mathrm{ug} / \mathrm{L}$ & $\mathrm{U}$ \\
\hline 858 & 858111REGUF & $11 / 1 / 90$ & REG & 4-Methyil-2-pentanone & 10 & ug/L & $\mathrm{U}$ \\
\hline 858 & 858116REGUF & $1 / 16 / 89$ & REG & 4-Methyl-2-pentanone & 10 & ug $/ \mathrm{L}$ & U \\
\hline 858 & 858425REGUF & $4 / 25 / 89$ & REG & t-Methyl-2-pentanone & 10 & ug $/ \mathrm{L}$ & $\mathrm{U}$ \\
\hline 858 & M01285 & $11 / 17 / 97$ & REG & Acetone & 10 & ug/L & $\mathrm{U}$ \\
\hline 858 & W00815 & $6 / 16 / 97$ & REG & Acetone & 10 & ug $/ \mathrm{L}$ & $\mathrm{U}$ \\
\hline 858 & M00078 & $11 / 14 / 96$ & REG & Acetone & 2 & ug/L & $=$ \\
\hline 858 & M00877 & $6 / 16 / 97$ & REG & Acetone & 4 & ug/L & $U$ \\
\hline 858 & S02772 & $3 / 6 / 95$ & REG & Acetone & 10 & ug $/ \mathrm{L}$ & $\mathrm{U}$ \\
\hline 858 & S04936 & $8 / 3 / 95$ & REG & Acetone & 10 & $\mathrm{ug} / \mathrm{L}$ & $\mathrm{U}$ \\
\hline 858 & 858215 REGUF & $2 / 15 / 91$ & REG & Acetone & 10 & ug $/ \mathrm{L}$ & $U$ \\
\hline 858 & 858516REGUF & $5 / 16 / 90$ & REG & Acetone & 10 & $u g / L$ & $\mathrm{U}$ \\
\hline 858 & 858111REGUF & $11 / 1 / 90$ & REG & Acetone & 10 & $u g / L$ & $\mathrm{U}$ \\
\hline 858 & 858116REGUF & $1 / 16 / 89$ & REG & Acetone & 10 & $u g / L$ & $\mathrm{U}$ \\
\hline 858 & 858425REGUF & $4 / 25 / 89$ & REG & Acetone & 10 & $\mathrm{ug} / \mathrm{L}$ & $\mathbf{U}$ \\
\hline 858 & M01285 & $11 / 17 / 97$ & REG & Benzene & 5 & $\mathrm{ug} / \mathrm{L}$ & $\mathrm{U}$ \\
\hline 858 & W00815 & $6 / 16 / 97$ & REG & Benzene & 5 & $\mathrm{ug} / \mathrm{L}$ & $\mathrm{U}$ \\
\hline 858 & M00078 & $11 / 14 / 96$ & REG & Benzene & 5 & ug/L & $\mathrm{U}$ \\
\hline 858 & M00877 & $6 / 16 / 97$ & REG & Benzene & 5 & $\mathrm{ug} / \mathrm{L}$ & U \\
\hline 858 & S02772 & $3 / 6 / 95$ & REG & Benzene & 5 & ug/L & $\mathrm{U}$ \\
\hline 858 & S04936 & $8 / 3 / 95$ & REG & Benzene & 5 & ug $/$ & $U$ \\
\hline 858 & 858215REGUF & $2 / 15 / 91$ & REG & Benzene & 5 & $\mathrm{ug} / \mathrm{L}$ & $\mathrm{U}$ \\
\hline 858 & 858516REGUF & $5 / 16 / 90$ & REG & Benzene & 5 & ug/L & $\mathrm{U}$ \\
\hline 858 & 858111 REGUF & $11 / 1 / 90$ & REG & Benzene & 5 & $\mathrm{ug} / \mathrm{L}$ & $\mathrm{U}$ \\
\hline 858 & 858116REGUF & $1 / 16 / 89$ & REG & Benzene & 5 & ug/L & $\mathrm{U}$ \\
\hline 858 & $858+25$ REGUF & $4 / 25 / 89$ & REG & Benzene & 5 & $\mathrm{ug} / \mathrm{L}$ & $\mathrm{U}$ \\
\hline 858 & M01285 & $11 / 17 / 97$ & REG & Bromodichloromethane & 5 & $\mathrm{ug} / \mathrm{L}$ & $\mathrm{U}$ \\
\hline 858 & W00815 & $6 / 16 / 97$ & REG & Bromodichloromethane & 5 & $u g / L$ & U \\
\hline 858 & M00078 & $11 / 14 / 96$ & REG & Bromodichloromethane & 5 & ug/L & $\mathrm{U}$ \\
\hline 858 & M00877 & $6 / 16 / 97$ & REG & Bromodichloromethane & 5 & $\mathrm{ug} / \mathrm{L}$ & $\mathrm{U}$ \\
\hline 858 & S02772 & $3 / 6 / 95$ & REG & Bromodichloromethane & 5 & ug $/ \mathrm{L}$ & $\mathrm{U}$ \\
\hline 858 & S04936 & $8 / 3 / 95$ & REG & Bromodichloromethane & 5 & $\mathrm{ug} / \mathrm{L}$ & $\mathrm{U}$ \\
\hline 858 & 858215REGUF & $2 / 15 / 91$ & REG & Bromodichloromethane & 5 & $\mathrm{ug} / \mathrm{L}$ & $\mathrm{U}$ \\
\hline 858 & 858516REGUF & $5 / 16 / 90$ & REG & Bromodichloromethane & 5 & ug/ & $\mathrm{U}$ \\
\hline 858 & 858111 REGUF & $11 / 1 / 90$ & REG & Bromodichloromethane & 5 & ug/L & $\mathrm{U}$ \\
\hline 858 & 858116REGUF & $1 / 16 / 89$ & REG & Bromodichloromethane & 5 & $\mathrm{ug} / \mathrm{L}$ & $\mathrm{U}$ \\
\hline 858 & 858425REGUF & $4 / 25 / 89$ & REG & Bromodichloromethane & 5 & ug/L & $\mathrm{U}$ \\
\hline 858 & M01285 & $11 / 17 / 97$ & REG & Bromoform & 5 & ug/L & $\mathrm{U}$ \\
\hline 858 & W00815 & $6 / 16 / 97$ & REG & Bromoform & 5 & $\mathrm{ug} / \mathrm{L}$ & $\mathrm{U}$ \\
\hline
\end{tabular}




\begin{tabular}{|c|c|c|c|c|c|c|c|}
\hline Station & Sample ID & Sample Date & Sample Type & Chemical & Results & Units & Qualifier \\
\hline 858 & M00078 & $11 / 14 / 96$ & REG & Bromoform & 5 & $\mathrm{ug} / \mathrm{L}$ & $\mathrm{U}$ \\
\hline 858 & M00877 & $6 / 16 / 97$ & REG & Bromoform & 5 & $\mathrm{ug} / \mathrm{L}$ & $\mathrm{U}$ \\
\hline 858 & S02772 & $3 / 6 / 95$ & REG & Bromoform & 5 & $\mathrm{ug} / \mathrm{L}$ & $\mathrm{U}$ \\
\hline 858 & S04936 & $8 / 3 / 95$ & REG & Bromoform & 5 & $u g / L$ & U \\
\hline 858 & 858215REGUF & $2 / 15 / 91$ & REG & Bromoform & 5 & $\mathrm{ug} / \mathrm{L}$ & U \\
\hline 858 & 858516REGUF & $5 / 16 / 90$ & REG & Bromoform & 5 & $\mathrm{ug} / \mathrm{L}$ & $\mathrm{U}$ \\
\hline 858 & 858111REGUF & $11 / 1 / 90$ & REG & Bromoform & 5 & ug/L & $\mathrm{U}$ \\
\hline 858 & 858116REGUF & $1 / 16 / 89$ & REG & Bromoform & 5 & ug $/ \mathrm{L}$ & U \\
\hline 858 & 858425REGUF & $4 / 25 / 89$ & REG & Bromoform & 5 & $u g / L$ & $\mathrm{U}$ \\
\hline 858 & M01285 & $11 / 17 / 97$ & REG & Bromomethane & 10 & ug $/ \mathrm{L}$ & U \\
\hline 858 & W00815 & $6 / 16 / 97$ & REG & Bromomethane & 10 & $\mathrm{ug} / \mathrm{L}$ & $\mathrm{U}$ \\
\hline 858 & M00078 & $11 / 14 / 96$ & REG & Bromomethane & 10 & $\mathrm{ug} / \mathrm{L}$ & $\mathrm{U}$ \\
\hline 858 & M00877 & $6 / 16 / 97$ & REG & Bromomethane & 10 & $u g / L$ & $\mathrm{U}$ \\
\hline 858 & S02772 & $3 / 6 / 95$ & REG & Bromomethane & 10 & $\mathrm{ug} / \mathrm{L}$ & U \\
\hline 858 & S04936 & $8 / 3 / 95$ & REG & Bromomethane & 10 & $u g / L$ & U \\
\hline 858 & 858215REGUF & $2 / 15 / 91$ & REG & Bromomethane & 10 & ug/L & $\mathrm{U}$ \\
\hline 858 & 858516REGUF & $5 / 16 / 90$ & REG & Bromomethane & 10 & $u g / L$ & $\mathrm{U}$ \\
\hline 858 & 85811IREGUF & $11 / 1 / 90$ & REG & Bromomethane & 10 & $\mathrm{ug} / \mathrm{L}$ & $\mathrm{U}$ \\
\hline 858 & 858116REGUF & $1 / 16 / 89$ & REG & Bromomethane & 10 & ug/L & $\mathrm{U}$ \\
\hline 858 & 858425REGUF & $4 / 25 / 89$ & REG & Bromomethane & 10 & ug/L & $\mathrm{U}$ \\
\hline 858 & M01285 & $11 / 17 / 97$ & REG & Carbon disulfide & 5 & $\mathrm{ug} / \mathrm{L}$ & $\mathrm{U}$ \\
\hline 858 & W00815 & $6 / 16 / 97$ & REG & Carbon disulfide & 5 & $\mathrm{ug} / \mathrm{L}$ & $\mathrm{U}$ \\
\hline 858 & M00078 & $11 / 14 / 96$ & REG & Carbon disulfide & 5 & $\mathrm{ug} / \mathrm{L}$ & $\mathrm{U}$ \\
\hline 858 & M00877 & $6 / 16 / 97$ & REG & Carbon disulfide & 5 & ug/L & U \\
\hline 858 & $\mathrm{~S} 02772$ & $3 / 6 / 95$ & REG & Carbon disulfide & 5 & $\mathrm{ug} / \mathrm{L}$ & U \\
\hline 858 & S04936 & $8 / 3 / 95$ & REG & Carbon disulfide & 2 & ug/L & J \\
\hline 858 & 858215REGUF & $2 / 15 / 91$ & REG & Carbon disulfide & 5 & $\mathrm{ug} / \mathrm{L}$ & U \\
\hline 858 & 858516REGUF & $5 / 16 / 90$ & REG & Carbon disulfide & 5 & ug/L & $\mathrm{U}$ \\
\hline 858 & 858111REGUF & $11 / 1 / 90$ & REG & Carbon disulfide & 5 & $u g / L$ & $\mathrm{U}$ \\
\hline 858 & 858116REGUF & $1 / 16 / 89$ & REG & Carbon disulfide & 5 & $\mathrm{ug} / \mathrm{L}$ & U \\
\hline 858 & 858425REGUF & $4 / 25 / 89$ & REG & Carbon disulfide & 5 & ug/L & U \\
\hline 858 & M01285 & $11 / 17 / 97$ & REG & Carbon tetrachloride & 5 & $\mathrm{ug} / \mathrm{L}$ & U \\
\hline 858 & W00815 & $6 / 16 / 97$ & REG & Carbon tetrachloride & 5 & ug $/ \mathrm{L}$ & $\mathrm{U}$ \\
\hline 858 & M00078 & $11 / 14 / 96$ & REG & Carbon tetrachloride & 5 & $u g / L$ & $\mathrm{U}$ \\
\hline 858 & M00877 & $6 / 16 / 97$ & REG & Carbon tetrachloride & 5 & ug/ $\mathrm{L}$ & $\mathrm{U}$ \\
\hline 858 & $\mathrm{~S} 02772$ & $3 / 6 / 95$ & REG & Carbon tetrachloride & 5 & ug/L & $\mathrm{U}$ \\
\hline 858 & S04936 & $8 / 3 / 95$ & REG & Carbon tetrachloride & 5 & ug/L & $\mathrm{U}$ \\
\hline 858 & 858215REGUF & $2 / 15 / 91$ & REG & Carbon tetrachloride & 5 & ug $/ \mathrm{L}$ & $\mathrm{U}$ \\
\hline 858 & 858516REGUF & $5 / 16 / 90$ & REG & Carbon tetrachloride & 5 & ug/L & $\mathrm{U}$ \\
\hline 858 & 858111REGUF & $11 / 1 / 90$ & REG & Carbon tetrachloride & 5 & ug $/ \mathrm{L}$ & $\mathrm{U}$ \\
\hline 858 & 858116REGUF & $1 / 16 / 89$ & REG & Carbon tetrachloride & 5 & ug/ & $\mathrm{U}$ \\
\hline 858 & 858425REGUF & $4 / 25 / 89$ & REG & Carbon tetrachloride & 5 & $\mathrm{ug} / \mathrm{L}$ & $\mathrm{U}$ \\
\hline 858 & $\mathrm{M} 01285$ & $11 / 17 / 97$ & REG & Chlorobenzene & 5 & ug $/ \mathrm{L}$ & $\mathrm{U}$ \\
\hline 858 & W00815 & $6 / 16 / 97$ & REG & Chlorobenzene & 5 & ug/L & $\mathrm{U}$ \\
\hline 858 & M00078 & $11 / 14 / 96$ & REG & Chlorobenzene & 5 & ug/L & $\mathrm{U}$ \\
\hline 858 & M00877 & $6 / 16 / 97$ & REG & Chlorobenzene & 5 & $\mathrm{ug} / \mathrm{L}$ & U \\
\hline 858 & S02772 & $3 / 6 / 95$ & REG & Chlorobenzene & 5 & ug $/ \mathrm{L}$ & U \\
\hline 858 & S04936 & $8 / 3 / 95$ & REG & Chlorobenzene & 5 & ug $\mathcal{L}$ & $\mathrm{U}$ \\
\hline 858 & 858215REGUF & $2 / 15 / 91$ & REG & Chlorobenzene & 5 & ug $/$ & $\mathrm{U}$ \\
\hline 858 & 858516REGUF & $5 / 16 / 90$ & REG & Chlorobenzene & 5 & ug $/ \mathrm{L}$ & $\mathrm{U}$ \\
\hline
\end{tabular}


Station Sample ID Sample Date Sample Type Chemical

\begin{tabular}{|c|c|c|c|c|}
\hline 858 & 858111 REGUF & $11 / 1 / 90$ & REG & Chlorobenzene \\
\hline 858 & 858116REGUF & $1 / 16 / 89$ & REG & Chlorobenzene \\
\hline 858 & 858425REGUF & $4 / 25 / 89$ & REG & Chlorobenzene \\
\hline 858 & M01285 & $11 / 17 / 97$ & REG & Chloroethane \\
\hline 858 & W00815 & $6 / 16 / 97$ & REG & Chloroethane \\
\hline 858 & M00078 & $11 / 14 / 96$ & REG & Cluloroethane \\
\hline 858 & M00877 & $6 / 16 / 97$ & REG & Chloroethane \\
\hline 858 & S02772 & $3 / 6 / 95$ & REG & Chloroethane \\
\hline 858 & S04936 & $8 / 3 / 95$ & REG & Chloroethane \\
\hline 858 & $858215 R E G U F$ & $2 / 15 / 91$ & REG & Chloroethane \\
\hline 858 & 8585I6REGUF & $5 / 16 / 90$ & REG & Chloroethane \\
\hline 858 & 858111REGUF & $11 / 1 / 90$ & REG & Chloroethane \\
\hline 858 & 858116REGUF & $1 / 16 / 89$ & REG & Chloroethane \\
\hline 858 & 858425REGUF & $4 / 25 / 89$ & REG & Chloroethane \\
\hline 858 & M01285 & $11 / 17 / 97$ & REG & Chloroform \\
\hline 858 & W00815 & $6 / 16 / 97$ & REG & Chloroform \\
\hline 858 & M00078 & $11 / 14 / 96$ & REG & Chloroform \\
\hline 858 & M00877 & $6 / 16 / 97$ & REG & Chloroform \\
\hline 858 & S02772 & $3 / 6 / 95$ & REG & Chloroform \\
\hline 858 & S04936 & $8 / 3 / 95$ & REG & Chloroform \\
\hline 858 & 858215REGUF & $2 / 15 / 91$ & REG & Chloroform \\
\hline 858 & 858516REGUF & $5 / 16 / 90$ & REG & Chloroform \\
\hline 858 & 858111REGUF & $11 / 1 / 90$ & REG & Chloroform \\
\hline 858 & 858116REGUF & $1 / 16 / 89$ & REG & Chloroform \\
\hline 858 & 858425REGUF & $4 / 25 / 89$ & REG & Chloroform \\
\hline 858 & M01285 & $11 / 17 / 97$ & REG & Chloromethane \\
\hline 858 & W00815 & $6 / 16 / 97$ & REG & Chloromethane \\
\hline 858 & M00078 & $11 / 14 / 96$ & REG & Chloromethane \\
\hline 858 & M00877 & $6 / 16 / 97$ & REG & Chloromethane \\
\hline 858 & S02772 & $3 / 6 / 95$ & REG & Chloromethane \\
\hline 858 & S04936 & $8 / 3 / 95$ & REG & Chloromethane \\
\hline 858 & 858215REGUF & $2 / 15 / 91$ & REG & Chloromethane \\
\hline 858 & 858516REGUF & $5 / 16 / 90$ & REG & Chloromethane \\
\hline 858 & 858111REGUF & $11 / 1 / 90$ & REG & Chloromethane \\
\hline 858 & 858116 REGUF & $1 / 16 / 89$ & REG & Chloromethane \\
\hline 858 & 858425REGUF & $4 / 25 / 89$ & REG & Chloromethane \\
\hline 858 & M01285 & $11 / 17 / 97$ & REG & cis-1,2-Dichloroethene \\
\hline 858 & M00078 & $11 / 14 / 96$ & REG & cis-1.2-Dichloroethene \\
\hline 858 & M00877 & $6 / 16 / 97$ & REG & cis-1.2-Dichloroethene \\
\hline 858 & S02772 & $3 / 6 / 95$ & REG & cis-1.2-Dichloroethene \\
\hline 858 & S04936 & $8 / 3 / 95$ & REG & cis-1.2-Dichloroethene \\
\hline 858 & M01285 & $11 / 17 / 97$ & REG & cis-1,3-Dichloropropen \\
\hline 858 & W00815 & $6 / 16 / 97$ & REG & cis-1.3-Dichloropropene \\
\hline 858 & M00078 & $11 / 14 / 96$ & REG & cis-1,3-Dichloropropene \\
\hline 858 & M00877 & $6 / 16 / 97$ & REG & cis-1.3-Dichloropropene \\
\hline 858 & S02772 & $3 / 6 / 95$ & REG & cis-1,3-Dichloropropene \\
\hline 858 & S04936 & $8 / 3 / 95$ & REG & cis-1,3-Dichloropropene \\
\hline 858 & 858215REGUF & $2 / 15 / 91$ & REG & cis-1,3-Dichloropropene \\
\hline 858 & 858516REGUF & $5 / 16 / 90$ & REG & cis-1,3-Dichloropropene \\
\hline 858 & 858111 REGUF & $11 / 1 / 90$ & REG & cis-1.3-Dichloropropene \\
\hline
\end{tabular}

Results Units Qualifier

\begin{tabular}{|c|c|c|}
\hline 5 & ug/L & $\overline{\mathrm{U}}$ \\
\hline 5 & ug/L & U \\
\hline 5 & $\mathrm{ug} / \mathrm{L}$ & $\mathrm{U}$ \\
\hline 10 & $\mathrm{ug} / \mathrm{L}$ & $\mathrm{U}$ \\
\hline 10 & $\mathrm{ug} / \mathrm{L}$ & $\mathrm{U}$ \\
\hline 10 & ug/L & $\mathrm{U}$ \\
\hline 10 & ug/L & $\mathrm{U}$ \\
\hline 10 & $\mathrm{ug} / \mathrm{L}$ & $\mathrm{U}$ \\
\hline 10 & ug/L & $\mathrm{U}$ \\
\hline 10 & $\mathrm{ug} / \mathrm{L}$ & $\mathrm{U}$ \\
\hline 10 & $\mathrm{ug} / \mathrm{L}$ & $U$ \\
\hline 10 & ug $/ \mathrm{L}$ & $\mathbf{U}$ \\
\hline 10 & ug $/ \mathrm{L}$ & $\mathrm{U}$ \\
\hline 10 & $\mathrm{ug} / \mathrm{L}$ & $\mathrm{U}$ \\
\hline 5 & $\mathrm{ug} / \mathrm{L}$ & $\mathrm{U}$ \\
\hline 5 & $\mathrm{ug} / \mathrm{L}$ & $\mathbf{U}$ \\
\hline 5 & $\mathrm{ug} / \mathrm{L}$ & $\mathrm{U}$ \\
\hline 5 & $\mathrm{ug} / \mathrm{L}$ & $\mathrm{U}$ \\
\hline 5 & ugl & $U$ \\
\hline 5 & ug $/ \mathrm{L}$ & $\mathrm{U}$ \\
\hline 5 & ug $/ \mathrm{L}$ & $U$ \\
\hline 5 & $\mathrm{ug} / \mathrm{L}$ & $U$ \\
\hline 5 & ug/L & $\mathrm{U}$ \\
\hline 5 & $\mathrm{ug} / \mathrm{L}$ & $\mathrm{U}$ \\
\hline 5 & $\mathrm{ug} / \mathrm{L}$ & $\mathbf{U}$ \\
\hline 10 & $\mathrm{ug} / \mathrm{L}$ & $\mathrm{U}$ \\
\hline 10 & $\mathrm{ug} / \mathrm{L}$ & $\mathrm{U}$ \\
\hline 10 & $\mathrm{ug} / \mathrm{L}$ & $\mathrm{U}$ \\
\hline 10 & ug/L & $\mathrm{U}$ \\
\hline 10 & ug/L & $\mathrm{U}$ \\
\hline 10 & $\mathrm{ug} / \mathrm{L}$ & $\mathrm{U}$ \\
\hline 10 & ug/l & $\mathrm{U}$ \\
\hline 10 & ug/L & $\mathrm{U}$ \\
\hline 10 & ug/ & $\mathrm{U}$ \\
\hline 10 & ug/L & $\mathrm{U}$ \\
\hline 10 & $\mathrm{ug} / \mathrm{L}$ & $\mathrm{U}$ \\
\hline 5 & ug/L & $\mathrm{U}$ \\
\hline 5 & ug/L & $\mathbf{U}$ \\
\hline 5 & $\mathrm{ug} / \mathrm{L}$ & $\mathrm{U}$ \\
\hline 5 & $\mathrm{ug} / \mathrm{L}$ & $\mathrm{U}$ \\
\hline 5 & $\mathrm{ug} / \mathrm{L}$ & $\mathrm{U}$ \\
\hline 5 & ug/L & $\mathrm{U}$ \\
\hline 5 & ug/L & $\mathrm{U}$ \\
\hline 5 & $\mathrm{ug} / \mathrm{L}$ & $\mathrm{U}$ \\
\hline 5 & $\mathrm{ug} / \mathrm{L}$ & $\mathrm{U}$ \\
\hline 5 & $\mathrm{ug} / \mathrm{L}$ & $\mathbf{U}$ \\
\hline 5 & $\mathrm{ug} / \mathrm{L}$ & $\mathrm{U}$ \\
\hline 5 & ug/L & $\mathrm{U}$ \\
\hline 5 & $\mathrm{ug} / \mathrm{L}$ & $\mathrm{U}$ \\
\hline 5 & ug/L & $\mathrm{U}$ \\
\hline
\end{tabular}


B-90

\begin{tabular}{|c|c|c|c|c|c|c|c|}
\hline Station & Sample ID & Sample Date & Sample Type & Chemical & Results & Units & Qualifier \\
\hline 8.58 & 858116REGUF & $1 / 16 / 89$ & REG & cis-1.3-Dichloropropene & 5 & ug/L & $\mathrm{U}$ \\
\hline 858 & $858+25$ REGUF & $4 / 25 / 89$ & REG & cis-1.3-Dichloropropene & 5 & $\mathrm{ug} / \mathrm{L}$ & $\mathrm{U}$ \\
\hline 858 & M01285 & $11 / 17 / 97$ & REG & Dibromochloromethane & 5 & $\mathrm{ug} / \mathrm{L}$ & $\mathrm{U}$ \\
\hline 858 & W00815 & $6 / 16 / 97$ & REG & Dibromochloromethane & 5 & $\mathrm{ug} / \mathrm{L}$ & $\mathrm{U}$ \\
\hline 8.58 & M00078 & $11 / 14 / 96$ & REG & Dibromochloromethane & 5 & ug/ $/$ & $\mathrm{U}$ \\
\hline 858 & M00877 & $6 / 16 / 97$ & REG & Dibromochloromethane & 5 & $\mathrm{ug} / \mathrm{L}$ & $\mathrm{U}$ \\
\hline 858 & S02772 & $3 / 6 / 95$ & REG & Dibromochloromethane & 5 & $\mathrm{ug} / \mathrm{L}$ & $\mathrm{U}$ \\
\hline 858 & S04936 & $8 / 3 / 95$ & REG & Dibromochloromethane & 5 & $\mathrm{ug} / \mathrm{L}$ & $\mathrm{U}$ \\
\hline 858 & 858215REGUF & $2 / 15 / 91$ & REG & Dibromochloromethane & 5 & ug/ & $\mathrm{U}$ \\
\hline 858 & 858516REGUF & $5 / 16 / 90$ & REG & Dibromochloromethane & 5 & $\mathrm{ug} / \mathrm{L}$ & $\mathrm{U}$ \\
\hline 858 & 858111REGUF & $11 / 1 / 90$ & REG & Dibromochloromethane & 5 & ug/L & $\mathrm{U}$ \\
\hline 858 & 858116REGUF & $1 / 16 / 89$ & REG & Dibromochloromethane & 5 & $\mathrm{ug} / \mathrm{L}$ & $\mathrm{U}$ \\
\hline 858 & 858425REGUF & $4 / 25 / 89$ & REG & Dibromochloromethane & 5 & ug/L & $\mathrm{U}$ \\
\hline 858 & M00078 & $11 / 14 / 96$ & REG & Dimethylbenzene & 5 & ug/L & $\mathrm{U}$ \\
\hline 858 & 858215REGUF & $2 / 15 / 91$ & REG & Dimethylbenzene & 5 & $\mathrm{ug} / \mathrm{L}$ & $\mathrm{U}$ \\
\hline 858 & 858516REGUF & $5 / 16 / 90$ & REG & Dimethylbenzene & 5 & ug/L & $\mathrm{U}$ \\
\hline 858 & 858111 REGUF & $11 / 1 / 90$ & REG & Dimethylbenzene & 5 & ug/L & $\mathrm{U}$ \\
\hline 858 & 858116REGUF & $1 / 16 / 89$ & REG & Dimethylbenzene & 5 & ug $/ \mathrm{L}$ & $\mathrm{U}$ \\
\hline 858 & 858425REGUF & $4 / 25 / 89$ & REG & Dimethylbenzene & 5 & $\mathrm{ug} / \mathrm{L}$ & $\mathbf{U}$ \\
\hline 858 & M01285 & $11 / 17 / 97$ & REG & Ethylbenzene & 5 & ug/L & $\mathrm{U}$ \\
\hline 858 & W00815 & $6 / 16 / 97$ & REG & Ethylbenzene & 5 & ug $/ \mathrm{L}$ & $\mathrm{U}$ \\
\hline 858 & M00078 & $11 / 14 / 96$ & REG & Ethylbenzene & 5 & $\mathrm{ug} / \mathrm{L}$ & $\mathrm{U}$ \\
\hline 858 & M00877 & $6 / 16 / 97$ & REG & Ethylbenzene & 5 & ug/L & U \\
\hline 858 & S02772 & $3 / 6 / 95$ & REG & Ethylbenzene & 5 & ugl & $\mathrm{U}$ \\
\hline 858 & S04936 & $8 / 3 / 95$ & REG & Ethylbenzene & 5 & ug/L & $\mathrm{U}$ \\
\hline 858 & 858215REGUF & $2 / 15 / 91$ & REG & Ethylbenzene & 5 & $\mathrm{ug} / \mathrm{L}$ & $\mathrm{U}$ \\
\hline 858 & 858516REGUF & $5 / 16 / 90$ & REG & Ethyllbenzene & 5 & ug/ & $\mathrm{U}$ \\
\hline 858 & 858111 REGUF & $11 / 1 / 90$ & REG & Ethylbenzene & 5 & ug $\Omega$ & $\mathbf{U}$ \\
\hline 858 & 858116REGUF & $1 / 16 / 89$ & REG & Ethylbenzene & 5 & $u g / L$ & $\mathbf{U}$ \\
\hline 858 & 858425REGUF & $4 / 25 / 89$ & REG & Ethylbenzene & 5 & ug/L & $\mathbf{U}$ \\
\hline 858 & M01285 & $11 / 17 / 97$ & REG & m.p-Xylene & 5 & ug/L & U \\
\hline 858 & W00815 & $6 / 16 / 97$ & REG & m.p-Xylene & 5 & $\mathrm{ug} / \mathrm{L}$ & $\mathrm{U}$ \\
\hline 858 & M00078 & $11 / 14 / 96$ & REG & m.p-Xylene & 5 & $\mathrm{ug} / \mathrm{L}$ & $\mathrm{U}$ \\
\hline 858 & M00877 & $6 / 16 / 97$ & REG & m.p-Xylene & 5 & $\mathrm{ug} / \mathrm{L}$ & $\mathrm{U}$ \\
\hline 858 & S02772 & $3 / 6 / 95$ & REG & m.p-Xylene & 5 & $\mathrm{ug} / \mathrm{L}$ & $\mathrm{U}$ \\
\hline 858 & S04936 & $8 / 3 / 95$ & REG & m.p-Xylene & 5 & ug $/ \mathrm{L}$ & U \\
\hline 858 & M01285 & $11 / 17 / 97$ & REG & Methylene chloride & 7 & $\mathrm{ug} / \mathrm{L}$ & B \\
\hline 858 & W00815 & $6 / 16 / 97$ & REG & Methylene chloride & 5 & $\mathrm{ug} / \mathrm{L}$ & $\mathrm{U}$ \\
\hline 8.58 & M00078 & $11 / 14 / 96$ & REG & Methỵlene chloride & 5 & ug $/ L$ & U \\
\hline 858 & M00877 & $6 / 16 / 97$ & REG & Methỵlene chloride & 7 & $\mathrm{ug} / \mathrm{L}$ & $=$ \\
\hline 858 & S02772 & $3 / 6 / 95$ & REG & Methylene chloride & 5 & ug/L &.$U$ \\
\hline 858 & S04936 & $8 / 3 / 95$ & REG & Methylene chloride & 5 & ug/L & U \\
\hline 858 & 858215REGUF & $2 / 15 / 91$ & REG & Methylene chloride & 5 & ug/L & U \\
\hline 858 & 858516REGUF & $5 / 16 / 90$ & REG & Methylene chloride & 5 & ug/L & $\mathrm{U}$ \\
\hline 858 & 858111 REGUF & $11 / 1 / 90$ & REG & Methylene chloride & 5 & ug/L & U \\
\hline 858 & $858116 \mathrm{REGUF}$ & $1 / 16 / 89$ & REG & Methylene chloride & 1 & ug/ & J \\
\hline 858 & 858425REGUF & $4 / 25 / 89$ & REG & Methylene chloride & 1 & ug/L & $\mathrm{J}$ \\
\hline 858 & M01285 & $11 / 17 / 97$ & REG & Styrene & 5 & $\mathrm{ug} / \mathrm{L}$ & $\mathrm{U}$ \\
\hline 858 & W00815 & $6 / 16 / 97$ & REG & Styrene & 5 & ug $/ \mathrm{L}$ & $\mathrm{U}$ \\
\hline 858 & M00078 & $11 / 14 / 96$ & REG & Styrene & 5 & ug/L & $\mathrm{U}$ \\
\hline
\end{tabular}


B-91

\begin{tabular}{|c|c|c|c|c|c|c|c|}
\hline Station & Sample ID & Sample Date & Sample Type & Chemical & Results & Units & Qualifier \\
\hline$\overline{858}$ & Mo0877 & $6 / 16 / 97$ & REG & Styrene & 5 & $\mathrm{ug} / \mathrm{L}$ & $\bar{U}$ \\
\hline 858 & S02772 & $3 / 6 / 95$ & REG & Styrene & 5 & $\mathrm{ug} / \mathrm{L}$ & $\mathrm{U}$ \\
\hline 858 & S04936 & $8 / 3 / 95$ & REG & Styrene & 5 & $\mathrm{ug} / \mathrm{L}$ & U \\
\hline 858 & 858215REGUF & $2 / 15 / 91$ & REG & Styrene & 5 & $\mathrm{ug} / \mathrm{L}$ & $\mathrm{U}$ \\
\hline 858 & 858516REGUF & $5 / 16 / 90$ & REG & Styrene & 5 & $\mathrm{ug} / \mathrm{L}$ & $\mathrm{U}$ \\
\hline 858 & 858111 REGUF & $11 / 1 / 90$ & REG & Styrene & 5 & $\mathrm{ug} / \mathrm{L}$ & U \\
\hline 858 & 858116 REGUF & $1 / 16 / 89$ & REG & Styrene & 5 & $\mathrm{ug} / \mathrm{L}$ & $\mathrm{U}$ \\
\hline 858 & 858425REGUF & $4 / 25 / 89$ & REG & Styrene & 5 & $\mathrm{ug} / \mathrm{L}$ & $\mathrm{U}$ \\
\hline 858 & M01285 & $11 / 17 / 97$ & REG & Tetrachloroethene & 5 & $\mathrm{ug} / \mathrm{L}$ & $\mathrm{U}$ \\
\hline 858 & W00815 & $6 / 16 / 97$ & REG & Tetrachloroethene & 5 & $\mathrm{ug} / \mathrm{L}$ & $\mathrm{U}$ \\
\hline 858 & M00078 & $11 / 14 / 96$ & REG & Tetrachloroethene & 5 & $\mathrm{ug} / \mathrm{L}$ & $\mathrm{U}$ \\
\hline 858 & M00877 & $6 / 16 / 97$ & REG & Tetrachloroethene & 5 & $\mathrm{ug} / \mathrm{L}$ & $\mathrm{U}$ \\
\hline 858 & $\mathrm{~S} 02772$ & $3 / 6 / 95$ & REG & Tetrachloroethene & 5 & $\mathrm{ug} / \mathrm{L}$ & $\mathrm{U}$ \\
\hline 858 & S04936 & $8 / 3 / 95$ & REG & Tetrachloroethene & 5 & $\mathrm{ug} / \mathrm{L}$ & $\mathrm{U}$ \\
\hline 858 & 858215REGUF & $2 / 15 / 91$ & REG & Tetrachloroethene & 5 & $\mathrm{ug} / \mathrm{L}$ & $\mathrm{U}$ \\
\hline 858 & 858516REGUF & $5 / 16 / 90$ & REG & Tetrachloroethene & 5 & $\mathrm{ug} / \mathrm{L}$ & $\mathrm{U}$ \\
\hline 858 & 858111REGUF & $11 / 1 / 90$ & REG & Tetrachloroethene & 5 & ug/L & $\mathrm{U}$ \\
\hline 858 & 858116REGUF & $1 / 16 / 89$ & REG & Tetrachloroethene & 5 & $\mathrm{ug} / \mathrm{L}$ & $\mathrm{U}$ \\
\hline 858 & 858425REGUF & $4 / 25 / 89$ & REG & Tetrachloroethene & 5 & $\mathrm{ug} / \mathrm{L}$ & $\mathrm{U}$ \\
\hline 858 & M01285 & $11 / 17 / 97$ & REG & Toluene & 5 & ug/L & $\mathrm{U}$ \\
\hline 858 & W00815 & $6 / 16 / 97$ & REG & Toluene & 5 & $\mathrm{ug} / \mathrm{L}$ & $\mathrm{U}$ \\
\hline 858 & M00078 & $11 / 14 / 96$ & REG & Toluene & 5 & ug/L & $\mathrm{U}$ \\
\hline 858 & M00877 & $6 / 16 / 97$ & REG & Toluene & 5 & ug/ & $\mathrm{U}$ \\
\hline 858 & S02772 & $3 / 6 / 95$ & REG & Toluene & 5 & $\mathrm{ug} / \mathrm{L}$ & $\mathrm{U}$ \\
\hline 858 & S04936 & $8 / 3 / 95$ & REG & Toluene & 5 & $\mathrm{ug} / \mathrm{L}$ & $\mathrm{U}$ \\
\hline 858 & 858215REGUF & $2 / 15 / 91$ & REG & Toluene & 5 & $u g / L$ & $\mathrm{U}$ \\
\hline 858 & 858516REGUF & $5 / 16 / 90$ & REG & Toluene & 2 & $\mathrm{ug} / \mathrm{L}$ & $J$ \\
\hline 858 & 858111 REGUF & $11 / 1 / 90$ & REG & Toluene & 5 & $\mathrm{ug} / \mathrm{L}$ & $\mathrm{U}$ \\
\hline 858 & 858116REGUF & $1 / 16 / 89$ & REG & Toluene & 5 & $\mathrm{ug} / \mathrm{L}$ & $\mathrm{U}$ \\
\hline 858 & 858425REGUF & $4 / 25 / 89$ & REG & Toluene & 5 & $\mathrm{ug} / \mathrm{L}$ & $\mathrm{U}$ \\
\hline 858 & M01285 & $11 / 17 / 97$ & REG & trans-1.2-Dichloroethe & 5 & $\mathrm{ug} / \mathrm{L}$ & $\mathrm{U}$ \\
\hline 858 & M00078 & $11 / 14 / 96$ & REG & trans-1.2-Dichloroethene & 5 & ug/L & $\mathrm{U}$ \\
\hline 858 & M00877 & $6 / 16 / 97$ & REG & trans-1.2-Dichloroethene & 5 & $\mathrm{ug} / \mathrm{L}$ & $\mathrm{U}$ \\
\hline 858 & $\mathrm{~S} 02772$ & $3 / 6 / 95$ & REG & trans-1.2-Dichloroethene & 5 & $\mathrm{ug} / \mathrm{L}$ & $\mathrm{U}$ \\
\hline 858 & S04936 & $8 / 3 / 95$ & REG & trans-1.2-Dichloroethene & 5 & $\mathrm{ug} / \mathrm{L}$ & $\mathrm{U}$ \\
\hline 858 & M01285 & $11 / 17 / 97$ & REG & trans-1.3-Dichloroprop & 5 & $\mathrm{ug} / \mathrm{L}$ & $\mathrm{U}$ \\
\hline 858 & W00815 & $6 / 16 / 97$ & REG & trans-1.3-Dichloropropene & 5 & $\mathrm{ug} / \mathrm{L}$ & $\mathrm{U}$ \\
\hline 858 & M00078 & $11 / 14 / 96$ & REG & trans-1.3-Dichloropropene & 5 & ug/L & $\mathrm{U}$ \\
\hline 858 & M00877 & $6 / 16 / 97$ & REG & trans-1.3-Dichloropropene & 5 & $\mathrm{ug} / \mathrm{L}$ & U \\
\hline 858 & S02772 & $3 / 6 / 95$ & REG & trans-1.3-Dichloropropene & 5 & $\mathrm{ug} / \mathrm{L}$ & $\mathrm{U}$ \\
\hline 858 & S04936 & $8 / 3 / 95$ & REG & trans-1.3-Dichloropropene & 5 & $\mathrm{ug} / \mathrm{L}$ & $\mathrm{U}$ \\
\hline 858 & 858215REGUF & $2 / 15 / 91$ & REG & trans-1.3-Dichloropropene & 5 & $\mathrm{ug} / \mathrm{L}$ & $\mathrm{U}$ \\
\hline 858 & 858516REGUF & $5 / 16 / 90$ & REG & trans-1.3-Dichloropropene & 5 & ug/L & $\mathrm{U}$ \\
\hline 858 & 85811 IREGUF & $11 / 1 / 90$ & REG & trans-1.3-Dichloropropene & 5 & $\mathrm{ug} / \mathrm{L}$ & $\mathrm{U}$ \\
\hline 858 & 858116 REGUF & $1 / 16 / 89$ & REG & trans-1.3-Dichloropropene & 5 & ug/L & $\mathrm{U}$ \\
\hline 858 & 858425REGUF & $4 / 25 / 89$ & REG & trans-1,3-Dichloropropene & 5 & ug/L & $\mathrm{U}$ \\
\hline 858 & M01285 & $11 / 17 / 97$ & REG & Trichloroethene & 5 & ug/L & $\mathrm{U}$ \\
\hline 858 & W00815 & $6 / 16 / 97$ & REG & Trichloroethene & 5 & ug/L & $\mathrm{U}$ \\
\hline 858 & M00078 & $11 / 14 / 96$ & REG & Trichloroethene & 5 & ug/L & $\mathrm{U}$ \\
\hline 858 & M00877 & $6 / 16 / 97$ & REG & Trichloroethene & 5 & $\mathrm{ug} / \mathrm{L}$ & $\mathrm{U}$ \\
\hline
\end{tabular}


Station Sample ID Sample Date Sample Type Chemical

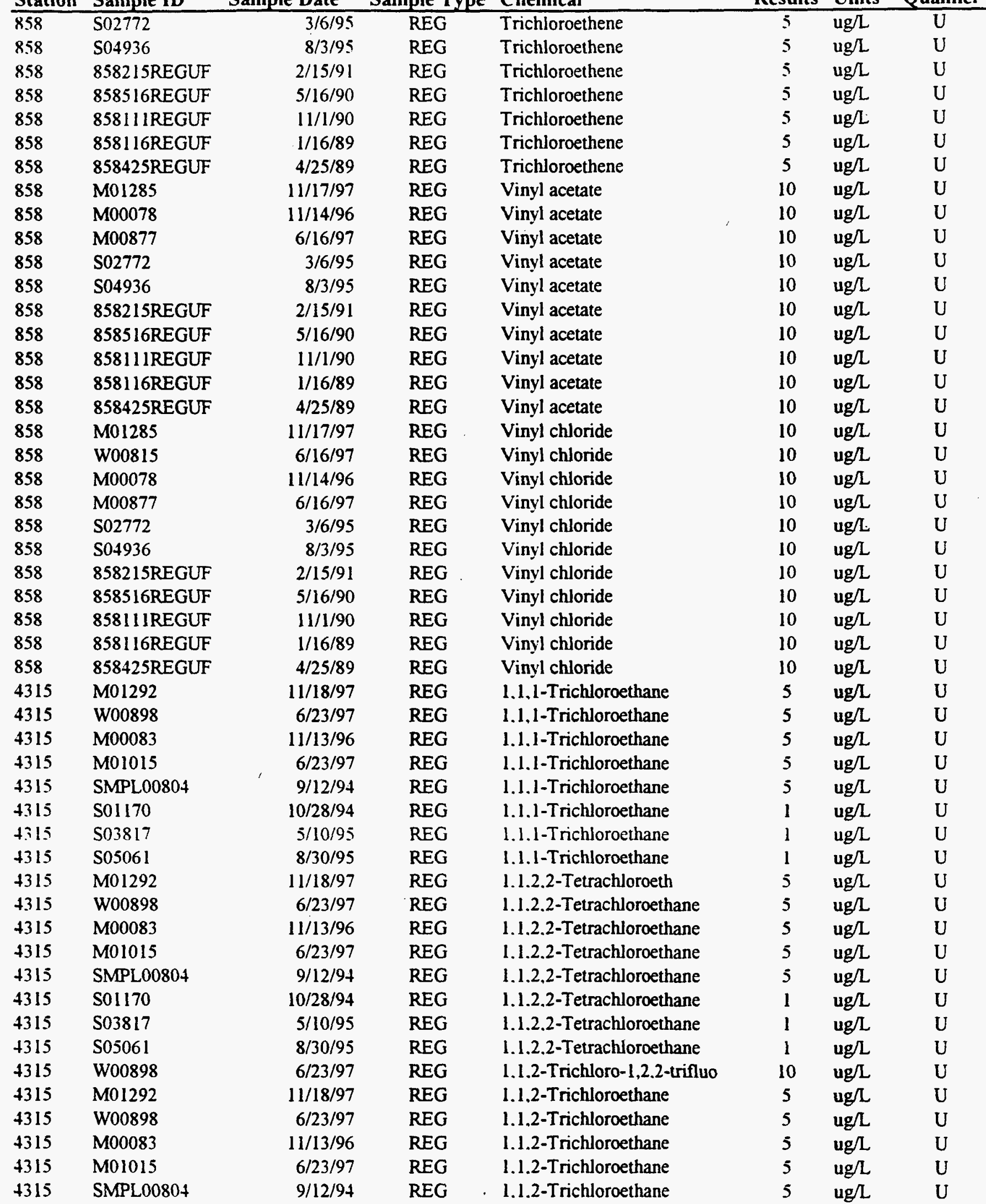


Station Sample ID Sample Date Sample Type Chemical

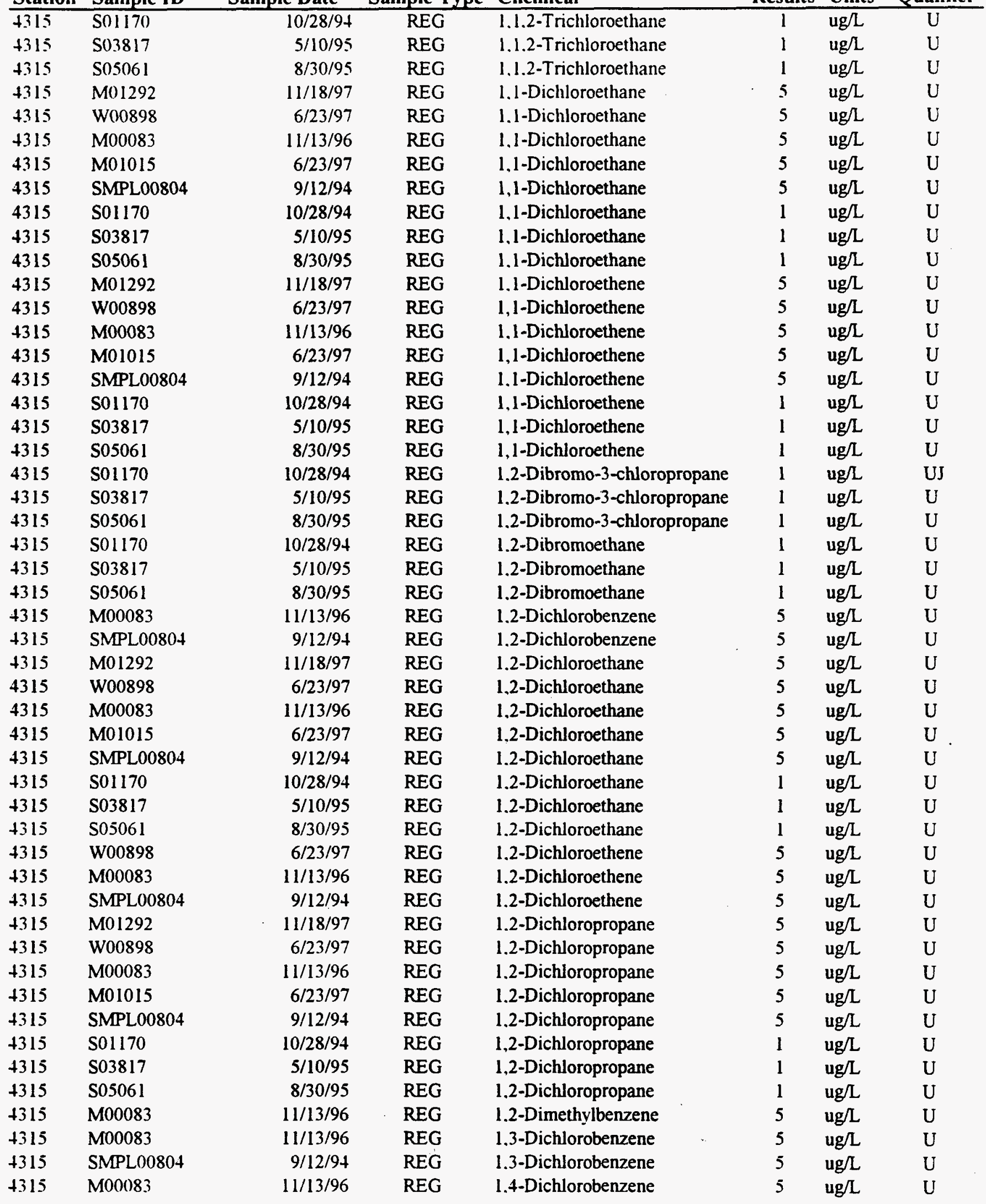


Station Sample ID Sample Date Sample Type Chemical

\begin{tabular}{|c|c|c|c|c|}
\hline 4315 & S03817 & $5 / 10 / 95$ & REG & Bromodichloromethane \\
\hline 4315 & S05061 & $8 / 30 / 95$ & $\mathrm{REG}$ & Bromodichloromethane \\
\hline 4315 & M01292 & $11 / 18 / 97$ & REG & Bromoform \\
\hline 4315 & W00898 & $6 / 23 / 97$ & REG & Bromoform \\
\hline 4315 & M00083 & $11 / 13 / 96$ & REG & Bromoform \\
\hline 4315 & M01015 & $6 / 23 / 97$ & REG & Bromoform \\
\hline 4315 & SMPL00804 & $9 / 12 / 94$ & REG & Bromoform \\
\hline 4315 & S01170 & $10 / 28 / 94$ & REG & Bromoform \\
\hline 4315 & S03817 & $5 / 10 / 95$ & REG & Bromoform \\
\hline 4315 & S05061 & $8 / 30 / 95$ & REG & Bromoform \\
\hline 4315 & M01292 & $11 / 18 / 97$ & REG & Bromomethane \\
\hline 4315 & W00898 & $6 / 23 / 97$ & REG & Bromomethane \\
\hline 4315 & M00083 & $11 / 13 / 96$ & REG & Bromomethane \\
\hline 4315 & M01015 & $6 / 23 / 97$ & REG & Bromomethane \\
\hline 4315 & SMPL00804 & $9 / 12 / 94$ & REG & Bromomethane \\
\hline 4315 & SO1170 & $10 / 28 / 94$ & REG & Bromomethane \\
\hline 4315 & S03817 & $5 / 10 / 95$ & REG & Bromomethane \\
\hline 4315 & S05061 & $8 / 30 / 95$ & REG & Bromomethane \\
\hline 4315 & M01292 & $11 / 18 / 97$ & REG & Carbon disulfide \\
\hline 4315 & W00898 & $6 / 23 / 97$ & REG & Carbon disulfide \\
\hline 4315 & M00083 & $11 / 13 / 96$ & REG & Carbon disulfide \\
\hline 4315 & M01015 & $6 / 23 / 97$ & REG & Carbon disulfide \\
\hline 4315 & SMPL00804 & $9 / 12 / 94$ & REG & Carbon disulfide \\
\hline 4315 & So1170 & $10 / 28 / 94$ & REG & Carbon disulfide \\
\hline 4315 & S03817 & $5 / 10 / 95$ & REG & Carbon disulfide \\
\hline 4315 & S05061 & $8 / 30 / 95$ & REG & Carbon disulfide \\
\hline 4315 & M01292 & $11 / 18 / 97$ & REG & Carbon tetrachloride \\
\hline 4315 & W00898 & $6 / 23 / 97$ & REG & Carbon tetrachloride \\
\hline 4315 & M00083 & $11 / 13 / 96$ & REG & Carbon tetrachloride \\
\hline 4315 & M01015 & $6 / 23 / 97$ & REG & Carbon tetrachloride \\
\hline 4315 & SMPL00804 & $9 / 12 / 94$ & REG & Carbon tetrachloride \\
\hline 4315 & Sol170 & $10 / 28 / 94$ & REG & Carbon tetrachloride \\
\hline 4315 & S03817 & $5 / 10 / 95$ & REG & Carbon tetrachloride \\
\hline 4315 & S05061 & $8 / 30 / 95$ & REG & Carbon tetrachloride \\
\hline 4315 & M01292 & $11 / 18 / 97$ & REG & Chlorobenzene \\
\hline 4315 & W00898 & $6 / 23 / 97$ & REG & Chlorobenzene \\
\hline 4315 & M00083 & $11 / 13 / 96$ & REG & Chlorobenzene \\
\hline 4315 & M01015 & $6 / 23 / 97$ & REG & Chlorobenzene \\
\hline+315 & SMPL00804 & $9 / 12 / 94$ & REG & Chlorobenzene \\
\hline 4315 & S01170 & $10 / 28 / 94$ & REG & Chlorobenzene \\
\hline 4315 & S03817 & $5 / 10 / 95$ & REG & Chlorobenzene \\
\hline 4315 & S05061 & $8 / 30 / 95$ & REG & Chlorobenzene \\
\hline 4315 & M01292 & $11 / 18 / 97$ & REG & Chloroethane \\
\hline 4315 & W00898 & $6 / 23 / 97$ & REG & Chloroethane \\
\hline 4315 & M00083 & $11 / 13 / 96$ & REG & Chloroethane \\
\hline 4315 & M01015 & $6 / 23 / 97$ & REG & Chloroethane \\
\hline 4315 & SMPL00804 & $9 / 12 / 94$ & REG & Chloroethane \\
\hline 4315 & S01170 & $10 / 28 / 94$ & REG & Chloroethane \\
\hline 4315 & S03817 & $5 / 10 / 95$ & REG & Chloroethane \\
\hline 4315 & S05061 & $8 / 30 / 95$ & REG & Chloroethane \\
\hline
\end{tabular}

Results Units Qualifier

$\begin{array}{ccc}1 & \mathrm{ug} / \mathrm{L} & \mathrm{U} \\ 1 & \mathrm{ug} / \mathrm{L} & \mathrm{U} \\ 5 & \mathrm{ug} / \mathrm{L} & \mathrm{U} \\ 5 & \mathrm{ug} / \mathrm{L} & \mathrm{U} \\ 5 & \mathrm{ug} / \mathrm{L} & \mathrm{U} \\ 5 & \mathrm{ug} / \mathrm{L} & \mathrm{U} \\ 5 & \mathrm{ug} / \mathrm{L} & \mathrm{U} \\ 1 & \mathrm{ug} / \mathrm{L} & \mathrm{U} \\ 1 & \mathrm{ug} / \mathrm{L} & \mathrm{U} \\ 1 & \mathrm{ug} / \mathrm{L} & \mathrm{U} \\ 10 & \mathrm{ug} / \mathrm{L} & \mathrm{U} \\ 10 & \mathrm{ug} / \mathrm{L} & \mathrm{U} \\ 10 & \mathrm{ug} / \mathrm{L} & \mathrm{U} \\ 10 & \mathrm{ug} / \mathrm{L} & \mathrm{U} \\ 10 & \mathrm{ug} / \mathrm{L} & \mathrm{U} \\ 1 & \mathrm{ug} / \mathrm{L} & \mathrm{U} \\ 1 & \mathrm{ug} / \mathrm{L} & \mathrm{U} \\ 1 & \mathrm{ug} / \mathrm{L} & \mathrm{U} \\ 5 & \mathrm{ug} / \mathrm{L} & \mathrm{U} \\ 5 & \mathrm{ug} / \mathrm{L} & \mathrm{U} \\ 5 & \mathrm{ug} / \mathrm{L} & \mathrm{U} \\ 5 & \mathrm{ug} / \mathrm{L} & \mathrm{U} \\ 5 & \mathrm{ug} / \mathrm{L} & \mathrm{U} \\ 1 & \mathrm{ug} / \mathrm{L} & \mathrm{U} \\ 1 & \mathrm{ug} / \mathrm{L} & \mathrm{U} \\ 1 & \mathrm{ug} / \mathrm{L} & \mathrm{U} \\ 5 & \mathrm{ug} / \mathrm{L} & \mathrm{U} \\ 5 & \mathrm{ug} / \mathrm{L} & \mathrm{U} \\ 5 & \mathrm{ug} / \mathrm{L} & \mathrm{U} \\ 5 & \mathrm{ug} / \mathrm{L} & \mathrm{U} \\ 5 & \mathrm{ug} / \mathrm{L} & \mathrm{U} \\ 1 & \mathrm{ug} / \mathrm{L} & \mathrm{U} \\ 1 & \mathrm{ug} / \mathrm{L} & \mathrm{U} \\ 1 & \mathrm{ug} / \mathrm{L} & \mathrm{U} \\ 5 & \mathrm{ug} / \mathrm{L} & \mathrm{U} \\ 5 & \mathrm{ug} / \mathrm{L} & \mathrm{U} \\ 5 & \mathrm{ug} / \mathrm{L} & \mathrm{U} \\ 1 & \mathrm{ug} / \mathrm{L} & \mathrm{u} \\ 1 & \mathrm{ug} / \mathrm{L} & \mathrm{U} \\ 5 & \mathrm{ug} / \mathrm{L} & \mathrm{U} \\ 5 & \mathrm{ug} / \mathrm{L} & \mathrm{U} \\ 1 & \mathrm{ug} / \mathrm{L} & \mathrm{U} \\ 1 & \mathrm{ug} / \mathrm{L} & \mathrm{U} \\ 1 & \mathrm{ug} / \mathrm{L} & \mathrm{U} \\ 10 & \mathrm{ug} / \mathrm{L} & \mathrm{U} \\ 10 & \mathrm{ug} / \mathrm{L} & \mathrm{U} \\ 10 & \mathrm{ug} / \mathrm{L} & \mathrm{U} \\ 10 & \mathrm{ug} / \mathrm{L} & \mathrm{U} \\ 10 & \mathrm{ug} / \mathrm{L} & \mathrm{U} \\ 1 & & \end{array}$




\begin{tabular}{|c|c|c|c|c|c|c|c|}
\hline Station & Sample ID & Sample Date & Sample Type & Chemical & Results & Units & Qualifier \\
\hline 4315 & M01292 & $11 / 18 / 97$ & REG & Chloroform & 5 & ug/L & $\bar{U}$ \\
\hline+315 & W00898 & $6 / 23 / 97$ & REG & Chloroform & 5 & $\mathrm{ug} / \mathrm{L}$ & $\mathrm{U}$ \\
\hline+315 & M00083 & $11 / 13 / 96$ & REG & Chloroform & 5 & $\mathrm{ug} / \mathrm{L}$ & $U$ \\
\hline 4315 & M01015 & $6 / 23 / 97$ & REG & Chloroform & 5 & ug/L & $\mathrm{U}$ \\
\hline 4315 & SMPL00804 & $9 / 12 / 94$ & REG & Chloroform & 5 & ug/L & $\mathrm{U}$ \\
\hline 4315 & S01170 & $10 / 28 / 94$ & REG & Chloroform & 1 & ug/L & $\mathrm{U}$ \\
\hline 4315 & S03817 & $5 / 10 / 95$ & REG & Chloroform & 1 & $\mathrm{ug} / \mathrm{L}$ & $\mathrm{U}$ \\
\hline 4315 & S05061 & $8 / 30 / 95$ & REG & Chloroform & 1 & $\mathrm{ug} / \mathrm{L}$ & $\mathrm{U}$ \\
\hline 4315 & M01292 & $11 / 18 / 97$ & REG & Chloromethane & 10 & ugh & $\mathrm{U}$ \\
\hline 4315 & W00898 & $6 / 23 / 97$ & REG & Chloromethane & 10 & ug/L & $\mathrm{U}$ \\
\hline 4315 & M00083 & $11 / 13 / 96$ & REG & Chloromethane & 10 & $\mathrm{ug} / \mathrm{L}$ & $\mathrm{U}$ \\
\hline 4315 & M01015 & $6 / 23 / 97$ & REG & Chloromethane & 10 & ug/L & $\mathrm{U}$ \\
\hline 4315 & SMPL00804 & $9 / 12 / 94$ & REG & Chloromethane & 10 & ug/L & $\mathrm{U}$ \\
\hline 4315 & S01170 & $10 / 28 / 94$ & REG & Chloromethane & 1 & ug/L & $\mathrm{U}$ \\
\hline 4315 & S03817 & $5 / 10 / 95$ & REG & Chloromethane & 1 & $\mathrm{ug} / \mathrm{L}$ & $\mathrm{U}$ \\
\hline 4315 & S05061 & $8 / 30 / 95$ & REG & Chloromethane & 1 & $\mathrm{ug} / \mathrm{L}$ & $\mathrm{U}$ \\
\hline 4315 & M01292 & $11 / 18 / 97$ & REG & cis-1,2-Dichloroethene & 5 & ug/L & $\mathbf{U}$ \\
\hline 4315 & M00083 & $11 / 13 / 96$ & REG & cis-1,2-Dichloroethene & 5 & ug/L & $\mathrm{U}$ \\
\hline 4315 & M01015 & $6 / 23 / 97$ & REG & cis-1,2-Dichloroethene & 5 & ug/L & $\mathrm{U}$ \\
\hline 4315 & SMPL00804 & $9 / 12 / 94$ & REG & cis-1,2-Dichloroethene & 5 & ug/L & $\mathrm{U}$ \\
\hline 4315 & S01170 & $10 / 28 / 94$ & REG & cis-1,2-Dichloroethene & 1 & ug/ & $\mathrm{U}$ \\
\hline 4315 & S03817 & $5 / 10 / 95$ & REG & cis-1,2-Dichloroethene & 1 & ug/ & $\mathrm{U}$ \\
\hline 4315 & S05061 & $8 / 30 / 95$ & REG & cis-1.2-Dichloroethene & 1 & ug $\Omega$ & $\mathrm{U}$ \\
\hline 4315 & M01292 & $11 / 18 / 97$ & REG & cis-1,3-Dichloropropen & 5 & $\mathrm{ug} / \mathrm{L}$ & $\mathbf{U}$ \\
\hline 4315 & W00898 & $6 / 23 / 97$ & REG & cis-1,3-Dichloropropene & 5 & $\mathrm{ug} / \mathrm{L}$ & $\mathrm{U}$ \\
\hline 4315 & M00083 & $11 / 13 / 96$ & REG & cis-1,3-Dichloropropene & 5 & ug/L & $\mathrm{U}$ \\
\hline 4315 & M01015 & $6 / 23 / 97$ & REG & cis-1,3-Dichloropropene & 5 & $\mathrm{ug} / \mathrm{L}$ & $\mathrm{U}$ \\
\hline 4315 & SMPL00804 & $9 / 12 / 94$ & REG & cis-1.3-Dichloropropene & 5 & $\mathrm{ug} / \mathrm{L}$ & $\mathrm{U}$ \\
\hline 4315 & S01170 & $10 / 28 / 94$ & REG & cis-1,3-Dichloropropene & 1 & $\mathrm{ug} / \mathrm{L}$ & $\mathrm{U}$ \\
\hline 4315 & S03817 & $5 / 10 / 95$ & REG & cis-1,3-Dichloropropene & 1 & ug/L & $\mathrm{U}$ \\
\hline 4315 & S05061 & $8 / 30 / 95$ & REG & cis-1,3-Dichloropropene & 1 & $\mathrm{ug} / \mathrm{L}$ & $\mathrm{U}$ \\
\hline 4315 & M01292 & $11 / 18 / 97$ & REG & Dibromochloromethane & 5 & ug/ & $\mathrm{U}$ \\
\hline 4315 & W00898 & $6 / 23 / 97$ & REG & Dibromochloromethane & 5 & $\mathrm{ug} / \mathrm{L}$ & $\mathrm{U}$ \\
\hline 4315 & M00083 & $11 / 13 / 96$ & REG & Dibromochloromethane & 5 & ug $/ \mathrm{L}$ & $\mathrm{U}$ \\
\hline 4315 & M01015 & $6 / 23 / 97$ & REG & Dibromochloromethane & 5 & $\mathrm{ug} / \mathrm{L}$ & $\mathrm{U}$ \\
\hline 4315 & .SMPL00804 & $9 / 12 / 94$ & REG & Dibromochloromethane & 5 & $\mathrm{ug} / \mathrm{L}$ & $\mathrm{U}$ \\
\hline 4315 & SOI170 & $10 / 28 / 94$ & REG & Dibromochloromethane & 1 & $\mathrm{ug} / \mathrm{L}$ & $\mathrm{U}$ \\
\hline+315 & S03817 & $5 / 10 / 95$ & REG & Dibromochloromethane & 1 & ug/L & $\mathrm{U}$ \\
\hline+315 & S05061 & $8 / 30 / 95$ & REG & Dibromochloromethane & 1 & ug/L & $\mathrm{U}$ \\
\hline+315 & M00083 & $11 / 13 / 96$ & REG & Dimethylbenzene & 5 & $\mathrm{ug} / \mathrm{L}$ & $\mathrm{U}$ \\
\hline 4315 & SMPL00804 & $9 / 12 / 94$ & REG & Dimethylbenzene & 5 & ug/ & U \\
\hline 4315 & SMPL00804 & $9 / 12 / 94$ & REG & Dimethylbenzene & 5 & ug/L & $\mathrm{U}$ \\
\hline 4315 & SMPL00804 & $9 / 12 / 94$ & REG & Dimethylbenzene & 5 & ug/L & $\mathrm{U}$ \\
\hline 4315 & M01292 & $11 / 18 / 97$ & REG & Ethylbenzene & 5 & ug/ & $\mathrm{U}$ \\
\hline+315 & W00898 & $6 / 23 / 97$ & REG & Ethylbenzene & 5 & ug/L & $\mathrm{U}$ \\
\hline 4315 & M00083 & $11 / 13 / 96$ & REG & Ethylbenzene & 5 & ug/ & $\mathrm{U}$ \\
\hline 4315 & M01015 & $6 / 23 / 97$ & REG & Ethylbenzene & 5 & ug/L & $\mathrm{U}$ \\
\hline 4315 & SMPL00804 & $9 / 12 / 94$ & REG & Ethyllbenzene & 5 & ug/L & $\mathrm{U}$ \\
\hline 4315 & S01170 & $10 / 28 / 94$ & REG & Ethylbenzene & 1 & ug/L & $\mathrm{U}$ \\
\hline+315 & S03817 & $5 / 10 / 95$ & REG & Ethylbenzene & 1 & ug/ & $\mathrm{U}$ \\
\hline
\end{tabular}




\begin{tabular}{|c|c|c|c|c|c|c|c|}
\hline Station & Sample ID & Sample Date & Sample Type & Chemical & Results & Units & Qualifier \\
\hline+315 & S05061 & $8 / 30 / 95$ & REG & Ethylbenzene & 1 & $u g / L$ & $\mathrm{U}$ \\
\hline 4315 & M01292 & $11 / 18 / 97$ & REG & m.p-Xylene & 5 & $\mathrm{ug} / \mathrm{L}$ & $\mathrm{U}$ \\
\hline+315 & W00898 & $6 / 23 / 97$ & REG & m.p-Xylene & 5 & $\mathrm{ug} / \mathrm{L}$ & $\mathrm{U}$ \\
\hline+315 & Mo0083 & $11 / 13 / 96$ & REG & m.p-Xylene & 5 & $\mathrm{ug} / \mathrm{L}$ & $\mathrm{U}$ \\
\hline 4315 & M01015 & $6 / 23 / 97$ & REG & m.p-Xylene & 5 & $u g / L$ & $\mathrm{U}$ \\
\hline+315 & M01292 & $11 / 18 / 97$ & REG & Methylene chloride & 19 & $\mathrm{ug} / \mathrm{L}$ & B \\
\hline 4315 & W00898 & $6 / 23 / 97$ & REG & Methylene chloride & 10 & $\mathrm{ug} / \mathrm{L}$ & $=$ \\
\hline 4315 & M00083 & $11 / 13 / 96$ & REG & Methylene chloride & 5 & $\mathrm{ug} / \mathrm{L}$ & $\mathrm{U}$ \\
\hline 4315 & M01015 & $6 / 23 / 97$ & REG & Methylene chloride & 7 & $\mathrm{ug} / \mathrm{L}$ & $=$ \\
\hline 4315 & SMPL00804 & $9 / 12 / 94$ & REG & Methylene chloride & 5 & $\mathrm{ug} / \mathrm{L}$ & $\mathrm{U}$ \\
\hline 4315 & S01170 & $10 / 28 / 94$ & REG & Methylene chloride & 1.2 & $\mathrm{ug} / \mathrm{L}$ & UJ \\
\hline 4315 & S03817 & $5 / 10 / 95$ & REG & Methylene chloride & 2 & $\mathrm{ug} / \mathrm{L}$ & $\mathbf{U}$ \\
\hline 4315 & S05061 & $8 / 30 / 95$ & REG & Methylene chloride & 2 & ug/L & $\mathrm{U}$ \\
\hline 4315 & M01292 & $11 / 18 / 97$ & REG & Styrene & 5 & $\mathrm{ug} / \mathrm{L}$ & $\mathrm{U}$ \\
\hline 4315 & W00898 & $6 / 23 / 97$ & REG & Styrene & 5 & $\mathrm{ug} / \mathrm{L}$ & $\mathrm{U}$ \\
\hline 4315 & M00083 & $11 / 13 / 96$ & REG & Styrene & 5 & $\mathrm{ug} / \mathrm{L}$ & $\mathrm{U}$ \\
\hline 4315 & M01015 & $6 / 23 / 97$ & REG & Styrene & 5 & $\mathrm{ug} / \mathrm{L}$ & $\mathbf{U}$ \\
\hline 4315 & SMPL00804 & $9 / 12 / 94$ & REG & Styrene & 5 & $\mathrm{ug} / \mathrm{L}$ & $\mathrm{U}$ \\
\hline 4315 & S01170 & $10 / 28 / 94$ & REG & Styrene & 1 & ug/L & $\mathbf{U}$ \\
\hline 4315 & S03817 & $5 / 10 / 95$ & REG & Styrene & 1 & $\mathrm{ug} / \mathrm{L}$ & $\mathrm{U}$ \\
\hline 43.15 & S05061 & $8 / 30 / 95$ & REG & Styrene & 1 & $\mathrm{ug} / \mathrm{L}$ & $\mathrm{U}$ \\
\hline 4315 & M01292 & $11 / 18 / 97$ & REG & Tetrachloroethene & 5 & $\mathrm{ug} / \mathrm{L}$ & $\mathbf{U}$ \\
\hline 4315 & W00898 & $6 / 23 / 97$ & REG & Tetrachloroethene & 5 & ug $/ \mathrm{L}$ & $\mathrm{U}$ \\
\hline 4315 & M00083 & $11 / 13 / 96$ & REG & Tetrachloroethene & 5 & $\mathrm{ug} / \mathrm{L}$ & $\mathrm{U}$ \\
\hline 4315 & M01015 & $6 / 23 / 97$ & REG & Tetrachloroethene & 5 & $\mathrm{ug} / \mathrm{L}$ & $\mathrm{U}$ \\
\hline 4315 & SMPL00804 & $9 / 12 / 94$ & REG & Tetrachloroethene & 5 & $u g / L$ & $\mathrm{U}$ \\
\hline 4315 & S01170 & $10 / 28 / 94$ & REG & Tetrachloroethene & 1 & $\mathrm{ug} / \mathrm{L}$ & $\mathrm{U}$ \\
\hline+315 & S03817 & $5 / 10 / 95$ & REG & Tetrachloroethene & 1 & $\mathrm{ug} / \mathrm{L}$ & $\mathrm{U}$ \\
\hline 4315 & S05061 & $8 / 30 / 95$ & REG & Tetrachloroethene & 1 & $\mathrm{ug} / \mathrm{L}$ & $\mathbf{U}$ \\
\hline 4315 & M01292 & $11 / 18 / 97$ & REG & Toluene & 5 & $\mathrm{ug} / \mathrm{L}$ & $U$ \\
\hline 4315 & W00898 & $6 / 23 / 97$ & REG & Toluene & 5 & $u g / L$ & $\mathrm{U}$ \\
\hline 4315 & M00083 & $11 / 13 / 96$ & REG & Toluene & 5 & $\mathrm{ug} / \mathrm{L}$ & $\mathrm{U}$ \\
\hline 4315 & M01015 & $6 / 23 / 97$ & REG & Toluene & 5 & $\mathrm{ug} / \mathrm{L}$ & $\mathrm{U}$ \\
\hline+315 & SMPL00804 & $9 / 12 / 94$ & REG & Toluene & 5 & $\mathrm{ug} / \mathrm{L}$ & U \\
\hline 4.315 & Sol170 & $10 / 28 / 94$ & REG & Toluene & 1 & $\mathrm{ug} / \mathrm{L}$ & $\mathrm{U}$ \\
\hline 4315 & S03817 & $5 / 10 / 95$ & REG & Toluene & 1 & $\mathrm{ug} / \mathrm{L}$ & U \\
\hline 4315 & S05061 & $8 / 30 / 95$ & REG & Toluene & 1 & $\mathrm{ug} / \mathrm{L}$ & $\mathrm{U}$ \\
\hline 4315 & M01292 & $11 / 18 / 97$ & REG & trans-1.2-Dichloroethe & 5 & $\mathrm{ug} / \mathrm{L}$ & $\mathrm{U}$ \\
\hline+315 & M00083 & $11 / 13 / 96$ & REG & trans-1.2-Dichloroethene & 5 & ug/L & $\mathrm{U}$ \\
\hline 4315 & M01015 & $6 / 23 / 97$ & REG & trans-1.2-Dichloroethene & 5 & $\mathrm{ug} / \mathrm{L}$ & $\mathrm{U}$ \\
\hline 4315 & SMPL00804 & $9 / 12 / 94$ & REG & trans-1.2-Dichloroethene & 5 & ug/L & $U$ \\
\hline 4315 & S01170 & $10 / 28 / 94$ & REG & trans-1.2-Dichloroethene & 1 & ug/L & $\mathrm{U}$ \\
\hline 4315 & S03817 & $5 / 10 / 95$ & REG & trans-1.2-Dichloroethene & 1 & ug/L & $U$ \\
\hline 4315 & S05061 & $8 / 30 / 95$ & REG & trans-1.2-Dichloroethene & 1 & $\mathrm{ug} / \mathrm{L}$ & $\mathrm{U}$ \\
\hline+315 & M01292 & $11 / 18 / 97$ & REG & trans-1,3-Dichloroprop & 5 & $\mathrm{ug} / \mathrm{L}$ & U \\
\hline 4315 & W00898 & $6 / 23 / 97$ & REG & trans-1,3-Dichloropropene & 5 & $\mathrm{ug} / \mathrm{L}$ & $\mathrm{U}$ \\
\hline 4315 & M00083 & $11 / 13 / 96$ & REG & trans-1.3-Dichloropropene & 5 & ug/L & $\mathrm{U}$ \\
\hline 4315 & M01015 & $6 / 23 / 97$ & REG & trans-1.3-Dichloropropene & 5 & ug/L & $\mathrm{U}$ \\
\hline 4315 & SMPL00804 & $9 / 12 / 94$ & REG & trans-1.3-Dichloropropene & 5 & ug/L & $\mathrm{U}$ \\
\hline 4315 & S01170 & $10 / 28 / 94$ & REG & trans-1,3-Dichloropropene & 1 & ug $/ \mathrm{L}$ & $\mathrm{U}$ \\
\hline
\end{tabular}




\begin{tabular}{|c|c|c|c|c|c|c|c|}
\hline Station & Sample ID & Sample Date & Sample Type & Chemical & Results & Units & Qualifier \\
\hline 4315 & 503817 & $5 / 10 / 95$ & REG & trans-1.i-Dichloropropene & 1 & ug/L & $\bar{U}$ \\
\hline 4315 & S05061 & $8 / 30 / 95$ & REG & trans-1.3-Dichloropropene & 1 & $u g / L$ & $\mathrm{U}$ \\
\hline 4315 & $\mathrm{M} 01292$ & $11 / 18 / 97$ & REG & Trichloroethene & 5 & $\mathrm{ug} / \mathrm{L}$ & $\mathrm{U}$ \\
\hline 4315 & Wo()898 & $6 / 23 / 97$ & REG & Trichloroethene & 5 & $\mathrm{ug} / \mathrm{L}$ & $\mathrm{U}$ \\
\hline 4315 & $\mathrm{M} 00083$ & $11 / 13 / 96$ & REG & Trichloroethene & 5 & $\mathrm{ug} / \mathrm{L}$ & $\mathrm{U}$ \\
\hline 4315 & M01015 & $6 / 23 / 97$ & REG & Trichloroethene & 5 & $\mathrm{ug} / \mathrm{L}$ & $\mathrm{U}$ \\
\hline 4315 & SMPL00804 & $9 / 12 / 94$ & REG & Trichloroethene & 5 & $\mathrm{ug} / \mathrm{L}$ & $\mathrm{U}$ \\
\hline 4315 & S01170 & $10 / 28 / 94$ & REG & Trichloroethene & 1 & $\mathrm{ug} / \mathrm{L}$ & $\mathrm{U}$ \\
\hline 4315 & S03817 & $5 / 10 / 95$ & REG & Trichloroethene & 1 & $\mathrm{ug} / \mathrm{L}$ & $\mathrm{U}$ \\
\hline 4315 & S05061 & $8 / 30 / 95$ & REG & Trichloroethene & 1 & $\mathrm{ug} / \mathrm{L}$ & U \\
\hline 4315 & M01292 & $11 / 18 / 97$ & REG & Vinyl acetate & 10 & $\mathrm{ug} / \mathrm{L}$ & $\mathrm{U}$ \\
\hline 4315 & M00083 & $11 / 13 / 96$ & REG & Vinyl acetate & 10 & $\mathrm{ug} / \mathrm{L}$ & $\mathrm{U}$ \\
\hline 4315 & M01015 & $6 / 23 / 97$ & REG & Vinyl acetate & 10 & ug/L & $\mathrm{U}$ \\
\hline 4315 & SMPL00804 & 9/12/94 & REG & Vinyl acetate & 10 & ug/L & $U$ \\
\hline 4315 & M01292 & $11 / 18 / 97$ & REG & Vinyl chloride & 10 & ug/L & $\mathrm{U}$ \\
\hline 4315 & W00898 & $6 / 23 / 97$ & REG & Vinyl chloride & 10 & $\mathrm{ug} / \mathrm{L}$ & $\mathrm{U}$ \\
\hline 4315 & M00083 & $11 / 13 / 96$ & REG & Vinyl chloride & 10 & $\mathrm{ug} / \mathrm{L}$ & $U$ \\
\hline 4315 & M01015 & $6 / 23 / 97$ & REG & Vinyl chloride & 10 & ug/L & $\mathrm{U}$ \\
\hline 4315 & SMPL00804 & $9 / 12 / 94$ & REG & Vinyl chloride & 10 & ug/L & $\mathrm{U}$ \\
\hline 4315 & S01170 & $10 / 28 / 94$ & REG & Vinyl chloride & 1 & ug/L & $\mathrm{U}$ \\
\hline 4315 & S03817 & $5 / 10 / 95$ & REG & Vinyl chloride & 1 & ug/L & $\mathrm{U}$ \\
\hline 4315 & S05061 & $8 / 30 / 95$ & REG & Vinyl chloride & 1 & ug/ & $U$ \\
\hline 4316 & M01298 & $11 / 18 / 97$ & REG & 1.1,1-Trichloroethane & 5 & $\mathrm{ug} / \mathrm{L}$ & $\mathrm{U}$ \\
\hline 4316 & W00900 & $6 / 24 / 97$ & REG & 1.1,1-Trichloroethane & 5 & $\mathrm{ug} / \mathrm{L}$ & $U$ \\
\hline 4316 & M00087 & $11 / 19 / 96$ & REG & 1.1,1-Trichloroethane & 5 & ug $/ \mathrm{L}$ & $\mathrm{U}$ \\
\hline 4316 & M01020 & $6 / 24 / 97$ & REG & 1.1,1-Trichloroethane & 5 & ug/L & $\mathrm{U}$ \\
\hline 4316 & SMPL00810 & 9/13/94 & REG & 1.1.1-Trichloroethane & 5 & $\mathrm{ug} / \mathrm{L}$ & $\mathrm{U}$ \\
\hline 4316 & S01183 & $11 / 3 / 94$ & REG & 1.1,1-Trichloroethane & 1 & $\mathrm{ug} / \mathrm{L}$ & $\mathrm{U}$ \\
\hline 4316 & S02266 & $1 / 12 / 95$ & REG & 1.1,1-Trichloroethane & 1 & $\mathrm{ug} / \mathrm{L}$ & $\mathbf{U}$ \\
\hline 4316 & S03828 & $5 / 8 / 95$ & REG & 1.1,1-Trichloroethane & 1 & $\mathrm{ug} / \mathrm{L}$ & $\mathbf{U}$ \\
\hline 4316 & S05070 & $8 / 30 / 95$ & REG & 1.1,1-Trichloroethane & 1 & $\mathrm{ug} / \mathrm{L}$ & $\mathrm{U}$ \\
\hline 4316 & M01298 & $11 / 18 / 97$ & REG & 1.1.2,2-Tetrachloroeth & 5 & $\mathrm{ug} / \mathrm{L}$ & $\mathrm{U}$ \\
\hline 4316 & W00900 & $6 / 24 / 97$ & REG & 1.1,2,2-Tetrachloroethane & 5 & ug $/ \mathrm{L}$ & $\mathrm{U}$ \\
\hline 4316 & M00087 & $11 / 19 / 96$ & REG & 1.1,2,2-Tetrachloroethane & 5 & $\mathrm{ug} / \mathrm{L}$ & $\mathrm{U}$ \\
\hline 4316 & $\mathrm{M} 01020$ & $6 / 24 / 97$ & REG & 1.1,2,2-Tetrachloroethane & 5 & $\mathrm{ug} / \mathrm{L}$ & $\mathrm{L}$ \\
\hline 4316 & SMPL00810 & 9/13/94 & REG & 1.1,2.2-Tetrachloroethane & 5 & $\mathrm{ug} / \mathrm{L}$ & $\mathrm{U}$ \\
\hline 4316 & S01183 & $11 / 3 / 94$ & REG & 1.1.2,2-Tetrachloroethane & 1 & ug $/ \mathrm{L}$ & $\mathrm{U}$ \\
\hline 4316 & S02266 & $1 / 12 / 95$ & REG & 1.1.2.2-Tetrachloroethane & 1 & $\mathrm{ug} / \mathrm{L}$ & $\mathrm{U}$ \\
\hline 4316 & S03828 & $5 / 8 / 95$ & REG & 1.1.2.2-Tetrachloroethane & 1 & $\mathrm{ug} / \mathrm{L}$ & $\mathrm{U}$ \\
\hline 4316 & S05070 & $8 / 30 / 95$ & REG & 1.1.2.2-Tetrachloroethane & 1 & $\mathrm{ug} / \mathrm{L}$ & $\mathbf{U}$ \\
\hline 4316 & W00900 & $6 / 24 / 97$ & REG & 1.1.2-Trichloro-1.2.2-trifluo & 10 & ug $/$ L & $\mathrm{U}$ \\
\hline 4316 & M01298 & $11 / 18 / 97$ & REG & 1.1,2-Trichloroethane & 5 & $\mathrm{ug} / \mathrm{L}$ & $\mathrm{U}$ \\
\hline 4316 & W00900 & $6 / 24 / 97$ & REG & 1.1.2-Trichloroethane & 5 & $\mathrm{ug} / \mathrm{L}$ & $\mathrm{U}$ \\
\hline 4316 & M00087 & $11 / 19 / 96$ & REG & 1.1,2-Trichloroethane & 5 & $\mathrm{ug} / \mathrm{L}$ & $\mathrm{U}$ \\
\hline 4316 & M01020 & $6 / 24 / 97$ & REG & 1.1,2-Trichloroethane & 5 & ug/ & $\mathrm{U}$ \\
\hline 4316 & SMPL00810 & 9/13/94 & REG & 1.1.2-Trichloroethane & 5 & $\mathrm{ug} / \mathrm{L}$ & $\mathrm{U}$ \\
\hline 4316 & S01183 & $11 / 3 / 94$ & REG & 1,1,2-Trichloroethane & 1 & $\mathrm{ug} / \mathrm{L}$ & $\mathrm{U}$ \\
\hline 4316 & S02266 & $1 / 12 / 95$ & REG & 1.1.2-Trichloroethane & 1 & ug $/$ & $U$ \\
\hline 4316 & S03828 & $5 / 8 / 95$ & REG & 1.1.2-Trichloroethane & 1 & $\mathrm{ug} / \mathrm{L}$ & U \\
\hline 4316 & S05070 & $8 / 30 / 95$ & REG & 1.1.2-Trichloroethane & 1 & ug/L & $\mathrm{U}$ \\
\hline
\end{tabular}


Station Sample ID Sample Date Sample Type Chemical

\begin{tabular}{|c|c|c|c|c|}
\hline & & & & \\
\hline 4316 & M01298 & $11 / 18 / 97$ & REG & 1.1-Dichloroethane \\
\hline+316 & W00900 & $6 / 24 / 97$ & REG & 1.1-Dichloroethane \\
\hline 4316 & M00087 & $11 / 19 / 96$ & REG & 1.1-Dichloroethane \\
\hline 4316 & $\mathrm{M} 01020$ & $6 / 24 / 97$ & REG & 1.1-Dichloroethane \\
\hline 4316 & SMPL00810 & $9 / 13 / 94$ & REG & 1.1-Dichloroethane \\
\hline 4316 & So1183 & $11 / 3 / 94$ & REG & 1.1-Dichloroethane \\
\hline 4316 & S02266 & $1 / 12 / 95$ & REG & 1.1-Dichloroethane \\
\hline 4316 & S03828 & $5 / 8 / 95$ & REG & 1,1-Dichloroethane \\
\hline 4316 & S05070 & $8 / 30 / 95$ & REG & 1.1-Dichloroethane \\
\hline 4316 & M01298 & $11 / 18 / 97$ & REG & 1.1-Dichloroethene \\
\hline 4316 & W00900 & $6 / 24 / 97$ & REG & 1.1-Dichloroethene \\
\hline 4316 & M00087 & $11 / 19 / 96$ & REG & 1.1-Dichloroethene \\
\hline 4316 & M01020 & $6 / 24 / 97$ & REG & 1,1-Dichloroethene \\
\hline 4316 & SMPL00810 & $9 / 13 / 94$ & REG & 1.1-Dichloroethene \\
\hline 4316 & S01183 & $11 / 3 / 94$ & REG & 1.1-Dichloroethene \\
\hline 4316 & S02266 & $1 / 12 / 95$ & REG & 1.1-Dichloroethene \\
\hline 4316 & S03828 & $5 / 8 / 95$ & REG & 1.1-Dichloroethene \\
\hline 4316 & S05070 & $8 / 30 / 95$ & REG & 1,1-Dichloroethene \\
\hline 4316 & S01183 & $11 / 3 / 94$ & REG & 1.2-Dibromo-3-chloropropane \\
\hline 4316 & S02266 & $1 / 12 / 95$ & REG & 1.2-Dibromo-3-chloropropane \\
\hline 4316 & S03828 & $5 / 8 / 95$ & REG & 1.2-Dibromo-3-chloropropane \\
\hline 4316 & S05070 & $8 / 30 / 95$ & REG & 1.2-Dibromo-3-chloropropane \\
\hline 4316 & S01183 & $11 / 3 / 94$ & REG & 1,2-Dibromoethane \\
\hline 4316 & S02266 & $1 / 12 / 95$ & REG & 1.2-Dibromoethane \\
\hline 4316 & S03828 & $5 / 8 / 95$ & REG & 1.2-Dibromoethane \\
\hline 4316 & S05070 & $8 / 30 / 95$ & REG & 1.2-Dibromoethane \\
\hline 4316 & M00087 & $11 / 19 / 96$ & REG & 1.2-Dichlorobenzene \\
\hline 4316 & SMPL00810 & $9 / 13 / 94$ & REG & 1.2-Dichlorobenzene \\
\hline 4316 & M01298 & $11 / 18 / 97$ & REG & 1,2-Dichloroethane \\
\hline 4316 & W00900 & $6 / 24 / 97$ & REG & 1,2-Dichloroethane \\
\hline 4316 & M00087 & $11 / 19 / 96$ & REG & 1.2-Dichloroethane \\
\hline+316 & M01020 & $6 / 24 / 97$ & REG & 1.2-Dichloroethane \\
\hline 4316 & SMPL00810 & $9 / 13 / 94$ & REG & 1,2-Dichloroethane \\
\hline 4316 & S01183 & $11 / 3 / 94$ & REG & 1.2-Dichloroethane \\
\hline 4316 & S02266 & $1 / 12 / 95$ & REG & 1.2-Dichloroethane \\
\hline 4316 & S03828 & $5 / 8 / 95$ & REG & 1.2-Dichloroethane \\
\hline 4316 & S05070 & $8 / 30 / 95$ & REG & 1.2-Dichloroethane \\
\hline 4316 & W00900 & $6 / 24 / 97$ & REG & 1.2-Dichloroethene \\
\hline 4316 & M00087 & $11 / 19 / 96$ & REG & 1.2-Dichloroethene \\
\hline+316 & SMPL00810 & $9 / 13 / 94$ & REG & 1.2-Dichloroethene \\
\hline 4316 & M01298 & $11 / 18 / 97$ & REG & 1.2-Dichloropropane \\
\hline 4316 & W00900 & $6 / 24 / 97$ & REG & 1.2-Dichloropropane \\
\hline+316 & M00087 & $11 / 19 / 96$ & REG & 1.2-Dichloropropane \\
\hline 4316 & M01020 & $6 / 24 / 97$ & REG & 1,2-Dichloropropane \\
\hline 4316 & SMPL00810 & $9 / 13 / 94$ & REG & 1.2-Dichloropropane \\
\hline+316 & S01183 & $11 / 3 / 94$ & REG & 1,2-Dichloropropane \\
\hline+316 & S02266 & $1 / 12 / 95$ & REG & 1.2-Dichloropropane \\
\hline 4316 & S03828 & $5 / 8 / 95$ & REG & 1.2-Dichloropropane \\
\hline+316 & S05070 & $8 / 30 / 95$ & REG & 1.2-Dichloropropane \\
\hline 4316 & M00087 & $11 / 19 / 96$ & REG & 1.2-Dimethỵlbenzene \\
\hline
\end{tabular}

Results Units Qualifier

$\begin{array}{lll}5 & \text { ug } \Omega & U \\ 5 & \text { ug } \Omega & U\end{array}$

5 ug $/$ U

5 ug/ U

5 ugl U

$1 \mathrm{ug} / \mathrm{U}$

1 ug $\Omega$ U

1 ug/ U

1 ug $\mathrm{U}$

5 ug $\Omega$ U

5 ug $/$ U

5 ug/ U

5 ug $/ \quad U$

5 ug/ U

$1 \mathrm{ug} / \mathrm{U}$

$1 \mathrm{ug} / \mathrm{U}$

1 ug/ U

$1 \mathrm{ug} / \mathrm{U}$

$1 \mathrm{ug} / \mathrm{L} U$

1 ug/ $\mathrm{U}$

1 ug/L U

1 ug/ $U$

1 ug L U

1 ug/ $\mathrm{U}$

1 ug/ U

1 ug/ $\mathrm{U}$

5 ug $\mathrm{L} \quad \mathrm{U}$

5 ug/ U

5 ug/ U

5 ug/ U

5 ug/ U

5 ug/ $\mathrm{U}$

5 ug/ $\mathrm{U}$

I ug/ $\mathrm{U}$

$0.63 \mathrm{ug} / \mathrm{L} \mathrm{J}$

1 ug/ $\mathrm{U}$

$0.62 \mathrm{ug} / \mathrm{L} \quad \mathrm{J}$

5 ug/ U

5 ug/ U

5 ug/ U

5 ug/L U

5 ug/ $\quad \mathrm{U}$

5 ug/ U

5 ug/ U

$5 \mathrm{ug} / \mathrm{L} U$

$1 \mathrm{ug} / \mathrm{L} U$

I ug/ $\mathrm{U}$

I ug/L U

1 ug/ $U$

5 ug/L U 


\begin{tabular}{|c|c|c|c|c|c|c|c|}
\hline Station & Sample ID & Sample Date & Sample Type & Chemical & Results & Units & Qualifier \\
\hline$\overline{4316}$ & M00087 & $11 / 19 / 96$ & REG & 1.3-Dichlorobenzene & 5 & $\mathrm{ug} / \mathrm{L}$ & $\bar{U}$ \\
\hline+316 & SMPL008 10 & $9 / 13 / 94$ & REG & 1.3-Dichlorobenzene & 5 & $\mathrm{ug} / \mathrm{L}$ & $\mathrm{U}$ \\
\hline 4316 & M00087 & $11 / 19 / 96$ & REG & 1.4-Dichlorobenzene & 5 & $\mathrm{ug} / \mathrm{L}$ & U \\
\hline 4316 & SMPL00810 & 9/13/94 & REG & 1.4-Dichlorobenzene & 5 & $\mathrm{ug} / \mathrm{L}$ & U \\
\hline 4316 & M01298 & $11 / 18 / 97$ & REG & 2-Butanone & 10 & $\mathrm{ug} / \mathrm{L}$ & $\mathrm{U}$ \\
\hline 4316 & W00900 & $6 / 24 / 97$ & REG & 2-Butanone & 10 & $\mathrm{ug} / \mathrm{L}$ & U \\
\hline 4316 & M00087 & $11 / 19 / 96$ & REG & 2-Butanone & 3 & $\mathrm{ug} / \mathrm{L}$ & $=$ \\
\hline 4316 & M01020 & $6 / 24 / 97$ & REG & 2-Butanone & 10 & $\mathrm{ug} / \mathrm{L}$ & U \\
\hline 4316 & SMPL00810 & $9 / 13 / 94$ & REG & 2-Butanone & 10 & $\mathrm{ug} / \mathrm{L}$ & U \\
\hline 4316 & S01183 & $11 / 3 / 94$ & REG & 2-Butanone & 5 & $\mathrm{ug} / \mathrm{L}$ & $\mathrm{U}$ \\
\hline 4316 & S02266 & $1 / 12 / 95$ & REG & 2-Butanone & 5 & $\mathrm{ug} / \mathrm{L}$ & J \\
\hline 4316 & S03828 & $5 / 8 / 95$ & REG & 2-Butanone & 5 & ug/L & $U$ \\
\hline 4316 & S05070 & $8 / 30 / 95$ & REG & 2-Butanone & 5 & $\mathrm{ug} / \mathrm{L}$ & $\mathrm{U}$ \\
\hline 4316 & M01298 & $11 / 18 / 97$ & REG & 2-Hexanone & 10 & ug/L & $\mathrm{U}$ \\
\hline 4316 & W00900 & $6 / 24 / 97$ & REG & 2-Hexanone & 10 & ug/L & $\mathrm{U}$ \\
\hline 4316 & M00087 & $11 / 19 / 96$ & REG & 2-Hexanone & 10 & ug/L & $\mathrm{U}$ \\
\hline 4316 & M01020 & $6 / 24 / 97$ & REG & 2-Hexanone & 10 & ug/L & U \\
\hline 4316 & SMPL00810 & $9 / 13 / 94$ & REG & 2-Hexanone & 10 & $\mathrm{ug} / \mathrm{L}$ & $\mathrm{U}$ \\
\hline 4316 & S01183 & $11 / 3 / 94$ & REG & 2-Hexanone & 5 & $\mathrm{ug} / \mathrm{L}$ & $\mathrm{U}$ \\
\hline 4316 & S02266 & $1 / 12 / 95$ & REG & 2-Hexanone & 5 & $\mathrm{ug} / \mathrm{L}$ & $\mathrm{U}$ \\
\hline 4316 & S03828 & $5 / 8 / 95$ & REG & 2-Hexanone & 5 & ug/L & $\mathrm{U}$ \\
\hline 4316 & S05070 & $8 / 30 / 95$ & REG & 2-Hexanone & 5 & $\mathrm{ug} / \mathrm{L}$ & $\mathrm{U}$ \\
\hline 4316 & M01298 & $11 / 18 / 97$ & REG & 4-Methyl-2-pentanone & 10 & $\mathrm{ug} / \mathrm{L}$ & U \\
\hline 4316 & W00900 & $6 / 24 / 97$ & REG & 4-Methil-2-pentanone & 10 & $\mathrm{ug} / \mathrm{L}$ & U \\
\hline 4316 & M00087 & $11 / 19 / 96$ & REG & 4-Methyl-2-pentanone & 10 & ug/L & U \\
\hline 4316 & Mo1020 & $6 / 24 / 97$ & REG & 4-Methyl-2-pentanone & 10 & $\mathrm{ug} / \mathrm{L}$ & U \\
\hline 4316 & SMPL008 10 & $9 / 13 / 94$ & REG & 4-Methyl-2-pentanone & 10 & $\mathrm{ug} / \mathrm{L}$ & $\mathrm{U}$ \\
\hline 4316 & S01183 & $11 / 3 / 94$ & REG & +-Methyl-2-pentanone & 5 & $\mathrm{ug} / \mathrm{L}$ & $\mathrm{U}$ \\
\hline 4316 & S02266 & $1 / 12 / 95$ & REG & +-Methyl-2-pentanone & 5 & $\mathrm{ug} / \mathrm{L}$ & $\mathrm{U}$ \\
\hline 4316 & S03828 & $5 / 8 / 95$ & REG & 4-Methyl-2-pentanone & 5 & ug/L & $\mathrm{U}$ \\
\hline 4316 & S05070 & $8 / 30 / 95$ & REG & 4-Methyl-2-pentanone & 5 & $\mathrm{ug} / \mathrm{L}$ & $\mathrm{U}$ \\
\hline 4316 & M01298 & $11 / 18 / 97$ & REG & Acetone & 10 & $u g / L$ & U \\
\hline 4316 & W00900 & $6 / 24 / 97$ & REG & Acetone & 10 & $\mathrm{ug} / \mathrm{L}$ & $\mathrm{U}$ \\
\hline 4316 & M00087 & $11 / 19 / 96$ & REG & Acetone & 2 & $\operatorname{ug} / \mathrm{L}$ & $=$ \\
\hline 4316 & M01020 & $6 / 24 / 97$ & REG & Acetone & 10 & $\mathrm{ug} / \mathrm{L}$ & $\mathrm{U}$ \\
\hline+316 & SMPL00810 & $9 / 13 / 94$ & REG & Acetone & 10 & $\mathrm{ug} / \mathrm{L}$ & U \\
\hline 4316 & S01183 & $11 / 3 / 94$ & REG & Acetone & 5 & $\mathrm{ug} / \mathrm{L}$ & $\mathrm{U}$ \\
\hline 4316 & S02266 & $1 / 12 / 95$ & REG & Acetone & 5 & $\mathrm{ug} / \mathrm{L}$ & $\mathrm{U}$ \\
\hline 4316 & S03828 & $5 / 8 / 95$ & REG & Acetone & 5.7 & ug/L & $=$ \\
\hline 4316 & S05070 & $8 / 30 / 95$ & REG & Acetone & 5 & $\mathrm{ug} / \mathrm{L}$ & $\mathrm{U}$ \\
\hline 4316 & M01298 & $11 / 18 / 97$ & REG & Benzene & 5 & $\mathrm{ug} / \mathrm{L}$ & $\mathrm{U}$ \\
\hline 4316 & W00900 & $6 / 24 / 97$ & REG & Benzene & 5 & ug/L & $\mathrm{U}$ \\
\hline 4316 & M00087 & $11 / 19 / 96$ & REG & Benzene & 5 & ug/ & $\mathrm{U}$ \\
\hline 4316 & M01020 & $6 / 24 / 97$ & REG & Benzene & 5 & ug/ & U \\
\hline 4316 & SMPL00810 & 9/13/94 & REG & Benzene & 5 & $\mathrm{ug} / \mathrm{L}$ & U \\
\hline 4316 & S01183 & $11 / 3 / 94$ & REG & Benzene & 1 & ug/L & $U$ \\
\hline 4316 & S02266 & $1 / 12 / 95$ & REG & Benzene & 1 & $\mathrm{ug} / \mathrm{L}$ & $\mathrm{U}$ \\
\hline 4316 & S03828 & $5 / 8 / 95$ & REG & Benzene & 1 & $u g / L$ & $\mathrm{U}$ \\
\hline 4316 & S05070 & $8 / 30 / 95$ & REG & Benzene & 1 & $\mathrm{ug} / \mathrm{L}$ & $\mathrm{U}$ \\
\hline 4316 & S01183 & $11 / 3 / 94$ & REG & Bromochloromethane & 1 & $u g / L$ & $\mathrm{U}$ \\
\hline
\end{tabular}


Station Sample ID Sample Date Sample Type Chemical

\begin{tabular}{|c|c|c|c|c|}
\hline 4316 & S02266 & $1 / 12 / 95$ & REG & Bromochloromethane \\
\hline 4.316 & S03828 & $5 / 8 / 95$ & REG & Bromochloromethane \\
\hline 4316 & S05070 & $8 / 30 / 95$ & REG & Bromochloromethane \\
\hline 4316 & M01298 & $11 / 18 / 97$ & REG & Bromodichloromethane \\
\hline 4316 & W00900 & $6 / 24 / 97$ & REG & Bromodichloromethane \\
\hline 4316 & M00087 & $11 / 19 / 96$ & REG & Bromodichloromethane \\
\hline 4316 & M01020 & $6 / 24 / 97$ & REG & Bromodichloromethane \\
\hline 4316 & SMPL00810 & $9 / 13 / 94$ & REG & Bromodichloromethane \\
\hline 4316 & S01183 & $11 / 3 / 94$ & REG & Bromodichloromethane \\
\hline 4316 & S02266 & $1 / 12 / 95$ & REG & Bromodichloromethane \\
\hline 4316 & S03828 & $5 / 8 / 95$ & REG & Bromodichloromethane \\
\hline 4316 & S05070 & $8 / 30 / 95$ & REG & Bromodichloromethane \\
\hline 4316 & M01298 & $11 / 18 / 97$ & REG & Bromoform \\
\hline 4316 & W00900 & $6 / 24 / 97$ & REG & Bromoform \\
\hline 4316 & M00087 & $11 / 19 / 96$ & REG & Bromoform \\
\hline 4316 & M01020 & $6 / 24 / 97$ & REG & Bromoform \\
\hline 4316 & SMPL00810 & $9 / 13 / 94$ & REG & Bromoform \\
\hline 4316 & S01183 & $11 / 3 / 94$ & REG & Bromoform \\
\hline 4316 & S02266 & $1 / 12 / 95$ & REG & Bromoform \\
\hline 4316 & S03828 & $5 / 8 / 95$ & REG & Bromoform \\
\hline 4316 & S05070 & $8 / 30 / 95$ & REG & Bromoform \\
\hline 4316 & M01298 & $11 / 18 / 97$ & REG & Bromomethane \\
\hline 4316 & W00900 & $6 / 24 / 97$ & REG & Bromomethane \\
\hline 4316 & M00087 & $11 / 19 / 96$ & REG & Bromomethane \\
\hline 4316 & M01020 & $6 / 24 / 97$ & REG & Bromomethane \\
\hline 4316 & SMPL00810 & $9 / 13 / 94$ & REG & Bromomethane \\
\hline 4316 & S01183 & $11 / 3 / 94$ & REG & Bromomethane \\
\hline 4316 & S02266 & $1 / 12 / 95$ & REG & Bromomethane \\
\hline 4316 & S03828 & $5 / 8 / 95$ & REG & Bromomethane \\
\hline 4316 & S05070 & $8 / 30 / 95$ & REG & Bromomethane \\
\hline 4316 & M01298 & $11 / 18 / 97$ & REG & Carbon disulfide \\
\hline 4316 & W00900 & $6 / 24 / 97$ & REG & Carbon disulfide \\
\hline 4316 & M00087 & $11 / 19 / 96$ & REG & Carbon disulfide \\
\hline 4316 & M01020 & $6 / 24 / 97$ & REG & Carbon disulfide \\
\hline 4316 & SMPL00810 & $9 / 13 / 94$ & REG & Carbon disulfide \\
\hline 4316 & S01 183 & $11 / 3 / 94$ & REG & Carbon disulfide \\
\hline 4316 & S02266 & $1 / 12 / 95$ & REG & Carbon disulfide \\
\hline 4316 & S03828 & $5 / 8 / 95$ & REG & Carbon disulfide \\
\hline 4316 & S05070 & $8 / 30 / 95$ & REG & Carbon disulfide \\
\hline 4316 & M01298 & $11 / 18 / 97$ & REG & Carbon tetrachloride \\
\hline 4316 & W00900 & $6 / 24 / 97$ & REG & Carbon tetrachloride \\
\hline 4316 & M00087 & $11 / 19 / 96$ & REG & Carbon tetrachloride \\
\hline+316 & M01020 & $6 / 24 / 97$ & REG & Carbon tetrachloride \\
\hline 4316 & SMPL00810 & $9 / 13 / 94$ & REG & Carbon tetrachloride \\
\hline 4316 & S01183 & $11 / 3 / 94$ & REG & Carbon tetrachloride \\
\hline 4316 & S02266 & $1 / 12 / 95$ & REG & Carbon tetrachloride \\
\hline 4316 & S03828 & $5 / 8 / 95$ & REG & Carbon tetrachloride \\
\hline 4316 & S05070 & $8 / 30 / 95$ & REG & Carbon tetrachloride \\
\hline 4316 & M01298 & $11 / 18 / 97$ & REG & Chlorobenzene \\
\hline 4316 & W00900 & $6 / 24 / 97$ & REG & Chlorobenzene \\
\hline
\end{tabular}

Results Units Qualifier 
B-101

Station Sample ID Sample Date Sample Type Chemical

\begin{tabular}{|c|c|c|c|c|c|c|c|}
\hline 4316 & M00087 & $11 / 19 / 96$ & REG & Chlorobenzene & 5 & $\mathrm{ug} / \mathrm{L}$ & $\bar{U}$ \\
\hline 4316 & M01020 & $6 / 24 / 97$ & REG & Chlorobenzene & 5 & $u g / L$ & $U$ \\
\hline+316 & SMPL00810 & $9 / 13 / 94$ & REG & Chlorobenzene & 5 & $\mathrm{ug} / \mathrm{L}$ & $U$ \\
\hline 4316 & S01183 & $11 / 3 / 94$ & REG & Chlorobenzene & 1 & ug $/$ & $\mathrm{U}$ \\
\hline 4316 & S02266 & $1 / 12 / 95$ & REG & Chlorobenzene & 1 & ug $/$ & $\mathrm{U}$ \\
\hline 4316 & S03828 & $5 / 8 / 95$ & REG & Chlorobenzene & 1 & $\mathrm{ug} / \mathrm{L}$ & $U$ \\
\hline 4316 & S05070 & $8 / 30 / 95$ & REG & Chlorobenzene & 1 & ug $/$ & $\mathrm{U}$ \\
\hline 4316 & M01298 & $11 / 18 / 97$ & REG & Chloroethane & 10 & $\mathrm{ug} / \mathrm{L}$ & $\mathrm{U}$ \\
\hline 4316 & W00900 & $6 / 24 / 97$ & REG & Chloroethane & 10 & $\mathrm{ug} / \mathrm{L}$ & $\mathrm{U}$ \\
\hline 4316 & M00087 & $11 / 19 / 96$ & REG & Chloroethane & 10 & ug $/ \mathrm{L}$ & $\mathrm{U}$ \\
\hline 4316 & M01020 & $6 / 24 / 97$ & REG & Chloroethane & 10 & $\mathrm{ug} / \mathrm{L}$ & $\mathrm{U}$ \\
\hline 4316 & SMPL00810 & $9 / 13 / 94$ & REG & Chloroethane & 10 & $u g / L$ & $\mathrm{U}$ \\
\hline 4316 & Sol183 & $11 / 3 / 94$ & REG & Chloroethane & 1 & ug/L & $\mathrm{U}$ \\
\hline 4316 & S02266 & $1 / 12 / 95$ & REG & Chloroethane & 1 & ug $/ \mathrm{L}$ & $\mathrm{U}$ \\
\hline 4316 & S03828 & $5 / 8 / 95$ & REG & Chloroethane & 1 & ug $/$ & $\mathrm{U}$ \\
\hline 4316 & S05070 & $8 / 30 / 95$ & REG & Chloroethane & 1 & $\mathrm{ug} / \mathrm{L}$ & $\mathrm{U}$ \\
\hline 4316 & M01298 & $11 / 18 / 97$ & REG & Chloroform & 5 & $\mathrm{ug} / \mathrm{L}$ & $\mathrm{U}$ \\
\hline 4316 & W00900 & $6 / 24 / 97$ & REG & Chloroform & 5 & ug $/ \mathrm{L}$ & $\mathrm{U}$ \\
\hline 4316 & M00087 & $11 / 19 / 96$ & REG & Chloroform & 5 & ug $/ \mathrm{L}$ & $\mathrm{U}$ \\
\hline 4316 & M01020 & $6 / 24 / 97$ & REG & Chloroform & 5 & $\mathrm{ug} / \mathrm{L}$ & $\mathrm{U}$ \\
\hline 4316 & SMPL00810 & $9 / 13 / 94$ & REG & Chloroform & 3 & ug $/ \mathrm{L}$ & $\mathrm{J}$ \\
\hline 4316 & S01183 & $11 / 3 / 94$ & REG & Chloroform & 1.2 & $\mathrm{ug} / \mathrm{L}$ & $\mathrm{U}$ \\
\hline 4316 & S02266 & $1 / 12 / 95$ & REG & Chloroform & 0.68 & ug $/ \mathrm{L}$ & $\mathrm{J}$ \\
\hline 4316 & S03828 & $5 / 8 / 95$ & REG & Chioroform & 1 & $\mathrm{ug} / \mathrm{L}$ & $\mathrm{U}$ \\
\hline 4316 & S05070 & $8 / 30 / 95$ & REG & Chloroform & 1 & ug $/ \mathrm{L}$ & $\mathrm{U}$ \\
\hline 4316 & M01298 & $11 / 18 / 97$ & REG & Chloromethane & 10 & ug $/ L$ & $\mathrm{U}$ \\
\hline 4316 & W00900 & $6 / 24 / 97$ & REG & Chloromethane & 10 & ug $/ \mathrm{L}$ & $\mathrm{U}$ \\
\hline 4316 & M00087 & $11 / 19 / 96$ & REG & Chioromethane & 10 & $\mathrm{ug} / \mathrm{L}$ & $\mathrm{U}$ \\
\hline 4316 & M01020 & $6 / 24 / 97$ & REG & Chloromethane & 10 & ug/L & $\mathrm{U}$ \\
\hline 4316 & SMPL00810 & 9/13/94 & REG & Chloromethane & 10 & $\mathrm{ug} / \mathrm{L}$ & $\mathrm{U}$ \\
\hline 4316 & S01183 & $11 / 3 / 94$ & REG & Chloromethane & 1 & $\mathrm{ug} / \mathrm{L}$ & $\mathrm{U}$ \\
\hline 4316 & S02266 & $1 / 12 / 95$ & REG & Chloromethane & 1 & ug/L & $\mathrm{U}$ \\
\hline 4316 & S03828 & $5 / 8 / 95$ & REG & Chloromethane & 3.7 & ug/ $/$ & $=$ \\
\hline 4316 & $\mathrm{~S} 05070$ & $8 / 30 / 95$ & REG & Chloromethane & 1 & $u g / \mathrm{L}$ & $\mathrm{U}$ \\
\hline 4316 & $\mathrm{M} 01298$ & $11 / 18 / 97$ & REG & cis-1.2-Dichloroethene & 5 & $\mathrm{ug} / \mathrm{L}$ & $\mathrm{U}$ \\
\hline 4316 & M00087 & $11 / 19 / 96$ & REG & cis-1.2-Dichloroethene & 5 & $\mathrm{ug} / \mathrm{L}$ & $\mathrm{U}$ \\
\hline 4316 & M01020 & $6 / 24 / 97$ & REG & cis-1.2-Dichloroethene & 5 & $\mathrm{ug} / \mathrm{L}$ & $\mathrm{U}$ \\
\hline 4316 & SMPL00810 & $9 / 13 / 94$ & REG & cis-1.2-Dichloroethene & 5 & $\mathrm{ug} / \mathrm{L}$ & $\mathrm{U}$ \\
\hline 4316 & S01183 & $11 / 3 / 94$ & REG & cis-1.2-Dichloroethene & 1 & ug $/ \mathrm{L}$ & $\mathrm{U}$ \\
\hline 4316 & S02266 & $1 / 12 / 95$ & REG & cis-1.2-Dichloroethene & 1 & $\mathrm{ug} / \mathrm{L}$ & $\mathrm{U}$ \\
\hline 4316 & S03828 & $5 / 8 / 95$ & REG & cis-1.2-Dichloroethene & 1 & $\mathrm{ug} / \mathrm{L}$ & $\mathrm{U}$ \\
\hline 4316 & S05070 & $8 / 30 / 95$ & REG & cis-1.2-Dichloroethene & 1 & $\mathrm{ug} / \mathrm{L}$ & $\mathrm{U}$ \\
\hline 4316 & M01298 & $11 / 18 / 97$ & REG & cis-1.3-Dichloropropen & 5 & $\mathrm{ug} / \mathrm{L}$ & $\mathrm{U}$ \\
\hline+316 & W00900 & $6 / 24 / 97$ & REG & cis-1,3-Dichloropropene & 5 & $\mathrm{ug} / \mathrm{L}$ & $\mathrm{U}$ \\
\hline 4316 & M00087 & $11 / 19 / 96$ & REG & cis-1.3-Dichloropropene & 5 & $\mathrm{ug} / \mathrm{L}$ & $\mathrm{U}$ \\
\hline 4316 & M01020 & $6 / 24 / 97$ & REG & cis-1,3-Dichloropropene & 5 & ug $/ \mathrm{L}$ & $\mathrm{U}$ \\
\hline 4316 & SMPL00810 & $9 / 13 / 94$ & REG & cis-1.3-Dichloropropene & 5 & ug/ $/$ & $\mathrm{U}$ \\
\hline+316 & S01183 & $11 / 3 / 94$ & REG & cis-1.3-Dichloropropene & 1 & $\mathrm{ug} / \mathrm{L}$ & $\mathrm{U}$ \\
\hline 4316 & S02266 & $1 / 12 / 95$ & REG & cis-1,3-Dichloropropene & 1 & ug $\Omega$ & $\mathrm{U}$ \\
\hline 4316 & S03828 & $5 / 8 / 95$ & REG & cis-1.3-Dichloropropene & 1 & ug $/ \mathrm{L}$ & $\mathrm{U}$ \\
\hline
\end{tabular}


B-102

Station Sample ID Sample Date Sample Type Chemical

\begin{tabular}{|c|c|c|c|c|c|c|c|}
\hline $4+316$ & 505070 & $8 / 30 / 95$ & REG & cis-1.3-Dichloropropene & 1 & ug $/ \mathrm{L}$ & $\mathrm{U}$ \\
\hline+316 & M01298 & $11 / 18 / 97$ & REG & Dibromochloromethane & 5 & $\mathrm{ug} / \mathrm{L}$ & $\mathrm{U}$ \\
\hline+316 & W00900 & $6 / 24 / 97$ & REG & Dibromochloromethane & 5 & ug $/ \mathrm{L}$ & $\mathrm{U}$ \\
\hline+316 & M00087 & $11 / 19 / 96$ & REG & Dibromochloromethane & 5 & $\mathrm{ug} / \mathrm{L}$ & $\mathrm{U}$ \\
\hline 4316 & M01020 & $6 / 24 / 97$ & REG & Dibromochloromethane & 5 & $u g / L$ & $\mathrm{U}$ \\
\hline 4316 & SMPL00810 & 9/13/94 & REG & Dibromochloromethane & 5 & ug/L & $\mathrm{U}$ \\
\hline 4316 & S01183 & $11 / 3 / 94$ & REG & Dibromochloromethane & 1 & ug $/ \mathrm{L}$ & $\mathrm{U}$ \\
\hline 4316 & S02266 & $1 / 12 / 95$ & REG & Dibromochloromethane & 1 & ug $/ \mathrm{L}$ & $\mathrm{U}$ \\
\hline 4316 & S03828 & $5 / 8 / 95$ & REG & Dibromochloromethane & 1 & ug $/$ & $\mathrm{U}$ \\
\hline 4316 & S05070 & $8 / 30 / 95$ & REG & Dibromochloromethane & 1 & ug $\Omega$ & $\mathrm{U}$ \\
\hline 4316 & M00087 & $11 / 19 / 96$ & REG & Dimethvlbenzene & 5 & $\mathrm{ug} / \mathrm{L}$ & $\mathrm{U}$ \\
\hline 4316 & SMPL00810 & $9 / 13 / 94$ & REG & Dimethylbenzene & 5 & $\mathrm{ug} / \mathrm{L}$ & $\mathrm{U}$ \\
\hline 4316 & SMPL00810 & $9 / 13 / 94$ & REG & Dimethylbenzene & 5 & ug/L & $\mathrm{U}$ \\
\hline 4316 & SMPL00810 & $9 / 13 / 94$ & REG & Dimethylbenzene & 5 & ug/L & $\mathrm{U}$ \\
\hline 4316 & M01298 & $11 / 18 / 97$ & REG & Ethylbenzene & 5 & ug/L & $\mathrm{U}$ \\
\hline 4316 & W00900 & $6 / 24 / 97$ & REG & Ethylbenzene & 5 & ug/L & $\mathrm{U}$ \\
\hline 4316 & M00087 & $11 / 19 / 96$ & REG & Ethylbenzene & 5 & ug/L & U \\
\hline 4316 & M01020 & $6 / 24 / 97$ & REG & Ethylbenzene & 5 & ug $/$ & $\mathrm{U}$ \\
\hline 4316 & SMPL00810 & $9 / 13 / 94$ & REG & Ethylbenzene & 5 & $\mathrm{ug} / \mathrm{L}$ & $\mathrm{U}$ \\
\hline 4316 & S01183 & $11 / 3 / 94$ & REG & Ethylbenzene & 1 & $\mathrm{ug} / \mathrm{L}$ & U \\
\hline 4316 & S02266 & $1 / 12 / 95$ & REG & Ethylbenzene & 1 & $\mathrm{ug} / \mathrm{L}$ & $\mathrm{U}$ \\
\hline 4316 & S03828 & $5 / 8 / 95$ & REG & Ethylbenzene & 1 & $\mathrm{ug} / \mathrm{L}$ & U \\
\hline 4316 & S05070 & $8 / 30 / 95$ & REG & Ethylbenzene & 1 & $\mathrm{ug} / \mathrm{L}$ & $\mathrm{U}$ \\
\hline 4316 & M01298 & $11 / 18 / 97$ & REG & m.p-Xylene & 5 & $\mathrm{ug} / \mathrm{L}$ & $\mathrm{U}$ \\
\hline 4316 & W00900 & $6 / 24 / 97$ & REG & m,p-Xylene & 5 & ug/ & $\mathrm{U}$ \\
\hline 4316 & M00087 & $11 / 19 / 96$ & REG & m.p-Xylene & 5 & ug/ & $\mathrm{U}$ \\
\hline 4316 & M01020 & $6 / 24 / 97$ & REG & m.p-Xylene & 5 & ug/L & $U$ \\
\hline 4316 & M01298 & $11 / 18 / 97$ & REG & Methylene chloride & 19 & ug/L & $\mathrm{B}$ \\
\hline 4316 & W00900 & $6 / 24 / 97$ & REG & Methylene chloride & 10 & ug/L & $=$ \\
\hline 4316 & M00087 & $11 / 19 / 96$ & REG & Methylene chloride & 5 & ug/L & $\mathrm{U}$ \\
\hline 4316 & M01020 & $6 / 24 / 97$ & REG & Methylene chloride & 5 & $\mathrm{ug} / \mathrm{L}$ & $\mathbf{U}$ \\
\hline+316 & SMPL00810 & 9/13/94 & REG & Methylene chloride & 5 & $\mathrm{ug} / \mathrm{L}$ & $\mathrm{U}$ \\
\hline 4316 & S01183 & $11 / 3 / 94$ & REG & Methylene chloride & 2 & $\mathrm{ug} / \mathrm{L}$ & UJ \\
\hline 4316 & S02266 & $1 / 12 / 95$ & REG & Methylene chloride & 2 & ug/L & $\mathbf{U}$ \\
\hline 4.316 & S03828 & $5 / 8 / 95$ & REG & Methylene chloride & 2 & $\mathrm{ug} / \mathrm{L}$ & $\mathrm{U}$ \\
\hline 4316 & S05070 & $8 / 30 / 95$ & REG & Methylene chloride & 2 & ug/L & $\mathrm{U}$ \\
\hline+316 & M01298 & $11 / 18 / 97$ & REG & Styrene & 5 & ug/ & $\mathrm{U}$ \\
\hline 4316 & W00900 & $6 / 24 / 97$ & REG & Sțrene & 5 & ug/L & $\mathrm{U}$ \\
\hline 4316 & M00087 & $11 / 19 / 96$ & REG & Styrene & 5 & ug/L & $\mathrm{U}$ \\
\hline 4316 & M01020 & $6 / 24 / 97$ & REG & Styrene & 5 & ug/ $\mathrm{L}$ & $\mathrm{U}$ \\
\hline+316 & SMPL00810 & $9 / 13 / 94$ & REG & Styrene & 5 & $\mathrm{ug} / \mathrm{L}$ & $\mathrm{U}$ \\
\hline 4316 & S01183 & $11 / 3 / 94$ & REG & Styrene & 1 & $\mathrm{ug} / \mathrm{L}$ & $\mathrm{U}$ \\
\hline 4316 & S02266 & $1 / 12 / 95$ & REG & Styrene & 1 & $\mathrm{ug} / \mathrm{L}$ & $\mathrm{U}$ \\
\hline 4316 & S03828 & $5 / 8 / 95$ & REG & Styrene & 1 & ug/ & $\mathrm{U}$ \\
\hline 4316 & S05070 & $8 / 30 / 95$ & REG & Styrene & 1 & ug/ & $\mathbf{U}$ \\
\hline 4316 & M01298 & $11 / 18 / 97$ & REG & Tetrachloroethene & 5 & $\mathrm{ug} / \mathrm{L}$ & $\mathrm{U}$ \\
\hline 4316 & W00900 & $6 / 24 / 97$ & REG & Tetrachloroethene & 5 & ug/L & $\mathrm{U}$ \\
\hline 4316 & M00087 & $11 / 19 / 96$ & REG & Tetrachloroethene & 5 & ug/L & $\mathrm{U}$ \\
\hline 4316 & M01020 & $6 / 24 / 97$ & REG & Tetrachloroethene & 5 & $\mathrm{ug} / \mathrm{L}$ & $\mathrm{U}$ \\
\hline 4316 & SMPL00810 & 9/13/94 & REG & Tetrachloroethene & 5 & ug $\Omega$ & $\mathbf{U}$ \\
\hline
\end{tabular}


B-103

\begin{tabular}{|c|c|c|c|c|c|c|c|}
\hline Station & Sample ID & Sample Date & Sample Type & Chemical & Results & Units & Qualifier \\
\hline 4316 & S01183 & $11 / 3 / 94$ & REG & Tetrachloroethene & 1 & ug/L & $\bar{U}$ \\
\hline 4316 & S02266 & $1 / 12 / 95$ & REG & Tetrachloroethene & 1 & ug $/$. & $\mathrm{U}$ \\
\hline 4316 & S03828 & $5 / 8 / 95$ & REG & Tetrachloroethene & 1 & ug $\Omega$ & $\mathrm{U}$ \\
\hline 4316 & S05070 & $8 / 30 / 95$ & REG & Tetrachloroethene & 1 & $\mathrm{ug} / \mathrm{L}$ & $\mathrm{U}$ \\
\hline+316 & M01298 & $11 / 18 / 97$ & REG & Toluene & 5 & ug $\Omega$ & $\mathrm{U}$ \\
\hline+316 & W00900 & $6 / 24 / 97$ & REG & Toluene & 5 & ug/L & $\mathrm{U}$ \\
\hline 4316 & M00087 & $11 / 19 / 96$ & REG & Toluene & 5 & $\mathrm{ug} /$ & $\mathrm{U}$ \\
\hline 4316 & M01020 & $6 / 24 / 97$ & REG & Toluene & 5 & $\mathrm{ug} / \mathrm{L}$ & $U$ \\
\hline 4316 & SMPL00810 & 9/13/94 & REG & Toluene & 5 & ug $/$ & $\mathrm{U}$ \\
\hline 4316 & S01183 & $11 / 3 / 94$ & REG & Toluene & 1 & ug $/$ & $\mathrm{U}$ \\
\hline 4316 & S02266 & $1 / 12 / 95$ & REG & Toluene & 1 & ug $/$ & $\mathrm{U}$ \\
\hline 4316 & S03828 & $5 / 8 / 95$ & REG & Toluene & 1 & ug $/$ & $\mathrm{U}$ \\
\hline 4316 & S05070 & $8 / 30 / 95$ & REG & Toluene & 1 & ug/L & $\mathrm{U}$ \\
\hline 4316 & M01298 & $11 / 18 / 97$ & REG & trans-1,2-Dichloroethe & 5 & ugl & $\mathrm{U}$ \\
\hline 4316 & M00087 & $11 / 19 / 96$ & REG & trans-1,2-Dichloroethene & 5 & ug/ & $\mathrm{U}$ \\
\hline 4316 & M01020 & $6 / 24 / 97$ & REG & trans-1,2-Dichloroethene & 5 & ug $/$ & $\mathrm{U}$ \\
\hline 4316 & SMPL00810 & $9 / 13 / 94$ & REG & trans-1.2-Dichloroethene & 5 & ug/ & $\mathrm{U}$ \\
\hline 4316 & S01183 & $11 / 3 / 94$ & REG & trans-1,2-Dichloroethene & 1 & ug/L & $\mathrm{U}$ \\
\hline 4316 & S02266 & $1 / 12 / 95$ & REG & trans-1,2-Dichloroethene & 1 & ug/ & $\mathrm{U}$ \\
\hline 4316 & S03828 & $5 / 8 / 95$ & REG & trans-1.2-Dichloroethene & 1 & ug/L & $\mathrm{U}$ \\
\hline 4316 & S05070 & $8 / 30 / 95$ & REG & trans-1.2-Dichloroethene & 1 & ug/ & $\mathrm{U}$ \\
\hline 4316 & M01298 & $11 / 18 / 97$ & REG & trans-1,3-Dichloroprop & 5 & ug $/ \mathrm{L}$ & $\mathrm{U}$ \\
\hline 4316 & W00900 & $6 / 24 / 97$ & REG & trans-1,3-Dichloropropene & 5 & ug/L & $\mathrm{U}$ \\
\hline 4316 & M00087 & $11 / 19 / 96$ & REG & trans-1.3-Dichloropropene & 5 & $\mathrm{ug} / \mathrm{L}$ & $\mathrm{U}$ \\
\hline 4316 & M01020 & $6 / 24 / 97$ & REG & trans-1,3-Dichloropropene & 5 & $\mathrm{ug} / \mathrm{L}$ & $\mathrm{U}$ \\
\hline 4316 & SMPL00810 & 9/13/94 & REG & trans-1.3-Dichloropropene & 5 & ug $/ \mathrm{L}$ & $\mathrm{U}$ \\
\hline 4316 & S01183 & 11/3/94 & REG & trans-1,3-Dichloropropene & 1 & ug $/ \mathrm{L}$ & $\mathrm{U}$ \\
\hline 4316 & S02266 & $1 / 12 / 95$ & REG & trans-1.3-Dichloropropene & 1 & ug/L & $\mathrm{U}$ \\
\hline 4316 & $\mathrm{~S} 03828$ & $5 / 8 / 95$ & REG & trans-1,3-Dichloropropene & 1 & ug/L & $\mathrm{U}$ \\
\hline 4316 & S05070 & $8 / 30 / 95$ & REG & trans-1,3-Dichloropropene & 1 & ug/L & U \\
\hline 4316 & M01298 & $11 / 18 / 97$ & REG & Trichloroethene & 5 & ug $/ \mathrm{L}$ & $\mathrm{U}$ \\
\hline+316 & W00900 & $6 / 24 / 97$ & REG & Trichloroethene & 5 & $\mathrm{ug} / \mathrm{L}$ & $\mathrm{U}$ \\
\hline 4316 & M00087 & $11 / 19 / 96$ & REG & Trichloroethene & 5 & ug $/ \mathrm{L}$ & $\mathrm{U}$ \\
\hline 4316 & M01020 & $6 / 24 / 97$ & REG & Trichloroethene & 5 & ug/L & $U$ \\
\hline 4316 & SMPL00810 & 9/13/94 & REG & Trichloroethene & 5 & $\mathrm{ug} / \mathrm{L}$ & $\mathrm{U}$ \\
\hline 4316 & S01183 & $11 / 3 / 94$ & REG & Trichloroethene & 1 & $\mathrm{ug} / \mathrm{L}$ & $\mathrm{U}$ \\
\hline 4316 & S02266 & $1 / 12 / 95$ & REG & Trichloroethene & 1 & ug/L & $\mathbf{U}$ \\
\hline 4316 & S03828 & $5 / 8 / 95$ & REG & Trichloroethene & 1 & ug/L & UJ \\
\hline+316 & S05070 & $8 / 30 / 95$ & REG & Trichloroethene & 1 & ug/L & $\mathbf{U}$ \\
\hline+316 & M01298 & $11 / 18 / 97$ & REG & Vinyl acetate & 10 & ug $/ \mathrm{L}$ & $\mathrm{U}$ \\
\hline+316 & M00087 & $11 / 19 / 96$ & REG & Vinyl acetate & 10 & ug/L & $\mathrm{U}$ \\
\hline 4316 & M01020 & $6 / 24 / 97$ & REG & Vinyl acetate & 10 & ug/L & $\mathrm{U}$ \\
\hline 4316 & SMPL00810 & 9/13/94 & REG & Vinyl acetate & 10 & ug $/ \mathrm{L}$ & $\mathrm{U}$ \\
\hline 4316 & M01298 & $11 / 18 / 97$ & REG & Vinyl chloride & 10 & ug/L & $\mathrm{U}$ \\
\hline+316 & W00900 & $6 / 24 / 97$ & REG & Vinyl chloride & 10 & ug/L & $\mathrm{U}$ \\
\hline 4316 & M00087 & $11 / 19 / 96$ & REG & Vinyl chloride & 10 & ug/L & U \\
\hline 4316 & M01020 & $6 / 24 / 97$ & REG & Vinyl chloride & 10 & $\mathrm{ug} / \mathrm{L}$ & $\mathbf{U}$ \\
\hline 4316 & SMPL00810 & $9 / 13 / 94$ & REG & Vinyl chloride & 10 & ug $/$ & $\mathbf{U}$ \\
\hline+316 & S01183 & $11 / 3 / 94$ & REG & Vinyl chloride & 1 & ug/L & $\mathrm{U}$ \\
\hline 4316 & S02266 & $1 / 12 / 95$ & REG & Vinyl chloride & $i$ & ug $/$ & $\mathrm{U}$ \\
\hline
\end{tabular}




\begin{tabular}{|c|c|c|c|c|c|c|c|}
\hline Station & Sample ID & Sample Date & Sample Type & Chemical & Results & Units & Qualifier \\
\hline 4316 & S03828 & $5 / 8 / 95$ & REG & Vinyl chloride & 1 & $\mathrm{ug} / \mathrm{L}$ & $\overline{\mathrm{U}}$ \\
\hline+316 & S05070) & $8 / 30 / 95$ & REG & Vinyl chloride & 1 & ug/L & U \\
\hline 4.317 & M01304 & $11 / 19 / 97$ & REG & 1.1.1-Trichloroethane & 5 & $\mathrm{ug} / \mathrm{L}$ & $\mathrm{U}$ \\
\hline 4317 & W00820 & $6 / 17 / 97$ & REG & I.1.1-Trichloroethane & 5 & $\mathrm{ug} / \mathrm{L}$ & $\mathrm{U}$ \\
\hline+317 & M00091 & $11 / 20 / 96$ & REG & 1.1.1-Trichloroethane & 5 & $\mathrm{ug} / \mathrm{L}$ & $\mathrm{U}$ \\
\hline 4317 & M00896 & $6 / 17 / 97$ & REG & 1.1.1-Trichloroethane & 5 & $\mathrm{ug} / \mathrm{L}$ & $\mathrm{U}$ \\
\hline 4317 & SMPL00816 & $9 / 12 / 94$ & REG & 1.1,1-Trichloroethane & 5 & $\mathrm{ug} / \mathrm{L}$ & $\mathrm{U}$ \\
\hline 4317 & S01876 & $12 / 15 / 94$ & REG & 1,1,1-Trichloroethane & 1 & $\mathrm{ug} / \mathrm{L}$ & $\mathrm{U}$ \\
\hline 4317 & S02345 & $1 / 25 / 95$ & REG & 1.1,1-Trichloroethane & 1 & ug/L & $\mathrm{U}$ \\
\hline 4317 & S03456 & $4 / 13 / 95$ & REG & 1.1,1-Trichloroethane & 1 & ug/L & $\mathrm{U}$ \\
\hline 4317 & M01304 & $11 / 19 / 97$ & REG & 1,1,2,2-Tetrachloroeth & 5 & ug $/ \mathrm{L}$ & $\mathrm{U}$ \\
\hline 4317 & W00820 & $6 / 17 / 97$ & REG & 1,1,2,2-Tetrachloroethane & 5 & $\mathrm{ug} / \mathrm{L}$ & $\mathrm{U}$ \\
\hline 4317 & M00091 & $11 / 20 / 96$ & REG & 1,1,2,2-Tetrachloroethane & 5 & $\mathrm{ug} / \mathrm{L}$ & $\mathrm{U}$ \\
\hline 4317 & M00896 & $6 / 17 / 97$ & REG & 1,1,2,2-Tetrachloroethane & 5 & $\mathrm{ug} / \mathrm{L}$ & $\mathrm{U}$ \\
\hline 4317 & SMPL00816 & $9 / 12 / 94$ & REG & 1,1,2,2-Tetrachloroethane & 5 & $\mathrm{ug} / \mathrm{L}$ & $\mathbf{U}$ \\
\hline 4317 & S01876 & $12 / 15 / 94$ & REG & 1,1,2,2-Tetrachloroethane & 1 & $\mathrm{ug} / \mathrm{L}$ & $\mathrm{U}$ \\
\hline 4317 & S02345 & $1 / 25 / 95$ & REG & 1,1,2,2-Tetrachloroethane & 1 & $\mathrm{ug} / \mathrm{L}$ & $\mathrm{U}$ \\
\hline 4317 & S03456 & $4 / 13 / 95$ & REG & 1,1,2,2-Tetrachloroethane & 1 & ug/L & $\mathrm{U}$ \\
\hline 4317 & W00820 & $6 / 17 / 97$ & REG & 1.1,2-Trichloro-1.2,2-trifluo & 10 & ug/L & $\mathrm{U}$ \\
\hline 4317 & M01304 & $11 / 19 / 97$ & REG & 1.1,2-Trichloroethane & 5 & ug/L & $\mathrm{U}$ \\
\hline 4317 & W00820 & $6 / 17 / 97$ & REG & 1,1,2-Trichloroethane & 5 & ug/L & $\mathrm{U}$ \\
\hline 4317 & M00091 & $11 / 20 / 96$ & REG & 1,1,2-Trichloroethane & 5 & ug/L & $\mathrm{U}$ \\
\hline 4317 & M00896 & $6 / 17 / 97$ & REG & 1,1,2-Trichloroethane & 5 & ug/ & U \\
\hline 4317 & SMPL00816 & $9 / 12 / 94$ & REG & 1.1,2-Trichloroethane & 5 & ug/h & $\mathrm{U}$ \\
\hline 4317 & S01876 & $12 / 15 / 94$ & REG & 1,1,2-Trichloroethane & 1 & ug $/ \mathrm{L}$ & $\mathrm{U}$ \\
\hline 4317 & S02345 & $1 / 25 / 95$ & REG & 1,1,2-Trichloroethane & 1 & $\mathrm{ug} / \mathrm{L}$ & $\mathrm{U}$ \\
\hline 4317 & S03456 & $4 / 13 / 95$ & REG & 1.1,2-Trichloroethane & 1 & $\mathrm{ug} / \mathrm{L}$ & $\mathrm{U}$ \\
\hline 4317 & M01304 & $11 / 19 / 97$ & REG & 1.1-Dichloroethane & 5 & ug/L & $\mathrm{U}$ \\
\hline 4317 & W00820 & $6 / 17 / 97$ & REG & 1,1-Dichloroethane & 5 & $\mathrm{ug} / \mathrm{L}$ & $\mathrm{U}$ \\
\hline 4317 & M00091 & $11 / 20 / 96$ & REG & 1,1-Dichloroethane & 5 & $\mathrm{ug} / \mathrm{L}$ & $\mathrm{U}$ \\
\hline+317 & M00896 & $6 / 17 / 97$ & REG & I.1-Dichloroethane & 5 & ug/ & $\mathrm{U}$ \\
\hline 4317 & SMPL00816 & $9 / 12 / 94$ & REG & 1,1-Dichloroethane & 5 & $\mathrm{ug} / \mathrm{L}$ & U \\
\hline 4317 & S01876 & $12 / 15 / 94$ & REG & 1.1-Dichloroethane & 1 & ug/L & $\mathrm{U}$ \\
\hline 4317 & S02345 & $1 / 25 / 95$ & REG & 1,1-Dichloroethane & 1 & ug $/$ & $\mathrm{U}$ \\
\hline+317 & $\mathrm{~S} 03+56$ & $4 / 13 / 95$ & REG & 1,1-Dichloroethane & 1 & ug/L & $\mathrm{U}$ \\
\hline+317 & M01304 & $11 / 19 / 97$ & REG & 1,1-Dichloroethene & 5 & $\mathrm{ug} / \mathrm{L}$ & $\mathrm{U}$ \\
\hline+317 & W00820 & $6 / 17 / 97$ & REG & 1.1-Dichloroethene & 5 & $\mathrm{ug} / \mathrm{L}$ & $\mathrm{U}$ \\
\hline+317 & M00091 & $11 / 20 / 96$ & REG & 1.1-Dichloroethene & 5 & ug/L & $\mathrm{U}$ \\
\hline 4317 & M00896 & $6 / 17 / 97$ & REG & 1.1-Dichloroethene & 5 & $\mathrm{ug} / \mathrm{L}$ & $\mathrm{U}$ \\
\hline 4317 & SMPL00816 & $9 / 12 / 94$ & REG & 1.1-Dichloroethene & 5 & $\mathrm{ug} / \mathrm{L}$ & $\mathrm{U}$ \\
\hline 4317 & S01876 & $12 / 15 / 94$ & REG & 1,1-Dichloroethene & 1 & ug/L & $\mathrm{U}$ \\
\hline 4317 & S02345 & $1 / 25 / 95$ & REG & 1.1-Dichloroethene & 1 & ug/ & $\mathrm{U}$ \\
\hline 4317 & S03456 & $4 / 13 / 95$ & REG & 1.1-Dichloroethene & 1 & ug $\Omega$ & $\mathrm{U}$ \\
\hline+317 & S01876 & $12 / 15 / 94$ & REG & 1.2-Dibromo-3-chloropropane & 1 & ug/h & $\mathrm{U}$ \\
\hline+317 & S02345 & $1 / 25 / 95$ & REG & 1,2-Dibromo-3-chloropropane & 1 & $\mathrm{ug} / \mathrm{L}$ & $\mathrm{U}$ \\
\hline 4317 & S03456 & $4 / 13 / 95$ & REG & 1.2-Dibromo-3-chloropropane & 1 & $\mathrm{ug} / \mathrm{L}$ & $\mathrm{U}$ \\
\hline 4317 & S01876 & $12 / 15 / 94$ & REG & 1.2-Dibromoethane & 1 & ug $/ \mathrm{L}$ & $\mathrm{U}$ \\
\hline 4317 & S02345 & $1 / 25 / 95$ & REG & 1.2-Dibromoethane & 1 & ug/ $/$ & $\mathrm{U}$ \\
\hline 4317 & S03456 & $4 / 13 / 95$ & REG & 1.2-Dibromoethane & 1 & $\mathrm{ug} / \mathrm{L}$ & $\mathrm{U}$ \\
\hline 4317 & M00091 & $11 / 20 / 96$ & REG & 1.2-Dichlorobenzene & 5 & ug $/ \mathrm{L}$ & $\mathrm{U}$ \\
\hline
\end{tabular}




\begin{tabular}{|c|c|c|c|c|c|c|c|}
\hline Station & Sample ID & Sample Date & Sample Type & Chemical & Results & Units & Qualifier \\
\hline 4.317 & SMPL00816 & $9 / 12 / 94$ & REG & 1.2-Dichlorobenzene & 5 & ug $\Omega$ & $\mathrm{U}$ \\
\hline 4.317 & M01304 & $11 / 19 / 97$ & REG & 1.2-Dichloroethane & 5 & $\mathrm{ug} / \mathrm{L}$ & $\mathrm{U}$ \\
\hline+317 & W00820 & $6 / 17 / 97$ & REG & 1.2-Dichloroethane & 5 & $\mathrm{ug} / \mathrm{L}$ & $\mathrm{U}$ \\
\hline+317 & M00091 & $11 / 20 / 96$ & REG & 1.2-Dichloroethane & 5 & $\mathrm{ug} / \mathrm{L}$ & $\mathrm{U}$ \\
\hline 4317 & M00896 & $6 / 17 / 97$ & REG & 1.2-Dichloroethane & 5 & ug/L & $\mathrm{U}$ \\
\hline 4317 & SMPL00816 & $9 / 12 / 94$ & REG & 1.2-Dichloroethane & 5 & $u g / L$ & U \\
\hline 4317 & S01876 & $12 / 15 / 94$ & REG & 1.2-Dichloroethane & 1 & ug $\Omega$ & $\mathrm{U}$ \\
\hline 4317 & S02345 & $1 / 25 / 95$ & REG & 1.2-Dichloroethane & 1 & ug $/ \mathrm{L}$ & $\mathrm{U}$ \\
\hline 4317 & S03456 & $4 / 13 / 95$ & REG & 1.2-Dichloroethane & 0.73 & ug $/ \mathrm{L}$ & J \\
\hline 4317 & W00820 & $6 / 17 / 97$ & REG & 1.2-Dichloroethene & 5 & $\mathrm{ug} / \mathrm{L}$ & $U$ \\
\hline 4317 & M00091 & $11 / 20 / 96$ & REG & 1.2-Dichloroethene & 5 & $\mathrm{ug} / \mathrm{L}$ & $\mathrm{U}$ \\
\hline+317 & SMPL00816 & $9 / 12 / 94$ & REG & 1.2-Dichloroethene & 5 & $\mathrm{ug} / \mathrm{L}$ & U \\
\hline 4317 & M01304 & $11 / 19 / 97$ & REG & 1,2-Dichloropropane & 5 & ug $/ \mathrm{L}$ & $\mathrm{U}$ \\
\hline+317 & W00820 & $6 / 17 / 97$ & REG & 1,2-Dichloropropane & 5 & $\mathrm{ug} / \mathrm{L}$ & $\mathrm{U}$ \\
\hline 4317 & M00091 & $11 / 20 / 96$ & REG & 1.2-Dichloropropane & 5 & $\mathrm{ug} / \mathrm{L}$ & $\mathrm{U}$ \\
\hline 4317 & M00896 & $6 / 17 / 97$ & REG & 1.2-Dichloropropane & 5 & $\mathrm{ug} / \mathrm{L}$ & U \\
\hline 4317 & SMPL00816 & $9 / 12 / 94$ & REG. & 1,2-Dichloropropane & 5 & $\mathrm{ug} / \mathrm{L}$ & $\mathrm{U}$ \\
\hline+317 & S01876 & $12 / 15 / 94$ & REG & 1,2-Dichloropropane & 1 & $\mathrm{ug} / \mathrm{L}$ & $\mathrm{U}$ \\
\hline 4317 & S02345 & $1 / 25 / 95$ & REG & 1.2-Dichloropropane & 1 & ug/L & $\mathrm{U}$ \\
\hline+317 & S03456 & $4 / 13 / 95$ & REG & 1.2-Dichloropropane & 1 & ug/L & $\mathrm{U}$ \\
\hline 4317 & M00091 & $11 / 20 / 96$ & REG & 1.2-Dimethylbenzene & 5 & $\mathrm{ug} / \mathrm{L}$ & $\mathrm{U}$ \\
\hline 4317 & M00091 & $11 / 20 / 96$ & REG & 1.3-Dichlorobenzene & 5 & $\mathrm{ug} / \mathrm{L}$ & $\mathrm{U}$ \\
\hline 4317 & SMPL00816 & $9 / 12 / 94$ & REG & 1.3-Dichlorobenzene & 5 & ug/L & $\mathrm{U}$ \\
\hline+317 & M00091 & $11 / 20 / 96$ & REG & 1,4-Dichlorobenzene & 5 & ug/L & $\mathrm{U}$ \\
\hline 4317 & SMPL00816 & $9 / 12 / 94$ & REG & 1,4-Dichlorobenzene & 5 & $\mathrm{ug} / \mathrm{L}$ & $\mathrm{U}$ \\
\hline 4317 & M01304 & $11 / 19 / 97$ & REG & 2-Butanone & 10 & ug/L & U \\
\hline+317 & W00820 & $6 / 17 / 97$ & REG & 2-Butanone & 10 & ug/L & $\mathrm{U}$ \\
\hline 4317 & M00091 & $11 / 20 / 96$ & REG & 2-Butanone & 2 & ug/L & $=$ \\
\hline 4317 & M00896 & $6 / 17 / 97$ & REG & 2-Butanone & 10 & $\mathrm{ug} / \mathrm{L}$ & $\mathrm{U}$ \\
\hline+317 & SMPL00816 & $9 / 12 / 94$ & REG & 2-Butanone & 10 & $\mathrm{ug} / \mathrm{L}$ & $\mathrm{U}$ \\
\hline+317 & S01876 & $12 / 15 / 94$ & REG & 2-Butanone & 5 & $\mathrm{ug} / \mathrm{L}$ & $\mathrm{U}$ \\
\hline 4317 & S02345 & $1 / 25 / 95$ & REG & 2-Butanone & 5 & $\mathrm{ug} / \mathrm{L}$ & $\mathrm{U}$ \\
\hline 4317 & S03456 & $4 / 13 / 95$ & REG & 2-Butanone & 5 & $\mathrm{ug} / \mathrm{L}$ & U \\
\hline 4317 & M01304 & $11 / 19 / 97$ & REG & 2-Hexanone & 10 & $\mathrm{ug} / \mathrm{L}$ & $\mathrm{U}$ \\
\hline 4317 & W00820 & $6 / 17 / 97$ & REG & 2-Hexanone & 10 & $\mathrm{ug} / \mathrm{L}$ & $\mathrm{U}$ \\
\hline 4317 & M00091 & $11 / 20 / 96$ & REG & 2-Hexanone & 10 & $\mathrm{ug} / \mathrm{L}$ & $\mathrm{U}$ \\
\hline 4317 & M00896 & $6 / 17 / 97$ & REG & 2-Hexanone & 10 & $\mathrm{ug} / \mathrm{L}$ & $\mathrm{U}$ \\
\hline 4317 & SMPL00816 & $9 / 12 / 94$ & REG & 2-Hexanone & 10 & $\mathrm{ug} / \mathrm{L}$ & U \\
\hline 4317 & S01876 & $12 / 15 / 94$ & REG & 2-Hexanone & 5 & $\mathrm{ug} / \mathrm{L}$ & $\mathrm{U}$ \\
\hline 4317 & S02345 & $1 / 25 / 95$ & REG & 2-Hexanone & 5 & $\mathrm{ug} / \mathrm{L}$ & $\mathrm{U}$ \\
\hline 4317 & S03456 & $4 / 13 / 95$ & REG & 2-Hexanone & 5 & $\mathrm{ug} / \mathrm{L}$ & $\mathrm{U}$ \\
\hline+317 & M01304 & $11 / 19 / 97$ & REG & 4-Methyl-2-pentanone & 10 & $\mathrm{ug} / \mathrm{L}$ & $\mathrm{U}$ \\
\hline 4317 & W00820 & $6 / 17 / 97$ & REG & 4-Methyl-2-pentanone & 10 & $\mathrm{ug} / \mathrm{L}$ & U \\
\hline 4317 & M00091 & $11 / 20 / 96$ & REG & 4-Methyl-2-pentanone & 10 & $\mathrm{ug} / \mathrm{L}$ & $\mathrm{U}$ \\
\hline 4317 & M00896 & $6 / 17 / 97$ & REG & 4-Methyl-2-pentanone & 10 & $\mathrm{ug} / \mathrm{L}$ & U \\
\hline+317 & SMPL00816 & $9 / 12 / 94$ & REG & 4-Methyl-2-pentanone & 10 & $\mathrm{ug} / \mathrm{L}$ & $\mathrm{U}$ \\
\hline 4317 & S01876 & $12 / 15 / 94$ & REG & 4-Methyl-2-pentanone & 5 & $\mathrm{ug} / \mathrm{L}$ & $\mathrm{U}$ \\
\hline 4317 & S02345 & $1 / 25 / 95$ & REG & 4-Methyl-2-pentanone & 5 & ug/L & $U$ \\
\hline 4317 & S03456 & $4 / 13 / 95$ & REG & 4-Methyl-2-pentanone & 5 & $\mathrm{ug} / \mathrm{L}$ & $U$ \\
\hline$\$ 317$ & M01304 & $11 / 19 / 97$ & REG & Acetone & 10 & $\mathrm{ug} / \mathrm{L}$ & $\mathrm{U}$ \\
\hline
\end{tabular}




\begin{tabular}{|c|c|c|c|c|c|c|c|}
\hline Station & Sample ID & Sample Date & Sample Type & Chemical & Results & Units & Qualifier \\
\hline 4317 & W00820 & $6 / 17 / 97$ & REG & Acetone & 10 & $\mathrm{ug} / \mathrm{L}$ & $\bar{U}$ \\
\hline 4317 & M00091 & $11 / 20 / 96$ & REG & Acetone & 2 & $\mathrm{ug} / \mathrm{L}$ & $=$ \\
\hline 4317 & M00896 & $6 / 17 / 97$ & REG & Acetone & 5 & $\mathrm{ug} / \mathrm{L}$ & $U$ \\
\hline 4.317 & SMPL00816 & $9 / 12 / 94$ & REG & Acetone & 10 & $\mathrm{ug} / \mathrm{L}$ & U \\
\hline 4317 & S01876 & $12 / 15 / 94$ & REG & Acetone & 5 & $\mathrm{ug} / \mathrm{L}$ & $\mathrm{U}$ \\
\hline 4317 & S02345 & $1 / 25 / 95$ & REG & Acetone & 6.7 & $\mathrm{ug} / \mathrm{L}$ & U \\
\hline 4317 & S03456 & $4 / 13 / 95$ & REG & Acetone & 3.8 & ug/L & $\mathbf{J}$ \\
\hline 4317 & M01304 & $11 / 19 / 97$ & REG & Benzene & 5 & $\mathrm{ug} / \mathrm{L}$ & U \\
\hline 4317 & W00820 & $6 / 17 / 97$ & REG & Benzene & 5 & $\mathrm{ug} / \mathrm{L}$ & $U$ \\
\hline 4317 & M00091 & $11 / 20 / 96$ & REG & Benzene & 5 & $\mathrm{ug} / \mathrm{L}$ & U \\
\hline 4317 & M00896 & $6 / 17 / 97$ & REG & Benzene & 5 & ug/L & U \\
\hline 4317 & SMPL00816 & $9 / 12 / 94$ & REG & Benzene & 5 & ug/ & $\mathrm{U}$ \\
\hline 4317 & S01876 & $12 / 15 / 94$ & REG & Benzene & 1 & ug/L & $\mathrm{U}$ \\
\hline 4317 & S02345 & $1 / 25 / 95$ & REG & Benzene & 1 & $\mathrm{ug} / \mathrm{L}$ & U \\
\hline 4317 & S03456 & $4 / 13 / 95$ & REG & Benzene & 1 & ug/L & $\mathrm{U}$ \\
\hline 4317 & S01876 & $12 / 15 / 94$ & REG & Bromochloromethane & 1 & ug/L & $\mathbf{U}$ \\
\hline 4317 & S02345 & $1 / 25 / 95$ & REG & Bromochloromethane & 1 & $\mathrm{ug} / \mathrm{L}$ & $\mathrm{U}$ \\
\hline 4317 & S03456 & $4 / 13 / 95$ & REG & Bromochloromethane & 1 & ug/ & $\mathbf{U}$ \\
\hline 4317 & M01304 & $11 / 19 / 97$ & REG & Bromodichloromethane & 5 & ug/ & $\mathrm{U}$ \\
\hline 4317 & W00820 & $6 / 17 / 97$ & REG & Bromodichloromethane & 5 & ug $\Omega$ & $\mathrm{U}$ \\
\hline 4317 & M00091 & $11 / 20 / 96$ & REG & Bromodichloromethane & 5 & $\mathrm{ug} / \mathrm{L}$ & $\mathrm{U}$ \\
\hline 4317 & M00896 & $6 / 17 / 97$ & REG & Bromodichloromethane & 5 & $\mathrm{ug} / \mathrm{L}$ & $\mathrm{U}$ \\
\hline 4317 & SMPL00816 & $9 / 12 / 94$ & REG & Bromodichloromethane & 5 & ug $/ \mathrm{L}$ & $\mathrm{U}$ \\
\hline 4317 & S01876 & $12 / 15 / 94$ & REG & Bromodichloromethane & 1 & $\mathrm{ug} / \mathrm{L}$ & $\mathrm{U}$ \\
\hline 4317 & S02345 & $1 / 25 / 95$ & REG & Bromodichloromethane & 1 & $\mathrm{ug} / \mathrm{L}$ & U \\
\hline 4317 & S03456 & 4/13/95 & REG & Bromodichloromethane & 1 & ug/ & $\mathrm{U}$ \\
\hline 4317 & M01304 & $11 / 19 / 97$ & REG & Bromoform & 5 & $\mathrm{ug} / \mathrm{L}$ & U \\
\hline 4317 & W00820 & $6 / 17 / 97$ & REG & Bromoform & 5 & ug/ & $\mathrm{U}$ \\
\hline 4317 & M00091 & $11 / 20 / 96$ & REG & Bromoform & 5 & ug/L & $\mathrm{U}$ \\
\hline 4317 & M00896 & $6 / 17 / 97$ & REG & Bromoform & 5 & $\mathrm{ug} / \mathrm{L}$ & $\mathrm{U}$ \\
\hline 4317 & SMPL00816 & $9 / 12 / 94$ & REG & Bromoform & 5 & ug $\Omega$ & $\mathrm{U}$ \\
\hline 4317 & S01876 & $12 / 15 / 94$ & REG & Bromoform & 1 & $\mathrm{ug} / \mathrm{L}$ & $\mathrm{U}$ \\
\hline 4317 & S02345 & $1 / 25 / 95$ & REG & Bromoform & 1 & $\mathrm{ug} / \mathrm{L}$ & U \\
\hline 4317 & S03456 & $4 / 13 / 95$ & REG & Bromoform & 1 & $u g / L$ & $\mathrm{U}$ \\
\hline 4317 & M01304 & $11 / 19 / 97$ & REG & Bromomethane & 10 & $\mathrm{ug} / \mathrm{L}$ & $\mathrm{U}$ \\
\hline 4317 & W00820 & $6 / 17 / 97$ & REG & Bromomethane & 10 & $u g / L$ & $\mathrm{U}$ \\
\hline 4317 & M00091 & $11 / 20 / 96$ & REG & Bromomethane & 10 & $\mathrm{ug} / \mathrm{L}$ & $U$ \\
\hline 4317 & M00896 & $6 / 17 / 97$ & REG & Bromomethane & 10 & ug/L & $\mathrm{U}$ \\
\hline 4317 & SMPL00816 & $9 / 12 / 94$ & REG & Bromomethane & 10 & $\mathrm{ug} / \mathrm{L}$ & $\mathrm{U}$ \\
\hline 4317 & S01876 & $12 / 15 / 94$ & REG & Bromomethane & 1 & $\mathrm{ug} / \mathrm{L}$ & $\mathrm{U}$ \\
\hline 4317 & S02345 & $1 / 25 / 95$ & REG & Bromomethane & 1 & $\mathrm{ug} / \mathrm{L}$ & $\mathrm{U}$ \\
\hline 4317 & S03456 & $4 / 13 / 95$ & REG & Bromomethane & 1 & $\mathrm{ug} / \mathrm{L}$ & $\mathrm{U}$ \\
\hline+317 & M01304 & $11 / 19 / 97$ & REG & Carbon disulfide & 5 & ug/L & $\mathrm{U}$ \\
\hline 4317 & W00820 & $6 / 17 / 97$ & REG & Carbon disulfide & 5 & $u g / L$ & $\mathrm{U}$ \\
\hline 4317 & M00091 & $11 / 20 / 96$ & REG & Carbon disulfide & 5 & ug/L & $\mathrm{U}$ \\
\hline 4317 & M00896 & $6 / 17 / 97$ & REG & Carbon disulfide & 5 & $\mathrm{ug} / \mathrm{L}$ & $\mathrm{U}$ \\
\hline 4317 & SMPL00816 & $9 / 12 / 94$ & REG & Carbon disulfide & 5 & $u g / L$ & $\mathrm{U}$ \\
\hline 4317 & S01876 & $12 / 15 / 94$ & REG & Carbon disulfide & 1 & ug/L & $\mathrm{U}$ \\
\hline+317 & S02345 & $1 / 25 / 95$ & REG & Carbon disulfide & 1 & ug $/ \mathrm{L}$ & $\mathrm{U}$ \\
\hline+317 & S03456 & $4 / 13 / 95$ & REG & Carbon disulfide & 1 & $\mathrm{ug} / \mathrm{L}$ & $\mathrm{U}$ \\
\hline
\end{tabular}


Station Sample ID Sample Date Sample Type Chemical

\begin{tabular}{|c|c|c|c|c|}
\hline 4317 & $\mathrm{M} 01304$ & $11 / 19 / 97$ & REG & Carbon tetrachloride \\
\hline 4317 & W00820 & $6 / 17 / 97$ & REG & Carbon tetrachloride \\
\hline 4317 & M00091 & $11 / 20 / 96$ & REG & Carbon tetrachloride \\
\hline 4.317 & M00896 & $6 / 17 / 97$ & REG & Carbon tetrachloride \\
\hline 4317 & SMPL00816 & $9 / 12 / 94$ & $\mathrm{REG}$ & Carbon tetrachloride \\
\hline 4317 & S01876 & $12 / 15 / 94$ & REG & Carbon tetrachloride \\
\hline 4317 & $\mathrm{~S} 02345$ & $1 / 25 / 95$ & REG & Carbon tetrachloride \\
\hline 4317 & S03456 & $4 / 13 / 95$ & REG & Carbon tetrachloride \\
\hline 4317 & M01304 & $11 / 19 / 97$ & REG & Chlorobenzene \\
\hline 4317 & W00820 & $6 / 17 / 97$ & REG & Chlorobenzene \\
\hline 4317 & M0009l & $11 / 20 / 96$ & REG & Chlorobenzene \\
\hline 4317 & M00896 & $6 / 17 / 97$ & REG & Chlorobenzene \\
\hline 4317 & SMPL00816 & $9 / 12 / 94$ & REG & Chlorobenzene \\
\hline 4317 & S01876 & $12 / 15 / 94$ & REG & Chlorobenzene \\
\hline 4317 & S02345 & $1 / 25 / 95$ & REG & Chlorobenzene \\
\hline 4317 & S03456 & $4 / 13 / 95$ & REG & Chlorobenzene \\
\hline 4317 & M01304 & $11 / 19 / 97$ & REG & Chloroethane \\
\hline 4317 & W00820 & $6 / 17 / 97$ & REG & Chloroethane \\
\hline 4317 & M00091 & $11 / 20 / 96$ & REG & Chloroethane \\
\hline 4317 & M00896 & $6 / 17 / 97$ & REG & Chloroethane \\
\hline 4317 & SMPL00816 & $9 / 12 / 94$ & REG & Chloroethane \\
\hline 4317 & S01876 & $12 / 15 / 94$ & REG & Chloroethane \\
\hline 4317 & S02345 & $1 / 25 / 95$ & REG & Chloroethane \\
\hline 4317 & S03456 & $4 / 13 / 95$ & REG & Chloroethane \\
\hline 4317 & M01304 & $11 / 19 / 97$ & REG & Chloroform \\
\hline 4317 & W00820 & $6 / 17 / 97$ & REG & Chloroform \\
\hline 4317 & M00091 & $11 / 20 / 96$ & REG & Chloroform \\
\hline+317 & M00896 & $6 / 17 / 97$ & REG & Chloroform \\
\hline 4317 & SMPL00816 & $9 / 12 / 94$ & REG & Chloroform \\
\hline 4317 & S01876 & $12 / 15 / 94$ & REG & Chloroform \\
\hline 4317 & S02345 & $1 / 25 / 95$ & REG & Chloroform \\
\hline 4317 & S03456 & $4 / 13 / 95$ & REG & Chloroform \\
\hline 4317 & M01304 & $11 / 19 / 97$ & REG & Chloromethane \\
\hline 4317 & W00820 & $6 / 17 / 97$ & REG & Chloromethane \\
\hline 4.317 & M0009l & $11 / 20 / 96$ & REG & Chloromethane \\
\hline+317 & M00896 & $6 / 17 / 97$ & REG & Chloromethane \\
\hline 4317 & SMPL00816 & $9 / 12 / 94$ & REG & Chloromethane \\
\hline 4317 & S01876 & $12 / 15 / 94$ & REG & Chloromethane \\
\hline 4317 & S02345 & $1 / 25 / 95$ & REG & Chloromethane \\
\hline 4317 & S03456 & $4 / 13 / 95$ & REG & Chloromethane \\
\hline 4317 & M01304 & $11 / 19 / 97$ & REG & cis-1,2-Dichloroethene \\
\hline 4317 & M00091 & $11 / 20 / 96$ & REG & cis-1,2-Dichloroethene \\
\hline 4317 & M00896 & $6 / 17 / 97$ & REG & cis-1.2-Dichloroethene \\
\hline 4317 & SMPL00816 & $9 / 12 / 94$ & REG & cis-1,2-Dichloroethene \\
\hline 4317 & S01876 & $12 / 15 / 94$ & REG & cis-1,2-Dichloroethene \\
\hline 4317 & S02345 & $1 / 25 / 95$ & REG & cis-1,2-Dichloroethene \\
\hline 4317 & S03456 & $4 / 13 / 95$ & REG & cis-1,2-Dichloroethene \\
\hline 4317 & M01304 & $11 / 19 / 97$ & REG & cis-1,3-Dichloropropen \\
\hline 4317 & W00820 & $6 / 17 / 97$ & REG & cis-1.3-Dichloropropene \\
\hline+317 & M00091 & $11 / 20 / 96$ & REG & cis-1.3-Dichloropropene \\
\hline
\end{tabular}

Results Units Qualifier

$\begin{array}{lll}5 & \text { ug/L } & U \\ 5 & \text { ug/L } & U \\ 5 & \text { ug } / & U \\ 5 & \text { ug } / \text { } & U \\ 5 & \text { ug } / & U\end{array}$

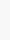

1

5

5

(5

1

10

10

10

10

10

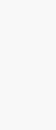

(5)

5

5

5

5

5

1

1

10

10

1

5

5

5

5

1

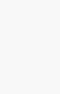

(

5

5


B-108 .

Station Sample ID Sample Date Sample Type Chemical

\begin{tabular}{|c|c|c|}
\hline 4317 & M00896 & $6 / 17 / 97$ \\
\hline 4317 & SMPL00816 & $9 / 12 / 94$ \\
\hline 4317 & S01876 & $12 / 15 / 94$ \\
\hline+317 & S02345 & $1 / 25 / 95$ \\
\hline 4317 & S03456 & $4 / 13 / 95$ \\
\hline 4.317 & $\mathrm{M} 01304$ & $11 / 19 / 97$ \\
\hline 4317 & W00820 & $6 / 17 / 97$ \\
\hline 4317 & M00091 & $11 / 20 / 96$ \\
\hline+317 & M00896 & $6 / 17 / 97$ \\
\hline 4317 & SMPL00816 & $9 / 12 / 94$ \\
\hline 4317 & S01876 & $12 / 15 / 94$ \\
\hline 4317 & S02345 & $1 / 25 / 95$ \\
\hline 4317 & S03456 & $4 / 13 / 95$ \\
\hline 4317 & M00091 & $11 / 20 / 96$ \\
\hline 4317 & SMPL00816 & $9 / 12 / 94$ \\
\hline 4317 & SMPL00816 & $9 / 12 / 94$ \\
\hline 4317 & SMPL00816 & $9 / 12 / 94$ \\
\hline 4317 & M01304 & $11 / 19 / 97$ \\
\hline 4317 & W00820 & $6 / 17 / 97$ \\
\hline 4317 & M00091 & $11 / 20 / 96$ \\
\hline+317 & M00896 & $6 / 17 / 97$ \\
\hline+317 & SMPL00816 & $9 / 12 / 94$ \\
\hline 4317 & S01876 & $12 / 15 / 94$ \\
\hline 4317 & S02345 & $1 / 25 / 95$ \\
\hline+317 & S03456 & $4 / 13 / 95$ \\
\hline 4317 & M01304 & $11 / 19 / 97$ \\
\hline 4317 & W00820 & $6 / 17 / 97$ \\
\hline+317 & M00091 & $11 / 20 / 96$ \\
\hline+317 & M00896 & $6 / 17 / 97$ \\
\hline 4317 & M01304 & $11 / 19 / 97$ \\
\hline 4317 & W00820 & $6 / 17 / 97$ \\
\hline+317 & M00091 & $11 / 20 / 96$ \\
\hline 4317 & M00896 & $6 / 17 / 97$ \\
\hline+317 & SMPL00816 & $9 / 12 / 94$ \\
\hline $4: 317$ & S01876 & $12 / 15 / 94$ \\
\hline 4317 & S02345 & $1 / 25 / 95$ \\
\hline 4317 & S03456 & $4 / 13 / 95$ \\
\hline+317 & M01304 & $11 / 19 / 97$ \\
\hline 4317 & W00820 & $6 / 17 / 97$ \\
\hline 4317 & M00091 & $11 / 20 / 96$ \\
\hline 4317 & M00896 & $6 / 17 / 97$ \\
\hline 4317 & SMPL00816 & $9 / 12 / 94$ \\
\hline 4317 & S01876 & $12 / 15 / 94$ \\
\hline 4317 & S02345 & $1 / 25 / 95$ \\
\hline 4317 & S03+56 & $4 / 13 / 95$ \\
\hline 4317 & M01304 & $11 / 19 / 97$ \\
\hline 4317 & W00820 & $6 / 17 / 97$ \\
\hline 4317 & M00091 & $11 / 20 / 96$ \\
\hline 4317 & M00896 & $6 / 17 / 97$ \\
\hline 4317 & SMPL00816 & $9 / 12 / 94$ \\
\hline
\end{tabular}

cis-1.3-Dichloropropene

cis-1.3-Dichloropropene

cis-1.3-Dichloropropene

cis-1.3-Dichloropropene

cis-1.3-Dichloropropene

Dibromochloromethane

Dibromochloromethane

Dibromochloromethane

Dibromochloromethane

Dibromochloromethane

Dibromochloromethane

Dibromochloromethane

Dibromochloromethane

Dimethylbenzene

Dimethylbenzene

Dimethylbenzene

Dimethylbenzene

Ethylbenzene

Ethylbenzene

Ethylbenzene

Ethylbenzene

Ethylbenzene

Ethylbenzene

Ethylbenzene

Ethylbenzene

m,p-Xylene

m,p-Xylene

m,p-Xylene

m,p-Xylene

Methylene chloride

Methylene chloride

Methylene chloride

Methylene chloride

Methylene chloride

Methylene chloride

Methylene chloride

Methylene chloride

Styrene

Styrene

Styrene

Styrene

Styrene

Styrene

Styrene

Styrene

Tetrachloroethene

Tetrachloroethene

Tetrachloroethene

Tetrachloroethene

Tetrachloroethene
Results Units Qualifier

\begin{tabular}{|c|c|c|}
\hline 5 & $\mathrm{ug} / \mathrm{L}$ & $\overline{\mathrm{U}}$ \\
\hline 5 & $\mathrm{ug} / \mathrm{L}$ & $\mathrm{U}$ \\
\hline 1 & $\mathrm{ug} / \mathrm{L}$ & $\mathrm{U}$ \\
\hline 1 & $\mathrm{ug} / \mathrm{L}$ & $\mathrm{U}$ \\
\hline 1 & ug/L & $\mathrm{U}$ \\
\hline 5 & ug/L & $\mathrm{U}$ \\
\hline 5 & ug/L & $\mathrm{U}$ \\
\hline 5 & $\mathrm{ug} / \mathrm{L}$ & $\mathrm{U}$ \\
\hline 5 & $\mathrm{ug} / \mathrm{L}$ & $\mathrm{U}$ \\
\hline 5 & $\mathrm{ug} / \mathrm{L}$ & $\mathrm{U}$ \\
\hline 1 & $\mathrm{ug} / \mathrm{L}$ & $\mathbf{U}$ \\
\hline 1 & $\mathrm{ug} / \mathrm{L}$ & $\mathrm{U}$ \\
\hline 1 & ug $/ \mathrm{L}$ & $\mathrm{U}$ \\
\hline 5 & ug $/$ & $\mathrm{U}$ \\
\hline 5 & $\mathrm{ug} / \mathrm{L}$ & $\mathbf{U}$ \\
\hline 5 & ug $/$ & $\mathrm{U}$ \\
\hline 5 & $\mathrm{ug} / \mathrm{L}$ & $\mathrm{U}$ \\
\hline 5 & ug $/ \mathrm{L}$ & $\mathrm{U}$ \\
\hline 5 & $\mathrm{ug} / \mathrm{L}$ & $\mathrm{U}$ \\
\hline 5 & $\mathrm{ug} / \mathrm{L}$ & $\mathrm{U}$ \\
\hline 5 & $\mathrm{ug} / \mathrm{L}$ & $\mathrm{U}$ \\
\hline 5 & $\mathrm{ug} / \mathrm{L}$ & $\mathbf{U}$ \\
\hline 1 & $\mathrm{ug} / \mathrm{L}$ & $\mathbf{U}$ \\
\hline 1 & $\mathrm{ug} / \mathrm{L}$ & $\mathrm{U}$ \\
\hline 1 & $\mathrm{ug} / \mathrm{L}$ & $\mathbf{U}$ \\
\hline 5 & $\mathrm{ug} / \mathrm{L}$ & $\mathrm{U}$ \\
\hline 5 & $\mathrm{ug} / \mathrm{L}$ & $\mathrm{U}$ \\
\hline 5 & $\mathrm{ug} / \mathrm{L}$ & $\mathrm{U}$ \\
\hline 5 & $\mathrm{ug} / \mathrm{L}$ & $\mathrm{U}$ \\
\hline 12 & ug/L & B \\
\hline 9 & $\mathrm{ug} / \mathrm{L}$ & $=$ \\
\hline 5 & $\mathrm{ug} / \mathrm{L}$ & $\mathrm{U}$ \\
\hline 8 & $\mathrm{ug} / \mathrm{L}$ & $=$ \\
\hline 5 & $\mathrm{ug} / \mathrm{L}$ & $\mathrm{U}$ \\
\hline 2 & $\mathrm{ug} / \mathrm{L}$ & $\mathrm{U}$ \\
\hline 2 & ug/L & $\mathrm{U}$ \\
\hline 2 & $\mathrm{ug} / \mathrm{L}$ & $\mathrm{U}$ \\
\hline 5 & $\mathrm{ug} / \mathrm{L}$ & $\mathrm{U}$ \\
\hline 5 & $\mathrm{ug} / \mathrm{L}$ & $\mathrm{U}$ \\
\hline 5 & ug/L & $\mathrm{U}$ \\
\hline 5 & $\mathrm{ug} / \mathrm{L}$ & $\mathrm{U}$ \\
\hline 5 & $u g / L$ & U \\
\hline 1 & $\mathrm{ug} / \mathrm{L}$ & $\mathrm{U}$ \\
\hline 1 & ug/L & $\mathrm{U}$ \\
\hline 1 & ug/L & $\mathrm{U}$ \\
\hline 5 & ug $/$ L & $\mathrm{U}$ \\
\hline 5 & $\mathrm{ug} / \mathrm{L}$ & $\mathrm{U}$ \\
\hline 5 & ug/L & $\mathrm{U}$ \\
\hline 5 & ug/ & $\mathrm{U}$ \\
\hline 5 & ug $/ \mathrm{L}$ & $\mathrm{U}$ \\
\hline
\end{tabular}




\begin{tabular}{|c|c|c|c|c|c|c|c|}
\hline Station & Sample ID & Sample Date & Sample Type & Chemical & Results & Units & Qualifier \\
\hline 4317 & S01876 & $12 / 15 / 94$ & REG & Tetrachloroethene & 1 & ug/L & $\overline{\mathrm{U}}$ \\
\hline 4317 & $\mathrm{~S} 023+5$ & $1 / 25 / 95$ & REG & Tetrachloroethene & 1 & $\mathrm{ug} / \mathrm{L}$ & $\mathrm{U}$ \\
\hline 4317 & S03456 & $4 / 13 / 95$ & REG & Tetrachloroethene & 1 & $\mathrm{ug} / \mathrm{L}$ & U \\
\hline 4317 & M01304 & $11 / 19 / 97$ & REG & Toluene & 5 & ug/L & U \\
\hline 4317 & W00820 & $6 / 17 / 97$ & REG & Toluene & 5 & ug $/ \mathrm{L}$ & $\mathrm{U}$ \\
\hline 4317 & M00091 & $11 / 20 / 96$ & REG & Toluene & 5 & $\mathrm{ug} / \mathrm{L}$ & U \\
\hline 4317 & M00896 & $6 / 17 / 97$ & REG & Toluene & 5 & $u g / L$ & U \\
\hline 4317 & SMPL00816 & $9 / 12 / 94$ & REG & Toluene & 5 & $\mathrm{ug} / \mathrm{L}$ & U \\
\hline 4317 & S01876 & $12 / 15 / 94$ & REG & Toluene & 1 & $\mathrm{ug} / \mathrm{L}$ & $U$ \\
\hline 4317 & S02345 & $1 / 25 / 95$ & REG & Toluene & 1 & $\mathrm{ug} / \mathrm{L}$ & $U$ \\
\hline 4317 & S03456 & $4 / 13 / 95$ & REG & Toluene & 1 & $\mathrm{ug} / \mathrm{L}$ & $\mathrm{U}$ \\
\hline 4317 & M01304 & $11 / 19 / 97$ & REG & trans-1.2-Dichloroethe & 5 & $\mathrm{ug} / \mathrm{L}$ & $\mathrm{U}$ \\
\hline 4317 & M00091 & $11 / 20 / 96$ & REG & trans-1,2-Dichloroethene & 5 & ug/L & $\mathrm{U}$ \\
\hline 4317 & M00896 & $6 / 17 / 97$ & REG & trans-1.2-Dichloroethene & 5 & $\mathrm{ug} / \mathrm{L}$ & $\mathrm{U}$ \\
\hline 4317 & SMPL00816 & $9 / 12 / 94$ & REG & trans-1,2-Dichloroethene & 5 & $\mathrm{ug} / \mathrm{L}$ & $\mathrm{U}$ \\
\hline 4317 & S01876 & $12 / 15 / 94$ & REG & trans-1.2-Dichloroethene & 1 & ug/L & $\mathrm{U}$ \\
\hline 4317 & S02345 & $1 / 25 / 95$ & REG & trans-1,2-Dichloroethene & 1 & $\mathrm{ug} / \mathrm{L}$ & U \\
\hline 4317 & S03456 & $4 / 13 / 95$ & REG & trans-1,2-Dichloroethene & 1 & ug $/ \mathrm{L}$ & $\mathrm{U}$ \\
\hline 4317 & M01304 & $11 / 19 / 97$ & REG & trans-1.3-Dichloroprop & 5 & $\mathrm{ug} / \mathrm{L}$ & $\mathrm{U}$ \\
\hline 4317 & W00820 & $6 / 17 / 97$ & REG & trans-1.3-Dichloropropene & 5 & $\mathrm{ug} / \mathrm{L}$ & $U$ \\
\hline 4317 & M00091 & $11 / 20 / 96$ & REG & trans-1,3-Dichloropropene & 5 & ug/L & $\mathrm{U}$ \\
\hline 4317 & M00896 & $6 / 17 / 97$ & REG & trans-1.3-Dichloropropene & 5 & $\mathrm{ug} / \mathrm{L}$ & $\mathrm{U}$ \\
\hline 4317 & SMPL00816 & $9 / 12 / 94$ & REG & trans-1,3-Dichloropropene & 5 & $\mathrm{ug} / \mathrm{L}$ & $\mathrm{U}$ \\
\hline 4317 & S01876 & $12 / 15 / 94$ & REG & trans-1.3-Dichloropropene & 1 & $\mathrm{ug} / \mathrm{L}$ & $\mathrm{U}$ \\
\hline 4317 & S02345 & $1 / 25 / 95$ & REG & trans-1,3-Dichloropropene & 1 & $\mathrm{ug} / \mathrm{L}$ & $\mathrm{U}$ \\
\hline 4317 & S03456 & $4 / 13 / 95$ & REG & trans-1.3-Dichloropropene & 1 & $\mathrm{ug} / \mathrm{L}$ & $U$ \\
\hline 4317 & M01304 & $11 / 19 / 97$ & REG & Trichloroethene & 5 & $\mathrm{ug} / \mathrm{L}$ & $\mathrm{U}$ \\
\hline 4317 & W00820 & $6 / 17 / 97$ & REG & Trichloroethene & 5 & ug/L & U \\
\hline 4317 & M00091 & $11 / 20 / 96$ & REG & Trichloroethene & 5 & ug/L & $\mathrm{U}$ \\
\hline 4317 & M00896 & $6 / 17 / 97$ & REG & Trichloroethene & 5 & ug/L & $\mathrm{U}$ \\
\hline 4317 & SMPL00816 & $9 / 12 / 94$ & REG & Trichloroethene & 5 & ug/L & $\mathrm{U}$ \\
\hline 4317 & S01876 & $12 / 15 / 94$ & REG & Trichloroethene & 1 & $\mathrm{ug} / \mathrm{L}$ & $\mathrm{U}$ \\
\hline 4317 & S02345 & $1 / 25 / 95$ & REG & Trichloroethene & 1 & $\mathrm{ug} / \mathrm{L}$ & $\mathrm{U}$ \\
\hline 4317 & S03456 & $4 / 13 / 95$ & REG & Trichloroethene & 1 & $\mathrm{ug} / \mathrm{L}$ & $\mathrm{U}$ \\
\hline 4317 & Mo1304 & $11 / 19 / 97$ & REG & Vinyl acetate & 10 & $\mathrm{ug} / \mathrm{L}$ & U \\
\hline 4317 & M00091 & $11 / 20 / 96$ & REG & Vinyl acetate & 10 & ug $/ \mathrm{L}$ & U \\
\hline 4317 & M00896 & $6 / 17 / 97$ & REG & Vinyl acetate & 10 & ug/L & $\mathrm{U}$ \\
\hline 4317 & SMPL00816 & $9 / 12 / 94$ & REG & Vinyl acetate & 10 & $\mathrm{ug} / \mathrm{L}$ & $\mathrm{U}$ \\
\hline 4317 & M01304 & $11 / 19 / 97$ & REG & Vinỵl chloride & 10 & $\mathrm{ug} / \mathrm{L}$ & $\mathrm{U}$ \\
\hline 4317 & W00820 & $6 / 17 / 97$ & REG & Vinỵl chloride & 10 & ug $\Omega$ & $\mathrm{U}$ \\
\hline 4317 & M00091 & $11 / 20 / 96$ & REG & Vinyl chloride & 10 & $\mathrm{ug} / \mathrm{L}$ & U \\
\hline 4317 & M00896 & $6 / 17 / 97$ & REG & Vinyl chloride & 10 & ug/ & $\mathrm{U}$ \\
\hline 4317 & SMPL00816 & $9 / 12 / 94$ & REG & Vinyl chloride & 10 & ug/L & $\mathrm{U}$ \\
\hline 4317 & S01876 & $12 / 15 / 94$ & REG & Vinyl chloride & 1 & $\mathrm{ug} / \mathrm{L}$ & $\mathrm{U}$ \\
\hline 4317 & S02345 & $1 / 25 / 95$ & REG & Vinyl chloride & 1 & $\mathrm{ug} / \mathrm{L}$ & U \\
\hline 4317 & S03456 & $4 / 13 / 95$ & REG & Vinỵl chloride & 1 & $\mathrm{ug} / \mathrm{L}$ & $\mathbf{U}$ \\
\hline
\end{tabular}


APPENDIX C

DATA QUALITY ASSESSMENT 
IWQP - ORNL RCRA WELLS

Project: $\quad$ ORNL Data Validation Task, Oak Ridge National Laboratory, Oak Ridge, Tennessee

Analysis: $\quad$ Volatile Organic Compounds (VOC) and Inorganics (Lead)

DRG Nos.: $\quad$ 9711L301 and 9711L480

No. of Samples: 26

Matrix: Water

Sample Nos.: M01225, M01229, M01233, M01236, M01250, M01251, M01244, M01245, M01279, M01280, M01254, M01255, M01232, M01239, M10308, M01256, M01267, M01273, M01285, M01286, M01292, M01295, M01298, M01304, M01305, and M01289

The data were validated following the criteria stated in or referred to in the Statement of Work and the EPA data validation functional guidelines.

The following QC criteria were considered in the validation process:

\section{$\underline{\text { OC Criteria }}$}

- Holding Time

- GC/MS Tuning

- Calibrations (VOC and Lead)

- Blanks

- Surrogate Recoveries

- Matrix Spike/Matrix Spike Duplicates

- Internal Standards Performance

- Laboratory Control Samples

- Case Narrative

A total of 26 water samples from wells at ORNL were collected between 11/10/97 through 11/13/97 and $11 / 17 / 97$ through 11/20/97. Twenty-two (22) samples were analyzed by EPA SW-846 method 8240 for RCRA volatile organic compounds (VOC) and represented 792 analyses (i.e., data points) and 4 samples were analyzed by SW-846 method $6010 \mathrm{~A}$ for lead and represented 4 data points. The analytical laboratory was RCRA LabNet of Lionville, Pennsylvania.

\section{Volatile Organic Compounds (VOC):}

The project produced valid results for $100 \%$ of the VOC data. There were no rejected data. Positive VOC results were observed in 7 samples ( $32 \%$ of the samples) and represented $1.9 \%$ of the total data points. Results for 2-butanone in M01233; 1,1-dichloroethane, 2-butanone in M01250; 2-butanone in M01251; and 2-butanone and tolune in M01245 were reported unqualified but estimated (J) because the observed values were between the detection levels and laboratory reporting levels. Positive VOC results reported unqualified that were greater than the laboratory reporting level 
included cis-1,2-dichloroethene, chloroform, 1,2-dichloroethane, carbon tetrachloride, and trichloroethene in M01250; trichloroethene in M01244; cis-1,2-dichloroethene in M01254; and cis1,2-dichloroethene in M01255. The highest analyte level observed was trichloroethene at $150 \mathrm{ug} / \mathrm{L}$ in M01250. All other positive VOC analytes were detected at levels slightly above the reporting level but less than $30 \mathrm{ug} / \mathrm{L}$.

Based on VOC continuing calibration \%D deviations, sample result for 2-butanone in M01255 was qualified as estimated $(J)$ and represented only $0.1 \%$ of the total data points. Based on VOC method blank levels observed, results for methylene chloride in all 22 samples, acetone in 14 samples, and chloromethane in 2 samples were qualified as not detected at the associated level (U) and the Contract Required Quantitation Levels (CRQL) were reported or elevated as required. These qualifications are not uncommon since these analytes occur as frequent laboratory contaminants. These were the only instances where data qualifications were required and represented $4.9 \%$ of the overall VOC data.

\section{Inorganics (Lead):}

The four samples analyzed for lead included M01308, M01232, M01239, and M01289. No deviations from the validation criteria required any qualifications of the lead sample data. The project produced valid results for $100 \%$ of the lead data. All lead results were not detected (U).

Based on the limited number of qualified data and no rejected data, the VOC and lead data are considered of high quality and acceptable for unlimited use. 
IWQP - ORNL RCRA WELLS - VOLATILE ORGANIC COMPOUND DATA

Project: $\quad$ ORNL Data Validation Task, Oak Ridge National Laboratory, Oak Ridge, Tennessee

Analysis: $\quad$ Volatile Organic Compounds (VOC)

DRG Nos.: $\quad$ 9706L193 and 9706L104

No. of Samples: 13

Matrix: Water

Sample Nos. (Station Nos.): $\quad$ M00823(835), M01012(837), M00836(841), M00843(842), M00847(843), M00853(843), M00858(844), M01018(846), M00870(857), M00877(858), M01015(4315), M01020(4316), and M00896(4317)

The data were validated following the criteria stated in or referred to in the Statement of Work and the EPA data validation functional guidelines.

The following QC criteria were considered in the validation process:

\section{QC Criteria}

- Holding Time

- GC/MS Tuning

- Calibrations

- Blanks

- Surrogate Recoveries

- Matrix Spike/Matrix Spike Duplicates

- Internal Standards Performance

- Case Narrative

\section{Volatiles Organic Compounds:}

A total of 13 water samples from 12 wells at ORNL were collected between 6/16/97 and 6/26/97. The samples were analyzed by EPA SW-846 method 8240 for RCRA volatile organic compounds (VOC) and represented 442 analytes (i.e. data points). The analytical laboratory was RCRA Labnet of Lionville, Pennsylvania.

The project produced valid results for $100 \%$ of the VOC data. There were no rejected data. Positive VOC results were observed in 5 samples (38\% of the samples) and represented $2.9 \%$ of the total data points. Results for 1,1-dichloroethane in M00843, trichloroethene in M00847, chloroform, and trichloroethene in M00853 were reported unqualified but estimated (J) because the observed values were between the detection levels and laboratory reporting levels. Positive VOC results reported unqualified that were greater than the laboratory reporting level included 2-butanone in M01012; trichloroethene in M00836; 1,2-dichloroethane, carbon tetrachloride, chloroform, trichloroethene, cis1,2-dichloroethene in M00843; cis-1,2-dichloroethene in M00847; and cis-1,2-dichloroethene in 


\section{C-6}

M00853. The highest analyte level observed was trichloroethene at $110 \mathrm{ug} / \mathrm{L}$ in $\mathrm{M} 00843$. All other positive VOC analytes were detected at levels slightly above the reporting level but less than $30 \mathrm{ug} / \mathrm{L}$.

Based on VOC method blank levels observed, results for methylene chloride in 12 samples and acetone in 5 samples were qualified as not detected at the associated level (U) and the Contract Required Quantitation Levels (CRQL) were reported or elevated as required. These qualifications are not uncommon since these analytes occur as frequent laboratory contaminants. These were the only instances where data qualifications were required and represented $3.8 \%$ of the overall VOC data.

Based on the limited number of qualified data and no rejected data, the VOC data are considered of high quality and acceptable for unlimited use. 


\section{APPENDIX D}

\section{LEAD SAMPLE RESULTS FOR HILLCUT TEST FACILITY,}

1988-1997 
D-3

Appendix D

Lead sample results for Hillcut Test Facility, 1988-1997

\begin{tabular}{|c|c|c|c|c|c|}
\hline Station & Sample date & Results & Units & Qual. & Det. Limit \\
\hline HCTANK1P & $12 / 21 / 95$ & 0.004 & $\mathrm{mg} / \mathrm{L}$ & $\mathrm{U}$ & 0 \\
\hline HCTANK1P & $1 / 18 / 96$ & 0.000004 & $\mathrm{mg} / \mathrm{L}$ & $\mathrm{U}$ & 0 \\
\hline HCTANK1P & $2 / 21 / 96$ & 0.014 & $\mathrm{mg} / \mathrm{L}$ & $=$ & 0 \\
\hline HCTANKIP & $3 / 19 / 96$ & 0.004 & $\mathrm{mg} / \mathrm{L}$ & $\mathrm{U}$ & 0 \\
\hline HCTANK1P & $5 / 10 / 96$ & 0.004 & $\mathrm{mg} / \mathrm{L}$ & $\mathrm{U}$ & 0 \\
\hline HCTANK1P & $7 / 2 / 96$ & 0.004 & $\mathrm{mg} / \mathrm{L}$ & $\mathrm{U}$ & 0 \\
\hline HCTANK1P & $11 / 5 / 97$ & 0.0227 & $\mathrm{mg} / \mathrm{L}$ & $\mathrm{U}$ & 0.0227 \\
\hline HCTANK2UP & $7 / 8 / 89$ & 0.05 & $\mathrm{mg} / \mathrm{L}$ & $\mathrm{U}$ & 0 \\
\hline HCTANK2UP & $7 / 8 / 89$ & 0.018 & $\mathrm{mg} / \mathrm{L}$ & $=$ & 0 \\
\hline HCTANK2UP & $7 / 8 / 89$ & 0.05 & $\mathrm{mg} / \mathrm{L}$ & $\mathrm{U}$ & 0 \\
\hline HCTANK2UP & $7 / 8 / 89$ & 0.004 & $\mathrm{mg} / \mathrm{L}$ & $\mathrm{U}$ & 0 \\
\hline HCTANK2UP & $9 / 12 / 89$ & 0.05 & $\mathrm{mg} / \mathrm{L}$ & $\mathrm{U}$ & 0 \\
\hline HCTANK2UP & $9 / 12 / 89$ & 0.004 & $\mathrm{mg} / \mathrm{L}$ & $\mathrm{U}$ & 0 \\
\hline HCTANK2UP & $9 / 12 / 89$ & 0.05 & $\mathrm{mg} / \mathrm{L}$ & $\mathrm{U}$ & 0 \\
\hline HCTANK2UP & $9 / 12 / 89$ & 0.004 & $\mathrm{mg} / \mathrm{L}$ & $\mathrm{U}$ & 0 \\
\hline HCTANK2UP & $10 / 26 / 89$ & 0.05 & $\mathrm{mg} / \mathrm{L}$ & $\mathrm{U}$ & 0 \\
\hline HCTANK2UP & $10 / 26 / 89$ & 0.004 & $\mathrm{mg} / \mathrm{L}$ & $\mathrm{U}$ & 0 \\
\hline HCTANK2UP & $10 / 26 / 89$ & 0.05 & $\mathrm{mg} / \mathrm{L}$ & $\mathrm{U}$ & 0 \\
\hline HCTANK2UP & $10 / 26 / 89$ & 0.004 & $\mathrm{mg} / \mathrm{L}$ & $\mathrm{U}$ & 0 \\
\hline HCTANK2UP & $2 / 17 / 90$ & 0.05 & $\mathrm{mg} / \mathrm{L}$ & $\mathrm{U}$ & 0 \\
\hline HCTANK2UP & $2 / 17 / 90$ & 0.004 & $\mathrm{mg} / \mathrm{L}$ & $\mathrm{U}$ & 0 \\
\hline HCTANK2UP & $2 / 17 / 90$ & 0.05 & $\mathrm{mg} / \mathrm{L}$ & U & 0 \\
\hline HCTANK2UP & $2 / 17 / 90$ & 0.004 & $\mathrm{mg} / \mathrm{L}$ & $\mathrm{U}$ & 0 \\
\hline HCTANK2UP & $9 / 15 / 94$ & 0.0008 & $\mathrm{mg} / \mathrm{L}$ & $\mathrm{U}$ & 0.003 \\
\hline HCTANK2UP & $9 / 15 / 94$ & 0.0008 & $\mathrm{mg} / \mathrm{L}$ & $\mathrm{U}$ & 0.003 \\
\hline HCTANK2UP & $3 / 10 / 95$ & 0.001 & $\mathrm{mg} / \mathrm{L}$ & $\mathbf{U}$ & 0.003 \\
\hline HCTANK2UP & $3 / 10 / 95$ & 0.001 & $\mathrm{mg} / \mathrm{L}$ & $\mathbf{U}$ & 0.003 \\
\hline HCTANK2UP & $10 / 2 / 95$ & 0.0008 & $\mathrm{mg} / \mathrm{L}$ & $\mathrm{U}$ & 0.0008 \\
\hline HCTANK2UP & $10 / 2 / 95$ & 0.0008 & $\mathrm{mg} / \mathrm{L}$ & $\mathbf{U}$ & 0.0008 \\
\hline HCTANK2UP & $12 / 21 / 95$ & 0.004 & $\mathrm{mg} / \mathrm{L}$ & $\mathrm{U}$ & 0 \\
\hline HCTANK2UP & $1 / 18 / 96$ & 0.000004 & $\mathrm{mg} / \mathrm{L}$ & $\mathbf{U}$ & 0 \\
\hline HCTANK2UP & $5 / 10 / 96$ & 0.004 & $\mathrm{mg} / \mathrm{L}$ & $\mathbf{U}$ & 0 \\
\hline HCTANK2UP & $7 / 2 / 96$ & 0.004 & $\mathrm{mg} / \mathrm{L}$ & $\mathbf{U}$ & 0 \\
\hline
\end{tabular}




\section{DISTRIBUTION}

1-3. C. S. Haase

4. S. A. Herron

5. R. R. Lee

6. T. E. Myrick

7. P. T. Owen

8. L. B. Raulston

9-11. D. D. Skipper

12. Central Research Library

13. File-EMEF DMC-RC

14. M. McDonald, Jacobs Engineering Group, 125 Broadway Ave., Oak Ridge, TN 37830

15. A. S. Will, Science Applications International Corporation, 800 Oak Ridge Turnpike, $10^{\text {th }}$ Floor, Oak Ridge, TN 37830 
M98003432

Report Number (14) ORNL/ER- 436

Publ. Date (11) 199802

Sponsor code (18) DOE/EM,XF

UC Category (19) $M C-2000, D O E / E R$

DOE 